\title{
Analytic Solutions for Flows Through Cascades
}

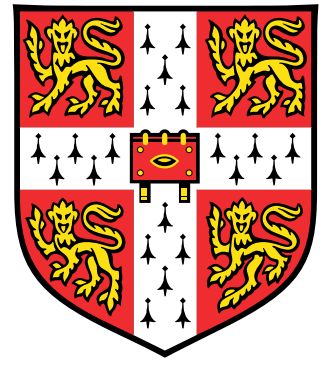

\section{Peter Jonathan Baddoo}

\author{
Supervisor: Dr. Lorna J. Ayton
}

Department of Applied Mathematics and Theoretical Physics

University of Cambridge

This thesis is submitted for the degree of

Doctor of Philosophy 



\section{Declaration}

This thesis is the result of my own work and includes nothing which is the outcome of work done in collaboration except as declared in the Preface and specified in the text. It is not substantially the same as any that I have submitted, or, is being concurrently submitted for a degree or diploma or other qualification at the University of Cambridge or any other University or similar institution except as declared in the Preface and specified in the text. I further state that no substantial part of my thesis has already been submitted, or, is being concurrently submitted for any such degree, diploma or other qualification at the University of Cambridge or any other University or similar institution except as declared in the Preface and specified in the text. It does not exceed the prescribed word limit for the relevant Degree Committee.

The analytic solution in chapter 2 has been published in Proceedings of the Royal Society $A$ as Baddoo and Ayton (2018b). Parts of chapter 3 were presented at the Aviation Forum of the American Institute of Aeronautics and Astronautics (Baddoo and Ayton, 2017, 2018a) and the final analysis is in press at the Journal of Fluid Mechanics (Baddoo and Ayton, 2020). A truncated version of chapter 5 has been published in the Proceedings of the 23rd International Congress on Acoustics (Baddoo and Ayton, 2019c) and an extended version is under review at the Journal of Fluid Mechanics (Baddoo and Ayton, 2019b). The derivation of the periodic Schwarz-Christoffel mappings mentioned in section 6.2.3 has been published in Proceedings of the Royal Society $A$ as Baddoo and Crowdy (2019). An extended version of chapter 6 is under review at Theoretical and Computational Fluid Dynamics (Baddoo and Ayton, 2019a). An early version of chapter 7 has been published in the Proceedings of the 25th AIAA/CEAS Aeroacoustics Conference (Baddoo and Ayton, 2019d).

This thesis is my own work, though I will exercise nosism throughout.

Peter Jonathan Baddoo

July 2019 



\begin{abstract}
Noise reduction is a principal aim of the aviation industry. Particular attention is devoted to reducing turbomachinery noise, which is an important component of overall aero-engine noise during the take-off and landing stages. This thesis therefore presents analytic investigations into turbomachinery flows with the aim that the resulting solutions be used by aircraft designers to produce quieter aircraft. In order to facilitate exact solutions, the turbine is unwrapped onto the two-dimensional plane, resulting in a periodic array of blades commonly referred to as a "cascade". Previous research has been restricted to the case where the cascade consists of a single impermeable row of flat plates at zero angle of attack. Consequently, this thesis considers three separate scenarios where the cascades consist of (i) blades with realistic geometry, (ii) blades with porosity gradients, and (iii) multiple blades per period window. In each case, we begin by solving the steady potential flow and proceed by investigating the effects of unsteady perturbations. This coupled approach provides analysis from both aerodynamic and aeroacoustic perspectives which is essential for achieving practical noise reductions. In order to find analytic solutions, sophisticated complex analysis is employed in the form of singular integral equations, Riemann-Hilbert problems, the Wiener-Hopf method and conformal mappings via the transcendental Schottky-Klein prime function. These methods are applied in the context of rigorous asymptotic expansions where the solution is expanded in terms of a small parameter such as the amplitude of an unsteady incident disturbance or the size of the blades. The aerodynamic analysis generates exact expressions for the surface velocity, drag, lift and deflection angle whilst the aeroacoustic solutions furnish exact expressions for the unsteady surface pressure, sound power output and far-field sound. These formulae are rapid to compute compared to CFD simulations currently used in industry and, moreover, they provide fresh insight into the roles played by blade spacing, geometry and porosity for turbomachinery noise and aerodynamics. Although the solutions in this thesis are applied to turbomachinery, they will also be useful in other applications such as solid mechanics, poroelasticity and biological fliers or swimmers operating in formation.
\end{abstract}



This thesis is dedicated to my grandparents

Paul, Janet, Michael and Barbara. 



\section{Acknowledgements}

I must first and foremost express my sincere gratitude to my supervisor, Dr. Lorna Ayton, for her unceasing support at every stage of my $\mathrm{PhD}$. Her mathematical and physical insight has been incredibly valuable, and she has always made time to answer my many queries. Thank you, Lorna, for your patience and dedication whilst supervising my PhD - I feel genuinely privileged to have been supervised by such a brilliant mathematician, and I am excited to see what you accomplish in the future!

The Department of Applied Mathematics and Theoretical Physical (DAMTP) has provided a stimulating environment for my $\mathrm{PhD}$ research. I have benefited from many scientific discussions with past and present members of the Waves group, including Nigel Peake, Ed Brambley, Stephen Cowley, Anastasia Kisil, David Baker, Owen Petrie and Benshuai Lyu. Thank you in particular to Georg Maierhofer, Matthew Priddin and Mungo Aitken for reading and commenting on drafts of thesis chapters and papers. Thanks also to my office mate, Will Earley, for helping me wrestle with Linux and for sharing late night pizzas in the department!

I am immensely indebted to my family for their support. Mum and Dad: thank you so much for all the opportunities you have given me through your hard work, dedication, and love. You have set an example for me in every aspect of life, and I aspire to be like you. Kate and Harriet: I'm so privileged to have you as my sisters and, despite being the eldest, I am continually learning from you. Your kindness and faith are inspirational to me. James: welcome to the family! In many ways you are the brother that I always hoped for, but your Catan record leaves something to be desired.

There are many other people who have not provided mathematical help (actually, some have proved more of a hindrance) but are nevertheless worthy of thanks. In particular, thank you to Jonno Saunders, Ed \& Clem Hinton, Matthew Johnson, Leander Crocker and Chris Smoove. Thank you to the congregation of St Andrew the Great for providing a warm and encouraging spiritual home for the last few years. I'm especially grateful for the friendship and fellowship of my housemates Clark Glasgow, Andy Gales and Jon Savage. Thank you for sitting politely through my incomprehensible ravings 
about the Schottky-Klein prime function (to be defined later!). I would also like to thank my teachers Steve Pedlow and Alan Walder for initiating this mathematical adventure.

I must also acknowledge the various sources of funding that have supported this work. My PhD was generously supported by a studentship from the Engineering and Physical Sciences Research Council. I'm also grateful to the Quarterly Journal of Applied Mathematics and Mechanics who provided funding to visit Lehigh University, where the ideas of chapter 4 were generated. Additionally, I must thank Jesus College, Cambridge for hosting me for the last few years and providing funding for travel to conferences.

Mathematics is sometimes described as "thinking God's thoughts after Him". Whilst my writing makes it unlikely that this will be the reader's experience, I hope that this thesis somehow reveals something of God's character and goodness. I'm truly grateful for the amazing gifts of science and mathematics, the privilege of using these tools to understand His creation, and how these disciplines ultimately point us to the gift of His Son. 


\section{Table of contents}

List of figures $\quad$ XV

List of tables $\quad$ xix

1 Introduction 1

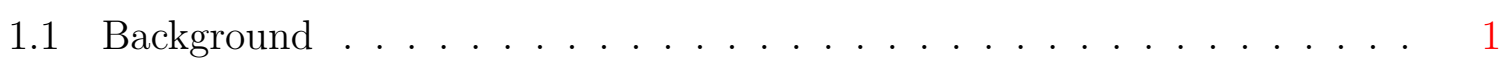

1.2 Methods of Complex Analysis . . . . . . . . . . . . . . . . 7

1.2.1 The Wiener-Hopf Method . . . . . . . . . . . . . 7

1.2.2 Riemann-Hilbert Problems and Singular Integral Equations . . . 10

1.2.3 Multiply Connected Conformal Mappings . . . . . . . . . . . . . . 13

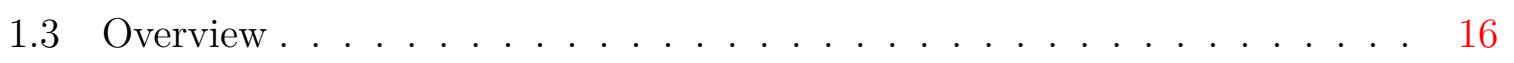

2 Potential Flow Through Cascades of Thin, Impermeable Aerofoils 21

2.1 Introduction . . . . . . . . . . . . . . . . . 21

2.2 Model Derivation . . . . . . . . . . . . . . . . . . . . . . . . . . . . . . . . . . . .

2.3 Direct Problem Solution . . . . . . . . . . . . . . . . 26

2.3.1 General Solution . . . . . . . . . . . . . . . 27

2.3.2 Far-Field Behaviour . . . . . . . . . . . . . . . 30

2.3.3 Endpoint Behaviour . . . . . . . . . . . . . . . . . 31

2.3.4 Final Complex Velocity . . . . . . . . . . . . . . . . . 33

2.4 Inverse Problem Solution . . . . . . . . . . . . . . . . . . . . 34

2.5 Results for Analytic Solution . . . . . . . . . . . . . . 36

2.5.1 Flow Field . . . . . . . . . . . . . . 36

2.5.2 Tangential Surface Velocity . . . . . . . . . . . . . . . . . . 38

2.5.3 Deflection Angle and Lift . . . . . . . . . . . . . . . . . 38

2.6 Numerical Solutions for Large Stagger . . . . . . . . . . . . . . . . 44

2.6.1 Upstream and Downstream Flow Decompositions . . . . . . . . . 48

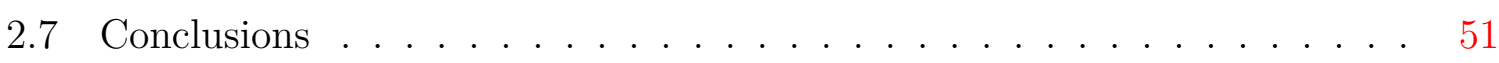


2.A Modified Plemelj Formulae . . . . . . . . . . . . . . . . 56

2.B Asymptotic Results at Endpoints . . . . . . . . . . . . . . . . 57

3 Scattering by Cascades of Aerofoils with Realistic Geometry 59

3.1 Introduction . . . . . . . . . . . . . . . . . . . . . . . 59

3.2 Modelling . . . . . . . . . . . . . . . . . . 60

3.2.1 Steady Flow . . . . . . . . . . . . . . . . . . 62

3.2.2 Unsteady Formulation . . . . . . . . . . . . . . . . . 65

3.2.3 Boundary Conditions . . . . . . . . . . . . . . . . . 67

3.3 Acoustic Scattered Solution _. . . . . . . . . . . . . . . 69

3.3.1 Solution to $\mathcal{O}(1)$ Problem . . . . . . . . . . . . . . . . . 71

3.3.2 Solution to $\mathcal{O}(\epsilon)$ Problem . . . . . . . . . . . . . . . . . . . 72

3.4 Inversion of Fourier Transform . . . . . . . . . . . . . . . . . . . . 80

3.4.1 Upstream Region (I) . . . . . . . . . . . . . . . . . . . . . . . 82

3.4.2 Inter-Blade Upstream Region (II) . . . . . . . . . . . . . . . 82

3.4.3 Inter-Blade Inner Region (III) . . . . . . . . . . . . . . . . . . 82

3.4.4 Inter-Blade Downstream Region (IV) . . . . . . . . . . . . . . 83

3.4.5 Downstream Region $(\mathrm{V}) \ldots \ldots . \ldots$. . . . . . . . 84

3.4.6 Fourier Inversion Discussion . . . . . . . . . . . . . . . . . . . . 84

3.5 Results ........................... 84

3.5.1 Validation ....................... 85

3.5.2 Effects of Realistic Geometry . . . . . . . . . . . . . . . 87

3.6 Conclusions . . . . . . . . . . . . . . . . . . . . . 95

3.A $\quad$ O $(1)$ Solution . . . . . . . . . . . . . . . . . . . . . . 98

3.B Solutions to Wiener-Hopf Problems . . . . . . . . . . . . . . . . 100

3.B.1 Details of Wiener-Hopf Kernel, $j(\gamma)$. . . . . . . . . . . . 100

3.B.2 Solution for $D_{1, \Sigma} \ldots \ldots \ldots \ldots$. . . . . . . . . . . . 101

3.B.3 Solution for $D_{1, \Delta} \ldots \ldots \ldots \ldots$. . . . . . . . . . . 111

3.B.4 Solution for $D_{1, \Gamma} \ldots \ldots \ldots \ldots \ldots$. . . . . . . . . . . 115

3.B.5 Solution for $D_{1, S} \ldots \ldots \ldots \ldots$

3.C Asymptotic Behaviour of Half-Range Fourier Integrals . . . . . . . . . . . 123

3.D List of Identities . . . . . . . . . . . . . . . . . . . . . . . . . 123

3.D.1 Radical Equation . . . . . . . . . . . . . . . . . . . . 124

3.D.2 Integrals . . . . . . . . . . . . . . . . . . . . . . . . . 125

3.D.3 Doubly-Infinite Sums . . . . . . . . . . . . . . . . . . 125

3.E Analysis of Source Terms . . . . . . . . . . . . . . . . . . 127

3.E.1 Exponential Decomposition of Source Terms . . . . . . . . . . 127 
3.E.2 Transformation of Source Terms . . . . . . . . . . . . . . . 131

3.E.3 Inversion of Source Terms . . . . . . . . . . . . . . . . . . . . . 134

3.F Resolving Resonance . . . . . . . . . . . . . . . . . . 135

3.F.1 Resonance in the $h_{1, S}$ Solution . . . . . . . . . . . . . . 135

3.F.2 Resonance in the $h_{1, \Gamma}$ Solution . . . . . . . . . . . . . . . . . . . . 139

3.F.3 Summary . . . . . . . . . . . . . . . . . . . . . . . 141

3.G Coefficients of Acoustic Potential Function . . . . . . . . . . . . . . . . 141

4 Potential Flow Through Cascades of Thin, Porous Aerofoils 147

4.1 Introduction . . . . . . . . . . . . . . . . . . . 147

4.2 Model Derivation . . . . . . . . . . . . . . . . . . . . . 149

4.3 Periodic Singular Integral Equation Solution . . . . . . . . . . . . . . 151

4.3.1 Application to the Cascade of Porous Aerofoils . . . . . . . . . . . 154

4.3.2 Validation . . . . . . . . . . . . . . . . . . . 154

4.4 Results . . . . . . . . . . . . . . . . . . 156

4.4.1 Deflection Angle and Lift . . . . . . . . . . . . . . . . . . . 156

4.4.2 Exact Solutions for Partially Porous Flat Plates . . . . . . . . . . 157

4.4.3 Results on Aerofoils with Continuous Porosity Distributions . . . 167

4.5 Conclusions . . . . . . . . . . . . . . . . . 170

4.A Alternative Solution Method via Substitution . . . . . . . . . . . . 172

4.B Uniqueness of Solutions to the Homogeneous, Periodic Riemann-Hilbert Problem . . . . . . . . . . . . . . . . . . . . . 174

5 Scattering by Cascades of Aerofoils with Complex Boundary Conditions $\quad 175$

5.1 Introduction . . . . . . . . . . . . . . . . . . 175

5.2 Mathematical Formulation . . . . . . . . . . . . . . . . . 177

5.2.1 Boundary Conditions . . . . . . . . . . . . . . . . . 180

5.3 Solution . . . . . . . . . . . . . . . . . . . . . . . 184

5.3.1 Inversion of Fourier Transform . . . . . . . . . . . . . . . . 187

5.4 Results . . . . . . . . . . . . . . . . . . . . . . . . . 189

5.4.1 Sound Generation . . . . . . . . . . . . . . . . . 189

5.4 .2 Sound Transmission . . . . . . . . . . . . . . . . . . . 193

5.5 Conclusions . . . . . . . . . . . . . . . . . . . . . 196

5.A Wiener-Hopf Solution . . . . . . . . . . . . . . 200

5.B Details of Fourier Inversion . . . . . . . . . . . . . . . . . . . . 209

5.C Factorisation of Kernel Function . . . . . . . . . . . . . . . . . 212 
5.C.1 Factorisation of Poles of $K \ldots \ldots \ldots \ldots$. . . . . . . 213

5.C.2 Factorisation of Zeros of $K \ldots \ldots \ldots \ldots . \ldots \ldots$

5.C.3 Full Factorisation of Kernel, $K$. . . . . . . . . . . . . . 217

6 Potential Flow Through Cascades with Multiple Aerofoils per Period 223

6.1 Introduction . . . . . . . . . . . . . . . . . . . . . . 223

6.2 Periodic Conformal Mappings . . . . . . . . . . . . . . . 225

6.2.1 Two Canonical Periodic Conformal Mappings . . . . . . . . . . . 226

6.2.2 The Periodic Joukowski Mapping . . . . . . . . . . . . . . . . . . 229

6.2.3 The Periodic Schwarz-Christoffel Formula . . . . . . . . . . . . . 234

6.3 The Calculus of Flows in Periodic Domains . . . . . . . . . . . . . . . . 235

6.3.1 Point Vortices . . . . . . . . . . . . . . . . . . 236

6.3 .2 Uniform Flow . . . . . . . . . . . . . . . . . . . . 238

6.3.3 Moving Objects . . . . . . . . . . . . . . . . . . . 242

6.3.4 The Kutta Condition . . . . . . . . . . . . . . . . . . . 246

6.4 Conclusions . . . . . . . . . . . . . . . . . . . 247

7 The Quasi-Periodic Compact Green's Function 251

7.1 Introduction . . . . . . . . . . . . . . . . . . . . . 251

7.2 Mathematical Modelling . . . . . . . . . . . . . . . 252

7.3 Compact Approximation without Background Flow . . . . . . . . . . . . 254

7.4 Compact Approximation with Background Flow . . . . . . . . . . . . . . 259

7.5 Conclusions . . . . . . . . . . . . . . . . . . . 261

8 Conclusion 263

8.1 Future Work by Chapter . . . . . . . . . . . . . . . . . . 264

$\begin{array}{lr}\text { References } & 267\end{array}$ 


\section{List of figures}

1.1 Comparison of turbofan and turbojet engines . . . . . . . . . . . 2

1.2 A modern Trent 1000 aero-engine . . . . . . . . . . . . . 3

1.3 A schematic diagram of a rotor-stator interaction. . . . . . . . . . . 5

1.4 The porous, compliant trailing edge of an owl's wing. . . . . . . . . . 6

1.5 An example of the domains of analyticity for a Wiener-Hopf problem. . . 9

1.6 Examples of closed and open contours for Riemann-Hilbert problems. . . 11

1.7 A conformal map between two triply connected domains. . . . . . . . . . 14

1.8 A quadruply connected circular domain. . . . . . . . . . . . 15

1.9 The five canonical conformal maps for a quadruply connected domain. . . 17

2.1 A schematic diagram of cascade of aerofoils for the potential flow problem. 24

2.2 Horizontal velocity and streamline plots for flat plates and three sets of NACA aerofoils. . . . . . . . . . . . . . . . . 37

2.3 Tangential surface velocity plots for flat plates and three sets of NACA aerofoils. . . . . . . . . . . . . . . . . . 39

2.4 Asymptotic behaviour of the deflection angle as a function of gap-to-chord ratio. . . . . . . . . . . . . . . . . . . 41

2.5 The contour of integration illustrating the relationship between circulation and deflection angle. . . . . . . . . . . . . . . . . . . . . 42

2.6 Asymptotic behaviour of the lift as a function of gap-to-chord ratio. . . . 43

2.7 The error convergence for the numerical method. . . . . . . . . . . . . . 49

2.8 Velocity potential and streamfunction contours for a range of geometries and angles of attack. . . . . . . . . . . . . . . . 52

2.9 Close-up of velocity potential and streamfunction for a range of geometries and angles of attack. . . . . . . . . . . . . . . . . . 53

3.1 Road map of solution method. . . . . . . . . . . . . . . . . . 61

3.2 An unsteady disturbance incident on a cascade of aerofoils . . . . . . . . . 62 
3.3 Illustration of the change of coordinate system used to simplify the application of boundary conditions. . . . . . . . . . . . . . . . . 63

3.4 Diagram showing parameters in the transformed $(\phi, \psi)$-space. . . . . . . 64

3.5 Schematic illustrating where each boundary condition is applied. . . . . . 67

3.6 Diagram indicating the different areas in the $(\phi, \psi)$-plane which require different regions of contour integration in the Fourier inversion. . . . . . . 81

$3.7 \quad$ Validations of surface pressure jump for flat plates. . . . . . . . . . . 86

3.8 Comparison to figure 11 of Posson et al. (2010b) . . . . . . . . . . . . . . 86

3.9 An example of the scattered pressure field. . . . . . . . . . . . 87

3.10 The unsteady lift for a range of blade geometries. . . . . . . . . . . . . . 90

3.11 Upstream and downstream sound power output as a function of nondimensional frequency for a range of aerofoil geometries. . . . . . . . . . . 93

3.1 The two-dimensional oblique Bravais lattice for source terms in the interblade region. . . . . . . . . . . . . . . . . . . . . 130

4.1 Schematic diagram of a cascade of porous aerofoils. . . . . . . . . . . . . 149

4.2 Comparison of bound vorticity for isolated aerofoils and a cascade of aerofoils for a range of junction locations. . . . . . . . . . . . . . . . . 159

4.3 Comparison of bound vorticity for isolated aerofoils and a cascade of aerofoils for a range of porosities. . . . . . . . . . . . . . 160

4.4 Drag for cascades of partially porous flat plates with a range of blade spacings. . . . . . . . . . . . . . . . . . . 163

4.5 Lift for cascades of partially porous flat plates with a range of blade spacings. 165

4.6 Lift-to-drag ratio for partially porous flat plates with a range of blade spacings. . . . . . . . . . . . . . . 166

4.7 Continuous porosity gradients used to approximate a partially porous distribution. . . . . . . . . . . . . . . . . 167

4.8 Bound vorticity for cascades of aerofoils with continuous porosity distributions. . . . . . . . . . . . . . . . . . . 168

4.9 Lift-to-drag ratio for cascades of aerofoils with continuous porosity distributions. . . . . . . . . . . . . . . . . . 169

$5.1 \quad$ A rectilinear cascade of flat plates with complex boundaries. . . . . . . 177 
5.2 A three-dimensional view of the cascade in the rotated dimensional $\left(x^{*}, y^{*}, z^{*}\right)$ coordinate system. The chordwise and spanwise background velocities are denoted by $U_{\infty}^{*}$ and $W_{\infty}^{*}$ respectively. The complex boundaries are illustrated by the "holes" on each blade, which may represent compliance, porosity, or impedance. . . . . . . . . . . . . . . . . . . 178

$5.3 \quad$ Schematic illustrating where each boundary condition is applied. . . . . . 184

5.4 Schematic diagram illustrating the solution method. The abbreviations "FT" and "IFT" stand for "Fourier transform" and "Inverse Fourier transform" respectively.

5.5 Diagram indicating the different regions in the $(x, y)$-plane which require different areas of contour integration in the Fourier inversion. . . . . . . . 187

5.6 Unsteady lift for a range of frequencies and porosities. . . . . . . . . . . . 191

5.7 Modal downstream sound power output for a cascade of porous blades at a range of frequencies. . . . . . . . . . . . . . . . . . . . . . . 194

5.8 Modal downstream sound power level for a cascade of porous blades at a range of frequencies. . . . . . . . . . . . . . . . . . . . . . . . 195

5.9 The total pressure field and reflection and transmission coefficients for a cascade of porous aerofoils. . . . . . . . . . . . . . . . . . . . 197

5.10 Reflection and transmission coefficients as a function of porosity coefficient, $C_{I I} \ldots \ldots \ldots \ldots \ldots \ldots \ldots$

5.1 Illustration of the locations of poles in the complex $\gamma$-plane, and the relevant contours of integration. . . . . . . . . . . . . . . . . . . . . 210

6.1 An example of a possible rotor-stator configuration amenable to the methods outlined in this chapter. . . . . . . . . . . . . . . . . . . . . . . . 224

6.2 An example of a periodic conformal mapping. . . . . . . . . . . . . . 225

6.3 A circular arc map for $M=2 \ldots \ldots \ldots \ldots$. . . . . . . . . . . . . . . . . . 27

6.4 The effect of the conformal mapping $H$ on a canonical circular domain for

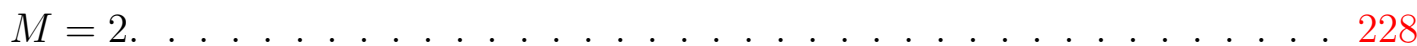

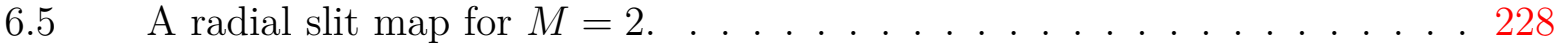

6.6 The effect of the conformal mapping $V$ on a canonical circular domain for

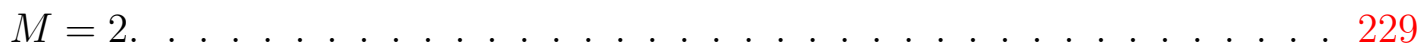

6.7 The effect of the conformal mapping $S$ on a canonical circular domain for

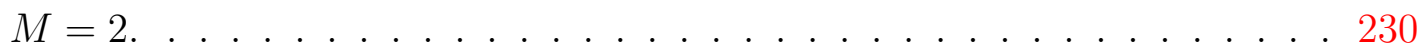

6.8 The procedure for constructing the non-periodic Joukowski mapping. . . . 231

6.9 The procedure for constructing the periodic Joukowski mapping. . . . . . 232 
6.10 Several examples of Joukowski-type aerofoils that are amenable to the periodic Joukowski formula. . . . . . . . . . . . . . . . . . . . . . . . . 232

6.11 Several examples of cascades of Joukowski-type aerofoils. . . . . . . . . . 233

6.12 An example of a periodic Schwarz-Christoffel mapping with real period. . 235

6.13 Streamlines induced by point vortices in a periodic array of slits. . . . . . 238

6.14 Streamlines for uniform flow through a periodic array of slits. . . . . . . . 241

6.15 Streamlines induced by a cascade of moving Joukowski aerofoils. . . . . . 245

6.16 Illustration of the application of the Kutta condition to periodic arrays of slits with and without point vortices. . . . . . . . . . . . . . . 248

6.17 Several examples of the flow past cascades of Joukowski-type aerofoils where the Kutta condition is enforced. . . . . . . . . . . . . . . . . . . . . 249

7.1 A cascade with multiple aerofoils per period window with quasi-periodic acoustic sources. . . . . . . . . . . . . . . . . . . . 252

7.2 An illustration of the reduction of the integrals to a single period window. 254

7.3 The contours of the Kirchhoff vectors for a cascade with three aerofoils per period window. . . . . . . . . . . . . . . . . 258 


\section{List of tables}

3.1 Summary of different cascade and aeroacoustics parameters used in results sections. . . . . . . . . . . . . . . . . . 85

3.2 A summary of the meaning of the coefficients of the source terms (3.142) in the downstream region. . . . . . . . . . . . . . . . . 129

5.1 Summary of possible boundary conditions. . . . . . . . . . . . . 183

5.2 Summary of parameters used in results section. . . . . . . . . . . 189 



\section{Chapter 1}

\section{Introduction}

\subsection{Background}

The theoretical and practical understanding of sound represents a fascinating challenge for researchers in fluid mechanics. When generated intentionally, sound can often be pleasant, such as in music or communication. Conversely, unwanted sound (termed "noise") and can negatively impact our physical and mental health, thereby reducing our overall quality of life. For example, the World Health Organisation estimates that over 1 million healthy life years are lost each year due to traffic-related noise in Western Europe alone (World Health Organisation, 2011). A large portion of traffic-related noise is due to aircraft and, accordingly, many researchers are investigating aircraft noise with the ultimate aim of controlling and subsequently eliminating sources of noise. In this thesis we analyse flows through aeroengines from two perspectives of practical significance: aerodynamic, i.e. the study of the motion of air and its interactions with solid objects, and aeroacoustic, i.e. the study of sound generation by air flows. Whilst the aerodynamic studies are valuable in their own right, their purpose as presented in this thesis is to facilitate the corresponding aeroacoustic studies. Discerning the aerodynamics is essential for investigating the far more complicated aeroacoustic flows, since the propagation of turbulence through the engine needs to be understood before aeroacoustic modelling can take place.

Work over the past few decades has yielded significant reductions in aircraft noise. Jet noise, which was previously the main contributor to overall aircraft noise, has been shrunk to a level comparable to that of turbomachinery noise (Peake and Parry, 2012). Early aero-engines were "turbojets", and consisted of a single stream of air accelerated through the engine core, as illustrated in figure 1.1a. Engines noise was consequently dominated by the mixing of the hot jet with the cold ambient air at the exhaust of 


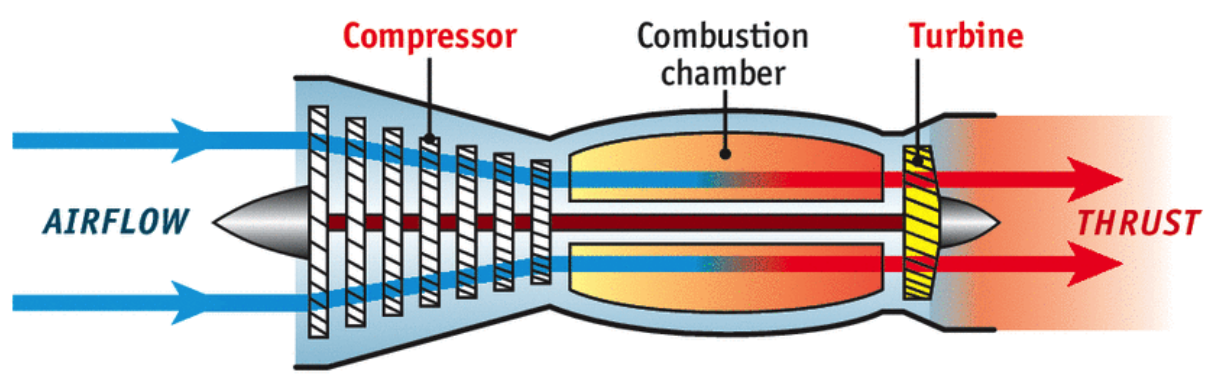

(a) A turbojet aero-engine.

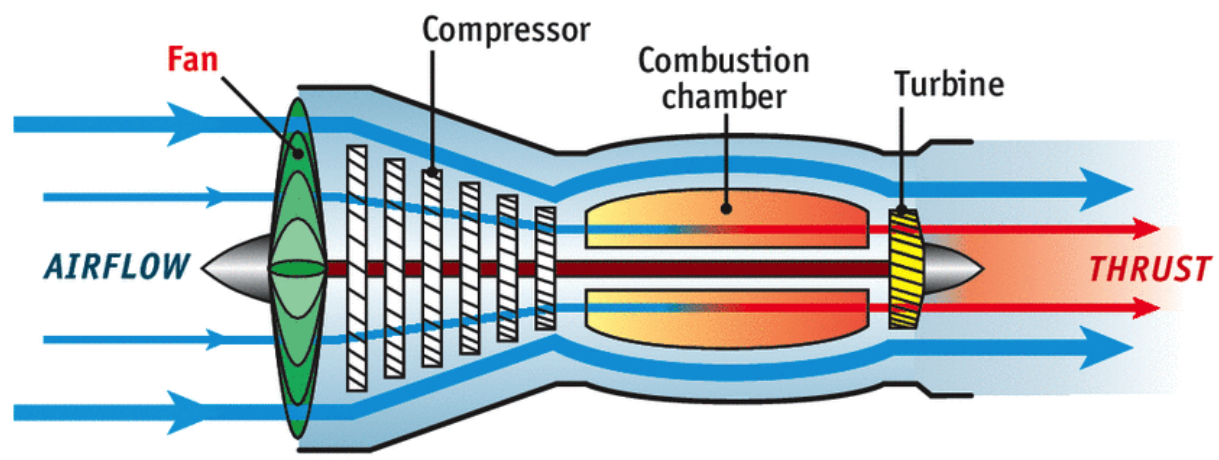

(b) A turbofan aero-engine.

Fig. 1.1 Comparison of turbofan and turbojet engines (The Economist, 2015).

the engine. Turbojets remain desirable at high speeds such as in supersonic flight, but efficiency and noise concerns mean that alternative "turbofan" engines have become more widespread in commercial aviation. Turbofan engines are designed such that the engine features two streams of air where part of the flow "bypasses" the core, as illustrated in figure 1.1b. The ratio between the mass flow rate of these two streams of air is called the bypass ratio: higher bypass ratios imply that more air bypasses the core.

The strong coupling of several environmental concerns greatly increases the complexity of designing aero-engines. Of primary concern are sustainability, greenhouse gas emissions, fuel efficiency, and noise emissions. These concerns were crystallised into specific targets in the European Commission's Flightpath 2050 report (ACARE, 2011), which aims for reductions of $65 \%$ in effective perceived noise level, $75 \%$ in $\mathrm{CO}_{2}$ emissions, and a $90 \%$ in $\mathrm{NO}_{\mathrm{x}}$ emissions, all relative to their respective levels in 2000. These concerns are magnified when combined with increasing global demand for air travel. In particular, the worldwide aircraft fleet is expected to double in size over the next 20-25 years. Using aircraft of current capacity, this growth would require approximately 1,300 new international airports. 


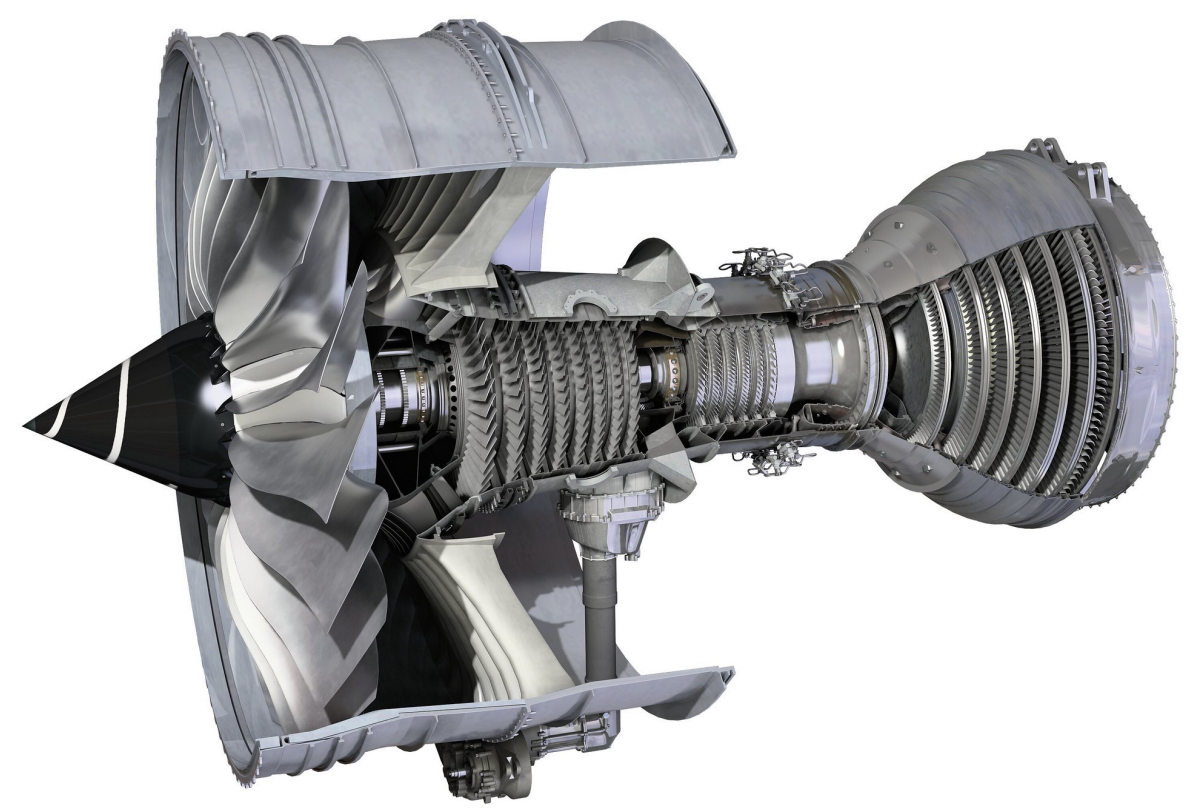

Fig. 1.2 A modern Trent 1000 aero-engine (Rolls-Royce, 2019)

Modern aero-engines are highly intricate and complicated machines with many complex physical processes occurring at a range of length scales; a modern Trent 1000 aero-engine is illustrated in figure 1.2. Such aero-engines are therefore extremely difficult to model mathematically. The design of the Trent 1000 is of note: the aero-engine has a high bypass ratio of 10:1 and consequently the fan generates over $85 \%$ of the engines thrust (Rolls-Royce, 2019). This is in contrast to the previous Trent 900, whose bypass ratio was only 8.7. There is also a low number of turbomachinery stages: the compressor consists of a single-stage low pressure (LP) compressor (the fan), an eight-stage intermediate pressure (IP) compressor, and a six-stage high pressure (HP) compressor (EASA, 2019). Similarly, the turbine consists of a six-stage LP turbine, a single-stage IP turbine and a single-stage HP turbine.

These features are part of broader design trends that promote the importance of turbomachinery noise in the near future. Firstly, aircraft designers are designing engines with higher and higher by-pass ratios through increasing the fan diameter and reducing the nacelle (Moreau, 2019). Accordingly, fan noise is expected to increase in future aircraft in contrast to jet noise, which is expected to decrease (Hughes, 2013). Secondly, future engine designs are likely to have reduced number of stages in the turbine. Consequently, the turbine rows will experience higher loading, thus generating more intense tonal and broad band noise. Moreover, these sources of noise are often shifted to frequency 
bands that result in a higher effective perceived noise level (Hultgren, 2010) leading to higher levels of annoyance. Thirdly, ambitions for lighter aero-engines are resulting in lighter and slimmer blades in turbomachinery stages (Saiz, 2008). Consequently, flutter and resonance are likely to play an increasingly important role in turbomachinery aerodynamics, which will surely impact the overall engine noise. The synergy of these design trends mean that turbomachinery noise is expected to become the dominant noise contribution in future aircraft.

Compressor and turbine stages typically consist of pairs of rotor and stators. Whilst the stators are stationary, the rotors move with a range of 2,700 RPM in LP stages to 13,400 RPM in HP stages (EASA, 2019). The role of the rotors is to accelerate the flow, while the purpose of the stators is to deflect the flow through a desired angle, thus increasing the pressure. "Rotor-stator noise" - the noise produced by unsteady wakes shed by the rotors interacting with the stators - is an important source of turbomachinery noise. As outlined by Peake and Parry (2012), there are a range of source of turbomachinery noise such as rotor self-noise, fan-vane interaction, and the interaction between the casing boundary layer and the rotors. However, rotor-stator noise is the largest source of broadband noise. One reason for the prominence of rotor-stator interaction is that the wakes create unsteady loading on the stator row, which in turn creates far field sound. In many practical scenarios, the flow through turbomachinery stages can be modelled as approximately 2-D since the flow is constrained to a nearly cylindrical surface of revolution. Consequently, we may "unwrap" the surface of revolution to obtain a periodic array of aerofoils in the plane, i.e. a "cascade". A typical rotor-stator interaction in a cascade is illustrated in figure 1.3.

The noise produced by rotor-stator interaction is well understood for stators modelled as flat plates at zero angle of attack (Glegg, 1999; Koch, 1971; Peake, 1992, 1993; Peake and Kerschen, 1995; Posson et al., 2010b). However, these models do not take into account the realistic and important effects of mean flow gradients refracting both the incident gust and scattered field. Surprisingly, the corresponding effects of realistic aerofoil geometry (including both camber and thickness) have not been investigated to the same extent, despite knowledge that realistic geometries can have significant impact on the upstream acoustic field (Evers and Peake, 2002; Peake and Kerschen, 1997, 2004). In each of these approaches, the flow is calculated by splitting the domain into inner regions around each leading edge, and matching with an outer solution. This approach is necessary due to the large gradients which occur at the leading edge, but the methods cannot reproduce the details of the flow field in the crucial inter-blade region. 


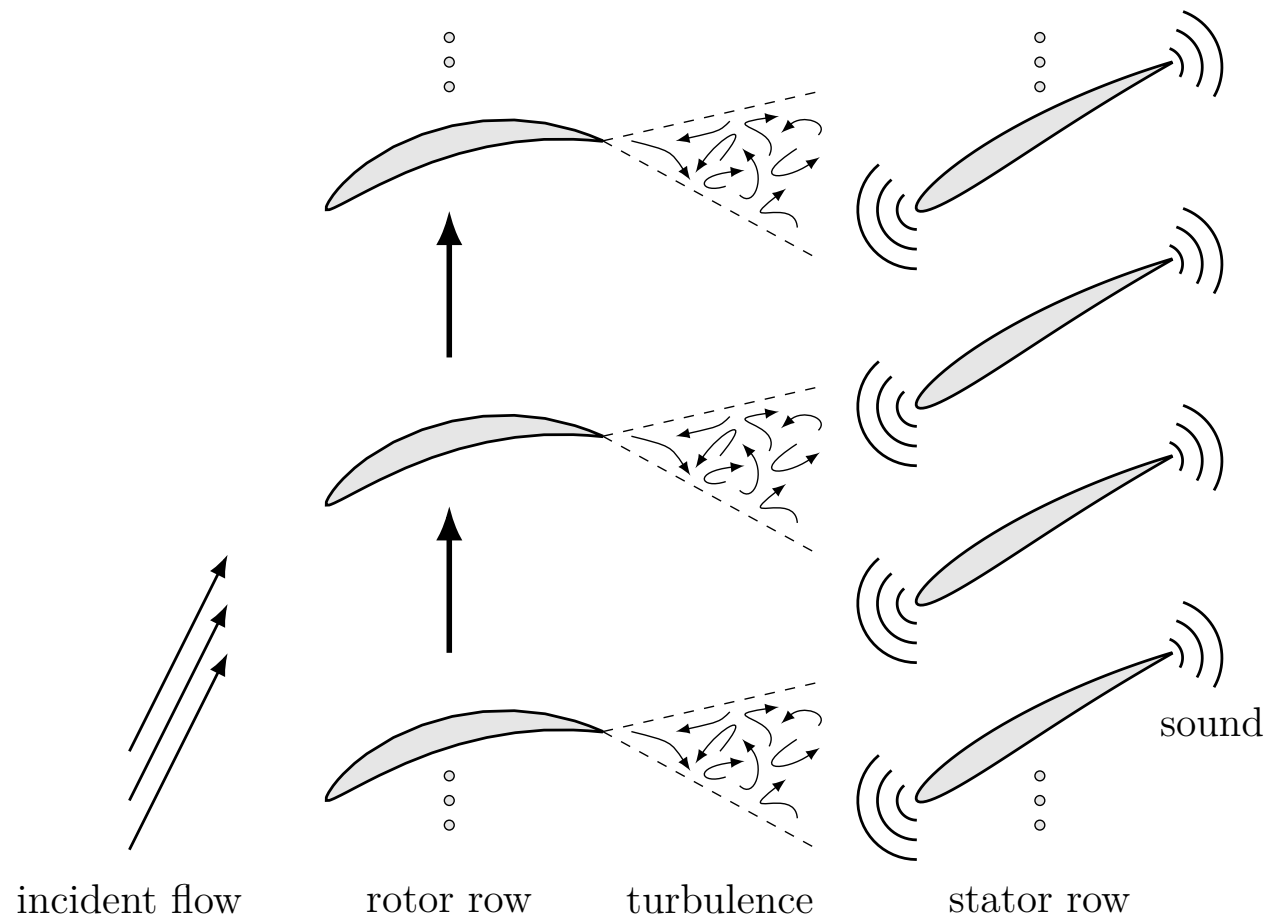

Fig. 1.3 A schematic diagram of a rotor-stator interaction.

Numerical simulations of the noise produced in rotor-stator interaction using computational aeroacoustics (CAA) codes can typically provide details of the unsteady field throughout the whole flow domain (Gea-Aguilera et al., 2016; Grace, 2016; Hixon et al., 2010; Verdon, 1993). However, these methods can be computationally costly, and an analytic expression is useful in elucidating the role of various parameters without the need for expensive computations. In this thesis we will extend the analysis of Glegg (1999) and Posson et al. (2010b), which only considered the influence of flat plates at zero angle of attack. In the former, Glegg found an analytic solution for the acoustic field outside of the flat plate blade row, and Posson et al. (2010b) extended this to calculate the unsteady field within the inter-blade region. Our new analytic solution, which is valid throughout the entire flow domain, extends these two works further and can be used to swiftly calculate the effects of geometry on the far-field acoustics and unsteady blade loading, as well as providing a benchmark to test CAA simulations against.

Many researchers in the aeroacoustics community are taking inspiration from the silent flight of owls in order to develop noise reducing technologies (Graham, 1934; Jaworski and Peake, 2020). It is generally believed that owls' wings possess three unique features that enable them to fly in effective silence. Firstly, there is a comb of stiff fibres along the leading edge of the wing. Secondly, the wing has a soft, velvety surface, resembling 


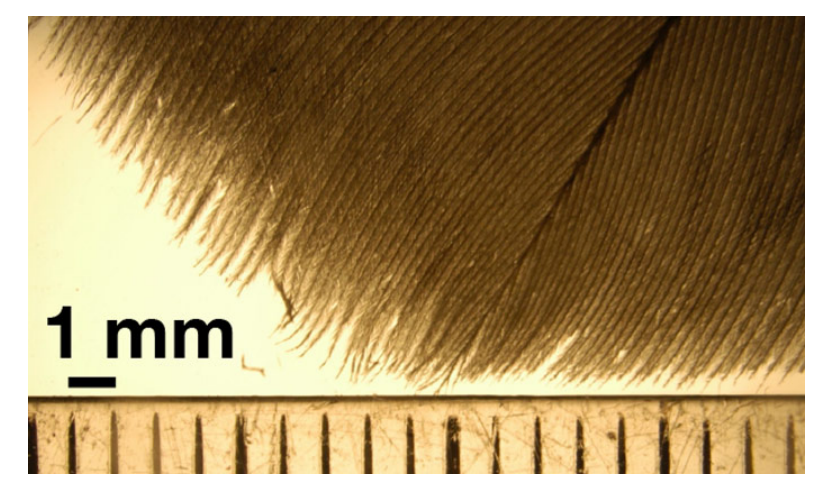

Fig. 1.4 The porous, compliant trailing edge of an owl's wing (Lehigh University, 2014).

a forest canopy Clark et al. (2016). Thirdly, the trailing edge of the wing is porous and flexible, as illustrated in figure 1.4. This last feature is believe to be particularly important in the owls' noise reduction mechanism, and has generated a range of analytic studies (Ayton, 2016; Jaworski and Peake, 2013; Kisil and Ayton, 2018). These works indicate that porous trailing edges can generate significant aeroacoustic gains, and these findings are supported by numerical (Cavalieri et al., 2016) and experimental (Geyer et al., 2010) studies. In spite of their aeroacoustic advantages, porous blades have not yet found relevance in turbomachinery, possibly due to adverse aerodynamic effects associated with the seepage through the blades. In this thesis we will present two studies (aerodynamic and aeroacoustic) that address this gap in the literature.

Current analytic models for turbomachinery are limited to modelling a single turbomachinery stage (i.e. a single rotor row or stator row). This is a severe restriction, as the interaction effects between adjacent blade rows are important in characterising sound generation and propagation, as illustrated in figure 1.3. The difficulty in the analytical treatment of multiple blade rows lies in the non-trivial topology induced by the inclusion of a second (or third or fourth) blade row. We will address these difficulties and present the first analytical treatment of blade rows with multiple aerofoils per period window. As is the theme of this thesis, this problems will be approached from both aerodynamic and aeroacoustic perspectives.

The scientific study of aeroacoustics begin with the theories of Sir James Lighthill (Lighthill, 1952, 1954) who considered a high speed turbulent flow in a fluid that is otherwise at rest. The principal contribution of these works was to rearrange the NavierStokes equations into a simple wave equation with quadrupole forcing. The so-called "Lighthill's wave equation" was then solved using Green's functions by Curle (1955), who also accounted for the presence of solid boundaries. The acoustic analogy was further 
extended by Ffowcs Williams and Hawkings (1969) to account for aeroacoustic sources that are in relative motion with respect to a solid boundary.

Analytic, numerical and experimental investigations each have their own comparative advantages in studying fluid mechanics. All three approaches must be considered for a truly holistic scientific analysis. Experimental studies are physically faithful and can consequently elucidate important physical features. Numerical studies are able to solve broad classes of equations with high degrees of accuracy. The aim of this thesis is to provide an analytic perspective that can be integrated into a comprehensive understanding of flows in periodic domains. Since the full Navier-Stokes equations are prohibitively difficult to solve, asymptotic approximations are often employed. For example, in this thesis we consider flows that are mostly inviscid and undergoing small amplitude perturbations. A huge advantage of this approach is that it enables the rapid evaluation of solutions since they are generally written in terms of easy-to-compute functions. Swift calculations are entirely necessary if the solution is to be employed in an optimisation loop, or used as a benchmark for high-fidelity numerical simulations. Furthermore, analytic solutions are useful for elucidating the structure of the solution, and their dependence on important physical parameters. These important features can often be obscured by numerical techniques. Finally, analytic solutions are straightforward to replicate by other researchers, in contrast to experimental and numerical studies.

Complex function theory has proved a powerful tool in understanding these fluid problems. The utility of complex function theory stems from its ability to solve two partial differential equations that arise frequently in aerodynamics and aeroacoustics, namely the Laplace and Helmholtz equations. In the context of this thesis, these two equations generally correspond to aerodynamics and aeroacoustics problems respectively. Of particular importance are the Wiener-Hopf technique, Riemann-Hilbert problems, and conformal mappings, for which we now provide a brief introduction.

\subsection{Methods of Complex Analysis}

This thesis relies heavily on techniques of complex analysis. In this section we provide a concise introduction to three of the most common methods used in this research.

\subsubsection{The Wiener-Hopf Method}

The Wiener-Hopf method (Wiener and Hopf, 1931) is an elegant technique that is commonly employed to solve particular linear partial differential equations (or, equivalently, 
integral equations) subject to mixed boundary conditions. The method has been applied to a broad range of fields in the physical sciences, such as elasticity, electromagnetism, finance, solid mechanics, geophysical fluid dynamics as outlined in Lawrie and Abrahams (2007). Most relevantly, the Wiener-Hopf method has proved a highly popular and successful technique for solving aeroacoustic problems. In this context, it has been used to analyse isolated aerofoils (Ayton and Peake, 2015), poroelastic edges (Jaworski and Peake, 2013), duct linings (Rienstra, 2007), cascade configurations (Glegg, 1999; Peake, 1992) and aerofoils with serrated edges (Ayton, 2018). Many mathematical details and examples of the technique are provided in Noble (1958), which has become the canonical text on Wiener-Hopf problems.

We now briefly describe the Wiener-Hopf procedure. By taking an appropriate integral transform of the relevant partial differential equation or integral equation, we obtain the identity

$$
\Phi_{+}(\gamma)-G(\gamma) \Phi_{-}(\gamma)=F(\gamma)
$$

in a strip

$$
\mathcal{S}=\left\{\gamma: \tau_{-}(s)<\Im[\gamma]<\tau_{+}(s),-\infty<s<\infty\right\}
$$

where $G(\gamma)$ is the known "kernel" of the Wiener-Hopf problem, $F$ is a known forcing function, and $\Phi_{+}$and $\Phi_{-}$are unknown functions to be found. In this context, the subscripts " \pm " indicate that the relevant function is analytic in an upper of lower half planes $\mathscr{L}_{ \pm}$defined as

$$
\mathscr{L}_{+}=\left\{\gamma: \Im[\gamma]>\tau_{-}\right\}, \quad \mathscr{L}_{-}=\left\{\gamma: \Im[\gamma]<\tau_{+}\right\}
$$

as illustrated in figure 1.5. The most challenging step in solving any Wiener-Hopf problem is factorising the kernel function $G$ into parts that have no poles or zeros in $\mathscr{L}_{ \pm}$ respectively. Assuming that this can be done, we write

$$
G(\gamma)=G_{-}(\gamma) G_{+}(\gamma)
$$

and rearrange (1.1) to obtain

$$
\frac{\Phi_{+}(\gamma)}{G_{+}(\gamma)}-G_{-}(\gamma) \Phi_{-}(\gamma)=\frac{F(\gamma)}{G_{+}(\gamma)}
$$




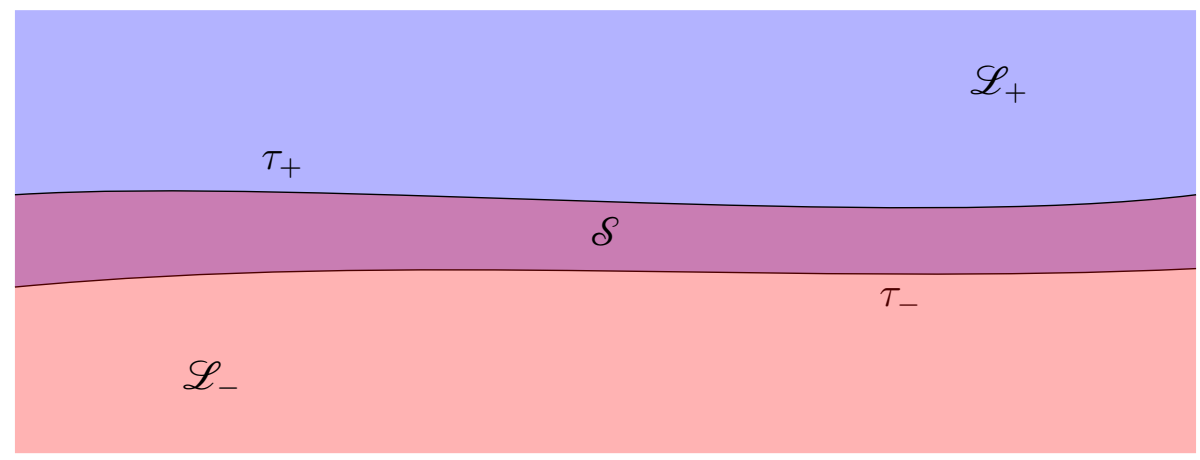

Fig. 1.5 An example of the domains of analyticity for a Wiener-Hopf problem. The blue region represents $\mathscr{L}_{+}$, the red region represents $\mathscr{L}_{-}$and the overlapping purple region represents the strip of analyticity $\mathcal{S}$.

We now additively split the right-hand side of (1.3) into parts that are analytic in $\mathscr{L}_{ \pm}$ respectively:

$$
\frac{F(\gamma)}{G_{+}(\gamma)}=C_{+}(\gamma)+C_{-}(\gamma)
$$

Consequently, we may rearrange (1.3) into the form

$$
\frac{\Phi_{+}(\gamma)}{G_{+}(\gamma)}-C_{+}(\gamma)=G_{-}(\gamma) \Phi_{-}(\gamma)+C_{-}(\gamma)
$$

We now perform the fundamental step in the Wiener-Hopf analysis. We have rearranged (1.1) into the form (1.4) so that the left-hand side is analytic in $\mathscr{L}_{+}$and the right-hand side is analytic in $\mathscr{L}_{-}$. Moreover, (1.4) states that the left- and right-hand sides agree in a strip. Consequently, each side of (1.4) is the analytic continuation of the other and consequently each side represents an entire function, E. Accordingly, we may rearrange (1.4) to obtain

$$
\begin{aligned}
& \Phi_{+}(\gamma)=G_{+}(\gamma)\left(E(\gamma)+C_{+}(\gamma)\right), \\
& \Phi_{-}(\gamma)=\frac{1}{G_{-}(\gamma)}\left(E(\gamma)-C_{-}(\gamma)\right)
\end{aligned}
$$


Now we aim to apply Liouville's theorem in order to obtain a polynomial bound on $E$. Typically, one shows that there are two positive integers, $p$ and $q$ such that

$$
\begin{array}{ll}
E(\gamma)=\mathcal{O}\left(|\gamma|^{p}\right), & \text { as }|\gamma| \rightarrow \infty, \quad \gamma \in \mathscr{L}_{+}, \\
E(\gamma)=\mathcal{O}\left(|\gamma|^{q}\right), & \text { as }|\gamma| \rightarrow \infty, \quad \gamma \in \mathscr{L}_{-} .
\end{array}
$$

Consequently, $E$ is bounded by a polynomial of order $\max (p, q)$. In most aeroacoustic applications, physical considerations imply that $p=q=0$ so that the entire function is a constant. Further physical arguments generally yield that the entire function vanishes (Ayton et al., 2016).

As mentioned in the above example, the prevailing difficulty in solving WienerHopf problems concerns the multiplicative factorisation of the kernel function. When analytical factorisations are impossible (which is often the case for matrix Wiener-Hopf problems), several alternative approaches are available. Firstly, the kernel may be factorised asymptotically, possibly using an approach similar to Crighton (2001). This method has been successfully applied to cascade aeroacoustics (Peake, 1992) when the frequency was assumed to be high. Secondly, a numerical scheme may be employed to iteratively factorise the kernel function (Kisil, 2015). Thirdly, in some cases an explicit kernel factorisation can be entirely obviated by employing the Unified Transform Method (Crowdy and Luca, 2014). Fortunately, the Wiener-Hopf problems encountered in this thesis have kernels that can be factorised analytically using the Weierstrass factorisation theorem (Ablowitz and Fokas, 2003), so the aforementioned alternative methods are not necessary.

\subsubsection{Riemann-Hilbert Problems and Singular Integral Equa- tions}

Riemann-Hilbert problems find significance in many areas of mathematics such as random matrices (Deift, 2000), inverse scattering (Zhou, 2005) and geophysical fluid dynamics (Moore, 2017). In a typical scalar Riemann-Hilbert problem, we seek to find a function $\Phi$ that is analytic in the entire plane except possibly on a smooth contour $L$ where $\Phi$ satisfies the relation

$$
\Phi_{+}(t)-G(t) \Phi_{-}(t)=F(t)
$$




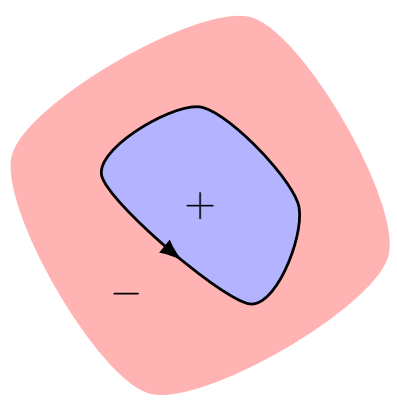

(a) Closed contour

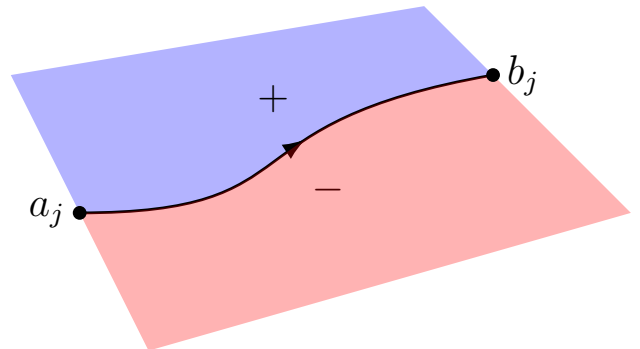

(b) Open contour

Fig. 1.6 Examples of closed and open contours, $L$, for Riemann-Hilbert problems. The blue region indicates the left-hand side $(+)$ of the contour and the red region indicates the right-hand side $(-)$ of the contour. The endpoints of the open counter are indicated by $a_{j}$ and $b_{j}$.

for $t \in L$. The contour $L$ may be open or closed, as illustrated in figure 1.6. Furthermore, when $L$ is open, it may also consist of the union of $M+1$ open contours such that $L=L_{0}+\cdots+L_{M}$. In this case, the endpoints of $L_{j}$ are denoted by $a_{j}$ and $b_{j}$, and the subscripts \pm refer to the limiting values of $\Phi$ when $z$ approaches the contour $L$ from the left- and right-hand sides respectively.

Note the striking similarity between the Riemann-Hilbert problem (1.5) and the Wiener-Hopf problem (1.1). The major difference is that, as opposed to being described on the strip $\mathcal{S}$, the Riemann-Hilbert problem (1.5) is defined on the contour L. Consequently, the Riemann-Hilbert problem is really a generalisation of the Wiener-Hopf problem. The Riemann-Hilbert problem (1.5) is defined on a contour and not in a strip and, accordingly, it can represent Wiener-Hopf problems by considering the contour as a subset of the strip. Conversely, the Wiener-Hopf technique does not extend to the case where the strip reduces to a contour, as the analytic continuation argument requires the overlapping region of agreement to constitute an open subset. Moreover, in the Riemann-Hilbert context, the function $F$ need only satisfy a Hölder condition on the contour $L$, as opposed to satisfying an analyticity requirement in the strip $\mathcal{S}$, which is far more restrictive. The relationship between Wiener-Hopf problems and Riemann-Hilbert problems has been explored in further detail by Kisil (2015).

Riemann-Hilbert problems are intimately connected with the theory of singular integral equations (Muskhelishvili, 1946). In order to see this, we note that any solution to $(1.5)$ can be written as a Cauchy-type integral

$$
\Phi(z)=\frac{1}{2 \pi \mathrm{i}} \int_{L} \frac{\phi(\tau)}{\tau-z} \mathrm{~d} \tau
$$


where $\phi$ is an unknown "density" function that satisfies a Hölder condition on $L$. When $z$ approaches $L$ from either side, the "Plemelj formulae" (Ablowitz and Fokas, 2003) yields the limiting behaviour

$$
\Phi_{ \pm}(t)= \pm \frac{1}{2} \phi(t)+\frac{1}{2 \pi \mathrm{i}} \int_{L} \frac{\phi(\tau)}{\tau-t} \mathrm{~d} \tau
$$

Substitution of the Plemelj formulae (1.6) into the original Riemann-Hilbert problem (1.5) yields the singular integral equation

$$
A(t) \phi(t)+\frac{B(t)}{\pi \mathrm{i}} f_{L} \frac{\phi(\tau)}{\tau-t} \mathrm{~d} \tau=f(t)
$$

where

$$
\frac{A(t)-B(t)}{A(t)+B(t)}=G(t), \quad f(t)=(A(t)+B(t)) F(t)
$$

Consequently, solving a Riemann-Hilbert problem is equivalent to solving its associated singular integral equation.

When solving Riemann-Hilbert problems, it is typical to first solve the homogeneous problem and then use this solution to solve the inhomogeneous problem. We neglect closed contours since they do not arise in the analysis in this thesis. The homogeneous Riemann-Hilbert problem concerns funding the unknown function $\Phi^{(H)}$ with the limiting values $\Phi_{ \pm}^{(H)}$ such that

$$
\Phi_{+}^{(H)}(t)-G(t) \Phi_{-}^{(H)}(t)=0
$$

for $t \in L$. The solution is given by Muskhelishvili (1946) as

$$
\Phi^{(H)}(z)=X(z) P(z),
$$

where $P$ is an arbitrary polynomial and

$$
X(z)=\prod_{j=0}^{M}\left(z-a_{j}\right)^{\alpha_{j}}\left(z-b_{j}\right)^{\beta_{j}} \exp \left[\frac{1}{2 \pi \mathrm{i}} \int_{L} \frac{\log (G(\tau))}{\tau-z} \mathrm{~d} \tau\right]
$$

where $\alpha_{j}$ and $\beta_{j}$ are integers chosen to satisfy

$$
-1<\alpha_{j}+\Re\left[-\frac{1}{2 \pi \mathrm{i}} \log \left(G\left(a_{j}\right)\right)\right]<1,
$$




$$
-1<\beta_{j}+\Re\left[\frac{1}{2 \pi \mathrm{i}} \log \left(G\left(b_{j}\right)\right)\right]<1 .
$$

In an analogous way to the selection of the entire function $E$ in the Wiener-Hopf method in section 3.B, the integers $\alpha_{j}$ and $\beta_{j}$ are often chosen subject to physical constraints. In the applications considered in this thesis, we enforce the Kutta condition at the trailing edges $\left(t=b_{j}\right)$ and restrict the singular nature of the pressure at the leading edges $\left(t=a_{j}\right)$.

We now use the solution to the homogeneous problem (1.7) to construct a class of solutions to the original inhomogeneous problem (1.5). It may be verified by the Plemelj formula that the function

$$
\Phi(z)=\frac{X(z)}{2 \pi \mathrm{i}} \int_{L} \frac{F(t)}{X_{+}(t)} \frac{\mathrm{d} t}{t-z}+X(z) P(z),
$$

satisfies the full Riemann-Hilbert problem (1.5).

\subsubsection{Multiply Connected Conformal Mappings}

During this thesis we are frequently required to solve Laplace's equation in complicated geometries. Conformal mapping are powerful tools for attacking such problems since they allow potential problems in complicated geometries to be reduced to potential problems in simple geometries (Ablowitz and Fokas, 2003). Accordingly, in fluid dynamical applications, it is often sufficient to construct the complex potential in a simple (typically circular) domain and then conformally map this solution to the complicated physical domain of interest. To this end, we relate the physical coordinate $z$ to the parametric coordinate $\zeta$ by the relation

$$
z=f(\zeta)
$$

where $f$ represents the conformal mapping function. A typical conformal map that may be of aerodynamic interest is illustrated in figure 1.7.

Although conformal mappings have been used for centuries, they have typically been restricted to simply connected geometries. A domain is "simply connected" if any simple closed curve can be continuously deformed to a point in the domain. A domain that is connected but not simply connected is "multiply connected". It was not until the early 2000s that substantial progress was made in conformal mapping theory for multiple connected domains. This link is predominantly attributed to new results on the "SchottkyKlein prime function" (sometimes referred to as the "prime function"), which has been 


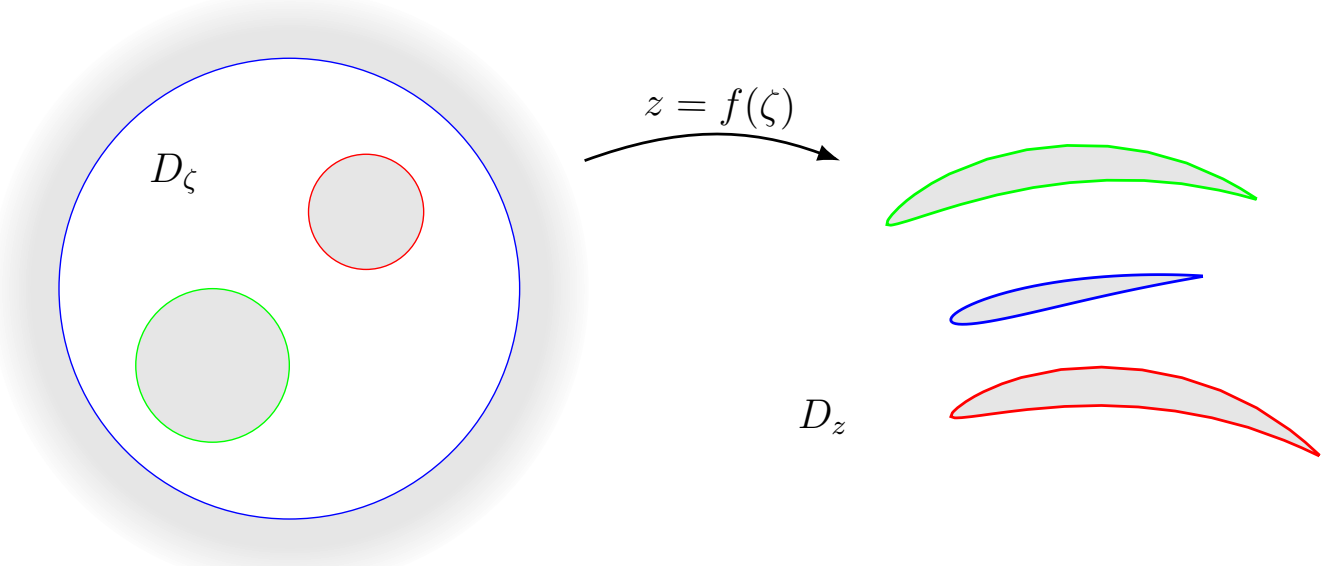

Fig. 1.7 A conformal map between two triply connected domains.

shown to be a fundamental object in understanding multiply connected domains (Crowdy, 2005, 2007, 2008b; Crowdy and Marshall, 2006). Whilst the prime function is intimately connected with the theory of Abelian functions and Riemann surfaces (Baker, 1897), for this thesis it is sufficient to view the prime function as a transcendental special function. In particular, the prime function is computable, as recent research has developed a novel numerical algorithm to swiftly and accurately compute the prime function for practical applications (Crowdy et al., 2016, https://github.com/ACCA-Imperial/SKPrime).

\subsubsection{The Canonical Circular Domain}

We define a canonical circular domain of connectivity $M+1$ as the interior of the unit disc with $M$ excised discs. The unit disc is labelled $C_{0}$ and the excised discs are labelled $\left\{C_{j} \mid j=1, \cdots, M\right\}$. We call the geometrical data associated with the circular domain the "conformal moduli", which may be expressed as $\left\{q_{j}, \delta_{j} \mid j=1, \cdots, M\right\}$ where the $q_{j}$ denote the radii of the excised circles and the $\delta_{j}$ denote the centres. An example of a quadruply connected canonical circular domain is illustrated in figure 1.8 .

\subsubsection{The Schottky-Klein prime function}

The prime function is a transcendental analytic function associated with a particular canonical circular domain, such as that illustrated in 1.8. For brevity, we suppress the dependence of the prime function on the conformal moduli $\left(q_{j}\right.$ and $\left.\delta_{j}\right)$ and write it as a bivariate function $\omega(\zeta, \alpha)$. 


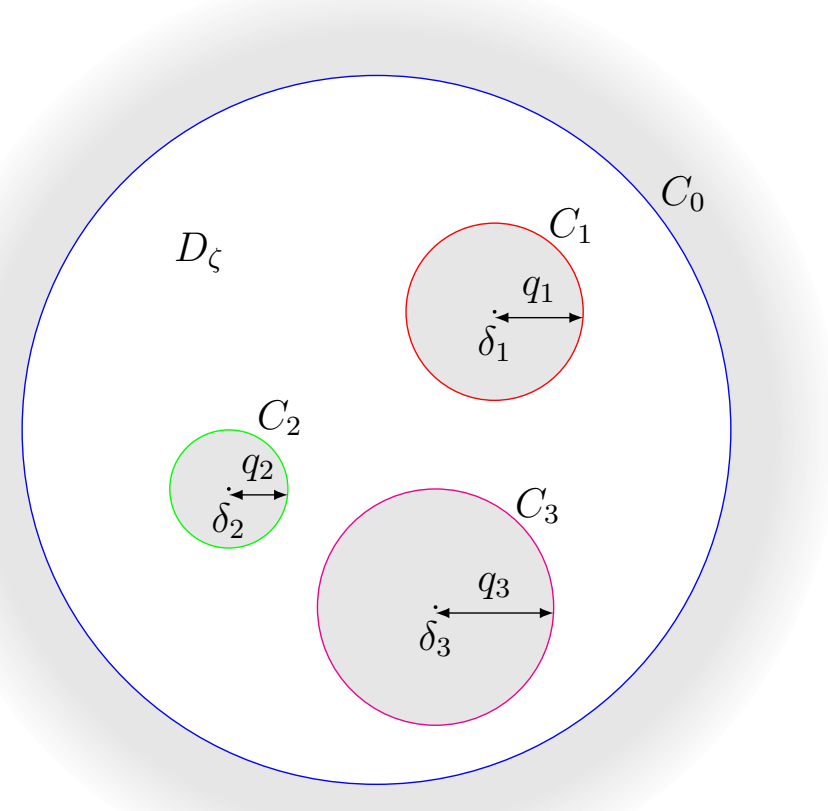

Fig. 1.8 A quadruply connected circular domain.

The general definition of the prime function is

$$
\omega(\zeta, \alpha)=(\zeta-\alpha) \prod_{\theta \in \Theta^{\prime \prime}} \frac{(\zeta-\theta(\alpha))(\alpha-\theta(\zeta))}{(\zeta-\theta(\zeta))(\alpha-\theta(\alpha))}
$$

where $\Theta^{\prime \prime}$ represents the Schottky group (which is the collection of all Möbius maps representing the reflections of excised circles in the unit disc) excluding the identity and all inverses (Crowdy and Marshall, 2006). This product formula may appear unwieldy, but in certain situations the infinite product may be written in more concise forms. For example, in the case $M=0$ the canonical circular domain is simply the unit disc and the prime function is therefore

$$
\omega(\zeta, \alpha)=\zeta-\alpha
$$

When $M=1$, the canonical circular domain is the annulus

$$
\zeta=r \mathrm{e}^{\mathrm{i} \theta}, \quad q<r<1,0<\theta<2 \pi .
$$


In this case, the infinite product (1.8) may be written as

$$
\omega(\zeta, \alpha)=-\frac{\alpha}{C^{2}} P(\zeta / \alpha, q)
$$

where

$$
P(\zeta, q) \equiv(1-\zeta) \prod_{k=1}^{\infty}\left(1-q^{2 k} \zeta\right)\left(1-q^{2 k} \zeta^{-1}\right)
$$

for constant $C$.

\subsubsection{The Canonical Conformal Maps}

Nehari (1952) identified five "canonical" multiply connected conformal mappings for a circular domain of connectivity $M$. The target domains of these canonical maps are

(I) $M+1$ radial slits,

(II) $M+1$ circular arcs,

(III) $M+1$ parallel slits,

(IV) A disc with $M$ circular arc slits, and,

(V) A concentric annulus with $M-1$ circular arc slits.

These canonical conformal maps are illustrated in figure 1.9. Later work by Crowdy and Marshall (2006) derived expressions for these conformal mappings explicitly in terms of the prime function, thereby demonstrating the role of the prime function as a fundamental object in analysing multiply connected domains. In chapter 6 we will present two new canonical multiply connected conformal mappings for periodic domains.

\subsection{Overview}

We now provide an overview of this thesis and emphasise the main results of each chapter. A list of symbols for each chapter is located immediately after the main body of work in each chapter, and before any appendices. All references may be found at the end of the thesis. 


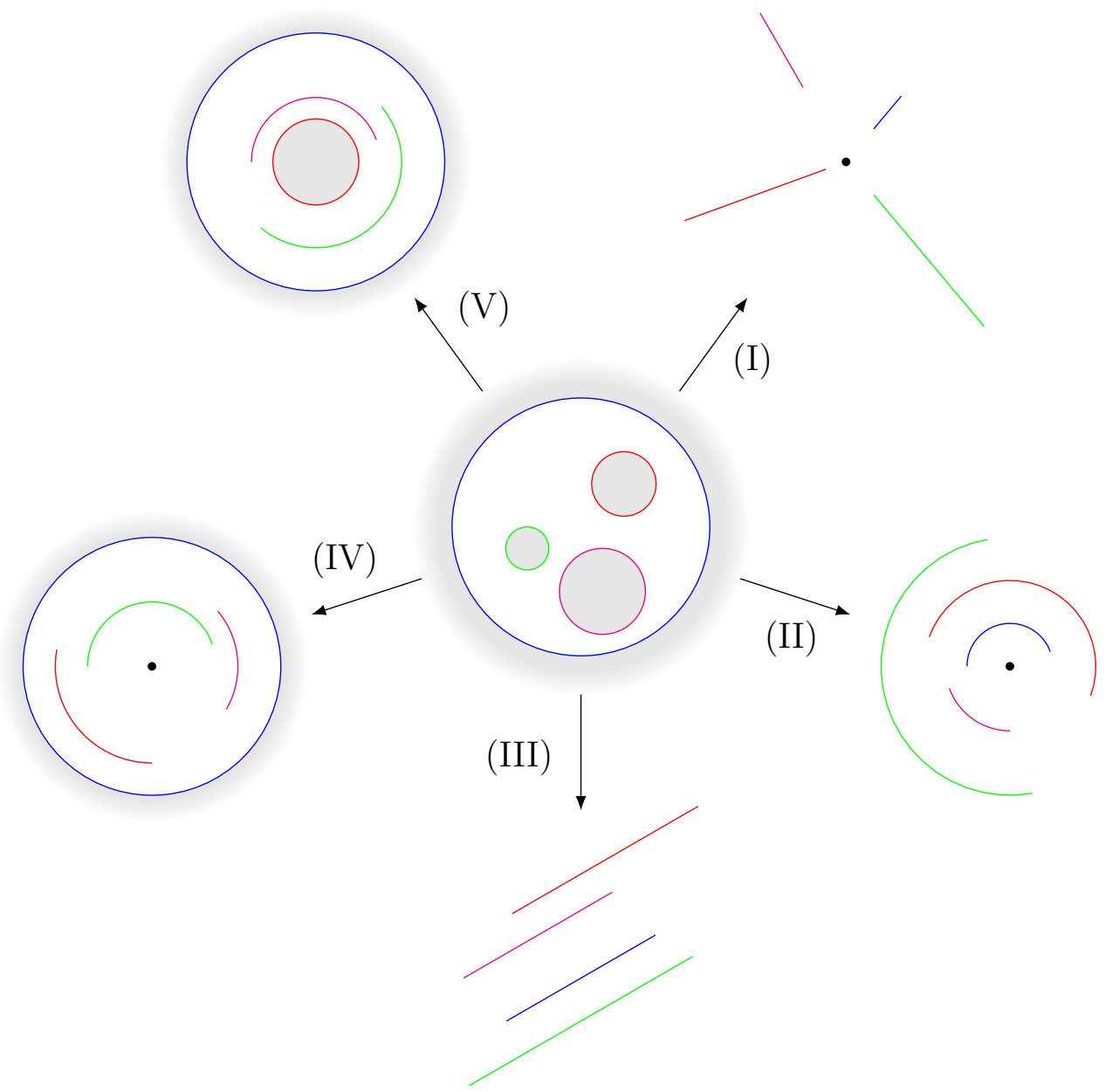

Fig. 1.9 The five canonical conformal maps for a quadruply connected domain. 


\section{Chapter 2}

We begin by solving the potential flow through a cascade of thin aerofoils. This analysis extends previous works (Rienstra, 1992; Thwaites, 1960) who calculated the flow field for an isolated aerofoil. As is typical in these analyses, we seek an asymptotic approximation where the aspect ratio of the aerofoils in the cascade are small, along with the angle of attack. Analytic solutions are available when the stagger $\chi$ is small via a Riemann-Hilbert approach (Gakhov, 1966; Muskhelishvili, 1946), which furnishes compact expressions for aerodynamically relevant quantities such as the lift, deflection angle, and surface velocity. The inverse problem - where the surface velocity is known and the aerofoil geometry must be found - is also solved in the small stagger case. Conversely, when the stagger is large, we adopt a numerical spectral collocation approach in order to determine the surface velocity distribution which, in turn, is used to calculate the flow field in the entire domain. In this case, it is more appropriate to view the problem from the perspective of a singular integral equation, and numerical solutions are found by expanding the solution in terms of weighted Chebyshev polynomials (Rienstra, 1992), and applying a numerical collocation scheme (Erdogan and Gupta, 1972). The scheme is observed to converge spectrally as the number of basis polynomials is increased.

\section{Chapter 3}

We now use the potential flow solution of chapter 2 to investigate the sound generation for a cascade of aerofoils with realistic geometry. This chapter extends previous analyses by Glegg (1999) and Posson et al. (2010b) who considered flat plates with uniform background flow. Nonlinear and viscous effects are ignored, except at the trailing edge of each blade, where steady and unsteady Kutta conditions are applied. The interaction is modelled as a convected, unsteady, vortical or entropic gust incident on an infinite rectilinear cascade of staggered aerofoils in a background flow that is uniform far away from the cascade. By applying Rapid Distortion Theory (RDT, Batchelor and Proudman (1954)) and transforming to an orthogonal coordinate system, we reduce the aerofoils to a cascade of flat plates. In order to proceed, we seek a perturbation expansion in terms of the disturbance of the background flow from uniform flow. The solution to the leading-order (flat plate) problem is already available (Glegg, 1999; Posson et al., 2010b), and becomes the forcing term in the first-order problem. The quasi-periodicity of the problem geometry allows the complicated source terms originating from the RDT formulation to be expanded in a Fourier-type basis. Moreover, we approximate the complex Neumann boundary condition data as a Fourier series, which allows the model to 
be solved analytically using the Wiener-Hopf method. The resulting expression is inverted to give the scattered acoustic potential function in the entire domain, i.e. a solution to the inhomogeneous convected Helmholtz equation with inhomogeneous boundary conditions in a cascade geometry. The solution significantly extends previous analytical work that is restricted to flat plates or only calculates the far-upstream radiation, and as such can give insight into the role played by blade geometry on the acoustic field, upstream, downstream and in the important inter-blade region of the cascade. We discuss various aeroacoustic results including the scattered pressure, surface pressure and sound power output for a range of geometries and angles of attack.

\section{Chapter 4}

We now move on to study cascades with porous aerofoils. In an analogous manner to chapter 2 , this chapter is an aerodynamic investigation and we calculate the potential flow through a cascade of porous aerofoils. Previous work (Hajian and Jaworski, 2017; Iosilevskii, 2011) has calculated the potential flow past an isolated aerofoil with a porosity gradient, and the purpose of this chapter is to extend these solutions to cascades of aerofoils. The cascade is modelled as a periodic array of bound vortex sheets whose strengths satisfy a periodic singular integral equation (SIE) coupled to a Darcy flow condition. We solve a more general canonical periodic SIE by adapting the classical method of Muskhelishvili (1946). We then apply the solution to the porous cascade problem. This method yields exact forms for the lift and deflection angle, as well as the complex velocity field. We investigate partially porous aerofoils in detail and find analytic expressions for the seepage drag for flat plates. We also present results for cambered aerofoils with continuous porosity distributions. These expressions elucidate the role played by porosity on aerodynamic performance, and show that the adverse effects of porosity can be mitigated by reducing the blade spacing.

\section{Chapter 5}

Continuing with the theme of porous aerofoils, we now explore the aeroacoustic performance of cascades of aerofoils with complex boundaries. The method is appropriate to model blades that are rigid, compliant, porous, or satisfy an impedance condition. Similarly to chapter 3, the solution builds on previous analyses into the aeroacoustic response of rigid cascades (Glegg, 1999; Peake, 1992, 1993; Posson et al., 2010b). In comparison to these analyses, the effect of modifying the boundary conditions is to modify the zeros of the kernel in the Wiener-Hopf analysis. Consequently, the complex boundaries have a significant effect on the modal structure of the cascade's inter-blade region, whilst the 
modes in the far-field regions are unchanged. The results demonstrate the considerable impact of small modifications to the boundary conditions and highlight the importance of the accurate modelling of aeroelastic effects when predicting sound transmission and generation in turbomachinery. Significant attention is devoted to ensuring that the kernel of the Wiener-Hopf equation has the appropriate behaviour in the far field.

\section{Chapter 6}

Having studied porous aerofoils in detail, we next consider cascades with multiple blades per period window. This chapter develops fundamental tools for this problem, which are then applied to investigate aerodynamic performance. The most effective tools for these problems are multiply connected conformal mappings (Baddoo and Crowdy, 2019; Crowdy, 2005; Crowdy and Marshall, 2006). We also present a calculus for calculating flow through periodic domains, which builds heavily on the calculus for non-periodic domains presented by Crowdy (2010). We present the periodic extension of the Joukowski mapping, which enables exact solutions for the potential flow through a cascade of thick aerofoils.

\section{Chapter 7}

Finally, we study the aeroacoustics of cascades with multiple blades per period. We derive a compact approximation (Howe, 2003) for the quasi-periodic Green's function using the results of the previous chapter. Consequently, the results are valid for an arbitrary number of aerofoil per period window. We approximate the quasi-periodic Green's function both with and without a low Mach number background flow.

\section{Chapter 8 - Conclusions}

In this chapter we summarise the major results of the thesis and suggest directions for future work. 


\section{Chapter 2}

\section{Potential Flow Through Cascades of Thin, Impermeable Aerofoils}

\section{$2.1 \quad$ Introduction}

Potential flow past a periodic array of bodies is commonplace in a large range of fluid mechanical problems. For example, the flow through a rotor cascade in aerodynamics (Hall and Thwaites, 1965), the flow through structured porous materials (Brenner, 1980), and the flow around large schools of fish (Liao, 2007). Within these applications it is not merely the potential flow through the structure that must be calculated but also the complicated interactions between unsteady perturbations to the flow (such as turbulence) and the structures themselves. To approach any of these complex unsteady interactions it is vital to have a clear understanding of the background steady flow as it can convect and distort the unsteady perturbations. However, there is a distinct lack of analytic solutions for uniform flow past periodic arrays.

Early research by Balsa (1977) considered the uniform flow past an array of cylinders which have small diameters compared to their separation distance through the use of asymptotic analysis. The development of a novel transform method known formally as the Unified Transform Method (Fokas, 2008) (but more commonly as the Fokas method) allowed Crowdy (2016) to extend Balsa (1977) to allow for arbitrary ratios of diameter to separation distance. By solving the problem of uniform flow around cylinders, one could perhaps use a conformal mapping from a periodic array of non-cylindrical structures, to the cylindrical array described by Crowdy (2016). Unfortunately, these mappings are often impossible to invert analytically and thus a numerical scheme must be implemented (Theodorsen and Garrick, 1979). 
Although there are already several ways to calculate the potential flow through a cascade of aerofoils, analytical solutions that elucidate the underlying physics are rare. One well-known solution for flat plates at angle of attack can be found by constructing a conformal map from a canonical circular domain to the cascade (Robinson and Laurmann, 1956, p. 149). Whilst this solution is exact, the expression for the conformal mapping is not invertible, so the velocity field may only be written implicitly. Another method that is ubiquitous in thin-aerofoil theory is the method of singularities: the aerofoil surface is modelled as a distribution of mass sources and vortices on the chord (for thin aerofoils) or the surface (for thick aerofoils). This theory has been applied to cascades on several occasions (Falcão, 1975; Schulten, 1982; Spurr and Allen, 1947), the latter of which is only valid for large chord-to-gap ratios. The vortex distribution is typically written as a Glauert sine series, which corresponds to a series of weighted Chebyshev polynomials in physical space. These methods (of conformal mappings and Glauert sine series) have been combined (Evers and Peake, 2002) to provide an analytical expression for the steady flow near the leading edges.

This chapter therefore presents two new solutions: an analytic solution that is valid when the stagger angle is small, and a numerical solution that is valid for arbitrary stagger angles.

The analytic solution is based on solving a Riemann-Hilbert problem (Muskhelishvili, 1946) for the potential flow through a periodic array of thin objects. This is an extension of the solution for an isolated aerofoil by Thwaites (1960), which is commonplace in aeroacoustic problems (Ayton and Peake, 2013). In addition to the direct problem, of obtaining the potential flow through a cascade of aerofoils with specified geometry, this approach allows us to also solve the inverse problem: given a desired flow around an aerofoil within the cascade, we can determine the geometry of each aerofoil within the cascade required to generate this specific flow. Following the analytic solution, we present a Chebyshev spectral collocation method to rapidly compute the flow through a cascade of aerofoils at arbitrary stagger angle. This method is an extension of the classical Glauert sine series expansion (Rienstra, 1992) to a cascade geometry. This new method is an improvement on previous similar analyses (Spurr and Allen, 1947), as discussed later. We use an ansatz that is inspired by the analytic solution to construct a singular integral equation for the bound vorticity along the chord. This singular integral equation is then solved by the method of Erdogan and Gupta (1972).

The layout of this chapter is as follows. In section 2.2 we set up the Riemann-Hilbert problem for the potential flow through a cascade of aerofoils. In section 2.3 we solve the direct problem by segregating the boundary conditions arising due to thickness, camber 
and angle of attack, and in section 2.4 we use equations derived in the direct problem to solve the inverse problem. In section 2.5 we present results for the analytic solution for a variety of geometries and angles of attack. We derive analytic forms for key aerodynamics parameters, such as the surface velocity, lift, and deflection angle. We also demonstrate the inverse problem for a particular class of aerofoil geometries. In section 2.6 we provide the mathematical details of the numerical scheme. This numerical method is validated against the analytic solution and the convergence is seen to be spectral (i.e. faster than any algebraic convergence). We also present an exponential decomposition of the flow in the upstream and downstream regions, which will be useful in the following aeroacoustic analysis. Finally, in section 2.7 we discuss the conclusions of the chapter.

\subsection{Model Derivation}

Consider an infinite cascade of thin aerofoils under the assumption of small disturbances in a two-dimensional, steady, incompressible flow. We non-dimensionalise lengths so that the semi-chord of each aerofoil is 1 . The extension to compressible flows may be achieved via a Prandtl-Glauert transformation (Kuethe and Chow, 1998). The flow is uniform far upstream and has angle of attack $\alpha$ relative to the chords of the aerofoils, which are inclined at stagger angle $\chi$. We assume that $\alpha, \chi=\mathcal{O}(\epsilon)$ where $\epsilon$ is a small parameter the order of the aspect ratio of the aerofoils. This arrangement is illustrated in figure 2.1. We write the complex potential for the total steady flow in the form of a series expansion in $\epsilon$ :

$$
w(z)=\phi(z)+\mathrm{i} \psi(z)=U_{\infty}\left(z \mathrm{e}^{-\mathrm{i}(\alpha+\chi)}+\epsilon w_{1}(z)+\mathcal{O}\left(\epsilon^{2}\right)\right),
$$

where $z=x+\mathrm{i} y$ and $w_{1}(z)$ is a function to be found subject to a no-flux boundary condition on the aerofoils' surfaces, appropriate edge conditions at the leading and trailing edges, and decay far upstream.

We denote the upper and lower boundaries of the $n^{\text {th }}$ aerofoil as $y_{s, n}^{ \pm}(t)$ respectively, so that

$$
y_{s, n}^{ \pm}(t)=\epsilon y^{ \pm}(t)+t \sin (\chi)+\mathrm{i} n \Delta,
$$

and $y_{s}^{ \pm}(t)= \pm y_{t h}(t)+y_{c}(t)$, where the subscripts $t h$ and $c$ denote thickness and camber respectively, $n \in \mathbb{Z}$ and $t \in[-1,1]$ parameterises the aerofoil chord. We restrict our analysis to bodies that have, at worst, parabolic noses, i.e. $y_{t h} \sim \sqrt{t}$ as $t \rightarrow-1$. This is in line with the NACA 4-digit aerofoil series that is commonplace in the literature. 


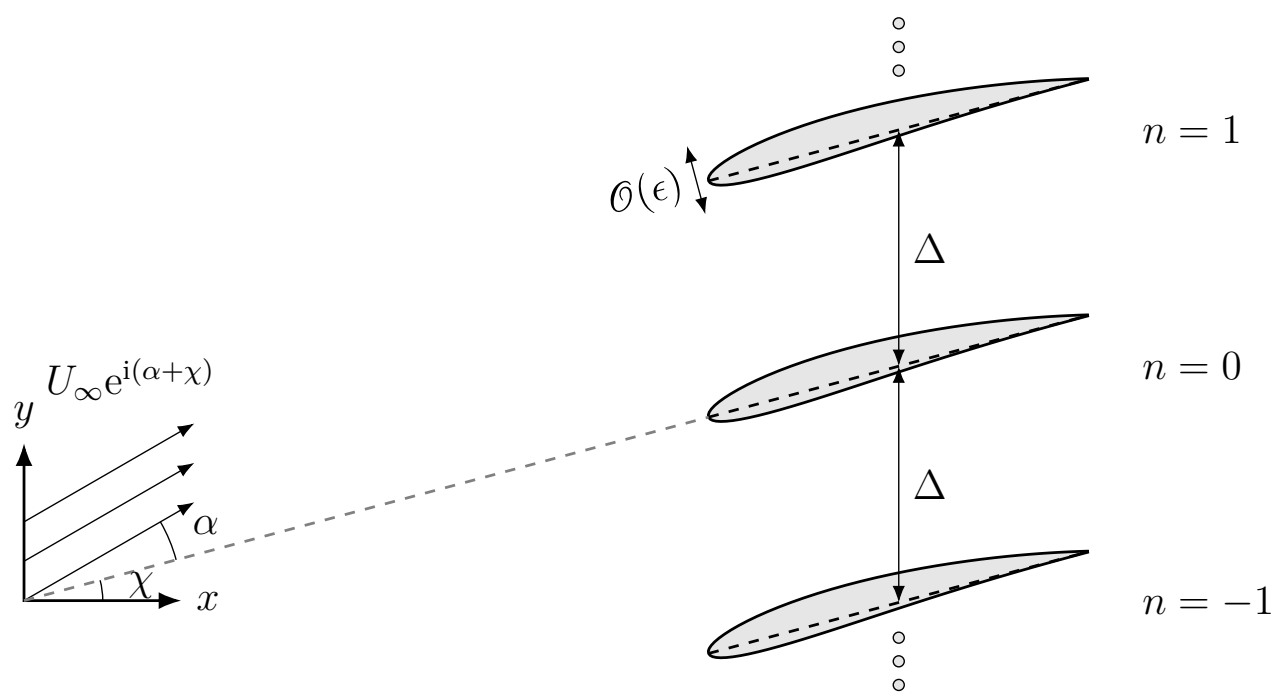

Fig. 2.1 A staggered cascade of aerofoils. The vertical spacing between the aerofoils is $\Delta$ and the upstream velocity is $U_{\infty}$, which is inclined at angle of attack $\alpha$. The stagger angle is $\chi$.

Any trailing-edge angle is permitted by the analysis, including cusped ends and finite angle trailing edges. In the latter case, we expect a stagnation point to form at the trailing edge. This can be proved via a conformal mapping for a semi-infinite wedge (Batchelor, 2000, p. 412). In practice, this means that our perturbation solution will have a log-singularity at the trailing edge to create a stagnation point when combined with the $\mathcal{O}(1)$ solution. Although this log-singularity is unavoidable for thick aerofoils, we may still enforce that the jump in velocity at the trailing edge vanishes, so the Kutta condition still holds. Additionally, we require that $y_{s}^{ \pm \prime}(t)$ satisfies a Hölder condition on $t \in(-1,1)$.

The no-flux boundary condition requires $\partial \operatorname{Re}[w] / \partial n=0$ on the boundary of the aerofoil, except at the sharp trailing edge where the outward normal derivative is undefined. Since the outward normal to the upper (lower) surface of each aerofoil is given by $\left(-\epsilon y_{s, n}^{ \pm \prime}(t), 1\right)$, the no-flux condition on the upper (lower) surface is $\left(-\epsilon y_{s, n}^{ \pm \prime}, 1\right) \cdot \nabla \operatorname{Re}[w]=0$, i.e.

$$
\begin{aligned}
0 & =\left(-y_{s}^{ \pm \prime}(t), 1\right) \cdot\left(1+\epsilon u^{ \pm}(t),(\alpha+\chi)+\epsilon v^{ \pm}(t)\right)+\mathcal{O}\left(\epsilon^{2}\right) \\
& =-y_{s}^{ \pm \prime}(t)+(\alpha+\chi)+\epsilon v^{ \pm}(t)+\mathcal{O}\left(\epsilon^{2}\right)
\end{aligned}
$$

where we have assumed that $y_{s}^{ \pm \prime}, u^{ \pm}, v^{ \pm}$are all $\mathcal{O}(1)$. This assumption proves to be valid everywhere except in an $\mathcal{O}\left(\epsilon^{2}\right)$ region close to the leading edge where a further 
asymptotic expansion would be required (Van Dyke, 1964), however this is beyond the scope of this chapter.

Since $\epsilon \ll 1$, we can use Taylor's theorem to apply the no-flux condition on the aerofoils' surface to their chords. Furthermore, since $\chi$ is small, we may approximate the staggered chord as a chord with no stagger. This approximation has an $\mathcal{O}\left(\epsilon^{2}\right)$ effect on the solution, and will be inverted at the end of section 2.3 in order to properly locate the leading- and trailing-edge stagnation points. Accordingly, an analytic, periodic function $f$ satisfies

$$
\epsilon f\left(t, y_{s, n}^{ \pm}(t)\right)=\epsilon f^{ \pm}(t)+\mathcal{O}\left(\epsilon^{2}\right)
$$

Combining (2.2) and (2.3) yields

$$
\epsilon v(t, \mathrm{i} n \Delta \pm)=y_{s, n}^{ \pm \prime}(t)-\alpha-\chi=\epsilon y_{c}^{\prime}(t)-\alpha \pm \epsilon y_{t h}^{\prime}(t)
$$

The superscript \pm here indicates the limiting value taken on the upper and lower sides of $L$ respectively, where $L$ is defined as the union of the unstaggered chords traversed from leading edge to trailing edge. Hence, by considering the complex velocity $\Phi(z)=w_{1}^{\prime}(z)=u(x, y)-\mathrm{i} v(x, y)$ either side of $L, \Phi$ must satisfy

$$
\Phi^{ \pm}(t)=u^{ \pm}(t)-\mathrm{i}\left(y_{c}^{\prime}(t)-\alpha \pm y_{t h}^{\prime}(t)\right)
$$

where $u^{ \pm}$are the unknown upper and lower tangential surface velocities. If we can solve for $\Phi(z)$, we can obtain the tangential surface velocities and the total complex potential, $w(z)$.

In addition to the no-flux condition, we must also enforce the Kutta condition by selecting the circulation such that the rear stagnation point is located at the trailing edges of the aerofoils. This is equivalent to specifying that the flows over the upper and lower surfaces are parallel to one another at the trailing edges (Clancy, 1978). Since this formulation does not permit any wakes, it is sufficient to impose the jump in tangential velocity to be zero at the trailing edges. At the leading edges, thin aerofoil theory (Abbott and Von Doenhoff, 1959) tells us that the fluid velocity scales as the inverse square root of the distance from the leading edges, so we permit square root singularities here. To fully summarise our problem, we are seeking a function $\Phi(z)$ such that

(I) $\Phi(z)$ satisfies Laplace's equation (is holomorphic) in $\mathbb{C} \backslash \bar{L}$,

(II) The boundary conditions in (2.5) are satisfied either side of $L$, 
(III) $\Phi(z)=\mathcal{O}\left(|z|^{-1 / 2}\right)$ as $z$ approaches the leading edge of each aerofoil,

(IV) $\Phi(t)$ has zero jump in tangential velocity as $t$ approaches the trailing edge along the chord, and,

(V) $\Phi(z) \rightarrow 0$ as $z \rightarrow-\infty$.

Once we know $\Phi(z)$ we can obtain the potential flow around the cascade correct to $\mathcal{O}(\epsilon)$. In the following section, we find a solution by interpreting (I-V) as a Riemann-Hilbert problem.

\subsection{Direct Problem Solution}

We seek to solve (I-V) as a Riemann-Hilbert problem (Muskhelishvili, 1946) but must first note several non-standard features of the formulation. First, the contour $L$ is unbounded, and is an infinite union of disjoint contours. Much of the analysis of Riemann-Hilbert problems is only applicable to problems defined on bounded, finite contours. However, we do not require all of the theory to solve this problem, and show in Appendix 2.A that, due to the periodicity of the problem, there is a modified form of the Plemelj formulae (2.52) which may be applied in this case. This modified form is used to find the "density function" and solve (I, II) in section 2.3.1. In contrast to the isolated aerofoil case, the Riemann-Hilbert formulation does not automatically ensure that $\Phi \rightarrow 0$ as $|z| \rightarrow \infty$, but the use of a fundamental solution in section 2.3.2 is sufficient to fix the upstream behaviour for $(\mathrm{V})$. Additionally, in section 2.3.3 we verify that the solution satisfies the edge conditions (III,IV) by considering the asymptotic behaviour near the endpoints, as given in Appendix 2.B.

A second non-standard feature of the problem is that only the imaginary data is prescribed on $L$, as opposed to the full value of $\Phi$. The unknown real data $u^{ \pm}(t)$ may be eliminated by employing the Schwarz reflection principle (Ablowitz and Fokas, 2003, p. 346). We write the complex velocity as

$$
\Phi(z)=\Phi_{t h}(z)+\Phi_{c, \alpha}(z)
$$

where

$$
\Phi_{t h}(z)=\frac{1}{2}(\Phi(z)+\bar{\Phi}(z)), \quad \quad \Phi_{c, \alpha}(z)=\frac{1}{2}(\Phi(z)-\bar{\Phi}(z))
$$


The overline " - " denotes the Schwarz conjugate (denoted in Ablowitz and Fokas (2003) as " "). For example, given an analytic function

$$
f(x, y)=g(x, y)+\mathrm{i} h(x, y)
$$

where $g$ and $h$ are real functions, the Schwarz conjugate is defined as

$$
\bar{f}(z)=g(x,-y)-\mathrm{i} h(x,-y)
$$

The functions defined in (2.6) additionally have the properties

$$
\Phi_{t h}(z)=\bar{\Phi}_{t h}(z), \quad \Phi_{c, \alpha}(z)=-\bar{\Phi}_{c, \alpha}(z)
$$

By taking the limiting value of $\Phi$ either side of $L$, we obtain

$$
\begin{aligned}
\Phi_{t h}^{+}(t)-\Phi_{t h}^{-}(t) & =-2 \mathrm{i} y_{t h}^{\prime}(t), \\
\Phi_{c, \alpha}^{+}(t)+\Phi_{c, \alpha}^{-}(t) & =-2 \mathrm{i} y_{c}^{\prime}(t)+2 \mathrm{i} \alpha,
\end{aligned}
$$

which are two Riemann-Hilbert problems. This formulation yields convenient boundary values on the chords that only take known imaginary values on $L$ and unknown real quantities, $u^{ \pm}(t)$, have been removed.

\subsubsection{General Solution}

Here we find the general solution for $\Phi$, satisfying (I, II) which we will later modify to set the upstream behaviour and satisfy the edge conditions (III, IV, V). We treat the thickness, camber, and angle of attack problems separately since the boundary condition is linear.

\subsubsection{Thickness Term, $\Phi_{t h}$}

Here we solve (2.7). This is a straightforward Riemann-Hilbert problem and the solution is

$$
\Phi_{t h}(z)=\frac{1}{2 \pi \mathrm{i}} \int_{L} \frac{-2 \mathrm{i} y_{t h}^{\prime}(\tau)}{\tau-z} \mathrm{~d} \tau
$$


which can easily be verified with the Modified Plemelj formulae (2.52). We use the result (2.50) from Appendix 2.A to write

$$
\Phi_{t h}(z)=-\frac{1}{\Delta} \int_{-1}^{1} y_{t h}^{\prime}(\tau) \operatorname{coth}\left(\frac{\pi(\tau-z)}{\Delta}\right) \mathrm{d} \tau
$$

\subsubsection{Camber Term, $\Phi_{c}$}

Now we solve for the camber term by splitting $\Phi_{c, \alpha}$ into two terms dependent on angle of attack and camber respectively. We decompose (2.8) into parts depending only on camber and write

$$
\Phi_{c}^{+}(t)+\Phi_{c}^{-}(t)=-2 \mathrm{i} y_{c}^{\prime}(t)
$$

The solution to this type of Riemann-Hilbert problem for bounded contours is detailed in p. 429 of Gakhov (1966). This solution relied on constructing a bounded, closed contour that connects the contours along which the Riemann-Hilbert problem is defined. This is not possible in our case, but the method may be adapted. We first need to solve the homogeneous equation to find the so-called fundamental solution, which we denote by $X(z)$. In general, the homogeneous equation is

$$
X^{+}(t)=G(t) X^{-}(t)
$$

and a solution is given by (43.2) of Gakhov (1966) as

$$
X(z)=\mathrm{e}^{\Pi(z)}, \quad \Pi(z)=\frac{1}{2 \pi \mathrm{i}} \int_{L} \frac{\log G(\tau)}{\tau-z} \mathrm{~d} \tau
$$

In our case, the homogeneous equation is

$$
X^{+}(t)+X^{-}(t)=0
$$

and we have $\log (G)=\pi \mathrm{i}(1+2 n)$. Therefore,

$$
\Pi(z)=\left(n+\frac{1}{2}\right) \int_{L} \frac{1}{\tau-z} \mathrm{~d} \tau=\left(n+\frac{1}{2}\right) \log \left(\frac{\sinh \left(\frac{\pi(z-1)}{\Delta}\right)}{\sinh \left(\frac{\pi(z+1)}{\Delta}\right)}\right) .
$$

More solutions to the homogeneous equation may be found by multiplying or dividing by polynomials whose the roots are the endpoints of the contours. This is useful when specifying whether the behaviour at either endpoint is permitted to be unbounded. In 
the periodic case, this non-uniqueness of solutions can be expressed as

$$
\begin{aligned}
X(z)=\left[\sinh \left(\frac{\pi(z+1)}{\Delta}\right)\right]^{\lambda} & {\left[\sinh \left(\frac{\pi(z-1)}{\Delta}\right)\right]^{\mu} \mathrm{e}^{\Pi(z)} } \\
= & {\left[\sinh \left(\frac{\pi(z+1)}{\Delta}\right)\right]^{\lambda-n-\frac{1}{2}}\left[\sinh \left(\frac{\pi(z-1)}{\Delta}\right)\right]^{\mu+n+\frac{1}{2}}, }
\end{aligned}
$$

where $\lambda$ and $\mu$ are integers chosen to satisfy

$$
-1<\lambda-n-\frac{1}{2}<1, \quad-1<\mu+n+\frac{1}{2}<1 .
$$

At this point we recall the conditions on the endpoint behaviour needed to satisfy the Kutta condition (IV) and leading-edge behaviour (III). There are two choices each for $\lambda$ and $\mu$ but we choose $\lambda=n, \mu=-n$ in order to satisfy (III) and (IV). Therefore,

$$
X(z)=\sqrt{\frac{\sinh \left(\frac{\pi(z-1)}{\Delta}\right)}{\sinh \left(\frac{\pi(z+1)}{\Delta}\right)}},
$$

and the limiting value either side of $L$, with this choice of branch, is

$$
X^{ \pm}(t)= \pm \mathrm{i} \sqrt{\frac{\sinh \left(\frac{\pi(1-t)}{\Delta}\right)}{\sinh \left(\frac{\pi(1+t)}{\Delta}\right)}} .
$$

Following Gakhov (1966), we now use the fundamental solution to solve the inhomogeneous problem. We write

$$
\Phi_{c}(z)=X(z) \Psi(z)
$$

so that $\Psi$ satisfies

$$
\Psi^{+}(t)-\Psi^{-}(t)=-\frac{2 \mathrm{i} y_{c}^{\prime}(t)}{X^{+}(t)}
$$

This is a Riemann-Hilbert problem of the form (2.10) and has solution

$$
\Psi(z)=\frac{1}{2 \pi \mathrm{i}} \int_{L} \frac{-2 \mathrm{i} y_{c}^{\prime}(\tau)}{X^{+}(\tau)(\tau-z)} \mathrm{d} \tau .
$$


Therefore, the solution for equation (2.11) is given by

$$
\Phi_{c}(z)=-\frac{X(z)}{\Delta} \int_{-1}^{1} \frac{y_{c}^{\prime}(\tau)}{X^{+}(\tau)} \operatorname{coth}\left(\frac{\pi(\tau-z)}{\Delta}\right) \mathrm{d} \tau .
$$

\subsubsection{Angle of Attack Term, $\Phi_{\alpha}$}

We decompose (2.8) into parts depending only on angle of attack and write

$$
\Phi_{\alpha}^{+}(t)+\Phi_{\alpha}^{-}(t)=2 \mathrm{i} \alpha
$$

which has the simple solution

$$
\Phi_{\alpha}(z)=\mathrm{i} \alpha
$$

\subsubsection{Far-Field Behaviour}

In this section we ensure that each solution decays at upstream infinity (V).

\subsubsection{Thickness Term, $\Phi_{t h}$}

Since $\operatorname{coth}(z) \rightarrow \pm 1$ as $z \rightarrow \pm \infty$, we find that $\Phi_{t h}(z) \rightarrow \pm \frac{1}{\Delta} \int_{-1}^{1} y_{t h}^{\prime}(\tau) \mathrm{d} \tau=0$ as $y_{t h}( \pm 1)=0$. Therefore, the upstream condition is already satisfied. Moreover, this result tells us that an unstaggered, thick cascade does not deflect a flow incident with zero angle of attack. This is what we would expect physically due to the symmetry of the problem, and we shall see later that this is not the case with non-zero angle of attack or camber.

\subsubsection{Camber Term, $\Phi_{c}$}

We note that

$$
X(z) \rightarrow \mathrm{e}^{\mp \frac{\pi}{\Delta}}
$$

as $z \rightarrow \pm \infty$. Applying this limit to (2.13) gives

$$
\Phi_{c}(z) \rightarrow-\frac{\mathrm{e}^{\frac{\pi}{\Delta}}}{\Delta} \int_{-1}^{1} \frac{y_{c}^{\prime}(\tau)}{X^{+}(\tau)} \mathrm{d} \tau
$$

as $z \rightarrow-\infty$. So the solution, in its current form, does not satisfy our upstream condition $(\mathrm{V})$. We note that the boundary value problem defined by (I) and (II) is only unique up 
to the addition of a function holomorphic on $\mathbb{C} \backslash L$ that takes real values on $L^{ \pm}$and has the correct endpoint behaviour (III, IV). If we have such a function, then we can add an arbitrary multiple of it to the current solution and the resulting function will satisfy the same boundary value problem, albeit with modified far-field behaviour. An appropriate function is the fundamental solution, $X(z)$, which is pure imaginary on $L$. Addition of imaginary multiples of the fundamental solution will not affect the imaginary parts of the boundary values. Therefore, the modified function

$$
\Phi_{c}(z)=-\frac{X(z)}{\Delta} \int_{-1}^{1} \frac{y_{c}^{\prime}(\tau)}{X^{+}(\tau)}\left(\operatorname{coth}\left(\frac{\pi(\tau-z)}{\Delta}\right)-1\right) \mathrm{d} \tau
$$

now has the correct upstream behaviour.

\subsubsection{Angle of Attack Term, $\Phi_{\alpha}$}

Similarly to the cambered case, we can use multiples of the fundamental solution to specify the correct upstream behaviour. The function with the correct upstream behaviour is

$$
\Phi_{\alpha}(z)=\mathrm{i} \alpha\left(1-X(z) \mathrm{e}^{-\frac{\pi}{\Delta}}\right)
$$

\subsubsection{Endpoint Behaviour}

In this section we verify that each solution possesses the correct behaviour at the leading and trailing edges, according to (III, IV).

\subsubsection{Thickness Term, $\Phi_{t h}$}

The tangential velocity may be calculated by applying the Plemelj formula:

$$
u_{t}^{ \pm}(t)=-\frac{1}{\Delta} \int_{-1}^{1} y_{t h}^{\prime}(\tau) \operatorname{coth}\left(\frac{\pi(\tau-t)}{\Delta}\right) \mathrm{d} \tau .
$$

The tangential velocity is identical either side of the aerofoil and therefore the Kutta condition (IV) is satisfied. This can be seen by the symmetry of the problem and indicates that there will be no lift and therefore no flow deflection far downstream. As stated earlier, we only permit thickness functions whose derivatives have, at worst, square root singularities at the leading edges. This is certainly the case for aerofoils with parabolic leading edges, such as NACA aerofoils, where $y_{t h}^{\prime}(x) \sim x^{-1 / 2}$. We refer to Appendix 2.B to explore the behaviour of our solution at the leading edges and show that it is consistent with condition (III). We write $f(t)=y_{t h}^{\prime}(t)$ for consistency with Appendix 
2.B. For the leading edges we have

$$
f(t)=\frac{\tilde{f}(t)}{(t+1)^{1 / 2}}
$$

where $\tilde{f}(t)$ satisfies a Hölder condition at $t=-1$. We apply equation (2.53c) so as $z \rightarrow-1+\mathrm{i} n \Delta$, with $z \notin L$,

$$
\Phi_{t h}(z) \sim \frac{\mathrm{e}^{\pi \mathrm{i} / 2}}{2 \mathrm{i} \sin (\pi / 2)} \cdot \frac{\tilde{f}(-1)}{(z+1)^{1 / 2}}+\Phi_{t 1}(z)+\Phi_{t 0}(z) .
$$

Since $\Phi_{t 0}(z)$ is bounded and tends to a definite limit as $z \rightarrow-1+\mathrm{i} n s$, this corresponds to an inverse square root singularity, which is permissible. For the trailing edge, we have already established that the horizontal velocity jump is identically zero on the aerofoil and hence the Kutta condition is satisfied.

\subsubsection{Camber Term, $\Phi_{c}$}

We now use equation (2.11) and the Plemelj formulae (2.52) to give

$$
u_{c}^{ \pm}(t)=\mp \frac{X^{+}(t)}{\Delta} f_{-1}^{1} \frac{y_{c}^{\prime}(\tau)}{X^{+}(\tau)}\left(\operatorname{coth}\left(\frac{\pi(\tau-t)}{\Delta}\right)-1\right) \mathrm{d} \tau .
$$

The Kutta condition states that the jump in horizontal velocity must be zero at the trailing edge, and this will only be the case if $u_{c}^{ \pm}(1)=0$. We apply the results of Appendix 2.B, where

$$
f(t)=\frac{y_{c}^{\prime}(t)}{X^{+}(t)}
$$

We are in the case of equation (2.53d) where $\gamma=1 / 2$, so

$$
f(t)=\frac{\tilde{f}(t)}{(t-1)^{1 / 2}},
$$

as $t \rightarrow 1$ where $\tilde{f}$ satisfies a Hölder condition at $t=1$. Therefore, as $t \rightarrow 1$ along the contour,

$$
\begin{aligned}
u_{c}^{+}(t)-u_{c}^{-}(t) & \sim-X^{+}(t)\left(\frac{\cot (\pi / 2)}{2 \mathrm{i}} \cdot \frac{\tilde{f}(1)}{(t-1)^{\gamma}}+\Phi_{c 1}(t)+\Phi_{c 0}(t)\right) \\
& =-X^{+}(t)\left(\Phi_{c 1}(t)+\Phi_{c 0}(t)\right) \rightarrow 0,
\end{aligned}
$$


since $\Phi_{c 0}(t)$ and $\Phi_{c 1}(t)$ are bounded at $t=1$. Therefore, the Kutta condition is satisfied.

We now consider the behaviour at the leading edges. We are therefore in the case of equation (2.53a), with $f(t)=\tilde{f}(t)$, so as $z \rightarrow-1+\mathrm{i} n \Delta$, with $z \notin L$,

$$
\begin{aligned}
\Phi_{c}(z) & \sim-X(z)\left(-\frac{f(-1)}{2 \pi \mathrm{i}} \log \left(\frac{1}{z+1}\right)+\Phi_{c 1}(z)+\Phi_{c 0}(z)\right) \\
& =-X(z)\left(\Phi_{c 1}(z)+\Phi_{c 0}(z)\right)
\end{aligned}
$$

Hence there is a square root singularity at the leading edge, which is permissible by (III).

\subsubsection{Angle of Attack Term, $\Phi_{\alpha}$}

Clearly (2.17) has the correct endpoint behaviour and satisfied (III, IV). The tangential surface velocity is given by

$$
u_{\alpha}^{ \pm}(t)=\mp \alpha \mathrm{ie}^{-\frac{\pi}{\Delta}} X^{+}(t)
$$

\subsubsection{Final Complex Velocity}

By summing the constituent parts of the problem, we get

$$
\begin{aligned}
\Phi(z)= & \mathrm{i} \alpha\left(1-\mathrm{e}^{-\frac{\pi}{\Delta}} \sqrt{\frac{\sinh \left(\frac{\pi(z-1)}{\Delta}\right)}{\sinh \left(\frac{\pi(z+1)}{\Delta}\right)}}\right)-\frac{1}{\Delta} \int_{-1}^{1} y_{t h}^{\prime}(\tau) \operatorname{coth}\left(\frac{\pi(\tau-z)}{\Delta}\right) \mathrm{d} \tau \\
& -\frac{1}{\mathrm{i} \Delta} \sqrt{\frac{\sinh \left(\frac{\pi(z-1)}{\Delta}\right)}{\sinh \left(\frac{\pi(z+1)}{\Delta}\right)}} \int_{-1}^{1} y_{c}^{\prime}(\tau) \sqrt{\frac{\sinh \left(\frac{\pi(1+\tau)}{\Delta}\right)}{\sinh \left(\frac{\pi(1-\tau)}{\Delta}\right)}}\left(\operatorname{coth}\left(\frac{\pi(\tau-z)}{\Delta}\right)-1\right) \mathrm{d} \tau .
\end{aligned}
$$

The solutions in this section are valid for small stagger angles $(\chi \ll 1)$. However, due to the approximation of the staggered chord as a horizontal line in section 2.2, the solution (2.20) suggests that the leading-edge stagnation points are at $-1+\mathrm{in} \Delta$ and the trailing-edge stagnation points are at $+1+\mathrm{i} n \Delta$. Conversely, the physical leading edge is located at $-\mathrm{e}^{\mathrm{i} \chi}$ and the physical trailing edge is located at $\mathrm{e}^{\mathrm{i} \chi}$. Consequently, we include multiples of $\mathrm{e}^{\mathrm{i} \chi}$ in appropriate places in (2.20) to perturb the stagnation points to the correct locations. This procedure only introduces $\mathcal{O}\left(\epsilon^{2}\right)$ errors, which are allowable in the current asymptotic regime. Therefore, the final solution for the complex potential 
with these singularities in the correct locations is given by

$$
\begin{gathered}
\Phi(z)=\mathrm{i} \alpha\left(1-\mathrm{e}^{-\frac{\pi}{\Delta} \mathrm{e}^{-\mathrm{i} \chi}} \sqrt{\frac{\sinh \left(\frac{\pi\left(z-\mathrm{e}^{\mathrm{i} \chi}\right)}{\Delta}\right)}{\sinh \left(\frac{\pi\left(z+\mathrm{e}^{\mathrm{i} \chi}\right)}{\Delta}\right)}}\right)-\frac{1}{\Delta} \int_{-1}^{1} y_{t h}^{\prime}(\tau) \operatorname{coth}\left(\frac{\pi\left(\tau \mathrm{e}^{\mathrm{i} \chi}-z\right)}{\Delta}\right) \mathrm{e}^{\mathrm{i} \chi} \mathrm{d} \tau \\
-\frac{1}{\mathrm{i} \Delta} \sqrt{\frac{\sinh \left(\frac{\pi\left(z-\mathrm{e}^{\mathrm{i} \chi}\right)}{\Delta}\right)}{\sinh \left(\frac{\pi\left(z+\mathrm{e}^{\mathrm{i} \chi}\right)}{\Delta}\right)}} \int_{-1}^{1} y_{c}^{\prime}(\tau) \sqrt{\frac{\sinh \left(\mathrm{e}^{\left.\mathrm{i} \chi \frac{\pi(1+\tau)}{\Delta}\right)}\right.}{\sinh \left(\mathrm{e}^{\left.\mathrm{i} \chi \frac{\pi(1-\tau)}{\Delta}\right)}\right.}\left(\operatorname{coth}\left(\frac{\pi\left(\tau \mathrm{e}^{\mathrm{i} \chi}-z\right)}{\Delta}\right)-1\right) \mathrm{e}^{\mathrm{i} \chi} \mathrm{d} \tau .}
\end{gathered}
$$

It should be noted that, in the asymptotic limit $\Delta \rightarrow \infty$, this matches the well-known case of the flow around a single aerofoil given by Thwaites (1960).

\subsection{Inverse Problem Solution}

We now consider the problem where the tangential velocities on the upper and lower surfaces of the aerofoils are specified and an appropriate aerofoil geometry must be found. This specification of tangential velocity is essential in blade design for axial-flow compressors in order to control boundary-layer growth and separation (Goldstein and Jerison, 1947). We may use equations (2.18) and (2.19) to find singular integral equations for the thickness, camber and angle of attack:

$$
\begin{aligned}
& -\frac{2}{\Delta} \int_{-1}^{1} y_{t h}^{\prime}(\tau) \operatorname{coth}\left(\frac{\pi(\tau-t)}{\Delta}\right) \mathrm{d} \tau=u^{+}(t)+u^{-}(t), \\
& -\frac{2 X^{+}(t)}{\Delta} f_{-1}^{1} \frac{y_{c}^{\prime}(\tau)-\alpha}{X^{+}(\tau)}\left(\operatorname{coth}\left(\frac{\pi(\tau-t)}{\Delta}\right)-1\right) \mathrm{d} \tau=u^{+}(t)-u^{-}(t) .
\end{aligned}
$$

where $u^{ \pm}(t)$ each satisfy a Hölder condition on $(-1,1)$, except possibly at the end points, where they have, at worst, integrable singularities. We solve these equations separately.

\subsubsection{Thickness Term}

We define the auxiliary function

$$
I_{t h}(z)=-\frac{1}{\Delta} \int_{-1}^{1} y_{t h}^{\prime}(\tau) \operatorname{coth}\left(\frac{\pi(\tau-z)}{\Delta}\right) \mathrm{d} \tau,
$$


so that

$$
I_{t h}^{+}(t)+I_{t h}^{-}(t)=u^{+}(t)+u^{-}(t)
$$

This Riemann-Hilbert problem is analogous to the one stated in equation (2.11) and therefore has the solution

$$
I_{t h}(z)=\frac{X(z)}{2 \mathrm{i} \Delta} \int_{-1}^{1} \frac{u^{+}(t)+u^{-}(t)}{X^{+}(t)} \operatorname{coth}\left(\frac{\pi(\tau-z)}{\Delta}\right) \mathrm{d} \tau .
$$

Taking the difference either side of $L$ and equating the expressions in (2.24) and (2.25) yields

$$
y_{t h}^{\prime}(t)=\frac{X^{+}(t)}{2 \Delta} \int_{-1}^{1} \frac{u^{+}(\tau)+u^{-}(\tau)}{X^{+}(\tau)} \operatorname{coth}\left(\frac{\pi(\tau-t)}{\Delta}\right) \mathrm{d} \tau,
$$

subject to the solvability condition

$$
\int_{-1}^{1} \frac{u^{+}(\tau)+u^{-}(\tau)}{X^{+}(\tau)} \mathrm{d} \tau=0
$$

This condition is necessary because it ensures that (2.24) matches (2.25) in the farfield. Moreover, it can be seen by integrating (2.26) that this condition guarantees that $y_{t h}(-1)=y_{t h}(+1)$. Physically, this condition can be viewed as a consequence of conservation of momentum: if (2.27) did not hold, then a non-lifting cascade would generate a non-zero deflection angle. In section 2.5.3 we prove that this is not possible.

\subsubsection{Camber and Angle of Attack Terms}

Similarly to the previous section, we define the auxiliary function

$$
I_{c, \alpha}(z)=-\frac{X(z)}{\Delta} \int_{-1}^{1} \frac{y_{c}^{\prime}(\tau)-\alpha}{X^{+}(\tau)}\left(\operatorname{coth}\left(\frac{\pi(\tau-z)}{\Delta}\right)-1\right) \mathrm{d} \tau,
$$

so that

$$
I_{c, \alpha}^{+}(t)-I_{c, \alpha}^{-}(t)=u^{+}(t)-u^{-}(t)
$$


This Riemann-Hilbert problem is the same as (2.7) and therefore has the solution, with correct far-field behaviour,

$$
I_{c, \alpha}(z)=\frac{1}{2 \mathrm{i} \Delta} \int_{-1}^{1}\left(u^{+}(\tau)-u^{-}(\tau)\right)\left(\operatorname{coth}\left(\frac{\pi(\tau-z)}{\Delta}\right)-1\right) \mathrm{d} \tau .
$$

Taking the sum either side of $L$ and equating the expressions in (2.28) and (2.29) yields

$$
y_{c}^{\prime}(t)-\alpha=\frac{1}{2 \Delta} f_{-1}^{1}\left(u^{+}(\tau)-u^{-}(\tau)\right)\left(\operatorname{coth}\left(\frac{\pi(\tau-t)}{\Delta}\right)-1\right) \mathrm{d} \tau .
$$

Both equations (2.26) and (2.30) may be substituted back into (2.22) and (2.23) respectively. By applying the Poincaré-Bertrand transformation formula (Muskhelishvili, 1946, $\S 23)$ it may be shown that the stated thickness, camber and angle of attack do, in fact, result in the correct velocity distributions. In each case, asymptotic analysis indicates that the singularities at the endpoints of the integrals are either square-root singularities or log singularities, when the aerofoils have thick trailing edges. Consequently, the integrals in (2.26) and (2.30) are integrable and the desired aerofoil shape can be found.

\subsection{Results for Analytic Solution}

In this section we give details of some of the aerodynamically relevant results using the analysis of section 2.3 .

\subsubsection{Flow Field}

We may use (2.21) to plot the velocity fields and streamlines in figure 2.2. The streamlines show good agreement with the aerofoil surfaces and the flow leaves the aerofoils' trailing edges smoothly, indicating that the Kutta condition is satisfied. The flow perturbation is greatest near the leading and trailing edges, where singularities and stagnation points develop, and in the inter-blade region. Away from the cascade, the flow becomes uniform exponentially fast, in contrast to the single-aerofoil case, where the flow becomes uniform only algebraically fast. 


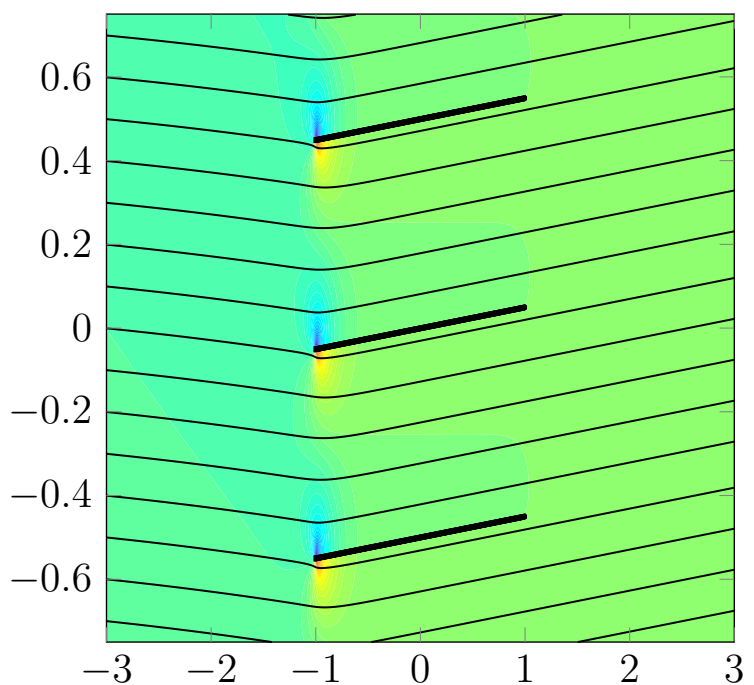

(a) Flat plates, $\alpha=-0.1, \quad \chi=0.05$, $\Delta=0.5$.

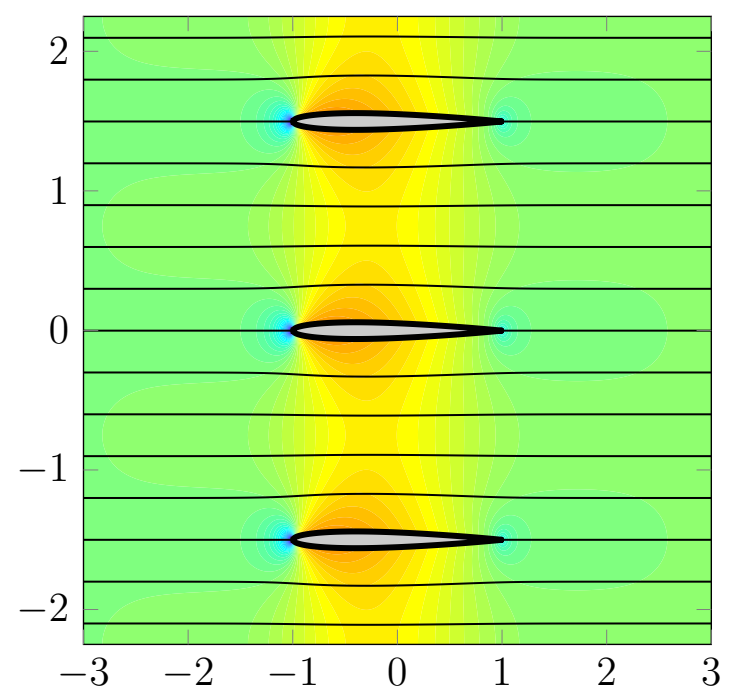

(c) NACA 0006, $\alpha=0, \chi=0, \Delta=1.5$.

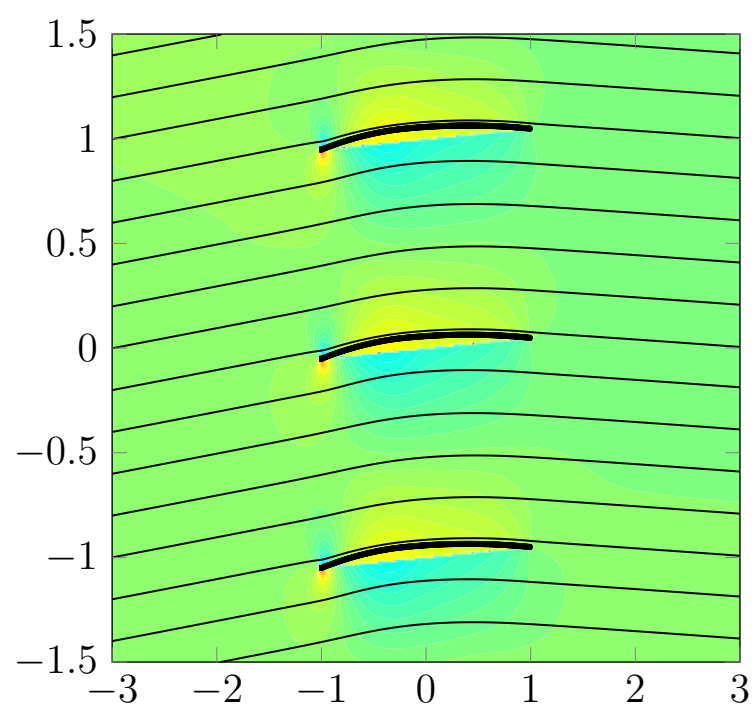

(b) NACA $3400, \alpha=0.05, \chi=0.05, \Delta=1$.

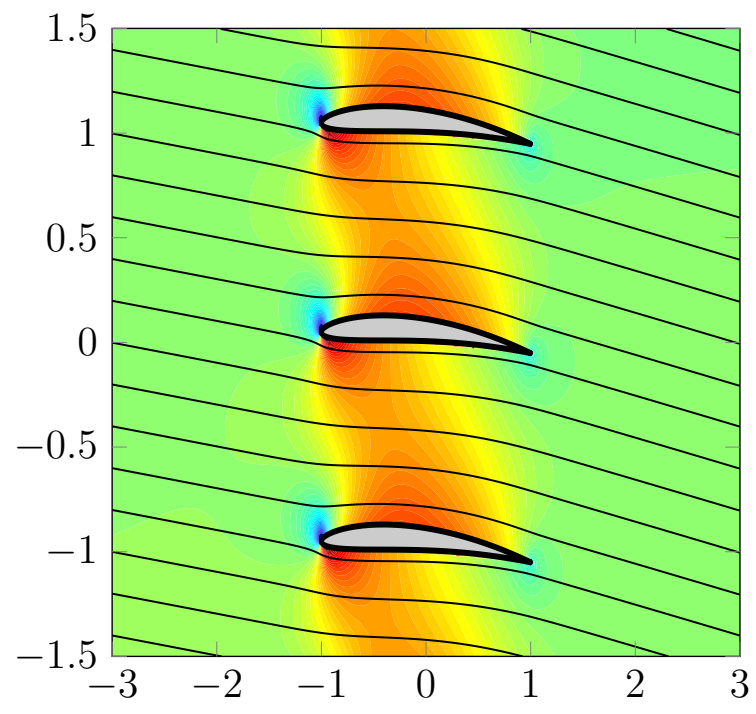

(d) NACA 3506, $\alpha=-0.05, \chi=-0.05$ $\Delta=1$.

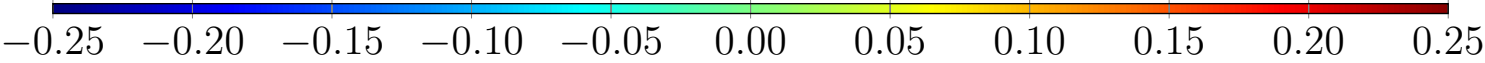

$$
\begin{aligned}
& \text { Horizontal velocity, } u(x, y)
\end{aligned}
$$

Fig. 2.2 Horizontal velocity and streamline plots for cascades of flat plates and three sets of NACA aerofoils. The streamlines are indicated in black and the background colour shows the horizontal velocity perturbation. 


\subsubsection{Tangential Surface Velocity}

We may sum the tangential velocity distributions found in section 2.3 to find the total distribution either side of each aerofoil:

$$
\begin{aligned}
u^{ \pm}(t) & = \pm \alpha \mathrm{e}^{-\frac{\pi}{\Delta}} \sqrt{\frac{\sinh \left(\frac{\pi(1-t)}{\Delta}\right)}{\sinh \left(\frac{\pi(1+t)}{\Delta}\right)}}-\frac{1}{\Delta} \int_{-1}^{1} y_{t h}^{\prime}(\tau) \operatorname{coth}\left(\frac{\pi(\tau-t)}{\Delta}\right) \mathrm{d} \tau \\
& \mp \frac{1}{\Delta} \sqrt{\frac{\sinh \left(\frac{\pi(1-t)}{\Delta}\right)}{\sinh \left(\frac{\pi(1+t)}{\Delta}\right)}} f_{-1}^{1} y_{c}^{\prime}(\tau) \sqrt{\frac{\sinh \left(\frac{\pi(1+\tau)}{\Delta}\right)}{\sinh \left(\frac{\pi(1-\tau)}{\Delta}\right)}}\left(\operatorname{coth}\left(\frac{\pi(\tau-t)}{\Delta}\right)-1\right) \mathrm{d} \tau .
\end{aligned}
$$

In figure 2.3 we plot this tangential surface velocity as a function of distance along the chord for a variety of aerofoil spacings and geometries.

The analytical solution is compared with a numerical solution (Bhimarasetty and Govardhan, 2010) which is obtained by using a conformal map to transform the cascade of aerofoils to a single object and the potential flow over this body is solved using a higher-order vortex panel method (Kuethe and Chow, 1998). This numerical method has shown good agreement with other numerical (Gostelow, 1984) and experimental results (Herrig et al., 1957). In figure 2.3, the agreement between the analytic and numerical solutions is almost exact in the cases of angle of attack and camber and the only significant deviations occur at the leading edge of the thick aerofoils, where our assumption of small gradient breaks down. In order to resolve this, a further expansion in the $\mathcal{O}\left(\epsilon^{2}\right)$ region and asymptotic matching is required, but this is beyond the scope of the chapter. The divergence from the numerical solution at the thick leading edge is also observed in the single aerofoil case at $\mathcal{O}(\epsilon)$. As verified by the asymptotic behaviour of the analytic solution close to the edges in section 2.3.3, the Kutta condition can be seen to be satisfied since the tangential velocities match at the trailing edge and the leading edge has an integrable singularity. In the cases of angle of attack and camber, the tangential surface velocity is equal magnitude but opposite sign either side of the aerofoils, whereas in the thickness case the upper and lower velocities match.

\subsubsection{Deflection Angle and Lift}

A primary purpose of aerofoil cascades in turbomachinery applications is to deflect the flow through a desired angle. Our analysis allows us to derive an analytic expression for the change in flow angle, denoted by $\delta \alpha$, for a given cascade geometry and inlet angle. 


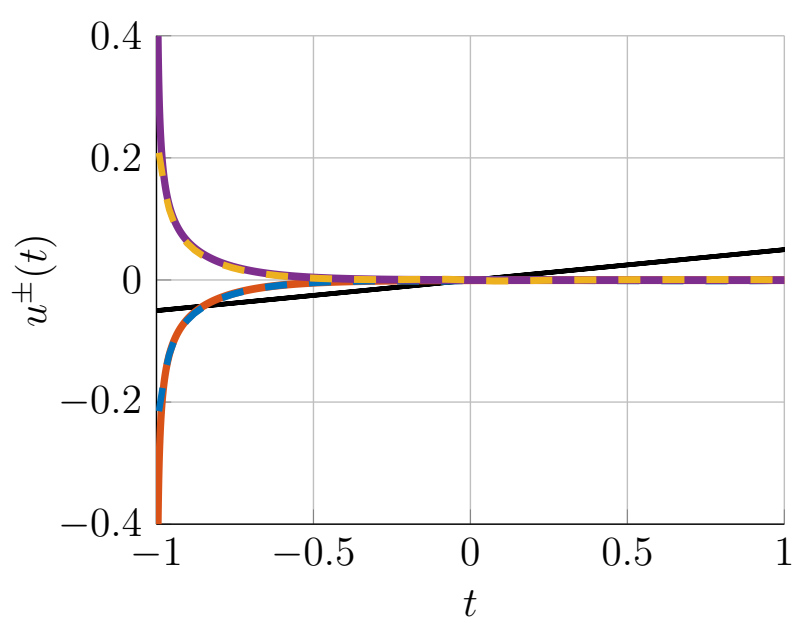

(a) Flat plates, $\alpha=-0.1, \quad \chi=0.05$, $\Delta=0.5$.

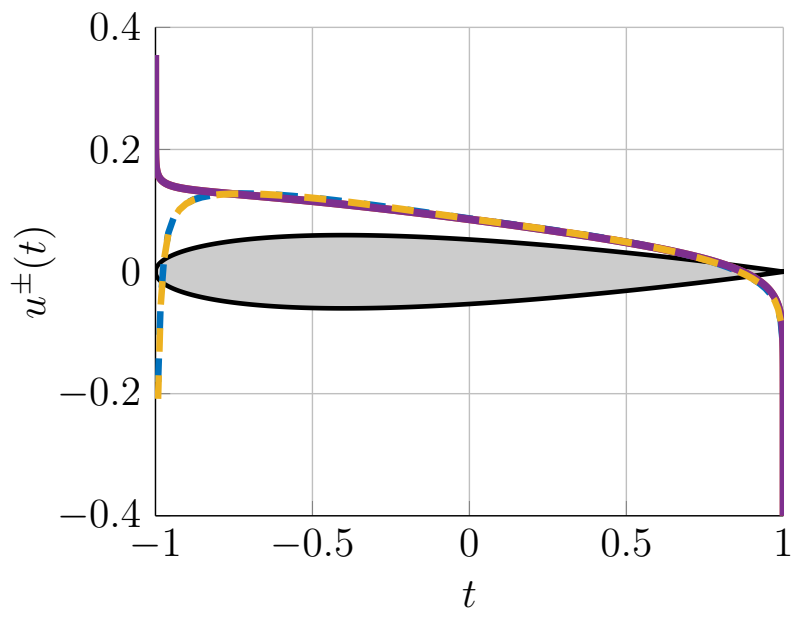

(c) NACA 0006, $\alpha=0, \chi=0, \Delta=1.5$.

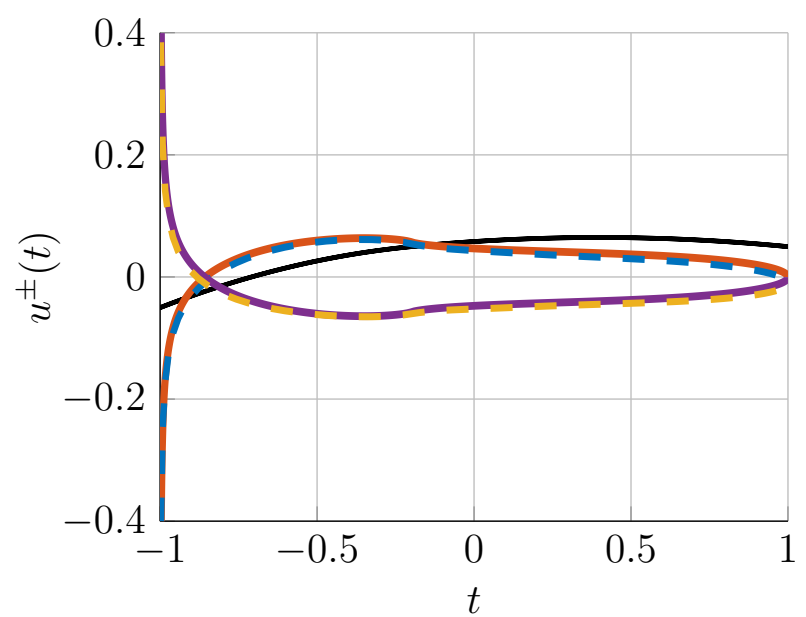

(b) NACA $3400, \alpha=0.05, \chi=0.05, \Delta=1$.

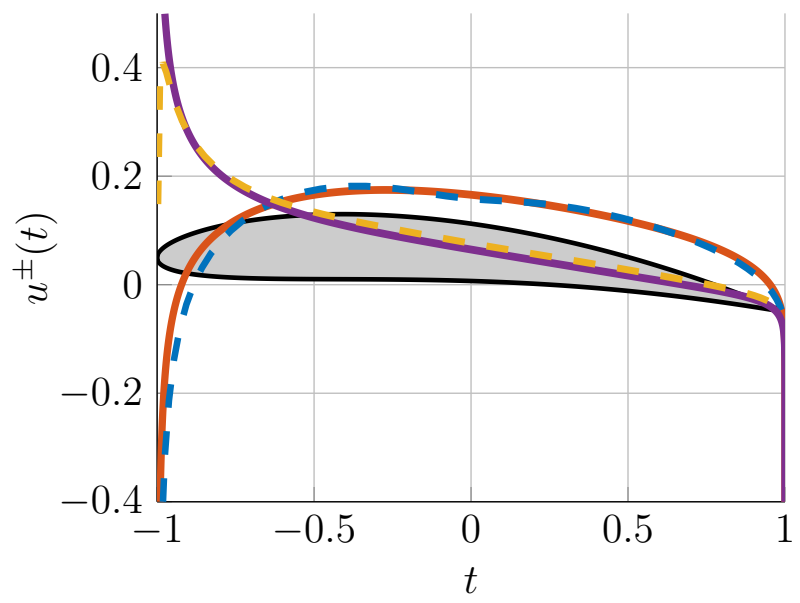

(d) NACA 3506, $\alpha=-0.05, \chi=-0.05$, $\Delta=1$

Fig. 2.3 Tangential surface velocity plots for flat plates and three sets of NACA aerofoils. Our analytical solution is given by the solid lines, corresponding to the upper and lower surfaces. The numerical solution is given by the dashed lines corresponding to the upper and lower surfaces.

Since the change in angle of attack is small, we have

$$
\delta \alpha=\operatorname{Im}[\bar{\Phi}(+\infty)-\bar{\Phi}(-\infty)]=-\Phi(+\infty)
$$


We can use our expression for the flow perturbation (2.21) to write

$$
\delta \alpha=2 \mathrm{e}^{-\frac{\pi}{\Delta}}\left\{-\alpha \sinh \left(\frac{\pi}{\Delta}\right)+\frac{1}{\Delta} \int_{-1}^{1} y_{c}^{\prime}(\tau) \sqrt{\frac{\sinh \left(\frac{\pi(1+\tau)}{\Delta}\right)}{\sinh \left(\frac{\pi(1-\tau)}{\Delta}\right)}} \mathrm{d} \tau\right\}
$$

where we have ignored $\mathcal{O}\left(\epsilon^{2}\right)$ terms for clarity. This equation may be used to choose the spacing, angle of attack or camber in order to achieve a desired deflection angle.

We may evaluate the deflection angle for the different limits of $\Delta$ : as the aerofoil spacing increases, $\Delta \rightarrow \infty$, we have

$$
\delta \alpha \sim \frac{2}{\Delta}\left(1-\frac{\pi}{\Delta}\right)\left(-\pi \alpha+f_{-1}^{1} \sqrt{\frac{1+\tau}{1-\tau}} y_{c}^{\prime}(\tau) \mathrm{d} \tau\right)+\mathcal{O}\left(\Delta^{-3}\right)
$$

and the deflection angle decays algebraically as the aerofoil spacing increases. This is consistent with the single blade case (Thwaites, 1960) where there is no flow deflection far downstream of the blade. Conversely, if we consider the limit of very close blades, $\Delta \rightarrow 0^{+}$, then

$$
\delta \alpha \sim-\alpha+y_{c}^{\prime}(1)
$$

The asymptotic behaviour of the integral in (2.32) is evaluated using Laplace's method. This equation states that the deflection angle will be equal to the difference between of the angle of the trailing edge of the mean line of the aerofoil and the angle of attack. In other words, the outlet angle is the angle of the camber at the trailing edge. The asymptotic behaviours of the deflection angle $(2.33,2.34)$ is plotted for a range of blade spacings in figure 2.4 .

The deflection angle is closely connected to the aerofoil lift; the Kutta-Joukowski theorem expresses the lift per unit span acting on the aerofoil as

$$
\mathscr{L}=-\rho_{\infty} U_{\infty} \Gamma
$$

The circulation is

$$
\Gamma=\oint_{C} \boldsymbol{u} \cdot \mathrm{d} \boldsymbol{t}=-\int_{-1}^{1}\left(\Phi^{+}(t)-\Phi^{-}(t)\right) \mathrm{d} t=-\int_{-1}^{1}\left(u^{+}(t)-u^{-}(t)\right) \mathrm{d} t
$$

where $u^{ \pm}$is given by (2.31). The above integral is complicated to calculate, but may be evaluated analytically using residue calculus. However, this intricacy can be avoided by 


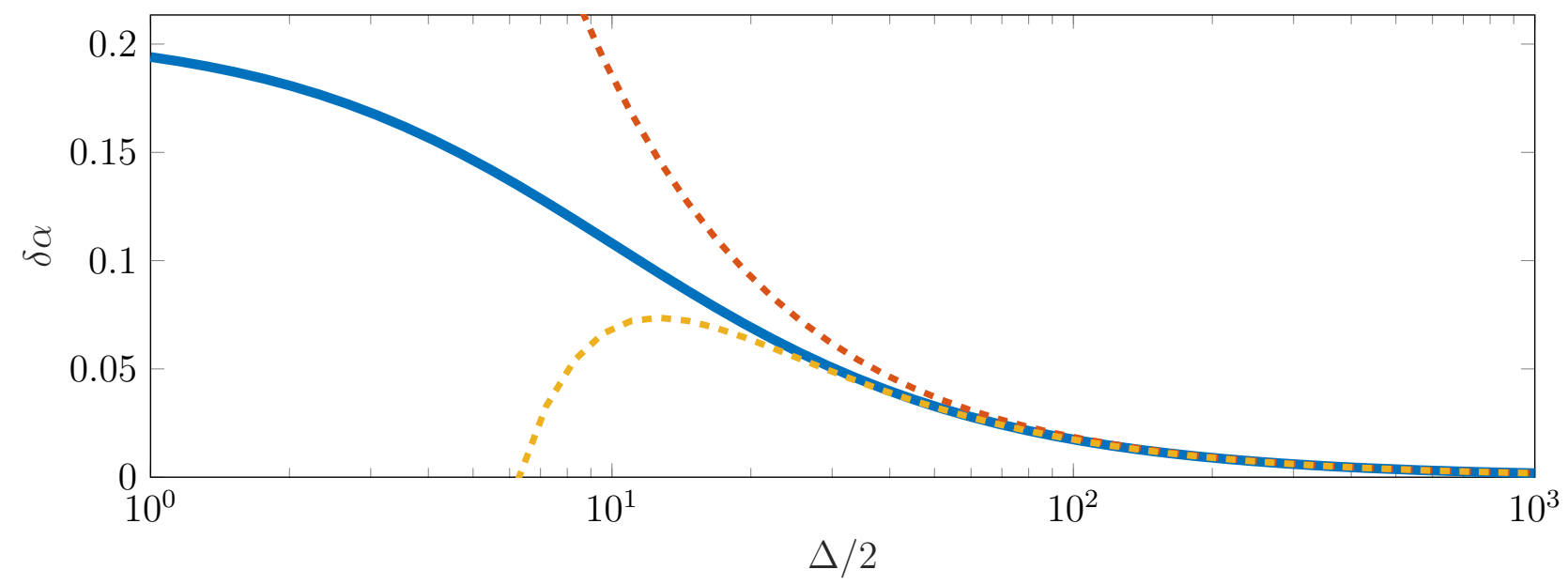

(a) The large $\Delta$ limit, as described in (2.33). The exact solution (2.32) is represented by the blue curve, the first-order approximation is the orange curve, and the second order approximation is the yellow curve.

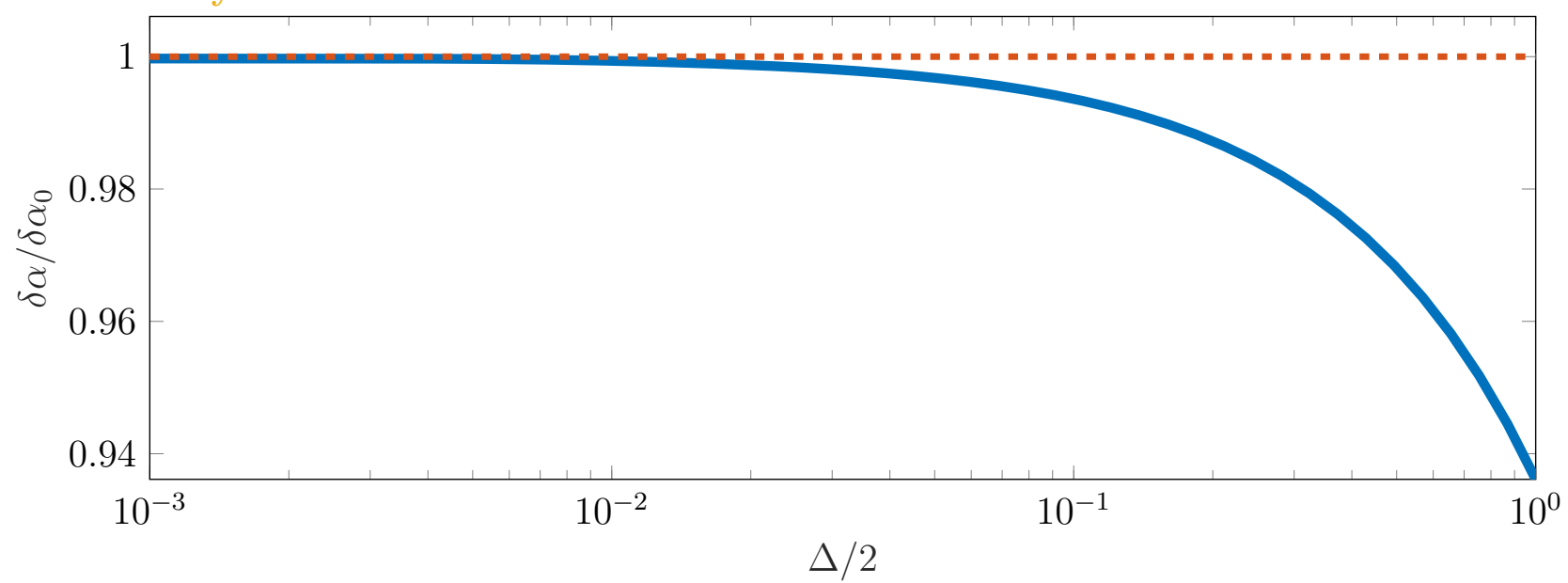

(b) The small $\Delta$ limit, as described in (2.34). The deflection angle is normalised by the deflection angle with zero spacing. The exact solution (2.32) is represented by the blue curve, the first-order approximation is the orange curve according to (2.34).

Fig. 2.4 Asymptotic behaviour for the deflection angle $\delta \alpha$ on an individual blade in a cascade of NACA 3506 aerofoils at angle of attack $5^{\circ}$ for large and small gap-to-chord ratio, $\Delta / 2$. 


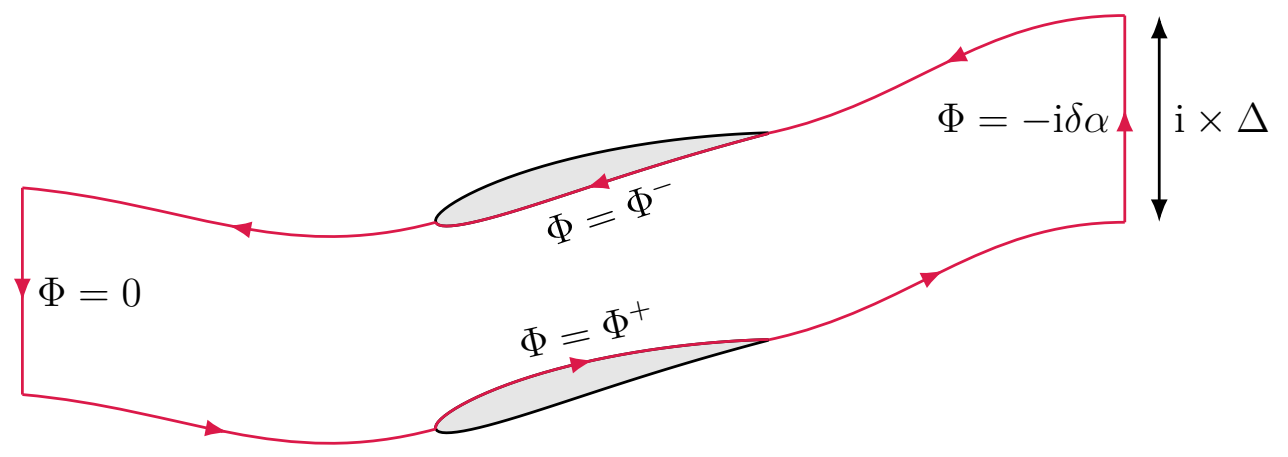

Fig. 2.5 The contour of integration illustrating the relationship between circulation and deflection angle.

expressing the lift in terms of the deflection angle. To this end, we integrate $\Phi$ along an appropriate contour, as illustrated in figure 2.5.

This contour consists of the streamline corresponding to the upper surface of an aerofoil, the streamline corresponding to the lower surface of the aerofoil directly above, and two vertical contours at upstream and downstream infinity of length $\Delta$. In this region, $\Phi$ is holomorphic and the resulting integral is zero by Cauchy's theorem. Due to the periodicity of $\Phi$, the contributions from the streamlines cancel out except on the aerofoil surface, which corresponds to the negative of the circulation, $-\Gamma$. The upstream vertical contribution vanishes and we are left with the downstream vertical contribution: $-\mathrm{i} \delta \alpha \times \mathrm{i} \Delta$. Rearranging the integral yields the expression

$$
\Gamma=\Delta \times \delta \alpha
$$

where the analytic expression of $\delta \alpha$ is given in equation (2.32). Substituting (2.36) into (2.35) yields an analytic expression for the lift. This relation may be understood as a result of conservation of momentum; the net change in momentum (the change in flow angle times the aerofoil spacing) must be equal to the force exerted on the fluid (the lift). The single aerofoil result for the lift given by Thwaites (1960) may be recovered from (2.33) and (2.36). In this limit the deflection angle decays, but the lift approaches a constant value. The asymptotic behaviour of the lift $(2.33,2.34$ and 2.36$)$ is plotted for a range of blade spacings in figure 2.6. 


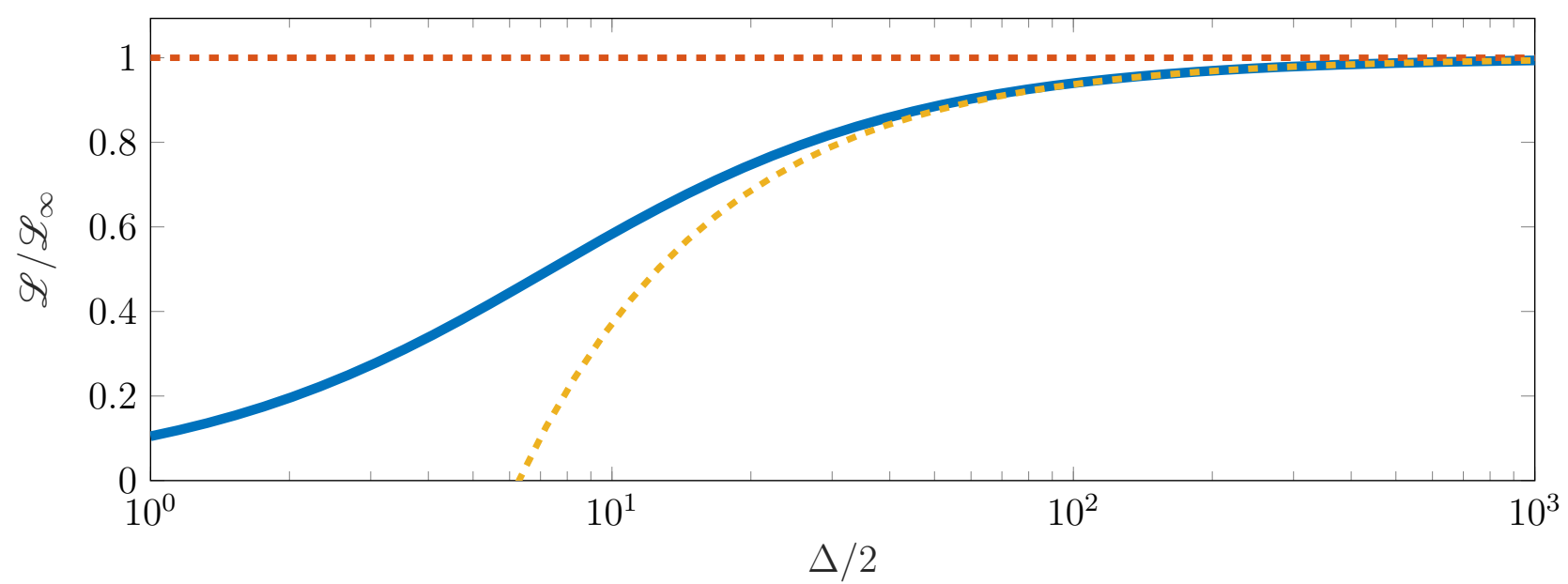

(a) The large $\Delta$ limit. The lift is normalised by the far-field lift, $\mathscr{L}_{\infty}$. The exact solution $(2.35)$ is represented by the blue curve, the first-order approximation is the orange curve, and the second order approximation is the yellow curve according to $(2.33,2.36)$.

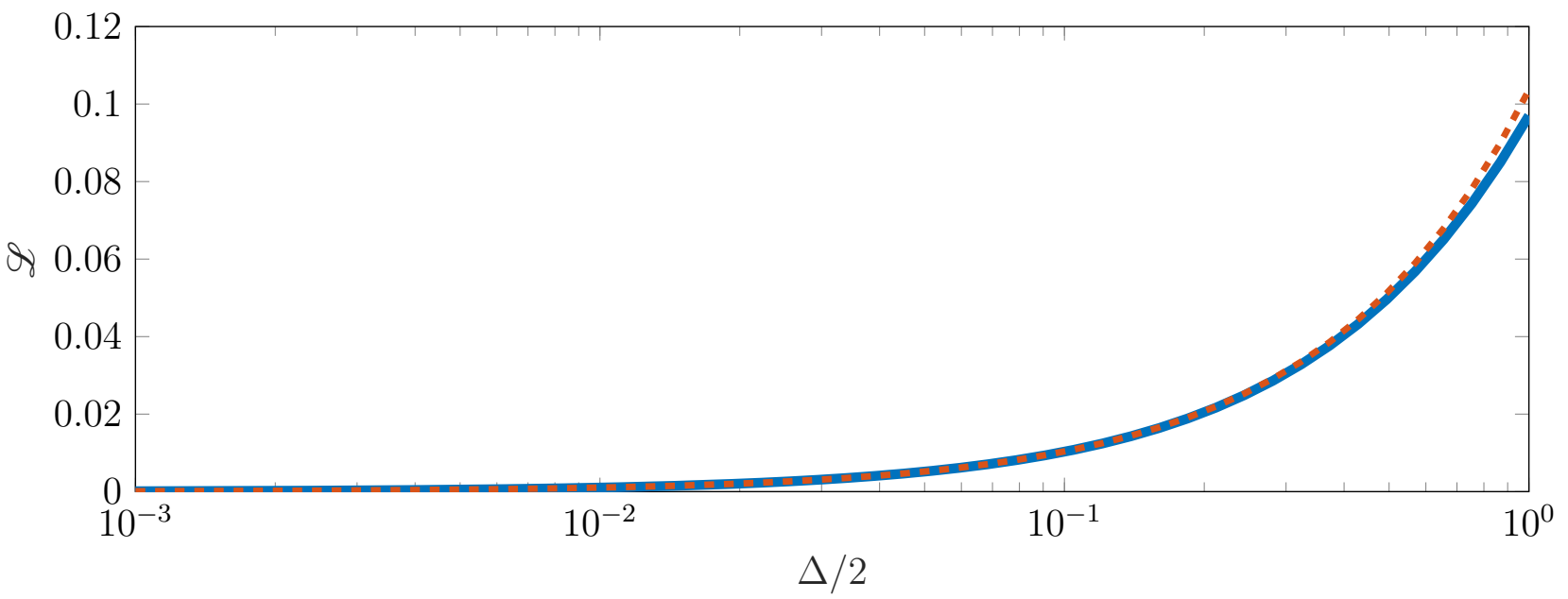

(b) The small $\Delta$ limit. The exact solution (2.35) is represented by the blue curve, the first-order approximation is the orange curve according to $(2.34,2.36)$.

Fig. 2.6 Asymptotic behaviour for the lift $\mathscr{L}$ on an individual blade in a cascade of NACA 3506 aerofoils at angle of attack $5^{\circ}$ for large and small gap-to-chord ratio $\Delta / 2$. 


\subsection{Numerical Solutions for Large Stagger}

When the stagger is large, the analytic approach presented in the previous section becomes invalid. The Schwarz reflection method of section 2.3 - which allowed the thickness and vorticity distribution to be decoupled - now breaks down, and the corresponding Riemann-Hilbert problem is not amenable to standard methods. Accordingly, we are precluded from deriving an analytic solution of the same form as (2.21) and a numerical solution is required. The small-stagger solution (2.21) provides insight into the form of the numerical solution, as well as being an essential benchmarking tool.

We rotate the coordinate system through the stagger angle $\chi$ so that the blades are each aligned with the horizontal axis and are separated by $d+\mathrm{i} s$ where

$$
d+\mathrm{i} s=\mathrm{i} \Delta \mathrm{e}^{\mathrm{i} \chi} .
$$

Informed by the solution to the small stagger problem (2.21), we consider an ansatz for the complex velocity of the form

$$
\Phi(z)=\frac{1}{2(d+\mathrm{i} s)} \int_{-1}^{1} \gamma(\tau)\left(\operatorname{coth}\left(\frac{\pi \mathrm{i}(\tau-z)}{d+\mathrm{i} s}\right)-1\right) \mathrm{d} \tau
$$

where $\gamma(\tau)$ represents a distribution of sources, sinks and vorticity arranged on the chord line. The problem is now to find $\gamma$, which we decompose into real and imaginary parts as

$$
\gamma(\tau)=\gamma_{\mathrm{r}}(\tau)+\mathrm{i} \gamma_{\mathrm{i}}(\tau)
$$

In a similar way to the unstaggered case, we may consider the limits of $\Phi(z)$ as $z \rightarrow t^{ \pm}$, (where $t \in[-1,1]$ ) via the Modified Plemelj formula (2.52). Taking the difference between the limits on the upper and lower sides, and equating imaginary parts yields

$$
\gamma_{\mathrm{i}}(t)=-2 y_{t h}^{\prime}(t)
$$

Conversely, summing $\Phi(z)$ either side of the chord lines the singular integral equation

$$
\Sigma u^{ \pm}(t)-2 \mathrm{i}\left(y_{c}^{\prime}(t)-\alpha\right)=\frac{1}{2(d+\mathrm{i} s)} \int_{-1}^{1} \gamma(\tau)\left(\operatorname{coth}\left(\frac{\pi \mathrm{i}(\tau-t)}{d+\mathrm{i} s}\right)-1\right) \mathrm{d} \tau
$$

We now consider the imaginary part of the (2.38). It is precisely at this point that we diverge from the small-stagger solution: in contrast to the previous section, the imaginary part of the right-hand side of (2.38) does not result in a simple expression amenable to 
typical Riemann-Hilbert methods. Instead, we have the singular integral equation

$$
4 \pi\left(y_{c}^{\prime}(t)-\alpha\right)=f_{-1}^{1}\left(-2 y_{t h}^{\prime}(\tau) K_{\mathrm{r}}(\tau, t)+\gamma_{\mathrm{r}}(\tau) K_{\mathrm{i}}(\tau, t)\right) \mathrm{d} \tau,
$$

where

$$
\begin{aligned}
& K_{\mathrm{r}}(\tau, t)=\operatorname{Re}\left[\frac{-\pi}{(d+\mathrm{i} s)}\left(\operatorname{coth}\left(\frac{\pi \mathrm{i}(\tau-t)}{d+\mathrm{i} s}\right)-1\right)\right], \\
& K_{\mathrm{i}}(\tau, t)=\operatorname{Im}\left[\frac{-\pi}{(d+\mathrm{i} s)}\left(\operatorname{coth}\left(\frac{\pi \mathrm{i}(\tau-t)}{d+\mathrm{i} s}\right)-1\right)\right] .
\end{aligned}
$$

We note that $K_{i}$ is a singular operator; as $\tau \rightarrow t$ we have

$$
K_{\mathrm{i}}(\tau, t) \sim \frac{1}{\tau-t}
$$

Consequently, we separate the singular and regular parts of $K_{\mathrm{i}}$ and write

$$
K_{\mathrm{i}}(\tau, t)=\frac{1}{\tau-t}+\tilde{K}_{\mathrm{i}}(\tau, t)
$$

where

$$
\tilde{K}_{\mathrm{i}}(\tau, t)=\left\{\begin{array}{cc}
\operatorname{Im}\left[\frac{\pi}{d+\mathrm{i} s}\right], & \text { for } t=\tau, \\
\operatorname{Im}\left[\frac{-\pi}{d+\mathrm{i} s}\left(\operatorname{coth}\left(\frac{\pi \mathrm{i}(\tau-t)}{d+\mathrm{i} s}\right)-1\right)-\frac{1}{\tau-t}\right], & \text { for } t \neq \tau .
\end{array}\right.
$$

Consequently, we may express (2.39) as the singular integral equation

$$
f_{-1}^{1} \frac{\gamma_{\mathrm{r}}(\tau)}{\tau-t} \mathrm{~d} \tau+\int_{-1}^{1} \gamma_{\mathrm{r}}(\tau) \tilde{K}_{\mathrm{i}}(\tau, t) \mathrm{d} \tau=f(t)
$$

where the forcing term $f$ is given by

$$
f(t)=4 \pi\left(y_{c}^{\prime}(t)-\alpha\right)-2 f_{-1}^{1} 2 y_{t h}^{\prime}(\tau) K_{r}(\tau, t) \mathrm{d} \tau
$$

Note that the second integral in (2.41) is not considered in the principal value sense because $\tilde{K}_{\mathrm{i}}$ is regular for all $(t, \tau) \in[-1,1]^{2}$.

This form of singular integral is amenable to numerical methods by expanding $\gamma_{\mathrm{r}}$ into a suitable series of basis functions. This method was first proposed by Erdogan and Gupta 
(1972), who suggested an expansion of the unknown function $\gamma_{\mathrm{r}}$ as a series of weighted Chebyshev polynomials. This expansion accurately captures the endpoint behaviour of $\gamma_{\mathrm{r}}$, which necessarily possesses a square-root singularity and zero at the leading and trailing edge respectively. The method may be extended to permit functions whose endpoint singularities and zeros are not square roots by considering instead an expansion in weighted Jacobi polynomials (Krenk, 1975). However, the weighted Chebyshev expansion is entirely appropriate for the present problem where we are predominantly interested in NACA 4-digit profiles, and the approach is widely used in isolated aerofoil analyses (Glauert, 1926; Rienstra, 1992).

Accordingly, we expand $\gamma_{\mathrm{r}}$ as a series of weighted Chebyshev polynomials in the form

$$
\gamma_{\mathrm{r}}(t)=\gamma_{\mathrm{r}, 0} \sqrt{\frac{1-t}{1+t}}+\sqrt{1-t^{2}} \sum_{n=1}^{\infty} \gamma_{\mathrm{r}, n} U_{n-1}(t),
$$

where $U_{n}$ represent the Chebyshev polynomials of the second kind and $\gamma_{\mathrm{r}, n}$ represent unknown coefficients. The problem is now to find these coefficients. The Chebyshev polynomials $U_{n}$ may be expressed as

$$
U_{n}(t)=\frac{\sin [(n+1) \theta]}{\sin (\theta)}, \quad t=\cos (\theta) .
$$

Moreover, they satisfy the orthogonality relation

$$
\int_{-1}^{1} \sqrt{1-\tau^{2}} U_{n}(\tau) U_{m}(\tau) \mathrm{d} \tau=\delta_{n, m} \frac{\pi}{2}
$$

In order to apply the expansion (2.42) to the singular integral equation (2.41), we use the finite Hilbert transforms

$$
\begin{gathered}
f_{-1}^{1} \sqrt{1-\tau^{2}} U_{n-1}(\tau) \cdot \frac{\mathrm{d} \tau}{\tau-t}=-\pi T_{n}(t), \\
f_{-1}^{1} \sqrt{\frac{1-\tau}{1+\tau}} \cdot \frac{\mathrm{d} \tau}{\tau-t}=-\pi,
\end{gathered}
$$

where $T_{n}$ are the Chebyshev polynomials of the first kind.

We now substitute (2.42) into (2.41) and apply the identities (2.43) and (2.44) to obtain

$$
-\pi \gamma_{\mathrm{r}, 0}-\pi \sum_{n=1}^{\infty} \gamma_{\mathrm{r}, n} T_{n}(t)+\int_{-1}^{1} \gamma_{\mathrm{r}}(\tau) \tilde{K}_{\mathrm{i}}(\tau, t) \mathrm{d} \tau=f(t)
$$


The remaining integral in the above expression is amenable to Chebyshev-Gauss quadrature. We note the quadrature rules (Olver et al., 2010, §3.5)

$$
\int_{-1}^{1} g(\tau) \sqrt{\frac{1-\tau}{1+\tau}} \mathrm{d} \tau \sim \sum_{m=1}^{M} w_{m}^{(1)} g\left(\tau_{m}^{(1)}\right), \quad \text { as } M \rightarrow \infty
$$

for

$$
\begin{aligned}
\tau_{k}^{(1)} & =\cos \left(\frac{2 m}{2 M+1} \pi\right), \\
w_{k}^{(1)} & =\frac{4 \pi}{2 M+1} \sin ^{2}\left(\frac{m}{2 M+1} \pi\right),
\end{aligned}
$$

and

$$
\int_{-1}^{1} g(\tau) \sqrt{1-\tau^{2}} \mathrm{~d} \tau \sim \sum_{m=1}^{M} w_{m}^{(2)} g\left(\tau_{m}^{(2)}\right), \quad \text { as } M \rightarrow \infty
$$

for

$$
\begin{aligned}
\tau_{k}^{(2)} & =\cos \left(\frac{m}{M+1} \pi\right), \\
w_{k}^{(2)} & =\frac{\pi}{M+1} \sin ^{2}\left(\frac{m}{M+1} \pi\right) .
\end{aligned}
$$

Consequently, we may express (2.45) as

$$
\begin{gathered}
-\pi \gamma_{\mathrm{r}, 0}-\pi \sum_{n=1}^{\infty} \gamma_{\mathrm{r}, n} T_{n}(t)+\sum_{m=1}^{\infty}\left[\gamma_{r, 0} w_{m}^{(1)} \tilde{K}_{\mathrm{i}}\left(t_{m}^{(1)}, t\right)\right. \\
\left.+w_{m}^{(2)} \tilde{K}_{\mathrm{i}}\left(t_{m}^{(2)}, t\right) \sum_{n=1}^{\infty} \gamma_{\mathrm{r}, n} U_{n-1}\left(t_{m}^{(2)}\right)\right] \\
=f(t) .
\end{gathered}
$$

We now truncate the infinite sums in (2.46) at $N$ and collocate at the zeros of $U_{N+1}$ to obtain an $(N+1) \times(N+1)$ linear system for the $N+1$ unknown coefficients $v_{n}$. The remaining matrix equation may easily be inverted in MATLAB to obtain the coefficients $\gamma_{\mathrm{r}, n}$.

\subsubsection{Validation of Numerical Scheme}

We now validate our numerical scheme for a range of examples. The analytic solutions found in section 2.3.1.2 are used as a benchmark for the numerical method. We consider 
two types of error: the maximum relative error

$$
\epsilon_{M}(N)=\max _{i} \frac{\left|\gamma_{\mathrm{r}, \mathrm{N}}\left(t_{i}\right)-\gamma_{\mathrm{r}}\left(t_{i}\right)\right|}{\left|\gamma_{\mathrm{r}}\left(t_{i}\right)\right|}
$$

and the $L^{2}$ relative error

$$
\epsilon_{L^{2}}(N)=\sqrt{\frac{\int_{-1}^{1}\left|\gamma_{\mathrm{r}, \mathrm{N}}(t)-\gamma_{\mathrm{r}}(t)\right|^{2} \mathrm{~d} t}{\int_{-1}^{1}\left|\gamma_{\mathrm{r}}(t)\right|^{2} \mathrm{~d} t}},
$$

where $\gamma_{\mathrm{r}, N}$ represents the approximate solution with $N$ Chebyshev polynomials, and $\gamma_{\mathrm{r}}$ represents the exact solution.

We observe that both errors converge exponentially with $N$ for all tested cases. For example, figure 2.7 shows the convergence for a range of parameters. The numerical results show that the convergence is generally faster for larger blade spacings. This suggests that the Chebyshev polynomial expansion could be improved by selecting an alternative set of orthogonal basis functions. However, this extension represents future work and the current scheme is sufficient for the present research. Moreover, we are operating in an asymptotic regime where $\mathcal{O}\left(\epsilon^{2}\right)$ errors are ignored, so usually a low number of basis polynomials are required.

\subsubsection{Upstream and Downstream Flow Decompositions}

We use the solution for $\gamma$ (2.42) to express the background flow (2.37) in a form that is amenable to the Wiener-Hopf method to be applied in chapter 3. Essentially, we wish to express the mean flow perturbation (2.37) in a Fourier-type basis, as this will yield a simple form in the spectral plane under a Fourier transform.

It is straightforward to show that

$$
\operatorname{coth}\left(\frac{\pi \mathrm{i}(\tau-z)}{d+\mathrm{i} s}\right)-1=-\frac{2}{1-\exp \left(\frac{2 \pi \mathrm{i}(\tau-z)}{d+\mathrm{i} s}\right)}
$$




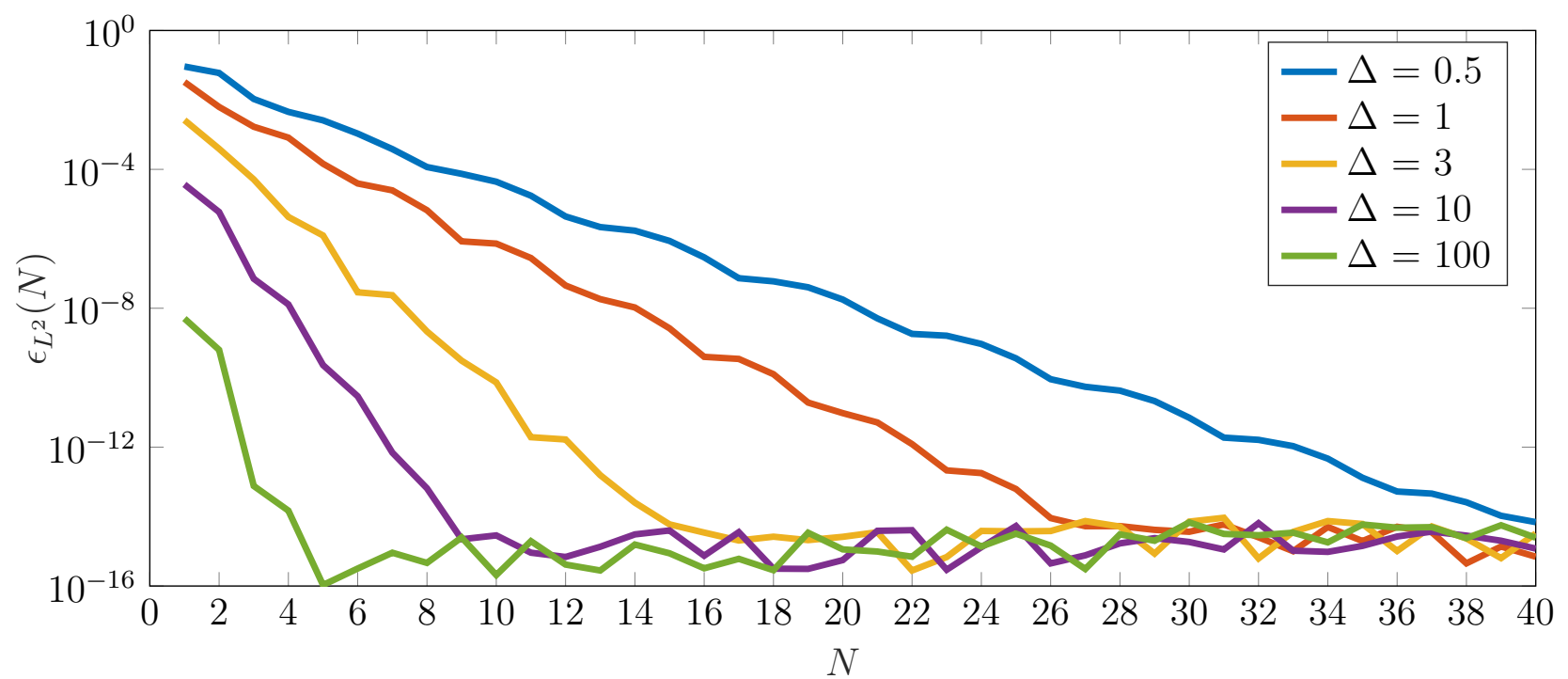

(a) The $L^{2}$ error for a cascade of flat plates at constant angle of attack.

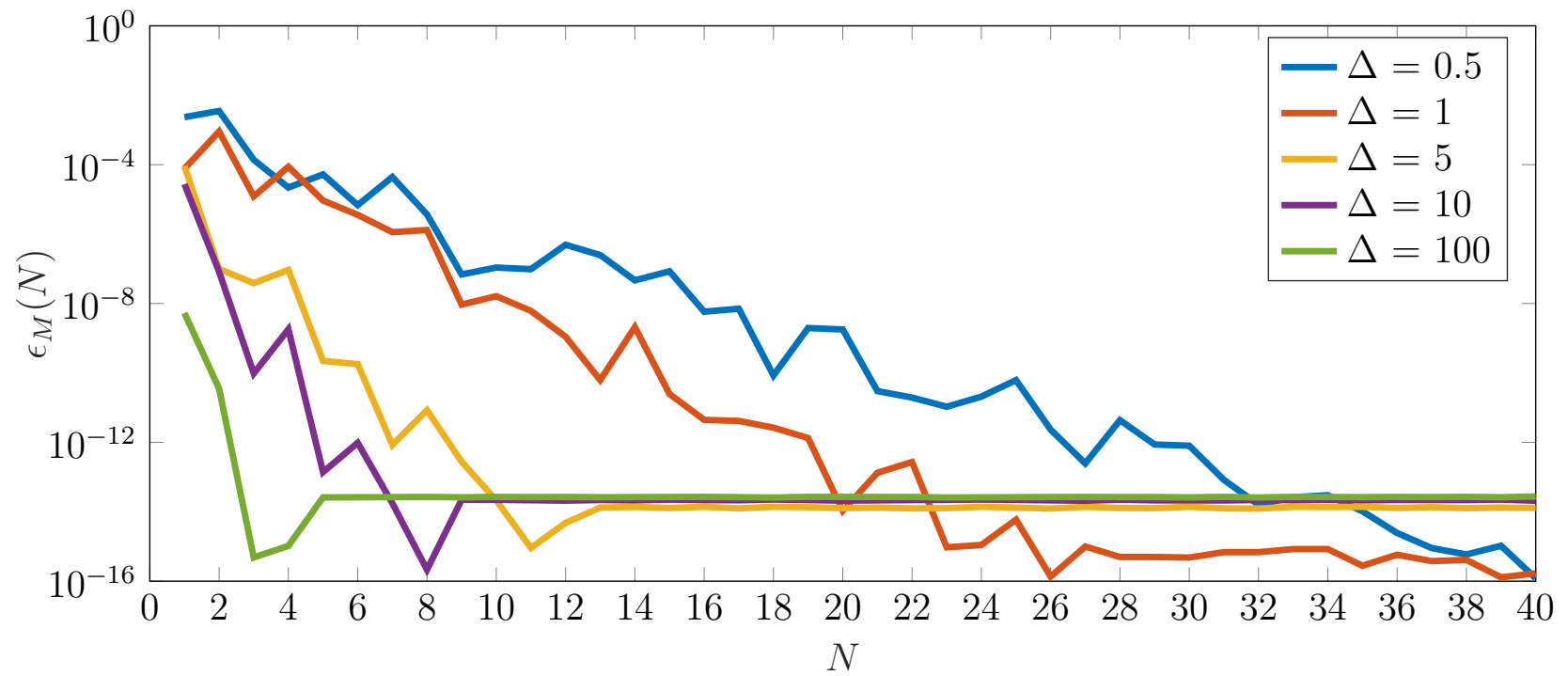

(b) The maximum relative error for a cascade of NACA 3506 aerofoils. The grid is a sequence of 100 linearly spaced points between -1 and 1 excluding the end points.

Fig. 2.7 The error convergence for the numerical method. 
The above series can be expanded as a geometric series depending on whether $z$ is upstream or downstream of the cascade:

$$
\operatorname{coth}\left(\frac{\pi \mathrm{i}(\tau-z)}{d+\mathrm{i} s}\right)-1=\left\{\begin{array}{cl}
2 \sum_{n=1}^{\infty} \exp \left(\frac{-2 \pi \mathrm{i} n(\tau-z)}{d+\mathrm{i} s}\right), & (x+1) s<y d, \\
-2 \sum_{n=0}^{\infty} \exp \left(\frac{2 \pi \mathrm{i} n(\tau-z)}{d+\mathrm{i} s}\right), & (x-1) s>y d .
\end{array}\right.
$$

Substituting this into the expression for the mean flow (2.37) yields the exponential representation

$$
w_{1}(z)=\left\{\begin{array}{cc}
\sum_{n=1}^{\infty} v_{n}^{+} \exp \left(\frac{2 \pi \mathrm{i} n z}{d+\mathrm{i} s}\right), & (x+1) s<y d, \\
-\sum_{n=0}^{\infty} v_{n}^{-} \exp \left(\frac{-2 \pi \mathrm{i} n z}{d+\mathrm{i} s}\right), & (x-1) s>y d
\end{array}\right.
$$

where the coefficients are given by

$$
v_{n}^{ \pm}=\frac{1}{d+\mathrm{i} s} \int_{-1}^{1} \gamma(\tau) \exp \left(\frac{\mp 2 \pi \mathrm{i} n \tau}{d+\mathrm{i} s}\right) \mathrm{d} \tau
$$

Analytical expressions for the above coefficients are available by using results for the finite Fourier transforms of weighted Chebyshev polynomials (Olver et al., 2010, Eq. 18.17.16), but it is also straightforward to integrate the above numerically using Gauss-Chebyshev quadrature. Note that $v_{0}$ is expressible in terms of the circulation $\Gamma$ as

$$
v_{0}^{-}=-\frac{\Gamma}{d+\mathrm{i} s}
$$

Integrating (2.47) yields analytic forms for the full velocity potential and streamfunction. We select the constant of integration so that the leading edge $z=-1$ corresponds to $w(-1)=0$. In the upstream region, $(x+1) s<y d$, we have

$$
\frac{w(z)}{U_{\infty}}=(1-\mathrm{i} \alpha)(z+1)+\frac{d+\mathrm{i} s}{2 \pi \mathrm{i}} \sum_{n=1}^{\infty} \frac{v_{n}^{+}}{n}\left[\exp \left(\frac{2 \pi \mathrm{i} n z}{d+\mathrm{i} s}\right)-\exp \left(\frac{-2 \pi \mathrm{i} n}{d+\mathrm{i} s}\right)\right],
$$

and in downstream region, $(x-1) s>y d$, we have

$$
\frac{w(z)}{U_{\infty}}=\left(1-\mathrm{i} \alpha-\frac{\Gamma}{d+\mathrm{i} s}\right)(z-1)
$$




$$
\begin{aligned}
& -\frac{1}{2 \pi \mathrm{i}} \int_{-1}^{1} \gamma(\tau)\left(\log \left(\frac{\sinh \left(\frac{\pi \mathrm{i}(\tau-1)}{d+\mathrm{i} s}\right)}{\sinh \left(\frac{\pi \mathrm{i}(\tau+1)}{d+\mathrm{i} s}\right)}\right)+\frac{2 \pi \mathrm{i}}{d+\mathrm{i} s}\right) \mathrm{d} \tau \\
& +\frac{d+\mathrm{i} s}{2 \pi \mathrm{i}} \sum_{n=1}^{\infty} \frac{v_{n}^{-}}{n}\left[\exp \left(\frac{-2 \pi \mathrm{i} n z}{d+\mathrm{i} s}\right)-\exp \left(\frac{-2 \pi \mathrm{i} n}{d+\mathrm{i} s}\right)\right] .
\end{aligned}
$$

There is no equivalent decomposition in the inter-blade region since the expressions in (2.47) are not available. In this region the velocities must be integrated with GaussChebyshev quadrature to find the velocity potential and streamfunction.

We plot the velocity potential and streamfunction for a range of geometries, stagger angles and spacings in figures 2.8 and 2.9. Note that the flow clearly satisfies the no-flux condition on the blades. Additionally, figure 2.9 shows that the flow exits the cascade smoothly, thus indicating that the Kutta condition is satisfied.

\subsection{Conclusions}

We have presented two methods to calculate the potential flow through an infinite cascade of aerofoils. We first presented an analytic solution using a Riemann-Hilbert method and next presented a numerical method using a weighted Chebyshev expansion. Elements of the solutions have been chosen to satisfy the appropriate edge conditions at the leading and trailing edges, as well as the correct upstream behaviour. Analytic expressions for the surface velocity, deflection angle, and lift have been calculated in terms of the angle of attack and aerofoil geometry and their asymptotic behaviour has been shown to match with well established results for single aerofoils. The expressions for surface velocity have been shown to agree well with numerical solutions which have been validated against experimental results.

The model and methods of solution are readily extendable to a variety of situations. For example, the generalisation to weakly compressible flows is straightforward, and follows swiftly by a Prandtl-Glauert transformation (Kuethe and Chow, 1998) provided the flow is not transonic. Also, multiple cascades may be easily included in the analysis to model, for example, multiple columns of swimmers in shoals of fish. Having elucidated the kernel of the singular integral operator, it is now plain to see that the flow perturbation approaches uniformity exponentially fast away from the cascade unlike the single aerofoil case, where the flow becomes uniform algebraically quickly away from the blade. This means that we may place a second cascade almost directly behind the first one and, taking the angle of attack experienced by the second cascade from equation 2.32, calculate the new flow field. 

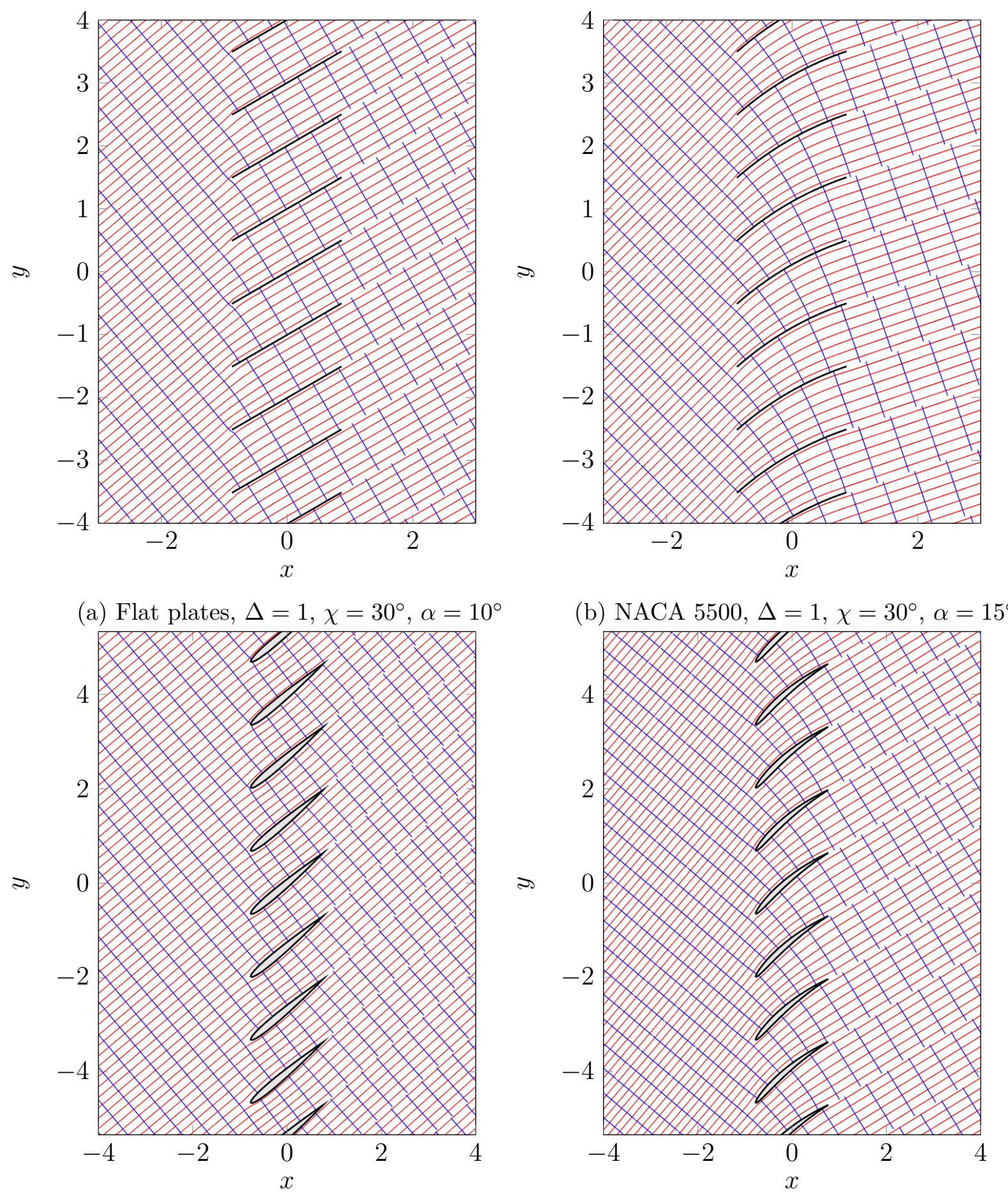

(c) NACA $0008, \Delta=4 / 3, \chi=40^{\circ}, \alpha=0^{\circ}$

(d) NACA 5506, $\Delta=4 / 3, \chi=40^{\circ}, \alpha=10^{\circ}$

Fig. 2.8 Contour lines for the velocity potential (blue) and streamfunction (red) for a range of geometries and angles of attack. 

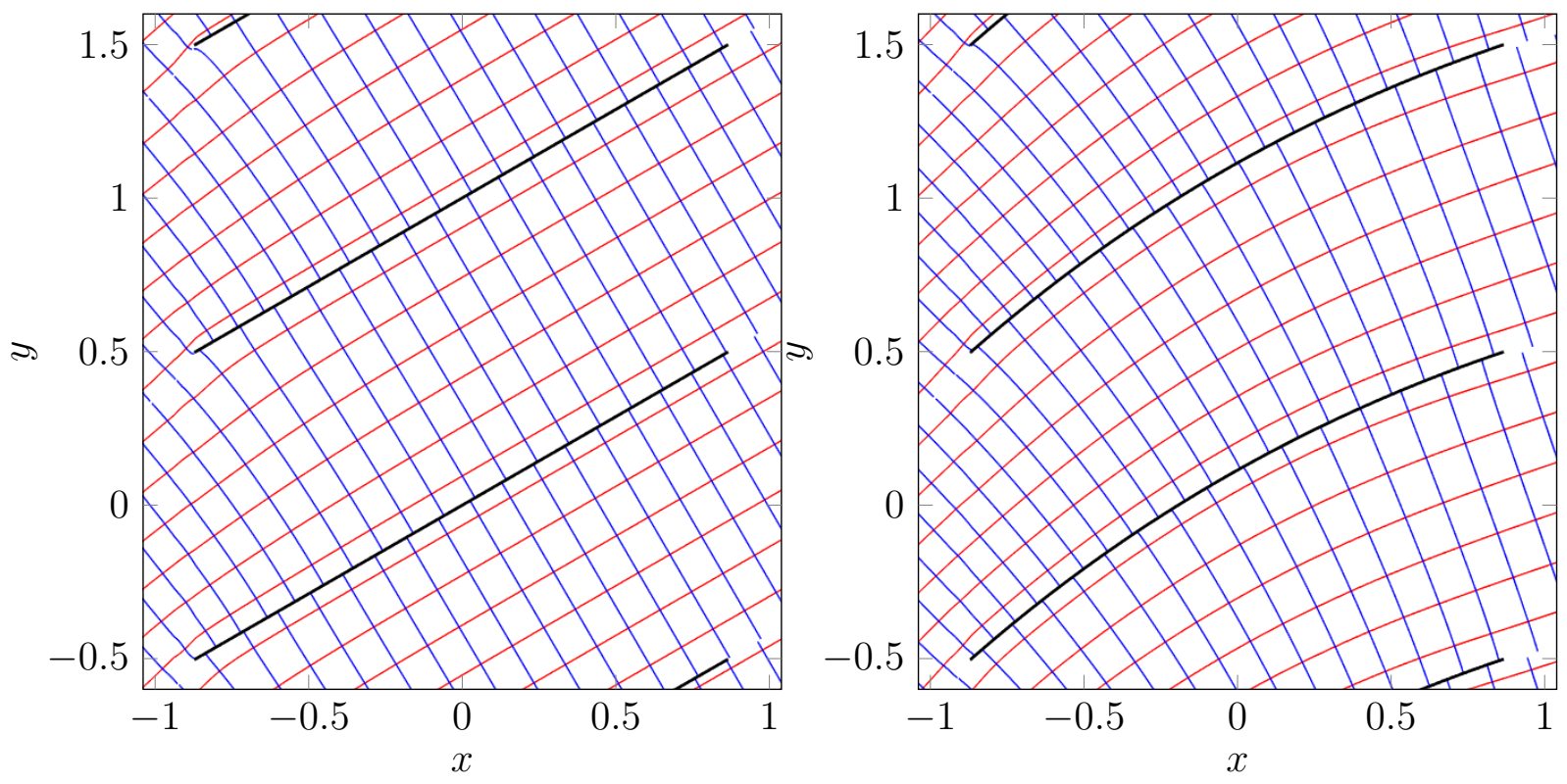

(a) Flat plates, $\Delta=1, \chi=30^{\circ}, \alpha=10^{\circ}$
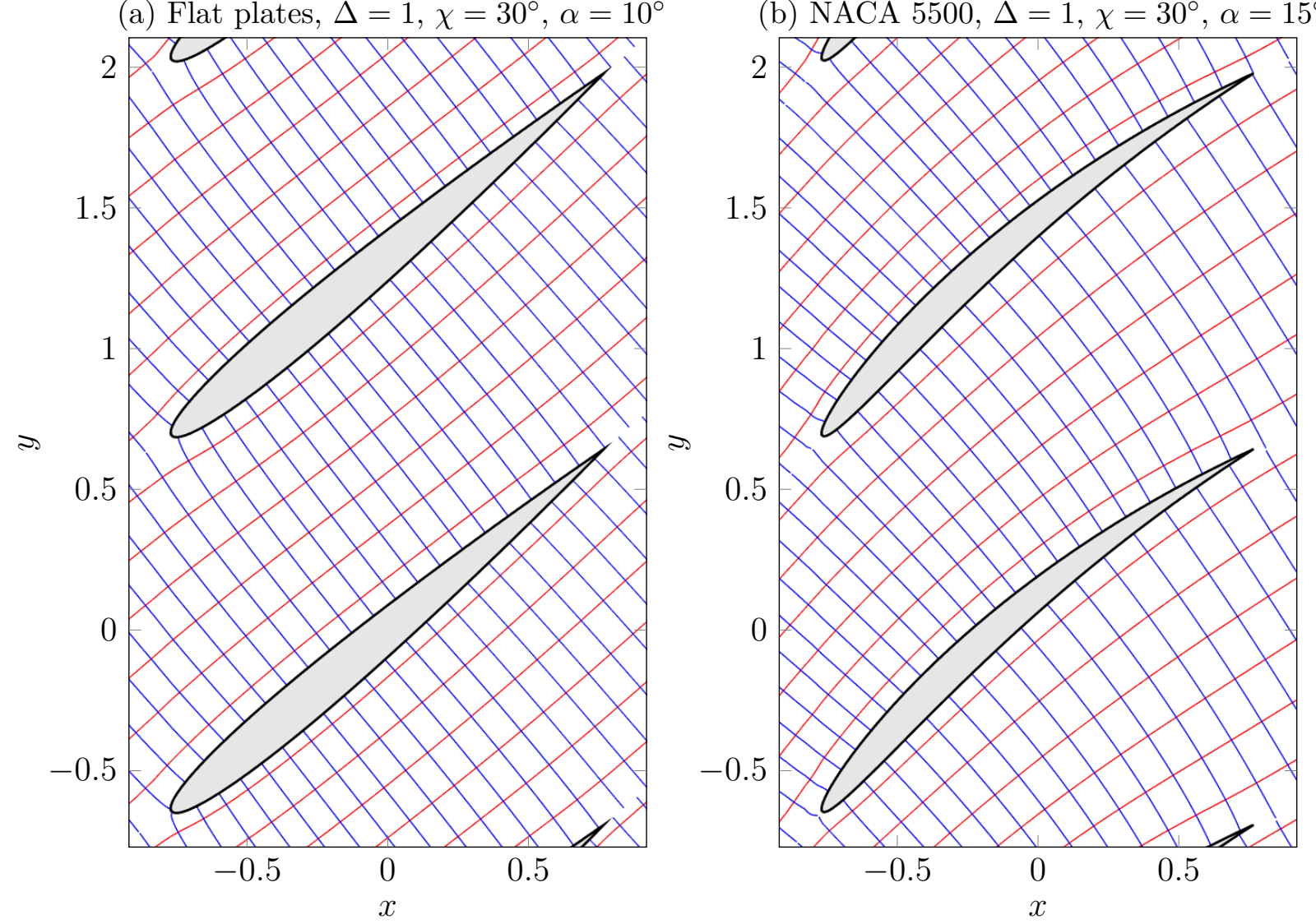

(c) NACA 0008, $\Delta=4 / 3, \chi=40^{\circ}, \alpha=0^{\circ}$

(d) NACA 5506, $\Delta=4 / 3, \chi=40^{\circ}, \alpha=10^{\circ}$

Fig. 2.9 Contour lines for the velocity potential (blue) and streamfunction (red) in the inter-blade region for a range of geometries and angles of attack. 
Although the solutions presented in this chapter are appropriate for the ensuing aeroacoustic analysis in chapter 3 , there are several limitations that may be relevant to other studies. The blades in this chapter are modelled as impermeable, with a solid body no-flux condition. This may not be realistic in biological applications if the aerofoils are modelling wings that may permit flow leakage. Consequently, in chapter 4 we will extend the analytic solution to permit porous aerofoils. The blade geometries in the present chapter are also required to have a small aspect ratio and the angle of attack must also be small. In many turbomachinery applications the incident angle will be large and the blades may have geometric profiles beyond those amenable to the methods in this chapter. These limitations will be addressed in chapter 6 where we derive potential flow solutions for cascades with arbitrary aerofoil shapes and angles of attack. Furthermore, the work in the present chapter cannot account for flow separation. This could be addressed using free streamline theory, where streamlines are assumed to emanate from the body to separate the outer inviscid region from the turbulent wake, such as in Wade (1967). Whilst we do not address flow separation fully, it could be modelled using the trajectories of point vortices presented in chapter 6 . 


\section{List of Symbols}

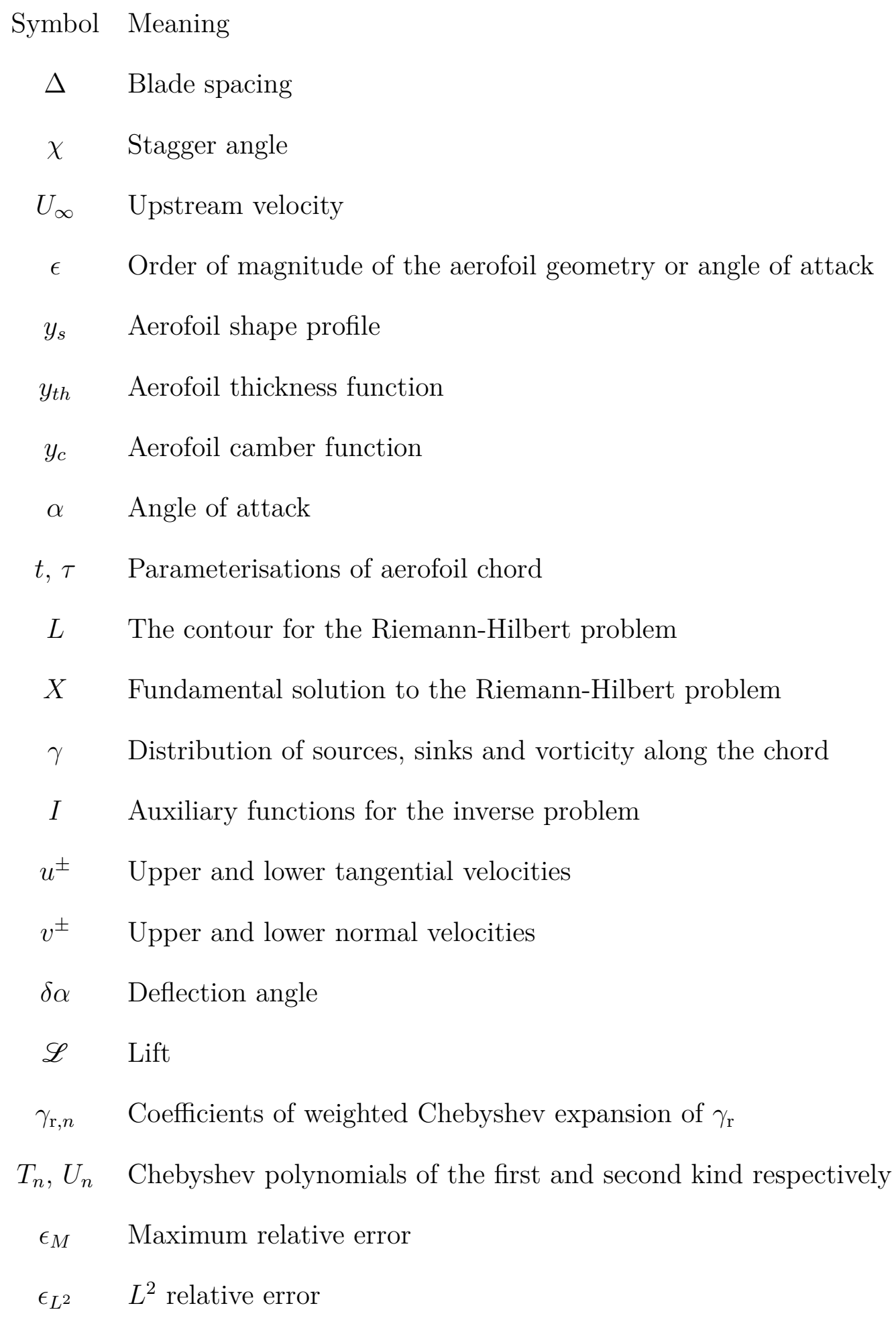




\section{A Modified Plemelj Formulae}

In this section we prove the Modified Plemelj formulae which is used in the solution of the Riemann-Hilbert problem in section 2.3. The traditional Plemelj formulae must be adapted to be suitable for unbounded domains, as found in the cascade problem.

Theorem 1. If $f(t)$ satisfies a Hölder condition on $L$ as defined in section 2.2, except possibly at the endpoints where it may have integrable singularities, and has period $\Delta \mathrm{i}$, then, for $z \notin L$,

$$
\Phi(z):=\frac{1}{2 \pi \mathrm{i}} \int_{L} \frac{f(\zeta)}{\zeta-z} \mathrm{~d} \zeta=\frac{1}{2 \mathrm{i} \Delta} \int_{-1}^{1} f(\tau) \operatorname{coth}\left(\frac{\pi(\tau-z)}{\Delta}\right) \mathrm{d} \tau .
$$

Proof. By parametrising $L$, we may write

$$
\frac{1}{2 \pi \mathrm{i}} \int_{L} \frac{f(\zeta)}{\zeta-z} \mathrm{~d} \zeta=\frac{1}{2 \pi \mathrm{i}} \sum_{n=-\infty}^{\infty} \int_{-1}^{1} \frac{f(\tau)}{(\tau+\mathrm{i} n \Delta)-z} \mathrm{~d} \tau
$$

We now use the dominated convergence theorem to interchange the orders of summation and integration for $z \notin L$. We write

$$
h_{N}(\tau, z):=f(\tau) \sum_{n=-N}^{N} \frac{1}{(\tau+\mathrm{i} n \Delta)-z}=f(\tau)\left(\frac{1}{\tau-z}+2 \sum_{n=1}^{N} \frac{(\tau-z)}{(\tau-z)^{2}+n^{2} \Delta^{2}}\right)
$$

and then

$$
\left|h_{N}(\tau, z)\right| \leq g(\tau, z):=|f(\tau)|\left(\frac{1}{|\tau-z|}+2 \sum_{n=1}^{\infty} \frac{|(\tau-z)|}{\left|(\tau-z)^{2}+n^{2} \Delta^{2}\right|}\right) .
$$

To complete the proof, we must show that $g$ is integrable. As $f$ satisfies the Hölder condition and possibly has integrable singularities at the end points, all that remains is to show that the bracketed term is bounded. Since $z \notin L$, we may write $z-\tau=r \mathrm{e}^{\mathrm{i} \theta}$, where $r$ and $\theta$ are functions of $\tau$. Moreover, $r \mathrm{e}^{\mathrm{i} \theta} \neq \pm \mathrm{i} n \Delta$ and therefore,

$$
g(\tau, z)=\frac{|f(\tau)|}{r}\left(1+2 \frac{r^{2}}{\Delta^{2}} \sum_{n=1}^{\infty} \frac{1}{\left|\frac{r^{2}}{\Delta^{2}} \mathrm{e}^{2 \mathrm{i} \theta}+n^{2}\right|}\right) .
$$

By the comparison test with $\frac{1}{n^{2}}$, this sum converges for all $\tau \in[-1,1]$, so $g(\tau, z)$ is bounded in the domain of integration and therefore integrable. By the dominated 
convergence theorem, we are free to interchange the order of limit and integral, so

$$
\begin{aligned}
\frac{1}{2 \pi \mathrm{i}} \int_{L} \frac{f(\zeta)}{\zeta-z} \mathrm{~d} \zeta & =\frac{1}{2 \pi \mathrm{i}} \int_{-1}^{1} f(\tau) \sum_{n=-\infty}^{\infty} \frac{1}{(\tau+\mathrm{i} n \Delta)-z} \mathrm{~d} \tau \\
& =\frac{1}{2 \mathrm{i} \Delta} \int_{-1}^{1} f(\tau) \operatorname{coth}\left(\frac{\pi(\tau-z)}{\Delta}\right) \mathrm{d} \tau
\end{aligned}
$$

where the last identity is obtained from a classical formula (Bromwich, 2005, p. 296).

Since, as we have shown above, we may split up the integral into its contributions from each chord, we have

$$
\frac{1}{2 \pi \mathrm{i}} f_{L} \frac{f(\zeta)}{\zeta-z} \mathrm{~d} \zeta=\frac{1}{2 \mathrm{i} \Delta} f_{-1}^{1} f(\tau) \operatorname{coth}\left(\frac{\pi(\tau-z)}{\Delta}\right) \mathrm{d} \tau,
$$

and the analogous result for the Plemelj formulae holds:

$$
\Phi^{ \pm}(t)= \pm \frac{f(t)}{2}+\frac{1}{2 \mathrm{i} \Delta} f_{-1}^{1} f(\tau) \operatorname{coth}\left(\frac{\pi(\tau-t)}{\Delta}\right) \mathrm{d} \tau .
$$

\section{B Asymptotic Results at Endpoints}

In this section, we consider the asymptotic behaviour of Cauchy-type integrals with coth kernels, which is necessary for the analysis of endpoint behaviour in section 2.3.3. We restrict our attention to the endpoints $\mp 1$, since the behaviour at $\mp 1+\mathrm{i} n \Delta$ will be identical by the periodicity of the kernel. We define

$$
\Phi(z)=\frac{1}{2 \mathrm{i} \Delta} \int_{-1}^{1} f(\tau) \operatorname{coth}\left(\frac{\pi(\tau-z)}{\Delta}\right) \mathrm{d} \tau=\Phi_{1}(z)+\frac{1}{2 \pi \mathrm{i}} \int_{-1}^{1} \frac{f(\tau)}{\tau-z} \mathrm{~d} \tau
$$

where $f(t)$ satisfies a Hölder condition on $(-1,1)$, except possibly at the ends where it satisfies

$$
f(t)=\frac{\tilde{f}(t)}{(t-c)^{\beta}}
$$

where $c$ is an endpoint of $L, \beta$ is a real constant, and $\tilde{f}(t)$ satisfies a Hölder condition near and at $c$. In our case the relevant parameters are $\beta=0,1 / 2$. We have removed the principal value part in (2.53) so that $\Phi_{1}$ is bounded and takes a definite value as $z \rightarrow c$ along any path. When $z=t$ on the contour, the remaining integral is considered in the principal value sense. In the following formulae, \pm correspond to taking $c=\mp 1$. The 
branch of $\log \frac{1}{z-c}$ is chosen to pass through the contour. Then the following limits, which can be deduced from the non-periodic analysis in (Muskhelishvili, 1946, §29), are valid:

1. $\beta=0$

We have the asymptotic behaviours:

(a) as $z \rightarrow c$, with $z$ not on the contour,

$$
\Phi(z) \sim \pm \frac{f(c)}{2 \pi \mathrm{i}} \cdot \log \left(\frac{1}{z-c}\right)+\Phi_{1}(z)+\Phi_{0}(z)
$$

(b) as $t \rightarrow c$, with $t$ on the contour,

$$
\Phi(t) \sim \pm \frac{f(c)}{2 \pi \mathrm{i}} \cdot \log \left(\frac{1}{t-c}\right)+\Phi_{1}(t)+\Psi_{0}(t)
$$

where $\Phi_{1}$ is a function that is analytic at $z=c, \Psi_{0}$ satisfies a Hölder condition near and at $t=c$, and $\Phi_{0}$ is a bounded function tending to a definite limit as $z \rightarrow c$.

2. $\underline{\beta \neq 0}$

We have the asymptotic behaviours:

(a) as $z \rightarrow c$, with $z$ not on the contour,

$$
\Phi(z) \sim \pm \frac{\mathrm{e}^{ \pm \beta \pi \mathrm{i}}}{2 \mathrm{i} \sin (\beta \pi)} \cdot \frac{\tilde{f}(c)}{(z-c)^{\beta}}+\Phi_{1}(z)+\Phi_{0}(z)
$$

(b) as $t \rightarrow c$, with $t$ on the contour,

$$
\Phi(t) \sim \pm \frac{\cot (\beta \pi)}{2 \mathrm{i}} \cdot \frac{\tilde{f}(c)}{(t-c)^{-\beta}}+\Phi_{1}(t)+\Psi_{0}(t)
$$

where $\Phi_{1}$ is a function that is analytic at $c, \Psi_{0}=o\left((t-c)^{-\beta}\right)$ as $t \rightarrow c$, and $\Phi_{0}=o\left((z-c)^{\beta}\right)$. 


\section{Chapter 3}

\section{Scattering by Cascades of Aerofoils with Realistic Geometry}

\subsection{Introduction}

In this chapter we present an analytic solution for rotor-stator interaction noise. The solution extends previous work by Glegg (1999) and Posson et al. (2010b) by accounting for blade geometry and angle of attack, thus enabling us to study the important effects of aerofoil profiles. Furthermore, we will find expressions for the acoustic potential function throughout the entire flow domain, and will therefore not be limited to upstream (Peake and Kerschen, 1997) and downstream (Peake and Kerschen, 2004) regions. We model a single Fourier component of turbulence incident on the cascade, which we represent as an unsteady convected gust. Accounting for blade geometry complicates the application of the boundary conditions but, by making use of an orthogonal coordinate transform, we are able to model the blades as flat plates in our parametric coordinate system. We seek a perturbation expansion in terms of the disturbance of the background flow from uniform flow in order to make analytic progress. In a similar way to the aforementioned works, the solution is derived using the Wiener-Hopf method. The resulting Fourier transform is inverted via contour integration to obtain the acoustic potential.

The model in the present chapter is subject to some asymptotic restrictions. Firstly, the aerofoils are assumed to be formally "thin", so that background flow gradients are $\mathcal{O}(\epsilon)$ perturbations from uniformity. Secondly, the frequency must satisfy $k \epsilon \ll 1$, where $k$ is the reduced frequency. Far from being restrictive, this asymptotic regime crucially accounts for the first few blade passing frequencies (BPFs), which significantly contribute to engine noise. Typically, the upstream rotors move and shed wakes which impinge on the cascade at the blade passing frequency; accurate modelling of the scattering of 
incident wakes of frequencies close to the BPFs is essential because they usually occur in the most sensitive frequency regime of human hearing (Dittmar, 1972). Additionally, the BPFs occur at large sound pressure levels, so they are usually the loudest tones, as well as the most annoying. Presently, other analytic cascade interaction models that account for aerofoil geometry (Evers and Peake, 2002; Peake and Kerschen, 1997, 2004) are restricted to high frequencies that cannot account for these critical interactions.

In section 3.2 we discuss the modelling assumptions and give some key details of the form of the background mean flow, which prove useful in later analysis. In section 3.2.2 the governing equations for the acoustic potential function are derived using Rapid Distortion Theory, as in Goldstein (1978), and the corresponding boundary conditions are derived. A perturbation expansion is used, where the small parameter $\epsilon$ is the order of magnitude of the distortion of the mean flow from uniform flow. This expansion yields two equations: one at $\mathcal{O}(1)$ and another at $\mathcal{O}(\epsilon)$. The leading order, $\mathcal{O}(1)$, equation is the flat-plate problem solved previously by Glegg (1999). In section 3.3.2, we solve the $\mathcal{O}(\epsilon)$ equation for the Fourier transform of the jump in potential either side of the blade. Whilst more complicated than the $\mathcal{O}(1)$ problem due to forcing terms arising from the mean flow distortion, this problem can be solved using a similar approach to that of Glegg (1999). In section 3.4 this Fourier transform is inverted, using a similar method to Posson et al. (2010b). In section 3.5 various aeroacoustic results are discussed. Finally, in section 3.6, we summarise our work and suggest future directions of research. We illustrate the important details of the procedure in a road map in figure 3.1.

\subsection{Modelling}

We consider a linear cascade of blades in a subsonic flow as illustrated in figure 3.2. The blades are separated by $d^{*}$ in the chordwise direction, $s^{*}$ in the normal direction and have infinite span in the $x_{3}^{*}$ direction. (Here $*$ denotes a dimensional quantity.) The aerofoils have semi-chord length $b^{*}$ in Cartesian coordinates $\left(x^{*}, y^{*}\right)$ and the coordinate origin is taken to be at the leading edge of the $0^{\text {th }}$ blade. We non-dimensionalise lengths by $b^{*}$. We assume that the blades have non-trivial geometry, such that the upper and lower surfaces of the blade, $\epsilon y_{s \pm}$, can be described by

$$
\epsilon y_{s \pm}(x)= \pm \epsilon y_{t h}(x)+\epsilon y_{c}(x)
$$

where the subscripts $c$ and $t h$ denote camber and thickness respectively and $\epsilon \ll 1$. We restrict our analysis to bodies that have, at worst, parabolic noses, i.e. $y_{t h} \sim \epsilon t h^{\prime} \sqrt{x}$ as 


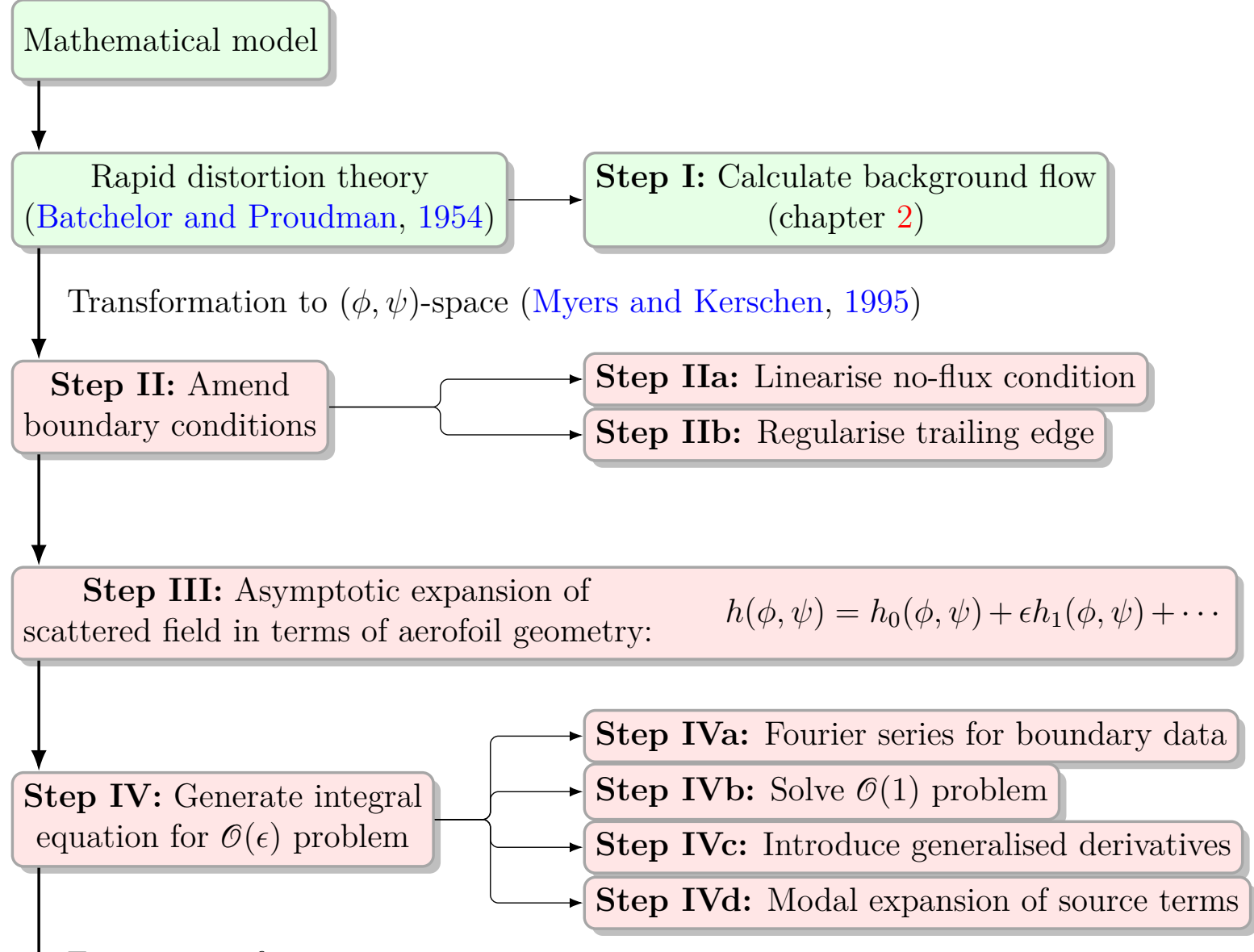

Fourier transform

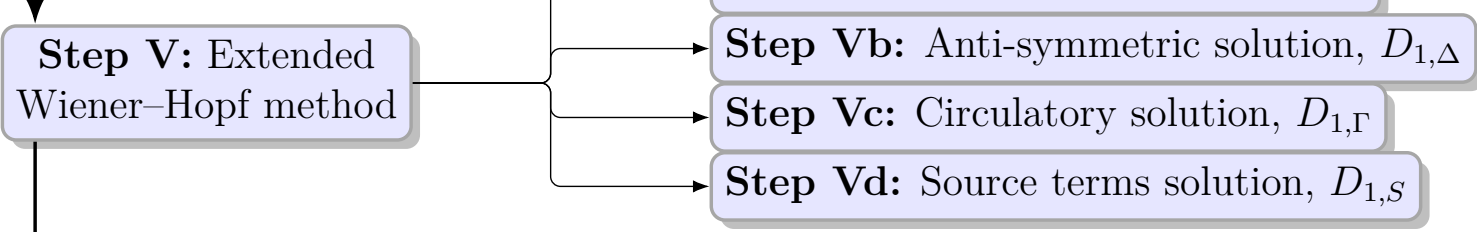

Step VI: Invert Fourier transform

Expression for $h(\phi, \psi)$

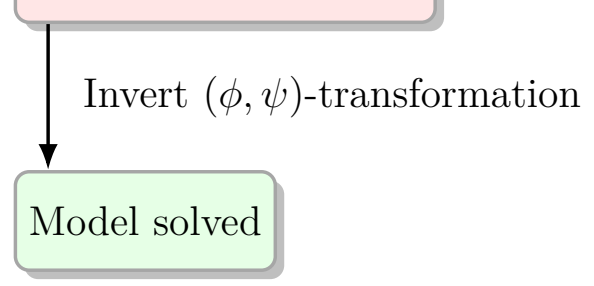

Fig. 3.1 Schematic diagram illustrating the solution method of the present chapter. The colour of each box represents the space in which the relevant analysis takes place: green denotes physical $(x, y, z)$-space; red, parametric $(\phi, \psi, z)$-space; and blue, spectral $\gamma$-space. 

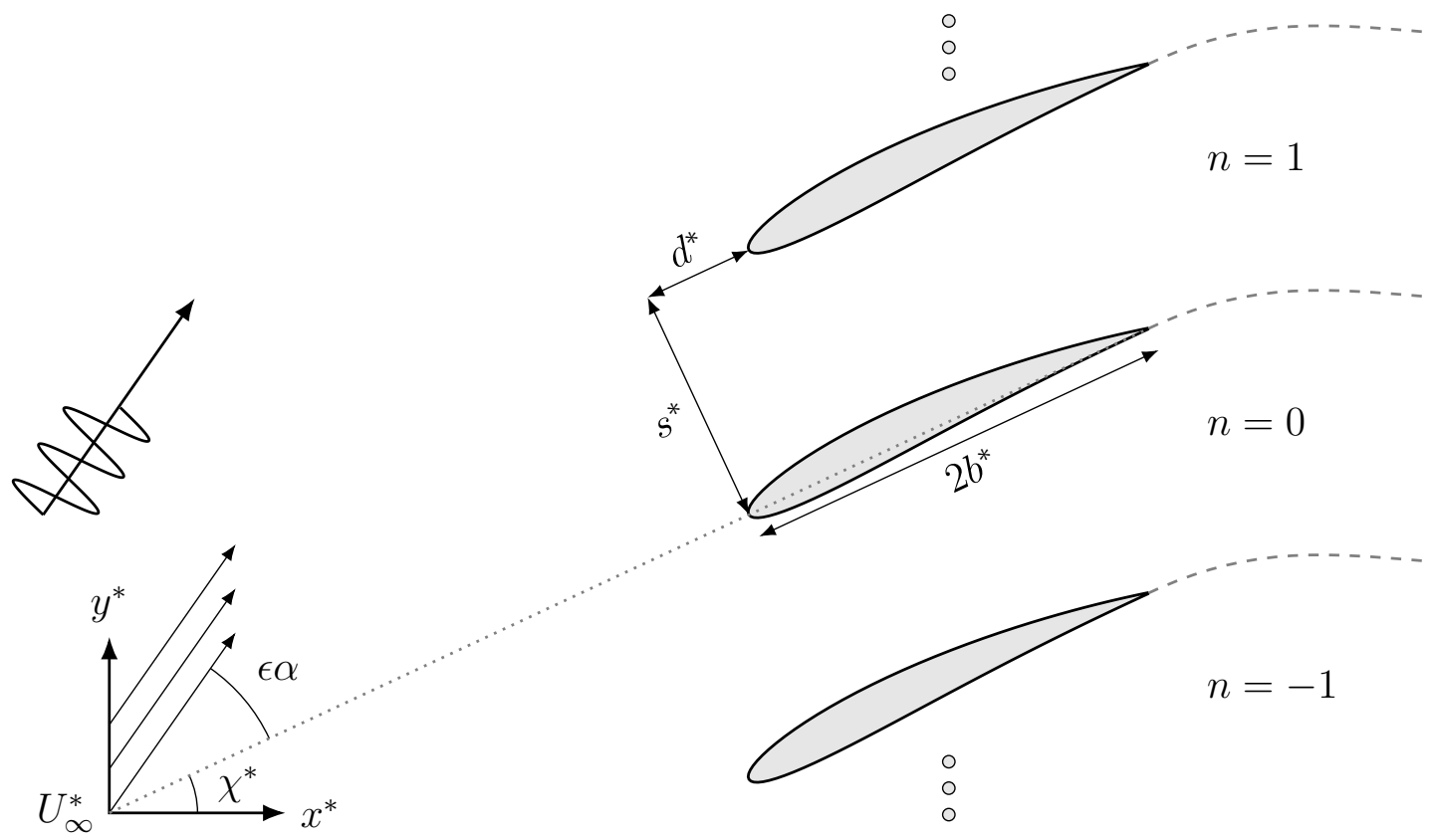

Fig. 3.2 A cascade of realistic aerofoils at stagger angle $\chi^{*}$ and incidence angle $\epsilon \alpha$ subject to a convected disturbance. The dotted line indicates the chordwise direction and the dashed line represents the unsteady wakes.

$x \rightarrow 0^{+}$where $t h=\epsilon t h^{\prime}$ is the maximum thickness of the aerofoil, which is common in analytical aerofoil interaction problems (Tsai (1992), Ayton and Peake (2013)). Additionally, we require that the camber is bounded and continuous. The steady mean flow far upstream is uniform with speed $U_{\infty}^{*}$ (which we will use to non-dimensionalise velocities). We also permit the upstream mean flow at infinity to be at a small angle of attack, $\epsilon \alpha$.

We denote the velocity potential and streamfunction of the steady background flow through the cascade as $\phi^{*}$ and $\psi^{*}$ respectively, which we non-dimensionalise as

$$
\phi=\frac{\phi^{*}}{U_{\infty}^{*} b^{*}}, \quad \psi=\beta_{\infty} \frac{\psi^{*}}{U_{\infty}^{*} b^{*}}, \quad x_{3}=\frac{x_{3}^{*}}{b^{*}}
$$

where $\beta_{\infty}=\sqrt{1-M_{\infty}^{2}}$ is the Prandtl-Glauert factor and $M_{\infty}$ is the mean flow Mach number far upstream. The density is non-dimensionalised with respect to the density far upstream $\rho^{*}$ and the pressure is non-dimensionalised with respect to $\rho^{*} U_{\infty}^{* 2}$

\subsubsection{Steady Flow}

Before we can approach the unsteady aeroacoustics, we must first solve the steady background flow.This is step I in the road map (figure 3.1), and the analysis was 


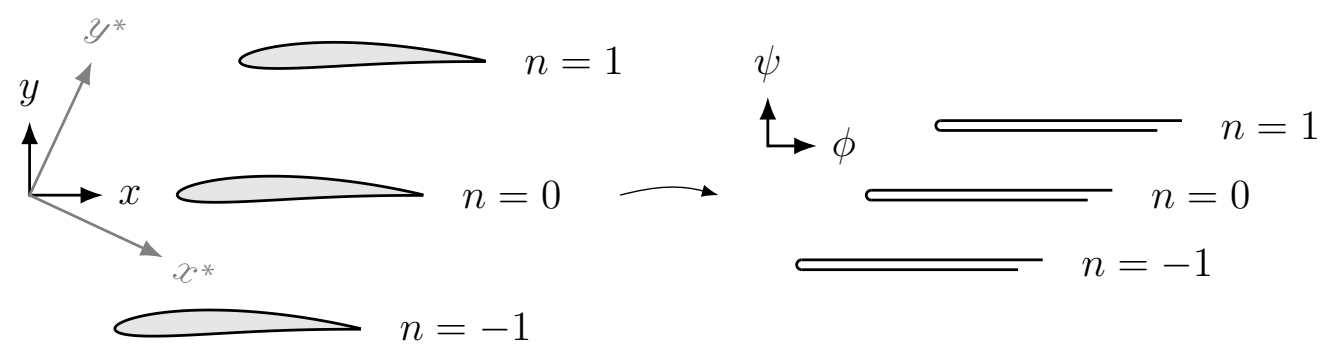

Fig. 3.3 Illustration of the change of coordinate system used to simplify the application of boundary conditions. The complex aerofoil surface is mapped to a flat plate.

carried out in chapter 2. To aid application of the boundary conditions in the unsteady problem, we will transform from non-dimensional Cartesian coordinates $(x, y)$ to potentialstreamline coordinates $(\phi, \psi)$ as is typical for realistic-geometry aerofoil interactions studied analytically (Ayton, 2017; Ayton and Peake, 2013; Myers and Kerschen, 1995, 1997; Peake and Kerschen, 1997, 2004). The relationship between coordinates is given by

$$
z_{\beta}=z_{\phi}+\epsilon F\left(z_{\phi}\right)
$$

where $z_{\beta}=x+\mathrm{i} \beta_{\infty} y$ are the non-dimensional coordinates in the Prandtl-Glauert space (where the flow is incompressible) and $z_{\phi}=\phi+\mathrm{i} \psi \cdot F\left(z_{\phi}\right)$ is the complex potential of the steady flow written as $q\left(z_{\phi}\right)-\mathrm{i} \mu\left(z_{\phi}\right)=F^{\prime}\left(z_{\phi}\right)$ where the amplitude of the steady flow is given by $1+\epsilon q$, in the direction making an angle $\epsilon \mu$ with the horizontal. Consequently, we may write the non-dimensional perturbation to horizontal uniform flow as (see chapter 2)

$$
q\left(z_{\beta}\right)-\mathrm{i} \mu\left(z_{\beta}\right)=-\mathrm{i} \alpha+\frac{1}{2(d+\mathrm{i} s)} \int_{0}^{2} \gamma(\tau)\left(\operatorname{coth}\left(\frac{\pi \mathrm{i}\left(\tau-z_{\beta}\right)}{d+\mathrm{i} s}\right)-1\right) \mathrm{d} \tau
$$

where $\gamma$ is a distribution of vortices, sources and sinks arranged on the aerofoil chord and $d$ and $s$ are the blade spacing in the Prandtl-Glauert space. In chapter 2 we presented a numerical scheme that quickly calculates $\gamma$.

By integrating expression (3.2), we obtain analytic forms for the streamfunction and velocity potential

$$
\phi\left(z_{\beta}\right)+\mathrm{i} \psi\left(z_{\beta}\right)=z_{\beta}(1-\mathrm{i} \epsilon \alpha)-\frac{\epsilon}{2 \pi \mathrm{i}} \int_{0}^{2} \gamma(\tau)\left(\log \left(\frac{\sinh \left(\frac{\pi \mathrm{i}\left(\tau-z_{\beta}\right)}{d+\mathrm{i} s}\right)}{\sinh \left(\frac{\pi \mathrm{i} \tau}{d+\mathrm{i} s}\right)}\right)+\frac{\pi \mathrm{i} z_{\beta}}{d+\mathrm{i} s}\right) \mathrm{d} \tau,
$$



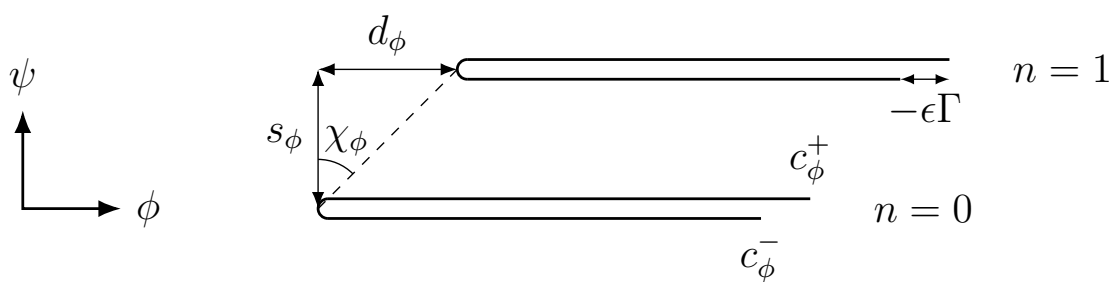

Fig. 3.4 Diagram showing parameters in the transformed $(\phi, \psi)$-space.

where the constant of integration has been chosen such that $F(0)=0$ and the logarithmic branch is orientated along the aerofoil wake. Consequently, (3.3) allows us to calculate the spacing between blades in the new coordinate space as

$$
d_{\phi}+\mathrm{i} s_{\phi}=\phi(d+\mathrm{i} s)+\mathrm{i} \psi(d+\mathrm{i} s)=(d+\epsilon \alpha s)+\mathrm{i}(s-\epsilon \alpha d) .
$$

We immediately note that the angle of attack affects the blade spacing in $(\phi, \psi)$-space and will accordingly influence the cut-on and cut-off frequencies of the acoustic modes. These new parameters are illustrated in figure 3.4 .

Exponential decompositions of the complex velocity field $q-\mathrm{i} \mu$ are available in the upstream and downstream regions, and are detailed in chapter 2. Such a decomposition is essential for the application of the Wiener-Hopf method in section 3.3.2.

We define the location of the upper/lower side of the image of the trailing edge in $(\phi, \psi)$-space as $\left(c_{\phi}^{ \pm}, 0\right)$. It is important to note that the image of the physical trailing edge is not unique, but the upper and lower sides are in fact mapped to two different points. Formula (3.3) yields analytic expressions for the trailing-edge images as

$$
c_{\phi}^{ \pm}=2(1-\mathrm{i} \epsilon \alpha)-\frac{\epsilon}{2 \pi \mathrm{i}} \int_{0}^{2} \gamma(\tau)\left(\log \left(\frac{\sinh \left(\frac{\pi \mathrm{i}(\tau-2)}{d+\mathrm{i} s}\right)}{\sinh \left(\frac{\pi \mathrm{i} \tau}{d+\mathrm{i} s}\right)}\right) \pm \pi \mathrm{i}+\frac{2 \pi \mathrm{i}}{d+\mathrm{i} s}\right) \mathrm{d} \tau,
$$

which are both real numbers since the physical trailing edge $\left(2,0^{ \pm}\right)$lies on the same streamline as the origin. The difference between the location of the upper and lower trailing edges in $(\phi, \psi)$-space is $\epsilon \Gamma$, which is the circulation around a single aerofoil, and may be calculated as

$$
\epsilon \Gamma=-\epsilon \int_{0}^{2} \gamma(\tau) \mathrm{d} \tau=-\epsilon \pi\left(\gamma_{\mathrm{r}, 0}+\frac{\gamma_{\mathrm{r}, 1}}{2}\right)
$$

where $\gamma_{\mathrm{r}, 0}$ and $\gamma_{\mathrm{r}, 1}$ are the first two coefficients of the weighted Chebyshev series defined in $(2.42)$. 


\subsubsection{Unsteady Formulation}

We now consider the unsteady interaction in the new, transformed coordinate system. The present analysis is restricted to inviscid linear disturbances of a two-dimensional irrotational compressible mean flow, using the formulation derived by Goldstein (1978) and further developed by Myers and Kerschen (1995). We assume that there are small amplitude vortical and entropic perturbations in the uniform flow far upstream of the cascade, which we denote by $\boldsymbol{v}^{\prime}$ and $s^{\prime}$ respectively. These disturbances are convected downstream by the mean flow and interact with the cascade, thus producing sound. Similar approaches have been employed (Evers and Peake, 2002; Peake and Kerschen, 1997, 2004) in modelling gust-cascade interaction. Unlike the flat plate case (Glegg, 1999), the mean flow modifies both the boundary conditions and governing equation. We will see that extra forcing terms are induced in the governing equation, while the boundary conditions include terms depending on the surface velocity.

The total unsteady velocity perturbation may be decomposed into the form (Goldstein, 1978)

$$
\boldsymbol{u}^{\prime}=\nabla G^{\prime}+\boldsymbol{v}^{\prime}
$$

where $\boldsymbol{v}^{\prime}$ satisfies a modified form of the linearised momentum equation. By employing the method of characteristics, $\boldsymbol{v}^{\prime}$ may be found exactly throughout the whole flow domain. The irrotational scattered field, $G^{\prime}$, then satisfies (Goldstein, 1978)

$$
\frac{\mathrm{D}_{0}}{\mathrm{D} t}\left(\frac{1}{c_{0}^{2}} \frac{\mathrm{D}_{0} G^{\prime}}{\mathrm{D} t}\right)-\frac{1}{\rho_{0}} \nabla \cdot\left(\rho_{0} \nabla G^{\prime}\right)=\frac{1}{\rho_{0}} \nabla \cdot\left(\rho_{0} \boldsymbol{v}^{\prime}\right)
$$

where the material derivative $\mathrm{D}_{0} / \mathrm{D} t$ is taken with respect to the local mean flow. The quantities $\rho_{0}$ and $c_{0}$ are the local values of density and speed of sound for the mean flow. The no-flux boundary condition on $G^{\prime}$ at any rigid surface is

$$
\frac{\partial G^{\prime}}{\partial n}=-\boldsymbol{v}^{\prime} \cdot \boldsymbol{n}
$$

where $\boldsymbol{n}$ is the unit normal vector.

The non-dimensional upstream velocity and entropy disturbances far upstream may be written as

$$
\boldsymbol{v}^{\prime} \rightarrow \nu\left[\begin{array}{c}
A_{t} \\
A_{n} \\
A_{s}
\end{array}\right] \mathrm{e}^{\mathrm{i} k\left(\phi+k_{n} \psi+k_{s} x_{3}-t\right)}
$$




$$
s^{\prime} \rightarrow 2 \nu B \mathrm{e}^{\mathrm{i} k\left(\phi+k_{n} \psi+k_{s} x_{3}-t\right)},
$$

subject to $A_{t}+A_{n} k_{n} \beta_{\infty}+A_{s} k_{s}=0$, where $k=\omega b^{*} / U_{\infty}^{*}$ is the reduced frequency and time is normalised by $b^{*} / U_{\infty}^{*}$. The amplitude of the disturbance, $\nu$, is assumed to be small $(\nu \ll \epsilon)$.

The transformation

$$
G^{\prime}(\phi, \psi)=h(\phi, \psi) \exp \left[\mathrm{i} k\left(k_{3} x_{3}-t-\delta M_{\infty}^{2} \phi\right)+\epsilon M_{\infty}^{2} q\right]
$$

allowed Kerschen and Myers (1987) to express Goldstein's wave equation as

$$
\begin{array}{r}
\frac{\partial^{2} h}{\partial \phi^{2}}+\frac{\partial^{2} h}{\partial \psi^{2}}+k^{2} w^{2} h+\epsilon(\gamma+1) M_{\infty}^{4} \delta\left\{q\left[\frac{\partial^{2} h}{\partial \psi^{2}}+2 \mathrm{i} k \delta \frac{\partial h}{\partial \phi}+k^{2}\left(w^{2}+\delta^{2}\right) h\right]\right. \\
\left.-\frac{\partial q}{\partial \phi}\left[\frac{\partial h}{\partial \phi}-\mathrm{i} k \delta h\right]\right\}-\epsilon 2 k^{2} w^{2} \beta_{\infty}^{2} q h=\epsilon k S(\phi, \psi) \mathrm{e}^{\mathrm{i} k \Omega}
\end{array}
$$

where

$$
\begin{gathered}
\Omega=\delta \phi+k_{n} \psi+\epsilon g(\phi, \psi), \quad w^{2}=\left(\delta M_{\infty}\right)^{2}-\left(k_{s} / \beta_{\infty}\right)^{2}, \quad \delta=1 / \beta_{\infty}^{2}, \quad \tilde{A}_{t}=A_{t}-B, \\
S(\phi, \psi)=2 \delta\left\{\mathrm{i}\left(\tilde{A}_{t}-k_{n} A_{n} \beta_{\infty}^{3}\right) q+\mathrm{i}\left(k_{n} \tilde{A}_{t} \beta_{\infty}+A_{n} \beta_{\infty}\right) \mu\right. \\
\left.+\frac{1}{k}\left[\tilde{A}_{t} M_{\infty}^{2} \frac{\partial q}{\partial \phi}+A_{n} M_{\infty}^{2} \beta_{\infty} \frac{\partial q}{\partial \psi}\right]\right\},
\end{gathered}
$$

and $g$ is Lighthill's Drift function, which represents the cumulative distortion of vortex filaments by the non-uniform mean flow:

$$
g(\phi, \psi)=\int_{-\infty}^{\phi}\left[\frac{1}{U_{0}^{2}(\eta, \psi)}-1\right] \mathrm{d} \eta=-2 \int_{-\infty}^{\phi} q(\eta, \psi) \mathrm{d} \eta
$$

The no-flux boundary condition applied to rigid surfaces (3.6d) becomes

$$
\frac{\partial h}{\partial \psi}+\epsilon M_{\infty}^{2} \frac{\partial q}{\partial \psi} h=-\left[\frac{A_{n}}{\beta_{\infty}}\left(1-\epsilon M_{\infty}^{2} q\right)-\epsilon 2 \tilde{A}_{t} \mu\right] \mathrm{e}^{\mathrm{i} k \Omega}
$$




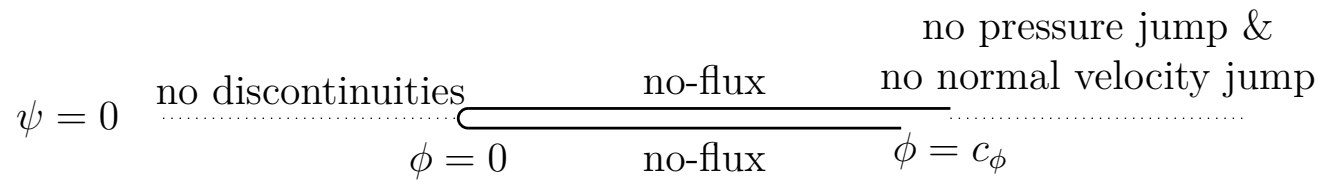

Fig. 3.5 Schematic illustrating where each boundary condition is applied.

applied on the body surface. We will use the modified pressure $p$, which is defined in terms of actual pressure $p^{\prime}$ as

$$
p=-p^{\prime} \mathrm{e}^{\mathrm{i} k\left(-k_{s} x_{3}+t\right)}=\frac{\partial}{\partial \phi}\left(h \mathrm{e}^{-\mathrm{i} k \delta \phi}\right) \mathrm{e}^{\mathrm{i} k \phi} .
$$

\subsubsection{Boundary Conditions}

We must specify an adequate number of boundary conditions in order for the problem to be well-posed and physically valid. The boundary conditions are applied to each streamline that corresponds to a blade surface, i.e. $\psi=n s_{\phi}$ for integer $n$. Upstream of the blade, we require that there are no discontinuities across this streamline. On the blade itself, the no-flux condition for a rigid surface (3.8) must be satisfied either side of the blade. Downstream of the blade, we require two conditions - firstly the jump in normal velocity either side of the wake must vanish, and secondly, there can be no pressure jump across the wake. Due to the disparity between the images of the upper and lower trailing edge, which is equal to the mean flow circulation, some care is required with these latter two boundary conditions. The full set of boundary conditions is summarised and illustrated in figure 3.5.

As previously mentioned in (3.5), there are two trailing edges $c_{\phi}^{ \pm}$in the parametric $(\phi, \psi)$-space. In the application of the boundary conditions, we define a single trailing edge at $c_{\phi}=c_{\phi}^{+}$. This produces $\mathcal{O}(\epsilon)$ errors, which will be corrected in our asymptotic expansion later.

In the following analysis, we use $\Delta_{n}$ and $\Sigma_{n}$ to denote the difference and sum of a given quantity either side of the $n^{\text {th }}$ aerofoil's streamline.

\subsubsection{Upstream Boundary Condition}

The condition of no upstream discontinuities is simply

$$
\Delta_{n}[h](\phi)=0, \quad \text { for } \phi<0 .
$$




\subsubsection{No-Flux Boundary Conditions}

The flow on each side of the blade must satisfy the rigid surface condition (3.8) which, after ignoring $\mathcal{O}\left(\epsilon^{2}\right)$ terms, becomes

$$
\begin{aligned}
\frac{\partial h^{ \pm}}{\partial \psi}(\phi) & +\epsilon M_{\infty}^{2} \frac{\partial q^{ \pm}}{\partial \psi}(\phi) h^{ \pm}(\phi) \\
& =-\left[\frac{A_{n}}{\beta_{\infty}}\left(1-\epsilon M_{\infty}^{2} q^{ \pm}(\phi)+\epsilon \mathrm{i} k g^{ \pm}(\phi)\right)-\epsilon 2 \tilde{A}_{t} \mu^{ \pm}(\phi)\right] \mathrm{e}^{\mathrm{i} k \delta \phi}, \quad 0<\phi<c_{\phi},
\end{aligned}
$$

where we have assumed that $\epsilon k \ll 1$. This linearisation corresponds to step IIa in the road map.

\subsubsection{Wake Boundary Conditions}

We now derive the boundary conditions to be applied on the aerofoil wake. This corresponds to step IIb in the road map. The lower trailing edge lags behind the upper trailing edge in $(\phi, \psi)$-space by a distance of $-\epsilon \Gamma$, as illustrated in figures 3.4 and 3.5. Consequently, the condition of continuity of normal velocity in terms of our acoustic potential function is

$$
\frac{\partial h^{+}}{\partial \psi}(\phi) \mathrm{e}^{-\mathrm{i} k M_{\infty}^{2} \delta \phi}-\frac{\partial h^{-}}{\partial \psi}(\phi+\epsilon \Gamma) \mathrm{e}^{-\mathrm{i} k M_{\infty}^{2} \delta(\phi+\epsilon \Gamma)}=0, \quad \text { for } \phi>c_{\phi}
$$

We may Taylor expand the second term about $\phi$ to give

$$
\frac{\partial h^{+}}{\partial \psi}(\phi)-\left(\frac{\partial h^{-}}{\partial \psi}(\phi)+\epsilon \Gamma \frac{\partial^{2} h^{-}}{\partial \phi \partial \psi}(\phi)\right)\left(1-\mathrm{i} k M_{\infty}^{2} \delta \epsilon \Gamma\right)=0,
$$

where we have assumed that $\epsilon k M_{\infty}^{2} \ll 1$ and ignored $\mathcal{O}\left(\epsilon^{2}\right)$ terms. We accordingly obtain the condition on the jump in normal velocity in $(\phi, \psi)$-space as

$$
\Delta_{n}\left[\frac{\partial h}{\partial \psi}\right](\phi)=\epsilon \Gamma\left(\frac{\partial^{2} h^{-}}{\partial \phi \partial \psi}(\phi)-\mathrm{i} k M_{\infty}^{2} \delta \frac{\partial h^{-}}{\partial \psi}(\phi)\right), \quad \text { for } \phi>c_{\phi}
$$

Using a similar method, we reformulate the pressure continuity condition in $(\phi, \psi)$-space. Due to the lag in the lower trailing edge location, we may write

$$
p^{\prime+}(\phi)-p^{\prime-}(\phi+\epsilon \Gamma)=0, \quad \text { for } \phi>c_{\phi} \text {. }
$$


Taylor expanding the latter term about $\phi$ and ignoring terms of $\mathcal{O}\left(\epsilon^{2}\right)$ yields

$$
p^{\prime+}(\phi)-p^{\prime-}(\phi)-\epsilon \Gamma \frac{\partial p^{-}}{\partial \phi}(\phi)=0
$$

By substituting (3.9) and cancelling terms, we obtain

$$
\frac{\partial}{\partial \phi}\left(\Delta_{n}[h](\phi) \mathrm{e}^{-\mathrm{i} k \delta \phi}\right) \mathrm{e}^{\mathrm{i} k \phi}-\epsilon \Gamma \frac{\partial}{\partial \phi}\left(\frac{\partial}{\partial \phi}\left(h^{-}(\phi) \mathrm{e}^{-\mathrm{i} k \delta \phi}\right) \mathrm{e}^{\mathrm{i} k \phi}\right)=0 .
$$

We may integrate the above expression to obtain the condition on the jump in acoustic potential in $(\phi, \psi)$-space as

$$
\Delta_{n}[h](\phi)=2 \pi \mathrm{i} P \mathrm{e}^{\mathrm{i} k \delta \phi}+\epsilon \Gamma\left(\frac{\partial h^{-}}{\partial \phi}(\phi)-\mathrm{i} k M_{\infty}^{2} \delta h^{-}(\phi)\right), \quad \text { for } \phi>c_{\phi},
$$

where $P$ is a constant of integration that will be determined by the Wiener-Hopf technique.

\subsection{Acoustic Scattered Solution}

We now seek a regular perturbation expansion in $\epsilon$ for $h$ as

$$
h(\phi, \psi) \sim h_{0}(\phi, \psi)+\epsilon h_{1}(\phi, \psi)+\cdots
$$

This corresponds to step III in the road map. We now separate the $\mathcal{O}(1)$ and $\mathcal{O}(\epsilon)$ problems in order to generate an integral equation for the $\mathcal{O}(\epsilon)$ problem (road map step IV). The asymptotic expansion results in a pair of equations for the first and second order expansions of $h$ :

$$
\begin{array}{ll}
\mathcal{O}(1): & \mathscr{L}\left(h_{0}\right)=0, \\
\mathcal{O}(\epsilon): & \mathscr{L}\left(h_{1}\right)=\mathbb{S}(\phi, \psi),
\end{array}
$$

where

$$
\mathscr{L}(f)=\frac{\partial^{2} f}{\partial \phi^{2}}+\frac{\partial^{2} f}{\partial \psi^{2}}+k^{2} w^{2} f
$$


and the forcing term is

$$
\begin{gathered}
\mathbb{S}(\phi, \psi)=k S(\phi, \psi) \mathrm{e}^{\mathrm{i} k \Omega}-(\gamma+1) M_{\infty}^{4} \delta\left\{q\left[\frac{\partial^{2} h_{0}}{\partial \psi^{2}}+2 \mathrm{i} k \delta \frac{\partial h_{0}}{\partial \phi}+k^{2}\left(w^{2}+\delta^{2}\right) h_{0}\right]\right. \\
\left.-\frac{\partial q}{\partial \phi}\left[\frac{\partial h_{0}}{\partial \phi}-\mathrm{i} k \delta h_{0}\right]\right\}+2 k^{2} w^{2} \beta_{\infty}^{2} q h_{0} .
\end{gathered}
$$

The condition of no discontinuities upstream of the cascade simply becomes

$$
\begin{aligned}
& \Delta_{0}\left[h_{0}\right](\phi)=0, \quad \text { for } \phi<0 \text {, } \\
& \Delta_{0}\left[h_{1}\right](\phi)=0, \quad \text { for } \phi<0 \text {. }
\end{aligned}
$$

We may use (3.15) to write the the no-flux conditions (3.11) in terms of our perturbation expansion. In order to simplify the Wiener-Hopf analysis, we separate the boundary data into symmetric and anti-symmetric parts. This enables us to express the boundary data striclty in terms of its contribution to the sum $\left(\Sigma_{0}\right)$ or difference $\left(\Delta_{0}\right)$ either side of the blade. To further facilitate the Wiener-Hopf analysis, we expand the boundary data in terms of a Fourier series (step IVa of the road map). The anti-symmetric boundary data becomes

$$
\begin{array}{ll}
\Delta_{0}\left[\frac{\partial h_{0}}{\partial \psi}\right](\phi)=0, & 0<\phi<c_{\phi}, \\
\Delta_{0}\left[\frac{\partial h_{1}}{\partial \psi}\right](\phi)=\sum_{l=-\infty}^{\infty} c_{\Delta, l} \mathrm{e}^{-\mathrm{i} \kappa_{l}^{-} \phi}, & 0<\phi<c_{\phi},
\end{array}
$$

where $\kappa_{l}^{-}=-k \delta+l \pi / c_{\phi}$. The Fourier coefficients are defined using (3.11) as

$$
c_{\Delta, l}=\frac{1}{c_{\phi}} \int_{0}^{c_{\phi}} \Delta_{0}\left[\frac{\partial h_{1}}{\partial \psi}\right](\phi) \cos \left(\frac{n \pi \phi}{c_{\phi}}\right) \mathrm{e}^{\mathrm{i} \kappa_{0}^{-} \phi} \mathrm{d} \phi,
$$

which also accounts for the $\epsilon M_{\infty}^{2} \frac{\partial q^{ \pm}}{\partial \psi}(\phi) h_{0}^{ \pm}(\phi)$ terms taken to the right-hand side of (3.11) which may be considered as known due to the perturbation expansion. Note that the periodicity of this Fourier series is $2 c_{\phi}$ to ensure a good approximation at the leading and trailing edges.

Similarly, we may write the symmetric data in the no-flux condition (3.11) as

$$
\Sigma_{0}\left[\frac{\partial h_{0}}{\partial \psi}\right](\phi)=-2 \frac{A_{2}}{\beta_{\infty}} \mathrm{e}^{\mathrm{i} k \delta \phi}, \quad 0<\phi<c_{\phi}
$$




$$
\Sigma_{0}\left[\frac{\partial h_{1}}{\partial \psi}\right](\phi)=\sum_{l=-\infty}^{\infty} c_{\Sigma, l} \mathrm{e}^{-\mathrm{i} \kappa_{l}^{-} \phi}, \quad 0<\phi<c_{\phi}
$$

where the Fourier coefficients are defined using (3.11) as

$$
c_{\Sigma, l}=\frac{1}{c_{\phi}} \int_{0}^{c_{\phi}} \Sigma_{0}\left[\frac{\partial h_{1}}{\partial \psi}\right](\phi) \cos \left(\frac{n \pi \phi}{c_{\phi}}\right) \mathrm{e}^{\mathrm{i} \kappa_{0}^{-} \phi} \mathrm{d} \phi .
$$

Combining the anti-symmetric (3.20.a, 3.20.b) and symmetric (3.21.a, 3.21.b) data yields the original no-flux condition (3.11).

By applying the perturbation expansion to continuity of normal velocity across the wake (3.13) and equating terms of equal order gives

$$
\begin{aligned}
& \Delta_{0}\left[\frac{\partial h_{0}}{\partial \psi}\right](\phi)=0 \\
& \Delta_{0}\left[\frac{\partial h_{1}}{\partial \psi}\right](\phi)=\Gamma\left(\frac{\partial^{2} h^{-}}{\partial \phi \partial \psi}(\phi)-\mathrm{i} k M_{\infty}^{2} \delta \frac{\partial h^{-}}{\partial \psi}(\phi)\right), \quad \phi>c_{\phi} .
\end{aligned}
$$

Using (3.15) allows us to write the continuity of pressure condition (3.14) as

$$
\begin{array}{ll}
\Delta_{0}\left[h_{0}\right](\phi)=2 \pi \mathrm{i} P_{0} \mathrm{e}^{-\mathrm{i} \kappa_{0}^{-} \phi}, & \phi>c_{\phi}, \\
\Delta_{0}\left[h_{1}\right](\phi)=2 \pi \mathrm{i} P_{1} \mathrm{e}^{-\mathrm{i} \kappa_{0}^{-} \phi}+\Gamma\left(\frac{\partial h^{-}}{\partial \phi}(\phi)-\mathrm{i} k M_{\infty}^{2} \delta h^{-}(\phi)\right), & \phi>c_{\phi},
\end{array}
$$

where $P_{0}$ and $P_{1}$ are constants of integration to be determined by the unsteady Kutta condition (Ayton et al., 2016).

\subsubsection{Solution to $\mathcal{O}(1)$ Problem}

The $\mathcal{O}(1)$ system of equations (3.16.a, 3.19.a, 3.20.a, 3.21.a, 3.22.a, 3.23.a) was considered by Glegg (1999) and Posson et al. (2010b). This corresponds to step IVb in the road map. The solution is written in terms of the $\phi$-Fourier transform of the jump in potential either side of the blade and wake:

$$
D_{0}(\gamma)=\frac{1}{2 \pi} \int_{0}^{\infty} \Delta_{0}[h](\phi) \mathrm{e}^{\mathrm{i} \gamma \phi} \mathrm{d} \phi
$$


and the solution is

$$
\begin{gathered}
D_{0}(\gamma)=\frac{-\mathrm{i} A_{n}}{\beta_{\infty}(2 \pi)^{2}\left(\gamma-\kappa_{0}^{-}\right) J_{+}(\gamma) J_{-}\left(\kappa_{0}^{-}\right)}-\sum_{n=0}^{\infty} \frac{\left(\mathscr{A}_{0, n}+\mathscr{C}_{0, n}\right) \mathrm{e}^{\mathrm{i}\left(\gamma-\theta_{n}^{-}\right) c_{\phi}}}{\mathrm{i}\left(\gamma-\kappa_{0}^{-}\right)\left(\gamma-\theta_{n}^{-}\right)} \cdot \frac{J_{-}\left(\theta_{n}^{-}\right)}{J_{-}(\gamma)} \\
-\sum_{n=0}^{\infty} \frac{\mathscr{B}_{0, n}}{\left(\gamma-\theta_{n}^{+}\right)} \cdot \frac{J_{+}\left(\theta_{n}^{+}\right)}{J_{+}(\gamma)}
\end{gathered}
$$

where all undefined terms may be found in the appendices. The function $D_{0}(\gamma)$ was found by Glegg (1999), and the Fourier transform was inverted to provide the acoustic field in the upstream and downstream regions. The acoustic field in the inter-blade region was found by Posson et al. (2010b) by choosing an appropriate contour of integration dependent on the region in the channel where the acoustic field was to be evaluated.

\subsubsection{Solution to $\mathcal{O}(\epsilon)$ Problem}

Having solved the $\mathcal{O}(1)$ equation, we now move on to the $\mathcal{O}(\epsilon)$ equation. In particular, we seek to generate an integral equation that will be amenable to the Wiener-Hopf method. Following Glegg (1999), we seek a solution using integral transforms. Currently, $h_{1}$ has a discontinuity across each blade and wake and consequently its derivatives are not integrable. Therefore, we introduce generalised derivatives (Lighthill, 1958) such that the Laplacian operator becomes

$$
\nabla^{2} h_{1}=\tilde{\nabla}^{2} h_{1}-\sum_{n=-\infty}^{\infty} \Delta_{n}\left[h_{1}\right](\phi) \delta^{\prime}\left(\psi-n s_{\phi}\right)-\sum_{n=-\infty}^{\infty} \Delta_{n}\left[\frac{\partial h_{1}}{\partial \psi}\right](\phi) \delta\left(\psi-n s_{\phi}\right),
$$

where $\tilde{\nabla}^{2}$ represents the Laplacian operator with discontinuities removed, the second term on the right represents the discontinuity of acoustic potential either side of the blade and wake, and the third term on the right represents the discontinuity of normal velocity either side of the blade and wake. This step corresponds to step IVc in the road map. Crucially, the above is expressed in the $(\phi, \psi)$-space, not in physical space.

In Glegg (1999), the last term in (3.25) disappears on the aerofoil surfaces because the blades are infinitesimally thin, so there is no jump in the normal velocity either side of the blade. However, although the blades are "thin" in our $(\phi, \psi)$-space, they have been converted from thick blades in physical space, and therefore will induce some mass flux in order to satisfy the no-flux condition in the physical domain. Also, in Glegg (1999), the last term in (3.25) disappears along the wake, because it is not a source of mass injection. Whilst this condition is true in our case in physical space, the mean flow circulation has induced a shift in the upper and lower trailing edges in the $(\phi, \psi)$-space, so some mass 
injection is required in the transformed space in order to have continuous normal velocity in the physical space.

The jump in the acoustic potential across a blade-streamline is unknown and must be found as part of the solution. Conversely, the jump $\psi$-derivative of the acoustic potential is known in terms of mean flow quantities and the $\mathcal{O}(1)$ solution.

As is typical in cascade acoustics problems, we note that the periodicity of the background flow and gust furnishes a quasi-periodic condition on the physical unsteady potential:

$$
G^{\prime}\left(\phi+n d_{\phi}, \psi+n s_{\phi}\right)=G^{\prime}(\phi, \psi) \mathrm{e}^{\mathrm{i} n \sigma_{\phi}},
$$

where $\sigma_{\phi}$ is the inter-blade phase angle of the gust in $(\phi, \psi)$-space so that $\sigma_{\phi}=k\left(d_{\phi}+k_{n} s_{\phi}\right)$. The inter-blade phase angle in the physical domain, $\sigma$, is obtained using (3.4). In terms of the modified potential, this quasi-periodic condition becomes

$$
h_{1}\left(\phi+n d_{\phi}, \psi+n s_{\phi}\right)=h_{1}(\phi, \psi) \mathrm{e}^{\mathrm{i} n \sigma_{\phi}^{\prime}}
$$

where the modified inter-blade phase angle is $\sigma_{\phi}^{\prime}=k\left(\delta d_{\phi}+k_{n} s_{\phi}\right)$. We will use this relation repeatedly to reduce the problem from an infinite cascade to a strip of finite height. For example, by inserting (3.26) into the expression for the generalised Laplacian (3.25), we may rewrite the $\mathcal{O}(\epsilon)$ governing equation (3.16.b) as

$$
\begin{aligned}
\frac{\partial^{2} h_{1}}{\partial \phi^{2}}+\frac{\partial^{2} h_{1}}{\partial \psi^{2}}+k^{2} w^{2} h_{1}=\mathbb{S}(\phi, \psi) & +\sum_{n=-\infty}^{\infty} \Delta_{0}\left[h_{1}\right]\left(\phi-n d_{\phi}\right) \delta^{\prime}\left(\psi-n s_{\phi}\right) \mathrm{e}^{\mathrm{i} n \sigma_{\phi}^{\prime}} \\
& +\sum_{n=-\infty}^{\infty} \Delta_{0}\left[\frac{\partial h_{1}}{\partial \psi}\right]\left(\phi-n d_{\phi}\right) \delta\left(\psi-n s_{\phi}\right) \mathrm{e}^{\mathrm{i} n \sigma_{\phi}^{\prime}}
\end{aligned}
$$

We define the Fourier integral transform and its inverse as

$$
\begin{aligned}
& F(\gamma, \mu)=\frac{1}{(2 \pi)^{2}} \int_{-\infty}^{\infty} \int_{-\infty}^{\infty} f(\phi, \psi) \mathrm{e}^{\mathrm{i} \gamma \phi+\mathrm{i} \mu \psi} \mathrm{d} \phi \mathrm{d} \psi, \\
& f(\phi, \psi)= \\
& \int_{-\infty}^{\infty} \int_{-\infty}^{\infty} F(\gamma, \mu) \mathrm{e}^{-\mathrm{i} \gamma \phi-\mathrm{i} \mu \psi} \mathrm{d} \gamma \mathrm{d} \mu .
\end{aligned}
$$

Applying the transform to the left-hand side of (3.27) yields

$$
\left(-\gamma^{2}-\mu^{2}+k^{2} w^{2}\right) H_{1}(\gamma, \mu)=s_{0}(\gamma, \mu)+\frac{1}{2 \pi} \sum_{n=-\infty}^{\infty}\left\{-\mathrm{i} \mu D_{1}(\gamma)+G(\gamma)\right\} \mathrm{e}^{\mathrm{i} n\left(\sigma_{\phi}^{\prime}+\gamma d_{\phi}+\mu s_{\phi}\right)}
$$


where

$$
\begin{aligned}
s_{0}(\gamma, \mu) & =\frac{1}{(2 \pi)^{2}} \int_{-\infty}^{\infty} \int_{-\infty}^{\infty} \mathbb{S}(\phi, \psi) \mathrm{e}^{\mathrm{i} \gamma \phi+\mathrm{i} \mu \psi} \mathrm{d} \phi \mathrm{d} \psi, \\
D_{1}(\gamma) & =\frac{1}{2 \pi} \int_{0}^{\infty} \Delta_{0}\left[h_{1}\right](\phi) \mathrm{e}^{\mathrm{i} \gamma \phi} \mathrm{d} \phi \\
G(\gamma) & =\frac{1}{2 \pi} \int_{0}^{\infty} \Delta_{0}\left[\frac{\partial h_{1}}{\partial \psi}\right](\phi) \mathrm{e}^{\mathrm{i} \gamma \phi} \mathrm{d} \phi .
\end{aligned}
$$

The first of these expressions (3.29) is known in terms of the background flow quantities and $\mathcal{O}(1)$ solution. The last of these expressions (3.31) is also known, and may be evaluated using the no-flux condition on the blade (3.21.b) and continuity of normal velocity either side of the wake (3.22.b, 3.53) to obtain

$$
\begin{aligned}
G(\gamma) & =\sum_{l=-\infty}^{\infty} \frac{c_{\Delta, l}\left((-1)^{l} \mathrm{e}^{\mathrm{i}\left(\gamma-\kappa_{0}^{-}\right) c_{\phi}}-1\right)}{2 \pi \mathrm{i}\left(\gamma-\kappa_{l}^{-}\right)} \\
& +\frac{\Gamma}{2 \pi}\left(\sum_{ \pm} \frac{ \pm \zeta_{\kappa_{0}^{-}} \mathscr{H}_{0, \kappa_{0}^{-}}^{ \pm \prime} \mathrm{e}^{\mathrm{i}\left(\gamma-\kappa_{0}^{-}\right) c_{\phi}}}{\gamma-\kappa_{0}^{-}}+\sum_{m=-\infty}^{\infty} \frac{\zeta_{m}^{-} \mathscr{H}_{0, m}^{\prime} \mathrm{e}^{\mathrm{i}\left(\gamma-\lambda_{m}^{-}\right) c_{\phi}}}{\gamma-\lambda_{m}^{-}}\right) .
\end{aligned}
$$

The problem is now to find $D_{1}(\gamma)$ - the Fourier transform of the jump in acoustic potential either side of the blade and wake. To do so, we must obtain an expression for the sum of the normal derivative in order to apply the relevant boundary conditions.

We now invert the Fourier transform in (3.28) to obtain an integral equation into which we may insert the known boundary data:

$$
\begin{aligned}
h_{1}(\phi, \psi) & =\int_{-\infty}^{\infty} \int_{-\infty}^{\infty} \frac{\mathrm{e}^{-\mathrm{i} \gamma \phi-\mathrm{i} \mu \psi}}{k^{2} w^{2}-\mu^{2}-\gamma^{2}} \times \\
& \left\{s_{0}(\gamma, \mu)+\frac{1}{2 \pi} \sum_{n=-\infty}^{\infty}\left[-\mathrm{i} \mu D_{1}(\gamma)+G(\gamma)\right] \mathrm{e}^{\mathrm{i} n\left(\sigma_{\phi}^{\prime}+\gamma d_{\phi}+\mu s_{\phi}\right)}\right\} \mathrm{d} \gamma \mathrm{d} \mu .
\end{aligned}
$$

The $\mu$ integral can be carried out using contour integration by writing $\zeta=\sqrt{k^{2} w^{2}-\gamma^{2}}$ where the branch cut is taken such that $\operatorname{Im}[\zeta]>0$ when $\gamma$ is in a strip in the spectral plane for the Wiener-Hopf method. This gives

$$
\begin{aligned}
h_{1}(\phi, \psi)=\int_{-\infty}^{\infty}\left\{s_{1}(\gamma)+\frac{1}{2} \sum_{n=-\infty}^{\infty}[\right. & -D_{1}(\gamma) \operatorname{sgn}\left(n s_{\phi}-\psi\right) \\
& \left.\left.+\frac{G(\gamma)}{\mathrm{i} \zeta}\right] \mathrm{e}^{\mathrm{i} n\left(\sigma_{\phi}^{\prime}+\gamma d_{\phi}\right)+\mathrm{i} \zeta\left|n s_{\phi}-\psi\right|}\right\} \mathrm{e}^{-\mathrm{i} \gamma \phi} \mathrm{d} \gamma
\end{aligned}
$$


where we have used the quasi-periodicity (3.26) of the source terms to write

$$
\begin{aligned}
s_{1}(\gamma, \psi) & =\frac{1}{4 \mathrm{i} \pi \zeta} \int_{0}^{s_{\phi}} S_{\phi}\left(\gamma, \psi_{1}\right) \sum_{n=-\infty}^{\infty} \mathrm{e}^{\mathrm{i} n\left(\sigma_{\phi}^{\prime}+\gamma d_{\phi}\right)+\mathrm{i} \zeta\left|\psi_{1}+n s_{\phi}-\psi\right|} \mathrm{d} \psi_{1}, \\
S_{\phi}\left(\gamma, \psi_{1}\right) & =\int_{-\infty}^{\infty} \mathbb{S}\left(\phi_{1}, \psi_{1}\right) \mathrm{e}^{\mathrm{i} \gamma \phi_{1}} \mathrm{~d} \phi_{1} .
\end{aligned}
$$

We differentiate (3.33) with respect to $\psi$ and take the limit $\psi \rightarrow 0^{ \pm}$to obtain

$$
\frac{\partial h_{1}^{ \pm}}{\partial \psi}(\phi)=2 \pi \int_{-\infty}^{\infty}\left\{-D_{1}(\gamma) j(\gamma)+G(\gamma)[k(\gamma) \mp 1]+S(\gamma)\right\} \mathrm{e}^{-\mathrm{i} \gamma \phi} \mathrm{d} \gamma
$$

where

$$
\begin{aligned}
j(\gamma) & =\frac{\mathrm{i} \zeta}{4 \pi} \sum_{n=-\infty}^{\infty} \mathrm{e}^{\mathrm{i} n\left(\sigma_{\phi}^{\prime}+\gamma d_{\phi}\right)+\mathrm{i} \zeta\left|n s_{\phi}\right|}=\frac{\zeta}{4 \pi} \cdot \frac{\sin \left(\zeta s_{\phi}\right)}{\cos \left(\zeta s_{\phi}\right)-\cos \left(\gamma d_{\phi}+\sigma_{\phi}^{\prime}\right)} \\
k(\gamma) & =\frac{1}{4 \pi} \sum_{\substack{n=-\infty \\
n \neq 0}}^{\infty} \operatorname{sgn}\left(n s_{\phi}\right) \mathrm{e}^{\mathrm{i} n\left(\sigma_{\phi}^{\prime}+\gamma d_{\phi}\right)+\mathrm{i} \zeta\left|n s_{\phi}\right|}=\frac{1}{4 \pi \mathrm{i}} \cdot \frac{\sin \left(\gamma d_{\phi}+\sigma_{\phi}^{\prime}\right)}{\cos \left(\zeta s_{\phi}\right)-\cos \left(\gamma d_{\phi}+\sigma_{\phi}^{\prime}\right)} \\
S(\gamma) & =-\frac{1}{8 \pi^{2}} \int_{0}^{s_{\phi}} S_{\phi}\left(\gamma, \psi_{1}\right) \sum_{n=-\infty}^{\infty} \operatorname{sgn}\left(\psi_{1}+n s_{\phi}\right) \mathrm{e}^{\mathrm{i} n\left(\sigma_{\phi}^{\prime}+d_{\phi} \gamma\right)} \mathrm{e}^{\mathrm{i} \zeta\left|\psi_{1}+n s_{\phi}\right|} \mathrm{d} \psi_{1} \\
& =-\frac{1}{8 \pi^{2}} \int_{0}^{s_{\phi}} S_{\phi}\left(\gamma, \psi_{1}\right) \sum_{ \pm} \frac{\mathrm{e}^{ \pm \mathrm{i} \zeta \psi_{1}}}{1-\mathrm{e}^{\mathrm{i}\left( \pm \zeta s_{\phi}+\sigma_{\phi}^{\prime}+\gamma d_{\phi}\right)}} \mathrm{d} \psi_{1}
\end{aligned}
$$

and we have used $(3.134,3.135,3.136)$ in the appendix to simplify the infinite sums. The analysis of the transforms of these source terms is carried out in appendix 3.E where the source terms are decomposed into Fourier-type series in the upstream, downstream and inter-blade regions. The modal expansion corresponds to step IVd in the road map.

We note that if we take the difference of formulae (3.35) and use contour integration to calculate the resulting integral, we recover the conditions for the jump in normal velocity (3.20.b) and (3.23.b), as expected. Conversely, summing formulae (3.35) gives the governing integral equation for the acoustic field

$$
\Sigma_{0}\left[\frac{\partial h_{1}}{\partial \psi}\right](\phi)=4 \pi \int_{-\infty}^{\infty}\left\{-D_{1}(\gamma) j(\gamma)+G(\gamma) k(\gamma)+S(\gamma)\right\} \mathrm{e}^{-\mathrm{i} \gamma \phi} \mathrm{d} \gamma
$$

We see that $h_{1}$ and its normal derivative $\partial h_{1} / \partial \psi$ can be expressed solely in terms of the unknown $D_{1}(\gamma)$. Thus our problem is now to find $D_{1}(\gamma)$ which solves the integral equation (3.39) subject to (3.19.b), (3.20.b) and (3.23.b). 
The linearity of the governing equation and boundary conditions allow the solution to be split into four parts:

$$
h_{1}(\phi, \psi)=h_{1, \Sigma}(\phi, \psi)+h_{1, \Delta}(\phi, \psi)+h_{1, \Gamma}(\phi, \psi)+h_{1, S}(\phi, \psi) \text {. }
$$

There are several reasons for this splitting, chiefly that each component can be broadly attributed to a feature of the background flow and distortion of the $\mathcal{O}(1)$ acoustic field. Firstly, $h_{1, \Sigma}$ depends predominantly on the value of the sum of the normal velocity either side of the blade. In chapter 2 , it was shown that this sum is a function of camber and angle of attack, and thickness terms generally do not appear. Therefore, $h_{1, \Sigma}$ may roughly be viewed as the influence of camber and angle of attack on the acoustic field. Secondly, $h_{1, \Delta}$ depends predominantly on the jump in normal velocity. In the flat plate case, this term disappears due to the symmetry of the blades, whereas in the present case this term can be viewed as a mass source required to satisfy the no-flux condition. Similarly, this jump was shown in chapter 2 to depend predominantly on aerofoil thickness, and therefore $h_{1, \Delta}$ may be roughly viewed as the influence of thickness on the acoustic field. However, the separation of geometrical effects is not total, as both $h_{1, \Sigma}$ and $h_{1, \Delta}$ include terms proportional to the drift function, $g$, which does not have a straightforward dependence on aerofoil geometry. Thirdly, $h_{1, \Gamma}$ arises due to the circulation induced on each blade by the background flow. The sum and jump in its normal velocity vanish on the blade, but it has a specified pressure jump (in $(\phi, \psi)$-space) across the wake. This jump is necessary to ensure no pressure jump in the physical $(x, y)$-space. This disparity arises due to the circulation around the blade in mean flow. Fourthly, and finally, $h_{1, S}$ depends on the source terms in the equation only. Similarly to $h_{1, \Gamma}$, the sum and jump in its normal velocity on the blades disappear, but unlike the previous solutions it has a forcing term in the governing Helmholtz equation. This forcing depends on the background flow, and the distortion of the $\mathcal{O}(1)$ solution by the background flow.

In addition to the above physical reasons, this splitting substantially reduces the mathematical complexity of the problem. Each component induces its own system of Wiener-Hopf problems, and these may be solved separately. The Kutta condition is enforced individually for each component. When the Fourier transform is inverted, the components may be inverted individually, which yields slightly more tractable expressions, each of which may be verified independently.

In the following sections, the governing equations and boundary conditions are stated for each of the four components and we solve for the relevant $D_{1, x}(\gamma)$ (defined in an analogous way to (3.30)). The Fourier transform of the jump in potential either side of the blade is solved using a similar Wiener-Hopf method to Glegg (1999) and the solution 
is stated in each case. The analysis is carried out in section 3.B of the appendices, where the definitions of all new parameters may be found.

\subsubsection{1 $h_{1, \Sigma}$ Problem and Solution (Road Map Step Va)}

The governing equation is

$$
\Sigma_{0}\left[\frac{\partial h_{1, \Sigma}}{\partial \psi}\right](\phi)=-4 \pi \int_{-\infty}^{\infty} D_{1, \Sigma}(\gamma) j(\gamma) \mathrm{e}^{-\mathrm{i} \gamma \phi} \mathrm{d} \gamma
$$

subject to

$$
\begin{aligned}
\Delta_{0}\left[h_{1, \Sigma}\right](\phi) & =0, & \phi<0 ; \\
\Sigma_{0}\left[\frac{\partial h_{1, \Sigma}}{\partial \psi}\right](\phi) & =\sum_{l=-\infty}^{\infty} c_{\Sigma, l} \mathrm{e}^{-\mathrm{i} \kappa_{l}^{-} \phi}, & 0<\phi<c_{\phi} ; \\
\Delta_{0}\left[\frac{\partial h_{1, \Sigma}}{\partial \psi}\right](\phi) & =0, & 0<\phi<c_{\phi} ; \\
\Delta_{0}\left[\frac{\partial h_{1, \Sigma}}{\partial \psi}\right](\phi) & =0, & \phi>c_{\phi} ; \\
\Delta_{0}\left[h_{1, \Sigma}\right](\phi) & =2 \pi \mathrm{i} P_{1, \Sigma} \mathrm{e}^{-\mathrm{i} \kappa_{0}^{-} \phi}, & \phi>c_{\phi} .
\end{aligned}
$$

This problem corresponds to the homogeneous Helmholtz equation with symmetric boundary conditions on each blade (3.21.b), along with some unknown vortex shedding into the wake. The decomposition of the boundary data into a Fourier series means that the method of solution is relevant to a range of applications where arbitrary boundary data is prescribed. The method of solution is described in appendix 3.B.2. The solution is

$$
\begin{aligned}
D_{1, \Sigma}(\gamma)= & \sum_{l=-\infty}^{\infty} \frac{\mathscr{T}_{1, \Sigma, l}}{\gamma-\kappa_{l}^{-}} \cdot \frac{1}{J_{+}(\gamma)}-\sum_{l=-\infty}^{\infty} \frac{\mathcal{S}_{1, \Sigma, l} \mathrm{e}^{\mathrm{i}\left(\gamma-\kappa_{l}^{-}\right) c_{\phi}}}{\mathrm{i}\left(\gamma-\kappa_{0}^{-}\right)\left(\gamma-\kappa_{l}^{-}\right)} \cdot \frac{J_{-}\left(\kappa_{l}^{-}\right)}{J_{-}(\gamma)} \\
& -\sum_{n=0}^{\infty} \frac{\mathscr{B}_{1, \Sigma, n}}{\gamma-\theta_{n}^{+}} \cdot \frac{J_{+}\left(\theta_{n}^{+}\right)}{J_{+}(\gamma)}-\sum_{n=0}^{\infty} \frac{\left(\mathscr{A}_{1, \Sigma, n}+\mathscr{C}_{1, \Sigma, n}\right) \mathrm{e}^{\mathrm{i} c_{\phi}\left(\gamma-\theta_{n}^{-}\right)}}{\mathrm{i}\left(\gamma-\kappa_{0}^{-}\right)\left(\gamma-\theta_{n}^{-}\right)} \cdot \frac{J_{-}\left(\theta_{n}^{-}\right)}{J_{-}(\gamma)} .
\end{aligned}
$$

\subsubsection{2 $h_{1, \Delta}$ Problem and Solution (Road Map Step Vb)}

The governing equation is

$$
\Sigma_{0}\left[\frac{\partial h_{1, \Delta}}{\partial \psi}\right](\phi)=4 \pi \int_{-\infty}^{\infty}\left\{-D_{1, \Delta}(\gamma) j(\gamma)+G_{\Delta}(\gamma) k(\gamma)\right\} \mathrm{e}^{-\mathrm{i} \gamma \phi} \mathrm{d} \gamma
$$


where

$$
G_{\Delta}(\gamma)=\sum_{l=-\infty}^{\infty} \frac{c_{\Delta, l}\left((-1)^{l} \mathrm{e}^{\mathrm{i}\left(\gamma-\kappa_{0}^{-}\right) c_{\phi}}-1\right)}{2 \pi \mathrm{i}\left(\gamma-\kappa_{l}^{-}\right)}
$$

This integral equation is solved subject to

$$
\begin{array}{rlr}
\Delta_{0}\left[h_{1, \Delta}\right](\phi) & =0, & \phi<0 ; \\
\Sigma_{0}\left[\frac{\partial h_{1, \Delta}}{\partial \psi}\right](\phi) & =0, & 0<\phi<c_{\phi} ; \\
\Delta_{0}\left[\frac{\partial h_{1, \Delta}}{\partial \psi}\right](\phi) & =\sum_{l=-\infty}^{\infty} q_{l} \mathrm{e}^{-\mathrm{i} \kappa_{l}^{-} \phi}, & 0<\phi<c_{\phi} ; \\
\Delta_{0}\left[\frac{\partial h_{1, \Delta}}{\partial \psi}\right](\phi) & =0, & \phi>c_{\phi} ; \\
\Delta_{0}\left[h_{1, \Delta}\right](\phi) & =2 \pi \mathrm{i} P_{1, \Delta} \mathrm{e}^{-\mathrm{i} \kappa_{0}^{-} \phi}, & \phi>c_{\phi} .
\end{array}
$$

This problem corresponds to the homogeneous Helmholtz equation with anti-symmetric boundary conditions along the blade. This term is not present in the flat plate case, but the blade geometry induces a difference between upper and lower surface velocities, which generates anti-symmetric terms in the boundary data (3.20.b). The method of solution is described in appendix 3.B.3. The solution is

$$
\begin{aligned}
D_{1, \Delta}(\gamma)= & \sum_{l=-\infty}^{\infty} \frac{\mathscr{T}_{1, \Delta, l}}{\gamma-\kappa_{l}^{-}} \cdot \frac{1}{J_{+}(\gamma)}-\sum_{l=-\infty}^{\infty} \frac{\mathcal{S}_{1, \Delta, l} \mathrm{e}^{\mathrm{i}\left(\gamma-\kappa_{l}^{-}\right) c_{\phi}}}{\mathrm{i}\left(\gamma-\kappa_{0}^{-}\right)\left(\gamma-\kappa_{l}^{-}\right)} \cdot \frac{J_{-}\left(\kappa_{l}^{-}\right)}{J_{-}(\gamma)} \\
& +\sum_{m=-\infty}^{\infty} \frac{\mathscr{R}_{1, \Delta, m}}{\gamma-\lambda_{m}^{-}} \cdot \frac{1}{J_{+}(\gamma)}-\sum_{m=-\infty}^{\infty} \frac{\mathscr{U}_{1, \Delta, m}}{\mathrm{i}\left(\gamma-\kappa_{0}^{-}\right)\left(\gamma-\lambda_{m}^{+}\right)} \cdot \frac{1}{J_{-}(\gamma)} \\
& -\sum_{n=0}^{\infty} \frac{\mathscr{B}_{1, \Delta, n}}{\gamma-\theta_{n}^{+}} \cdot \frac{J_{+}\left(\theta_{n}^{+}\right)}{J_{+}(\gamma)}-\sum_{n=0}^{\infty} \frac{\left(\mathscr{A}_{1, \Delta, n}+\mathscr{C}_{1, \Delta, n}\right) \mathrm{e}^{\mathrm{i} c_{\phi}\left(\gamma-\theta_{n}^{-}\right)}}{\mathrm{i}\left(\gamma-\kappa_{0}^{-}\right)\left(\gamma-\theta_{n}^{-}\right)} \cdot \frac{J_{-}\left(\theta_{n}^{-}\right)}{J_{-}(\gamma)}
\end{aligned}
$$

\subsubsection{3 $h_{1, \Gamma}$ Problem and Solution (Road Map Step Vc)}

The governing equation is

$$
\Sigma_{0}\left[\frac{\partial h_{1, \Gamma}}{\partial \psi}\right](\phi)=4 \pi \int_{-\infty}^{\infty}\left\{-D_{1, \Gamma}(\gamma) j(\gamma)+G_{\Gamma}(\gamma) k(\gamma)\right\} \mathrm{e}^{-\mathrm{i} \gamma \phi} \mathrm{d} \gamma
$$


where

$$
G_{\Gamma}(\gamma)=\frac{\Gamma}{2 \pi}\left(\sum_{m=-\infty}^{\infty} \frac{\zeta_{m}^{-} \mathscr{H}_{0, m}^{\prime} \mathrm{e}^{\mathrm{i}\left(\gamma-\lambda_{m}^{-}\right) c_{\phi}}}{\gamma-\lambda_{m}^{-}}+\sum_{ \pm} \frac{ \pm \zeta_{\kappa_{0}^{-}} \mathscr{H}_{0, \kappa_{0}^{-}}^{ \pm \prime} \mathrm{e}^{\mathrm{i}\left(\gamma-\kappa_{0}^{-}\right) c_{\phi}}}{\gamma-\kappa_{0}^{-}}\right)
$$

This integral equation is solved subject to

$$
\begin{array}{rlrl}
\Delta_{0}\left[h_{1, \Gamma}\right](\phi) & =0, & \phi<0 ; \\
\Sigma_{0}\left[\frac{\partial h_{1, \Gamma}}{\partial \psi}\right](\phi) & =0, & 0<\phi<c_{\phi} ; \\
\Delta_{0}\left[\frac{\partial h_{1, \Gamma}}{\partial \psi}\right](\phi) & =0, & 0<\phi<c_{\phi} ; \\
\Delta_{0}\left[\frac{\partial h_{1, \Gamma}}{\partial \psi}\right](\phi) & = & \\
\Gamma\left(\sum_{ \pm} \mp \mathrm{i} \zeta_{\kappa_{0}^{-}} \mathscr{H}_{0, \kappa_{0}^{-}}^{ \pm} \mathrm{e}^{-\mathrm{i} \kappa_{0}^{-} \phi}-\mathrm{i} \sum_{m=-\infty}^{\infty} \zeta_{m}^{-} \mathscr{H}_{0, m}^{\prime} \mathrm{e}^{-\mathrm{i} \lambda_{m}^{-} \phi}\right) & \phi>c_{\phi} ; \\
\Delta_{0}\left[h_{1, \Gamma}\right](\phi) & = & \\
2 \pi \mathrm{i} P_{1, \Gamma} \mathrm{e}^{-\mathrm{i} \kappa_{0}^{-} \phi}+\Gamma\left(\mathscr{H}_{0, \kappa_{0}^{ \pm}}^{ \pm \prime} \mathrm{e}^{-\mathrm{i} \kappa_{0}^{-} \phi}+\sum_{m=-\infty}^{\infty} \mathscr{H}_{0, m}^{\prime} \mathrm{e}^{-\mathrm{i} \lambda_{m}^{-} \phi}\right), & \phi>c_{\phi} .
\end{array}
$$

This problem corresponds to the homogeneous Helmholtz equation with homogeneous boundary conditions on the blade and prescribed data along the wake (3.22.b, 3.23.b) that necessitates no flux and no pressure jump across the wake in physical space. In this case it happens that this prescribed data can be expressed in a Fourier series of the acoustic modes of the cascade, but any type of Fourier series could have been considered. However, the acoustic potential function is not fully specified along the wake, and the unknown constant $P_{1, \Gamma}$ still needs to be determined via the Wiener-Hopf method to specify the strength of vortex shedding into the wake. The method of solution is described in appendix 3.B.4. The solution is

$$
\begin{aligned}
D_{1, \Gamma}(\gamma)= & -\frac{\Gamma}{2 \pi \mathrm{i}} \cdot \sum_{m=-\infty}^{\infty} \frac{\left(\lambda_{m}^{-}-\kappa_{0}^{-}\right) \mathscr{H}_{0, m}^{\prime} \mathrm{e}^{\mathrm{i}\left(\gamma-\lambda_{m}^{-}\right) c_{\phi}}}{\left(\gamma-\kappa_{0}^{-}\right)\left(\gamma-\lambda_{m}^{-}\right)} \cdot \frac{J_{-}\left(\lambda_{m}^{-}\right)}{J_{-}(\gamma)} \\
& -\sum_{m=-\infty}^{\infty} \frac{\mathscr{U}_{1, \Gamma, m} \mathrm{e}^{\mathrm{i} \gamma c_{\phi}}}{\mathrm{i}\left(\gamma-\kappa_{0}^{-}\right)\left(\gamma-\lambda_{m}^{+}\right)} \cdot \frac{1}{J_{-}(\gamma)}-\sum_{n=0}^{\infty} \frac{\mathscr{B}_{1, \Gamma, n}}{\gamma-\theta_{n}^{+}} \cdot \frac{J_{+}\left(\theta_{n}^{+}\right)}{J_{+}(\gamma)} \\
& -\sum_{n=0}^{\infty} \frac{\mathscr{C}_{1, \Gamma, n} \mathrm{e}^{\mathrm{i} c_{\phi}\left(\gamma-\theta_{n}^{-}\right)}}{\mathrm{i}\left(\gamma-\kappa_{0}^{-}\right)\left(\gamma-\theta_{n}^{-}\right)} \cdot \frac{J_{-}\left(\theta_{n}^{-}\right)}{J_{-}(\gamma)}
\end{aligned}
$$




\subsubsection{4 $h_{1, S}$ Problem and Solution (Road Map Step Vd)}

The governing equation is

$$
\Sigma_{0}\left[\frac{\partial h_{1, S}}{\partial \psi}\right](\phi)=4 \pi \int_{-\infty}^{\infty}\left\{-D_{1, S}(\gamma) j(\gamma)+S(\gamma)\right\} \mathrm{e}^{-\mathrm{i} \gamma \phi} \mathrm{d} \gamma
$$

subject to homogeneous boundary conditions analogous to those in (3.41.b), (3.41.c) and (3.41.f). This problem is the inhomogeneous Helmholtz equation subject to homogeneous boundary conditions in a cascade geometry. The only requirements on the source terms are that they can be expanded in the upstream and downstream regions as series of the form (3.140) and (3.142) respectively. Consequently, this method of solution can be applied to other problems that involve forcing terms in a cascade geometry, such as in Guzman Inigo et al. (2019). By linearity, this solution can be combined with any of $h_{1, \Sigma}, h_{1, \Delta}$ or $h_{1, \Gamma}$ to generate a solution to the inhomogeneous Helmholtz equation with inhomogeneous boundary conditions. The boundary conditions for $h_{1, S}$ are given by

$$
\begin{aligned}
\Delta_{0}\left[h_{1, S}\right](\phi) & =0, & \phi<0 ; \\
\Sigma_{0}\left[\frac{\partial h_{1, S}}{\partial \psi}\right](\phi) & =0, & 0<\phi<c_{\phi} ; \\
\Delta_{0}\left[\frac{\partial h_{1, S}}{\partial \psi}\right](\phi) & =0, & 0<\phi<c_{\phi} ; \\
\Delta_{0}\left[\frac{\partial h_{1, S}}{\partial \psi}\right](\phi) & =0, & \phi<c_{\phi} ; \\
\Delta_{0}\left[h_{1, S}\right](\phi) & =2 \pi \mathrm{i} P_{1, S} \mathrm{e}^{-\mathrm{i} \kappa_{0}^{-} \phi}, & \phi>c_{\phi} .
\end{aligned}
$$

The method of solution is described in appendix 3.B.5 The solution is

$$
\begin{aligned}
D_{1, S}(\gamma)= & \sum_{m=-\infty}^{\infty} \frac{\mathscr{R}_{1, S, m}}{\gamma-\lambda_{m}^{-}} \cdot \frac{1}{J_{+}(\gamma)}-\sum_{m=-\infty}^{\infty} \frac{\mathscr{U}_{1, S, m}}{\mathrm{i}\left(\gamma-\kappa_{0}^{-}\right)\left(\gamma-\lambda_{m}^{+}\right)} \cdot \frac{\mathrm{e}^{\mathrm{i} \gamma c_{\phi}}}{J_{-}(\gamma)} \\
& -\sum_{n=0}^{\infty} \frac{\mathscr{B}_{1, S, n}}{\gamma-\theta_{n}^{+}} \cdot \frac{J_{+}\left(\theta_{n}^{+}\right)}{J_{+}(\gamma)}-\sum_{n=0}^{\infty} \frac{\left(\mathscr{A}_{1, S, n}+\mathscr{C}_{1, S, n}\right) \mathrm{e}^{\mathrm{i} c_{\phi}\left(\gamma-\theta_{n}^{-}\right)}}{\mathrm{i}\left(\gamma-\kappa_{0}^{-}\right)\left(\gamma-\theta_{n}^{-}\right)} \cdot \frac{J_{-}\left(\theta_{n}^{-}\right)}{J_{-}(\gamma)} .
\end{aligned}
$$

\subsection{Inversion of Fourier Transform}

In this section, we invert the Fourier transforms of the $\mathcal{O}(\epsilon)$ acoustic field found in section 3.3.2. This corresponds to step VI in the road map. Since $D_{1}$ is now known, the Fourier inversion integral in (3.33) can now be computed analytically. The integral is computed using residue calculus; this approach is similar to the inversion of the $\mathcal{O}(1)$ problem done 


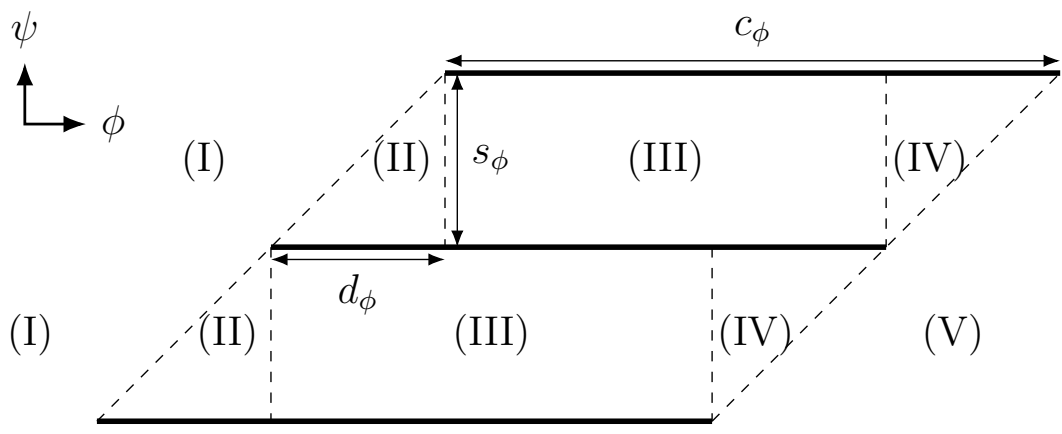

Fig. 3.6 Diagram indicating the different areas in the $(\phi, \psi)$-plane which require different regions of contour integration in the Fourier inversion.

in Posson et al. (2010b), except there are a large number of additional modes arising in the $\mathcal{O}(\epsilon)$ case.

The acoustic field is given by (3.33), which we rewrite as

$$
\begin{aligned}
h(\phi, \psi)=\int_{-\infty}^{\infty}( & \left\{\int_{0}^{s_{\phi}} \epsilon S_{\phi}\left(\gamma, \psi_{1}\right) I_{S}\left(\gamma, \psi, \psi_{1}\right) \mathrm{d} \psi_{1}\right\} \\
& \left.+D(\gamma) I_{D}(\gamma, \psi)+\epsilon G(\gamma) I_{G}(\gamma, \psi)\right) \mathrm{e}^{-\mathrm{i} \gamma \phi} \mathrm{d} \gamma
\end{aligned}
$$

where

$$
\begin{aligned}
I_{D}(\gamma, \psi) & =\frac{\cos \left(\zeta\left(\psi-s_{\phi}\right)\right)-\mathrm{e}^{\mathrm{i}\left(d_{\phi} \gamma+\sigma_{\phi}^{\prime}\right)} \cos (\zeta \psi)}{2\left(\cos \left(s_{\phi} \zeta\right)-\cos \left(d_{\phi} \gamma+\sigma_{\phi}^{\prime}\right)\right.} \\
I_{G}(\gamma, \psi) & =\frac{\sin \left(\zeta\left(\psi-s_{\phi}\right)\right)-\mathrm{e}^{\mathrm{i}\left(d_{\phi} \gamma+\sigma_{\phi}^{\prime}\right)} \sin (\zeta \psi)}{2 \zeta\left(\cos \left(s_{\phi} \zeta\right)-\cos \left(d_{\phi} \gamma+\sigma_{\phi}^{\prime}\right)\right)} \\
I_{S}\left(\gamma, \psi, \psi_{1}\right) & =\frac{\sin \left(\zeta\left(\left|\psi-\psi_{1}\right|-s_{\phi}\right)\right)-\mathrm{e}^{\mathrm{i}\left(d_{\phi} \gamma+\sigma_{\phi}^{\prime}\right) \operatorname{sgn}\left(\psi-\psi_{1}\right)} \sin \left(\zeta\left|\psi-\psi_{1}\right|\right)}{4 \pi \zeta\left(\cos \left(s_{\phi} \zeta\right)-\cos \left(d_{\phi} \gamma+\sigma_{\phi}^{\prime}\right)\right)}
\end{aligned}
$$

the derivations of which are provided in equations (3.137, 3.138,3.139) of the appendix. We proceed to evaluate this integral analytically.

The Fourier inversion is achieved by splitting the $(\phi, \psi)$-plane into five regions, as illustrated in figure 3.6. In a similar way to Posson et al. (2010b), the closure of the contour for each term is determined by the location in the $(\phi, \psi)$-plane. We calculate the acoustic potential function in the strip $0<\psi<s_{\phi}$, which gives the function in the entire domain via (3.26). We combine $\mathcal{O}(1)$ and $\mathcal{O}(\epsilon)$ terms to give the full solution up to $\mathcal{O}\left(\epsilon^{2}\right)$. After considerable calculation, cancellation and simplification, we obtain the following 
expressions, where the new constants and functions are defined in 3.G of the appendix. Functions denoted by $\mathbb{I}$ represent the inhomogeneous functions required by the source terms.

\subsubsection{Upstream Region (I)}

In the upstream region,

$$
\begin{aligned}
h(\phi, \psi) & =\sum_{m=-\infty}^{\infty} \mathscr{H}_{m}^{+} \exp \left[-\mathrm{i}\left(\lambda_{m}^{+} \phi-\zeta_{m}^{+} \psi\right)\right] \\
& +\sum_{M_{m}^{+}} \epsilon \mathbb{S}_{P, M_{m}^{+}}^{+} \mathbb{I}_{P, M_{m}^{+}}^{+}(\phi, \psi) .
\end{aligned}
$$

\subsubsection{Inter-Blade Upstream Region (II)}

In the inter-blade upstream region,

$$
\begin{aligned}
h(\phi, \psi)=\sum_{m=-\infty}^{\infty}\left\{\left(\mathscr{H}_{a, m}^{+} \cos \left(\psi \zeta_{m}^{+}\right)+\mathscr{H}_{G, a, m}^{+} \sin \left(\zeta_{m}^{+} \psi\right)\right) \exp \left[-\mathrm{i} \lambda_{m}^{+} \phi\right]\right. \\
\left.+\left(\mathscr{H}_{\mathscr{R}_{, a, m}^{-}}^{-} \cos \left(\psi \zeta_{m}^{-}\right)+\mathscr{H}_{G, a, m}^{-} \exp \left[\mathrm{i} \zeta_{m}^{-} \psi\right]\right) \exp \left[-\mathrm{i} \lambda_{m}^{-} \phi\right]\right\} \\
+\sum_{n=0}^{\infty}\left(\mathscr{H}_{\theta^{+}, a, n} \exp \left[-\mathrm{i} \theta_{n}^{+} \phi\right]+\mathscr{H}_{\theta^{-}, a, n} \exp \left[-\mathrm{i} \theta_{n}^{-} \phi\right]\right) \cos (n \psi) \\
+\sum_{l=-\infty}^{\infty}\left\{\left(\mathscr{H}_{S, a, l}+\mathscr{H}_{\Sigma, a, l}\right) \cos \left(\zeta_{\kappa_{l}^{-}}\left(\psi-s_{\phi}\right)\right)+\mathscr{H}_{S, b, l} \cos \left(\zeta_{\kappa_{l}^{-}} \psi\right)\right. \\
\left.+\left(\mathscr{H}_{S, c, l}+\mathscr{H}_{\Delta, a, l}\right) \sin \left(\zeta_{\kappa_{l}^{-}}\left(\psi-s_{\phi}\right)\right)+\mathscr{H}_{S, d, l} \sin \left(\zeta_{\kappa_{l}^{-}} \psi\right)\right\} \\
\times \exp \left[-\mathrm{i} \kappa_{l}^{-} \phi\right] \\
+\sum_{l_{1}, l_{2}} \epsilon \mathbb{S}_{l_{1}, l_{2}}^{i} \mathbb{I}_{l_{1}, l_{2}}^{i}(\phi, \psi) .
\end{aligned}
$$

\subsubsection{Inter-Blade Inner Region (III)}

In the inter-blade inner region,

$$
h(\phi, \psi)=\sum_{n=0}^{\infty}\left\{\left(\mathscr{H}_{\theta^{+}, a, n}+\mathscr{H}_{\theta^{+}, b, n}\right) \exp \left[-\mathrm{i} \theta_{n}^{+} \phi\right]\right.
$$




$$
\begin{aligned}
& \left.+\left(\mathscr{H}_{\theta^{-}, a, n}+\mathscr{H}_{\theta^{-}, b, n}\right) \exp \left[-\mathrm{i} \theta_{n}^{-} \phi\right]\right\} \cos (n \psi) \\
& +\sum_{l=-\infty}^{\infty}\left\{\left(\mathscr{H}_{S, a, l}+\mathscr{H}_{\Sigma, a, l}\right) \cos \left(\zeta_{\kappa_{l}^{-}}\left(\psi-s_{\phi}\right)\right)+\left(\mathscr{H}_{S, b, l}+\mathscr{H}_{\Sigma, b, l}\right) \cos \left(\zeta_{\kappa_{l}^{-}} \psi\right)\right. \\
& \left.+\left(\mathscr{H}_{S, c, l}+\mathscr{H}_{\Delta, a, l}\right) \sin \left(\zeta_{\kappa_{l}^{-}}\left(\psi-s_{\phi}\right)\right)+\left(\mathscr{H}_{S, d, l}+\mathscr{H}_{\Delta, b, l}\right) \sin \left(\zeta_{\kappa_{l}} \psi\right)\right\} \\
& \quad \times \exp \left[-\mathrm{i} \kappa_{l}^{-} \phi\right] \\
& +\sum_{l_{1}, l_{2}} \epsilon \mathbb{S}_{l_{1}, l_{2}}^{i} \mathbb{I}_{l_{1}, l_{2}}^{i}(\phi, \psi) .
\end{aligned}
$$

\subsubsection{Inter-Blade Downstream Region (IV)}

In the inter-blade downstream region,

$$
\begin{aligned}
& h(\phi, \psi)=\sum_{m=-\infty}^{\infty}\left\{\left(\mathscr{H}_{b, m}^{-} \cos \left(\left(\psi-s_{\phi}\right) \zeta_{m}^{-}\right)+\mathscr{H}_{G, b, m}^{-} \sin \left(\left(\psi-s_{\phi}\right) \zeta_{m}^{-}\right)\right.\right. \\
& \left.+\mathscr{H}_{m, b}^{-} \exp \left[-\mathrm{i} \zeta_{m}^{-} \psi\right]\right) \exp \left[-\mathrm{i} \lambda_{m}^{-} \phi\right] \\
& +\left(\mathscr{H}_{\mathcal{U}, b, m}^{+} \cos \left(\left(\psi-s_{\phi}\right) \zeta_{m}^{+}\right)+\mathscr{H}_{G, b, m}^{+} \exp \left[-\mathrm{i} \zeta_{m}^{+} \psi\right]\right) \exp \left[-\mathrm{i} \lambda_{m}^{+} \phi\right] \\
& \left.\left.+\left(\lambda_{m}^{-}\left(\psi-s_{\phi}\right)-\zeta_{m}^{-} \phi\right)\right) \mathscr{H}_{\Gamma, m, b}^{-} \mathrm{e}^{-\mathrm{i}\left(\lambda_{m}^{-} \phi+\zeta_{m}^{-} \psi\right)}\right\} \\
& +\sum_{n=0}^{\infty}\left(\mathscr{H}_{\theta^{+}, b, n} \exp \left[-\mathrm{i} \theta_{n}^{+} \phi\right]+\mathscr{H}_{\theta^{-}, b, n} \exp \left[-\mathrm{i} \theta_{n}^{-} \phi\right]\right) \cos (n \psi) \\
& +\sum_{l=-\infty}^{\infty}\left\{\mathscr{H}_{S, a, l} \cos \left(\zeta_{\kappa_{l}^{-}}\left(\psi-s_{\phi}\right)\right)+\left(\mathscr{H}_{S, b, l}+\mathscr{H}_{\Sigma, b, l}\right) \cos \left(\zeta_{\kappa_{l}^{-}} \psi\right)\right. \\
& \left.+\mathscr{H}_{S, c, l} \sin \left(\zeta_{\kappa_{l}^{-}}\left(\psi-s_{\phi}\right)\right)+\left(\mathscr{H}_{S, d, l}+\mathscr{H}_{\Delta, b, l}\right) \sin \left(\zeta_{\kappa_{l}^{-}} \psi\right)\right\} \\
& \times \exp \left[-\mathrm{i} \kappa_{l}^{-} \phi\right] \\
& +\left(\sum_{ \pm} \mathscr{H}_{\Gamma, \kappa_{0}^{-}, \pm, b}^{-} \exp \left[\mp \mathrm{i} \zeta_{\kappa_{0}^{-}} \psi\right]+\mathscr{H}_{\kappa_{0}^{-}, b}^{-} \cos \left(\left(\psi-s_{\phi}\right) \zeta_{\kappa_{0}^{-}}\right)\right) \exp \left[-\mathrm{i} \kappa_{0}^{-} \phi\right] \\
& +\sum_{l_{1}, l_{2}} \epsilon \mathbb{S}_{l_{1}, l_{2}}^{i} \mathbb{I}_{l_{1}, l_{2}}^{i}(\phi, \psi) \text {. }
\end{aligned}
$$




\subsubsection{Downstream Region (V)}

In the downstream region,

$$
\begin{aligned}
h(\phi, \psi) & =\sum_{m=-\infty}^{\infty} \mathscr{H}_{m}^{-} \exp \left[-\mathrm{i}\left(\tilde{\lambda}_{m}^{-} \phi+\tilde{\zeta}_{m}^{-} \psi\right)\right] \\
& +\sum_{M_{m}^{-}} \epsilon \mathbb{S}_{P, M_{m}^{-}}^{-} \mathbb{I}_{P, M_{m}^{-}}^{-}(\phi, \psi)+\sum_{N_{\phi, n}^{-}} \epsilon \mathbb{S}_{N, N_{\phi, n}^{-}}^{-} \mathbb{I}_{N, N_{\phi, n}^{-}}^{-}(\phi, \psi) \\
& +\sum_{ \pm} \epsilon \mathbb{S}_{N, \kappa_{0}^{-}, \pm}^{-} \mathbb{I}_{N, \kappa_{0}^{-}, \pm}^{-}(\phi, \psi)+\sum_{ \pm} \mathscr{H}_{\kappa_{0}^{-}, \pm}^{-} \mathrm{e}^{-\mathrm{i}\left(\kappa_{0}^{-} \phi \pm \zeta_{\kappa_{0}^{-}} \psi\right)}, \\
& +\sum_{m=-\infty}^{\infty} \mathscr{H}_{\Gamma, \lambda_{m}^{-}}^{-} \psi \exp \left[-\mathrm{i}\left(\tilde{\lambda}_{m}^{-} \phi+\tilde{\zeta}_{m}^{-} \psi\right)\right] .
\end{aligned}
$$

\subsubsection{Fourier Inversion Discussion}

It may be verified by hand that the acoustic potential satisfies the boundary conditions of no discontinuities upstream (3.19.b), the specified normal velocity on either side of the blade (3.20.b, 3.21.b), the specified jump in normal velocity (in $(\phi, \psi)$-space) across the wake (3.22.b) and the specified jump in acoustic potential across the wake (3.23.b). The pressure jump also vanishes (except in the $h_{1, \Gamma}$ case, where a finite jump is permitted), at the trailing edge, signifying the fulfilment of the unsteady Kutta condition. It can also be verified that the function satisfies the inhomogeneous convected Helmholtz equation, and has the correct forcing due to the source terms. In section 3.5 it will also be observed that $h$ is continuous across the different regions.

\subsection{Results}

In this section we present results to validate our solution against existing methods and codes, as well as demonstrating the versatility of our method by plotting results for a range of geometries and aeroacoustic parameters. Due to the large range of available variables, it is impossible to present a comprehensive parameter study. We have therefore chosen a range of illustrative cases (table 3.1), and for these we will present key unsteady features exhibited by our solution that highlight the importance of accurately modelling realistic aerofoil geometry. 
Case

\begin{tabular}{cccccccc} 
Parameter & Symbol & A & B & C & D & E & F \\
\hline gap-to-chord ratio & $\Delta / 2$ & 1 & 1 & $\sec \left(20^{\circ}\right)$ & 0.6 & 0.8 & 0.6 \\
stagger angle & $\chi$ & $0^{\circ}$ & $0^{\circ}$ & $20^{\circ}$ & $30^{\circ}$ & $40^{\circ}$ & $30^{\circ}$ \\
Mach number & $M$ & 0.5 & 0.5 & 0.2 & 0.3 & 0.3 & 0.3 \\
non-dimensional frequency & $k$ & $5 \pi / 4$ & $13 \pi / 4$ & 5 & 7.2 & - & - \\
spanwise wave number & $k_{s}$ & 0 & 0 & 0 & 0 & 0 & 0.1 \\
inter-blade phase angle & $\sigma_{\phi}$ & $5 \pi / 2$ & $13 \pi / 2$ & $3 \pi / 4$ & $\pi / 4$ & $3 \pi / 4$ & -
\end{tabular}

Table 3.1 Summary of different cascade and aeroacoustics parameters used in results sections. Cases A and B are found in Hall (1997) and case C may be found in Posson et al. (2010b).

\subsubsection{Validation}

We first present comparisons to well-known solutions for cascades of flat plates at zero angle of attack. This is the asymptotic limit of our solution where $\epsilon=0$, and therefore our solution should match other analytic and numerical models that cannot account for blade geometry. The previously mentioned Wiener-Hopf method (Posson et al., 2010b) uses a similar method to the present chapter but does not take into account blade geometry. The mode-matching technique (Bouley et al., 2017) matches modal expressions in the upstream, downstream and inter-blade domains by enforcing conservation laws. The LINSUB code (implemented here by Hall (1997)) makes use of a semi-numerical model due to Smith (1973) and compares favourably to experimental results.

We present two qualitative validations: firstly, we plot the surface pressure as predicted by the four methods in figures $3.7 \mathrm{a}$ and $3.7 \mathrm{~b}$. The agreement for both frequencies is excellent, and there are no discernible differences between the methods. The cascades considered in cases A and B have zero stagger angle. Consequently, we also present a second validation for a staggered cascade. In figure 3.8 we compare the pressure fields for flat plates to the field found using the method of Posson et al. (2010b). Again, the agreement is excellent and there are no discernible differences between the methods. 


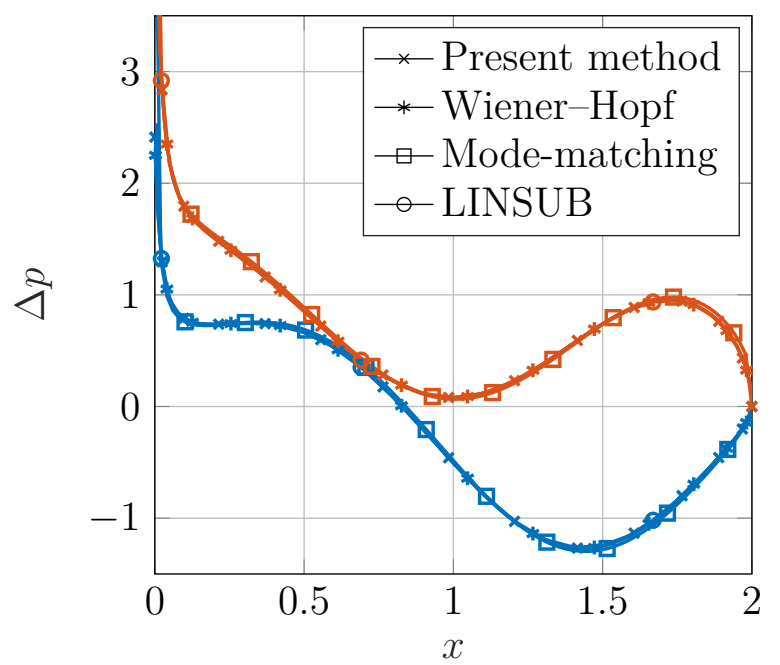

(a) Case A

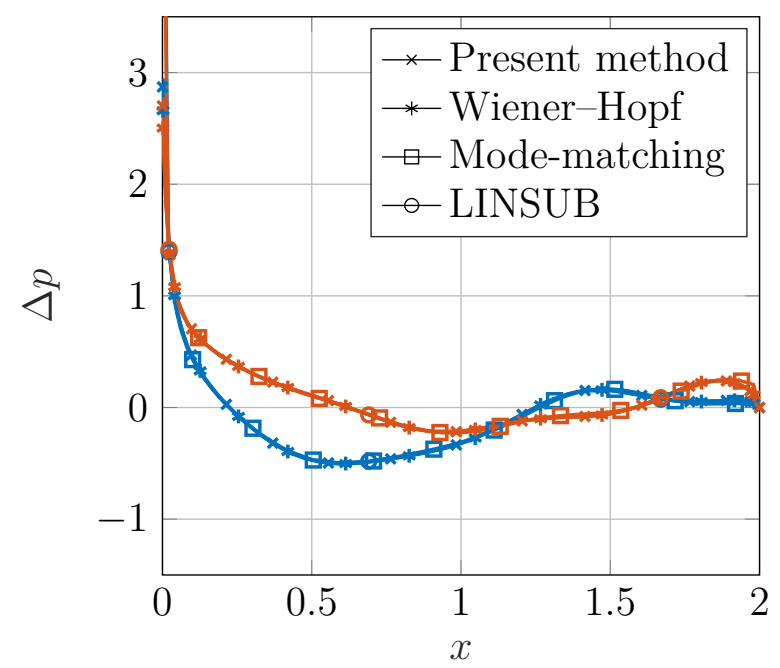

(b) Case B

Fig. 3.7 Validations of surface pressure jump for flat plates. The real ( - ) and imaginary (_-) parts of the blade pressure jump calculated by the present method are compared to the Wiener-Hopf method (Posson et al., 2010b), a mode-matching technique (Bouley et al., 2017), and a numerical method (Hall, 1997). The cascade and aeroacoustic parameters are in table 3.1.

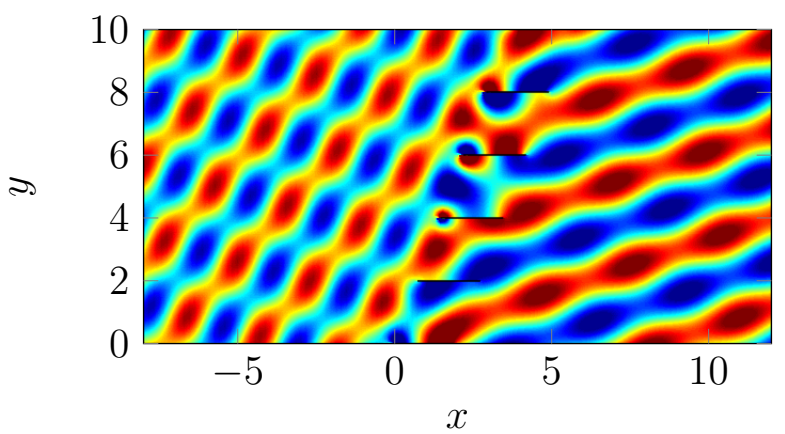

(a) Present method

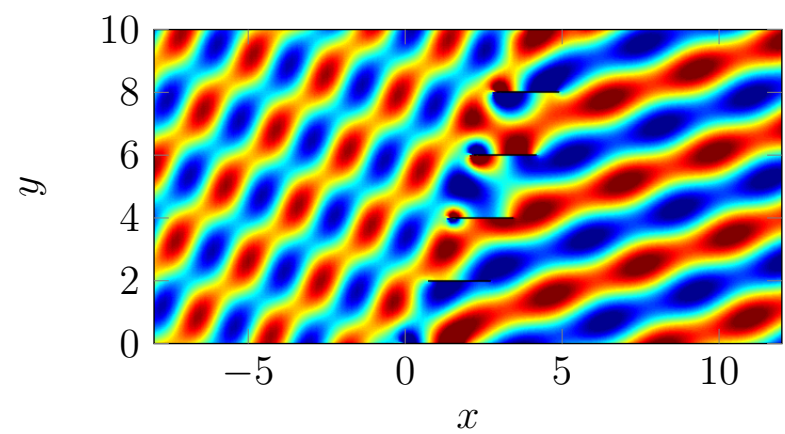

(b) Method of Posson et al. (2010b)

Fig. 3.8 Comparison to figure 11 of Posson et al. (2010b). The geometrical and aeroacoustic parameters correspond to case $\mathrm{C}$ in table 3.1. The color maps are the same in each figure. 


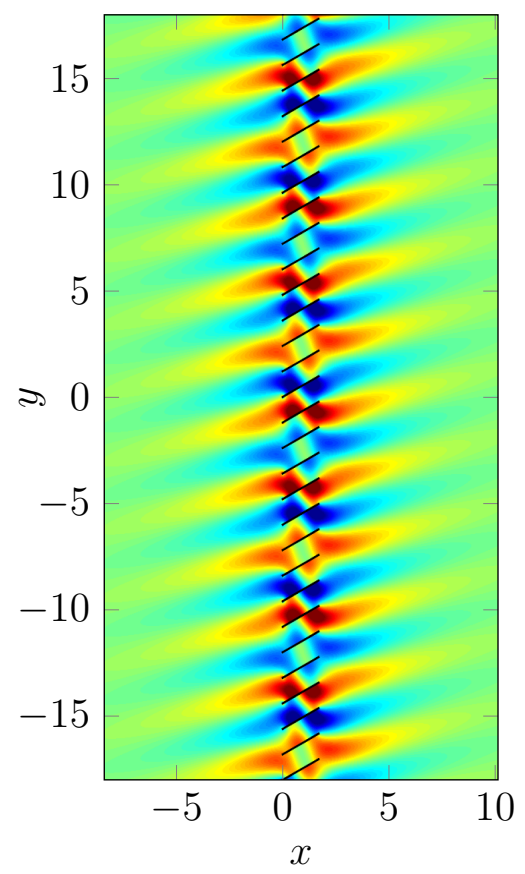

(a) $\alpha=0$, flat plates

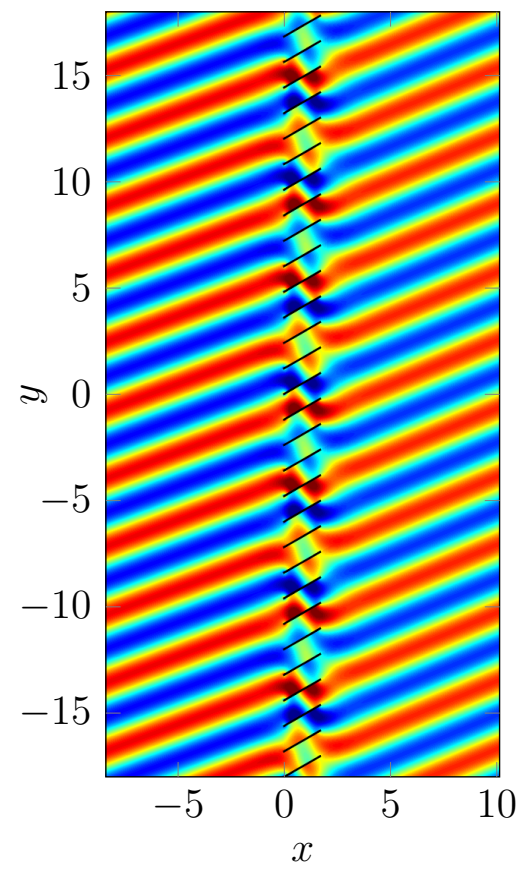

(b) $\alpha=-3^{\circ}$, flat plates

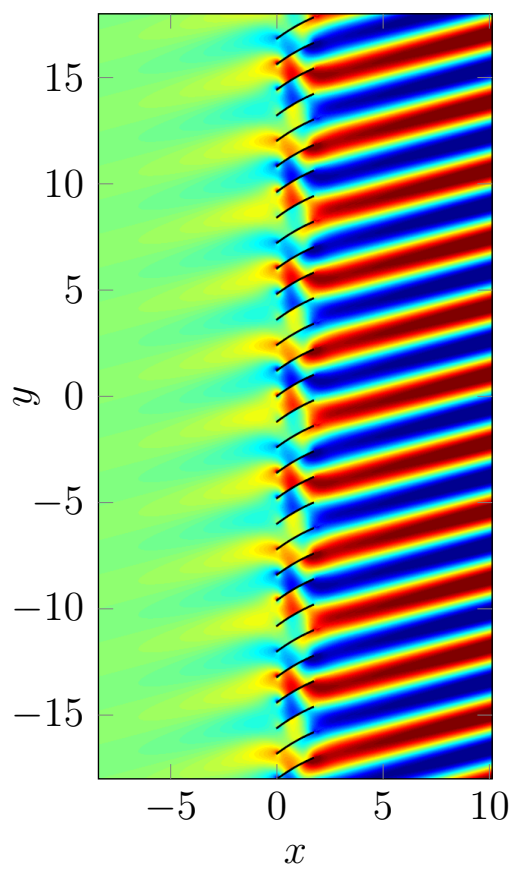

(c) $\alpha=0^{\circ}$, NACA 3500

$$
\begin{array}{lllllll}
\hline-1.5 & -1 & -0.5 & 0 & 0.5 & 1 & 1.5 \\
& & \text { Normalised pressure, } p(x, y) & &
\end{array}
$$

Fig. 3.9 Scattered pressure field for cascade and aeroacoustic parameters defined in case D in table 3.1.

\subsubsection{Effects of Realistic Geometry}

We now illustrate the versatility of the solution by calculating key aeroacoustic properties for a variety of cascade geometries. The results in this section use aerofoils from the NACA four-digit series.

\subsubsection{An Illustrative Example}

We now present an illustrative example that demonstrates the importance of accounting for blade geometry in gust-cascade interaction noise. We show that even subtle differences in aerofoil geometry and angle of attack can have a strong effect on the scattered pressure field. It is sufficient to consider a single example where the cascade and aeroacoustic parameters are defined in case D in table 3.1.

The scattered pressure field is strongly affected by the inlet and outlet angle of the flow, as illustrated in figure 3.9. We consider a system where a mode is close to cut-on so that the effects of aerofoil geometry are most noticeable. In particular, the 
non-dimensional frequency is 0.1 below that of the nearest cut-on frequency. In the first case, the blades are flat plates at zero angle of attack (figure 3.9a), and the modes are cut-off in both the upstream and downstream regions. Modifying the inlet angle changes the cut-on frequency of the modes in the upstream region, as illustrated in figure $3.9 \mathrm{~b}$. As the inlet angle is reduced to $-3^{\circ}$, the upstream mode becomes cut-on and the pressure field no longer decays exponentially in the far-field upstream. Mathematically, this difference is caused by modifications in blade spacing in the drift coordinates (3.4). Generally, increasing the angle of attack also increases the cut-on frequency so that modes that would otherwise be cut-on are evanescent. The downstream modes are also cut-on since the cascade deflected the flow.

Further differences appear when we introduce camber, such as in figure 3.9c. Aerodynamically, an effect of camber is to change the outlet angle of the flow by channelling the flow through a curved duct. In particular, the trailing-edge angle plays an important role in determining the overall deflection angle achieved by the cascade (Baddoo and Ayton, 2018b). In contrast to angle of attack, camber does not affect the modes in the upstream region: these are uniquely determined by $\lambda_{m}^{+}$in (3.55). Camber does, however, affect the modes in the downstream region through generating circulation around individual blades. Further details are available in appendix 3.F, but for now it is sufficient to note that the cut-on frequencies are modified by increasing the flow deflection. For example, in figure $3.9 \mathrm{c}$ we plot the scattered pressure field for a cascade of NACA 3500 aerofoils at zero angle of attack. This subtle change in geometry means that the mode in the downstream region is now cut-on. Moreover, the angle of the scattered pressure wave is not the same as that predicted by the solution for flat plates at angle of attack in figure 3.9b. The original flat plate solution (Glegg, 1999; Posson et al., 2010b) is not able to account for these effects, which we have shown are significant.

\subsubsection{Unsteady Lift}

Having found the acoustic potential field throughout the entire domain, we may now calculate the unsteady loading on each blade. The unsteady loading is defined as the integral of the unsteady pressure over the blade surface:

$$
C_{p}=\oint p^{\prime}(s) \mathrm{d} s
$$

We rewrite this integral in $(\phi, \psi)$-space as

$$
C_{p}=-\int_{0}^{c_{\phi}} \Delta_{0}\left[p \sqrt{1+\left(\epsilon y_{s}^{\prime}\right)^{2}}(1-\epsilon q)\right](\phi) \mathrm{d} \phi
$$


where $\epsilon y_{s}$ corresponds to the blade surface (3.1) and we have ignored time and spanwise dependence. The terms in the square root are expressible in terms of the surface velocity of the background flow on the blade. In contrast to the unsteady lift integral for flat plates (Glegg, 1999), the integral (3.45) may not be computed analytically. However, the pressure $p$ possesses a square-root singularity at the leading edge and a square-root zero at the trailing edge. Accordingly, the integral (3.45) may be rapidly computed using a modified version of Chebyshev-Gauss quadrature.

In figure 3.10 we plot the unsteady lift as a function of aerofoil geometry for a range of parameters. Consistent with previous analyses for loaded cascades (Fang and Atassi, 1993), we observe that the mean loading greatly affects the unsteady loading in figures $3.10 \mathrm{a}$ and $3.10 \mathrm{~b}$. The mean loading is generally increased by the inclusion of angle of attack (figure 3.10a) and camber (figure 3.10b). The effect appears to be more significant for angle of attack, and in particular, the location of the peak just below $k=4$ in figure $3.10 \mathrm{a}$ is modified. This effect is attributed to the change in the cut-on frequency of the upstream acoustic modes. Conversely, the effect of aerofoil camber in figure 3.10b around $k=4$ is noticeable but not as significant. Camber appears to shift the location of the second peak around $k \approx 7$, possibly by distorting the duct modes by creating a curved channel. Finally, thickness has a less significant effect on the unsteady loading insofar as the cut-on frequencies of the modes are unchanged, as illustrated in figure 3.10c. The inclusion of aerofoil thickness does not change the locations of the peaks in unsteady lift, although it does affect the amplitude at the peaks.

\subsubsection{Sound Power Output}

The sound power from a source, bounded by a volume $S$, is given by

$$
W=\int_{S} \boldsymbol{I} \cdot \boldsymbol{n} \mathrm{d} S
$$

where $\boldsymbol{I}$ is the acoustic intensity vector and $\boldsymbol{n}$ is the unit normal pointing out of the volume containing the sources.

We now define the surface $S$. Similarly to Glegg (1999), $S$ must enclose all the sources in the system. Accordingly, we consider a circular fan with $B$ blades and, consequently, the incoming disturbance and radiated field will repeat with $B$ blades. Equivalently, the inter-blade phase angle (in physical space) must be of the form $\sigma=2 \pi l / B$ for an integer $l$. 


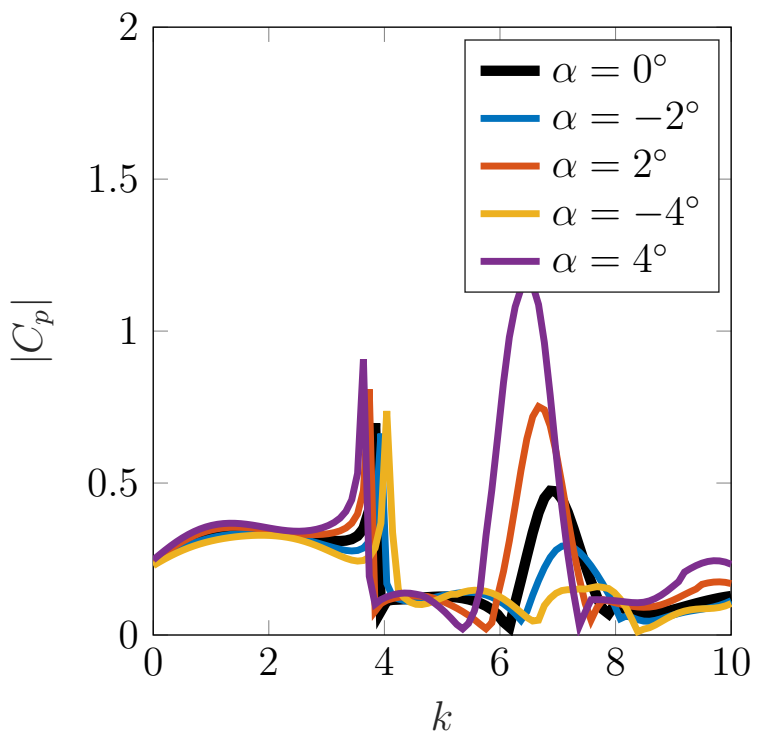

(a)

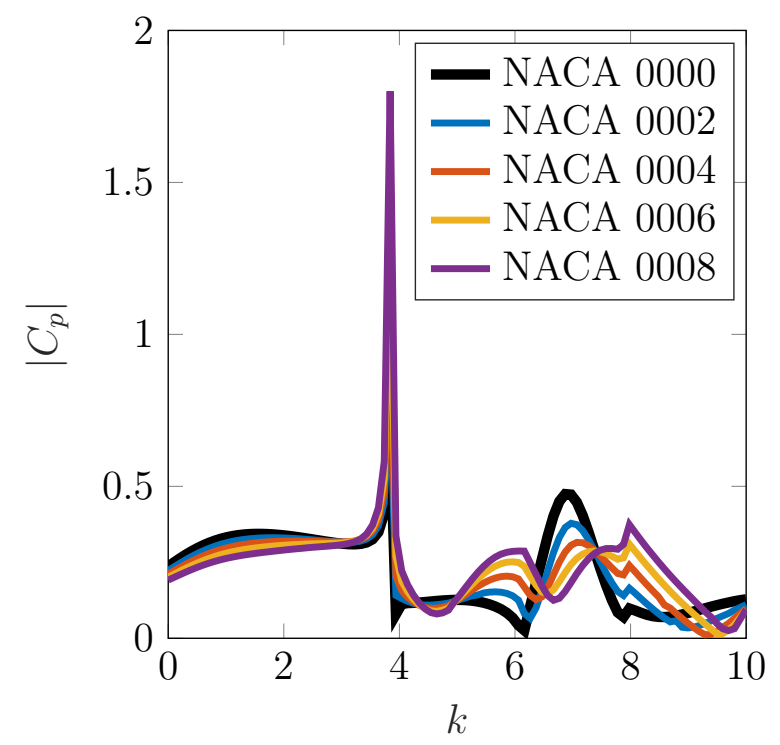

(c)

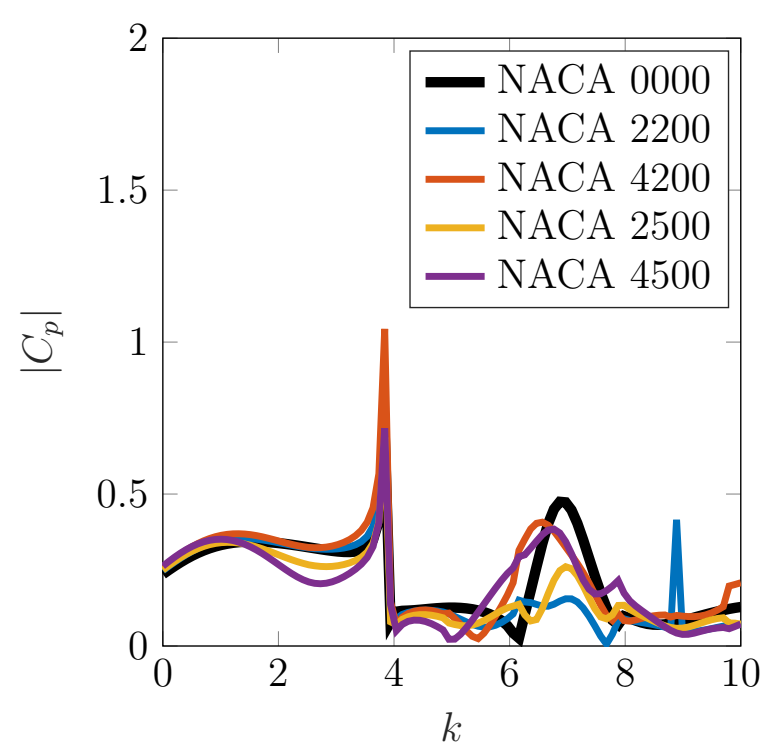

(b)

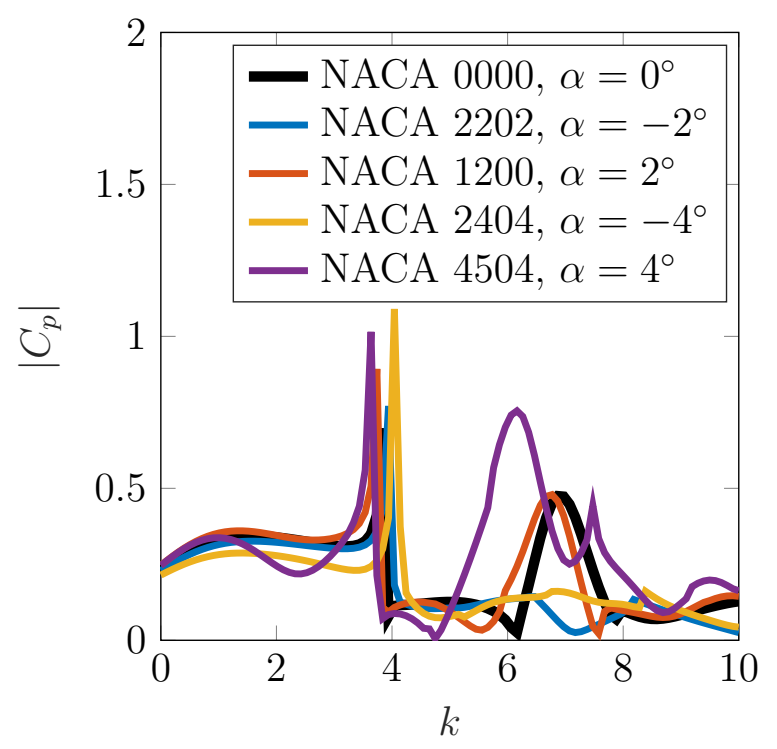

(d)

Fig. 3.10 The unsteady lift for a range of blade geometries. The graphs correspond to varying (a) the angle of attack, (b) the camber, (c) the thickness, and (d) all of the above. The parameters are defined in case $\mathrm{F}$ in table 3.1 with $k_{n}=1 / 2$. 
The upstream and downstream surfaces $S_{ \pm}$are defined in the physical plane along the lines

$$
s x-d \beta_{\infty} y=\text { constant. }
$$

We now translate these lines into $(\phi, \psi)$-space. The first point is to note that, in (3.3), as $x \rightarrow \mp \infty$ we have

$$
\log \left(\frac{\sinh \left(\frac{\pi \mathrm{i}\left(\tau-z_{\beta}\right)}{d+\mathrm{i} s}\right)}{\sinh \left(\frac{\pi \mathrm{i} \tau}{d+\mathrm{i} s}\right)}\right) \sim \mp \frac{\pi \mathrm{i} z_{\beta}}{d+\mathrm{i} s}
$$

and, therefore,

$$
(\phi+\mathrm{i} \psi)\left(z_{\beta}\right) \sim z_{\beta}\left(1-\mathrm{i} \alpha+H(\mp 1) \frac{\epsilon \Gamma}{d+\mathrm{i} s}\right)
$$

where the final term corresponds to the turning angle of the flow. Consequently, we have

$$
\begin{aligned}
x & \sim R_{1}^{ \pm} \phi-R_{2}^{ \pm} \psi, \\
\beta_{\infty} y & \sim R_{2}^{ \pm} \phi+R_{1}^{ \pm} \psi,
\end{aligned}
$$

where

$$
\begin{aligned}
R_{1}^{ \pm} & =\frac{\Delta^{2}+d H(\mp 1) \epsilon \Gamma}{|(d+\mathrm{i} s)(1-\mathrm{i} \alpha)+H(\mp 1) \epsilon \Gamma|^{2}}, \\
R_{2}^{ \pm} & =\frac{\alpha \Delta^{2}+s H(\mp 1) \epsilon \Gamma}{|(d+\mathrm{i} s)(1-\mathrm{i} \alpha)+H(\mp 1) \epsilon \Gamma|^{2}} .
\end{aligned}
$$

Accordingly, the upstream and downstream surface lines in $(\phi, \psi)$-space are

$$
\left(s R_{1}^{ \pm}-d R_{2}^{ \pm}\right) \phi-\left(d R_{1}^{ \pm}+s R_{2}^{ \pm}\right) \psi=\text { constant }
$$

and the outward pointing unit normal to these lines is given by

$$
\boldsymbol{n}_{\phi}^{ \pm}= \pm \frac{\left(-s R_{1}^{ \pm}+d R_{2}^{ \pm}, d R_{1}^{ \pm}+s R_{2}^{ \pm}, 0\right)}{\Delta \sqrt{\left(R_{1}^{ \pm}\right)^{2}+\left(R_{2}^{ \pm}\right)^{2}}}
$$


The (non-dimensional) acoustic intensity is given by (4.3) in Myers and Kerschen (1995) as

$$
\boldsymbol{I}=\left\langle\left[\frac{p^{\prime}}{\rho_{0}}+\nabla G^{\prime} \cdot \boldsymbol{U}_{0}\right]\left(\rho_{0} \nabla G^{\prime}+\rho \boldsymbol{U}_{0}\right)\right\rangle
$$

where $\langle\cdot\rangle$ denotes the expected value and $\boldsymbol{U}_{0}$ is the non-dimensional background flow, and $\rho_{0}$ is the non-dimensional local density value for the mean flow. By applying the definition of scattered pressure (Myers and Kerschen, 1995, 2.1c), the term in square brackets may be written as $-\partial G^{\prime} / \partial t$. We may also write $p^{\prime}=\rho / M_{0}^{2}$, (where $M_{0}$ is the local Mach number for the mean flow) and by following the approaches of sections 3.8 and 17.5 of Glegg and Devenport (2017), we obtain

$$
\boldsymbol{I}=-\rho_{0}\left\langle\frac{\partial G^{\prime}}{\partial t}\left(\nabla G^{\prime}-M_{0}^{2} \boldsymbol{U}_{0} \frac{\mathrm{D}_{0} G^{\prime}}{\mathrm{D} t}\right)\right\rangle
$$

Since the real part of the potential function $G^{\prime}$ is assumed, we may write

$$
\begin{aligned}
\frac{\partial G^{\prime}}{\partial t} & =\left|\frac{\partial G^{\prime}}{\partial t}\right| \cos \left(\theta_{1}-k t\right), \\
\nabla G^{\prime}-M_{0}^{2} \boldsymbol{U}_{0} \frac{\mathrm{D} G^{\prime}}{\mathrm{D} t} & =\left|\nabla G^{\prime}-M_{0}^{2} \boldsymbol{U}_{0} \frac{\mathrm{D} G^{\prime}}{\mathrm{D} t}\right| \cos \left(\theta_{2}-k t\right),
\end{aligned}
$$

where $\theta_{j}$ refers to the phase of the term on the left-hand side of each equation. The modulus terms may be taken outside of the expected value, and by using

$$
\left\langle\cos \left(\theta_{1}-k t\right) \cos \left(\theta_{2}-k t\right)\right\rangle=\frac{1}{2}\left\langle\cos \left(\theta_{1}+\theta_{2}-2 k t\right)+\cos \left(\theta_{1}-\theta_{2}\right)\right\rangle=\frac{1}{2} \cos \left(\theta_{1}-\theta_{2}\right),
$$

we may express the acoustic intensity as

$$
\boldsymbol{I}=-\frac{\rho_{0}}{2} \mathbb{R e}\left[\frac{\partial G^{\prime}}{\partial t} \overline{\left(\nabla G^{\prime}-M_{0}^{2} \boldsymbol{U}_{0} \frac{\mathrm{D}_{0} G^{\prime}}{\mathrm{D} t}\right)}\right]
$$

We may now integrate the acoustic intensity according to 3.46 in the $(\phi, \psi)$-plane by using the expression for the modified acoustic potential function found in 3.4.

In figure 3.11, we plot the upstream and downstream sound power outputs for a range of blade geometries. Consistent with numerical and experimental studies by Gea-Aguilera et al. (2016) and Fang and Atassi (1993), aerofoil camber and angle of attack (figures $3.11 \mathrm{a} \& 3.11 \mathrm{~b})$ introduce significant aeroacoustic effects. In each case, we see that the effects of geometry perturb the sound power output from the flat plate solution. In 

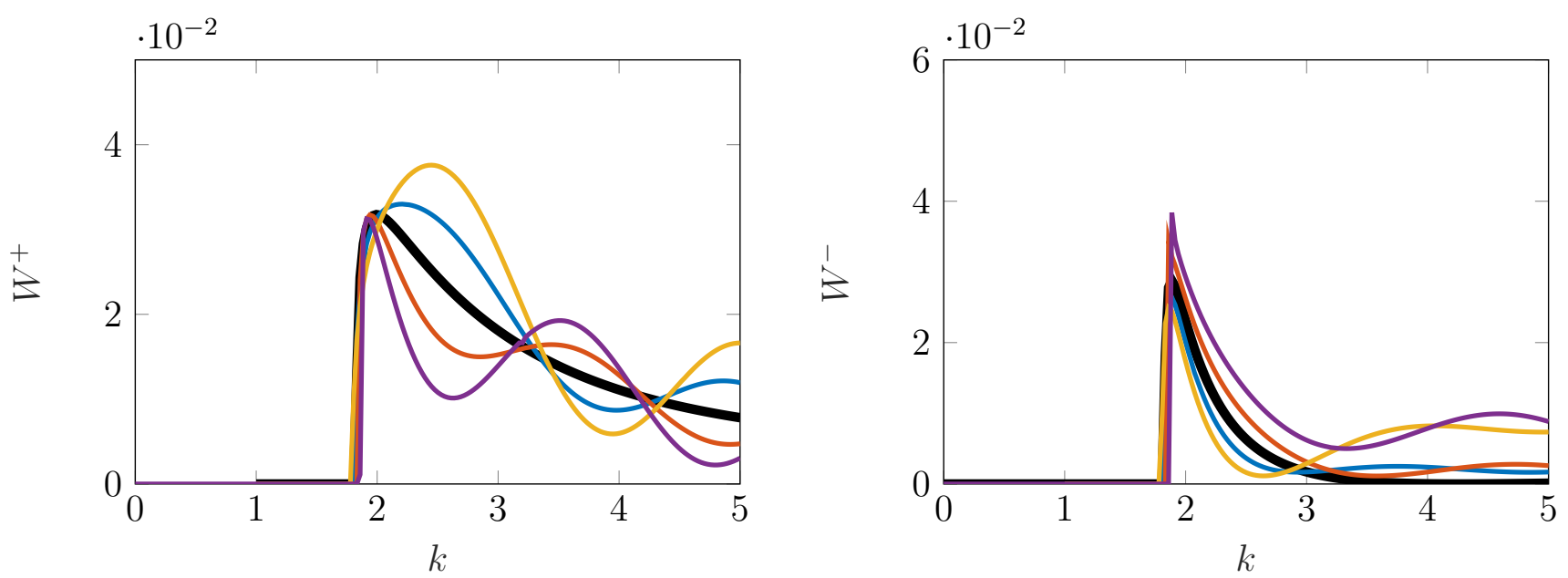

(a) Flat plates with varying angle of attack. The colours correspond to $\alpha=0(\boldsymbol{-}), \alpha=-2^{\circ}$ $(-), \alpha=2^{\circ}(-), \alpha=-4^{\circ}(-)$, and $\alpha=4^{\circ}(-)$.
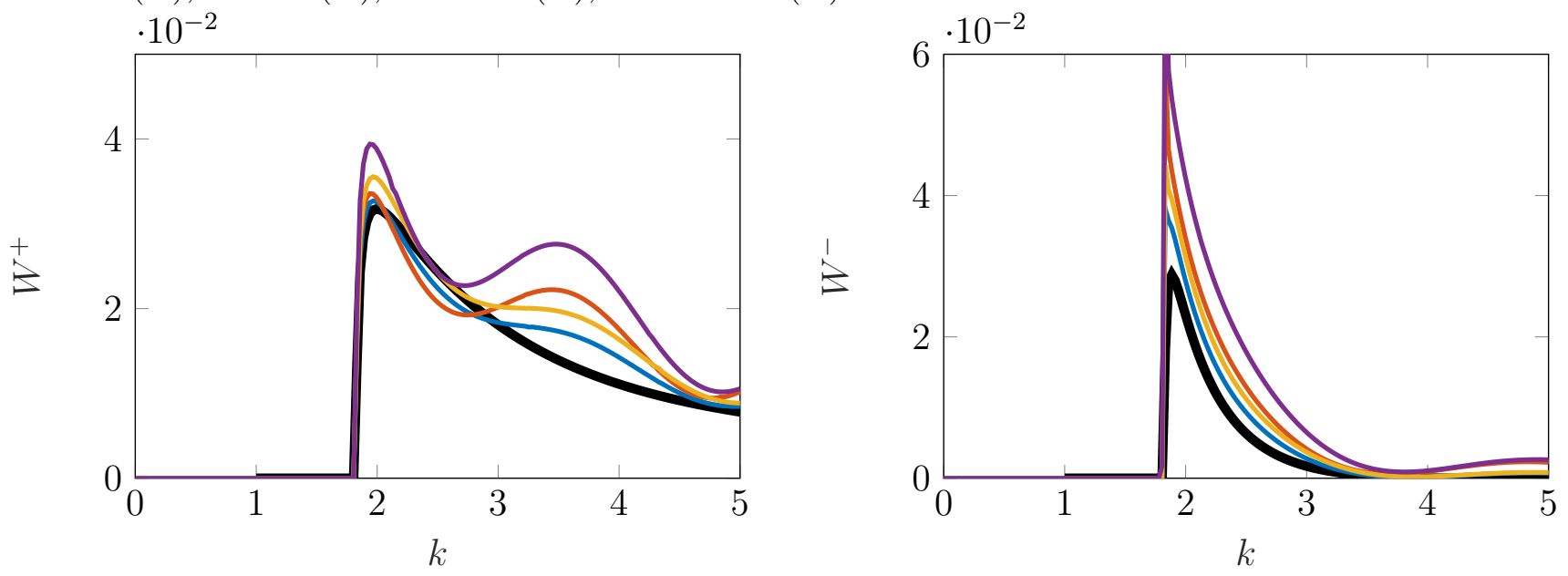

(b) Zero angle of attack with varying camber. The colours correspond to flat plates (-), NACA $1200(-)$, NACA $2200(-)$, NACA 1400 (-), and NACA $2400(-)$.

Fig. 3.11 Upstream (left) and downstream (right) sound power output as a function of non-dimensional frequency for a range of aerofoil geometries. The aeroacoustic parameters are case $\mathrm{E}$ in table 3.1, except for the non-dimensional frequency, which is the independent variable here. 
the upstream sound power output we observe new local maxima and minima, which we attribute to the new modes in the inter-blade channel that are not present in the $\mathcal{O}(1)$ solution. Alternatively, these maxima and minima may also exist in the flat plate solution, but the effect of aerofoil geometry is to change the frequency at which these peaks appear. As the frequency increases, the plots in figure 3.11 leave the domain of validity as the assumption $k \epsilon \ll 1$ breaks down. The downstream sound power output is general increased by the inclusion of camber, which is to be expected since the camber deflects the flow downstream. There are subtle changes in the downstream cut-on frequency for camber as indicated in figure 3.11b.

We attribute these observations to the strong influence of angle of attack and camber on the background flow. In particular, the mean loading on symmetrical aerofoils is generally much smaller than the loading on asymmetrical aerofoils, even at large stagger angles as illustrated in chapter 2. When the blades are loaded, the cut-on frequencies are different in the upstream and downstream regions (Fang and Atassi, 1993) due to the distortion of the acoustic field by the background flow. This is observed most clearly in figures 3.11a and 3.11b. In figure 3.11a, the angle of attack is varied and the cut-on frequencies are slightly changed in both the upstream and downstream regions since the flow is distorted in both regions. In terms of our solution expansion, for loaded blades the contributions from the $h_{1, \Gamma}$ term - which is proportional to the circulation - are far larger for loaded aerofoils. Additionally, the source terms are generally larger for cambered aerofoils, and accordingly the $h_{1, S}$ term has a significant contribution.

In all the results presented, we see that the inclusion of realistic geometry can strongly affect sound power predictions. The largest difference between the flat plate case and the solution accounting for full aerofoil geometry (figure 3.11) shows a difference in sound power level of approximately $4 \mathrm{~dB}$. This is a significant amount, and we attribute these effects predominantly to the inclusion of camber, which substantially deflects the flow. Moreover, camber distorts the inter-blade channel, and consequently modifies the duct modes. The results indicate that, for fan blade design purposes, camber should certainly be appropriately modelled in aeroacoustic predictive tools. The present method represents a powerful design tool to this end, and could be applied in industrial settings to rapidly and accurately compute the steady and unsteady flow through a cascade of realistic aerofoils. 


\subsection{Conclusions}

In this chapter we have presented an analytic expression for the acoustic field resulting from an unsteady vortical or entropic gust impinging on a rectilinear cascade of thin aerofoils in background flow at small angle of attack. In particular, we have solved the inhomogeneous convected Helmholtz equation with inhomogeneous Neumann boundary conditions in a cascade geometry. The resulting expression is valid in the upstream, interblade, and downstream regions, and represents a considerable extension to existing theory, by including the effects of realistic aerofoil geometry. Key aeroacoustic features, such as the pressure field, sound power output and unsteady surface pressure may be evaluated swiftly using MATLAB codes. The solution is validated against previous analytical and numerical approaches and is seen to give very good agreement. 


\section{List of Symbols}

Symbol Meaning

$\epsilon \quad$ Order of magnitude of the background flow perturbation

$q, \mu \quad$ Background flow horizontal and vertical perturbations

$M_{\infty} \quad$ Upstream background flow Mach number

$\phi, \psi \quad$ Velocity potential and streamfunction of background flow

$d_{\phi}, s_{\phi} \quad$ Horizontal and vertical blade spacings in $(\phi, \psi)$-space

$\chi_{\phi} \quad$ Stagger angle in $(\phi, \psi)$-space

$\Delta_{\phi} \quad$ Distance between adjacent leading edges in $(\phi, \psi)$-space

$c_{\phi}^{ \pm} \quad$ Length of upper and lower aerofoil surfaces in $(\phi, \psi)$-space

$\alpha \quad$ Angle of attack

$y_{s} \quad$ Aerofoil shape function

$h \quad$ Modified acoustic potential function

$p \quad$ Modified pressure

$\beta \quad$ Prandtl-Glauert factor

$k \quad$ Reduced frequency

$\Delta_{n}[f] \quad$ Jump of $f$ across $\psi=n s_{\phi}$

$\Sigma_{n}[f] \quad$ Sum of $f$ across $\psi=n s_{\phi}$

$\gamma \quad$ Spectral parameter in Fourier plane

$\zeta(\gamma) \quad \sqrt{k^{2} w^{2}-\gamma^{2}}$

$D_{x} \quad$ Fourier transform of $\Delta_{0}\left[h_{x}\right]$

$\mathscr{M}^{ \pm} \quad$ Upper/lower half-plane in $\gamma$-space

$\gamma \quad$ Bound vorticity distribution for background flow

$\Gamma \quad$ Background flow circulation 
j $\quad$ Kernel of Wiener-Hopf problem

$J_{ \pm} \quad$ Multiplicative splitting of the kernel $j$

$\theta_{n}^{ \pm} \quad$ Zeros of $J_{\mp}$

$\lambda_{m}^{ \pm} \quad$ Poles of $J_{\mp}$

$\kappa_{l}^{-} \quad-k \delta+l \pi / c_{\phi}$

$c_{\Sigma, l} \quad$ Fourier series coefficients of sum in normal velocity either side of each aerofoil

$c_{\Delta, l} \quad$ Fourier series coefficients of jump in normal velocity either side of each aerofoil

$\mathbb{S} \quad$ Source terms

$S \quad$ Fourier transform of source terms

\section{Subscripts and superscripts}

$0 \quad$ Leading-order terms

$1 \quad$ First-order terms

$\pm \quad$ Either terms located in $\mathscr{M}^{ \pm}$or analytic in $\mathscr{M}^{ \pm}$

$\Sigma \quad$ Terms with symmetric boundary conditions

$\Delta \quad$ Terms with anti-symmetric boundary conditions

$\Gamma \quad$ Terms proportional to circulation

$S \quad$ Terms corresponding to source terms 


\section{A $\mathcal{O}(1)$ Solution}

We may write the $\mathcal{O}(1)$ problem as

$$
\frac{\partial^{2} h_{0}}{\partial \phi^{2}}+\frac{\partial^{2} h_{0}}{\partial \psi^{2}}+k^{2} w^{2} h_{0}=0
$$

subject to no discontinuities upstream:

$$
\Delta_{0}\left[h_{0}\right](\phi)=0, \quad \phi<0,
$$

no-flux across the blade:

$$
\begin{array}{ll}
\Sigma_{0}\left[\frac{\partial h_{0}}{\partial \psi}\right](\phi)=-2 \frac{A_{n}}{\beta_{\infty}} \mathrm{e}^{\mathrm{i} k \delta \phi}, & 0<\phi<c_{\phi}, \\
\Delta_{0}\left[\frac{\partial h_{0}}{\partial \psi}\right](\phi)=0, & 0<\phi<c_{\phi}
\end{array}
$$

no-flux across the wake:

$$
\Delta_{0}\left[\frac{\partial h_{0}}{\partial \psi}\right](\phi)=0, \quad \phi>c_{\phi}
$$

and no pressure jump across the wake:

$$
\Delta_{0}\left[h_{0}\right](\phi)=2 \pi \mathrm{i} P_{0} \mathrm{e}^{\mathrm{i} k \delta \phi}, \quad \phi>c_{\phi} .
$$

The solution is given in terms of the Fourier transform of the jump in acoustic potential either side of the blade streamline in (3.24). In that equation, $\mathscr{A}_{0, n}, \mathscr{B}_{0, n}$ and $\mathscr{C}_{0, n}$ are defined analogously to section 3.B.2 with

$$
\mathscr{T}_{0,0}=-2 \frac{A_{n}}{\beta_{\infty}}, \quad \quad \mathscr{T}_{0, l}=0, \quad l \neq 0
$$

The Fourier transform of the acoustic field may be inverted in the downstream region (Glegg, 1999, §5.1) to obtain the value of the acoustic potential along the wake for insertion into the boundary conditions (3.22.b) and (3.23.b). In a strip in the downstream region 
$\left(0<\psi<s_{\phi}, s_{\phi}\left(\phi-c_{\phi}\right)<d_{\phi} \psi\right)$, we have

$$
\begin{aligned}
h_{0}(\phi, \psi)= & \sum_{m=-\infty}^{\infty} \mathscr{H}_{0, m}^{-} \mathrm{e}^{-\mathrm{i}\left(\lambda_{m}^{-} \phi+\zeta_{m}^{-} \psi\right)} \\
& +\sum_{ \pm} \mathscr{H}_{0, \kappa_{0}^{-}, \pm}^{-} \mathrm{e}^{-\mathrm{i}\left(\kappa_{0}^{-} \phi \pm \zeta_{\kappa_{0}^{-}} \psi\right)}
\end{aligned}
$$

where

$$
\begin{aligned}
\mathscr{H}_{0, m}^{-} & =\frac{-\pi \zeta_{m}^{-} D_{0}^{(2,4)}\left(\lambda_{m}^{-}\right)}{\Delta_{\phi} \sqrt{k^{2} w^{2}-f_{m}^{2}}}, \\
\mathscr{H}_{0, \kappa_{0}^{-}, \pm}^{-} & =\frac{\pi \mathrm{i} P_{0}}{2} \cdot \frac{\mathrm{e}^{\mathrm{i}\left(d_{\phi} \kappa_{0}^{-}+\sigma_{\phi}^{\prime}\right)}-\mathrm{e}^{ \pm \mathrm{i} s_{\phi} \zeta_{\kappa_{0}^{-}}}}{\cos \left(d_{\phi} \kappa_{0}^{-}+\sigma_{\phi}^{\prime}\right)-\cos \left(s_{\phi} \zeta_{\kappa_{0}^{-}}\right)}
\end{aligned}
$$

Along the lower side of the wake $\left(\psi=0^{-}\right)$we have

$$
\begin{aligned}
h_{0}^{-}(\phi) & =\sum_{ \pm} \mathscr{H}_{0, \kappa_{0}^{-}, \pm}^{-} \mathrm{e}^{-\mathrm{i}\left(\kappa_{0}^{-} \phi+d_{\phi} \kappa_{0}^{-}+\sigma_{\phi}^{\prime} \pm s_{\phi} \zeta_{\kappa_{0}^{-}}\right)}+\sum_{m=-\infty}^{\infty} \mathscr{H}_{0, m}^{-} \mathrm{e}^{-\mathrm{i} \lambda_{m}^{-} \phi} \\
\frac{\partial h_{0}^{-}}{\partial \psi}(\phi) & =\sum_{ \pm} \mp \mathrm{i} \zeta_{\kappa_{0}^{-}} \mathscr{H}_{0, \kappa_{0}^{-}, \pm}^{-} \mathrm{e}^{-\mathrm{i}\left(\kappa_{0}^{-} \phi+d_{\phi} \kappa_{0}^{-}+\sigma_{\phi}^{\prime} \pm s_{\phi} \zeta_{\kappa_{0}^{-}}\right)}-\mathrm{i} \sum_{m=-\infty}^{\infty} \zeta_{m}^{-} \mathscr{H}_{0, m}^{-} \mathrm{e}^{-\mathrm{i} \lambda_{m}^{-} \phi}
\end{aligned}
$$

where we have used the quasi-periodicity condition (3.26).

Finally, we define the constants

$$
\begin{aligned}
\mathscr{H}_{0, \kappa_{0}^{-}}^{ \pm \prime} & =-\mathrm{i}\left(\kappa_{0}^{-}+k M_{\infty}^{2} \delta\right) \mathscr{H}_{0, \kappa_{0}^{-}, \pm}^{-} \mathrm{e}^{-\mathrm{i}\left(d_{\phi} \kappa_{0}^{-}+\sigma_{\phi}^{\prime} \pm s_{\phi} \zeta_{\kappa_{0}^{-}}\right)} \\
\mathscr{H}_{0, m}^{\prime} & =-\mathrm{i}\left(\lambda_{m}^{-}+k M_{\infty}^{2} \delta\right) \mathscr{H}_{0, m}^{-},
\end{aligned}
$$

so that in the normal velocity continuity condition (3.13) we may insert

$$
\frac{\partial^{2} h^{-}}{\partial \phi \partial \psi}(\phi)-\mathrm{i} k M_{\infty}^{2} \delta \frac{\partial h^{-}}{\partial \psi}(\phi)=\sum_{ \pm} \mp \mathrm{i} \zeta_{\kappa_{0}^{-}} \mathscr{H}_{0, \kappa_{0}^{-}}^{ \pm \prime} \mathrm{e}^{-\mathrm{i} \kappa_{0}^{-} \phi}-\mathrm{i} \sum_{m=-\infty}^{\infty} \zeta_{m}^{-} \mathscr{H}_{0, m}^{\prime} \mathrm{e}^{-\mathrm{i} \lambda_{m}^{-} \phi}
$$

and in the pressure continuity condition (3.14) we may insert

$$
\frac{\partial h^{-}}{\partial \phi}(\phi)-\mathrm{i} k M_{\infty}^{2} \delta h^{-}(\phi)=\sum_{ \pm} \mathscr{H}_{0, \kappa_{0}^{-}}^{ \pm \prime} \mathrm{e}^{-\mathrm{i} \kappa_{0}^{-} \phi}+\sum_{m=-\infty}^{\infty} \mathscr{H}_{0, m}^{\prime} \mathrm{e}^{-\mathrm{i} \lambda_{m}^{-} \phi},
$$




\section{B Solutions to Wiener-Hopf Problems}

In this section we provide the details of the solutions to the integral equations (3.40.a), (3.41.a), (3.42.a) and (3.43.a) subject to the relevant boundary conditions. This corresponds to step $\mathrm{V}$ of the road map. The techniques used to solve each integral equation are quite similar. Therefore, we will provide the most details for the first solution $\left(h_{1, \Sigma}\right)$ and refer back to these for the subsequent solutions $\left(h_{1, \Delta}, h_{1, \Gamma}\right.$ and $\left.h_{1, S}\right)$.

We begin by providing some crucial details of the Wiener-Hopf kernel.

\section{B.1 Details of Wiener-Hopf Kernel, $j(\gamma)$}

Similarly to Glegg (1999), the multiplicative splitting of the Wiener-Hopf kernel is given by

$$
j(\gamma)=\frac{\zeta}{4 \pi}\left\{\frac{\sin \left(\zeta s_{\phi}\right)}{\cos \left(\zeta s_{\phi}\right)-\cos \left(\gamma d_{\phi}+\sigma_{\phi}^{\prime}\right)}\right\}=J_{+}(\gamma) J_{-}(\gamma)
$$

where

$$
\begin{aligned}
& J_{+}(\gamma)=\frac{k w \sin \left(k w s_{\phi}\right)}{4 \pi\left(\cos \left(k w s_{\phi}\right)-\cos \left(\sigma_{\phi}^{\prime}\right)\right)} \cdot \frac{\prod_{n=0}^{\infty}\left(1-\gamma / \theta_{n}^{-}\right)}{\prod_{m=-\infty}^{\infty}\left(1-\gamma / \lambda_{m}^{-}\right)} \mathrm{e}^{\Phi}, \\
& J_{-}(\gamma)=\frac{\prod_{m=0}^{\infty}\left(1-\gamma / \theta_{n}^{+}\right)}{\prod_{m=-\infty}^{\infty}\left(1-\gamma / \lambda_{m}^{+}\right)} \mathrm{e}^{-\Phi},
\end{aligned}
$$

and

$$
\Phi=\frac{-\mathrm{i} \gamma}{\pi}\left(s_{\phi} \log \left(2 \cos \left(\chi_{\phi}\right)+\chi_{\phi} d_{\phi}\right)\right.
$$

The zeros of $J_{ \pm}$are given by the duct modes

$$
\theta_{n}^{ \pm}= \pm \sqrt{k^{2} w^{2}-\left(\frac{n \pi}{s_{\phi}}\right)^{2}}
$$

and the poles are given by the acoustic modes

$$
\lambda_{m}^{ \pm}=-f_{m} \sin \left(\chi_{\phi}\right) \pm \cos \left(\chi_{\phi}\right) \sqrt{k^{2} w^{2}-f_{m}^{2}}, \quad f_{m}=\frac{\sigma_{\phi}^{\prime}-2 \pi m}{\Delta_{\phi}}
$$


The derivatives of $J^{ \pm}$evaluated at the duct modes are

$$
J_{ \pm}^{\prime}\left(\theta_{n}^{\mp}\right)=\frac{-\theta_{n}^{\mp} s_{\phi}\left(1+\delta_{n, 1}\right)}{4 \pi\left(1-(-1)^{n} \cos \left(d_{\phi} \theta_{n}^{\mp}+\sigma_{\phi}^{\prime}\right)\right) J_{\mp}\left(\theta_{n}^{\mp}\right)} .
$$

It may be demonstrated using standard results of the Gamma function (Peake, 1992, Appendix B), that the split kernel has asymptotic behaviour

$$
J^{ \pm}(\gamma)=\mathcal{O}\left(\gamma^{1 / 2}\right), \quad \text { as }|\gamma| \rightarrow \infty \text { in } \mathscr{M}^{ \pm}
$$

During the Wiener-Hopf analysis it is necessary to perform additive splittings of functions involving the Wiener-Hopf kernel. To expedite this processes, we define the auxiliary functions

$$
\tilde{J}_{ \pm}\left(\gamma, \eta^{ \pm}\right)=\frac{J_{-}(\gamma)}{\gamma-\eta^{ \pm}}
$$

Note that $\tilde{J}_{ \pm}$is not analytic in $\mathscr{M}^{ \pm}$since it possesses a simple pole at $\gamma=\eta^{ \pm}$. Accordingly, we use pole removal to obtain the additive splittings

$$
\left[\tilde{J}_{ \pm}\left(\gamma, \eta^{ \pm}\right)\right]_{ \pm}=\frac{J_{ \pm}(\gamma)-J_{ \pm}\left(\eta^{ \pm}\right)}{\gamma-\eta^{ \pm}}, \quad\left[\tilde{J}_{ \pm}\left(\gamma, \eta^{ \pm}\right)\right]_{\mp}=\frac{J_{ \pm}\left(\eta^{ \pm}\right)}{\gamma-\eta^{ \pm}}
$$

We now present the solutions for the Wiener-Hopf equations for the $D_{1, \Sigma}, D_{1, \Delta}, D_{1, \Gamma}$ and $D_{1, S}$ contributions.

\section{B.2 Solution for $D_{1, \Sigma}$}

The integral equation and boundary conditions for $\Delta_{0}\left[h_{1, \Sigma}\right]$ and $D_{1, \Sigma}$ are summarised in (3.40.a-3.40.f). In a similar way to Glegg (1999), we split this problem into four coupled problems which are amenable to the Wiener-Hopf method. We write

$$
\Delta_{0}\left[h_{1, \Sigma}\right](\phi)=\Delta_{0}\left[h_{1, \Sigma}^{(1)}\right](\phi)+\Delta_{0}\left[h_{1, \Sigma}^{(2)}\right](\phi)+\Delta_{0}\left[h_{1, \Sigma}^{(3)}\right](\phi)+\Delta_{0}\left[h_{1, \Sigma}^{(4)}\right](\phi),
$$

and its Fourier transform

$$
D_{1, \Sigma}(\gamma)=D_{1, \Sigma}^{(1)}(\gamma)+D_{1, \Sigma}^{(2)}(\gamma)+D_{1, \Sigma}^{(3)}(\gamma)+D_{1, \Sigma}^{(4)}(\gamma)
$$


where each $\Delta_{0}\left[h_{1, \Sigma}^{(n)}\right]$ and $D_{1, \Sigma}^{(n)}$ satisfy a semi-infinite integral equation of the form

$$
f_{1, \Sigma}^{(n)}(\phi)=-4 \pi \int_{-\infty}^{\infty} D_{1, \Sigma}^{(n)}(\gamma) j(\gamma) \mathrm{e}^{-\mathrm{i} \gamma \phi} \mathrm{d} \gamma
$$

for $n=1,2,3,4$. The corresponding boundary values are

$$
\begin{array}{rlrl}
f_{1, \Sigma}^{(1)}(\phi) & =\sum_{l=-\infty}^{\infty} c_{\Sigma, l} \mathrm{e}^{-\mathrm{i} \kappa_{l}^{-} \phi}, & \phi>0 ; \\
\Delta_{0}\left[h_{1, \Sigma}^{(1)}\right](\phi) & =0, & & \phi<0 ; \\
f_{1, \Sigma}^{(2)}(\phi) & =0, & & \phi<c_{\phi} ; \\
\Delta_{0}\left[h_{1, \Sigma}^{(1)}\right](\phi)+\Delta_{0}\left[h_{1, \Sigma}^{(2)}\right](\phi) & =2 \pi \mathrm{i} P_{1, \Sigma}^{(2)} \mathrm{e}^{-\mathrm{i} \kappa_{0}^{-} \phi}, & & \phi>c_{\phi} ; \\
f_{1, \Sigma}^{(3)}(\phi) & =0, & & \phi>0 ; \\
\Delta_{0}\left[h_{1, \Sigma}^{(2)}\right](\phi)+\Delta_{0}\left[h_{1, \Sigma}^{(3)}\right](\phi)+\Delta_{0}\left[h_{1, \Sigma}^{(4)}\right](\phi) & =0, & & \phi<0 ; \\
f_{1, \Sigma}^{(4)}(\phi) & =0, & & \phi<c_{\phi} ; \\
\Delta_{0}\left[h_{1, \Sigma}^{(3)}\right](\phi)+\Delta_{0}\left[h_{1, \Sigma}^{(4)}\right](\phi) & =2 \pi \mathrm{i} P_{1, \Sigma}^{(4)} \mathrm{e}^{-\mathrm{i} \kappa_{0}^{-} \phi}, & \phi>c_{\phi} ;
\end{array}
$$

where $P_{1, \Sigma}^{(2)}$ and $P_{1, \Sigma}^{(4)}$ are two constants of integration that will be specified to enforce the Kutta condition. Summing the four above conditions results in the original boundary conditions and, consequently, we may apply the Wiener-Hopf method to each semiinfinite integral equation and sum the resulting contributions to obtain a solution to the original equations.

\section{B.2.1 Solution to First Wiener-Hopf Problem $-D_{1, \Sigma}^{(1)}$}

In this section we solve the integral equation (3.62) for $n=1$,

$$
f_{1, \Sigma}^{(1)}(\phi)=-4 \pi \int_{-\infty}^{\infty} D_{1, \Sigma}^{(1)}(\gamma) j(\gamma) \mathrm{e}^{-\mathrm{i} \gamma \phi} \mathrm{d} \gamma
$$


subject to (3.62.a) and (3.62.b):

$$
f_{1, \Sigma}^{(1)}(\phi)=\sum_{l=-\infty}^{\infty} c_{\Sigma, l} \mathrm{e}^{-\mathrm{i} \kappa_{l}^{-} \phi}, \quad \phi>0 ; \quad \Delta_{0}\left[h_{1, \Sigma}^{(1)}\right](\phi)=0, \quad \phi<0 .
$$

Taking a Fourier transform of (3.66.a) gives

$$
F_{1, \Sigma,-}^{(1)}(\gamma)+F_{1, \Sigma,+}^{(1)}(\gamma)=-4 \pi D_{1, \Sigma,+}^{(1)}(\gamma) j(\gamma)
$$

where

$$
\begin{gathered}
F_{1, \Sigma,-}^{(1)}(\gamma)=\frac{1}{2 \pi} \int_{-\infty}^{0} f_{1, \Sigma}^{(1)}(\phi) \mathrm{e}^{\mathrm{i} \gamma \phi} \mathrm{d} \phi, \quad F_{1, \Sigma,+}^{(1)}(\gamma)=\frac{1}{2 \pi} \int_{0}^{\infty} f_{1, \Sigma}^{(1)}(\phi) \mathrm{e}^{\mathrm{i} \gamma \phi} \mathrm{d} \phi \\
D_{1, \Sigma,+}^{(1)}(\gamma)=\int_{0}^{\infty} \Delta_{0}\left[h_{1, \Sigma}^{(1)}\right](\phi) \mathrm{e}^{\mathrm{i} \gamma \phi} \mathrm{d} \phi .
\end{gathered}
$$

We may use (3.54) to multiplicatively factorise $j=J_{-} J_{+}$and express the Wiener-Hopf equation (3.67) as

$$
\frac{F_{1, \Sigma,-}^{(1)}(\gamma)}{4 \pi J_{-}(\gamma)}+\frac{F_{1, \Sigma,+}^{(1)}(\gamma)}{4 \pi J_{-}(\gamma)}=-D_{1, \Sigma,+}^{(1)}(\gamma) J_{+}(\gamma)
$$

Since we have decomposed the boundary data into a Fourier series, we are able to analytically calculate its half-range Fourier transform. Applying the first relation in (3.66.b) results in

$$
F_{1, \Sigma,+}^{(1)}(\gamma)=\frac{1}{2 \pi \mathrm{i}} \sum_{l=-\infty}^{\infty} \frac{c_{\Sigma, l}}{\gamma-\kappa_{l}^{-}}
$$

The next step in the Wiener-Hopf analysis is to additively factorise the left-hand side of (3.69). The first term is already analytic in the lower half plane but the second term is not analytic in either half plane. Therefore, we apply the typical technique of pole removal (Noble, 1958) to obtain

$$
\frac{F_{1, \Sigma,+}^{(1)}(\gamma)}{J_{-}(\gamma)}=\underbrace{\frac{1}{2 \pi \mathrm{i}} \sum_{l=-\infty}^{\infty} \frac{c_{\Sigma, l}}{\gamma-\kappa_{l}^{-}} \frac{1}{J_{-}\left(\kappa_{l}^{-}\right)}}_{+}+\underbrace{\frac{1}{2 \pi \mathrm{i}} \sum_{l=-\infty}^{\infty} \frac{c_{\Sigma, l}}{\gamma-\kappa_{l}^{-}}\left\{\frac{1}{J_{-}(\gamma)}-\frac{1}{J_{-}\left(\kappa_{l}^{-}\right)}\right\}}_{-},
$$


where the underbrace \pm denotes that the function is analytic in the upper or lower half-plane respectively. Therefore, (3.69) becomes

$$
\begin{aligned}
& \frac{F_{1, \Sigma,-}^{(1)}(\gamma)}{4 \pi J_{-}(\gamma)}+\frac{1}{8 \pi^{2} \mathrm{i}} \sum_{l=-\infty}^{\infty} \frac{c_{\Sigma, l}}{\gamma-\kappa_{l}^{-}}\left\{\frac{1}{J_{-}(\gamma)}-\frac{1}{J_{-}\left(\kappa_{l}^{-}\right)}\right\} \\
& =-D_{1, \Sigma,+}^{(1)}(\gamma) J_{+}(\gamma)-\frac{1}{8 \pi^{2} \mathrm{i}} \sum_{l=-\infty}^{\infty} \frac{c_{\Sigma, l}}{\gamma-\kappa_{l}^{-}} \frac{1}{J_{-}\left(\kappa_{l}^{-}\right)}
\end{aligned}
$$

We may now apply the standard Wiener-Hopf argument: since the left- and right-hand sides of (3.70) are analytic in $\mathscr{M}^{\mp}$ respectively, and they agree on a strip, each side defines the analytic continuation of the other. Therefore, equation (3.70) defines an entire function, $E_{1}(\gamma)$. By appealing to typical arguments that are justified in section 3.C, as $|\gamma| \rightarrow \infty$ in $\mathscr{M}^{-}$, the left-hand side of (3.70) decays due to (3.126.a) and (3.56). Similarly, as $|\gamma| \rightarrow \infty$ in $\mathscr{M}^{+}$, the right-hand side of (3.70) is bounded due to (3.126.b) and (3.56). Therefore, $E_{1}(\gamma)$ is bounded in the entire plane so Liouville's theorem tells us that it must be a constant and, since $E_{1}(\gamma)$ decays in $\mathscr{M}^{-}$, this constant must be zero. Therefore, we may rearrange the right-hand side of (3.70) to obtain

$$
D_{1, \Sigma,+}^{(1)}(\gamma)=\sum_{l=-\infty}^{\infty} \frac{\mathscr{T}_{1, \Sigma, l}}{\left(\gamma-\kappa_{l}^{-}\right)} \cdot \frac{1}{J_{+}(\gamma)}
$$

where

$$
\mathscr{T}_{1, \Sigma, l}=-\frac{c_{\Sigma, l}}{8 \pi^{2} \mathrm{i}} \cdot \frac{1}{J_{-}\left(\kappa_{l}^{-}\right)}
$$

\section{B.2.2 Solution to Second Wiener-Hopf Problem $-D_{1, \Sigma}^{(2)}$}

In this section we solve the integral equation (3.62) for $n=2$,

$$
f_{1, \Sigma}^{(2)}(\phi)=-4 \pi \int_{-\infty}^{\infty} D_{1, \Sigma}^{(2)}(\gamma) j(\gamma) \mathrm{e}^{-\mathrm{i} \gamma \phi} \mathrm{d} \gamma
$$

subject to (3.63.a) and (3.63.b):

$$
f_{1, \Sigma}^{(2)}(\phi)=0, \quad \phi<c_{\phi} ; \quad \Delta_{0}\left[h_{1, \Sigma}^{(1)}\right](\phi)+\Delta_{0}\left[h_{1, \Sigma}^{(2)}\right](\phi)=2 \pi \mathrm{i} P_{1, \Sigma}^{(2)} \mathrm{e}^{-\mathrm{i} \kappa_{0}^{-} \phi}, \quad \phi>c_{\phi} .
$$

Taking the Fourier transform of (3.72.a) gives

$$
F_{1, \Sigma,+}^{(2)}(\gamma)=-4 \pi\left(D_{1, \Sigma,-}^{(2)}(\gamma)+D_{1, \Sigma,+}^{(2)}\right) j(\gamma)
$$


where

$$
\begin{gathered}
F_{1, \Sigma,+}^{(2)}(\gamma)=\frac{1}{2 \pi} \int_{c_{\phi}}^{\infty} f_{1, \Sigma}^{(2)}(\phi) \mathrm{e}^{\mathrm{i} \gamma \phi} \mathrm{d} \phi \\
=\frac{\mathrm{e}^{\mathrm{i} \gamma c_{\phi}}}{2 \pi} \int_{0}^{\infty} f_{1, \Sigma}^{(2)}\left(\phi+c_{\phi}\right) \mathrm{e}^{\mathrm{i} \phi \gamma} \mathrm{d} \phi=\mathrm{e}^{\mathrm{i} \gamma c_{\phi}} F_{1, \Sigma,+}^{*(2)}(\gamma), \\
\begin{aligned}
D_{1, \Sigma,+}^{(2)}(\gamma)=\frac{1}{2 \pi} \int_{c_{\phi}}^{\infty} \Delta_{0}\left[h_{1, \Sigma}^{(2)}\right](\phi) \mathrm{e}^{\mathrm{i} \gamma \phi} \mathrm{d} \phi \\
=\frac{\mathrm{e}^{\mathrm{i} \gamma c_{\phi}}}{2 \pi} \int_{0}^{\infty} \Delta_{0}\left[h_{1, \Sigma}^{(2)}\right]\left(\phi+c_{\phi}\right) \mathrm{e}^{\mathrm{i} \phi \gamma} \mathrm{d} \phi=\mathrm{e}^{\mathrm{i} \gamma c_{\phi}} D_{1, \Sigma,+}^{*(2)}(\gamma), \\
D_{1, \Sigma,-}^{(2)}(\gamma)=\frac{1}{2 \pi} \int_{-\infty}^{c_{\phi}} \Delta_{0}\left[h_{1, \Sigma}^{(2)}\right](\phi) \mathrm{e}^{\mathrm{i} \gamma \phi} \mathrm{d} \phi \\
=\frac{\mathrm{e}^{\mathrm{i} \gamma c_{\phi}}}{2 \pi} \int_{-\infty}^{0} \Delta_{0}\left[h_{1, \Sigma}^{(2)}\right]\left(\phi+c_{\phi}\right) \mathrm{e}^{\mathrm{i} \phi \gamma} \mathrm{d} \phi=\mathrm{e}^{\mathrm{i} \gamma c_{\phi}} D_{1, \Sigma,-}^{*(2)}(\gamma) .
\end{aligned}
\end{gathered}
$$

Factoring out the $\mathrm{e}^{\mathrm{i} \gamma c_{\phi}}$ dependence and dividing by $J_{+}$transforms the Wiener-Hopf equation (3.73) to

$$
\frac{F_{1, \Sigma,+}^{*(2)}(\gamma)}{4 \pi J_{+}(\gamma)}=-\left(D_{1, \Sigma,-}^{*(2)}(\gamma)+D_{1, \Sigma,+}^{*(2)}(\gamma)\right) J_{-}(\gamma)
$$

We may use the downstream boundary condition for this problem (3.72.b) to write

$$
D_{1, \Sigma,+}^{*(2)}(\gamma)=-\frac{P_{1, \Sigma}^{*(2)}}{\gamma-\kappa_{0}^{-}}-\frac{1}{2 \pi} \int_{0}^{\infty} \Delta_{0}\left[h_{1, \Sigma}^{(1)}\right]\left(\phi+c_{\phi}\right) \mathrm{e}^{\mathrm{i} \gamma \phi} \mathrm{d} \phi
$$

where $P_{1, \Sigma}^{*(2)}=P_{1, \Sigma}^{(2)} \mathrm{e}^{-\mathrm{i} \kappa_{0}^{-} c_{\phi}}$. To calculate the remaining integral, we use the inversion formula for the Fourier transform:

$$
\Delta_{0}\left[h_{1, \Sigma}^{(1)}\right](\phi)=\int_{-\infty-\mathrm{i} \tau_{1}}^{\infty-\mathrm{i} \tau_{1}} D_{1, \Sigma}^{(1)}(\gamma) \mathrm{e}^{-\mathrm{i} \gamma \phi} \mathrm{d} \gamma
$$

for some sufficiently small $\tau_{1}>0$. By substituting this representation into our desired integral in (3.76), we obtain

$$
\frac{1}{2 \pi} \int_{0}^{\infty} \Delta_{0}\left[h_{1, \Sigma}^{(1)}\right]\left(\phi+c_{\phi}\right) \mathrm{e}^{\mathrm{i} \gamma \phi} \mathrm{d} \phi=\frac{1}{2 \pi} \int_{0}^{\infty} \int_{-\infty-\mathrm{i} \tau_{1}}^{\infty-\mathrm{i} \tau_{1}} D_{1, \Sigma}^{(1)}\left(\gamma_{1}\right) \mathrm{e}^{-\mathrm{i} \gamma_{1}\left(\phi+c_{\phi}\right)} \mathrm{d} \gamma_{1} \mathrm{e}^{\mathrm{i} \gamma \phi} \mathrm{d} \phi .
$$


Rearranging the order of integration and computing the resulting $\phi$-integral gives

$$
\frac{1}{2 \pi} \int_{0}^{\infty} \Delta_{0}\left[h_{1, \Sigma}^{(1)}\right]\left(\phi+c_{\phi}\right) \mathrm{e}^{\mathrm{i} \gamma \phi} \mathrm{d} \phi=\frac{1}{2 \pi \mathrm{i}} \int_{-\infty-\mathrm{i} \tau_{1}}^{\infty-\mathrm{i} \tau_{1}} \frac{D_{1, \Sigma}^{(1)}\left(\gamma_{1}\right) \mathrm{e}^{-\mathrm{i} \gamma_{1} c_{\phi}}}{\gamma_{1}-\gamma} \mathrm{d} \gamma_{1}
$$

Inspection of the asymptotic behaviour of (3.71) determines that, since $d_{\phi}<c_{\phi}$, this integral can be closed in $\mathscr{M}^{-}$. Since there are no branches in the integrand, the integral will consist of the residues of simple poles; $D_{1, \Sigma}^{(1)}$ possesses simple poles at $\gamma=\kappa_{l}^{-}, \theta_{n}^{-}$. Accordingly, we obtain

$$
\begin{aligned}
& \frac{1}{2 \pi \mathrm{i}} \int_{-\infty-\mathrm{i} \tau_{1}}^{\infty-\mathrm{i} \tau_{1}} \frac{1}{J_{+}\left(\gamma_{1}\right)} \sum_{l=-\infty}^{\infty} \frac{\mathscr{T}_{1, \Sigma, l}}{\left(\gamma_{1}-\kappa_{l}^{-}\right)} \cdot \frac{\mathrm{e}^{-\mathrm{i} \gamma_{1} c_{\phi}}}{\gamma_{1}-\gamma} \mathrm{d} \gamma_{1} \\
& \quad=\sum_{l=-\infty}^{\infty} \frac{\mathscr{T}_{1, \Sigma, l}}{J_{+}\left(\kappa_{l}^{-}\right)} \cdot \frac{\mathrm{e}^{-\mathrm{i} \kappa_{l}^{-} c_{\phi}}}{\gamma-\kappa_{l}^{-}}+\sum_{n=0}^{\infty}\left\{\sum_{l=-\infty}^{\infty} \frac{\mathscr{T}_{1, \Sigma, l}}{\left(\theta_{n}^{-}-\kappa_{l}^{-}\right)}\right\} \cdot \frac{\mathrm{e}^{-\mathrm{i} \theta_{n}^{-} c_{\phi}}}{J_{+}^{\prime}\left(\theta_{n}^{-}\right)\left(\gamma-\theta_{n}^{-}\right)},
\end{aligned}
$$

and, therefore,

$$
\begin{aligned}
\frac{1}{2 \pi \mathrm{i}} \int_{-\infty-\mathrm{i} \tau_{1}}^{\infty-\mathrm{i} \tau_{1}} \frac{D_{1, \Sigma}^{(1)}\left(\gamma_{1}\right) \mathrm{e}^{-\mathrm{i} \gamma_{1} c_{\phi}}}{\gamma_{1}-\gamma} \mathrm{d} \gamma_{1} & =\sum_{l=-\infty}^{\infty} \frac{\mathcal{S}_{1, \Sigma, l} \mathrm{e}^{-\mathrm{i} \kappa_{l}^{-} c_{\phi}}}{\mathrm{i}\left(\kappa_{0}^{-}-\kappa_{l}^{-}\right)\left(\gamma-\kappa_{l}^{-}\right)} \\
& +\sum_{n=0}^{\infty} \frac{\mathscr{A}_{1, \Sigma, n} \mathrm{e}^{-\mathrm{i} \theta_{n}^{-} c_{\phi}}}{\mathrm{i}\left(\theta_{n}^{-}-\kappa_{0}^{-}\right)\left(\gamma-\theta_{n}^{-}\right)}
\end{aligned}
$$

where the constants $\mathcal{S}_{1, \Sigma, l}$ and $\mathscr{A}_{1, \Sigma, n}$ are defined as

$$
\mathcal{S}_{1, \Sigma, l}=\frac{\mathrm{i}\left(\kappa_{0}^{-}-\kappa_{l}^{-}\right)}{J_{+}\left(\kappa_{l}^{-}\right)} \mathscr{T}_{1, \Sigma, l}, \quad \mathscr{A}_{1, \Sigma, n}=\frac{\mathrm{i}\left(\theta_{n}^{-}-\kappa_{0}^{-}\right)}{J_{+}^{\prime}\left(\theta_{n}^{-}\right)} \cdot \sum_{l=-\infty}^{\infty} \frac{\mathscr{T}_{1, \Sigma, l}}{\theta_{n}^{-}-\kappa_{l}^{-}} .
$$

Finally, we combine the integral with (3.76) to obtain the expression

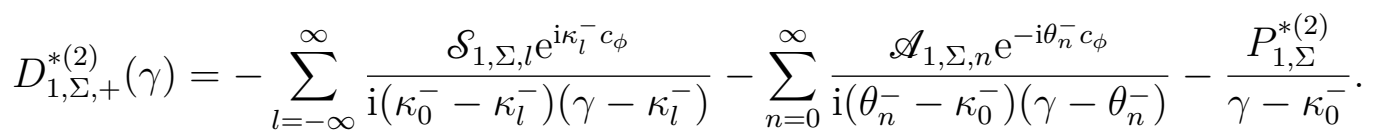

We may now substitute this representation for $D_{1, \Sigma,+}^{*(2)}$ into the Wiener-Hopf equation (3.75) to obtain

$$
\begin{aligned}
\frac{F_{1, \Sigma,+}^{*(2)}(\gamma)}{4 \pi J_{+}(\gamma)}= & -J_{-}(\gamma) D_{1, \Sigma,-}^{*(2)}(\gamma)+P_{1, \Sigma}^{*(2)} \tilde{J}_{-}\left(\gamma, \kappa_{0}^{-}\right) \\
& +\sum_{l=-\infty}^{\infty} \frac{\mathcal{S}_{1, \Sigma, l} \mathrm{e}^{\mathrm{i} \kappa_{l}^{-} c_{\phi}}}{\mathrm{i}\left(\kappa_{0}^{-}-\kappa_{l}^{-}\right)} \tilde{J}_{-}\left(\gamma, \kappa_{l}^{-}\right)+\sum_{n=0}^{\infty} \frac{\mathscr{A}_{1, \Sigma, n} \mathrm{e}^{-\mathrm{i} \theta_{n}^{-} c_{\phi}}}{\mathrm{i}\left(\theta_{n}^{-}-\kappa_{0}^{-}\right)} \tilde{J}_{-}\left(\gamma, \theta_{n}^{-}\right)
\end{aligned}
$$


where we have used the auxiliary function $\tilde{J}$ introduced in (3.57).

The left-hand side of the above equation is analytic in the upper half plane, but there are terms on the right-hand side which are not analytic in either plane. Therefore, we use the additive splitting (3.58) to obtain

$$
\begin{array}{r}
\frac{F_{1, \Sigma,+}^{*(2)}(\gamma)}{4 \pi J_{+}(\gamma)}-\sum_{l=-\infty}^{\infty} \frac{\mathcal{S}_{1, \Sigma, l} \mathrm{e}^{-\mathrm{i} \kappa_{l}^{-} c_{\phi}}}{\mathrm{i}\left(\kappa_{0}^{-}-\kappa_{l}^{-}\right)}\left[\tilde{J}_{-}\left(\gamma, \kappa_{l}^{-}\right)\right]_{+}-\sum_{n=0}^{\infty} \frac{\mathscr{A}_{1, \Sigma, n} \mathrm{e}^{-\mathrm{i} \theta_{n}^{-} c_{\phi}}}{\mathrm{i}\left(\theta_{n}^{-}-\kappa_{0}^{-}\right)}\left[\tilde{J}_{-}\left(\gamma, \theta_{n}^{-}\right)\right]_{+} \\
-P_{1, \Sigma}^{*(2)}\left[\tilde{J}_{-}\left(\gamma, \kappa_{0}^{-}\right)\right]_{+}=-J_{-}(\gamma) D_{1, \Sigma,-}^{*(2)}(\gamma)+\sum_{l=-\infty}^{\infty} \frac{\mathcal{S}_{1, \Sigma, l} \mathrm{e}^{-\mathrm{i} \kappa_{l}^{-} c_{\phi}}}{\mathrm{i}\left(\kappa_{0}^{-}-\kappa_{l}^{-}\right)}\left[\tilde{J}_{-}\left(\gamma, \kappa_{l}^{-}\right)\right]_{-} \\
+\sum_{n=0}^{\infty} \frac{\mathscr{A}_{1, \Sigma, n} \mathrm{e}^{-\mathrm{i} \theta_{n}^{-} c_{\phi}}}{\mathrm{i}\left(\theta_{n}^{-}-\kappa_{0}^{-}\right)}\left[\tilde{J}_{-}\left(\gamma, \theta_{n}^{-}\right)\right]_{+}+P_{1, \Sigma}^{*(2)}\left[\tilde{J}_{-}\left(\gamma, \kappa_{0}^{-}\right)\right]_{+} .
\end{array}
$$

In a similar way to section 3.B.2.1, we now apply the standard Wiener-Hopf argument. In contrast to the previous section where the edge condition constrained the leading-edge pressure singularity, we now apply the unsteady Kutta condition (Ayton et al., 2016) which tells us that the intensity of the wake is such that both the velocity and pressure are bounded at the trailing edge. Practically, this means that $\Delta_{0}\left[h_{1, \Sigma}\right]\left(c_{\phi}\right) \neq 0$ and its derivatives are finite. Consequently, the left-hand side of (3.78) is bounded. In a similar way to before, integration by parts can be applied to show that $D_{1, \Sigma,-}^{*(2)}(\gamma)=\mathscr{O}(1 / \gamma)$ as $|\gamma| \rightarrow \infty$ in $\mathscr{M}^{-}$. Therefore, in this limit the right-hand side of (3.78) tends to a constant. Analytic continuation, Liouville's theorem, and the fact that the left-hand side of (3.78) decays in $\mathscr{M}^{+}$, indicates that this constant must be zero. This allows us to find an appropriate value of $\Delta_{0}\left[h_{1, \Sigma}^{(2)}\right]\left(c_{\phi}\right)$ that satisfies the Kutta condition. If we multiply the left-hand side of (3.78) by $\gamma$ and let $|\gamma| \rightarrow \infty$ in $\mathscr{M}^{+}$, the left-hand side of (3.78) indicates that

$$
P_{1, \Sigma}^{*(2)}=-\sum_{l=-\infty}^{\infty} \frac{\mathcal{S}_{1, \Sigma, l} \mathrm{e}^{-\mathrm{i} \kappa_{l}^{-} c_{\phi}}}{\mathrm{i}\left(\kappa_{0}^{-}-\kappa_{l}^{-}\right)} \cdot \frac{J_{-}\left(\kappa_{l}^{-}\right)}{J_{-}\left(\kappa_{0}^{-}\right)}-\sum_{n=0}^{\infty} \frac{\mathscr{A}_{1, \Sigma, n} \mathrm{e}^{-\mathrm{i} \theta_{n}^{-} c_{\phi}}}{\mathrm{i}\left(\theta_{n}^{-}-\kappa_{0}^{-}\right)} \cdot \frac{J_{-}\left(\theta_{n}^{-}\right)}{J_{-}\left(\kappa_{0}^{-}\right)} .
$$

So that, after substituting in the downstream representation (3.77) and the expression for the pressure constant (3.79), the right-hand side of (3.78) yields

$$
D_{1, \Sigma}^{(2)}(\gamma)=-\sum_{l=-\infty}^{\infty} \frac{\mathcal{S}_{1, \Sigma, l} \mathrm{e}^{\mathrm{i}\left(\gamma-\kappa_{l}^{-}\right) c_{\phi}}}{\mathrm{i}\left(\gamma-\kappa_{0}^{-}\right)\left(\gamma-\kappa_{l}^{-}\right)} \cdot \frac{J_{-}\left(\kappa_{l}^{-}\right)}{J_{-}(\gamma)}-\sum_{n=0}^{\infty} \frac{\mathscr{A}_{1, \Sigma, n} \mathrm{e}^{\mathrm{i}\left(\gamma-\theta_{n}^{-}\right) c_{\phi}}}{\mathrm{i}\left(\gamma-\kappa_{0}^{-}\right)\left(\gamma-\theta_{n}^{-}\right)} \cdot \frac{J_{-}\left(\theta_{n}^{-}\right)}{J_{-}(\gamma)}
$$

It should be noted that the only poles of $D_{1, \Sigma}^{(2)}$ in $\mathscr{M}^{+}$are at the zeros of $J_{-}$where $\gamma=\theta_{n}^{+}$. 


\section{B.2.3 Solutions to Third and Fourth Wiener-Hopf Problems $-D_{1, \Sigma}^{(3)}, D_{1, \Sigma}^{(4)}$}

Since the boundary conditions (3.64.b) and (3.65.b) for $\Delta_{0}\left[h_{1, \Sigma}^{(3)}\right]$ and $\Delta_{0}\left[h_{1, \Sigma}^{(4)}\right]$ are coupled, we must solve for them simultaneously. Taking a Fourier Transform of (3.62) and applying the boundary conditions for $n=3,4$ gives

$$
\begin{aligned}
& F_{1, \Sigma,-}^{(3)}(\gamma)=-4 \pi\left(D_{1, \Sigma,-}^{(3)}(\gamma)+D_{1, \Sigma,+}^{(3)}(\gamma)\right) j(\gamma), \\
& F_{1, \Sigma,+}^{*(4)}(\gamma)=-4 \pi\left(D_{1, \Sigma,-}^{*(4)}(\gamma)+D_{1, \Sigma,+}^{*(4)}(\gamma)\right) j(\gamma)
\end{aligned}
$$

where $F_{1, \Sigma,-}^{(3)}, D_{1, \Sigma,-}^{(3)}$ and $D_{1, \Sigma,+}^{(3)}$ are defined in an analogous way to (3.68.a) and (3.68.b), and $F_{1, \Sigma,+}^{*(4)}, D_{1, \Sigma,+}^{*(4)}$ and $D_{1, \Sigma,-}^{*(4)}$ are defined in an analogous way to (3.74.a), (3.74.b) and (3.74.c). Using a similar approach to section 3.B.2.2, we may show that the upstream boundary condition (3.64.a) is equivalent to

$$
D_{1, \Sigma,-}^{(3)}(\gamma)=\frac{1}{2 \pi \mathrm{i}} \int_{-\infty+\mathrm{i} \tau_{0}}^{\infty+\mathrm{i} \tau_{0}} \frac{D_{1, \Sigma}^{(2)}\left(\gamma_{1}\right)+D_{1, \Sigma}^{(4)}\left(\gamma_{1}\right)}{\gamma_{1}-\gamma} \mathrm{d} \gamma_{1}
$$

for sufficiently small $\tau_{0}>0$. We now assume that the only poles of $D_{1, \Sigma,-}^{(4)}$ in $\mathscr{M}^{+}$are at $\gamma=\theta_{n}^{+}$. An equivalent assumption was made by Glegg (1999), and it turns out that the same result is achieved by matching upstream and downstream solutions across the inter-blade region (Peake, 1993). This assumption, and sufficiently fast decay, allows us to close the above integral in $\mathscr{M}^{+}$and evaluate it as

$$
D_{1, \Sigma,-}^{(3)}(\gamma)=-\sum_{n=0}^{\infty} \frac{\mathscr{B}_{1, \Sigma, n}}{\gamma-\theta_{n}^{+}}
$$

where $\mathscr{B}_{1, \Sigma, n}$ are the residues of $D_{1, \Sigma,-}^{(2)}\left(\gamma_{1}\right)+D_{1, \Sigma,-}^{(4)}\left(\gamma_{1}\right)$ in $\gamma=\theta_{n}^{+}$. The residues of $D_{1, \Sigma,-}^{(4)}$ are currently unknown, but the residues of $D_{1, \Sigma,-}^{(2)}$ are given by

$$
\begin{aligned}
D_{r, \Sigma, k}^{(2)}=\frac{-\mathrm{e}^{\mathrm{i} \theta_{k}^{+} c_{\phi}}}{\mathrm{i}\left(\theta_{k}^{+}-\kappa_{0}^{-}\right) J_{-}^{\prime}\left(\theta_{k}^{+}\right)}\left\{\sum_{l=-\infty}^{\infty} \frac{\mathcal{S}_{1, \Sigma, l} \mathrm{e}^{-\mathrm{i} \kappa_{l}^{-} \phi_{c}}}{\theta_{k}^{+}-\kappa_{l}^{-}} \cdot J_{-}\left(\kappa_{l}^{-}\right)\right. \\
\left.+\sum_{n=0}^{\infty} \frac{\mathcal{A}_{1, \Sigma, n} \mathrm{e}^{-\mathrm{i} \theta_{n}^{-} c_{\phi}}}{\theta_{k}^{+}-\theta_{n}^{-}} \cdot J_{-}\left(\theta_{n}^{-}\right)\right\} .
\end{aligned}
$$


We may now substitute (3.82) into (3.80) and use the factorisations (3.54) and (3.58) to obtain the Wiener-Hopf equation

$$
\frac{F_{1, \Sigma,-}^{(3)}(\gamma)}{4 \pi J_{-}(\gamma)}+\sum_{n=0}^{\infty} \frac{\mathscr{B}_{1, \Sigma, n}}{\gamma-\theta_{n}^{+}}\left[\tilde{J}_{+}\left(\gamma, \theta_{n}^{+}\right)\right]_{-}=D_{1, \Sigma,+}^{(3)}(\gamma) J_{+}(\gamma)-\sum_{n=0}^{\infty} \frac{\mathscr{B}_{1, \Sigma, n}}{\gamma-\theta_{n}^{+}}\left[\tilde{J}_{+}\left(\gamma, \theta_{n}^{+}\right)\right]_{+}
$$

We appeal to similar edge conditions as those in section 3.B.2.1, and consequently apply the typical Wiener-Hopf argument to obtain

$$
D_{1, \Sigma,+}^{(3)}(\gamma)=\sum_{n=0}^{\infty} \frac{\mathscr{B}_{1, \Sigma, n}}{\gamma-\theta_{n}^{+}} \cdot\left\{1-\frac{J_{+}\left(\theta_{n}^{+}\right)}{J_{+}(\gamma)}\right\}
$$

When we combine this expression with the upstream representation (3.82) we obtain

$$
D_{1, \Sigma}^{(3)}(\gamma)=-\sum_{n=0}^{\infty} \frac{\mathscr{B}_{1, \Sigma, n}}{\gamma-\theta_{n}^{+}} \cdot \frac{J_{+}\left(\theta_{n}^{+}\right)}{J_{+}(\gamma)} .
$$

The residues $\mathscr{B}_{1, \Sigma, n}$ still need to be determined; we now move onto the solution for $D_{1, \Sigma}^{(4)}$. In a similar way to section 3.B.2.2, we may invert the Fourier transform for the downstream boundary condition (3.65.b) to write

$$
D_{1, \Sigma,+}^{*(4)}(\gamma)=-\frac{P_{1, \Sigma}^{*(4)}}{\gamma-\kappa_{0}^{-}}-\frac{1}{2 \pi \mathrm{i}} \int_{-\infty-\mathrm{i} \tau_{1}}^{\infty+\mathrm{i} \tau_{1}} \sum_{n=0}^{\infty} \frac{\mathscr{B}_{1, \Sigma, n}}{\left(\gamma_{1}-\gamma\right)\left(\gamma_{1}-\theta_{n}^{+}\right)}\left\{1-\frac{J_{+}\left(\theta_{n}^{+}\right)}{J_{+}\left(\gamma_{1}\right)}\right\} \mathrm{e}^{-\mathrm{i} \gamma_{1} c_{\phi}} \mathrm{d} \gamma_{1} .
$$

This integral can be closed in $\mathscr{M}^{-}$to obtain

$$
D_{1, \Sigma,+}^{*(4)}(\gamma)=-\frac{P_{1, \Sigma}^{*(4)}}{\gamma-\kappa_{0}^{-}}-\sum_{n=0}^{\infty} \frac{\mathscr{C}_{1, \Sigma, n} \mathrm{e}^{-\mathrm{i} \theta_{n}^{-} c_{\phi}}}{\mathrm{i}\left(\theta_{n}^{-}-\kappa_{0}^{-}\right)\left(\gamma-\theta_{n}^{-}\right)}
$$

where

$$
\mathscr{C}_{1, \Sigma, n}=\sum_{k=0}^{\infty} \frac{\mathrm{i}\left(\theta_{n}^{-}-\kappa_{0}^{-}\right)}{\left(\theta_{k}^{+}-\theta_{n}^{-}\right)} \cdot \frac{J_{+}\left(\theta_{k}^{+}\right)}{J_{+}^{\prime}\left(\theta_{n}^{-}\right)} \cdot \mathscr{B}_{1, \Sigma, k} .
$$

If we truncate the series, we may write this system of equations in matrix form:

$$
\mathscr{C}_{1, \Sigma}=\mathbf{L} \mathscr{B}_{1, \Sigma}
$$

where

$$
\{\mathbf{L}\}_{n, m}=\frac{\mathrm{i}\left(\theta_{n}^{-}-\kappa_{0}^{-}\right)}{\left(\theta_{m}^{+}-\theta_{n}^{-}\right)} \cdot \frac{J_{+}\left(\theta_{m}^{+}\right)}{J_{+}^{\prime}\left(\theta_{n}^{-}\right)} .
$$


We now use the factorisations (3.54) and (3.58) to write the Wiener-Hopf equation (3.81) in the form

$$
\begin{gathered}
\frac{F_{1, \Sigma,+}^{*(4)}(\gamma)}{4 \pi J_{+}(\gamma)}+P_{1, \Sigma}^{*(4)}\left[\tilde{J}_{-}\left(\gamma, \kappa_{0}^{-}\right)\right]_{+}+\sum_{n=0}^{\infty} \frac{\mathscr{C}_{1, \Sigma, n} \mathrm{e}^{-\mathrm{i} \theta_{n}^{-} c_{\phi}}}{\mathrm{i}\left(\theta_{n}^{-}-\kappa_{0}^{-}\right)}\left[\tilde{J}_{-}\left(\gamma, \theta_{n}^{-}\right)\right]_{+} \\
=D_{1, \Sigma,-}^{*(4)}(\gamma) J_{-}(\gamma)-P_{1, \Sigma}^{*(4)}\left[\tilde{J}_{-}\left(\gamma, \kappa_{0}^{-}\right)\right]_{-}-\sum_{n=0}^{\infty} \frac{\mathscr{C}_{1, \Sigma, n} \mathrm{e}^{-\mathrm{i} c_{\phi} \theta_{n}^{-}}}{\mathrm{i}\left(\theta_{n}^{-}-\kappa_{0}^{-}\right)}\left[\tilde{J}_{-}\left(\gamma, \theta_{n}^{-}\right)\right]_{-} .
\end{gathered}
$$

A similar argument to section 3.B.2.2 and application of the unsteady Kutta condition yields

$$
P_{1, \Sigma}^{*(4)}=-\sum_{n=0}^{\infty} \frac{\mathscr{C}_{1, \Sigma, n} \mathrm{e}^{-\mathrm{i} \theta_{n}^{-} c_{\phi}}}{\mathrm{i}\left(\theta_{n}^{-}-\kappa_{0}^{-}\right)} \cdot \frac{J_{-}\left(\theta_{n}^{-}\right)}{J_{-}\left(\kappa_{0}^{-}\right)}
$$

Therefore, rearranging (3.86) and applying the downstream boundary condition (3.84) yields

$$
D_{1, \Sigma}^{(4)}(\gamma)=-\sum_{n=0}^{\infty} \frac{\mathscr{C}_{1, \Sigma, n} \mathrm{e}^{\mathrm{i} c_{\phi}\left(\gamma-\theta_{n}^{-}\right)}}{\mathrm{i}\left(\theta_{n}^{-}-\kappa_{0}^{-}\right)\left(\gamma-\theta_{n}^{-}\right)} \cdot \frac{J_{-}\left(\theta_{n}^{-}\right)}{J_{-}(\gamma)} .
$$

As expected from our previous assumption, $D_{1, \Sigma}^{(4)}$ only posseses poles in $\mathscr{M}^{+}$at $\gamma=\theta_{n}^{+}$. We calculate the residues at these points as

$$
\mathscr{B}_{1, \Sigma, n}=D_{1, \Sigma, n}-\sum_{m=0}^{\infty} \frac{\mathscr{C}_{1, \Sigma, n} \mathrm{e}^{\mathrm{i}\left(\theta_{n}^{+}-\theta_{m}^{-}\right) c_{\phi}}}{\mathrm{i}\left(\theta_{n}^{+}-\kappa_{0}^{-}\right)\left(\theta_{n}^{+}-\theta_{m}^{-}\right)} \cdot \frac{J_{-}\left(\theta_{m}^{-}\right)}{J_{-}^{\prime}\left(\theta_{n}^{+}\right)},
$$

or, in matrix form,

$$
\mathscr{B}_{1, \Sigma}=\mathbf{D}_{r, \Sigma}+\mathbf{F} \mathscr{C}_{1, \Sigma}
$$

where

$$
\{\mathbf{F}\}_{n, m}=-\frac{\mathrm{e}^{\mathrm{i}\left(\theta_{n}^{+}-\theta_{m}^{-}\right) c_{\phi}}}{\mathrm{i}\left(\theta_{n}^{+}-\kappa_{0}^{-}\right)\left(\theta_{n}^{+}-\theta_{m}^{-}\right)} \cdot \frac{J_{-}\left(\theta_{m}^{-}\right)}{J_{-}^{\prime}\left(\theta_{n}^{+}\right)} .
$$

In a similar way to Glegg (1999), the pair of matrix equations (3.85) and (3.88) may be combined and solved to give expressions for $\mathscr{B}_{1, \Sigma, n}$ and $\mathscr{C}_{1, \Sigma, n}$. The solution for $D_{1, \Sigma}$ is now complete. 


\section{B.3 Solution for $D_{1, \Delta}$}

The integral equation and boundary conditions for $\Delta_{0}\left[h_{1, \Delta}\right]$ and $D_{1, \Delta}$ are summarised in (3.41.a-3.41.f). Similarly to section 3.B.2, we split this problem into four coupled problems in an analogous way to (3.59) and (3.60). Consequently, each $\Delta_{0}\left[h_{1, \Delta}^{(n)}\right]$ and $D_{1, \Delta}^{(n)}$ satisfy a semi-infinite integral equation of the form

$$
f_{1, \Delta}^{(n)}(\phi)=4 \pi \int_{-\infty}^{\infty}\left\{-D_{1, \Delta}(\gamma) j(\gamma)+\left(\delta_{n, 1} G_{\Delta}^{(1)}(\gamma)+\delta_{n, 2} G_{\Delta}^{(2)}(\gamma)\right) \cdot k(\gamma)\right\} \mathrm{e}^{-\mathrm{i} \gamma \phi} \mathrm{d} \gamma
$$

for $n=1,2,3,4$, where

$$
G_{\Delta}^{(1)}(\gamma)=-\sum_{l=-\infty}^{\infty} \frac{c_{\Delta, l}}{2 \pi \mathrm{i}\left(\gamma-\kappa_{l}^{-}\right)}, \quad G_{\Delta}^{(2)}(\gamma)=\sum_{l=-\infty}^{\infty} \frac{(-1)^{l} c_{\Delta, l}}{2 \pi \mathrm{i}\left(\gamma-\kappa_{l}^{-}\right)} \mathrm{e}^{\mathrm{i}\left(\gamma-\kappa_{0}^{-}\right) c_{\phi}}
$$

The corresponding boundary values are

$$
\begin{array}{rlrl}
f_{1, \Delta}^{(1)}(\phi) & =0, & & \phi>0 ; \\
\Delta_{0}\left[h_{1, \Delta}^{(1)}\right](\phi) & =0, & & \phi<0 ; \\
f_{1, \Delta}^{(2)}(\phi) & =0, & & \phi<c_{\phi} ; \\
\Delta_{0}\left[h_{1, \Delta}^{(1)}\right](\phi)+\Delta_{0}\left[h_{1, \Delta}^{(2)}\right](\phi) & =2 \pi \mathrm{i} P_{1, \Delta}^{(2)} \mathrm{e}^{-\mathrm{i} \kappa_{0}^{-} \phi}, & & \phi>c_{\phi} ; \\
f_{1, \Delta}^{(3)}(\phi) & =0, & \\
\Delta_{0}\left[h_{1, \Delta}^{(2)}\right](\phi)+\Delta_{0}\left[h_{1, \Delta}^{(3)}\right](\phi)+\Delta_{0}\left[h_{1, \Delta}^{(4)}\right](\phi) & =0, & & \phi>0 ; \\
f_{1, \Delta}^{(4)}(\phi) & =0, & & \phi<0 ; \\
\Delta_{0}\left[h_{1, \Delta}^{(3)}\right](\phi)+\Delta_{0}\left[h_{1, \Delta}^{(4)}\right](\phi) & =2 \pi \mathrm{i} P_{1, \Delta}^{(4)} \mathrm{e}^{-\mathrm{i} \kappa_{0}^{-} \phi}, & & \phi>c_{\phi} ;
\end{array}
$$

where $P_{1, \Delta}^{(2)}$ and $P_{1, \Delta}^{(4)}$ are two constants of integration that will be specified to enforce the Kutta condition. Summing the four above boundary values results in the original integral equation. Consequently, we may apply the Wiener-Hopf method to each individual 
integral equation and sum the resulting contributions to obtain a solution to the original integral equation.

\section{B.3.1 Solution to First Wiener-Hopf Problem $-D_{1, \Delta}^{(1)}$}

Taking a Fourier transform of (3.89) for $n=1$, using the kernel splitting relation (3.54), and applying (3.90.a) and (3.90.b) gives

$$
\frac{F_{1, \Delta,-}^{(1)}(\gamma)}{4 \pi J_{-}(\gamma)}=-D_{1, \Delta,+}^{(1)}(\gamma) J_{+}(\gamma)+G_{\Delta}^{(1)}(\gamma) \frac{k(\gamma)}{J_{-}(\gamma)}
$$

Now we note that the only poles of $G_{\Delta}^{(1)}(\gamma) k(\gamma)$ in $\mathscr{M}^{-}$are at $\gamma=\kappa_{l}^{-}, \lambda_{m}^{-}$. Therefore, we may apply pole removal to remove the singularities at these locations and write

$$
\begin{aligned}
\frac{k(\gamma) G_{\Delta}^{(1)}(\gamma)}{J_{-}(\gamma)}= & \underbrace{\sum_{m=-\infty}^{\infty} \frac{k_{r, m}^{-} G_{\Delta}^{(1)}\left(\lambda_{m}^{-}\right)}{\left(\gamma-\lambda_{m}^{-}\right) J_{-}\left(\lambda_{m}^{-}\right)}-\sum_{l=-\infty}^{\infty} \frac{k\left(\kappa_{l}^{-}\right) c_{\Delta, l}}{2 \pi \mathrm{i}\left(\gamma-\kappa_{l}^{-}\right) J_{-}\left(\kappa_{l}^{-}\right)}}_{+} \\
& +\underbrace{\frac{k(\gamma) G_{\Delta}^{(1)}(\gamma)}{J_{-}(\gamma)}-\sum_{m=-\infty}^{\infty} \frac{k_{r, m}^{-} G_{\Delta}^{(1)}\left(\lambda_{m}^{-}\right)}{\left(\gamma-\lambda_{m}^{-}\right) J_{-}\left(\lambda_{m}^{-}\right)}+\sum_{l=-\infty}^{\infty} \frac{k\left(\kappa_{l}^{-}\right) c_{\Delta, l}}{2 \pi \mathrm{i}\left(\gamma-\kappa_{l}^{-}\right) J_{-}\left(\kappa_{l}^{-}\right)}}_{-},
\end{aligned}
$$

where $k_{r, m}^{-}$are the residues of $k$ at $\gamma=\lambda_{m}^{-}$. Therefore, (3.94) becomes

$$
\begin{array}{r}
\frac{F_{1, \Delta,-}^{(1)}(\gamma)}{4 \pi J_{-}(\gamma)}-\frac{G_{\Delta}^{(1)}(\gamma) k(\gamma)}{J_{-}(\gamma)}+\sum_{m=-\infty}^{\infty} \frac{k_{r, m}^{-} G_{\Delta}^{(1)}\left(\lambda_{m}^{-}\right)}{\left(\gamma-\lambda_{m}^{-}\right) J_{m}\left(\lambda_{m}^{-}\right)}-\sum_{l=-\infty}^{\infty} \frac{k\left(\kappa_{0}^{-}\right) c_{\Delta, l}}{2 \pi \mathrm{i}\left(\gamma-\kappa_{l}^{-}\right) J_{-}\left(\kappa_{l}^{-}\right)}= \\
-D_{1, \Delta,+}^{(1)}(\gamma) J_{+}(\gamma)+\sum_{m=-\infty}^{\infty} \frac{k_{r, m}^{-} G_{\Delta}^{(1)}\left(\lambda_{m}^{-}\right)}{\left(\gamma-\lambda_{m}^{-}\right) J_{-}\left(\lambda_{m}^{-}\right)}-\sum_{l=-\infty}^{\infty} \frac{k\left(\kappa_{l}^{-}\right) c_{\Delta, l}}{2 \pi \mathrm{i}\left(\gamma-\kappa_{l}^{-}\right) J_{-}\left(\kappa_{l}^{-}\right)}
\end{array}
$$

We now appeal to an argument similar to that in section 3.B.2.1 to conclude that each side of the above expression is equivalent to a constant that must vanish. Consequently, we obtain the following expression

$$
D_{1, \Delta}^{(1)}(\gamma)=\sum_{l=-\infty}^{\infty} \frac{\mathscr{T}_{1, \Delta, l}}{\left(\gamma-\kappa_{l}^{-}\right)} \cdot \frac{1}{J_{+}(\gamma)}+\sum_{m=-\infty}^{\infty} \frac{\mathscr{R}_{1, \Delta, m}}{\left(\gamma-\lambda_{m}^{-}\right)} \cdot \frac{1}{J_{+}(\gamma)}
$$


where

$$
\mathscr{R}_{1, \Delta, m}=\frac{k_{r, m}^{-} G_{\Delta}^{(1)}\left(\lambda_{m}^{-}\right)}{J_{-}\left(\lambda_{m}^{-}\right)}, \quad \mathscr{T}_{1, \Delta, l}=\frac{c_{\Delta, l} k\left(\kappa_{l}^{-}\right)}{2 \pi \mathrm{i} J_{-}\left(\kappa_{l}^{-}\right)}
$$

\section{B.3.2 Solution to Second Wiener-Hopf Problem $-D_{1, \Delta}^{(2)}$}

Taking a Fourier transform of (3.89) for $n=2$, using the kernel splitting relation (3.54), and applying the analogous form of the upstream boundary values (3.91.a) gives

$$
\frac{F_{1+}^{*(2)}(\gamma)}{4 \pi J_{+}(\gamma)}=-\left(D_{1, \Delta-}^{*(2)}(\gamma)+D_{1, \Delta+}^{*(2)}(\gamma)\right) J_{-}(\gamma)+G_{\Delta}^{*(2)}(\gamma) \frac{k(\gamma)}{J_{+}(\gamma)}
$$

where $F_{1, \Delta,+}^{*(2)}, D_{1, \Delta,-}^{*(2)}$ and $D_{1, \Delta,+}^{*(2)}$ are defined analogously to (3.74.a), (3.74.b) and (3.74.c) respectively, and $G_{\Delta}^{*(2)}(\gamma)=G_{\Delta}^{(2)}(\gamma) \mathrm{e}^{-\mathrm{i} c_{\phi} \gamma}$. Using a similar approach to section 3.B.2.2, we may use the downstream boundary values (3.91.b) to write

$$
D_{1, \Delta,+}^{*(2)}(\gamma)=-\frac{P_{1, \Delta}^{*(2)}}{\gamma-\kappa_{l}^{-}}-\frac{1}{2 \pi \mathrm{i}} \int_{-\infty-\mathrm{i} \tau_{1}}^{\infty-\mathrm{i} \tau_{1}} \frac{D_{1, \Delta}^{(1)}\left(\gamma_{1}\right) \mathrm{e}^{-\mathrm{i} \gamma_{1} c_{\phi}}}{\gamma_{1}-\gamma} \mathrm{d} \gamma_{1} .
$$

This integral can be closed in $\mathscr{M}^{-}$. Inspection of (3.97) determines that the only poles of $D_{1, \Delta}^{(1)}$ in $\mathscr{M}^{-}$are at $\gamma=\kappa_{l}^{-}, \theta_{n}^{-}$. By noting the identities

$$
\begin{gathered}
\frac{1}{2 \pi \mathrm{i}} \int_{-\infty-\mathrm{i} \tau_{1}}^{\infty-\mathrm{i} \tau_{1}} \frac{1}{J_{+}\left(\gamma_{1}\right)} \sum_{l=-\infty}^{\infty} \frac{\mathscr{T}_{1, \Delta, l}}{\left(\gamma_{1}-\kappa_{l}^{-}\right)} \cdot \frac{\mathrm{e}^{-\mathrm{i} \gamma_{1} c_{\phi}}}{\gamma_{1}-\gamma} \mathrm{d} \gamma_{1}= \\
\sum_{l=-\infty}^{\infty} \frac{\mathscr{T}_{1, \Delta, l}}{J_{+}\left(\kappa_{l}^{-}\right)} \cdot \frac{\mathrm{e}^{-\mathrm{i} \kappa_{l}^{-} c_{\phi}}}{\gamma-\kappa_{l}^{-}}+\sum_{n=0}^{\infty}\left\{\sum_{l=-\infty}^{\infty} \frac{\mathscr{T}_{1, \Delta, l}}{\theta_{n}^{-}-\kappa_{l}^{-}}\right\} \cdot \frac{\mathrm{e}^{-\mathrm{i} \theta_{n}^{-} c_{\phi}}}{J_{+}^{\prime}\left(\theta_{n}^{-}\right)\left(\gamma-\theta_{n}^{-}\right)}, \\
\frac{1}{2 \pi \mathrm{i}} \int_{-\infty-\mathrm{i} \tau_{1}}^{\infty-\mathrm{i} \tau_{1}} \frac{1}{J_{+}\left(\gamma_{1}\right)} \sum_{m=-\infty}^{\infty} \frac{\mathscr{R}_{1, \Delta, m}}{\gamma_{1}-\lambda_{m}^{-}} \cdot \frac{\mathrm{e}^{-\mathrm{i} \gamma_{1} c_{\phi}}}{\gamma_{1}-\gamma} \mathrm{d} \gamma_{1}= \\
\sum_{n=0}^{\infty}\left\{\sum_{m=-\infty}^{\infty} \frac{\mathscr{R}_{1, \Delta, m}}{\theta_{n}^{-}-\lambda_{m}^{-}}\right\} \frac{\mathrm{e}^{-\mathrm{i} \theta_{n}^{-} c_{\phi}}}{J^{\prime}\left(\theta_{n}^{-}\right)\left(\gamma-\theta_{n}^{-}\right)},
\end{gathered}
$$


we conclude that

$$
\begin{aligned}
\frac{1}{2 \pi \mathrm{i}} \int_{-\infty-\mathrm{i} \tau_{1}}^{\infty-\mathrm{i} \tau_{1}} D_{1, \Delta}^{(1)}\left(\gamma_{1}\right) \frac{\mathrm{e}^{-\mathrm{i} \gamma_{1} c_{\phi}}}{\gamma_{1}-\gamma} \mathrm{d} \gamma_{1} & =\sum_{l=-\infty}^{\infty} \frac{\mathcal{S}_{l} \mathrm{e}^{-\mathrm{i} \kappa_{l}^{-} c_{\phi}}}{\mathrm{i}\left(\kappa_{0}^{-}-\kappa_{l}^{-}\right)\left(\gamma-\kappa_{l}^{-}\right)} \\
& +\sum_{n=0}^{\infty} \frac{\mathscr{A}_{n} \mathrm{e}^{-\mathrm{i} \theta_{n}^{-} c_{\phi}}}{\mathrm{i}\left(\theta_{n}^{-}-\kappa_{0}^{-}\right)\left(\gamma-\theta_{n}^{-}\right)},
\end{aligned}
$$

where

$$
\begin{aligned}
\mathcal{S}_{1, \Delta, l} & =\frac{\mathrm{i}\left(\kappa_{0}^{-}-\kappa_{l}^{-}\right)}{J_{+}\left(\kappa_{l}^{-}\right)} \mathscr{T}_{1, \Delta, l}, \\
\mathscr{A}_{1, \Delta, n} & =\frac{\mathrm{i}\left(\theta_{n}^{-}-\kappa_{0}^{-}\right)}{J_{+}^{\prime}\left(\theta_{n}^{-}\right)}\left(\sum_{m=-\infty}^{\infty} \frac{\mathscr{R}_{1, \Delta, m}}{\theta_{n}^{-}-\lambda_{m}^{-}}+\sum_{l=-\infty}^{\infty} \frac{\mathscr{T}_{1, \Delta, l}}{\left.\theta_{n}^{-}-\kappa_{l}^{-}\right)}\right) .
\end{aligned}
$$

Combining these expressions with (3.99) obtains the identity

$$
D_{1, \Delta,+}^{*(2)}(\gamma)=-\sum_{l=-\infty}^{\infty} \frac{\mathcal{S}_{1, \Delta, l} \mathrm{e}^{-\mathrm{i} \kappa_{l}^{-} c_{\phi}}}{\mathrm{i}\left(\kappa_{0}^{-}-\kappa_{l}^{-}\right)\left(\gamma-\kappa_{l}^{-}\right)}-\sum_{n=0}^{\infty} \frac{\mathscr{A}_{1, \Delta, n} \mathrm{e}^{-\mathrm{i} \theta_{n}^{-} c_{\phi}}}{\mathrm{i}\left(\theta_{n}^{-}-\kappa_{l}^{-}\right)\left(\gamma-\theta_{n}^{-}\right)}-\frac{P_{1, \Delta}^{*(2)}}{\gamma-\kappa_{0}^{-}}
$$

Substitution of (3.100) into (3.98) and application of the additive factorisation (3.58), and an analogous version of (3.95), yields

$$
\begin{aligned}
& \frac{F_{1, \Delta,+}^{*(2)}(\gamma)}{4 \pi J_{+}(\gamma)}-\sum_{l=-\infty}^{\infty} \frac{\mathcal{S}_{1, \Delta, l} \mathrm{e}^{-\mathrm{i} \kappa_{l}^{-} c_{\phi}}}{\mathrm{i}\left(\kappa_{0}^{-}-\kappa_{l}^{-}\right)}\left[\tilde{J}_{-}\left(\gamma, \kappa_{l}^{-}\right)\right]_{+}-\sum_{n=0}^{\infty} \frac{\mathscr{A}_{1, \Delta, n} \mathrm{e}^{-\mathrm{i} \theta_{n}^{-} c_{\phi}}}{\mathrm{i}\left(\theta_{n}^{-}-\kappa_{0}^{-}\right)}\left[\tilde{J}_{-}\left(\gamma, \theta_{n}^{-}\right)\right]_{+} \\
& -\frac{k(\gamma) G_{\Delta}^{*(2)}(\gamma)}{J_{+}(\gamma)}+\sum_{m=-\infty}^{\infty} \frac{k_{r, m}^{+} G_{\Delta}^{*(2)}\left(\lambda_{m}^{+}\right)}{\left(\gamma-\lambda_{m}^{+}\right) J_{+}\left(\lambda_{m}^{+}\right)}-P_{1, \Delta}^{*(2)}\left[\tilde{J}_{-}\left(\gamma, \kappa_{0}^{-}\right)\right]_{+} \\
= & -J_{-}(\gamma) D_{1, \Delta,-}^{*(2)}(\gamma)+\sum_{l=-\infty}^{\infty} \frac{\mathcal{S}_{1, \Delta, l} \mathrm{e}^{-\mathrm{i} \kappa_{l}^{-} c_{\phi}}}{\mathrm{i}\left(\kappa_{0}^{-}-\kappa_{l}^{-}\right)}\left[\tilde{J}_{-}\left(\gamma, \kappa_{l}^{-}\right)\right]_{-} \\
& +\sum_{n=0}^{\infty} \frac{\mathscr{A}_{1, \Delta, n} \mathrm{e}^{-\mathrm{i} \theta_{n}^{-} c_{\phi}}}{\mathrm{i}\left(\theta_{n}^{-}-\kappa_{0}^{-}\right)}\left[\tilde{J}_{-}\left(\gamma, \theta_{n}^{-}\right)\right]_{-}+\sum_{m=-\infty}^{\infty} \frac{k_{r, m}^{+} G_{\Delta}^{*(2)}\left(\lambda_{m}^{+}\right)}{\left(\gamma-\lambda_{m}^{+}\right) J_{+}\left(\lambda_{m}^{+}\right)}+P_{1, \Delta}^{*(2)}\left[\tilde{J}_{-}\left(\gamma, \kappa_{0}^{-}\right)\right]_{-} .
\end{aligned}
$$

By applying the unsteady Kutta condition via a similar argument to section 3.B.2.2, we conclude that each side of the above equation must be equal to zero. Multiplying by $\gamma$ 
and letting $|\gamma| \rightarrow \infty$ in $\mathscr{M}^{+}$, the left-hand side of (3.102) gives

$$
\begin{aligned}
P_{1, \Delta}^{*(2)}= & -\sum_{l=-\infty}^{\infty} \frac{\mathcal{S}_{1, \Delta, l} \mathrm{e}^{-\mathrm{i} \kappa_{l}^{-} c_{\phi}}}{\mathrm{i}\left(\kappa_{0}^{-}-\kappa_{l}^{-}\right)} \cdot \frac{J_{-}\left(\kappa_{l}^{-}\right)}{J_{-}\left(\kappa_{0}^{-}\right)}-\sum_{n=0}^{\infty} \frac{\mathscr{A}_{1, \Delta, n} \mathrm{e}^{-\mathrm{i} \theta_{n}^{-} c_{\phi}}}{\mathrm{i}\left(\theta_{n}^{-}-\kappa_{0}^{-}\right)} \cdot \frac{J_{-}\left(\theta_{n}^{-}\right)}{J_{-}\left(\kappa_{0}^{-}\right)} \\
& +\sum_{m=-\infty}^{\infty} \frac{\mathcal{U}_{1, \Delta, m}}{\mathrm{i}\left(\lambda_{m}^{+}-\kappa_{0}^{-}\right)} \cdot \frac{1}{J_{-}\left(\kappa_{0}^{-}\right)},
\end{aligned}
$$

where

$$
\mathcal{U}_{1, \Delta, m}=\mathrm{i}\left(\lambda_{m}^{+}-\kappa_{0}^{-}\right) \cdot \frac{k_{r, m}^{+} G_{\Delta}^{*(2)}\left(\lambda_{m}^{+}\right)}{J_{+}\left(\lambda_{m}^{+}\right)} .
$$

Substituting in the downstream boundary data (3.100), rearranging the right-hand side of (3.102), and applying the pressure representation (3.103) gives the final expression

$$
\begin{aligned}
D_{1, \Delta}^{(2)}(\gamma)= & -\sum_{l=-\infty}^{\infty} \frac{\mathcal{S}_{1, \Delta, l} \mathrm{e}^{\mathrm{i}\left(\gamma-\kappa_{l}^{-}\right) c_{\phi}}}{\mathrm{i}\left(\gamma-\kappa_{0}^{-}\right)\left(\gamma-\kappa_{l}^{-}\right)} \cdot \frac{J_{-}\left(\kappa_{l}^{-}\right)}{J_{-}(\gamma)}-\sum_{n=0}^{\infty} \frac{\mathscr{A}_{1, \Delta, n} \mathrm{e}^{\mathrm{i}\left(\gamma-\theta_{n}^{-}\right) c_{\phi}}}{\mathrm{i}\left(\gamma-\kappa_{0}^{-}\right)\left(\gamma-\theta_{n}^{-}\right)} \cdot \frac{J_{-}\left(\theta_{n}^{-}\right)}{J_{-}(\gamma)} \\
& -\sum_{m=-\infty}^{\infty} \frac{\mathscr{U}_{1, \Delta, m} \mathrm{e}^{\mathrm{i} \gamma c_{\phi}}}{\mathrm{i}\left(\gamma-\kappa_{0}^{-}\right)\left(\gamma-\lambda_{m}^{+}\right)} \cdot \frac{1}{J_{-}(\gamma)} .
\end{aligned}
$$

\section{B.3.3 Solution to Third and Fourth Wiener-Hopf Problems $-D_{1, \Delta}^{(3)}, D_{1, \Delta}^{(4)}$} The method of solution for $D_{1, \Delta}^{(3)}$ and $D_{1, \Delta}^{(4)}$ is identical to section 3.B.2.3 except that the residues of $D_{1, \Delta}^{(2)}$ at $\gamma=\theta_{n}^{+}$are given by

$$
\begin{aligned}
D_{r, \Delta, k}^{(2)}=\frac{-\mathrm{e}^{\mathrm{i} \theta_{k}^{+} c_{\phi}}}{\mathrm{i}\left(\theta_{k}^{+}-\kappa_{0}^{-}\right) J_{-}^{\prime}\left(\theta_{k}^{+}\right)} & \left\{\sum_{l=-\infty}^{\infty} \frac{\mathcal{S}_{1, \Delta, l} \mathrm{e}^{-\mathrm{i} \kappa_{l}^{-} c_{\phi}}}{\left(\theta_{k}^{+}-\kappa_{l}^{-}\right)} \cdot J_{-}\left(\kappa_{l}^{-}\right)\right. \\
& \left.+\sum_{n=0}^{\infty} \frac{\mathscr{A}_{1, \Delta, n} \mathrm{e}^{-\mathrm{i} \theta_{n}^{-} c_{\phi}}}{\left(\theta_{k}^{+}-\theta_{n}^{-}\right)} \cdot J_{-}\left(\theta_{n}^{-}\right)+\sum_{m=-\infty}^{\infty} \frac{\mathcal{U}_{1, \Delta, m}}{\theta_{k}^{+}-\lambda_{m}^{+}}\right\} .
\end{aligned}
$$

Consequently, analogous relations to (3.83) and (3.87) may be derived, and the calculations of $\mathscr{B}_{1, \Delta, n}$ and $\mathscr{C}_{1, \Delta, n}$ are achieved via (3.85) and (3.88).

\section{B.4 Solution for $D_{1, \Gamma}$}

The integral equation and boundary values for $\Delta h_{1, \Gamma}$ and $D_{1, \Gamma}$ are summarised in (3.42.a-3.42.f). Similarly to section 3.B.2, we split this problem into four coupled 
problems, such as in (3.59) and (3.60), resulting in the semi-infinite integral equations

$$
f_{1, \Gamma}^{(n)}(\phi)=4 \pi \int_{-\infty}^{\infty}\left\{-D_{1, \Gamma}^{(n)}(\gamma) j(\gamma)+\delta_{2, n} G_{\Gamma}(\gamma) k(\gamma)\right\} \mathrm{e}^{-\mathrm{i} \gamma \phi} \mathrm{d} \gamma
$$

for $n=1,2,3,4$. The corresponding boundary values are given by

$$
\begin{aligned}
& f_{1, \Gamma}^{(1)}(\phi)=0, \\
& \phi>0 \\
& \Delta_{0}\left[h_{1, \Gamma}^{(1)}\right](\phi)=0, \\
& \phi<0 \\
& f_{1, \Gamma}^{(2)}(\phi)=0 \\
& \phi<c_{\phi} ; \\
& \Delta_{0}\left[h_{1, \Gamma}^{(1)}\right](\phi)+\Delta_{0}\left[h_{1, \Gamma}^{(2)}\right](\phi)= \\
& 2 \pi \mathrm{i} P_{1, \Gamma}^{(2)} \mathrm{e}^{-\mathrm{i} \kappa_{0}^{-} \phi}+\Gamma\left(\sum_{ \pm} \mathscr{H}_{0, \kappa_{0}^{-}}^{ \pm \prime} \mathrm{e}^{-\mathrm{i} \kappa_{0}^{-} \phi}+\sum_{m=-\infty}^{\infty} \mathscr{H}_{0, m}^{\prime} \mathrm{e}^{-\mathrm{i} \lambda_{m}^{-} \phi}\right), \quad \phi>c_{\phi} ; \\
& f_{1, \Gamma}^{(3)}(\phi)=0, \\
& \phi>0 \text {; } \\
& \Delta_{0}\left[h_{1, \Gamma}^{(2)}\right](\phi)+\Delta_{0}\left[h_{1, \Sigma}^{(3)}\right]+\Delta_{0}\left[h_{1, \Gamma}^{(4)}\right](\phi)=0, \quad \phi<0 ; \\
& f_{1, \Gamma}^{(4)}(\phi)=0 \\
& \phi<c_{\phi} \\
& \Delta_{0}\left[h_{1, \Gamma}^{(3)}\right](\phi)+\Delta_{0}\left[h_{1, \Sigma}^{(4)}\right](\phi)=2 \pi \mathrm{i} P_{1, \Gamma}^{(4)} \mathrm{e}^{-\mathrm{i} \kappa_{0}^{-} \phi}, \quad \phi>c_{\phi} ;
\end{aligned}
$$

where, as before, $P_{1, \Gamma}^{(2)}$ and $P_{1, \Gamma}^{(4)}$ are two constants of integration that will be specified to enforce the Kutta condition.

\section{B.4.1 Solution to First Wiener-Hopf Problem $-D_{1, \Gamma}^{(1)}$}

By applying a similar argument to section 3.B.2.1, we conclude that

$$
D_{1, \Gamma}^{(1)}(\gamma)=0
$$




\section{B.4.2 Solution to Second Wiener-Hopf Problem $-D_{1, \Gamma}^{(2)}$}

Taking the Fourier transform of (3.104) for $n=2$, using the kernel splitting property (3.54), and applying the upstream boundary values (3.106.a) yields

$$
\frac{F_{1, \Gamma,+}^{*(2)}(\gamma)}{4 \pi J_{+}(\gamma)}=-\left(D_{1, \Gamma,-}^{*(2)}(\gamma)+D_{1, \Gamma,+}^{*(2)}(\gamma)\right) J_{-}(\gamma)+G_{\Gamma}^{*}(\gamma) \frac{k(\gamma)}{J_{+}(\gamma)}
$$

where $F_{1, \Gamma,+}^{*(2)}, D_{1, \Gamma,-}^{*(2)}$ and $D_{1, \Gamma,+}^{*(2)}$ are defined analogously to (3.74.a), (3.74.b) and (3.74.c) respectively. The solution to the first integral equation (3.109) means that $\Delta_{0}\left[h_{1, \Gamma}^{(1)}\right](\phi) \equiv$ 0 . Therefore, we may use the downstream boundary values (3.106.b) to write

$$
D_{1, \Gamma,+}^{*(2)}(\gamma)=-\frac{P_{1, \Gamma}^{*(2)}}{\gamma-\kappa_{0}^{-}}-\frac{\Gamma}{2 \pi \mathrm{i}}\left(\sum_{ \pm} \frac{\mathscr{H}_{0, \kappa_{0}^{ \pm}}^{ \pm \prime} \mathrm{e}^{-\mathrm{i} \kappa_{0}^{-} c_{\phi}}}{\gamma-\kappa_{0}^{-}}+\sum_{m=-\infty}^{\infty} \frac{\mathscr{H}_{0, m}^{\prime} \mathrm{e}^{-\mathrm{i} \lambda_{m}^{-} c_{\phi}}}{\gamma-\lambda_{m}^{-}}\right)
$$

Consequently, we use pole removal to obtain the additive splitting

$$
J_{-}(\gamma) D_{1, \Gamma,+}^{*(2)}(\gamma)=\left[J_{-}(\gamma) D_{1, \Gamma,+}^{*(2)}(\gamma)\right]_{+}+\left[J_{-}(\gamma) D_{1, \Gamma,+}^{*(2)}(\gamma)\right]_{-}
$$

where

$$
\begin{aligned}
& {\left[J_{-}(\gamma) D_{1, \Gamma,+}^{*(2)}(\gamma)\right]_{ \pm}=-P_{1, \Gamma}^{*(2)}\left[\tilde{J}_{-}\left(\gamma, \kappa_{0}^{-}\right)\right]_{ \pm} } \\
- & \frac{\Gamma}{2 \pi \mathrm{i}}\left(\sum_{ \pm} \mathscr{H}_{0, \kappa_{0}^{-}}^{ \pm \prime} \mathrm{e}^{-\mathrm{i} \kappa_{0}^{-} c_{\phi}}\left[\tilde{J}_{-}\left(\gamma, \kappa_{0}^{-}\right)\right]_{ \pm}+\sum_{m=-\infty}^{\infty} \mathscr{H}_{0, m}^{\prime} \mathrm{e}^{-\mathrm{i} \lambda_{m}^{-} c_{\phi}}\left[\tilde{J}_{-}\left(\gamma, \lambda_{m}^{-}\right)\right]_{ \pm}\right) .
\end{aligned}
$$

We may also use pole removal to obtain the additive splitting

$$
\begin{aligned}
G_{\Gamma}^{*}(\gamma) \frac{k(\gamma)}{J_{+}(\gamma)} & =\underbrace{G_{\Gamma}^{*}(\gamma) \frac{k(\gamma)}{J_{+}(\gamma)}-\sum_{m=-\infty}^{\infty} \frac{\mathscr{U}_{1, \Gamma, m}}{\mathrm{i}\left(\lambda_{m}^{+}-\kappa_{0}^{-}\right)\left(\gamma-\lambda_{m}^{+}\right)}}_{+} \\
& +\underbrace{\sum_{m=-\infty}^{\infty} \frac{\mathcal{U}_{1, \Gamma, m}}{\mathrm{i}\left(\lambda_{m}^{+}-\kappa_{0}^{-}\right)\left(\gamma-\lambda_{m}^{+}\right)}}_{-},
\end{aligned}
$$

where

$$
\mathscr{U}_{1, \Gamma, m}=\frac{\Gamma \zeta_{m}^{+}\left(\lambda_{m}^{+}-\kappa_{0}^{-}\right)}{8 \pi^{2} \Delta_{\phi} \sqrt{k^{2} w^{2}-f_{m}}} \cdot \frac{1}{J_{+}\left(\lambda_{m}^{+}\right)}
$$




$$
\times\left(\sum_{ \pm} \frac{ \pm \zeta_{\kappa_{0}^{-}} \mathscr{H}_{0, \kappa_{0}^{-}}^{ \pm \prime} \mathrm{e}^{-\mathrm{i} \kappa_{0}^{-} c_{\phi}}}{\lambda_{m}^{+}-\kappa_{0}^{-}}+\sum_{k=-\infty}^{\infty} \frac{\zeta_{k}^{-} \mathscr{H}_{0, k}^{\prime} \mathrm{e}^{-\mathrm{i} \lambda_{k}^{-} c_{\phi}}}{\lambda_{m}^{+}-\lambda_{k}^{-}}\right)
$$

Substituting the additive splitting (3.111) and wake coefficient (3.112) into the WienerHopf equation (3.110) yields

$$
\begin{aligned}
& \frac{F_{1, \Gamma,+}^{*(2)}(\gamma)}{4 \pi J_{+}(\gamma)}-G_{\Gamma}^{*}(\gamma) \frac{k(\gamma)}{J_{+}(\gamma)}+\sum_{m=-\infty}^{\infty} \frac{\mathscr{U}_{1, \Gamma, m}}{\mathrm{i}\left(\lambda_{m}^{+}-\kappa_{0}^{-}\right)\left(\gamma-\lambda_{m}^{+}\right)}+\left[J_{-}(\gamma) D_{1, \Gamma,+}^{*(2)}(\gamma)\right]_{+} \\
& =-D_{1, \Gamma,-}^{*(2)}(\gamma) J_{-}(\gamma)-\left[J_{-}(\gamma) D_{1, \Gamma,+}^{*(2)}(\gamma)\right]_{-}+\sum_{m=-\infty}^{\infty} \frac{\mathscr{U}_{1, \Gamma, m}}{\mathrm{i}\left(\lambda_{m}^{+}-\kappa_{0}^{-}\right)\left(\gamma-\lambda_{m}^{+}\right)}
\end{aligned}
$$

Following section 3.B.2.2, we apply the standard Wiener-Hopf method and obtain

$$
\begin{aligned}
P_{1, \Gamma}^{*(2)} & =\sum_{m=-\infty}^{\infty} \frac{\mathscr{U}_{1, \Delta, m}}{\mathrm{i}\left(\lambda_{m}^{+}-\kappa_{0}^{-}\right)} \cdot \frac{1}{J_{-}\left(\kappa_{0}^{-}\right)} \\
& -\frac{\Gamma}{2 \pi \mathrm{i}}\left(\sum_{ \pm} \mathscr{H}_{0, \kappa_{0}^{-}}^{ \pm \prime} \mathrm{e}^{-\mathrm{i} \kappa_{0}^{-} c_{\phi}}+\sum_{m=-\infty}^{\infty} \mathscr{H}_{0, m}^{\prime} \mathrm{e}^{-\mathrm{i} \lambda_{m}^{-} c_{\phi}} \cdot \frac{J_{-}\left(\lambda_{m}^{-}\right)}{J_{-}\left(\kappa_{0}^{-}\right)}\right)
\end{aligned}
$$

and

$$
\begin{aligned}
& D_{1, \Gamma,-}^{*(2)}(\gamma)=\sum_{m=-\infty}^{\infty} \frac{\mathscr{U}_{1, \Gamma, m}}{\mathrm{i}\left(\lambda_{m}^{+}-\kappa_{0}^{-}\right)\left(\gamma-\lambda_{m}^{+}\right)} \cdot \frac{1}{J_{-}(\gamma)}+P_{1, \Gamma}^{*(2)} \frac{\left[\tilde{J}_{-}\left(\gamma, \kappa_{0}^{-}\right)\right]_{-}}{J_{-}(\gamma)} \\
& +\frac{\Gamma}{2 \pi \mathrm{i}}\left(\sum_{ \pm} \mathscr{H}_{0, \kappa_{0}^{-}}^{ \pm \prime} \mathrm{e}^{-\mathrm{i} \kappa_{0}^{-} c_{\phi}} \frac{\left[\tilde{J}_{-}\left(\gamma, \kappa_{0}^{-}\right)\right]_{-}}{J_{-}(\gamma)}+\sum_{m=-\infty}^{\infty} \mathscr{H}_{0, m}^{\prime} \mathrm{e}^{-\mathrm{i} \lambda_{m}^{-} c_{\phi}} \frac{\left[\tilde{J}_{-}\left(\gamma, \lambda_{m}^{-}\right)\right]_{-}}{J_{-}(\gamma)}\right) .
\end{aligned}
$$

Consequently, substituting in the downstream representation (3.111) and (3.113) results in

$$
\begin{aligned}
D_{1, \Gamma}^{(2)}(\gamma)=-\frac{\Gamma}{2 \pi \mathrm{i}} \sum_{m=-\infty}^{\infty} \frac{J_{-}\left(\lambda_{m}^{-}\right)}{J_{-}(\gamma)} \cdot \frac{\left(\lambda_{m}^{-}-\kappa_{0}^{-}\right) \mathscr{H}_{0, m}^{\prime} \mathrm{e}^{\mathrm{i}\left(\gamma-\lambda_{m}^{-}\right) c_{\phi}}}{\left(\gamma-\kappa_{0}^{-}\right)\left(\gamma-\lambda_{m}^{-}\right)} \\
-\sum_{m=-\infty}^{\infty} \frac{\mathcal{U}_{1, \Gamma, m} \mathrm{e}^{\mathrm{i} \gamma c_{\phi}}}{\mathrm{i}\left(\gamma-\kappa_{0}^{-}\right)\left(\gamma-\lambda_{m}^{+}\right)} \cdot \frac{1}{J_{-}(\gamma)} .
\end{aligned}
$$




\section{B.4.3 Solution to Third and Fourth Wiener-Hopf problems $-D_{1, \Gamma}^{(3)}, D_{1, \Gamma}^{(4)}$}

The solution to the coupled 3rd and 4th Wiener-Hopf equations is identical to that in previous sections except

$$
\begin{aligned}
& D_{r, \Delta, k}^{(2)}=\frac{-\mathrm{e}^{\mathrm{i} \theta_{k}^{+} c_{\phi}}}{\mathrm{i}\left(\theta_{k}^{+}-\kappa_{0}^{-}\right) J_{-}^{\prime}\left(\theta_{k}^{+}\right)}\left\{\sum_{m=-\infty}^{\infty} \frac{\mathscr{U}_{1, \Gamma, m}}{\theta_{k}^{+}-\lambda_{m}^{+}}\right. \\
&\left.+\frac{\Gamma}{2 \pi} \sum_{m=-\infty}^{\infty} \frac{\lambda_{m}^{-}-\kappa_{0}^{-}}{\theta_{k}^{+}-\lambda_{m}^{-}} J_{-}\left(\lambda_{m}^{-}\right) \mathscr{H}_{0, m}^{\prime} \mathrm{e}^{-\mathrm{i} \lambda_{m}^{-} c_{\phi}}\right\} .
\end{aligned}
$$

\section{B.5 Solution for $D_{1, S}$}

The integral equation and boundary values for $\Delta_{0}\left[h_{1, S}\right]$ and $D_{1, S}$ are summarised in section 3.3.2.4. We perform an analogous splitting to that in (3.59) and (3.60). The integral equations satisfied by each $\Delta_{0}\left[h_{1, S}^{(n)}\right]$ and $D_{1, S}^{(n)}$ are

$$
f_{1, S}^{(n)}(\phi)=4 \pi \int_{-\infty}^{\infty}\left\{-D_{1, S}(\gamma) j(\gamma)+\delta_{n, 1} S^{(1)}(\gamma)+\delta_{n, 2} S^{(2)}(\gamma)\right\} \mathrm{e}^{-\mathrm{i} \gamma \phi} \mathrm{d} \gamma
$$

for $n=1,2,3,4$, where

$$
S^{(1)}(\gamma)=S^{u}(\gamma)+S^{i,(1)}(\gamma), \quad S^{(2)}(\gamma)=S^{d}(\gamma)+S^{i,(2)}(\gamma)
$$

which are defined in (3.150), (3.151) and (3.152).

The (homogeneous) boundary values are given by

$$
\begin{aligned}
f_{1, S}^{(1)}(\phi) & =0, & & \phi>0 ; \\
\Delta_{0}\left[h_{1, S}^{(1)}\right](\phi) & =0, & & \phi<0 ; \\
f_{1, S}^{(2)}(\phi) & =0, & & \phi<c_{\phi} ; \\
\Delta_{0}\left[h_{1, S}^{(1)}\right](\phi)+\Delta_{0}\left[h_{1, S}^{(2)}\right](\phi) & =2 \pi \mathrm{i} P_{1, S}^{(2)} \mathrm{e}^{-\mathrm{i} \kappa_{0}^{-} \phi}, & & \phi>c_{\phi} ; \\
f_{1, S}^{(3)}(\phi) & =0, & & \\
\Delta_{0}\left[h_{1, S}^{(2)}\right](\phi)+\Delta_{0}\left[h_{1, S}^{(3)}\right](\phi)+\Delta_{0}\left[h_{1, S}^{(4)}\right](\phi) & =0, & & \phi>0 ;
\end{aligned}
$$




$$
\begin{aligned}
& f_{1, S}^{(4)}(\phi)=0, \quad \phi<c_{\phi} ; \\
& \Delta_{0}\left[h_{1, S}^{(4)}\right](\phi)+\Delta_{0}\left[h_{1, S}^{(3)}\right](\phi)=2 \pi \mathrm{i} P_{1, S}^{(4)} \mathrm{e}^{-\mathrm{i} \kappa_{0}^{-} \phi}, \quad \phi>c_{\phi} ;
\end{aligned}
$$

where $P_{1, S}^{(2)}$ and $P_{1, S}^{(4)}$ are two constants of integration that will be specified to enforce the Kutta condition.

\section{B.5.1 Solution to First Wiener-Hopf Problem $-D_{1, S}^{(1)}$}

Taking a Fourier transform of (3.114) for $n=1$, applying the boundary values (3.115.a) and (3.115.b), and utilising the kernel splitting (3.54) results in

$$
\frac{F_{1, S,-}^{(1)}(\gamma)}{4 \pi J_{-}(\gamma)}=-D_{1, S,+}^{(1)}(\gamma) J_{+}(\gamma)+\frac{S^{(1)}(\gamma)}{J_{-}(\gamma)}
$$

where $F_{1, S,-}^{(1)}$ and $D_{1, S,+}^{(1)}$ are defined in an analogous way to section 3.B.2.1. We now note that the only poles of $S^{(1)}$ in $\mathscr{M}^{-}$are where $\gamma=\lambda_{m}^{-}, \kappa_{l}^{-}$. Therefore, we may apply pole removal to obtain the additive factorisation

$$
\begin{aligned}
\frac{S^{(1)}(\gamma)}{J_{-}(\gamma)} & =\underbrace{\sum_{m=-\infty}^{\infty} \frac{S_{m}^{u, r,(1)}}{\left(\gamma-\lambda_{m}^{-}\right) J_{-}\left(\lambda_{m}^{-}\right)}+\sum_{l=-\infty}^{\infty} \frac{\sum_{l_{2}=-\infty}^{\infty} f_{l, l_{2}} \mathscr{F}_{l_{2}}\left(\kappa_{l}^{-}\right)}{\left(\gamma-\kappa_{l}^{-}\right) J_{-}\left(\kappa_{l}^{-}\right)}}_{+} \\
& +\underbrace{\frac{S^{(1)}(\gamma)}{J_{-}(\gamma)}-\sum_{m=-\infty}^{\infty} \frac{S_{m}^{u, r,(1)}}{\left(\gamma-\lambda_{m}^{-}\right) J_{-}\left(\lambda_{m}^{-}\right)}-\sum_{l=-\infty}^{\infty} \frac{\sum_{l_{2}=-\infty}^{\infty} f_{l, l_{2}} \mathscr{F}_{l_{2}}\left(\kappa_{l}^{-}\right)}{\left(\gamma-\kappa_{l}^{-}\right) J_{-}\left(\kappa_{l}^{-}\right)}}_{-}
\end{aligned}
$$

where

$$
S_{m}^{u, r,(1)}=S_{m}^{u, r}+\sum_{l_{1}, l_{2}} f_{l_{1}, l_{2}} \frac{\mathscr{F}_{l_{2}, m}^{r,-}}{\left(\lambda_{m}^{-}-\kappa_{l_{1}}^{-}\right)},
$$

and $\mathscr{F}_{l_{2}, m}^{r,-}$ are the residues of $\mathscr{F}_{l_{2}}(\gamma)$ at the acoustic modes $\gamma=\lambda_{m}^{-}$, defined in (3.153).

Therefore, (3.119) becomes

$$
\begin{aligned}
& \frac{F_{-}^{(1)}(\gamma)}{4 \pi J_{-}(\gamma)}-\frac{S^{(1)}(\gamma)}{J_{-}(\gamma)}+\sum_{m=-\infty}^{\infty} \frac{S_{m}^{u, r,(1)}}{\left(\gamma-\lambda_{m}^{-}\right) J_{-}\left(\lambda_{m}^{-}\right)}+\sum_{l=-\infty}^{\infty} \frac{\sum_{l_{2}=-\infty}^{\infty} f_{l, l_{2}} \mathscr{F}_{l_{2}}\left(\kappa_{l}^{-}\right)}{\left(\gamma-\kappa_{l}^{-}\right) J_{-}\left(\kappa_{l}^{-}\right)} \\
= & -D_{1, S,+}^{(1)}(\gamma) J_{+}(\gamma)+\sum_{m=-\infty}^{\infty} \frac{S_{m}^{u, r,(1)}}{\left(\gamma-\lambda_{m}^{-}\right) J_{-}\left(\lambda_{m}^{-}\right)}+\sum_{l=-\infty}^{\infty} \frac{\sum_{l_{2}=-\infty}^{\infty} f_{l, l_{2}} \mathscr{F}_{l_{2}}\left(\kappa_{l}^{-}\right)}{\left(\gamma-\kappa_{l}^{-}\right) J_{-}\left(\kappa_{l}^{-}\right)}
\end{aligned}
$$


We now apply the typical Wiener-Hopf argument of section 3.B.2.1 and conclude that

$$
D_{1, S}^{(1)}(\gamma)=\sum_{l=-\infty}^{\infty} \frac{\mathscr{T}_{1, S, l}}{\gamma-\kappa_{l}^{-}} \cdot \frac{1}{J_{+}(\gamma)}+\sum_{m=-\infty}^{\infty} \frac{\mathscr{R}_{1, S, m}}{\gamma-\lambda_{m}^{-}} \cdot \frac{1}{J_{+}(\gamma)},
$$

where

$$
\mathscr{R}_{1, S, m}=\frac{S_{m}^{u, r,(1)}}{J_{-}\left(\lambda_{m}^{-}\right)}, \quad \mathscr{T}_{1, S, l}=\frac{\sum_{l_{2}=-\infty}^{\infty} f_{l, l_{2}} \mathscr{F}_{l_{2}}\left(\kappa_{l}^{-}\right)}{J_{-}\left(\kappa_{l}^{-}\right)}
$$

\section{B.5.2 Solution to Second Wiener-Hopf Problem $-D_{1, S}^{(2)}$}

Taking a Fourier transform of (3.114) for $n=2$, applying the boundary value (3.116.a), and utilising the kernel splitting (3.54) results in

$$
\frac{F_{1, S,+}^{*(2)}(\gamma)}{4 \pi J_{+}(\gamma)}=-\left(D_{1, S,-}^{*(2)}(\gamma)+D_{1, S,+}^{*(2)}(\gamma)\right) J_{-}(\gamma)+\frac{S^{*(2)}(\gamma)}{J_{+}(\gamma)}
$$

where $F_{1, S,+}^{*(2)}, D_{1, S, \pm}^{*(2)}$ and $S^{*(2)}$ are defined in an analogous way to section 3.B.2.2. Similarly to section 3.B.3.2, we may use the downstream boundary values (3.116.b) to write

$$
D_{1, S,+}^{*(2)}(\gamma)=-\sum_{l=-\infty}^{\infty} \frac{\mathcal{S}_{1, S, l} \mathrm{e}^{-\mathrm{i} \kappa_{l}^{-} c_{\phi}}}{\mathrm{i}\left(\kappa_{0}^{-}-\kappa_{l}^{-}\right)\left(\gamma-\kappa_{l}^{-}\right)}-\sum_{n=0}^{\infty} \frac{\mathscr{A}_{1, S, n} \mathrm{e}^{-\mathrm{i} \theta_{n}^{-} c_{\phi}}}{\mathrm{i}\left(\theta_{n}^{-}-\kappa_{l}^{-}\right)\left(\gamma-\theta_{n}^{-}\right)}-\frac{P_{1, S}^{*(2)}}{\gamma-\kappa_{0}^{-}}
$$

where $\mathscr{A}_{1, S, n}$ and $\mathcal{S}_{1, S, l}$ are defined in an analogous way to section 3.B.3. Substitution of (3.123) into (3.122), application of the additive factorisation (3.58), and an analogous version of (3.120) yields

$$
\begin{aligned}
& \frac{F_{1, S,+}^{*(2)}(\gamma)}{4 \pi J_{+}(\gamma)}-\sum_{l=-\infty}^{\infty} \frac{\mathcal{S}_{1, S, l} \mathrm{e}^{-\mathrm{i} \kappa_{l}^{-} c_{\phi}}}{\mathrm{i}\left(\kappa_{0}^{-}-\kappa_{l}^{-}\right)}\left[\tilde{J}_{-}\left(\gamma, \kappa_{l}^{-}\right)\right]_{+}-\sum_{n=0}^{\infty} \frac{\mathscr{A}_{1, S, n} \mathrm{e}^{-\mathrm{i} \theta_{n}^{-} c_{\phi}}}{\mathrm{i}\left(\theta_{n}^{-}-\kappa_{0}^{-}\right)}\left[\tilde{J}_{-}\left(\gamma, \theta_{n}^{-}\right)\right]_{+} \\
& -\frac{S^{*(2)}(\gamma)}{J_{+}(\gamma)}+\sum_{m=-\infty}^{\infty} \frac{S_{m}^{* d, r,(2)}}{\left(\gamma-\lambda_{m}^{+}\right) J_{+}\left(\lambda_{m}^{+}\right)}-P_{1, S}^{*(2)}\left[\tilde{J}_{-}\left(\gamma, \kappa_{0}^{-}\right)\right] \\
= & \sum_{l=-\infty}^{\infty} \frac{\mathcal{S}_{1, S, l} \mathrm{e}^{-\mathrm{i} \kappa_{l}^{-} c_{\phi}}}{\mathrm{i}\left(\kappa_{0}^{-}-\kappa_{l}^{-}\right)\left(\gamma-\kappa_{l}^{-}\right)}\left[\tilde{J}_{-}\left(\gamma, \kappa_{l}^{-}\right)\right]_{-}+\sum_{n=0}^{\infty} \frac{\mathscr{A}_{1, S, n} \mathrm{e}^{-\mathrm{i} \theta_{n}^{-} c_{\phi}}}{\mathrm{i}\left(\theta_{n}^{-}-\kappa_{0}^{-}\right)}\left[\tilde{J}_{-}\left(\gamma, \theta_{n}^{-}\right)\right]_{-} \\
& -J_{-}(\gamma) D_{1, S,-}^{*(2)}(\gamma)+\sum_{m=-\infty}^{\infty} \frac{S_{m}^{* d, r,(2)}}{\left(\gamma-\lambda_{m}^{+}\right) J_{+}\left(\lambda_{m}^{+}\right)}+P_{1, S}^{*(2)}\left[\tilde{J}_{-}\left(\gamma, \kappa_{0}^{-}\right)\right]_{-} .
\end{aligned}
$$


By applying a similar argument to section 3.B.2.2, we conclude that each side of the above equation must be equal to zero. Multiplying both sides by $\gamma$ and letting $|\gamma| \rightarrow \infty$ in $\mathscr{M}^{+}$, the left-hand side of (3.124) gives the value of the wake coefficient

$$
\begin{aligned}
P_{1, S}^{*(2)}= & -\sum_{l=-\infty}^{\infty} \frac{\mathcal{S}_{1, S, l} \mathrm{e}^{-\mathrm{i} \kappa_{l}^{-} c_{\phi}}}{\mathrm{i}\left(\kappa_{0}^{-}-\kappa_{l}^{-}\right)} \cdot \frac{J_{-}\left(\kappa_{l}^{-}\right)}{J_{-}\left(\kappa_{0}^{-}\right)}-\sum_{n=0}^{\infty} \frac{\mathscr{A}_{1, S, n} \mathrm{e}^{-\mathrm{i} \theta_{n}^{-} c_{\phi}}}{\mathrm{i}\left(\theta_{n}^{-}-\kappa_{0}^{-}\right)} \cdot \frac{J_{-}\left(\theta_{n}^{-}\right)}{J_{-}\left(\kappa_{0}^{-}\right)} \\
& +\sum_{m=-\infty}^{\infty} \frac{\mathcal{U}_{1, S, m}}{\mathrm{i}\left(\lambda_{m}^{+}-\kappa_{0}^{-}\right)} \cdot \frac{1}{J_{-}\left(\kappa_{0}^{-}\right)},
\end{aligned}
$$

where

$$
\mathscr{U}_{1, S, m}=\mathrm{i}\left(\lambda_{m}^{+}-\kappa_{0}^{-}\right) \cdot \frac{S_{m}^{* d, r,(2)}}{J_{+}\left(\lambda_{m}^{+}\right)}
$$

Substituting the downstream boundary data (3.123) and the wake coefficient (3.125) into the Wiener-Hopf equation (3.124) gives the final expression

$$
\begin{aligned}
D_{1, S}^{(2)}(\gamma)= & -\sum_{l=-\infty}^{\infty} \frac{\delta_{1, S, l} \mathrm{e}^{\mathrm{i}\left(\gamma-\kappa_{l}^{-}\right) c_{\phi}}}{\mathrm{i}\left(\gamma-\kappa_{0}^{-}\right)\left(\gamma-\kappa_{l}^{-}\right)} \cdot \frac{J_{-}\left(\kappa_{l}^{-}\right)}{J_{-}(\gamma)}-\sum_{n=0}^{\infty} \frac{\mathscr{A}_{1, S, n} \mathrm{e}^{\mathrm{i}\left(\gamma-\theta_{n}^{-}\right) c_{\phi}}}{\mathrm{i}\left(\gamma-\kappa_{0}^{-}\right)\left(\gamma-\theta_{n}^{-}\right)} \cdot \frac{J_{-}\left(\theta_{n}^{-}\right)}{J_{-}(\gamma)} \\
& -\sum_{m=-\infty}^{\infty} \frac{\mathscr{U}_{1, S, m} \mathrm{e}^{\mathrm{i} \gamma c_{\phi}}}{\mathrm{i}\left(\gamma-\kappa_{0}^{-}\right)\left(\gamma-\lambda_{m}^{+}\right)} \cdot \frac{1}{J_{-}(\gamma)} .
\end{aligned}
$$

Similarly to previous sections, the only poles of $D_{1, S}^{*(2)}(\gamma)$ in the upper half plane are at the zeros of $J_{-}(\gamma)$.

\section{B.5.3 Solution to Third and Fourth Wiener-Hopf Problems $-D_{1, S}^{(3)}, D_{1, S}^{(4)}$}

The solution to the coupled 3rd and 4th Wiener-Hopf equations is identical to that in previous sections, except

$$
\begin{aligned}
D_{r, S, k}^{(2)}=\frac{-\mathrm{e}^{\mathrm{i} \theta_{k}^{+} c_{\phi}}}{\mathrm{i}\left(\theta_{k}^{+}-\kappa_{0}^{-}\right) J_{-}^{\prime}\left(\theta_{k}^{+}\right)} & \left\{\sum_{l=-\infty}^{\infty} \frac{\mathcal{S}_{1, S, l} \mathrm{e}^{-\mathrm{i} \kappa_{l}^{-} c_{\phi}}}{\theta_{k}^{+}-\kappa_{l}^{-}} \cdot J_{-}\left(\kappa_{l}^{-}\right)\right. \\
& \left.+\sum_{n=0}^{\infty} \frac{\mathscr{A}_{1, S, n} \mathrm{e}^{-\mathrm{i} \theta_{n}^{-} c_{\phi}}}{\theta_{k}^{+}-\theta_{n}^{-}} \cdot J_{-}\left(\theta_{n}^{-}\right)+\sum_{m=-\infty}^{\infty} \frac{\mathcal{U}_{1, S, m}}{\theta_{k}^{+}-\lambda_{m}^{+}}\right\} .
\end{aligned}
$$




\section{C Asymptotic Behaviour of Half-Range Fourier Integrals}

Before we may apply the Wiener-Hopf argument, we must determine the asymptotic behaviour of the half range Fourier transforms terms using the physical leading-edge condition. Here we consider the symmetric solution $h_{1, \Sigma}$, although the approach can be generalised to each of the other components. Firstly, the singularity of the velocity field at the leading edge $(\phi=0)$ can be, at worst, integrable. Therefore, we can write

$$
\Sigma_{0}\left[\frac{\partial h_{1, \Sigma}}{\partial \psi}\right](\phi) \sim A \phi^{\eta}, \quad \text { as } \phi \rightarrow 0^{-},
$$

for some constant $A$ and $-1<\eta<0$. By adapting result (1.74) from (Noble, 1958, p. $36)$, we determine that

$$
F_{1, \Sigma,-}^{(1)}(\gamma) \sim-\frac{A}{2 \pi} \Gamma(\eta+1) \mathrm{e}^{\frac{1}{2} \pi \mathrm{i}(\eta+1)} \gamma^{-(\eta+1)}, \quad \text { as }|\gamma| \rightarrow \infty \text { in } \mathscr{M}^{-},
$$

where $\Gamma$ here refers to the Gamma function.

We also assume that $\Delta_{0}\left[h_{1, \Sigma}\right]$ does not admit singular behaviour at the leading edge: this assumption is physically supported since, if it did not hold, the pressure at the leading edge would be non-integrable and result in an infinite force there. Therefore, $\Delta_{0}\left[h_{1, \Sigma}\right](0)$ is finite. Consequently, we may apply integration by parts to obtain

$D_{1, \Sigma,+}^{(1)}(\gamma) \sim-\frac{\Delta_{0}\left[h_{1, \Sigma}^{(1)}\right](0)}{2 \pi \mathrm{i} \gamma}-\frac{1}{2 \pi \mathrm{i} \gamma} \int_{0}^{\infty} \Delta_{0}\left[h_{1, \Sigma}^{(1)}\right](\phi) \mathrm{e}^{\mathrm{i} \gamma \phi} \mathrm{d} \phi, \quad$ as $|\gamma| \rightarrow \infty$ in $\mathscr{M}^{+}$,

and Riemann-Lebesgue lemma tells us that the second term in the above expression is $a\left(\gamma^{-1}\right)$.

\section{D List of Identities}

In this section we present some identities that are used throughout the chapter. 


\section{D.1 Radical Equation}

Consider the equation

$$
s_{\phi} \zeta= \pm\left(\sigma_{\phi}^{\prime}+d_{\phi} \gamma-2 m \pi\right)
$$

We may express $\left(\sigma_{\phi}^{\prime}-2 m \pi\right) / \Delta_{\phi}=f_{m}$ to write

$$
s_{\phi} \zeta= \pm\left(d_{\phi} \gamma+\Delta_{\phi} f_{m}\right)
$$

which has solutions

$$
\begin{aligned}
\gamma=\lambda_{m}^{ \pm} & =-\frac{d_{\phi}}{\Delta_{\phi}} f_{m} \pm \frac{\sqrt{4 d_{\phi}^{2} \Delta_{\phi}^{2} f_{m}^{2}-4\left(\Delta_{\phi}^{2} f_{m}^{2}-s_{\phi}^{2} k^{2} w^{2}\right)\left(d_{\phi}^{2}+s_{\phi}^{2}\right)}}{2\left(d_{\phi}^{2}+s_{\phi}^{2}\right)} \\
& =-\frac{d_{\phi}}{\Delta_{\phi}} f_{m} \pm \frac{s_{\phi}}{\Delta_{\phi}} \sqrt{k^{2} w^{2}-f_{m}^{2}}
\end{aligned}
$$

We can see that $\lambda_{m}^{+}$must correspond to the "+" equation, because the branch cut on the right-hand side gives positive imaginary part. Therefore, the roots of (3.127) are

$$
s_{\phi} \zeta_{m}^{ \pm}= \pm\left(\sigma_{\phi}^{\prime}+d_{\phi} \lambda_{m}^{ \pm}-2 m \pi\right)
$$

where

$$
\zeta_{m}^{ \pm}=\sqrt{k^{2} w^{2}-\lambda_{m}^{ \pm 2}}= \pm \frac{s_{\phi}}{\Delta_{\phi}} f_{m}+\frac{d_{\phi}}{\Delta_{\phi}} \sqrt{k^{2} w^{2}-f_{m}^{2}}
$$

This solution gives us several expressions that are useful for simplification at various points in the analysis:

$$
\begin{aligned}
d_{\phi} \zeta_{m}^{ \pm} \pm s_{\phi} \lambda_{m}^{ \pm} & =\Delta_{\phi} \sqrt{k^{2} w^{2}-f_{m}^{2}} \\
\Delta_{\phi}^{2}\left(\gamma-\lambda_{m}^{-}\right)\left(\gamma-\lambda_{m}^{+}\right) & =\left(s_{\phi} \zeta+d_{\phi} \gamma+\sigma_{\phi}^{\prime}-2 m \pi\right)\left(-s_{\phi} \zeta+d_{\phi} \gamma+\sigma_{\phi}^{\prime}-2 m \pi\right) \\
\lambda_{m}^{+}-\lambda_{m}^{-} & =2 \frac{s_{\phi}}{\Delta_{\phi}} \sqrt{k^{2} w^{2}-f_{m}^{2}}
\end{aligned}
$$

Accordingly, we have the following expressions for some relevant residues:

$$
\operatorname{Res}\left[\frac{1}{1-\mathrm{e}^{\mathrm{i}\left(\mp s_{\phi} \zeta+d_{\phi} \gamma+\sigma_{\phi}^{\prime}\right)}}, \lambda_{m}^{ \pm}\right]=\frac{\mathrm{i} \zeta_{m}^{ \pm}}{\Delta_{\phi} \sqrt{k^{2} w^{2}-f_{m}^{2}}} .
$$




\section{D.2 Integrals}

We note the integrals

$$
\begin{aligned}
\frac{1}{2 \pi} \int_{-\infty}^{\infty} \frac{\mathrm{i} \mu}{k^{2} w^{2}-\mu^{2}-\gamma^{2}} \mathrm{e}^{\mathrm{i} \mu\left(n s_{\phi}-\psi\right)} \mathrm{d} \mu & =\frac{1}{2} \operatorname{sgn}\left(n s_{\phi}-\psi\right) \mathrm{e}^{\mathrm{i} \zeta\left|n s_{\phi}-\psi\right|}, \\
\frac{1}{2 \pi} \int_{-\infty}^{\infty} \frac{1}{k^{2} w^{2}-\mu^{2}-\gamma^{2}} \mathrm{e}^{\mathrm{i} \mu\left(n s_{\phi}-\psi\right)} \mathrm{d} \mu & =\frac{1}{2 \mathrm{i} \zeta} \mathrm{e}^{\mathrm{i} \zeta\left|n s_{\phi}-\psi\right|},
\end{aligned}
$$

where $\zeta=\sqrt{k^{2} w^{2}-\gamma^{2}}$. These may be calculated via contour integration and specifying the branch of $\zeta$ to have positive imaginary part.

\section{D.3 Doubly-Infinite Sums}

For $0<\psi_{1}<s_{\phi}$, we have the following identities

$$
\begin{aligned}
j(\gamma) & =\frac{\mathrm{i} \zeta}{4 \pi} \sum_{n=-\infty}^{\infty} \mathrm{e}^{\mathrm{i} n\left(\sigma_{\phi}^{\prime}+d_{\phi} \gamma\right)+\mathrm{i} \zeta|n| s_{\phi}} \\
& =\frac{\mathrm{i} \zeta}{4 \pi}\left(\sum_{n=0}^{\infty} \mathrm{e}^{\mathrm{i} n\left(\zeta s_{\phi}-d_{\phi} \gamma-\sigma_{\phi}^{\prime}\right)}+\sum_{n=1}^{\infty} \mathrm{e}^{\mathrm{i} n\left(\zeta s_{\phi}+d_{\phi} \gamma+\sigma_{\phi}^{\prime}\right)}\right) \\
& =\frac{1}{4 \pi}\left(\frac{1}{1-\mathrm{e}^{\mathrm{i}\left(\zeta s_{\phi}-d_{\phi} \gamma-\sigma_{\phi}^{\prime}\right)}}-\frac{1}{1-\mathrm{e}^{-\mathrm{i}\left(s_{\phi} \zeta+d_{\phi} \gamma+\sigma_{\phi}^{\prime}\right)}}\right) \\
& =\frac{\zeta}{4 \pi} \cdot \frac{\sin \left(\zeta s_{\phi}\right)}{\cos \left(\zeta s_{\phi}\right)-\cos \left(\gamma d_{\phi}+\sigma_{\phi}^{\prime}\right)},
\end{aligned}
$$




$$
\begin{aligned}
& k(\gamma)=\frac{1}{4 \pi} \sum_{\substack{n=-\infty \\
n \neq 0}}^{\infty} \operatorname{sgn}\left(n s_{\phi}\right) \mathrm{e}^{\mathrm{i} n\left(\sigma_{\phi}^{\prime}+d_{\phi} \gamma\right)+\mathrm{i} \zeta\left|n s_{\phi}\right|} \\
& =\frac{1}{4 \pi}\left(-\sum_{n=1}^{\infty} \mathrm{e}^{\mathrm{i} n\left(s_{\phi} \zeta-d_{\phi} \gamma-\sigma_{\phi}^{\prime}\right)}+\sum_{n=1}^{\infty} \mathrm{e}^{\mathrm{i} n\left(s_{\phi} \zeta+d_{\phi} \gamma+\sigma_{\phi}^{\prime}\right)}\right) \\
& =\frac{\mathrm{i}}{4 \pi}\left(\frac{1}{1-\mathrm{e}^{-\mathrm{i}\left(s_{\phi} \zeta-d_{\phi} \gamma-\sigma_{\phi}^{\prime}\right)}}-\frac{1}{1-\mathrm{e}^{-\mathrm{i}\left(s_{\phi} \zeta+d_{\phi} \gamma+\sigma_{\phi}^{\prime}\right)}}\right) \\
& =\frac{1}{4 \pi \mathrm{i}} \cdot \frac{\sin \left(\gamma d_{\phi}+\sigma_{\phi}^{\prime}\right)}{\cos \left(\zeta s_{\phi}\right)-\cos \left(\gamma d_{\phi}+\sigma_{\phi}^{\prime}\right)}, \\
& \sum_{n=-\infty}^{\infty} \operatorname{sgn}\left(\psi_{1}+n s_{\phi}\right) \mathrm{e}^{\mathrm{i} n\left(\sigma_{\phi}^{\prime}+d_{\phi} \gamma\right)} \mathrm{e}^{\mathrm{i} \zeta\left|\psi_{1}+n s_{\phi}\right|} \\
& =-\mathrm{e}^{-\mathrm{i} \zeta \psi_{1}} \sum_{n=1}^{\infty} \mathrm{e}^{\mathrm{i} n\left(s_{\phi} \zeta-d_{\phi} \gamma-\sigma_{\phi}^{\prime}\right)}+\mathrm{e}^{\mathrm{i} \zeta \psi_{1}} \sum_{n=0}^{\infty} \mathrm{e}^{\mathrm{i} n\left(s_{\phi} \zeta+d_{\phi} \gamma+\sigma_{\phi}^{\prime}\right)} \\
& =\frac{\mathrm{e}^{-\mathrm{i} \zeta \psi_{1}}}{1-\mathrm{e}^{\mathrm{i}\left(d_{\phi} \gamma+\sigma_{\phi}^{\prime}-s_{\phi} \zeta\right)}}+\frac{\mathrm{e}^{\mathrm{i} \zeta \psi_{1}}}{1-\mathrm{e}^{\mathrm{i}\left(d_{\phi} \gamma+\sigma_{\phi}^{\prime}+s_{\phi} \zeta\right)}}, \\
& I_{D}(\gamma, \psi)=-\frac{1}{2} \sum_{n=-\infty}^{\infty} \operatorname{sgn}\left(n s_{\phi}-\psi\right) \mathrm{e}^{\mathrm{i} n\left(\sigma_{\phi}^{\prime}+\gamma d_{\phi}\right)+\mathrm{i} \zeta\left|n s_{\phi}-\psi\right|} \\
& =\frac{1}{2} \cdot\left(\frac{\mathrm{e}^{\mathrm{i} \zeta \psi}}{1-\mathrm{e}^{\mathrm{i}\left(s_{\phi} \zeta-d_{\phi} \gamma-\sigma_{\phi}^{\prime}\right)}}+\frac{\mathrm{e}^{-\mathrm{i} \zeta \psi}}{1-\mathrm{e}^{-\mathrm{i}\left(s_{\phi} \zeta+d_{\phi} \gamma+\sigma_{\phi}^{\prime}\right)}}\right) \\
& =\frac{\cos (\zeta(\psi-s))-\mathrm{e}^{\mathrm{i} d \gamma+\mathrm{i} \sigma} \cos (\zeta \psi)}{2(\cos (s \zeta)-\cos (d \gamma+\sigma)}, \\
& I_{G}(\gamma, \psi)=\frac{1}{2 \mathrm{i} \zeta} \sum_{n=-\infty}^{\infty} \mathrm{e}^{\mathrm{i} n\left(\sigma_{\phi}^{\prime}+\gamma d_{\phi}\right)+\mathrm{i} \zeta\left|n s_{\phi}-\psi\right|} \\
& =\frac{\mathrm{i}}{2 \zeta} \cdot\left(\frac{\mathrm{e}^{\mathrm{i} \zeta \psi}}{1-\mathrm{e}^{\mathrm{i}\left(s_{\phi} \zeta-d_{\phi} \gamma-\sigma_{\phi}^{\prime}\right)}}-\frac{\mathrm{e}^{-\mathrm{i} \zeta \psi}}{1-\mathrm{e}^{-\mathrm{i}\left(s_{\phi} \zeta+d_{\phi} \gamma+\sigma_{\phi}^{\prime}\right)}}\right) \\
& =\frac{\sin (\zeta(\psi-s))-\mathrm{e}^{\mathrm{i} d \gamma+\mathrm{i} \sigma} \sin (\zeta \psi)}{2 \zeta(\cos (s \zeta)-\cos (d \gamma+\sigma))}, \\
& I_{S}\left(\gamma, \psi, \psi_{1}\right)=\frac{1}{4 \pi \mathrm{i} \zeta} \sum_{n=-\infty}^{\infty} \mathrm{e}^{\mathrm{i} n\left(\sigma_{\phi}^{\prime}+\gamma d_{\phi}\right)+\mathrm{i} \zeta\left|\psi_{1}+n s_{\phi}-\psi\right|} \\
& =\frac{1}{4 \pi \mathrm{i} \zeta} \cdot\left(\mathrm{e}^{\mathrm{i} \zeta\left|\psi_{1}-\psi\right|}-\frac{\mathrm{e}^{\mathrm{i} \zeta\left(\psi_{1}-\psi\right)}}{1-\mathrm{e}^{-\mathrm{i}\left(\zeta s_{\phi}+d_{\phi} \gamma+\sigma_{\phi}^{\prime}\right)}}-\frac{\mathrm{e}^{-\mathrm{i} \zeta\left(\psi_{1}-\psi\right)}}{1-\mathrm{e}^{-\mathrm{i}\left(\zeta s_{\phi}-d_{\phi} \gamma-\sigma_{\phi}^{\prime}\right)}}\right) \\
& =\frac{\sin \left(\zeta\left(\left|\psi-\psi_{1}\right|-s_{\phi}\right)\right)-\mathrm{e}^{\mathrm{i}\left(d_{\phi} \gamma+\sigma_{\phi}^{\prime}\right) \operatorname{sgn}\left(\psi-\psi_{1}\right)} \sin \left(\zeta\left|\psi-\psi_{1}\right|\right)}{4 \pi \zeta\left(\cos \left(s_{\phi} \zeta\right)-\cos \left(d_{\phi} \gamma+\sigma_{\phi}^{\prime}\right)\right)} .
\end{aligned}
$$

It should be noted that, although these sums are strictly only valid in the Wiener-Hopf strip where $\operatorname{Im}[\zeta]>0$, they also represent the analytic continuation of the sums from the strip to the entire complex plane. 


\section{E Analysis of Source Terms}

In this section, we present our novel, quasi-analytic method of decomposing the source terms arising in the Rapid Distortion Theory framework. The inclusion of the source terms in the modified Helmholtz equation is an essential feature of the analysis and is generalisable to similar problems. Since the solution terms satisfies the quasi-periodicity relation (3.26), we may restrict our analysis to a single infinite channel of height $s_{\phi}$, and extrapolate to the entire domain. For example, in (3.38), we used the quasi-periodic relation to reduce the transformed source terms from an integral in the entire space to an integral along a channel. We now split the region of interest into the upstream $\left(\phi s_{\phi}<\psi d_{\phi}\right)$, downstream $\left(\phi s_{\phi}>\psi d_{\phi}+c_{\phi} s_{\phi}\right)$ and inter-blade $\left(\psi d_{\phi}<\phi s_{\phi}<\psi d_{\phi}+c_{\phi} s_{\phi}\right)$ regions.

We will first outline our decomposition of the relevant source terms into exponential functions. Then we will analyse the Fourier transformation of the decompositions in each of the three regions. Finally, we provide some details of how we invert the Fourier transform and obtain the final acoustic field.

\section{E.1 Exponential Decomposition of Source Terms}

In this section we perform the exponential decomposition of the source terms. This step is necessary in order to know the exact structure of the transformed source terms in the complex plane, which is a requirement for the application of the Wiener-Hopf method.

In each region (upstream, downstream and inter-blade), we decompose the source terms into Fourier series $(3.141,3.143,3.146)$. We denote the total source contribution to (3.38) by

$$
S(\gamma)=S^{u}(\gamma)+S^{d}(\gamma)+S^{i}(\gamma)
$$

where $u, d, i$ correspond to the upstream, downstream and inter-blade regions as defined in section 3.E.2.

\section{E.1.1 Upstream Region Decomposition}

In the upstream region, the source terms satisfy the quasi-periodic relation (3.26). Accordingly, we may express the upstream source terms as a series of exponential 
functions in the form

$$
\mathbb{S}^{u}(\phi, \psi)=\sum_{M_{m}^{+}} \mathbb{S}_{P, M_{m}^{+}}^{+} \mathrm{e}^{-\frac{2 m \pi \mathrm{i}}{\Delta_{\phi}^{2}}\left(d_{\phi} \phi+s_{\phi} \psi\right)} \mathrm{e}^{\mathrm{i} M_{m}^{+}\left(\psi d_{\phi}-\phi s_{\phi}\right)} \mathrm{e}^{\mathrm{i} k k_{n} \psi} \mathrm{e}^{-\mathrm{i} \kappa_{0}^{-} \phi},
$$

which we write as

$$
\mathbb{S}^{u}(\phi, \psi)=\sum_{M_{m}^{+}} \mathbb{S}_{P, M_{m}^{+}}^{+} \exp \left[-\mathrm{i} \tilde{M}_{m}^{+} \phi\right] \exp \left[\mathrm{i}\left(\Delta_{\phi} f_{m}+d_{\phi} \tilde{M}_{m}^{+}\right) \frac{\psi}{s_{\phi}}\right],
$$

where

$$
\tilde{M}_{m}^{+}=s_{\phi} M_{m}^{+}+\kappa_{0}^{-}+\frac{2 \pi d_{\phi} m}{\Delta_{\phi}^{2}}
$$

\section{E.1.2 Downstream Region Decomposition}

The source terms in the downstream region take a more complicated form than those in the upstream region. This increased complexity can be attributed to two factors. Firstly, the perturbation to the background velocity in the horizontal direction, $q$, decays in the upstream region, whereas in the downstream region $q$ tends to a constant value as a result of the cascade deflecting the flow. Consequently, some source terms that vanish in the upstream region do not vanish in the downstream region. These terms cause resonance with the $\mathcal{O}(1)$ solution, and this issue is resolved in section 3.F. Secondly, the source terms in the downstream region possess contributions that do not automatically satisfy the quasi-periodic relation (3.26). For example, the $\kappa_{0}^{-}$mode in (3.50) does not satisfy (3.26) and, therefore, neither does $q h_{0}$ in (3.18). Consequently, we express the downstream source terms as

$$
\begin{aligned}
\mathbb{S}^{d}(\phi, \psi) & =\sum_{M_{m}^{-}} \mathbb{S}_{P, M_{m}^{-}}^{-} \mathrm{e}^{-\frac{2 m \pi \mathrm{i}}{\Delta_{\phi}^{2}}\left(d_{\phi}\left(\phi-c_{\phi}\right)+s_{\phi} \psi\right)} \mathrm{e}^{\mathrm{i} M_{m}^{-}\left(\psi d_{\phi}-\left(\phi-c_{\phi}\right) s_{\phi}\right)} \mathrm{e}^{\mathrm{i} k k_{n} \psi} \mathrm{e}^{-\mathrm{i} \kappa_{0}^{-}\left(\phi-c_{\phi}\right)} \\
& +\sum_{m=-\infty}^{\infty} \mathbb{S}_{P, \lambda_{m}^{-}}^{-} \mathrm{e}^{-\mathrm{i}\left(\lambda_{m}^{-}\left(\phi-c_{\phi}\right)+\zeta_{m}^{-} \psi\right)} \\
& +\sum_{N_{\phi, n}^{-}} \mathbb{S}_{N, N_{\phi, n}^{-}}^{-} \mathrm{e}^{-\mathrm{i}\left(N_{\phi, n}^{-}\left(\phi-c_{\phi}\right)+N_{\psi, n}^{-} \psi\right)}+\sum_{ \pm} \mathbb{S}_{N, \kappa_{0}^{-}, \pm}^{-} \mathrm{e}^{-\mathrm{i}\left(\kappa_{0}^{-}\left(\phi-c_{\phi}\right) \pm \zeta_{\kappa_{0}} \psi\right)}
\end{aligned}
$$

The role of the coefficients is summarised in table 3.2. We rewrite (3.142) as

$$
\mathbb{S}^{d}(\phi, \psi)=\sum_{M_{m}^{-}} \mathbb{S}_{P, M_{m}^{-}}^{-} \exp \left[-\mathrm{i} \tilde{M}_{m}^{-}\left(\phi-c_{\phi}\right)\right] \exp \left[\mathrm{i}\left(\Delta_{\phi} f_{m}+d_{\phi} \tilde{M}_{m}^{-}\right) \frac{\psi}{s_{\phi}}\right]
$$




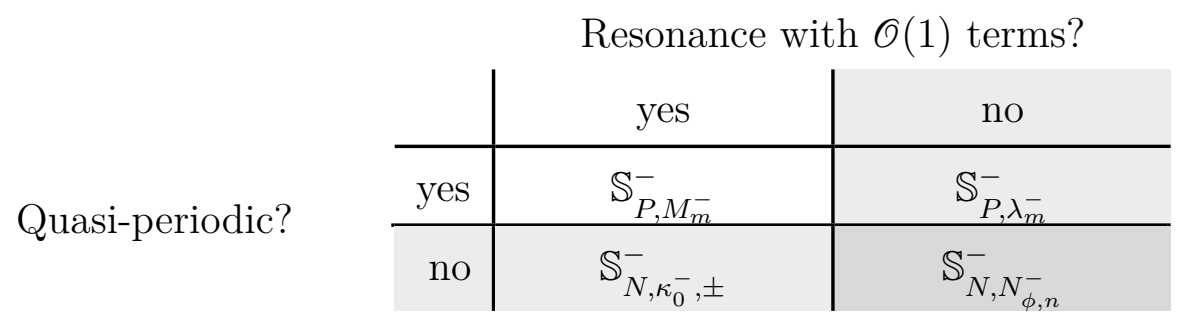

Table 3.2 A summary of the meaning of the coefficients of the source terms (3.142) in the downstream region.

$$
\begin{aligned}
& +\sum_{m=-\infty}^{\infty} \mathbb{S}_{P, \lambda_{m}^{-}}^{-} \exp \left[-\mathrm{i} \lambda_{m}^{-}\left(\phi-c_{\phi}\right)\right] \exp \left[-\mathrm{i} \zeta_{m}^{-} \psi\right] \\
& +\sum_{N_{\phi, n}^{-}} \mathbb{S}_{N, N_{\phi, n}^{-}}^{-} \exp \left[-\mathrm{i} N_{\phi, n}^{-}\left(\phi-c_{\phi}\right)\right] \exp \left[-\mathrm{i} N_{\psi, n}^{-} \psi\right] \\
& +\sum_{ \pm} \mathbb{S}_{N, \kappa_{0}^{-}, \pm}^{-} \exp \left[-\mathrm{i} \kappa_{0}^{-}\left(\phi-c_{\phi}\right)\right] \exp \left[\mp \mathrm{i} \zeta_{\kappa_{0}^{-}} \psi\right]
\end{aligned}
$$

where

$$
\tilde{M}_{m}^{-}=s_{\phi} M_{m}^{-}+\kappa_{0}^{-}+\frac{2 \pi d_{\phi} m}{\Delta_{\phi}^{2}}
$$

\section{E.1.3 Inter-Blade Region Decomposition}

We seek to express the source terms in the inter-blade region in a similar way to previous sections. Unfortunately, the decaying Fourier series representation used for the background flow in previous sections is not applicable since the kernel of the background cascade flow cannot be expanded as a sum of exponentials. Therefore, we seek a Fourier series representation of the entire source terms in the inter-blade region. Strictly speaking, this representation is only necessary for the mean flow terms since we have the exponential form of the acoustic field in this region. However, the structure of the $\mathcal{O}(1)$ acoustic field in the inter-blade region is highly complex, so we choose to decompose the entire source terms using this method.

A straightforward 2-D Fourier series where the period is the inter-blade region will break down. This is because the source terms are discontinuous either side of the blade in $(\phi, \psi)$-space and, more importantly, posses (integrable) singularities at the leading edge of every blade. If we were to take a naive Fourier series where the period window is simply $\mathscr{P}$ of figure 3.1, we would not be able to accurately capture the behaviour at the leading and trailing edges. 


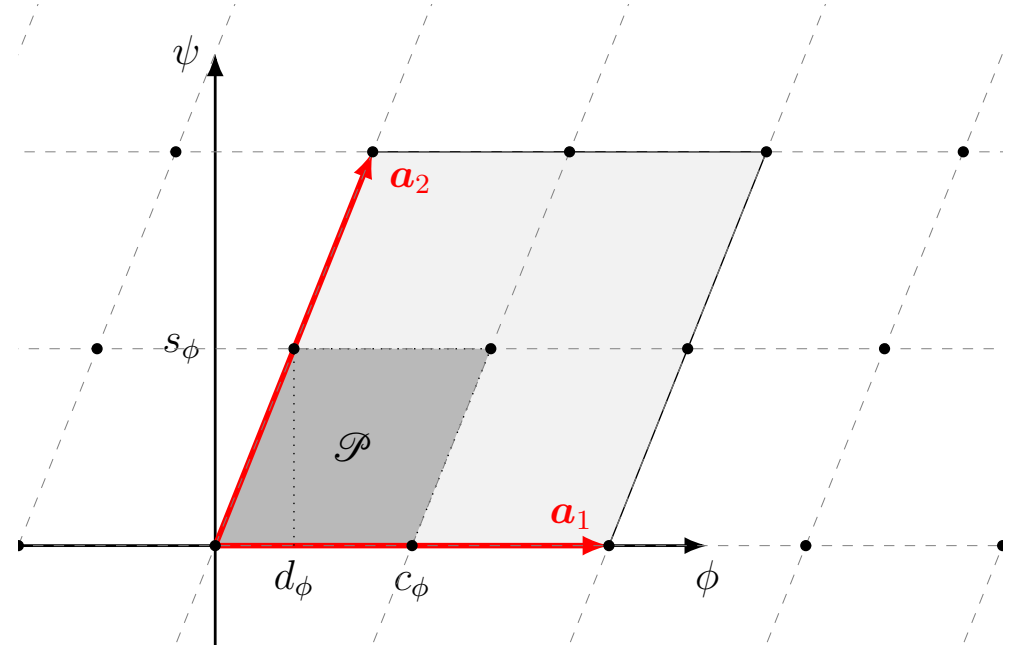

Fig. 3.1 The two-dimensional oblique Bravais lattice for source terms in the inter-blade region.

We parametrise every point in the plane $\boldsymbol{z}=(\phi, \psi)$ as

$$
\boldsymbol{z}=x_{1} \frac{\boldsymbol{a}_{1}}{\left|\boldsymbol{a}_{1}\right|}+x_{2} \frac{\boldsymbol{a}_{2}}{\left|\boldsymbol{a}_{2}\right|},
$$

where $\boldsymbol{a}_{1}=\left(2 c_{\phi}, 0\right)$ and $\boldsymbol{a}_{2}=\left(2 d_{\phi}, 2 s_{\phi}\right)$ are the periods of the extended source terms.

Rearranging (3.144) results in

$$
x_{1}=\phi_{1}-\frac{d_{\phi}}{s_{\phi}} \psi, \quad x_{2}=\frac{\Delta_{\phi}}{s_{\phi}} \psi_{1},
$$

and consequently, the source terms in the inter-blade region may be written in a Fourier series as

$$
\mathbb{S}^{i}(\phi, \psi)=\sum_{l_{1}, l_{2}} \mathbb{S}_{l_{1}, l_{2}}^{i} \exp \left[-\pi \mathrm{i} l_{1} \frac{\phi}{c_{\phi}}\right] \exp \left[-\pi \mathrm{i} \frac{\psi}{s_{\phi}}\left(l_{2}-\frac{l_{1} d_{\phi}}{c_{\phi}}\right)\right] \exp \left[\mathrm{i}\left(k k_{n} \psi-\kappa_{0}^{-} \phi\right)\right],
$$

which reduces to

$$
\mathbb{S}^{i}(\phi, \psi)=\sum_{l_{1}, l_{2}} \mathbb{S}_{l_{1}, l_{2}}^{i} \exp \left[-\mathrm{i} \kappa_{l_{1}}^{-} \phi\right] \exp \left[\mathrm{i}\left(\Delta_{\phi} f_{l_{2} / 2}+d_{\phi} \kappa_{l_{1}}^{-}\right) \frac{\psi}{s_{\phi}}\right]
$$


where the coefficients $\mathbb{S}_{l_{1}, l_{2}}^{i}$ are defined by

$$
\mathbb{S}_{l_{1}, l_{2}}^{i}=\frac{1}{\left|\boldsymbol{a}_{\mathbf{1}}\right|} \frac{1}{\left|\boldsymbol{a}_{\mathbf{2}}\right|} \int_{0}^{\left|\boldsymbol{a}_{\mathbf{2}}\right|} \int_{0}^{\left|\boldsymbol{a}_{\mathbf{1}}\right|} \mathbb{S}^{i}\left(\phi\left(x_{1}, x_{2}\right), \psi\left(x_{1}, x_{2}\right)\right) \mathrm{e}^{2 \pi \mathrm{i}\left(l_{1} \frac{x_{1}}{\left|\boldsymbol{a}_{\mathbf{1}}\right|}+l_{2} \frac{x_{2}}{\left|\boldsymbol{a}_{\mathbf{2}}\right|}\right)} \mathrm{d} x_{1} \mathrm{~d} x_{2} .
$$

\section{E.2 Transformation of Source Terms}

We segregate the integral (3.38) into upstream, downstream and inter-blade contributions, as in the previous section, and write

$$
S(\gamma)=S^{u}(\gamma)+S^{i}(\gamma)+S^{d}(\gamma)
$$

where

$$
S^{x}(\gamma)=-\frac{1}{8 \pi^{2}} \int_{0}^{s_{\phi}} S_{\phi}^{x}\left(\gamma, \psi_{1}\right) \sum_{ \pm} \frac{\mathrm{e}^{ \pm \mathrm{i} \zeta \psi_{1}}}{1-\mathrm{e}^{\mathrm{i}\left( \pm \zeta s_{\phi}+\sigma_{\phi}^{\prime}+\gamma d_{\phi}\right)}} \mathrm{d} \psi_{1}
$$

for $x=u, i, d$, and $S_{\phi}^{x}$ are defined as

$$
\begin{aligned}
& S_{\phi}^{u}(\gamma)=\int_{-\infty}^{\psi_{1} d_{\phi} / s_{\phi}} \mathbb{S}\left(\phi_{1}, \psi_{1}\right) \mathrm{e}^{\mathrm{i} \gamma \phi_{1}} \mathrm{~d} \phi_{1}, \\
& S_{\phi}^{i}(\gamma)=\int_{\psi_{1} d_{\phi} / s_{\phi}}^{\psi_{1} d_{\phi} / s_{\phi}+c_{\phi}} \mathbb{S}\left(\phi_{1}, \psi_{1}\right) \mathrm{e}^{\mathrm{i} \gamma \phi_{1}} \mathrm{~d} \phi_{1}, \\
& S_{\phi}^{d}(\gamma)=\int_{\psi_{1} d_{\phi} / s_{\phi}+c_{\phi}}^{\infty} \mathbb{S}\left(\phi_{1}, \psi_{1}\right) \mathrm{e}^{\mathrm{i} \gamma \phi_{1}} \mathrm{~d} \phi_{1} .
\end{aligned}
$$

\section{E.2.1 Upstream Region Transformation}

We may used the upstream decomposition of the source terms (3.141) to evaluate the upstream Fourier transform of the source terms as

$$
S_{\phi}^{u}\left(\gamma, \phi_{1}\right)=\sum_{m=-\infty}^{\infty} \mathbb{S}_{P, M_{m}^{+}}^{+} \frac{\mathrm{e}^{\mathrm{i}\left(d_{\phi} \gamma-2 m \pi+\sigma_{\phi}^{\prime}\right) \psi / s_{\phi}}}{\mathrm{i}\left(\gamma-\tilde{M}_{m}^{+}\right)}
$$

Therefore we may calculate the constituent parts of the transformed source terms in (3.148) as

$$
S^{u}(\gamma)=-\frac{s_{\phi}}{4 \pi^{2} \Delta_{\phi}^{2}} \sum_{m=-\infty}^{\infty} \frac{\mathbb{S}_{P, M_{m}^{+}}^{+}}{\gamma-\tilde{M}_{m}^{+}} \cdot \frac{d_{\phi} \gamma-2 m \pi+\sigma_{\phi}^{\prime}}{\left(\gamma-\lambda_{m}^{-}\right)\left(\gamma-\lambda_{m}^{+}\right)}
$$


The residues of $S^{u}(\gamma)$ at $\gamma=\lambda_{m}^{-}$are given by

$$
S_{m}^{u, r}=-\frac{s_{\phi} \zeta_{m}^{-}}{8 \pi^{2} \Delta_{\phi} \sqrt{k^{2} w^{2}-f_{m}^{2}}} \cdot \frac{\mathbb{S}_{P, M_{m}^{+}}^{+}}{\lambda_{m}^{-}-\tilde{M}_{m}^{+}},
$$

where we have used the identities (3.131) and (3.128).

\section{E.2.2 Downstream Region Transformation}

Similarly, we may used the downstream decomposition of the source terms (3.143) to evaluate the downstream Fourier transform of the source terms as

$$
\begin{aligned}
S_{\phi}^{d}\left(\gamma, \phi_{1}\right)= & -\sum_{m=-\infty}^{\infty} \mathbb{S}_{P, M_{m}^{-}}^{-} \frac{\mathrm{e}^{\mathrm{i}\left(d_{\phi} \gamma-2 m \pi+\sigma_{\phi}^{\prime}\right) \psi / s_{\phi}}}{\mathrm{i}\left(\gamma-\tilde{M}_{m}^{-}\right)} \mathrm{e}^{\mathrm{i} \gamma c_{\phi}} \\
& -\sum_{m=-\infty}^{\infty} \mathbb{S}_{P, \lambda_{m}^{-}}^{-} \frac{\mathrm{e}^{\mathrm{i}\left(d_{\phi} \gamma-2 m \pi+\sigma_{\phi}^{\prime}\right) \psi / s_{\phi}}}{\mathrm{i}\left(\gamma-\lambda_{m}^{-}\right)} \mathrm{e}^{\mathrm{i} \gamma c_{\phi}} \\
& -\sum_{n} \mathbb{S}_{N, N_{\phi, n}^{-}}^{-} \frac{\mathrm{e}^{\mathrm{i}\left(d_{\phi}\left(\gamma-N_{\phi, n}^{-}\right) s_{\phi}-N_{\psi, n}^{-}\right) \psi / s_{\phi}}}{\mathrm{i}\left(\gamma-N_{\phi, n}^{-}\right)} \mathrm{e}^{\mathrm{i} \gamma c_{\phi}} \\
& -\sum_{ \pm} \mathbb{S}_{N, \kappa_{0}^{-}, \pm}^{-} \frac{\mathrm{e}^{\mathrm{i}\left(d_{\phi}\left(\gamma-\kappa_{0}^{-}\right) \pm s_{\phi} \zeta_{\kappa_{0}^{-}}\right) \psi / s_{\phi}}}{\mathrm{i}\left(\gamma-\kappa_{0}^{-}\right)} \mathrm{e}^{\mathrm{i} \gamma c_{\phi}} .
\end{aligned}
$$

Therefore, we may calculate (3.148) as

$$
\begin{aligned}
S^{d}(\gamma) & =\frac{s_{\phi}}{4 \pi^{2} \Delta_{\phi}^{2}} \sum_{m=-\infty}^{\infty} \frac{\mathbb{S}_{P, M_{m}^{-}}^{-}}{\gamma-\tilde{M}_{m}^{-}} \cdot \frac{d_{\phi} \gamma-2 m \pi+\sigma_{\phi}^{\prime}}{\left(\gamma-\lambda_{m}^{-}\right)\left(\gamma-\lambda_{m}^{+}\right)} \mathrm{e}^{\mathrm{i} \gamma_{\phi}} \\
& +\frac{s_{\phi}}{4 \pi^{2} \Delta_{\phi}^{2}} \sum_{m=-\infty}^{\infty} \mathbb{S}_{P, \lambda_{m}^{-}}^{-} \frac{d_{\phi} \gamma-2 m \pi+\sigma_{\phi}^{\prime}}{\left(\gamma-\lambda_{m}^{-}\right)^{2}\left(\gamma-\lambda_{m}^{+}\right)} \mathrm{e}^{\mathrm{i} \gamma c_{\phi}} \\
& +\frac{s_{\phi}}{8 \pi^{2}} \sum_{ \pm} \frac{\mathbb{S}_{N, \kappa_{0}^{-}, \pm}^{-}}{\gamma-\kappa_{0}^{-}} \sum_{ \pm^{\prime}} \frac{\mathrm{e}^{\mathrm{i}\left(d_{\phi}\left(\gamma-\kappa_{0}^{-}\right)-s_{\phi}\left( \pm \zeta_{\kappa_{0}^{-}} \mp^{\prime} \zeta\right)\right)}-1}{d_{\phi}\left(\gamma-\kappa_{0}^{-}\right)-s_{\phi}\left( \pm \zeta_{\kappa_{0}^{-}} \mp^{\prime} \zeta\right)} \cdot \frac{1}{1-\mathrm{e}^{\mathrm{i}\left( \pm^{\prime} \zeta s_{\phi}+\gamma d_{\phi}+\sigma_{\phi}^{\prime}\right)}} \mathrm{e}^{\mathrm{i} \gamma c_{\phi}} \\
& +\frac{s_{\phi}}{8 \pi^{2}} \sum_{N_{\phi, n}^{-}} \frac{\mathbb{S}_{N, N_{\phi, n}^{-}}^{-}}{\gamma-N_{\phi, n}^{-}} \sum_{ \pm} \frac{\mathrm{e}^{\mathrm{i}\left(d_{\phi}\left(\gamma-N_{\phi, n}^{-}\right)-s_{\phi}\left(N_{\psi, n}^{-} \mp \zeta\right)\right)}-1}{d_{\phi}\left(\gamma-N_{\phi, n}^{-}\right)-s_{\phi}\left(N_{\psi, n}^{-} \mp \zeta\right)} \cdot \frac{1}{1-\mathrm{e}^{\mathrm{i}\left( \pm \zeta s_{\phi}+\gamma d_{\phi}+\sigma_{\phi}^{\prime}\right)}} \mathrm{e}^{\mathrm{i} \gamma c_{\phi}} .
\end{aligned}
$$


Using (3.132), we calculate the residues of $S^{d}(\gamma)$ at $\gamma=\lambda_{m}^{+}$as

$$
\begin{aligned}
S_{m}^{d, r} & =\frac{s_{\phi} \zeta_{m}^{+}}{8 \pi^{2} \Delta_{\phi} \sqrt{k^{2} w^{2}-f_{m}^{2}}} \cdot \frac{\mathbb{S}_{P, M_{m}^{-}}^{-}}{\lambda_{m}^{+}-\tilde{M}_{m}^{-}} \mathrm{e}^{\mathrm{i} c_{\phi} \lambda_{m}^{+}} \\
& +\frac{s_{\phi} \zeta_{m}^{+}}{8 \pi^{2} \Delta_{\phi} \sqrt{k^{2} w^{2}-f_{m}^{2}}} \cdot \frac{\mathbb{S}_{P, \lambda_{m}^{-}}^{-}}{\lambda_{m}^{+}-\lambda_{m}^{-}} \mathrm{e}^{\mathrm{i} c_{\phi} \lambda_{m}^{+}} \\
& +\frac{s_{\phi}}{8 \pi^{2}} \sum_{ \pm} \frac{\mathbb{S}_{N, \kappa_{0}^{-}, \pm}^{-}}{\lambda_{m}^{+}-\kappa_{0}^{-}} \cdot \frac{\mathrm{e}^{\mathrm{i}\left(d_{\phi}\left(\lambda_{m}^{+}-\kappa_{0}^{-}\right)-s_{\phi}\left( \pm \zeta_{\kappa_{0}^{-}}+\zeta_{m}^{+}\right)\right)}-1}{d_{\phi}\left(\lambda_{m}^{+}-\kappa_{0}^{-}\right)-s_{\phi}\left( \pm \zeta_{\kappa_{0}^{-}}+\zeta_{m}^{+}\right)} \cdot \frac{\zeta_{m}^{+}}{\mathrm{i} \Delta_{\phi} \sqrt{k^{2} w^{2}-f_{m}^{2}}} \mathrm{e}^{\mathrm{i} c_{\phi} \lambda_{m}^{+}} \\
& +\frac{s_{\phi}}{8 \pi^{2}} \sum_{N_{\phi, n}^{-}} \frac{\mathbb{S}_{N, N_{\phi, n}^{-}}^{-}}{\lambda_{m}^{+}-N_{\phi, n}^{-}} \cdot \frac{\mathrm{e}^{\mathrm{i}\left(d_{\phi}\left(\lambda_{m}^{+}-N_{\phi, n}^{-}\right)-s_{\phi}\left(N_{\psi, n}^{-}+\zeta_{m}^{+}\right)\right)}-1}{d_{\phi}\left(\lambda_{m}^{+}-N_{\phi, n}^{-}\right)-s_{\phi}\left(N_{\psi, n}^{-}+\zeta_{m}^{+}\right)} \cdot \frac{\zeta_{m}^{+}}{\mathrm{i} \Delta_{\phi} \sqrt{k^{2} w^{2}-f_{m}^{2}}} \mathrm{e}^{\mathrm{i} c_{\phi} \lambda_{m}^{+}} .
\end{aligned}
$$

\section{E.2.3 Inter-Blade Region Transformation}

We may calculate the inter-blade Fourier transform by substituting in our exponential expression (3.146) to obtain

$$
S_{\phi}^{i}\left(\gamma, \psi_{1}\right)=S_{\phi}^{i,(1)}\left(\gamma, \psi_{1}\right)+S_{\phi}^{i,(2)}\left(\gamma, \psi_{1}\right)
$$

where

$$
\begin{aligned}
& S_{\phi}^{i,(1)}\left(\gamma, \psi_{1}\right)=\sum_{l_{1}, l_{2}} \mathbb{S}_{l_{1}, l_{2}}^{i} \frac{-1}{\mathrm{i}\left(\gamma-\kappa_{l_{1}}^{-}\right)} \exp \left[\mathrm{i}\left(d_{\phi} \gamma+\sigma_{\phi}^{\prime}-\pi l_{2}\right) \frac{\psi_{1}}{s_{\phi}}\right], \\
& S_{\phi}^{i,(2)}\left(\gamma, \psi_{1}\right)=\sum_{l_{1}, l_{2}} \mathbb{S}_{l_{1}, l_{2}}^{i} \frac{\mathrm{e}^{\mathrm{i}\left(\gamma-\kappa_{l_{1}}^{-}\right) c_{\phi}}}{\mathrm{i}\left(\gamma-\kappa_{l_{1}}^{-}\right)} \exp \left[\mathrm{i}\left(d_{\phi} \gamma+\sigma_{\phi}^{\prime}-\pi l_{2}\right) \frac{\psi_{1}}{s_{\phi}}\right] .
\end{aligned}
$$

Using these representations, the $\psi_{1}$ integral in (3.148) may now be performed to obtain

$$
S^{i}(\gamma)=S^{i,(1)}(\gamma)+S^{i,(2)}(\gamma)
$$

where

$$
S^{i,(1)}(\gamma)=\sum_{l_{1}, l_{2}} \mathbb{S}_{l_{1}, l_{2}}^{i} \frac{\mathscr{F}_{l_{2}}(\gamma)}{\left(\gamma-\kappa_{l_{1}}^{-}\right)}, \quad S^{i,(2)}(\gamma)=-\sum_{l_{1}, l_{2}} \mathbb{S}_{l_{1}, l_{2}}^{i} \mathrm{e}^{\left(\gamma-\kappa_{l_{1}}^{-}\right) c_{\phi}} \frac{\mathscr{F}_{l_{2}}(\gamma)}{\left(\gamma-\kappa_{l_{1}}^{-}\right)},
$$

and

$$
\mathscr{F}_{l_{2}}(\gamma)=\frac{s_{\phi}}{8 \pi^{2}} \sum_{ \pm} \frac{(-1)^{l_{2}} \mathrm{e}^{\mathrm{i}\left(d_{\phi} \gamma+\sigma_{\phi}^{\prime} \pm s_{\phi} \zeta\right)}-1}{ \pm s_{\phi} \zeta+d_{\phi} \gamma+\sigma_{\phi}^{\prime}-\pi l_{2}} \cdot \frac{1}{1-\mathrm{e}^{\mathrm{i}\left( \pm \zeta s_{\phi}+\gamma d_{\phi}+\sigma_{\phi}^{\prime}\right)}}
$$


When $l_{2}=2 m$ is even, this reduces to

$$
\mathscr{F}_{l_{2}}(\gamma)=\frac{-s_{\phi}}{4 \pi^{2} \Delta_{\phi}^{2}} \cdot \frac{d_{\phi} \gamma+\sigma_{\phi}^{\prime}-2 m \pi}{\left(\gamma-\lambda_{m}^{+}\right)\left(\gamma-\lambda_{m}^{-}\right)}
$$

Consequently, the residues of $\mathscr{F}_{l_{2}}$ at $\lambda_{m}^{ \pm}$are

$$
\mathscr{F}_{l_{2}, m}^{r, \pm}=\frac{s_{\phi}}{8 \pi^{2}} \cdot \frac{\zeta_{m}^{ \pm}}{\Delta_{\phi} \sqrt{k^{2} w^{2}-f_{m}^{2}}} \times \begin{cases}\frac{2}{\pi \mathrm{i}\left(2 m-l_{2}\right)}, & l_{2} \text { odd }, \\ -\delta_{l_{2}, 2 m}, & l_{2} \text { even } .\end{cases}
$$

\section{E.3 Inversion of Source Terms}

When we invert the Fourier transform in section 3.4, we encounter integrals of the form

$$
\begin{aligned}
I(\gamma, \psi)=\int_{0}^{s_{\phi}} \mathrm{e}^{\mathrm{i} a(\gamma) \psi_{1} / s_{\phi}}\left\{\mathrm{e}^{\mathrm{i} \zeta\left|\psi_{1}-\psi\right|}-\frac{\mathrm{e}^{\mathrm{i} \zeta\left(\psi_{1}-\psi\right)}}{1-\mathrm{e}^{-\mathrm{i}\left(\zeta s_{\phi}+\gamma d_{\phi}+\sigma_{\phi}^{\prime}\right)}}-\frac{\mathrm{e}^{-\mathrm{i} \zeta\left(\psi_{1}-\psi\right)}}{1-\mathrm{e}^{\mathrm{i}\left(-\zeta s_{\phi}+\gamma d_{\phi}+\sigma_{\phi}^{\prime}\right)}}\right\} \mathrm{d} \psi_{1} \\
=\frac{\mathrm{i} s_{\phi}}{a(\gamma)-s_{\phi} \zeta}\left[\mathrm{e}^{\mathrm{i} \zeta \psi}-\mathrm{e}^{\mathrm{i} \psi a(\gamma) / s_{\phi}}+\frac{\mathrm{e}^{\mathrm{i}\left(a(\gamma)-\left(s_{\phi}-\psi\right) \zeta\right)}-\mathrm{e}^{\mathrm{i} \psi \zeta}}{1-\mathrm{e}^{\mathrm{i}\left(-s_{\phi} \zeta+d_{\phi} \gamma+\sigma_{\phi}^{\prime}\right)}}\right] \\
+\frac{\mathrm{i} s_{\phi}}{a(\gamma)+s_{\phi} \zeta}\left[\mathrm{e}^{\mathrm{i} \psi a(\gamma) / s_{\phi}}-\mathrm{e}^{\mathrm{i}\left(a(\gamma)+\left(s_{\phi}-\psi\right) \zeta\right)}+\frac{\mathrm{e}^{\mathrm{i}\left(a(\gamma)+\zeta\left(s_{\phi}-\psi\right)\right)}-\mathrm{e}^{-\mathrm{i} \psi \zeta}}{1-\mathrm{e}^{-\mathrm{i}\left(\zeta s_{\phi}+\gamma d_{\phi}+\sigma_{\phi}^{\prime}\right)}}\right]
\end{aligned}
$$

for some linear functions $a(\gamma)$. Note that the residues of $I$ at $\gamma=\lambda_{m}^{ \pm}$are

$$
\operatorname{Res}\left[I, \lambda_{m}^{ \pm}\right]=\frac{\mathrm{i} s_{\phi} \zeta_{m}^{ \pm}}{a\left(\lambda_{m}^{ \pm}\right) \mp s_{\phi} \zeta_{m}^{ \pm}} \cdot \frac{\mathrm{e}^{\mathrm{i}\left(a\left(\lambda_{m}^{ \pm}\right) \mp s_{\phi} \zeta_{m}^{ \pm}\right)}-1}{\Delta_{\phi} \sqrt{k^{2} w^{2}-f_{m}^{2}}} \mathrm{e}^{ \pm \mathrm{i} \zeta_{m}^{ \pm} \psi}
$$

In the special case $a(\gamma)=\mathrm{i}\left(\sigma_{\phi}^{\prime}-\pi l_{2}+d_{\phi} \gamma\right)$, we have

$$
\begin{aligned}
& I(\gamma, \psi) \\
& =\int_{0}^{s_{\phi}} \mathrm{e}^{\mathrm{i}\left(\sigma_{\phi}^{\prime}-\pi l_{2}+d_{\phi} \gamma\right) \psi_{1} / s_{\phi}}\left\{\mathrm{e}^{\mathrm{i} \zeta\left|\psi_{1}-\psi\right|}-\frac{\mathrm{e}^{\mathrm{i} \zeta\left(\psi_{1}-\psi\right)}}{1-\mathrm{e}^{-\mathrm{i}\left(\zeta s_{\phi}+\gamma d_{\phi}+\sigma_{\phi}^{\prime}\right)}}-\frac{\mathrm{e}^{-\mathrm{i} \zeta\left(\psi_{1}-\psi\right)}}{1-\mathrm{e}^{\mathrm{i}\left(-\zeta s_{\phi}+\gamma d_{\phi}+\sigma_{\phi}^{\prime}\right)}}\right\} \mathrm{d} \psi_{1} \\
& =-2 \pi \zeta\left\{\frac{s_{\phi}^{2}}{\Delta_{\phi}^{2}} \cdot \frac{\mathrm{e}^{\mathrm{i}\left(\sigma_{\phi}^{\prime}+d_{\phi} \gamma-\pi l_{2}\right) \psi / s_{\phi}}}{\left(\gamma-\lambda_{l_{2} / 2}^{+}\right)\left(\gamma-\lambda_{l_{2} / 2}^{-}\right)}\right. \\
& \left.+\sum_{ \pm} \frac{ \pm s_{\phi}}{2 \zeta} \cdot \frac{\mathrm{e}^{ \pm \mathrm{i} \zeta \psi}}{1-\mathrm{e}^{\mathrm{i}\left( \pm s_{\phi} \zeta-d_{\phi} \gamma-\sigma_{\phi}^{\prime}\right)}} \cdot \frac{\left((-1)^{l_{2}}-1\right)}{ \pm s_{\phi} \zeta-\sigma_{\phi}^{\prime}-d_{\phi} \gamma+\pi l_{2}}\right\} .
\end{aligned}
$$

During the inversion of the Fourier transform, the ensuing $\gamma$-integral is then closed in the upper or lower half plane, depending on which region of the physical domain is under 
consideration. In particular, the residues of the function in the curly brackets at $\gamma=\lambda_{m}^{ \pm}$ are given by

$$
\frac{s_{\phi} \mathrm{e}^{\mathrm{i} \zeta_{m}^{ \pm} \psi}}{2 \Delta_{\phi} \sqrt{k^{2} w^{2}-f_{m}^{2}}} \times \begin{cases}\frac{2}{\pi \mathrm{i}\left(2 m-l_{2}\right)}, & l_{2} \text { odd }, \\ -\delta_{l_{2}, 2 m}, & l_{2} \text { even } .\end{cases}
$$

\section{F Resolving Resonance}

In this section we observe that the regular perturbation expansion (3.15) breaks down when $\phi=\mathcal{O}\left(\epsilon^{-1}\right)$ in the downstream region. In particular, the $\mathcal{O}(\epsilon)$ solution grows to $\mathcal{O}(1)$ and therefore has the same magnitude as the leading-order solution. Consequently, the series (3.15) is no longer an asymptotic expansion of the solution. The growth of the $\mathcal{O}(\epsilon)$ solution is caused by resonance between the $\mathcal{O}(\epsilon)$ solution and terms in the $\mathcal{O}(1)$ solution. We now show how to regularise our solution by appealing to a multiple-scales argument so that the solution is valid in a larger region up to $\phi=\mathcal{O}\left(\epsilon^{-2}\right)$.

We first consider the resonance arising in the source term solution $h_{1, S}$, and then apply a similar argument to regularise the resonance arising in the $h_{1, \Gamma}$ solution.

\section{F.1 Resonance in the $h_{1, S}$ Solution}

In the downstream region we have

$$
\begin{aligned}
h_{1, S}(\phi, \psi) & =\sum_{m=-\infty}^{\infty} \mathscr{H}_{S, m}^{-} \exp \left[-\mathrm{i}\left(\lambda_{m}^{-} \phi+\zeta_{m}^{-} \psi\right)\right] \\
& +\sum_{M_{m}^{-}} \mathbb{S}_{P, M_{m}^{-}}^{-} \mathbb{I}_{P, M_{m}^{-}}^{-}(\phi, \psi)+\sum_{m=-\infty}^{\infty} \mathbb{S}_{P, \lambda_{m}^{-}}^{-} \mathbb{I}_{P, \lambda_{m}^{-}}^{-}(\phi, \psi) \\
& +\sum_{N_{\phi, n}^{-}} \mathbb{S}_{N, N_{\phi, n}^{-}}^{-} \mathbb{I}_{N, N_{\phi, n}^{-}}^{-}(\phi, \psi)+\sum_{ \pm} \mathbb{S}_{N, \kappa_{0}^{-}, \pm}^{-} \mathbb{I}_{N, \kappa_{0}^{-}, \pm}^{-}(\phi, \psi) \\
& +\sum_{ \pm} \mathscr{H}_{1, S, \kappa_{0}^{-}, \pm}^{-} \mathrm{e}^{-\mathrm{i}\left(\kappa_{0}^{-} \phi \pm \zeta_{\kappa_{0}^{-}} \psi\right)}
\end{aligned}
$$

where the coefficients are defined as

$$
\begin{aligned}
\mathscr{H}_{S, m}^{-}= & -\frac{\pi}{\Delta_{\phi}} \sum_{m=-\infty}^{\infty} \frac{\zeta_{m}^{-} D_{1, S}^{(2,4)}\left(\lambda_{m}^{-}\right)}{\sqrt{k^{2} w^{2}-f_{m}^{2}}}+ \\
& -\sum_{M_{m}^{-}} \mathbb{S}_{P, M_{m}^{-}}^{-} \frac{s_{\phi}}{\Delta_{\phi}} \cdot \frac{\exp \left[\mathrm{i} \lambda_{m}^{-} c_{\phi}\right]}{2 \sqrt{k^{2} w^{2}-f_{m}^{2}}\left(\tilde{M}_{m}^{-}-\lambda_{m}^{-}\right)}
\end{aligned}
$$




$$
\begin{aligned}
+ & \mathbb{S}_{P, \lambda_{m}^{-}}^{-} \frac{1}{4\left(k^{2} w^{2}-f_{m}^{2}\right)} \exp \left[\mathrm{i} \lambda_{m}^{-} c_{\phi}\right] \\
& -\sum_{N_{\phi, n}^{-}} \mathbb{S}_{N, N_{\phi, n}^{-}}^{-} \frac{\mathrm{i} s_{\phi}\left(\mathrm{e}^{\mathrm{i}\left(d_{\phi}\left(\lambda_{m}^{-}-N_{\phi, n}^{-}\right)+s_{\phi}\left(\zeta_{m}^{-}-N_{\psi, n}^{-}\right)\right)}-1\right)}{d_{\phi}\left(\lambda_{m}^{-}-N_{\phi, n}^{-}\right)+s_{\phi}\left(\zeta_{m}^{-}-N_{\psi, n}^{-}\right)} \frac{\exp \left[\mathrm{i} \lambda_{m}^{-} c_{\phi}\right]}{2\left(\lambda_{m}^{-}-N_{\phi, n}^{-}\right) \Delta_{\phi} \sqrt{k^{2} w^{2}-f_{m}^{2}}} \\
& -\sum_{ \pm} \mathbb{S}_{N, \kappa_{0}^{-}, \pm}^{-} \frac{\mathrm{i} s_{\phi}\left(\mathrm{e}^{\mathrm{i}\left(d_{\phi}\left(\lambda_{m}^{-}-\kappa_{0}^{-}\right)+s_{\phi}\left(\zeta_{m}^{-} \pm \zeta_{\kappa_{0}^{-}}\right)\right)}-1\right)}{d_{\phi}\left(\lambda_{m}^{-}-\kappa_{0}^{-}\right)+s_{\phi}\left(\zeta_{m}^{-} \pm \zeta_{\kappa_{0}^{-}}\right)} \frac{\exp \left[\mathrm{i} \lambda_{m}^{-} c_{\phi}\right]}{2\left(\lambda_{m}^{-}-\kappa_{0}^{-}\right) \Delta_{\phi} \sqrt{k^{2} w^{2}-f_{m}^{2}}} \\
& +\frac{4 \pi^{2}}{\zeta_{m}^{-}} \sum_{l_{1}, l_{2}} \frac{\mathbb{S}_{l_{1}, l_{2}}^{i} \mathscr{F}_{l_{2}, m}^{r,-}}{\lambda_{m}^{-}-\kappa_{l_{1}}^{-}} \mathrm{e}^{\mathrm{i}\left(\lambda_{m}^{-}-\kappa_{l_{1}}^{-}\right) c_{\phi}}, \\
\mathscr{H}_{1, S, \kappa_{0}^{-}, \pm}^{-} & =\frac{\pi \mathrm{i} P_{1, S}}{2} \cdot \frac{\mathrm{e}^{\mathrm{i}\left(d_{\phi} \kappa_{0}^{-}+\sigma_{\phi}^{\prime}\right)}-\mathrm{e}^{ \pm \mathrm{i} s_{\phi} \zeta_{\kappa_{0}^{-}}}}{\cos \left(d_{\phi} \kappa_{0}^{-}+\sigma_{\phi}^{\prime}\right)-\cos \left(s_{\phi} \zeta_{\kappa_{0}^{-}}\right)},
\end{aligned}
$$

and the inhomogeneous functions are defined as

$$
\begin{aligned}
& \mathbb{I}_{P, M_{m}^{-}}^{-}(\phi, \psi)=-\frac{s_{\phi}^{2}}{\Delta_{\phi}^{2}} \cdot \frac{\exp \left[-\mathrm{i}\left(\tilde{M}_{m}^{-}\left(\phi-c_{\phi}\right)-\left(\Delta_{\phi} f_{m}+d_{\phi} \tilde{M}_{m}^{-}\right) \frac{\psi}{s_{\phi}}\right)\right]}{\left(\tilde{M}_{m}^{-}-\lambda_{m}^{+}\right)\left(\tilde{M}_{m}^{-}-\lambda_{m}^{-}\right)}, \\
& \mathbb{I}_{N, N_{\phi, n}^{-}}^{-}(\phi, \psi)=\sum_{ \pm} \frac{1}{\zeta\left(N_{\phi, n}^{-}\right) \pm N_{\psi, n}^{-}} \cdot\left(\mathrm{e}^{-\mathrm{i} N_{\psi, n}^{-} \psi}-\mathrm{e}^{\mathrm{i}\left( \pm \zeta\left(N_{\phi, n}^{-}\right) \psi+H(\mp 1)\left(\zeta\left(N_{\phi, n}^{-}\right)-N_{\psi, n}^{-}\right) s_{\phi}\right)}\right. \\
& \left.\mp \frac{\mathrm{e}^{-\mathrm{i}\left(s_{\phi} N_{\psi, n}^{-} \pm\left(s_{\phi}-\psi\right) \zeta\left(N_{\phi, n}^{-}\right)\right)}-\mathrm{e}^{ \pm \mathrm{i} \zeta\left(N_{\phi, n}^{-}\right) \psi}}{1-\mathrm{e}^{-\mathrm{i}\left(\zeta\left(N_{\phi, n}^{-}\right) \mp d_{\phi} N_{\phi, n}^{-} \mp \sigma_{\phi}^{\prime}\right)}}\right) \frac{\exp \left[-\mathrm{i} N_{\phi, n}^{-}\left(\phi-c_{\phi}\right)\right]}{2 \zeta\left(N_{\phi, n}^{-}\right)}, \\
& \mathbb{I}_{P, \lambda_{m}^{-}}^{-}(\phi, \psi)=\left(s_{\phi}\left(\phi-c_{\phi}\right)-d_{\phi} \psi\right) \frac{\exp \left[-\mathrm{i}\left(\lambda_{m}^{-}\left(\phi-c_{\phi}\right)+\zeta_{m}^{-} \psi\right)\right]}{2 \mathrm{i} \Delta_{\phi} \sqrt{k^{2} w^{2}-f_{m}^{2}}}, \\
& \mathbb{I}_{N, \kappa_{0}^{-}, \pm}^{-}(\phi, \psi)=\left\{ \pm \mathrm{e}^{ \pm \mathrm{i} \zeta_{\kappa_{0}^{-}} \psi}\left(\psi-\frac{s_{\phi}}{1-\mathrm{e}^{\mathrm{i}\left(\mp s_{\phi} \zeta_{\kappa_{0}^{-}}+d_{\phi} \kappa_{0}^{-}+\sigma_{\phi}^{\prime}\right)}}\right)\right. \\
& \left.-\frac{\mathrm{e}^{-\mathrm{i} H(\mp 1) \zeta_{\kappa_{0}^{-}} s_{\phi}}}{2 \mathrm{i} \zeta_{\kappa_{0}^{-}}}\left[\mathrm{e}^{ \pm \mathrm{i} \zeta_{\kappa_{0}^{-}}\left(\psi-H(\mp 1) s_{\phi}\right)}-\mathrm{e}^{\mp \mathrm{i} \zeta_{\kappa_{0}^{-}}\left(\psi-H(\mp 1) s_{\phi}\right)} \frac{1-\mathrm{e}^{-\mathrm{i}\left(-s_{\phi} \zeta_{\kappa_{0}^{-}}+d_{\phi} \kappa_{0}^{-}+\sigma_{\phi}^{\prime}\right)}}{1-\mathrm{e}^{-\mathrm{i}\left(s_{\phi} \zeta_{\kappa_{0}^{-}}+d_{\phi} \kappa_{0}^{-}+\sigma_{\phi}^{\prime}\right)}}\right]\right\} \\
& \times \frac{\exp \left[-\mathrm{i}\left(\kappa_{0}^{-}\left(\phi-c_{\phi}\right)\right)\right]}{2 \zeta_{\kappa_{0}^{-}}} .
\end{aligned}
$$


It may be verified that applying the Helmholtz operator (3.17) to the inhomogeneous functions yields

$$
\begin{aligned}
\mathscr{L}\left(\mathbb{I}_{P, M_{m}^{-}}^{-}\right) & =\exp \left[-\mathrm{i}\left(\tilde{M}_{m}^{-}\left(\phi-c_{\phi}\right)-\left(\Delta_{\phi} f_{m}+d_{\phi} \tilde{M}_{m}^{-}\right) \frac{\psi}{s_{\phi}}\right)\right], \\
\mathscr{L}\left(\mathbb{I}_{P, \lambda_{m}^{-}}^{-}\right) & =\exp \left[-\mathrm{i}\left(\lambda_{m}^{-}\left(\phi-c_{\phi}\right)+\zeta_{m}^{-} \psi\right)\right], \\
\mathscr{L}\left(\mathbb{I}_{N, N_{\phi, n}^{-}}^{-}\right) & =\exp \left[-\mathrm{i} N_{\phi, n}^{-}\left(\phi-c_{\phi}\right)+N_{\psi, n}^{-} \psi\right], \\
\mathscr{L}\left(\mathbb{I}_{N, \kappa_{0}^{-}, \pm}^{-}\right) & =\exp \left[-\mathrm{i}\left(\kappa_{0}^{-}\left(\phi-c_{\phi}\right) \pm \zeta_{\kappa_{0}^{-}} \psi\right)\right],
\end{aligned}
$$

so that (3.154) is in fact the solution to the inhomogeneous Helmholtz equation.

We now combine the $h_{1, S}$ solution (3.154) with the $\mathcal{O}(1)$ solution $h_{0}$ (3.50) to write

$$
\begin{aligned}
h_{0}(\phi, \psi)+\epsilon h_{1, S}(\phi, \psi) & =\sum_{m=-\infty}^{\infty}\left(\mathscr{H}_{0, m}^{-}+\epsilon \mathscr{H}_{\Gamma, m}^{-}\right) \exp \left[-\mathrm{i}\left(\lambda_{m}^{-} \phi+\zeta_{m}^{-} \psi\right)\right] \\
& +\sum_{M_{m}^{-}} \epsilon \mathbb{S}_{P, M_{m}^{-}}^{-} \mathbb{I}_{P, M_{m}^{-}}^{-}(\phi, \psi)+\sum_{m=-\infty}^{\infty} \epsilon \mathbb{S}_{P, \lambda_{m}^{-}}^{-} \mathbb{I}_{P, \lambda_{m}^{-}}^{-}(\phi, \psi) \\
& +\sum_{N_{\phi, n}^{-}} \epsilon \mathbb{S}_{N, N_{\phi, n}^{-}}^{-} \mathbb{I}_{N, N_{\phi, n}^{-}}^{-}(\phi, \psi)+\sum_{ \pm} \epsilon \mathbb{S}_{N, \kappa_{0}^{-}, \pm}^{-} \mathbb{I}_{N, \kappa_{0}^{-}, \pm}^{-}(\phi, \psi) \\
& +\sum_{ \pm}\left(\mathscr{H}_{0, \kappa_{0}^{-}, \pm}^{-}+\epsilon \mathscr{H}_{1, S, \kappa_{0}^{-}, \pm}^{-}\right) \mathrm{e}^{-\mathrm{i}\left(\kappa_{0}^{-} \phi \pm \zeta_{\kappa_{0}^{-}} \psi\right)} .
\end{aligned}
$$

We note that the $\mathbb{I}_{P, \lambda_{m}^{-}}^{-}$terms are proportional to $\epsilon \phi$. These are secular terms (Van Dyke, 1964), and present a problem for our asymptotic series: the expansion (3.15) breaks down when $\phi=\mathcal{O}\left(\epsilon^{-1}\right)$. Moreover, the solution does not satisfy the Sommerfeld radiation condition as the acoustic field is not bounded in the far-field when even a single mode is cut-on. Consequently, we need a way of regularising these terms.

We now combine the resonant $\mathbb{I}_{P, \lambda_{m}^{-}}^{-}$terms with the terms on line (3.156) and write

$$
\begin{aligned}
\tilde{h}_{0, S}(\phi, \psi)=\sum_{m=-\infty}^{\infty} & \left(\mathscr{H}_{0, m}^{-}+\epsilon \mathscr{H}_{S, m}^{-}\right)\left\{1-\mathrm{i} \epsilon\left(s_{\phi}\left(\phi-c_{\phi}\right)-d_{\phi} \psi\right) O_{S, m}\right\} \\
& \times \exp \left[-\mathrm{i}\left(\lambda_{m}^{-} \phi+\zeta_{m}^{-} \psi\right)\right],
\end{aligned}
$$

where

$$
O_{S, m}=\frac{\mathbb{S}_{P, \lambda_{m}^{-}}^{-} \mathrm{e}^{\mathrm{i} \lambda_{m}^{-} c_{\phi}}}{2 \Delta_{\phi} \sqrt{k^{2} w^{2}-f_{m}^{2}} \mathscr{H}_{0, m}^{-}},
$$


and we have ignored $\mathcal{O}\left(\epsilon^{2}\right)$ terms. We now note that, when $s_{\phi}\left(\phi-c_{\phi}\right)-d_{\phi} \psi=\mathcal{O}(1)$, we may express the term in the curly brackets in (3.157) as

$$
1-\mathrm{i} \epsilon\left(s_{\phi}\left(\phi-c_{\phi}\right)-d_{\phi} \psi\right) O_{S, m}=\exp \left[-\mathrm{i} \epsilon\left(s_{\phi}\left(\phi-c_{\phi}\right)-d_{\phi} \psi\right) O_{S, m}\right]+\mathcal{O}\left(\left(\epsilon O_{S, m}\right)^{2}\right),
$$

In particular, the above relation is exact along the matching line $s_{\phi}\left(\phi-c_{\phi}\right)-d_{\phi} \psi$ where the downstream region meets the lower triangular region. Consequently, we may express (3.157) as

$$
\tilde{h}_{0, S}(\phi, \psi)=\sum_{m=-\infty}^{\infty}\left(\mathscr{H}_{0, m}^{-}+\epsilon \mathscr{H}_{S, m}^{-}\right) \exp \left[-\mathrm{i}\left(\tilde{\lambda}_{m, S}^{-} \phi+\tilde{\zeta}_{m, S}^{-} \psi\right)\right] \exp \left[\mathrm{i} \epsilon s_{\phi} c_{\phi} O_{S, m}\right]
$$

where we have introduced the modified modes

$$
\begin{aligned}
& \tilde{\lambda}_{S, m}^{-}=\lambda_{m}^{-}+\epsilon s_{\phi} O_{S, m}, \\
& \tilde{\zeta}_{S, m}^{-}=\zeta_{m}^{-}-\epsilon d_{\phi} O_{S, m} .
\end{aligned}
$$

Although we have apparently performed an illegal manoeuvre in regularising (3.157), the resulting function satisfies the required inhomogeneous Helmholtz equation up to $\mathcal{O}\left(\phi \epsilon^{2}\right)$. Applying the Helmholtz operator to $\tilde{h}_{0, S}$ as defined in (3.158) yields

$$
\begin{aligned}
\mathscr{L}\left(\tilde{h}_{0, S}\right)=\sum_{m=-\infty}^{\infty}\left(\mathscr{H}_{0, m}^{-}+\epsilon \mathscr{H}_{S, m}^{-}\right)\left\{2 \epsilon O_{S, m}\left(d_{\phi} \zeta_{m}^{-}-s_{\phi} \lambda_{m}^{-}\right)+\mathcal{O}\left(\epsilon^{2}\right)\right\} \\
\times \exp \left[-\mathrm{i}\left(\tilde{\lambda}_{S, m}^{-} \phi+\tilde{\zeta}_{S, m}^{-} \psi\right)\right] \exp \left[\mathrm{i} \epsilon s_{\phi} c_{\phi} O_{S, m}\right] \\
=\sum_{m=-\infty}^{\infty} \epsilon \mathbb{S}_{P, \lambda_{m}^{-}}^{-} \exp \left[-\mathrm{i}\left(\lambda_{m}^{-}\left(\phi-c_{\phi}\right)+\zeta_{m}^{-} \psi\right)\right]+\mathcal{O}\left(\phi \epsilon^{2}\right)
\end{aligned}
$$

which is the form of the desired forcing terms.

We now note that (3.158) is exactly the solution that would have been obtained if a multiple-scales perturbation expansion had been applied to the downstream region as opposed to the regular perturbation series (3.15). In that case, we would have written

$$
\tilde{h}_{0, S}(\phi, \psi)=H_{0}(\phi, \Phi, \psi)+\epsilon H_{1}(\phi, \Phi, \psi)+\cdots
$$

where $\Phi=\epsilon \phi$, and consequently obtained the solution (3.158). Accordingly, we have obtained the multiple-scales solution albeit via a circuitous route. The solution could be further expanded into the region $\phi=\mathcal{O}\left(\epsilon^{-2}\right)$ via a second multiple-scales type argument, but it is not necessary in the present work. 


\section{F.2 Resonance in the $h_{1, \Gamma}$ solution}

We now apply a similar procedure to regularise terms in the $h_{1, \Gamma}$ problem so that the solution is valid in the region $\phi=\mathcal{O}\left(\epsilon^{-1}\right)$. In the downstream region, we have

$$
\begin{aligned}
h_{1, \Gamma}(\phi, \psi) & =\sum_{m=-\infty}^{\infty} \mathscr{H}_{\Gamma, m}^{-} \exp \left[-\mathrm{i}\left(\lambda_{m}^{-} \phi+\zeta_{m}^{-} \psi\right)\right] \\
& -\sum_{m=-\infty}^{\infty} \Gamma \mathscr{H}_{0, m}^{\prime}\left(s_{\phi}\left(\phi-c_{\phi}\right)-d_{\phi} \psi\right) \frac{\zeta_{m}^{-} \exp \left[-\mathrm{i}\left(\lambda_{m}^{-} \phi+\zeta_{m}^{-} \psi\right)\right]}{s_{\phi} \Delta_{\phi} \sqrt{k^{2} w^{2}-f_{m}^{2}}} \\
& -\sum_{m=-\infty}^{\infty} \Gamma \mathscr{H}_{0, m}^{\prime} \frac{\psi}{s_{\phi}} \exp \left[-\mathrm{i}\left(\lambda_{m}^{-} \phi+\zeta_{m}^{-} \psi\right)\right] \\
& +\sum_{ \pm} \mathscr{H}_{1, \Gamma, \kappa_{0}^{-}, \pm}^{-} \mathrm{e}^{-\mathrm{i}\left(\kappa_{0}^{-} \phi \pm \zeta_{\kappa_{0}^{-}} \psi\right)}
\end{aligned}
$$

where

$$
\begin{aligned}
\mathscr{H}_{\Gamma, m}^{-}= & -\frac{\pi}{\Delta_{\phi}} \sum_{m=-\infty}^{\infty} \frac{\zeta_{m}^{-} D_{1, \Gamma}^{(2,4)}\left(\lambda_{m}^{-}\right)}{\sqrt{k^{2} w^{2}-f_{m}^{2}}} \\
& -\sum_{ \pm} \Gamma \mathscr{H}_{0, \kappa_{0}^{-}}^{ \pm \prime} \frac{ \pm \mathrm{i} \zeta_{\kappa_{0}^{-}} \mathrm{e}^{\mathrm{i}\left(\lambda_{m}^{-}-\kappa_{0}^{-}\right) c_{\phi}}}{2\left(\lambda_{m}^{-}-\kappa_{0}^{-}\right)} \frac{1}{\Delta_{\phi} \sqrt{k^{2} w^{2}-f_{m}^{2}}} \\
& -\sum_{n=-\infty}^{\infty}\left[\frac{\mathrm{i} \mathrm{e}^{\mathrm{i}\left(\lambda_{m}^{-}-\lambda_{n}^{-}\right) c_{\phi}}}{2\left(\lambda_{m}^{-}-\lambda_{n}^{-}\right)} \Gamma \mathscr{H}_{0, n}^{\prime}\left\{\zeta_{n}^{-}+\zeta_{m}^{-} \frac{\lambda_{n}^{-}-\kappa_{0}^{-}}{\lambda_{m}^{-}-\kappa_{0}^{-}} \cdot \frac{J_{-}\left(\lambda_{n}^{-}\right)}{J_{-}\left(\lambda_{m}^{-}\right)}\right\}\right] \frac{1}{\Delta_{\phi} \sqrt{k^{2} w^{2}-f_{m}^{2}}} \\
& -\sum_{m=-\infty}^{\infty} \Gamma \mathscr{H}_{0, m}^{\prime} \frac{\left(\left(\left(s_{\phi} \lambda_{m}^{-}\right)^{2}-\left(d_{\phi} \zeta_{m}^{-}\right)^{2}\right) \cot \left(s_{\phi} \zeta_{m}^{-}\right)-s_{\phi} / \zeta_{m}^{-}\left(\left(\zeta_{m}^{-}\right)^{2}+\left(\lambda_{m}^{-}\right)^{2}\right)\right)}{2 \mathrm{i} \Delta_{\phi}^{2}\left(k^{2} w^{2}-f_{m}^{2}\right)} \\
& -\Gamma \mathscr{H}_{0, m}^{\prime}\left\{\frac{\zeta_{m}^{-}}{\lambda_{m}^{-}-\kappa_{0}^{-}}-\frac{\lambda_{m}^{-}}{\zeta_{m}^{-}}+\zeta_{m}^{-} \frac{J_{-}^{\prime}\left(\lambda_{m}^{-}\right)}{J_{-}\left(\lambda_{m}^{-}\right)}\right\} \frac{\mathrm{i}}{2 \Delta_{\phi} \sqrt{k^{2} w^{2}-f_{m}^{2}}} \\
+ & \Gamma \mathscr{H}_{0, m}^{\prime} \frac{s_{\phi} \lambda_{m}^{-} \mathrm{e}^{\mathrm{i} s_{\phi} \lambda_{m}^{-}}+d_{\phi} \zeta_{m}^{-} \mathrm{e}^{-\mathrm{i} s_{\phi} \zeta_{m}^{-}}}{2 \mathrm{i} \sin \left(s_{\phi} \zeta_{m}^{-}\right)} \frac{1}{\Delta_{\phi} \sqrt{k^{2} w^{2}-f_{m}^{2}}}, \\
\mathscr{H}_{1, \Gamma, \kappa_{0}^{-}, \pm}^{-} & \frac{\pi \mathrm{i} P_{1, \Gamma}}{2} \cdot \frac{\mathrm{e}^{\mathrm{i}\left(d_{\phi} \kappa_{0}^{-}+\sigma_{\phi}^{\prime}\right)}-\mathrm{e}^{ \pm \mathrm{i} s_{\phi} \zeta_{\kappa_{0}^{-}}^{-}}}{\cos \left(d_{\phi} \kappa_{0}^{-}+\sigma_{\phi}^{\prime}\right)-\cos \left(s_{\phi} \zeta_{\kappa_{0}^{-}}\right)} \\
+ & \frac{\Gamma}{2} \mathscr{H}_{0, \kappa_{0}^{-}}^{ \pm \prime} \frac{\mathrm{e}^{\mathrm{i}\left(d_{\phi} \kappa_{0}^{-}+\sigma_{\phi}^{\prime}\right)}-\mathrm{e}^{ \pm \mathrm{i} s_{\phi} \zeta_{\kappa_{0}^{-}}}}{\cos \left(d_{\phi} \kappa_{0}^{-}+\sigma_{\phi}^{\prime}\right)-\cos \left(s_{\phi} \zeta_{\kappa_{0}^{-}}\right)} \cdot
\end{aligned}
$$


In a similar way to the previous section, we combine the $h_{1, \Gamma}$ solution with the $h_{0}$ solution to write

$$
\begin{aligned}
h_{0}(\phi, \psi)+\epsilon h_{1, \Gamma}(\phi, \psi) & =\sum_{m=-\infty}^{\infty}\left(\mathscr{H}_{0, m}^{-}+\epsilon \mathscr{H}_{\Gamma, m}^{-}\right) \exp \left[-\mathrm{i}\left(\lambda_{m}^{-} \phi+\zeta_{m}^{-} \psi\right)\right] \\
& -\sum_{m=-\infty}^{\infty} \epsilon \Gamma \mathscr{H}_{0, m}^{\prime}\left(s_{\phi}\left(\phi-c_{\phi}\right)-d_{\phi} \psi\right) \frac{\zeta_{m}^{-} \exp \left[-\mathrm{i}\left(\lambda_{m}^{-} \phi+\zeta_{m}^{-} \psi\right)\right]}{s_{\phi} \Delta_{\phi} \sqrt{k^{2} w^{2}-f_{m}^{2}}} \\
& -\sum_{m=-\infty}^{\infty} \epsilon \Gamma \mathscr{H}_{0, m}^{\prime} \frac{\psi}{s_{\phi}} \exp \left[-\mathrm{i}\left(\lambda_{m}^{-} \phi+\zeta_{m}^{-} \psi\right)\right] \\
& +\sum_{ \pm}\left(\mathscr{H}_{0, \kappa_{0}^{-}, \pm}^{-}+\epsilon \mathscr{H}_{1, \Gamma, \kappa_{0}^{-}, \pm}^{-}\right) \mathrm{e}^{-\mathrm{i}\left(\kappa_{0}^{-} \phi \pm \zeta_{\kappa_{0}^{-}} \psi\right)}
\end{aligned}
$$

Again, we note that the terms on line (3.161) are proportional to $\epsilon \phi$ and, accordingly, the asymptotic expansion (3.15) breaks down when $\phi=\mathcal{O}\left(\epsilon^{-1}\right)$. We combine these resonant terms with the terms on line (3.160) and write

$$
\begin{aligned}
\tilde{h}_{0, \Gamma}(\phi, \psi)=\sum_{m=-\infty}^{\infty} & \left(\mathscr{H}_{0, m}^{-}+\epsilon \mathscr{H}_{\Gamma, m}^{-}\right)\left\{1-\mathrm{i} \epsilon\left(s_{\phi}\left(\phi-c_{\phi}\right)-d_{\phi} \psi\right) O_{\Gamma, m}\right\} \\
& \times \exp \left[-\mathrm{i}\left(\lambda_{m}^{-} \phi+\zeta_{m}^{-} \psi\right)\right],
\end{aligned}
$$

where

$$
O_{\Gamma, m}=-\frac{\Gamma \zeta_{m}^{-}\left(\lambda_{m}^{-}+k M_{\infty}^{2} \delta\right)}{s_{\phi} \Delta_{\phi} \sqrt{k^{2} w^{2}-f_{m}^{2}}},
$$

and we have ignored $\mathcal{O}\left(\epsilon^{2}\right)$ terms and used the definition (3.51.b). Similarly to the previous section, we may express (3.163) as

$$
\tilde{h}_{0, \Gamma}(\phi, \psi)=\sum_{m=-\infty}^{\infty}\left(\mathscr{H}_{0, m}^{-}+\epsilon \mathscr{H}_{\Gamma, m}^{-}\right) \exp \left[-\mathrm{i}\left(\tilde{\lambda}_{\Gamma, m}^{-} \phi+\tilde{\zeta}_{\Gamma, m}^{-} \psi\right)\right] \exp \left[\mathrm{i} \epsilon s_{\phi} c_{\phi} O_{\Gamma, m}\right]
$$

where we have introduced the modified modes

$$
\begin{aligned}
& \tilde{\lambda}_{\Gamma, m}^{-}=\lambda_{m}^{-}+\epsilon s_{\phi} O_{\Gamma, m}, \\
& \tilde{\zeta}_{\Gamma, m}^{-}=\zeta_{m}^{-}-\epsilon d_{\phi} O_{\Gamma, m} .
\end{aligned}
$$


When combined with the term on line (3.162), the ensuing function satisfies the homogeneous Helmholtz equation up to $\mathcal{O}\left(\phi \epsilon^{2}\right)$. Moreover, we have

$$
\Delta_{0}\left[\tilde{h}_{0, \Gamma}\right](\phi)=0
$$

and the jump in acoustic potential (3.42.f) is obtained exclusively by the term on line (3.162).

\section{F.3 Summary}

In conclusion, we can resolve the resonance terms by modifying the terms proportional to $\mathrm{e}^{-\mathrm{i}\left(\lambda_{m}^{-} \phi+\zeta_{m}^{-} \psi\right)}$. In this context, we change the acoustic modes $\lambda_{m}^{-}$and $\zeta_{m}^{-}$to

$$
\begin{aligned}
& \tilde{\lambda}_{m}^{-}=\lambda_{m}^{-}+\epsilon s_{\phi}\left(O_{S, m}+O_{\Gamma, m}\right), \\
& \tilde{\zeta}_{m}^{-}=\zeta_{m}^{-}-\epsilon d_{\phi}\left(O_{S, m}+O_{\Gamma, m}\right),
\end{aligned}
$$

and rescale that part of the solution by a factor of $\exp \left[\mathrm{i} \epsilon s_{\phi} c_{\phi}\left(O_{S, m}+O_{\Gamma, m}\right)\right]$.

\section{G Coefficients of Acoustic Potential Function}

In this section we define the coefficients of the acoustic potential function found in section 3.4. In this section we use the notation

$$
\begin{aligned}
& D_{n, x}^{(i, j)}=D_{n, x}^{(i)}+D_{n, x}^{(j)} \\
& D^{(i, j)}=D_{0}^{(i, j)}+\epsilon\left(D_{1, \Sigma}^{(i, j)}+D_{1, \Delta}^{(i, j)}+D_{1, \Gamma}^{(i, j)}+D_{1, S}^{(i, j)}\right) .
\end{aligned}
$$

In the upstream region, we define

$$
\mathscr{H}_{m}^{+}=\mathscr{H}_{0, m}^{+}+\epsilon\left(\mathscr{H}_{\Sigma, m}^{+}+\mathscr{H}_{\Delta, m}^{+}+\mathscr{H}_{\Gamma, m}^{+}+\mathscr{H}_{S, m}^{+}\right)
$$

where

$$
\begin{aligned}
\mathscr{H}_{n, x, m}^{+} & =\frac{\pi \zeta_{m}^{+}}{\Delta_{\phi} \sqrt{k^{2} w^{2}-f_{m}^{2}}} D_{n, x}^{(1,3)}\left(\lambda_{m}^{+}\right), \quad \text { for } n=0 \text { and } n=1, x=\Sigma, \Gamma \\
\mathscr{H}_{\Delta, m}^{+} & =\frac{\pi \zeta_{m}^{+}}{\Delta_{\phi} \sqrt{k^{2} w^{2}-f_{m}^{2}}} D_{1, \Delta}^{(1,3)}\left(\lambda_{m}^{+}\right)+\frac{1}{\Delta_{\phi} \sqrt{k^{2} w^{2}-f_{m}^{2}}} \sum_{l=-\infty}^{\infty} \frac{c_{\Delta, l}}{2\left(\lambda_{m}^{+}-\kappa_{l}^{-}\right)}
\end{aligned}
$$




$$
\begin{aligned}
\mathscr{H}_{S, m}^{+} & =\frac{\pi \zeta_{m}^{+}}{\Delta_{\phi} \sqrt{k^{2} w^{2}-f_{m}^{2}}} D_{1, S}^{(1,3)}\left(\lambda_{m}^{+}\right)-\frac{4 \pi^{2}}{\zeta_{m}^{+}} \sum_{l_{1}, l_{2}} \frac{\mathbb{S}_{l_{1}, l_{2}}^{i} \mathscr{F}_{l_{2}, m}^{r,+}}{\lambda_{m}^{+}-\kappa_{l_{1}}^{-}} \\
& +\sum_{\tilde{M}_{m}^{+}} \frac{s_{\phi}}{2 \Delta_{\phi} \sqrt{k^{2} w^{2}-f_{m}^{2}}\left(\tilde{M}_{m}^{+}-\lambda_{m}^{+}\right)} .
\end{aligned}
$$

In the downstream region, we define

$$
\mathscr{H}_{m}^{-}=\left\{\mathscr{H}_{0, m}^{-}+\epsilon\left(\mathscr{H}_{\Sigma, m}^{-}+\mathscr{H}_{\Delta, m}^{-}+\mathscr{H}_{\Gamma, m}^{-}+\mathscr{H}_{S, m}^{-}\right)\right\} \exp \left[\mathrm{i} \epsilon s_{\phi} c_{\phi}\left(O_{S, m}+O_{\Gamma, m}\right)\right],
$$

where

$$
\begin{aligned}
& \mathscr{H}_{n, x, m}^{-}=-\frac{\pi \zeta_{m}^{-}}{\Delta_{\phi} \sqrt{k^{2} w^{2}-f_{m}^{2}}} D_{n, x}^{(2,4)}\left(\lambda_{m}^{-}\right), \quad \text { for } n=0 \text { and } n=1, x=\Sigma, \\
& \mathscr{H}_{\Delta, m}^{-}=-\frac{\pi \zeta_{m}^{-}}{\Delta_{\phi} \sqrt{k^{2} w^{2}-f_{m}^{2}}} D_{1, \Delta}^{(2,4)}\left(\lambda_{m}^{-}\right)-\frac{1}{\Delta_{\phi} \sqrt{k^{2} w^{2}-f_{m}^{2}}} \sum_{l=-\infty}^{\infty} \frac{c_{\Delta, l} \mathrm{e}^{\mathrm{i}\left(\lambda_{m}^{-}-\kappa_{l}^{-}\right) c_{\phi}}}{2\left(\lambda_{m}^{+}-\kappa_{l}^{-}\right)},
\end{aligned}
$$

and $\mathscr{H}_{S, m}^{-}$and $\mathscr{H}_{\Gamma, m}^{-}$are defined in section 3.F.

The coefficients responsible for enforcing the boundary conditions on the blades are given by

$$
\begin{aligned}
\mathscr{H}_{\theta^{+}, b, m} & =\pi \mathrm{i} \mathscr{B}_{n} \cdot \frac{\mathrm{e}^{\mathrm{i}\left(d_{\phi} \theta_{n}^{+}+\sigma_{\phi}^{\prime}\right)}}{\cos \left(d_{\phi} \theta_{n}^{+}+\sigma_{\phi}^{\prime}\right)-(-1)^{n}}, \\
\mathscr{H}_{\theta^{-}, a, m} & =-\pi \frac{\mathscr{A}_{n}+\mathscr{C}_{n}}{\theta_{n}^{-}-\kappa_{0}^{-}} \cdot \frac{(-1)^{n+1}}{\cos \left(d_{\phi} \theta_{n}^{-}+\sigma_{\phi}^{\prime}\right)-(-1)^{n}}, \\
\mathscr{H}_{\theta^{-}, b, m} & =-\pi \frac{\mathscr{A}_{n}+\mathscr{C}_{n}}{\theta_{n}^{-}-\kappa_{0}^{-}} \cdot \frac{\mathrm{e}^{\mathrm{i}\left(d_{\phi} \theta_{n}^{-}+\sigma_{\phi}^{\prime}\right)}}{\cos \left(d_{\phi} \theta_{n}^{-}+\sigma_{\phi}^{\prime}\right)-(-1)^{n}}, \\
\mathscr{H}_{\Sigma, a, l} & =-\pi \mathrm{i} \frac{\mathscr{T}_{l}}{J_{+}\left(\kappa_{l}^{-}\right)} \cdot \frac{-1}{\cos \left(d_{\phi} \kappa_{l}^{-}+\sigma_{\phi}^{\prime}\right)-\cos \left(s_{\phi} \zeta_{\kappa_{l}^{-}}\right)}, \\
\mathscr{H}_{\Sigma, b, l} & =-\pi \mathrm{i} \frac{\mathscr{T}_{l}}{J_{+}\left(\kappa_{l}^{-}\right)} \cdot \frac{\mathrm{e}^{\mathrm{i}\left(d_{\phi} \kappa_{l}^{-}+\sigma_{\phi}^{\prime}\right)}}{\cos \left(d_{\phi} \kappa_{l}^{-}+\sigma_{\phi}^{\prime}\right)-\cos \left(s_{\phi} \zeta_{\kappa_{l}^{-}}\right)}, \\
\mathscr{H}_{\Delta, a, l} & =\frac{-c_{\Delta, l}}{2 \zeta_{\kappa_{l}^{-}}\left(\cos \left(d_{\phi} \kappa_{l}^{-}+\sigma_{\phi}^{\prime}\right)-\cos \left(s_{\phi} \zeta_{\kappa_{l}^{-}}\right)\right)}, \\
\mathscr{H}_{\Delta, b, l} & =\frac{c_{\Delta, l} \mathrm{e}^{\mathrm{i}\left(d_{\phi} \kappa_{l}^{-}+\sigma_{\phi}^{\prime}\right)}}{2 \zeta_{\kappa_{l}^{-}}\left(\cos \left(d_{\phi} \kappa_{l}^{-}+\sigma_{\phi}^{\prime}\right)-\cos \left(s_{\phi} \zeta_{\kappa_{l}^{-}}\right)\right)},
\end{aligned}
$$


where

$$
\begin{aligned}
\mathscr{T}_{l} & =\mathscr{T}_{0, l}+\epsilon\left(\mathscr{T}_{1, \Sigma, l}+\mathscr{T}_{1, \Delta, l}+\mathscr{T}_{1, \Gamma, l}+\mathscr{T}_{1, S, l}\right), \\
\mathscr{A}_{n} & =\mathscr{A}_{0, n}+\epsilon\left(\mathscr{A}_{1, \Sigma, n}+\mathscr{A}_{1, \Delta, n}+\mathscr{A}_{1, \Gamma, n}+\mathscr{A}_{1, S, n}\right), \\
\mathscr{B}_{n} & =\mathscr{B}_{0, n}+\epsilon\left(\mathscr{B}_{1, \Sigma, n}+\mathscr{B}_{1, \Delta, n}+\mathscr{B}_{1, \Gamma, n}+\mathscr{B}_{1, S, n}\right), \\
\mathscr{C}_{n} & =\mathscr{C}_{0, n}+\epsilon\left(\mathscr{C}_{1, \Sigma, n}+\mathscr{C}_{1, \Delta, n}+\mathscr{C}_{1, \Gamma, n}+\mathscr{C}_{1, S, n}\right) .
\end{aligned}
$$

The coefficients responsible for enforcing the Kutta condition in the inter-blade downstream region are

$$
\mathscr{H}_{\kappa_{0}^{-}, b}^{-}=\mathscr{H}_{0, \kappa_{0}^{-}, b}^{-}+\epsilon\left(\mathscr{H}_{1, \Sigma, \kappa_{0}^{-}, b}^{-}+\mathscr{H}_{1, \Delta, \kappa_{0}^{-}, b}^{-}+\mathscr{H}_{\Gamma, \kappa_{0}^{-}, \pm, b}^{-}+\mathscr{H}_{1, S, \kappa_{0}^{-}, b}^{-}\right)
$$

where

$$
\mathscr{H}_{n, x, \kappa_{0}^{-}, b}^{-}=-\pi \mathrm{i} P_{n, x} \cdot \frac{\cos \left(s_{\phi} \zeta_{\kappa_{0}^{-}}\right)}{\cos \left(d_{\phi} \kappa_{0}^{-}+\sigma_{\phi}^{\prime}\right)-\cos \left(s_{\phi} \zeta_{\kappa_{0}^{-}}\right)}
$$

The coefficients responsible for enforcing the Kutta condition in the downstream region are

$$
\mathscr{H}_{\kappa_{0}^{-}, \pm}^{-}=\mathscr{H}_{0, \kappa_{0}^{-}, \pm}^{-}+\epsilon\left(\mathscr{H}_{1, \Sigma, \kappa_{0}^{-}, \pm}^{-}+\mathscr{H}_{1, \Delta, \kappa_{0}^{-}, \pm}^{-}+\mathscr{H}_{1, \Gamma, \kappa_{0}^{-}, \pm}^{-}+\mathscr{H}_{1, S, \kappa_{0}^{-}, \pm}^{-}\right)
$$

where

$$
\mathscr{H}_{n, x, \kappa_{0}^{-}, \pm}^{-}=\frac{\pi \mathrm{i} P_{n, x}}{2} \cdot \frac{\mathrm{e}^{\mathrm{i}\left(d_{\phi} \kappa_{0}^{-}+\sigma_{\phi}^{\prime}\right)}-\mathrm{e}^{ \pm \mathrm{i} s_{\phi} \zeta_{\kappa_{0}^{-}}}}{\cos \left(d_{\phi} \kappa_{0}^{-}+\sigma_{\phi}^{\prime}\right)-\cos \left(s_{\phi} \zeta_{\kappa_{0}^{-}}\right)}, \quad \text { for } n=0 \text { and } n=1, x=\Sigma, \Delta
$$

and $\mathscr{H}_{1, \Gamma, \kappa_{0}^{-}, \pm}^{-}$and $\mathscr{H}_{1, S, \kappa_{0}^{-}, \pm}^{-}$are defined in (3.159) and (3.155).

Further coefficients are defined as

$$
\begin{aligned}
\mathscr{H}_{S, a, l}=-\sum_{l_{2}=-\infty}^{\infty} & \frac{s_{\phi}^{2} \epsilon \mathbb{S}_{l, l_{2}}^{i}}{\Delta_{\phi}^{2}\left(\lambda_{l_{2} / 2}^{+}-\kappa_{l}^{-}\right)\left(\lambda_{l_{2} / 2}^{-}-\kappa_{l}^{-}\right)} \\
& \times \frac{1-(-1)^{l_{2}}}{2\left(\cos \left(s_{\phi} \zeta_{\kappa_{l}^{-}}\right)-\cos \left(d_{\phi} \kappa_{l}^{-}+\sigma_{\phi}^{\prime}\right)\right)}, \\
\mathscr{H}_{S, b, l}=\sum_{l_{2}=-\infty}^{\infty} & \frac{s_{\phi}^{2} \in \mathbb{S}_{l, l_{2}}^{i} \mathrm{e}^{\mathrm{i}\left(d_{\phi} \kappa_{l}^{-}+\sigma_{\phi}^{\prime}\right)}}{\Delta_{\phi}^{2}\left(\lambda_{l_{2} / 2}^{+}-\kappa_{l}^{-}\right)\left(\lambda_{l_{2} / 2}^{-}-\kappa_{l}^{-}\right)} \\
& \times \frac{1-(-1)^{l_{2}}}{2\left(\cos \left(s_{\phi} \zeta_{\kappa_{l}^{-}}\right)-\cos \left(d_{\phi} \kappa_{l}^{-}+\sigma_{\phi}^{\prime}\right)\right)},
\end{aligned}
$$




$$
\begin{aligned}
& \mathscr{H}_{S, c, l}=\sum_{l_{2}=-\infty}^{\infty} \frac{s_{\phi}^{2} \epsilon \mathbb{S}_{l, l_{2}}^{i}}{\Delta_{\phi}^{2}\left(\lambda_{l_{2} / 2}^{+}-\kappa_{l}^{-}\right)\left(\lambda_{l_{2} / 2}^{-}-\kappa_{l}^{-}\right)} \\
& \times \frac{1-(-1)^{l_{2}}}{2\left(\cos \left(s_{\phi} \zeta_{\kappa_{l}^{-}}\right)-\cos \left(d_{\phi} \kappa_{l}^{-}+\sigma_{\phi}^{\prime}\right)\right)} \cdot \frac{\mathrm{i}\left(\pi l_{2}-d_{\phi} \kappa_{l}^{-}-\sigma_{\phi}^{\prime}\right)}{s_{\phi} \zeta_{\kappa_{l}^{-}}}, \\
& \mathscr{H}_{S, d, l}=-\sum_{l_{2}=-\infty}^{\infty} \frac{s_{\phi}^{2} \epsilon \mathbb{S}_{l, l_{2}}^{i} \mathrm{e}^{\mathrm{i}\left(d_{\phi} \kappa_{l}^{-}+\sigma_{\phi}^{\prime}\right)}}{\Delta_{\phi}^{2}\left(\lambda_{l_{2} / 2}^{+}-\kappa_{l}^{-}\right)\left(\lambda_{l_{2} / 2}^{-}-\kappa_{l}^{-}\right)} \\
& \times \frac{1-(-1)^{l_{2}}}{2\left(\cos \left(s_{\phi} \zeta_{\kappa_{l}^{-}}\right)-\cos \left(d_{\phi} \kappa_{l}^{-}+\sigma_{\phi}^{\prime}\right)\right)} \cdot \frac{\mathrm{i}\left(\pi l_{2}-d_{\phi} \kappa_{l}^{-}-\sigma_{\phi}^{\prime}\right)}{s_{\phi} \zeta_{\kappa_{l}^{-}}}, \\
& \mathscr{H}_{\mathscr{U}, b, m}^{+}=\pi^{2} \frac{4 \epsilon \mathscr{U}_{1, S, m} J_{+}\left(\lambda_{m}^{+}\right)}{\left(\lambda_{m}^{+}-\kappa_{0}^{-}\right) \zeta_{m}^{+} \sin \left(s_{\phi} \zeta_{m}^{+}\right)} \cdot \mathrm{e}^{\mathrm{i} \lambda_{m}^{+} c_{\phi}}, \\
& \mathscr{H}_{\mathscr{R}, a, m}^{-}=-\pi^{2} \mathrm{i} \frac{4 \epsilon \mathscr{R}_{1, S, m} J_{-}\left(\lambda_{m}^{-}\right)}{\zeta_{m}^{-} \sin \left(s_{\phi} \zeta_{m}^{-}\right)} \cdot \mathrm{e}^{-\mathrm{i} s_{\phi} \zeta_{m}^{-}}, \\
& \mathscr{H}_{G, a, m}^{-}=\frac{\mathrm{ie}^{-\mathrm{i} s_{\phi} \zeta_{m}^{-}}}{\Delta_{\phi} \sqrt{k^{2} w^{2}-f_{m}^{2}}} \cdot \sum_{l=-\infty}^{\infty} \frac{\epsilon \mathcal{C}_{\Delta, l}}{2\left(\lambda_{m}^{-}-\kappa_{l}^{-}\right)}, \\
& \mathscr{H}_{G, a, m}^{+}=\frac{\mathrm{e}^{\mathrm{i} s_{\phi} \zeta_{m}^{+}}}{\Delta_{\phi} \sqrt{k^{2} w^{2}-f_{m}^{2}}} \cdot \frac{1}{\sin \left(s_{\phi} \zeta_{m}^{+}\right)} \cdot \sum_{l=-\infty}^{\infty} \frac{c_{\Delta, l}}{2\left(\lambda_{m}^{+}-\kappa_{l}^{-}\right)}, \\
& \mathscr{H}_{G, b, m}^{-}=\frac{1}{\sin \left(s_{\phi} \zeta_{m}^{-}\right) \Delta_{\phi} \sqrt{k^{2} w^{2}-f_{m}^{2}}} \cdot \sum_{l=-\infty}^{\infty} \frac{\epsilon c_{\Delta, l} \mathrm{e}^{\mathrm{i}\left(\lambda_{m}^{-}-\kappa_{l}^{-}\right) c_{\phi}}}{2\left(\lambda_{m}^{-}-\kappa_{l}^{-}\right)} \\
& +\frac{i \in \Gamma}{\Delta_{\phi} \sqrt{k^{2} w^{2}-f_{m}^{2}} \sin \left(\zeta_{m}^{-} s_{\phi}\right)} \\
& \times\left(\sum_{n \neq m} \zeta_{n}^{-} \mathscr{H}_{0, n}^{\prime} \frac{\mathrm{e}^{\mathrm{i}\left(\lambda_{m}^{-}-\lambda_{n}^{-}\right) c_{\phi}}}{2\left(\lambda_{m}^{-}-\lambda_{n}^{-}\right)}+\sum_{ \pm} \pm \zeta_{\kappa_{0}^{-}} \mathscr{H}_{0, \kappa_{0}^{-}}^{ \pm \prime} \frac{\mathrm{e}^{\mathrm{i}\left(\lambda_{m}^{-}-\kappa_{0}^{-}\right) c_{\phi}}}{2\left(\lambda_{m}^{-}-\kappa_{0}^{-}\right)}\right) \\
& -\frac{\epsilon \Gamma \mathscr{H}_{0, m}^{\prime}}{2 \Delta_{\phi} \sqrt{k^{2} w^{2}-f_{m}^{2}} \sin \left(\zeta_{m}^{-} s_{\phi}\right)}\left(c_{\phi} \zeta_{m}^{-}-\mathrm{i} \frac{\lambda_{m}^{-}}{\zeta_{m}^{-}}\right), \\
& \mathscr{H}_{G, b, m}^{+}=\frac{-\mathrm{ie}^{\mathrm{i} s_{\phi} \zeta_{m}^{+}}}{\sin \left(s_{\phi} \zeta_{m}^{+}\right) \Delta_{\phi} \sqrt{k^{2} w^{2}-f_{m}^{2}}} \cdot \sum_{l=-\infty}^{\infty} \frac{\epsilon c_{\Delta, l} \mathrm{e}^{\mathrm{i}\left(\lambda_{m}^{+}-\kappa_{l}^{-}\right) c_{\phi}}}{2\left(\lambda_{m}^{+}-\kappa_{l}^{-}\right)} \\
& +\frac{\epsilon \Gamma \mathrm{e}^{\mathrm{i} s_{\phi} \zeta_{m}^{+}}}{\Delta_{\phi} \sqrt{k^{2} w^{2}-f_{m}^{2}} \sin \left(s_{\phi} \zeta_{m}^{+}\right)} \\
& \times\left(\sum_{n=-\infty}^{\infty} \zeta_{n}^{-} \mathscr{H}_{0, n}^{\prime} \frac{\mathrm{e}^{\mathrm{i}\left(\lambda_{m}^{+}-\lambda_{n}^{-}\right) c_{\phi}}}{2\left(\lambda_{m}^{+}-\lambda_{n}^{-}\right)}+\sum_{ \pm} \mp \zeta_{\kappa_{0}^{-}} \mathscr{H}_{0, \kappa_{0}^{-}}^{ \pm \prime} \frac{\mathrm{e}^{\mathrm{i}\left(\lambda_{m}^{+}-\kappa_{0}^{-}\right) c_{\phi}}}{2\left(\lambda_{m}^{+}-\kappa_{0}^{-}\right)}\right), \\
& \mathscr{H}_{a, m}^{+}=\frac{-\pi \mathrm{i}}{\Delta_{\phi} \sqrt{k^{2} w^{2}-f_{m}^{2}}} \cdot \frac{\zeta_{m}^{+} \mathrm{e}^{\mathrm{i} s_{\phi} \zeta_{m}^{+}}}{\sin \left(s_{\phi} \zeta_{m}^{+}\right)} D^{(1,3)}\left(\lambda_{m}^{+}\right),
\end{aligned}
$$




$$
\begin{aligned}
\mathscr{H}_{b, m}^{-} & =\pi \mathrm{i} \frac{\zeta_{m}^{-}}{\Delta_{\phi} \sqrt{k^{2} w^{2}-f_{m}^{2}}} \cdot \frac{D^{(2,4)}\left(\lambda_{m}^{-}\right)}{\sin \left(s_{\phi} \zeta_{m}^{-}\right)} \\
& +\frac{-\epsilon \Gamma \zeta_{m}^{-}}{\Delta_{\phi} \sqrt{k^{2} w^{2}-f_{m}^{2}} \sin \left(s_{\phi} \zeta_{m}^{-}\right)} \sum_{n \neq m} \mathscr{H}_{0, n}^{\prime} \frac{\mathrm{e}^{\mathrm{i}\left(\lambda_{m}^{-}-\lambda_{n}^{-}\right) c_{\phi}}}{2\left(\lambda_{m}^{-}-\lambda_{n}^{-}\right)} \cdot \frac{\lambda_{n}^{-}-\kappa_{0}^{-}}{\lambda_{m}^{-}-\kappa_{0}^{-}} \cdot \frac{J_{-}\left(\lambda_{n}^{-}\right)}{J_{-}\left(\lambda_{m}^{-}\right)} \\
& +\frac{\epsilon \Gamma \zeta_{m}^{-}}{\Delta_{\phi} \sqrt{k^{2} w^{2}-f_{m}^{2}} \sin \left(s_{\phi} \zeta_{m}^{-}\right)} \frac{\mathscr{H}_{0, m}^{\prime}}{2}\left(\frac{1}{\lambda_{m}^{-}-\kappa_{0}^{-}}+\frac{J_{-}^{\prime}\left(\lambda_{m}^{-}\right)}{J_{-}\left(\lambda_{m}^{-}\right)}-\mathrm{i} c_{\phi}\right) \\
\mathscr{H}_{m, b}^{-} & =\epsilon \Gamma \mathscr{H}_{0, m}^{\prime} \mathrm{e}^{\mathrm{i} \zeta_{m}^{-} s_{\phi}} \\
& \times \frac{\left(\left(s_{\phi} \lambda_{m}^{-}\right)^{2}-\left(d_{\phi} \zeta_{m}^{-}\right)^{2}\right) \cos \left(s_{\phi} \zeta_{m}^{-}\right)-\frac{s_{\phi}}{\zeta_{m}^{-}}\left(\left(\zeta_{m}^{-}\right)^{2}+\left(\lambda_{m}^{-}\right)^{2}\right) \sin \left(s_{\phi} \zeta_{m}^{-}\right)}{4 \sin \left(s_{\phi} \zeta_{m}^{-}\right)^{2} \Delta_{\phi}^{2}\left(k^{2} w^{2}-f_{m}^{2}\right)} \\
\mathscr{H}_{\Gamma, \kappa_{0}^{-}, \pm, b}^{-} & =\frac{-\epsilon \Gamma \mathscr{H}_{0, \kappa_{0}^{-}}^{ \pm \prime} \mathrm{e}^{ \pm \mathrm{i} \zeta_{\kappa_{0}^{-}}^{-} s_{\phi}}}{2\left(\cos \left(d_{\phi} \kappa_{0}^{-}+\sigma_{\phi}^{\prime}\right)-\cos \left(s_{\phi} \zeta_{\kappa_{0}^{-}}\right)\right)}, \\
\mathscr{H}_{\Gamma, \lambda_{m}^{-}}^{-} & =-\mathscr{H}_{0, m}^{\prime} \frac{\epsilon \Gamma}{s_{\phi}} \exp \left[\mathrm{i} \epsilon s_{\phi} c_{\phi}\left(O_{S, m}+O_{\Gamma, m}\right)\right] \\
\mathscr{H}_{\Gamma, m, b}^{-}= & \frac{-\epsilon \Gamma \mathscr{H}_{0, m}^{\prime} \mathrm{i}}{2 \sin \left(s_{\phi} \zeta_{m}^{-}\right) \Delta_{\phi} \sqrt{k^{2} w^{2}-f_{m}^{2}}} \mathrm{e}^{\mathrm{i} \zeta_{m}^{-} s_{\phi}}, \\
\mathscr{H}_{\Gamma, \lambda_{m}^{-}}^{-} & =-\mathscr{H}_{0, m}^{\prime} \frac{\epsilon \Gamma}{s_{\phi}} \exp \left[\mathrm{i} \epsilon s_{\phi} c_{\phi}\left(O_{S, m}+O_{\Gamma, m}\right)\right]
\end{aligned}
$$

The inhomogeneous functions in the upstream and inter-blade regions are

$$
\begin{aligned}
\mathbb{I}_{P, M_{m}^{+}}^{+}(\phi, \psi) & =-\frac{s_{\phi}^{2}}{\Delta_{\phi}^{2}} \cdot \frac{\exp \left[-\mathrm{i}\left(\phi \tilde{M}_{m}^{+}-\frac{\psi}{s_{\phi}}\left(\Delta_{\phi} f_{m}+d_{\phi} \tilde{M}_{m}^{+}\right)\right)\right]}{\left(\tilde{M}_{m}^{+}-\lambda_{m}^{+}\right)\left(\tilde{M}_{m}^{+}-\lambda_{m}^{-}\right)}, \\
\mathbb{I}_{l_{1}, l_{2}}^{i}(\phi, \psi) & =-\frac{s_{\phi}^{2}}{\Delta_{\phi}^{2}} \cdot \frac{\exp \left[-\mathrm{i}\left(\kappa_{l_{1}}^{-} \phi-\frac{\psi}{s_{\phi}}\left(\Delta_{\phi} f_{l_{2} / 2}+d_{\phi} \kappa_{l_{1}}^{-}\right)\right)\right]}{\left(\kappa_{l_{1}}^{-}-\lambda_{l_{2} / 2}^{+}\right)\left(\kappa_{l_{1}}^{-}-\lambda_{l_{2} / 2}^{-}\right)} .
\end{aligned}
$$

Finally, the inhomogeneous functions in the downstream region are defined in section 3.F. 



\section{Chapter 4}

\section{Potential Flow Through Cascades of Thin, Porous Aerofoils}

\subsection{Introduction}

As mentioned in the Introduction, a promising direction in aeroacoustic research is to take inspiration from the silent flight of owls (Graham, 1934). Amongst other features, it is known that the porous trailing edge of an owl's wing is responsible for its surprisingly effective noise reduction capabilities. This realisation has been a substantial source of research into the effects of porosity on trailing-edge noise, from experimental (Chen et al., 2012; Geyer et al., 2010), numerical (Cavalieri et al., 2016; Leclaire et al., 2001) and analytic (Jaworski and Peake, 2013; Kisil and Ayton, 2018) perspectives. However, despite being aeroacoustically favourable, porosity gradients typically have adverse aerodynamic affects, and consequently the aeroacoustic gains afforded by porous trailing edges must be held in tension with the associated aerodynamic losses. This delicate balancing act has resulted in further research into the aerodynamic effects of isolated porous aerofoils (Hajian and Jaworski, 2017; Iosilevskii, 2011, 2013). Accordingly, in this chapter we conduct a novel investigation into the aerodynamic effects of porosity gradients for aerofoils in a cascade formation.

Despite their well-known aeroacoustic properties, porous trailing edges have not yet been applied to reduce turbomachinery noise. The potential flow through a cascade of thin impermeable objects was solved analytically in chapter 2 by recasting traditional thin aerofoil theory (Thwaites, 1960) as a Riemann-Hilbert problem. In this chapter, we extend this analysis to consider a cascade of porous aerofoils. This aerodynamic study is a necessary first step towards more sophisticated investigations involving the acoustic emissions generated by a porous cascade. However, the results are not just relevant to 
turbomachinery; there are also implications for natural formation flight, where a group of flyers or swimmers operate in a periodic arrangement. The compliant porous wing of a bird, for example, must be modelled in a different way to a rigid wing.

Similar to previous analyses (Hajian and Jaworski, 2017), we model the cascade as a bound vortex sheet. We consider a perturbation expansion where the blade geometry, angle of attack and stagger angle are assumed to be small. We also ignore blade thickness, as small thickness profiles do not affect the pressure jump across the blade and, therefore, blade thickness decouples from the effects of porosity, except in the definition of the porosity coefficient. For simplicity and tractability, the porosity is modelled as a Darcy condition across the wing. This modelling assumption assumes that the flow though the pores in the wing is a Stokes flow (i.e. low Reynolds number), which breaks down when the leading edge becomes porous, and higher order terms must be included in the boundary condition (Ergun, 1952). For this reason, we shall restrict our attention to aerofoils whose leading edges are impermeable.

During the solution of the porous cascade problem, we are faced with a periodic singular integral equation (SIE) for the bound vorticity. Analytic solutions to this equation could not be found in the literature, so, in a similar way to chapter 2 , we adapt classical approaches (Gakhov, 1966; Muskhelishvili, 1946) to find a new solution. The solution reduces to the original non-periodic solution when the period window becomes large. The resulting solution may be applied to other problems featuring periodic SIEs, such as periodic cracks in elasticity problems (Ioakmidis and Theocaris, 1977; Mikhlin, 1957; Schmueser and Comninou, 1979). This SIE is only solvable when the stagger angle is small and, consequently, this chapter is restricted to asymptotically small stagger angles, analogous to the analysis in chapter 2 .

The layout of this chapter is as follows: in section 4.2 we lay out the mathematical model and derive the periodic SIE for the bound vorticity. In section 4.3 we solve a more general periodic SIE by adapting typical approaches. This solution is then applied to the special case of porous aerofoils, and is validated against the isolated porous aerofoil and rigid cascade solutions. In section 4.4, we explore the role of porosity on aerodynamic performance, and observe the crucial role of the porosity at the trailing edge on the deflection angle. The case of partially porous flat plates, where the aerofoil consists of a rigid forward section and permeable aft section, is considered and analytic expressions are generated for the seepage drag and lift. We also investigate cascades with continuous porosity gradients, and consider the effects of camber. Finally, in section 4.5 we present the conclusions of the chapter. 


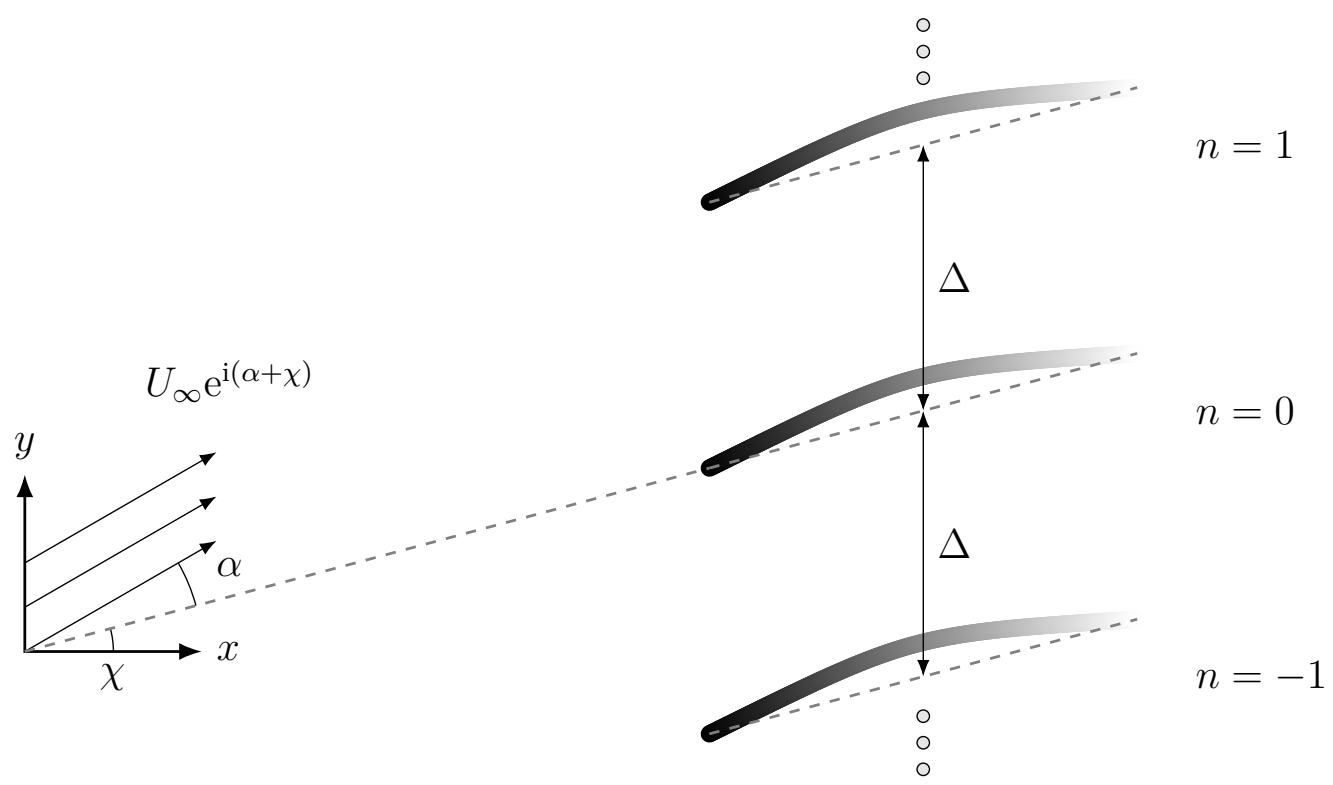

Fig. 4.1 An infinite staggered cascade of porous aerofoils. The vertical spacing between the aerofoils is $\Delta$, the stagger angle is $\chi$, and the upstream velocity is $U_{\infty}$, which is inclined at angle of attack $\alpha$. The colour gradient of the aerofoils represents the porosity gradient.

\subsection{Model Derivation}

In this section we present the mathematical model and derive the periodic singular integral equation for the bound vorticity around a single aerofoil in the cascade. From this we can then calculate the potential flow through the entire cascade. We ignore aerofoil thickness in this formulation, except when it is encoded into the porosity gradient, because, as shown in chapter 2, aerofoil thickness does not induce a velocity (pressure) jump across the aerofoil, and therefore will not play a role in the porous analysis. We consider an infinite cascade of infinitesimally thin aerofoils under the assumption of small disturbances in a two-dimensional, steady, incompressible flow. Similarly to chapter 2, the flow is uniform far upstream (with speed $U_{\infty}$ ) and has angle of attack $\alpha$ relative to the chords of the aerofoils, which are inclined at stagger angle $\chi$. Length scales are normalised so that each aerofoil has unit semi-chord. We assume that $\alpha, \chi=\mathcal{O}(\epsilon)$ where $\epsilon$ is a small parameter of the order of the aspect ratio of the aerofoils. As in the analytic solution in chapter 2, the stagger angle disappears from the analysis and we do not consider stagger further in this chapter. We also permit each blade to have small, $\mathcal{O}(\epsilon)$, camber. Our model is illustrated in figure 4.1. 
From chapter 2, the complex velocity of the perturbation to uniform flow may be expressed as

$$
u(z)-\mathrm{i} v(z)=\frac{1}{2 \mathrm{i} \Delta} \int_{-1}^{1} \gamma(\tau)\left(\operatorname{coth}\left(\frac{\pi(\tau-z)}{\Delta}\right)-1\right) \mathrm{d} \tau
$$

where $\gamma$ represents the tangential velocity jump across the blade, otherwise known as the bound vorticity.

Following Hajian and Jaworski (2017), we write the vertical surface velocity as

$$
v^{ \pm}(t)=v_{s}(t)+y_{c, \alpha}^{\prime}(t)
$$

where $v_{s}$ represents the seepage velocity through the aerofoil, $y_{c, \alpha}^{\prime}$ represents the gradient of the camber line and the superscript \pm represents the limiting value taken from the upper or lower side of the aerofoil respectively. When $v_{s}=0$ we recover the typical boundary condition of thin aerofoil theory for impermeable wings encountered in chapter 2. However, when the aerofoils are porous, $v_{s}$ is unknown and must be found as part of the problem.

We may express the seepage velocity in terms of the bound vorticity distribution around a single aerofoil by assuming a Darcy-type velocity distribution, where the seepage velocity is proportional to the pressure jump across the aerofoil (Lifanov et al., 2009):

$$
v_{s}(t)=\rho_{\infty} U_{\infty} C R(t) \gamma(t)
$$

Here $R$ is the porosity distribution, $\rho_{\infty}$ is the (constant) flow density, and $C$ is the porosity coefficient (Hajian and Jaworski, 2017). The Darcy flow formulation is only valid when the pore-level Reynolds number is sufficiently small and there is Stokes flow through the pores (Weidenfeld and Manela, 2016, Appendix A). This formulation therefore breaks down when the seepage velocity becomes too large. In this case, the Darcy model should be replaced with a nonlinear relationship between seepage velocity and pressure jump such as the Ergun model (Ergun, 1952), as suggested in Bae and Moon (2011). However, this modelling is beyond the scope of the present research and for the moment we focus on the Darcy flow condition.

We now close the problem for $\gamma(t)$ by applying the Modified Plemelj formulae for cascades (appendix 2.A of chapter 2) to (4.1), which yields another expression for the upper and lower vertical velocities, this time in terms of the bound vorticity distribution, 
$\gamma:$

$$
v^{ \pm}(t)=\frac{1}{2 \Delta} \int_{-1}^{1} \gamma(\tau)\left(\operatorname{coth}\left(\frac{\pi(\tau-t)}{\Delta}\right)-1\right) \mathrm{d} \tau
$$

By substituting (4.3) and (4.4) into (4.2), we obtain the singular integral equation for the bound vorticity:

$$
\rho_{\infty} U_{\infty} C R(t) \gamma(t)-\frac{1}{2 \Delta} \int_{-1}^{1} \gamma(\tau)\left(\operatorname{coth}\left(\frac{\pi(\tau-t)}{\Delta}\right)-1\right) \mathrm{d} \tau=-U_{\infty} y_{c, \alpha}^{\prime}(t)
$$

We now normalise the velocity with respect to $U_{\infty}$, and define the non-dimensional porosity distribution $\psi(x)=2 \rho_{\infty} U_{\infty} C R(x)$ to obtain the singular integral equation

$$
\psi(t) \gamma(t)-\frac{1}{\Delta} \int_{-1}^{1} \gamma(\tau)\left(\operatorname{coth}\left(\frac{\pi(\tau-t)}{\Delta}\right)-1\right) \mathrm{d} \tau=-2 y_{c, \alpha}^{\prime}(t) .
$$

\subsection{Periodic Singular Integral Equation Solution}

In this section we present the solution to the canonical SIE with periodic kernel by adapting a classical approach for non-periodic kernels (Muskhelishvili, 1946, Part V). As discussed in chapter 2, the usual methods, such as those in Muskhelishvili (1946) and Gakhov (1966) are restricted to contours that are bounded and the union of finitely many curves. In our case, the contour is not bounded and is the union of (countably) infinitely many curves. However, due to the periodicity, the technique of Muskhelishvili (1946) may be adapted. We only provide details of the solution to the dominant part of the SIE in the case of open contours with continuous coefficients (analogous to (Muskhelishvili, 1946, $\S 107)$ ), but all pertinent results, such as those on the full SIE, special ends, the adjoint equation, and discontinuous coefficients also follow in a similar way to the non-periodic case. In fact, the non-periodic case can be recovered in the limit $|\Delta| \rightarrow \infty$.

The periodic SIE may be written in the general form

$$
A(t) \gamma(t)+\frac{B(t)}{\mathrm{i} \Delta} f_{L} \gamma(\tau)\left(\operatorname{coth}\left(\frac{\pi(\tau-t)}{\Delta}\right)-1\right) \mathrm{d} \tau=f(t)
$$

for $t \in L$ where $L=L_{1}+L_{2}+\cdots+L_{p}$ is a finite union of open contours, whose ends are denoted by $\left\{c_{k} \mid k=1, \ldots, 2 k\right\}$, in no particular order. Furthermore, we specify that $A, B$ and $f$ satisfy a Hölder condition on $L$ and $A^{2}(t) \neq B^{2}(t)$ for all $t$.

Similar problems have been considered previously, but the solution to the general SIE has not been presented. For example, Mikhlin (1957) considered a periodic array 
of holes in an elastic medium. That problem is different to the one considered in the present research as it considered closed contours, and consequently required solution via conformal mappings. Other problems involving cracks (Ioakmidis and Theocaris, 1977; Schmueser and Comninou, 1979) also derive similar SIEs, but then resort to numerical solutions. Previous work in chapter 2 only considered the case $A \equiv 0, B \equiv 1$, so the corresponding analysis presented here is far more general.

Following the typical approach of solving SIEs for open contours (Muskhelishvili, 1946, §107), we transform the canonical SIE (4.7) into a Riemann-Hilbert problem by introducing the auxiliary function

$$
\Phi(z)=\frac{1}{2 \mathrm{i} \Delta} \int_{L} \gamma(\tau)\left(\operatorname{coth}\left(\frac{\pi(\tau-z)}{\Delta}\right)-1\right) \mathrm{d} \tau .
$$

Incidentally, $\Phi$ represents the complex velocity of the perturbed field in aerodynamic applications. We note that, unlike the non-periodic case, $\Phi$ does not always decay as $|z| \rightarrow \infty$

We may now rewrite (4.7) in terms of $\Phi$ by applying the modified Plemelj formulae (appendix 2.A of chapter 2) to obtain a Riemann-Hilbert problem in the canonical form

$$
\Phi^{+}(t)-G(t) \Phi^{-}(t)=f^{*}(t)
$$

where

$$
G(t)=\frac{A(t)-B(t)}{A(t)+B(t)}, \quad f^{*}(t)=\frac{f(t)}{A(t)+B(t)}
$$

We note that the auxiliary function $\Phi(z)$ defined in (4.8) possesses two essential qualities: it has period $\Delta \mathrm{i}$ and it decays as $z \rightarrow-\infty$. Consequently, we write $\Phi(z)=X(z) \Psi(z)$, where both $X(z)$ and $\Psi(z)$ have period is, but $X(z)$ tends to a constant whereas $\Psi(z)$ must vanish as $z \rightarrow-\infty$. Moreover, we specify that $X(z)$ is the fundamental solution, i.e. it satisfies the homogeneous Riemann-Hilbert problem defined by

$$
X^{+}(t)-G(t) X^{-}(t)=0 .
$$

There are infinitely many solutions to the above equation, which can be written in the general form

$$
X(z)=\prod_{k=1}^{2 p}\left[\sinh \left(\pi\left(z-c_{k}\right) / \Delta\right)\right]^{\lambda_{k}} \exp \left[\frac{1}{2 \mathrm{i} \Delta} \int_{L} \log (G(\tau)) \operatorname{coth}\left(\frac{\pi(\tau-z)}{\Delta}\right) \mathrm{d} \tau\right]
$$


where $\lambda_{k}$ are specially chosen integers (Muskhelishvili, 1946, §79). It can be verified that this choice of $X(z)$ satisfies (4.10) and has appropriate periodicity. Similarly to the non-periodic case, the values of $\lambda_{k}$ are determined by consideration of the behaviour of $\Phi$ at the ends of $L$. These are usually specified by physical considerations, such as the Kutta condition, and result in the classification of $c_{k}$ into special ends, where the solution is bounded, and non-special ends, where the solution may be unbounded. Our constraint that $X(z)$ tends to a constant as $z \rightarrow-\infty$ introduces the further condition $\sum_{k=1}^{2 p} \lambda_{k}=0$, i.e. the index of the class of solutions must vanish.

In the non-periodic case, polynomial multiples of the fundamental solution (4.11) are solutions to the homogeneous Riemann-Hilbert problem (4.10) (see (Muskhelishvili, 1946, $\S 79$ ), for example). However, we have specified that the solutions to the homogeneous problem must be bounded, so polynomial multiples are not permitted. In appendix 4.B we prove that every solution, bounded as $|z| \rightarrow \infty$, to the periodic, homogeneous RiemannHilbert problem is a multiple of (4.11): the solution is unique up to a multiplicative constant.

Similarly to Muskhelishvili (1946), we now define

$$
Z(t)=(A(t)+B(t)) X^{+}(t)=(A(t)-B(t)) X^{-}(t)
$$

Substitution into the original Riemann-Hilbert problem (4.9) now yields

$$
\Psi^{+}(t)-\Psi^{-}(t)=\frac{f(t)}{Z(t)}
$$

which has the solution, with appropriate periodicity and upstream decay,

$$
\Psi(z)=\frac{1}{2 \mathrm{i} \Delta} \int_{L} \frac{f(\tau)}{Z(\tau)}\left(\operatorname{coth}\left(\frac{\pi(\tau-z)}{\Delta}\right)-1\right) \mathrm{d} \tau
$$

Now we may use the modified Plemelj formulae to obtain $\gamma$ as

$$
\begin{aligned}
\gamma(t) & =\Phi^{+}(t)-\Phi^{-}(t) \\
& =A^{*}(t) f(t)-\frac{B^{*}(t) Z(t)}{\mathrm{i} \Delta} f_{L} \frac{f(\tau)}{Z(\tau)}\left(\operatorname{coth}\left(\frac{\pi(\tau-t)}{\Delta}\right)-1\right) \mathrm{d} \tau,
\end{aligned}
$$

where

$$
A^{*}(t)=\frac{A(t)}{A(t)^{2}-B(t)^{2}}, \quad \quad B^{*}=\frac{B(t)}{A(t)^{2}-B(t)^{2}}
$$


This completes the solution of the canonical periodic singular integral equation (4.7).

\subsubsection{Application to the Cascade of Porous Aerofoils}

We may now apply the solution found in (4.14) to the particular case of a cascade of porous aerofoils. By comparison of (4.6) with (4.7), we obtain

$$
\begin{array}{cc}
A(t)=\psi(t), & B(t)=-\mathrm{i}, \\
f(t)=-2 y_{c, \alpha}^{\prime}(t), & G(t)=\frac{\psi(t)+\mathrm{i}}{\psi(t)-\mathrm{i}} .
\end{array}
$$

The contour $L$ is taken to be the slit $[-1,1]$ and we set $c_{1}=-1, c_{2}=1, \lambda_{1}=0, \lambda_{2}=0$. We note that, by taking $t \rightarrow \pm 1$ in (4.12), the original fundamental solution possesses an integrable singularity at -1 and a zero at 1 . Therefore, the requisite leading and trailing edge behaviours are fulfilled, and there is no need to multiply by an entire, periodic function as suggested in the previous section. However, in other applications, such as elasticity, singularities at both the leading and trailing edges must be enforced, so a different treatment of the end points would be required.

The function $Z$ then becomes

$$
Z(t)=\sqrt{1+\psi^{2}(t)} \exp \left[\frac{\pi}{\Delta} f_{-1}^{1} k(\tau) \operatorname{coth}\left(\frac{\pi(\tau-t)}{\Delta}\right) \mathrm{d} \tau\right]
$$

where, similarly to Hajian and Jaworski (2017), we define $k(t)=(1 / \pi) \cot ^{-1} \psi(t)$.

The bound vorticity (4.14) is then given by

$$
\gamma(t)=-\frac{2 \psi(t) y_{c, \alpha}^{\prime}(t)}{1+\psi^{2}(t)}-\frac{2 Z(t)}{\Delta\left(1+\psi^{2}(t)\right)} f_{-1}^{1} \frac{y_{c, \alpha}^{\prime}(\tau)}{Z(\tau)}\left(\operatorname{coth}\left(\frac{\pi(\tau-t)}{\Delta}\right)-1\right) \mathrm{d} \tau,
$$

and the complex potential is

$$
u(z)-\mathrm{i} v(z)=\Phi(z)=-\frac{X(z)}{\mathrm{i} \Delta} \cdot \int_{-1}^{1} \frac{y_{c, \alpha}^{\prime}(\tau)}{Z(\tau)}\left(\operatorname{coth}\left(\frac{\pi(\tau-z)}{\Delta}\right)-1\right) \mathrm{d} \tau .
$$

\subsubsection{Validation}

Here we present a comparison of our solution to other relevant solutions. We compare our solution to previous results for an isolated aerofoil with a porosity gradient, and a cascade of aerofoils with rigid boundaries. 


\subsubsection{Comparison to an Isolated Aerofoil}

Firstly, we show that our solution agrees with the isolated aerofoil case of Hajian and Jaworski (2017) when the spacing between blades in the cascade is large. Taking $\Delta \rightarrow \infty$, the fundamental solution (4.12) becomes

$$
Z_{\infty}(t)=\sqrt{1+\psi^{2}(t)} \exp \left[f_{-1}^{1} \frac{k(\tau)}{\tau-t} \mathrm{~d} \tau\right]
$$

where the subscript $\infty$ is used to denote the limit of large spacing. Similarly, taking this limit in the solution (4.16), and noting the asymptotic behaviour of coth, yields

$$
\gamma_{\infty}(t)=-\frac{2 \psi(t) y_{c, \alpha}^{\prime}(t)}{1+\psi^{2}(t)}-\frac{2 Z_{\infty}(t)}{1+\psi^{2}(t)} \int_{-1}^{1} \frac{y_{c, \alpha}^{\prime}(\tau)}{Z_{\infty}(\tau)(\tau-t)} \mathrm{d} \tau
$$

Comparing with the isolated aerofoil solution, we see that (4.18) is identical to (3.7) in Hajian and Jaworski (2017) subject to a constant prefactor and (4.19) is identical to (3.8) in Hajian and Jaworski (2017), subject to the change from vorticity to pressure.

\subsubsection{Comparison to a Rigid Cascade}

Now we compare the solution for a cascade of porous aerofoil to the solution for a cascade of rigid aerofoils obtained in chapter 4 . Taking $\psi \rightarrow 0$ is equivalent to $k \rightarrow 1 / 2$, and therefore (4.15) becomes

$$
Z_{\text {rigid }}(t)=\exp \left[\frac{\pi}{2 \Delta} \int_{-1}^{1} \operatorname{coth}\left(\frac{\pi(\tau-t)}{\Delta}\right) \mathrm{d} \tau\right]=\sqrt{\frac{\sinh (\pi(1-t) / \Delta)}{\sinh (\pi(1+t) / \Delta)}}
$$

which is equivalent to the fundamental solution defined in section 2.3.1.2 of chapter 2 . Using this relation, and taking the rigid limit in the bound vorticity equation (4.16), we obtain

$$
\gamma_{\text {rigid }}(t)=-\frac{2 Z_{\text {rigid }}(t)}{\Delta} f_{-1}^{1} \frac{y_{c, \alpha}^{\prime}(\tau)}{Z_{\text {rigid }}(\tau)}\left(\operatorname{coth}\left(\frac{\pi(\tau-t)}{\Delta}\right)-1\right) \mathrm{d} \tau
$$

which is equivalent to equation (2.31) of chapter 2 . 


\subsection{Results}

Having demonstrated the validity of our solution, we now present results on the effects of the porosity gradient and blade spacing on aerodynamic performance.

\subsubsection{Deflection Angle and Lift}

One of the primary functions of cascades in turbomachinery is to turn the flow through a desired angle. Intuition suggests that any porosity will reduce the flow deflection, and consequently be detrimental to aerodynamics performance. The solution in section 4.3 provides a way to precisely measure the effects of porosity on aerodynamic performance, which could be used by designers attempting to balance the aeroacoustic benefits of porous aerofoils with their aerodynamic limitations.

The deflection angle $\delta \alpha$ is calculated by taking the imaginary part of the complex potential (4.17) as $z \rightarrow \infty$ :

$$
\delta \alpha=2 \exp \left[-\frac{\pi}{\Delta} \int_{-1}^{1} k(\tau) \mathrm{d} \tau\right] \int_{-1}^{1} \frac{y_{c, \alpha}^{\prime}(\tau)}{Z(\tau)} \cdot \frac{\mathrm{d} \tau}{\Delta}
$$

which is related to the non-dimensional lift by equation 2.36 of chapter 2 .

From the above formula, we argue that the porosity at the trailing edge has a dominant effect on the deflection angle of the cascade. Asymptotic analysis of (4.15) reveals that $Z(t)$ possess a zero at the trailing edge $(t=1)$. The strength of this zero depends critically on the porosity at the trailing edge: in the rigid case there is a square root zero, but in the porous case the strength of the zero is $k(1)$. Consequently, the major contribution from the integral in (4.22) will be close to the trailing edge, and will be strongly influenced by $k(1)$, the trailing edge porosity. Since $k<1 / 2$, the strength of this zero is always reduced by porosity, and the dominant contribution from the trailing edge will also be reduced.

Major aeroacoustic gains can be made by tuning the porosity of the trailing edge Ayton (2016); Kisil and Ayton (2018), but the above formula indicates that a substantial reduction in deflection angle is unavoidable. The deflection angle can be increased by reducing the blade spacing, but this is not always possible in practical situations when the blade spacing is subject to other limitations. 


\subsubsection{Exact Solutions for Partially Porous Flat Plates}

The aeroacoustic benefits of porous aerofoils are well documented (Ayton, 2016; Cavalieri et al., 2016; Crighton and Leppington, 1970; Jaworski and Peake, 2013). Particular attention has been given to partially porous aerofoils since they are more aerodynamically favourable than fully porous aerofoils. The mechanism for sound reduction in partially porous wings is elucidated by the iterative method implemented in Kisil and Ayton (2018), which reveals that interference between scattering from the impermeable-permeable junction and the trailing edge rotates the far-field directivity for the acoustic field. Consistent with experimental results Geyer and Sarradj (2014), this research suggests that the impermeable-permeable junction plays a more important role than previously believed. Consequently, the aerodynamic modelling of partially porous aerofoils that possess such junctions is a crucial step in understanding how to harmonise aerodynamic and aeroacoustic requirements.

The aerodynamics of partially porous aerofoils was first investigated by Iosilevskii (2011) and Iosilevskii (2013) and extended in Hajian and Jaworski (2017). We now investigate partially porous aerofoils in a cascade arrangement. The porosity gradient is

$$
\psi(t)=\psi_{0} H(t-a)
$$

i.e. the aerofoil is rigid, except for a finite porous extension along $[a, 1]$. We refer to the regions $[-1, a]$ and $[a, 1]$ as the forward and aft parts respectively.

Although this porosity distribution is not Hölder continuous, the solution (4.16) remains valid since the classical results on discontinuous coefficients (Muskhelishvili, 1946) can be imported from the non-periodic case.

In the case of flat plates at angle of attack $\alpha$, the singular integrals in (4.17) and (4.16) can be calculated analytically to give exact solutions. Using $y_{c, \alpha}^{\prime}=-\alpha$ and applying contour integration yields the bound vorticity (4.16) as

$$
\gamma(t)=\alpha \frac{2 \mathrm{e}^{-(1+a) \frac{\pi}{2 \Delta}} \mathrm{e}^{-(1-a) \frac{\pi k}{\Delta}}}{\sqrt{1+\psi(t)^{2}}}\left|\frac{\sinh (\pi(t-a) / \Delta)}{\sinh (\pi(t+1) / \Delta)}\right|^{1 / 2}\left|\frac{\sinh (\pi(t-1) / \Delta)}{\sinh (\pi(t-a) / \Delta)}\right|^{k},
$$


and the perturbed complex velocity (4.17) as

$$
\begin{aligned}
u(z)-\mathrm{i} v(z)=\alpha\left(\mathrm{e}^{-(1+a) \frac{\pi}{2 \Delta}} \mathrm{e}^{-(1-a) \frac{\pi k}{\Delta}}\right. & \left(\frac{\sinh (\pi(z-a) / \Delta)}{\sinh (\pi(z+1) / \Delta)}\right)^{1 / 2} \\
& \left.\times\left(\frac{\sinh (\pi(z-1) / \Delta)}{\sinh (\pi(z-a) / \Delta)}\right)^{k}-1\right),
\end{aligned}
$$

where $k=(1 / \pi) \cot ^{-1} \psi_{0}$.

We note that as the porosity along the aft section increases $\left(\psi_{0} \rightarrow \infty\right)$, the velocity jump across the permeable section has the asymptotic behaviour

$$
\gamma(t) \sim \alpha \frac{2 \mathrm{e}^{-(1+a) \frac{\pi}{2 \Delta}}}{\psi_{0}}\left|\frac{\sinh (\pi(t-a) / \Delta)}{\sinh (\pi(t+1) / \Delta)}\right|^{1 / 2} .
$$

Consequently, $\gamma(t)=\mathcal{O}\left(\psi_{0}^{-1}\right)$, and the porous section effectively vanishes as it becomes fully permeable.

For a cascade of partially porous flat plates with permeable length $a$, the maximum velocity jump is given by

$$
\begin{aligned}
\gamma\left(t_{\max }\right)=\alpha \frac{2 \mathrm{e}^{-(1+a) \frac{\pi}{2 \Delta}} \mathrm{e}^{-(1-a) \frac{\pi k}{\Delta}}}{\sqrt{1+\psi_{0}^{2}}} \frac{(2 k)^{k}}{(1-2 k)^{k-1 / 2}}\left|\frac{\sinh (\pi(1-a) / \Delta)}{\sinh (2 \pi / \Delta)}\right|^{1 / 2} \\
\times\left|\frac{\sinh (2 \pi / \Delta)}{\sinh (\pi(1+a) / \Delta)}\right|^{k},
\end{aligned}
$$

which is attained when

$$
t_{\max }=\frac{\Delta}{2 \pi} \log \left(\frac{\mathrm{e}^{2 \pi / \Delta} \sinh (\pi(a+1) / \Delta)+2 k \sinh (\pi(1-a) / \Delta)}{\sinh (\pi(a+1) / \Delta)+2 k \mathrm{e}^{2 \pi / \Delta} \sinh (\pi(1-a) / \Delta)}\right) .
$$

The pressure jump is also maximised at this point.

We now present results for the velocity jump across the chord (which is related to the pressure jump via $\gamma=-[p] / 2$ ) for a variety of permeable lengths, porosities, and blade spacings in figures 4.2 and 4.3. Since the velocity jump is small, the logarithmic values are plotted. However, the reader should not be misled by this scale, which may seem to suggest that the aerodynamic performance can be improved with porosity (see figure $4.2 \mathrm{~b}$, for example, where the pressure jump along the aft section is greater than when the aerofoil is rigid). Any aerodynamic gains made along the aft section are more than cancelled out by the corresponding losses along the rigid forward section, which is 


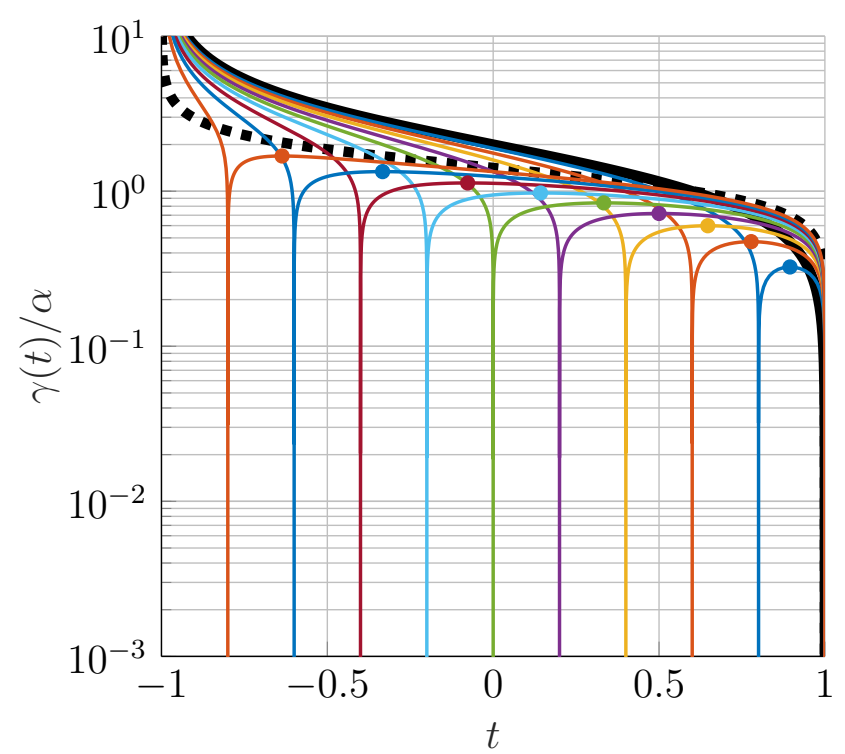

(a)

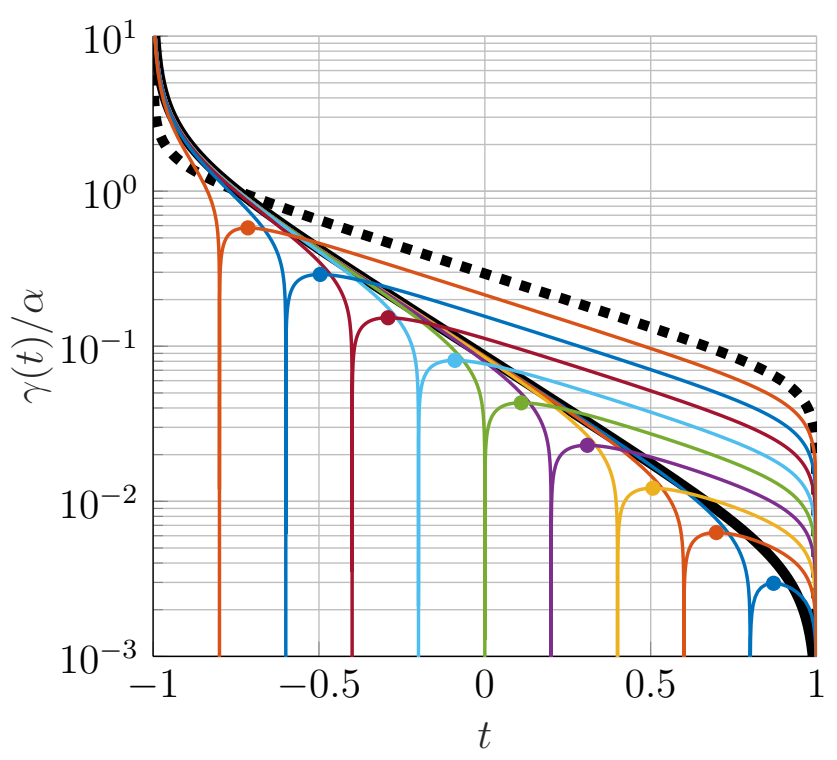

(b)

Fig. 4.2 Plots of bound vorticity for permeable lengths $a=-1,-0.8, \ldots, 0.8,1$ and porosity $\psi=1$ as a function of distance along the chord, $t$ for a) an isolated aerofoil, and b) a cascade of aerofoils with $\Delta=1$. The colour corresponding to each $a$ is clear from $\gamma(a)=0$. The case $a=1$, when the blade is fully rigid, is denoted by the solid black line, whereas the case $a=-1$, when the blade is fully permeable, is denoted by the dashed black line. The maximum velocity jump on the permeable aft section is marked by the solid dots.

illustrated more clearly in figure 4.2a. We shall return to the aerodynamic performance in the following section.

In figure 4.2 we plot the bound vorticity as a function of distance along the chord. The plots exhibit the difference in behaviour between isolated aerofoils and cascades. In the isolated case, there is a regime of $a$ where porosity increases the velocity jump across the blade. Comparing figures $4.2 \mathrm{a}$ and $4.2 \mathrm{~b}$ shows that an effect of reducing the blade spacing is to increase this regime where porosity increases the velocity jump. However, the magnitude of this increase is small and is offset considerably by the decrease in velocity jump close to the leading edge, also illustrated in figure 4.2. The apparent spikes are when the bound vorticity becomes zero at the junction. Physically, this zero is necessary to enforce continuity of seepage velocity (4.3): any other values result in jumps in the normal surface velocity. Later we will discuss smooth transitions from impermeable to permeable that do not exhibit this zero.

In figure 4.3 we plot the velocity jump for fixed $a$ and a variety of porosities. Similarly to figure 4.2 , it is observed that increasing blade spacing increases the region of $t$ where 


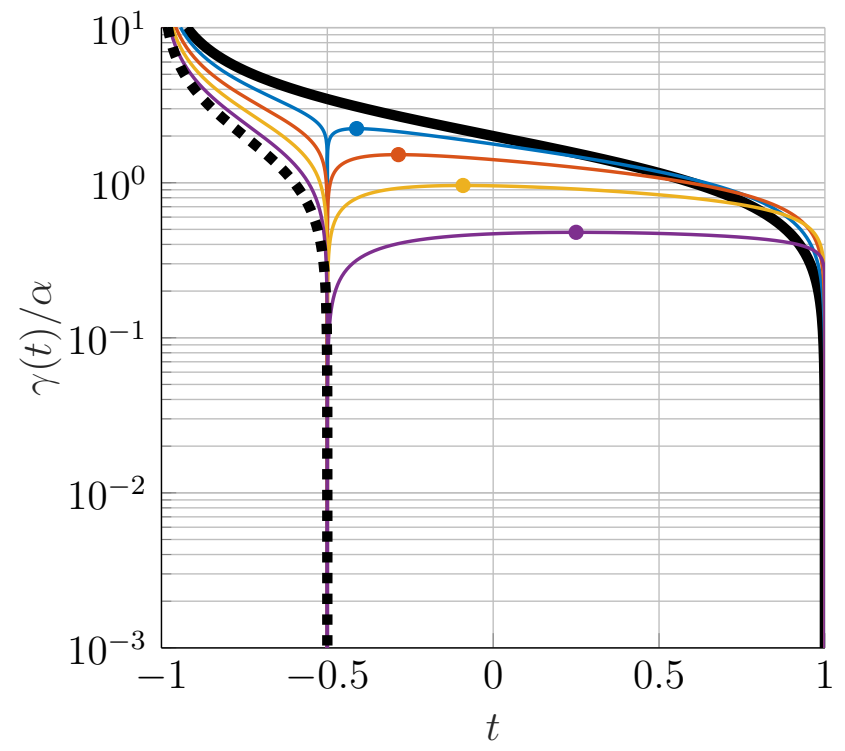

(a) Isolated aerofoil, $\Delta=\infty, a=-1 / 2$

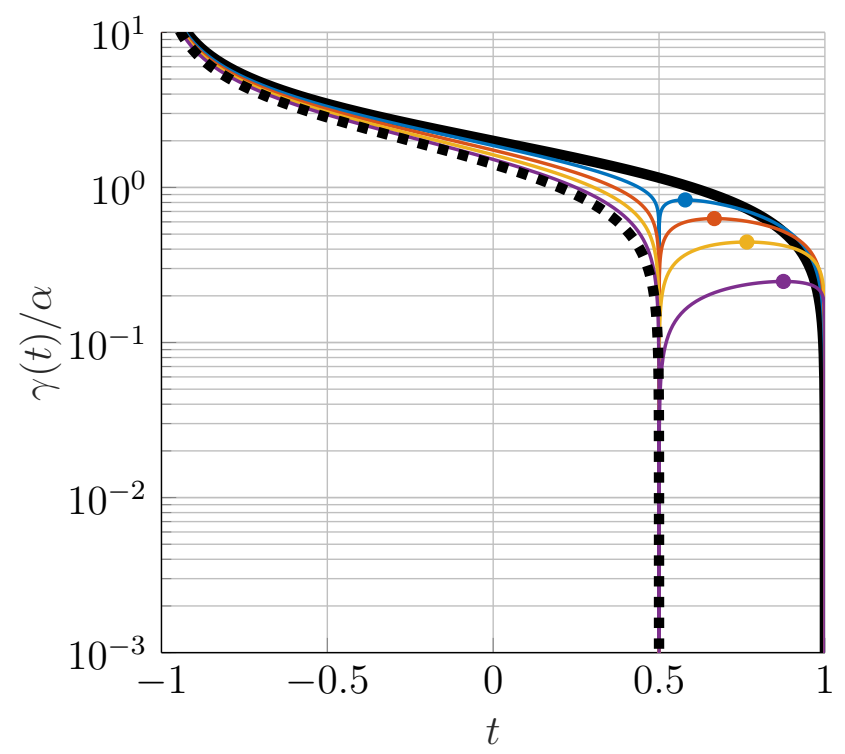

(c) Isolated aerofoil, $\Delta=\infty, a=1 / 2$

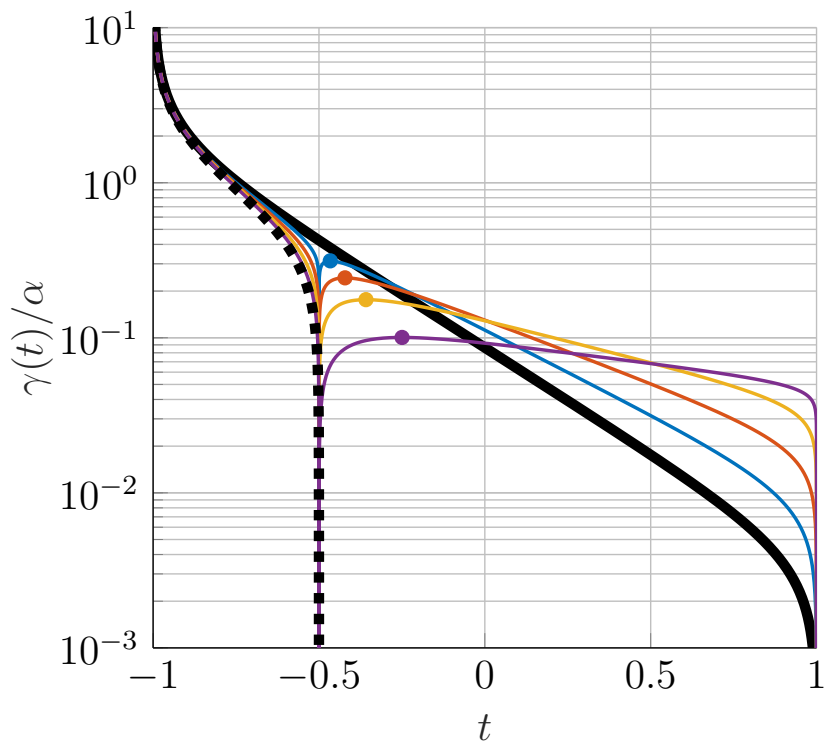

(b) Cascade of aerofoils, $\Delta=1, a=-1 / 2$

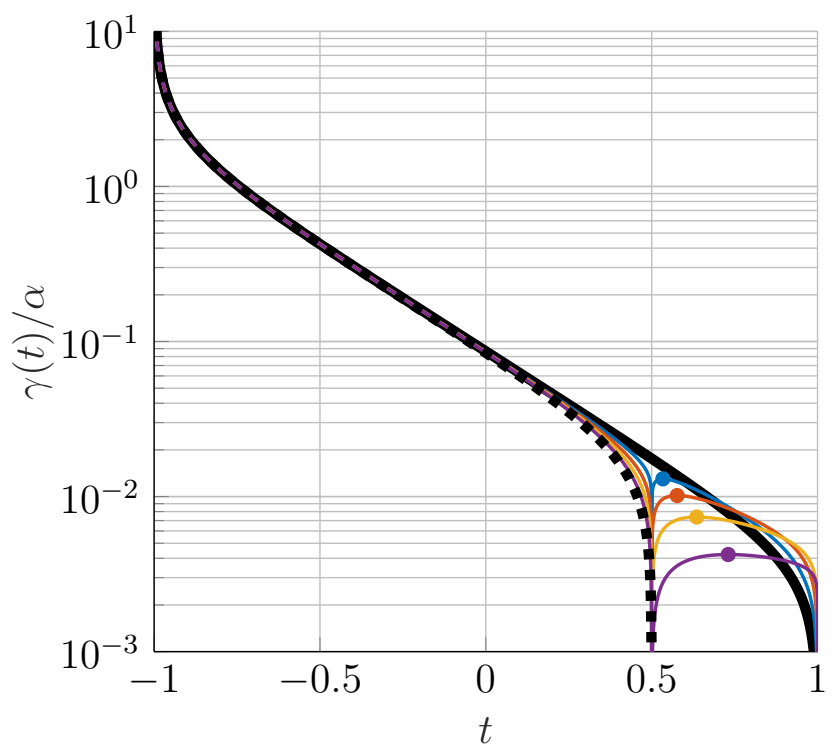

(d) Cascade of aerofoils, $\Delta=1, a=1 / 2$

Fig. 4.3 Plots of bound vorticity as a function of distance along the chord, $t$, for a variety of permeable lengths, blade spacings and porosities: a) isolated aerofoil, $a=-1 / 2, \mathrm{~b}$ ) cascade of aerofoils, $\Delta=1, a=-1 / 2$, b) isolated aerofoil, $a=1 / 2$, d) cascade of aerofoils, $\Delta=1, a=1 / 2$. The porosities are $k=0.1$ (blue), 0.2 (orange), 0.3 (yellow), 0.4 (purple). The case $k=0.5$, when the blade is fully rigid, is denoted by the solid black line, whereas the case $k=0$, when the aft part of the blade is fully permeable, is denoted by the dashed black line. The maximum velocity jump on the permeable aft section is marked by the solid dots. 
the velocity jump increases. Here it is also clear that increasing the blade spacing shifts the maximum value of $\gamma$ to the left.

\subsubsection{Lift and Drag}

We now find analytic expressions for the lift and drag on a partially porous flat plate in a cascade. The lift on an isolated partially porous aerofoil was found by Iosilevskii (2011) and the analysis was extended by Iosilevskii (2013) to evaluate the seepage drag.

Similarly to the isolated aerofoil case, there are two forces acting on each aerofoil in the cascade: the normal force and the leading-edge suction force. The components of these forces are resolved to give the lift and drag. For an isolated, rigid aerofoil, the forces are calculated using Blasius' theorem, which leads to the Kutta-Joukowski theorem, namely that the only force on the aerofoil is normal to the flow direction. For rigid cascades, a similar procedure yields that the net force on an individual aerofoil acts normal to the average of the upstream and downstream velocities (Robinson and Laurmann, 1956, p. 148).

When considering rigid flat plates, the normal force and leading-edge suction force are carefully balanced to cancel the drag force consistent with the Kutta-Joukowski theorem (see p. 107 of Katz and Plotkin (2009), for example). Alternatively, the full force calculation is achieved by properly integrating the singularity arising in Blasius' theorem at the leading edge (see p. 131 of Katz and Plotkin (2009), for example). However, the Kutta-Joukowski theorem no longer applies when the plates are porous and we expect the drag to be non-zero.

For the cascade, the mean of the upstream and downstream velocities is

$$
\mathbf{U}_{m}=\frac{1}{2}\left(\mathbf{U}_{1}+\mathbf{U}_{2}\right)=\left(1, \alpha+\frac{\delta \alpha}{2}\right),
$$

where $\mathbf{U}_{1}$ is the upstream velocity and $\mathbf{U}_{2}$ is the downstream velocity. The generalised Kutta-Joukowski theorem states that the only force on a cascade of impermeable aerofoils operates in the direction normal to $\mathbf{U}_{m}$, and we define the drag to be perpendicular to this vector. In general $\delta \alpha$ can be calculated by (4.22), or by sending $z \rightarrow \infty$ in the complex velocity expression (4.26).

When the leading edge of the aerofoil is rigid, the leading-edge suction force is

$$
F_{s}=\frac{\pi}{4}\left(\lim _{t \rightarrow-1} \gamma(t) \sqrt{1-t}\right)^{2}=\frac{\alpha^{2} \Delta}{2}\left(1-\mathrm{e}^{-4 \pi / \Delta}\right)^{2 k}\left(1-\mathrm{e}^{-2 \pi(1+a) / \Delta}\right)^{1-2 k} .
$$


We now calculate the non-dimensional normal force, which is equivalent to the lift up to $\mathcal{O}\left(\epsilon^{2}\right)$, using

$$
\mathscr{L}=F_{n}=\frac{1}{2} \int_{-1}^{1}\left(p^{+}(t)-p^{-}(t)\right) \mathrm{d} t=\Delta \alpha\left(1-\mathrm{e}^{-(1+a) \frac{\pi}{\Delta}} \mathrm{e}^{-(1-a) \frac{2 \pi k}{\Delta}}\right)
$$

By resolving the leading-edge suction force and normal force into the direction parallel to the velocity mean flow, we obtain an expression for the seepage drag:

$$
\begin{aligned}
\mathscr{D} & =-F_{s}+\left(\alpha+\frac{\delta \alpha}{2}\right) F_{n} \\
& =\frac{\Delta \alpha^{2}}{2}\left[1-\mathrm{e}^{-(1+a) \frac{2 \pi}{\Delta}} \mathrm{e}^{-(1-a) \frac{4 \pi k}{\Delta}}-\left(1-\mathrm{e}^{-4 \pi / \Delta}\right)^{2 k}\left(1-\mathrm{e}^{-2 \pi(1+a) / \Delta}\right)^{1-2 k}\right] .
\end{aligned}
$$

Note that the drag for a partially porous isolated aerofoil (Iosilevskii, 2013, equation (21)) is recovered in the limit $\Delta \rightarrow \infty$.

For a given blade spacing $\Delta$ and permeable length $a$, the maximum seepage drag is

$$
\begin{aligned}
\mathscr{D}_{\max } & =\frac{\Delta \alpha^{2}}{2}\left(1-\left[\frac{(1-a) 2 \pi}{\Delta \log \left(\frac{1-\mathrm{e}^{-4 \pi / \Delta}}{1-\mathrm{e}^{-2 \pi(1+a) / \Delta}}\right)}+1\right] \mathrm{e}^{-(1+a) 2 \pi / \Delta} \mathscr{M}\right), \\
\mathscr{M} & =\left(\left(\mathrm{e}^{2 \pi(1+a) / \Delta}-1\right) \cdot \frac{\log \left(\frac{1-\mathrm{e}^{-4 \pi / \Delta}}{1-\mathrm{e}^{-2 \pi(1+a) / \Delta}}\right)}{\log \left(\mathrm{e}^{-(1-a) 2 \pi / \Delta}\right)}\right) \frac{-(1-a) 2 \pi}{\Delta \log \left(\frac{\mathrm{e}^{-(1-a) 2 \pi / \Delta}}{\left(1-\mathrm{e}^{-4 \pi / \Delta}\right)\left(1-\mathrm{e}^{-2 \pi(1+a) / \Delta}\right)}\right)},
\end{aligned}
$$

which is attained when

$$
k_{\max }=\frac{\log \left(\left(\mathrm{e}^{2 \pi(1+a) / \Delta}-1\right) \cdot \frac{\log \left(\frac{1-\mathrm{e}^{-4 \pi / \Delta}}{1-\mathrm{e}^{-2 \pi(1+a) / \Delta}}\right)}{\log \left(\mathrm{e}^{-(1-a) 2 \pi / \Delta}\right)}\right)}{2 \log \left(\frac{\mathrm{e}^{-(1-a) 2 \pi / \Delta}}{\left(1-\mathrm{e}^{-4 \pi / \Delta}\right)\left(1-\mathrm{e}^{-2 \pi(1+a) / \Delta}\right)}\right)} .
$$

We now present figures illustrating the drag, lift and drag-to-lift ratio. The colour scales are chosen such that it is aerodynamically favourable to operate in blue regions of the colour plots.

In figure 4.4 we plot the drag coefficient as a function of permeable length $a$ and porosity $k$. The white line indicates the maximum drag possible for a given $a$, as given in equations (4.32) and (4.33). The thin blue boundaries on the top and bottom of figures 4.4 show that the drag vanishes for zero and infinite porosity. For zero porosity, the Kutta-Joukowski theorem (generalised for cascades) states that the drag on individual impermeable blades is zero. On the other hand, for infinite porosity, there is no pressure 


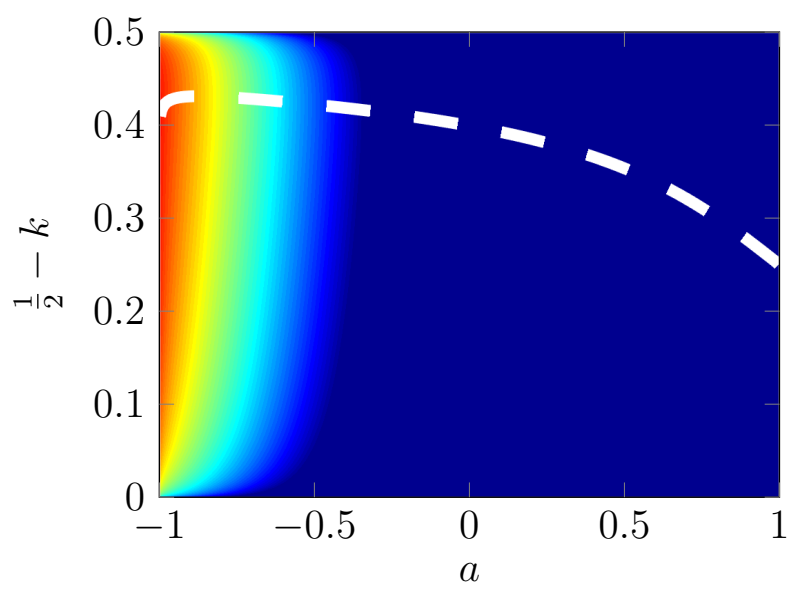

(a)

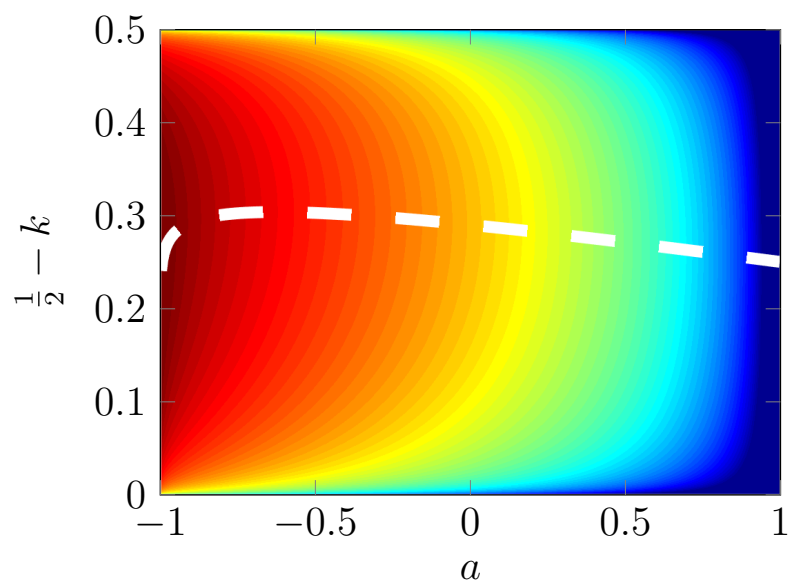

(c)

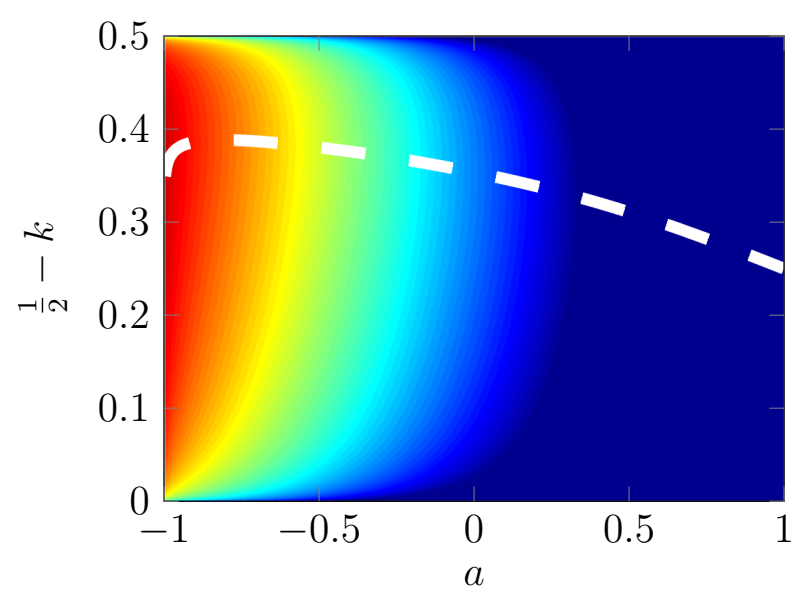

(b)

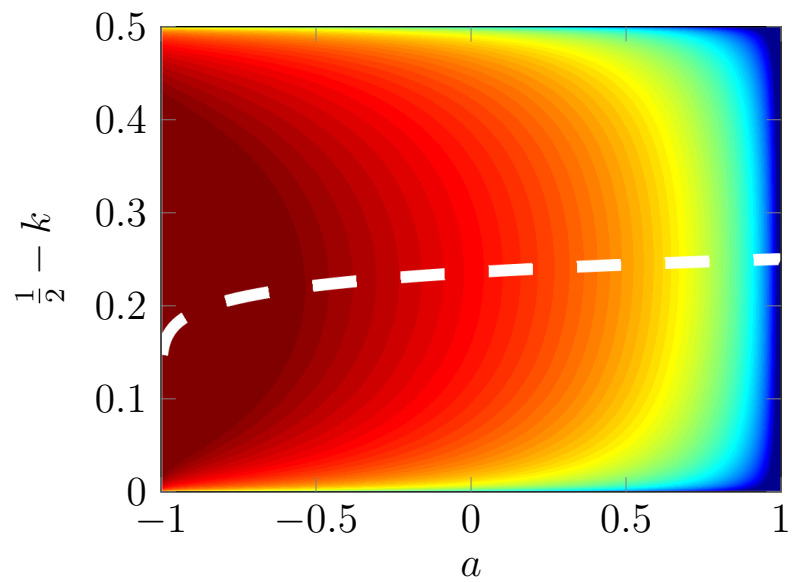

(d)

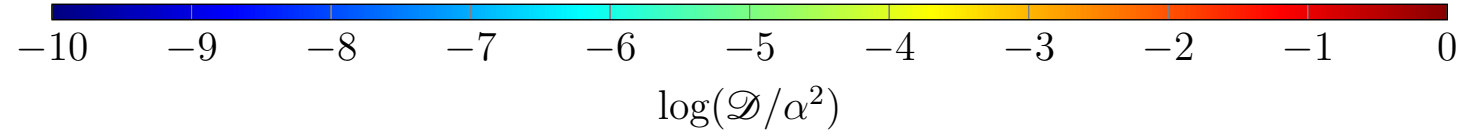

Fig. 4.4 Drag as a function of permeable length $a$ and porosity function $k$ for a) $\Delta=1$, b) $\Delta=3$, c) $\Delta=10$, d) $\Delta=\infty$. The white line indicates the maximum drag for a given impermeable length. 
jump across the aft, permeable part of wing, and the aerofoil behaves as if it were a rigid plate truncated at $a$, and the Kutta-Joukowski theorem applies again.

As described in Iosilevskii (2013), the seepage drag exhibits singular behaviour as $a \rightarrow-1$; there is a discontinuity in the seepage drag when the porosity of a uniform plate is varied from 0 (when the seepage drag vanishes) to an infinitesimally small value (when the seepage drag is finite). This discontinuity can be attributed to the discontinuity in leading-edge suction: for a uniformly porous flat plate, $\gamma=\mathcal{O}\left((1+t)^{-k}\right)$ as $t \rightarrow-1$, but since $k<1 / 2$, the leading-edge suction force (4.29) vanishes discontinuously for even the smallest value of porosity. Another perspective is that the seepage velocity (4.3) is proportional to the bound vorticity, so an infinite vorticity corresponds to an infinite seepage velocity. Since the vorticity is infinite at the leading edge, if the aerofoil is porous there then the seepage velocity will also be infinite there. Correspondingly, the pore-level Reynolds number is infinite and the Stokes flow assumption necessary for the Darcy flow through the pores is not applicable. Consequently, the results should not be trusted when the leading edge is porous and a more sophisticated porosity model is required, such as that proposed by Ergun (1952).

Figure 4.4 also illustrates the role played by blade spacing on the seepage drag. For small spacings (figures $4.4 \mathrm{a}$ and $4.4 \mathrm{~b}$ ), the maximum drag is always attained for $0<k<1 / 4$ (except for a very small region near $a=-1$ ) whereas for large spacings (figures c, d) the maximum drag is attained for $1 / 4<k<1 / 2$. Increasing the blade spacing also expands the area of significant drag, as indicated by the expanding red region. Consequently, decreasing blade spacing can mitigate against the negative aerodynamic effects associated with porosity.

Similarly to the single blade case (Iosilevskii, 2013), extending the permeable part forwards carries a severe drag penalty, due to the large pressure jump that the porosity is reducing. Conversely, the effect of the permeable part in the after direction is negligible, since the pressure jump is small here, owing to the Kutta condition.

We now investigate the effects of porosity on aerofoil lift. Equation (4.30) elucidates the symmetrical relationship between $a$ and $k$ : the lift $\mathscr{L}$ is unaffected by interchanging these two parameters. This parity is illustrated in figure 4.5, which plots the reduction in lift from the rigid case due to porosity and permeable length. The white dashed line indicates the reflective symmetry between $a$ and $k$. The effects on lift can be attributed to the influence of porosity on the pressure jump across the blade. On a rigid blade, the leading-edge singularity creates a large pressure jump near the front of the aerofoil, whereas the Kutta condition enforces a small pressure jump near the trailing edge. The effect of porosity is to reduce the pressure jump, and consequently the effects of porosity 


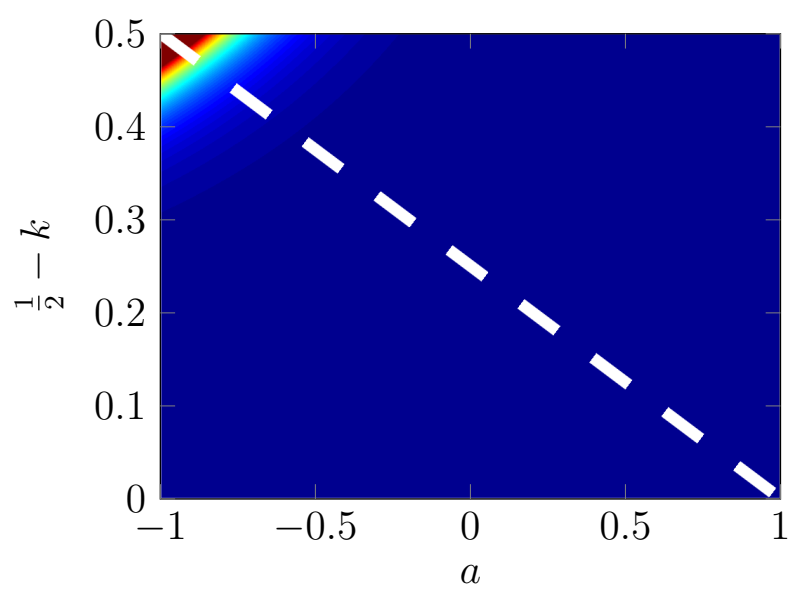

(a)

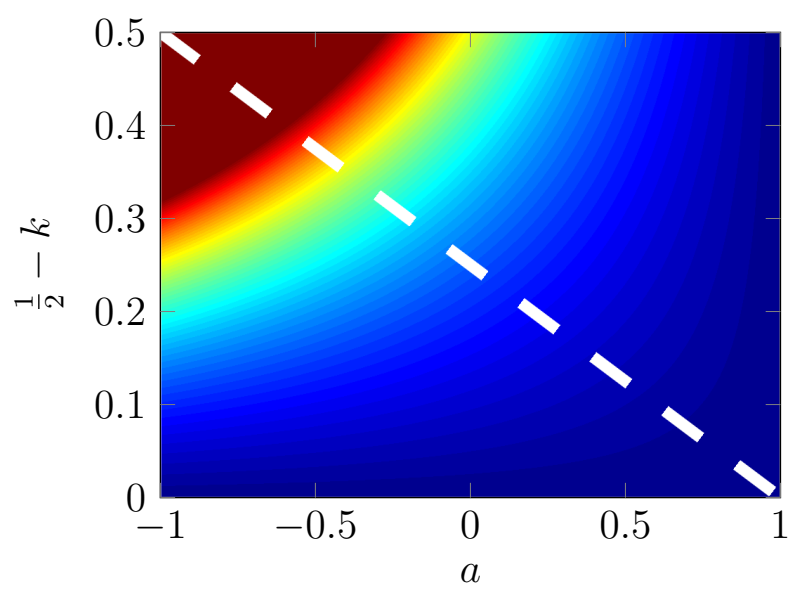

(c)

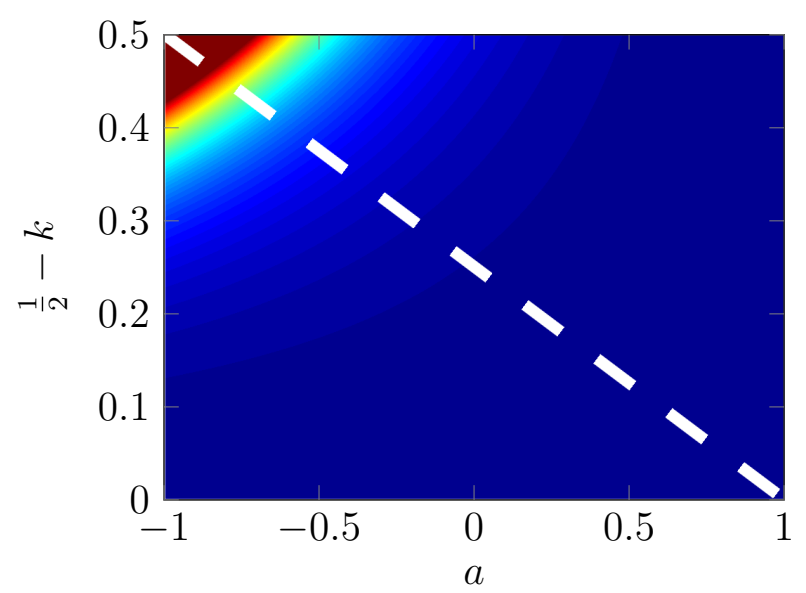

(b)

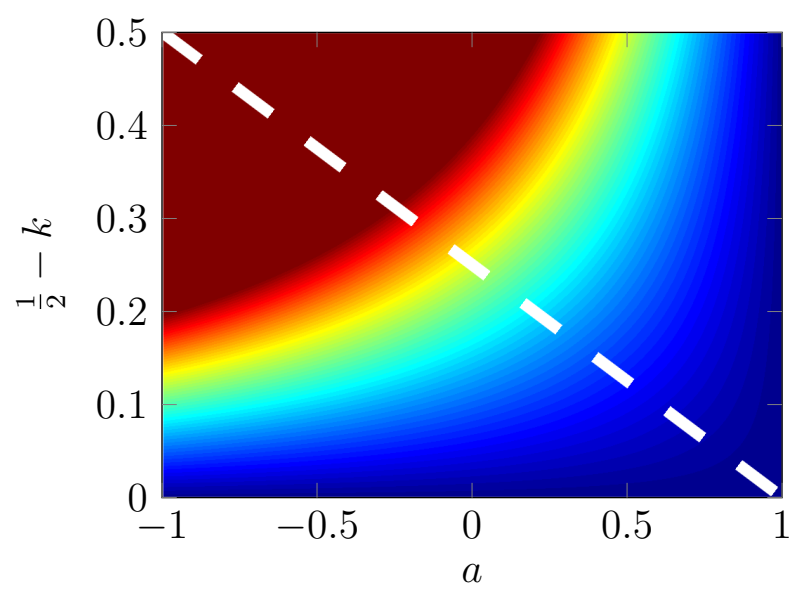

(d)

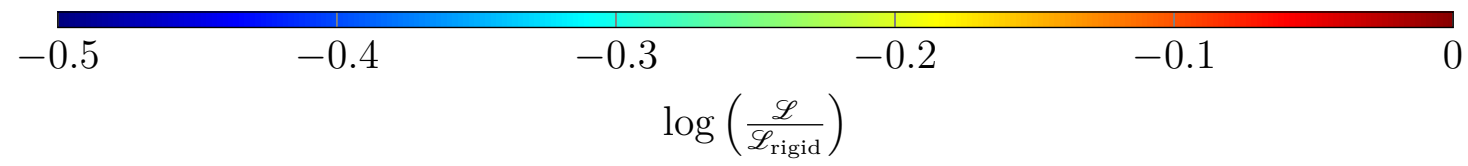

Fig. 4.5 Normalised lift as a function of permeable length and porosity a) $\Delta=1$, b) $\Delta=3$, c) $\Delta=10$, d) $\Delta=\infty$. The dashed white line indicates the line of symmetry between $k$ and $a$.

are most significant near the leading edge where there is a large reduction in pressure jump. This is observed through the sharp increase in lift reduction as the permeable part is extended into the forward direction. The blade spacing effectively reduces the pressure jump by spreading out the lift between the blades. Consequently, the effects of porosity and permeable length on the lift reduction are mitigated by reducing the blade spacing.

We finally explore the effects of porosity on lift-to-drag ratio in figure 4.6. No analytic expressions are available for the minimum lift-to-drag ratio, such as in equations (4.32) 


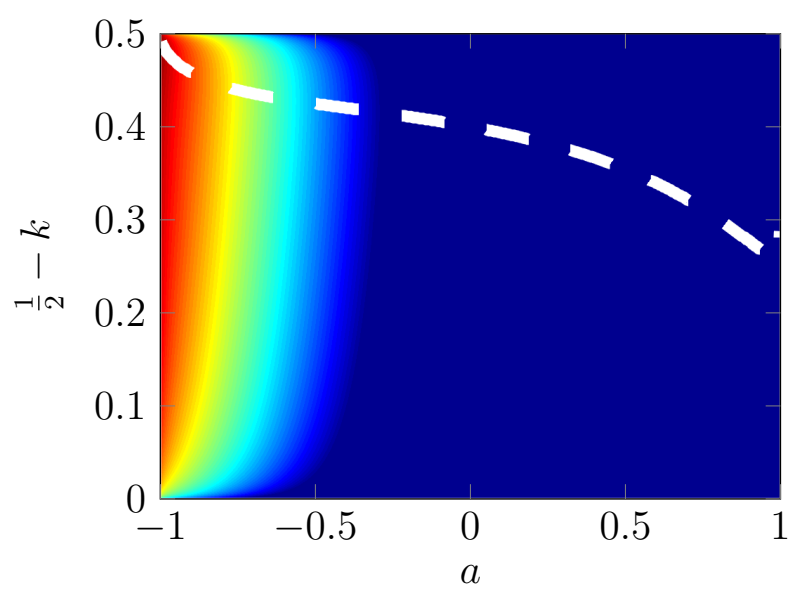

(a)

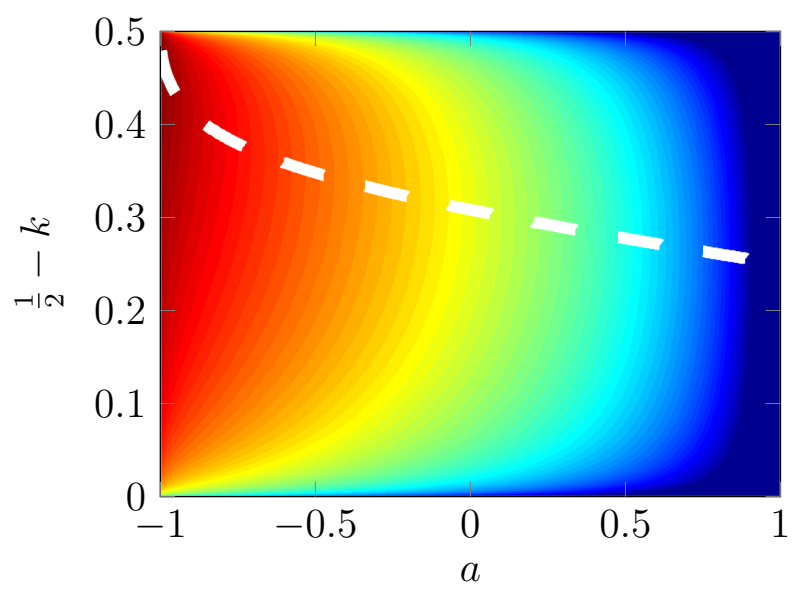

(c)

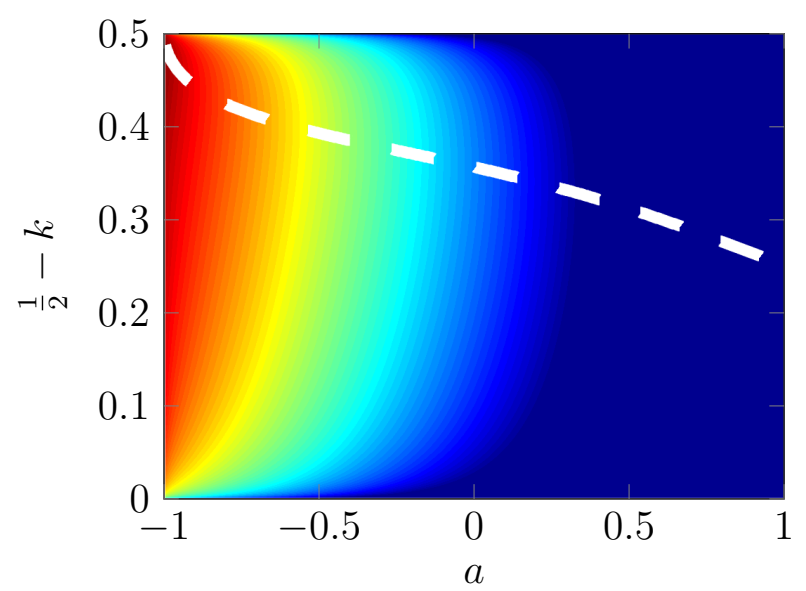

(b)

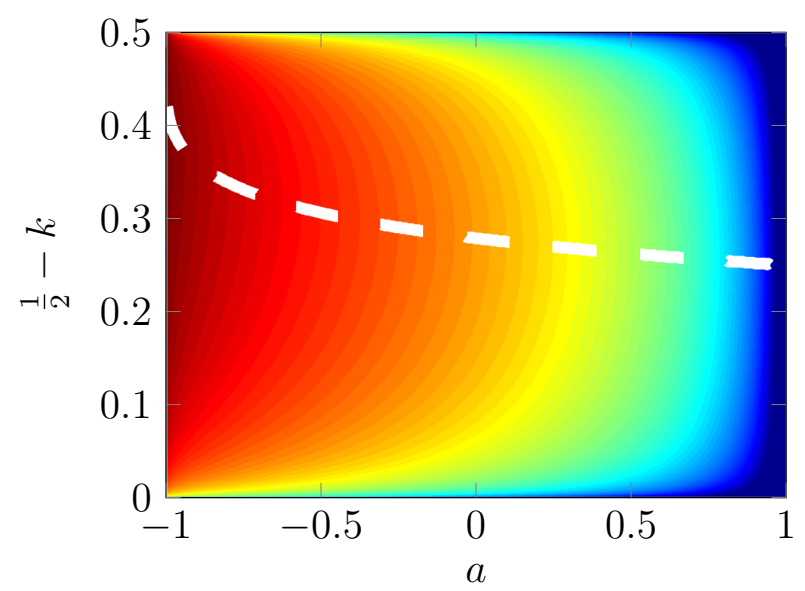

(d)

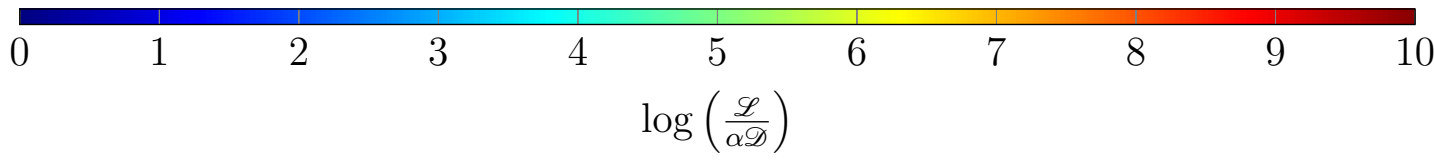

Fig. 4.6 Lift-to-drag ratio as a function of permeable length and porosity for a) $\Delta=1$, b) $\Delta=3$, c) $\Delta=10$, d) $\Delta=\infty$. The white line indicates the minimum lift-to-drag ratio for a given impermeable length.

and (4.33), so the minima are found numerically and plotted by the dashed white line. Similar conclusions to those found for the seepage drag may be applied, namely that the aerodynamic performance rapidly deteriorates as the permeable part is extended into the forward direction, and that blade spacing can be reduced to mitigate the effects of porosity. 


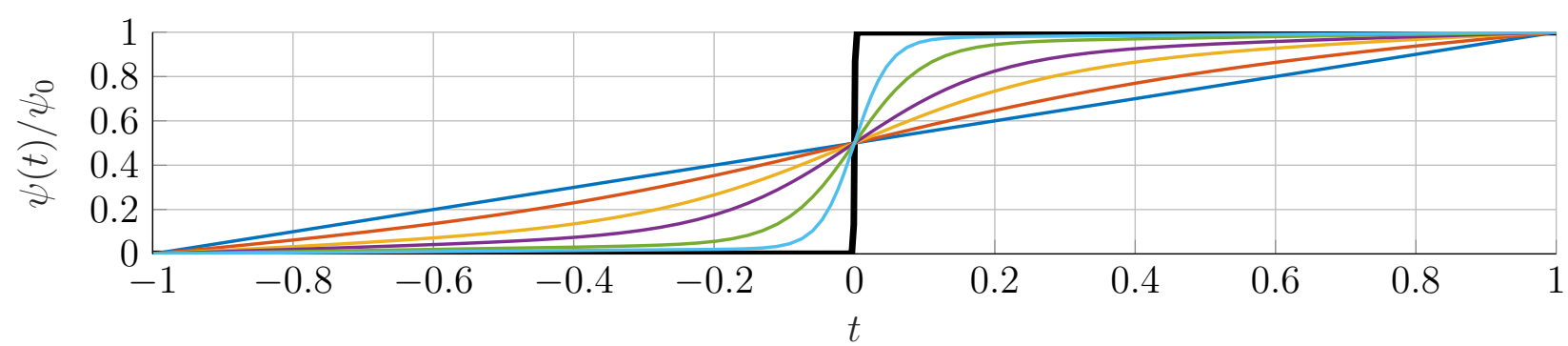

Fig. 4.7 The porosity gradient (4.34) used to approximate a partially porous distribution with junction at $a=0$. The curves correspond to $\mathrm{b}=0$ (dark blue), 0.5 (orange), 0.7 (yellow), 0.8 (purple), 0.9 (green), 0.95 (light blue), 1 (black).

\subsubsection{Results on Aerofoils with Continuous Porosity Distribu- tions}

Porosity gradients are rarely discontinuous in the natural world. For example, a bird's wing will exhibit a smooth transition from a impermeable leading edge to a porous trailing edge (Graham, 1934), the porosity gradients are rarely discontinuous. This smooth transition from rigid to porous may affect the aeroacoustic and aerodynamic performance of the aerofoil. Moreover, it is believed that discontinuities in porosity are a secondary source of aeroacoustic scattering and are, therefore, undesirable. Consequently, we now investigate the effects of a continuously varying porosity gradient. This section also illustrates the versatility of the solution to consider a range of porosity profiles. We choose a porosity gradient of

$$
\begin{array}{r}
\psi(t)=\frac{\psi_{0}}{2}((1+t) \\
(1-b)+b(1+\tanh ((a-t) /(b-1))) \\
+\frac{t(1-t)}{2}(1+\tanh ((a+1) /(b-1))) \\
+\frac{t(1+t)}{2}(1-\tanh ((a-1) /(b-1))),
\end{array}
$$

such that $b=0$ corresponds to a linear porosity gradient that is rigid at the leading edge and $b=1$ corresponds to the partially porous case with junction at $t=a$. This porosity distribution is illustrated in figure 4.7.

We consider aerofoils at angle of attack $\alpha$ with parabolic camber $\beta$ such that the geometrical profile may be written as

$$
y_{c, \alpha}^{\prime}(t)=-\beta t-\alpha .
$$




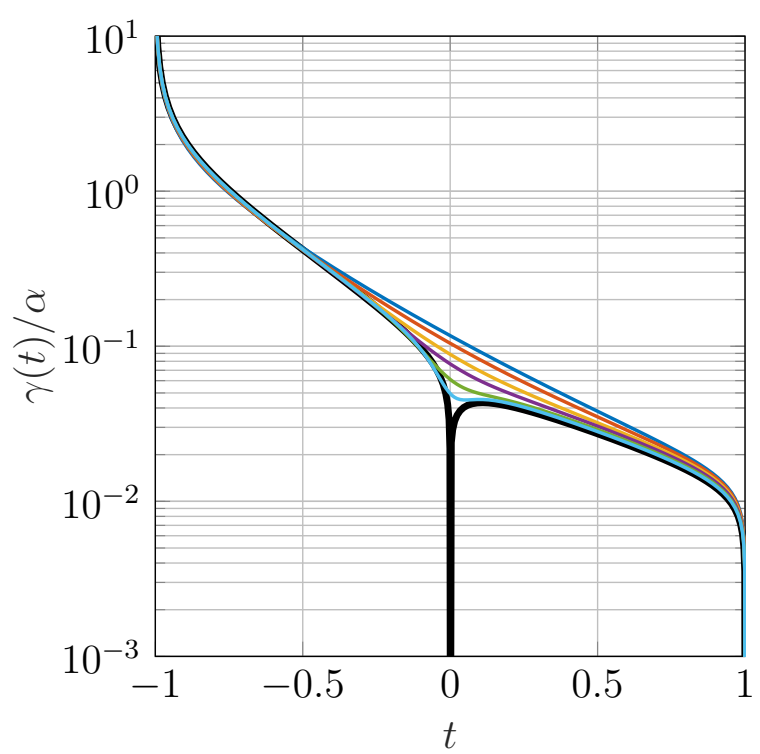

(a)

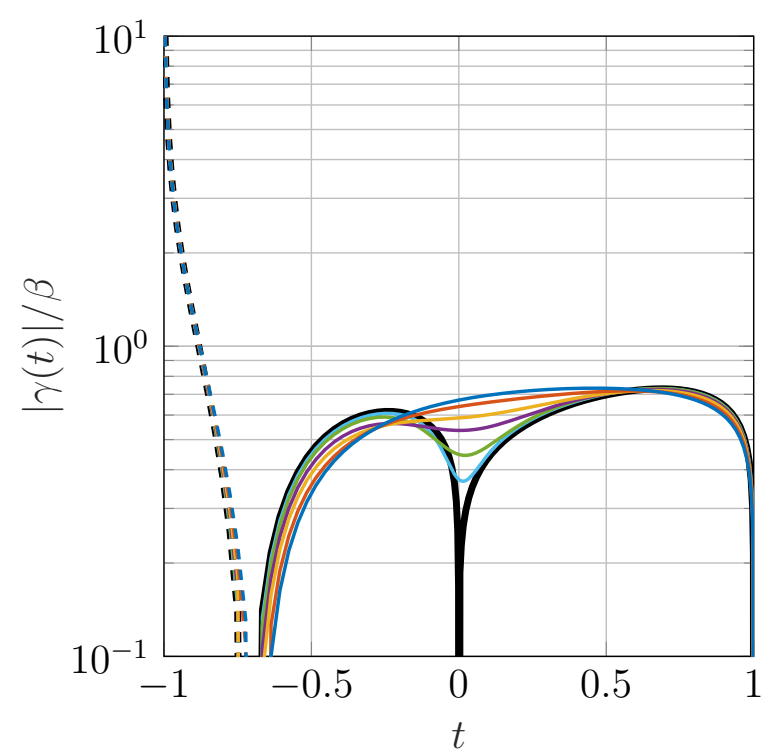

(b)

Fig. 4.8 Plots of bound vorticity for a cascade of aerofoils with continuous porosity gradients (4.34) with $\psi_{0}=1$ and blade spacing $\Delta=1$. a) The bound vorticity for a flat plate at angle of attack $\alpha$; b) The absolute value of bound vorticity for an aerofoil with parabolic camber at zero angle of attack. The solid curves indicate $\gamma>0$ and the dashed curves denote $\gamma<0$. The colours correspond to those defined in figure 4.7.

The linearity of this profile allows us to separate the effects of angle of attack and camber, which are illustrated in figures $4.8 \mathrm{a}$ and $4.8 \mathrm{~b}$. The singular integrals in $(4.15)$ and (4.16) have been integrated numerically using MATLAB. An effect of a smoother junction results in a smoother change in vorticity distribution, which no longer vanishes at $t=a$. Unlike the case of angle of attack, the vorticity distribution becomes negative for some sections for aerofoils with parabolic camber; the effective angle of attack at the leading edge of the aerofoil is negative. However, as for angle of attack, the effect of porosity is to reduce the lift on the cascade by reducing the positive section of the bound vorticity, where the effective angle of attack is positive.

We now derive an expression for the leading-edge suction for aerofoils with arbitrary porosity gradients. We first note that

$$
\lim _{t \rightarrow-1}(Z(t) \sqrt{1-t})=\sqrt{\frac{\Delta}{\pi}} \sqrt{\sinh (2 \pi / \Delta)} \lim _{t \rightarrow-1}\left(Z(t) \sqrt{\frac{\sinh (\pi(1-t) / \Delta)}{\sinh (\pi(1+t) / \Delta)}}\right) .
$$




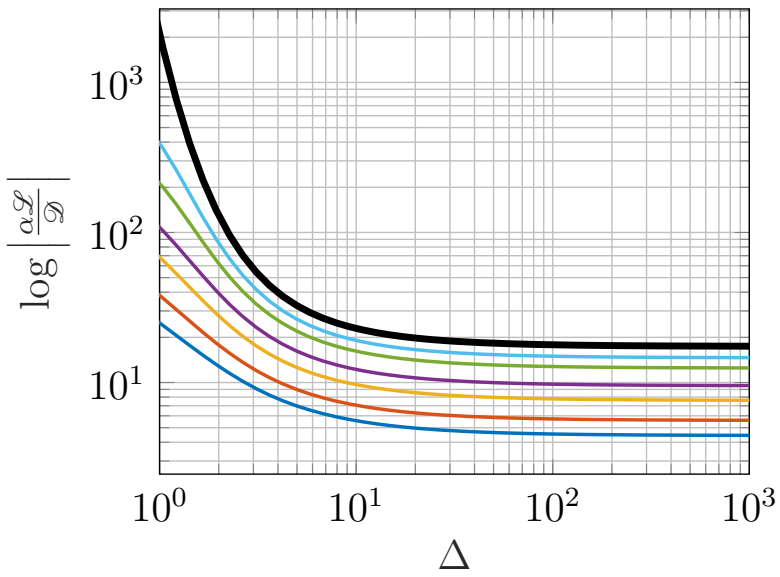

(a) Flat plates at angle of attack $\alpha$

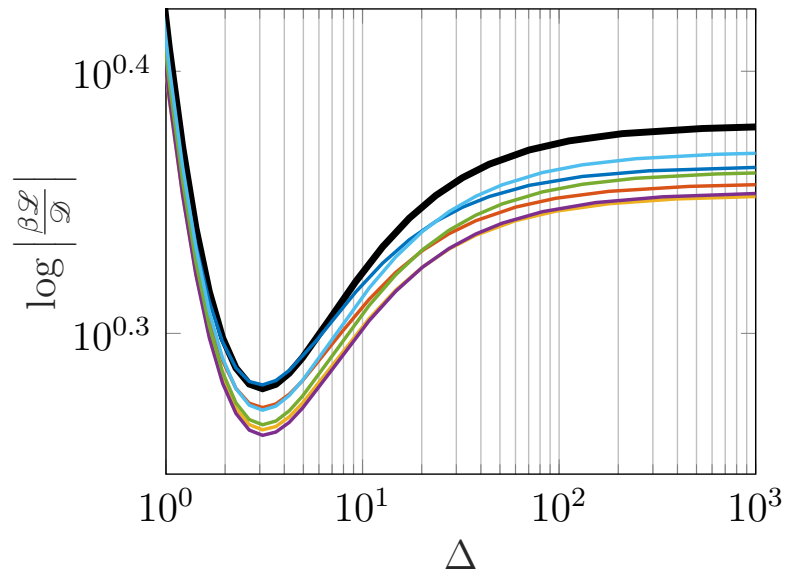

(b) Aerofoils with parabolic camber at zero angle of attack.

Fig. 4.9 Plots of the lift-to-drag ratio for cascades of aerofoils with continuous porosity gradients (4.34) with $\psi_{0}=1$ and a range of blade spacings. The colours correspond to those defined in figure 4.7 .

Consequently, using the definition of $Z$ in (4.15) and the second identity in (4.20), we may write

$$
\lim _{t \rightarrow-1}(Z(t) \sqrt{1-t})=\sqrt{\frac{\Delta}{\pi}} \sqrt{\sinh (2 \pi / \Delta)} \exp \left[\frac{\pi}{\Delta} \int_{-1}^{1}\left(k(\tau)-\frac{1}{2}\right) \operatorname{coth}\left(\frac{\pi(\tau+1)}{\Delta}\right) \mathrm{d} \tau\right] .
$$

If the leading edge is rigid (as we have assumed in this chapter) then $k(-1)=1 / 2$ and the above integral exists since the singularities at $\tau=-1$ are integrable. Consequently, for an arbitrary porosity gradient with rigid leading edge, the leading-edge suction force is given by

$$
\begin{aligned}
F_{s} & =\frac{\pi}{4}\left(\lim _{t \rightarrow-1} Z(t) \sqrt{1-t}\right)^{2} \cdot\left\{\frac{2}{\Delta} \int_{-1}^{1} \frac{y_{c, \alpha}^{\prime}(\tau)}{Z(\tau)}\left(\operatorname{coth}\left(\frac{\pi(\tau+1)}{\Delta}\right)-1\right) \mathrm{d} \tau\right\}^{2} \\
& =\frac{\sinh (2 \pi / \Delta)}{\Delta} \exp \left[\frac{2 \pi}{\Delta} \int_{-1}^{1}\left(k(\tau)-\frac{1}{2}\right) \operatorname{coth}\left(\frac{\pi(\tau+1)}{\Delta}\right) \mathrm{d} \tau\right] \\
& \times\left\{\int_{-1}^{1} \frac{y_{c, \alpha}^{\prime}(\tau)}{Z(\tau)}\left(\operatorname{coth}\left(\frac{\pi(\tau+1)}{\Delta}\right)-1\right) \mathrm{d} \tau\right\}^{2} .
\end{aligned}
$$


We use this result to calculate the drag on a single aerofoil in the cascade as

$$
\mathscr{D}=-F_{s}-\int_{-1}^{1} \gamma(\tau)\left(y_{c, \alpha}^{\prime}(\tau)-\frac{\delta \alpha}{2}\right) \mathrm{d} \tau,
$$

where the last term is the normal force resolved in the direction of the drag. The lift can be calculated from the deflection angle formula (4.22).

In figure 4.9 we illustrate the effect of blade spacing on the lift-to-drag ratio. Both the lift and drag vanish as $\Delta \rightarrow 0$, but the drag converges faster than the lift thus resulting in a infinite lift-to-drag ratio. The porosity plays a strong role in the lift-to-drag ratio for flat plates for all blade spacings, as illustrated in figure 4.9a. Conversely, in the case of aerofoils with parabolic camber, as the blade spacing vanishes, the lift-to-drag ratios become indistinguishable for different porosity profile in figure $4.9 \mathrm{~b}$.

\subsection{Conclusions}

We have presented a solution for the potential flow past a cascade of aerofoils with porosity gradients. This extends previous research that has only considered isolated aerofoils (Hajian and Jaworski, 2017) with porosity gradients, or a cascade of rigid aerofoils (chapter 2). By constructing a general singular integral equation, an analytic solution for the potential flow is found. In fact, the solution to this periodic singular integral equation has further applications beyond the present research, including applications to elasticity problems involving periodic arrays of cracks (Ioakmidis and Theocaris, 1977; Mikhlin, 1957; Schmueser and Comninou, 1979). This chapter forms an aerodynamic foundation upon which more sophisticated aeroacoustic analyses of porous cascades can be undertaken. The expression for the steady mean flow paves the way for a Rapid Distortion Theory analysis, possibly by adapting the approach of chapter 3 .

This research leads to an analytic expression of the deflection angle for a cascade of arbitrarily shaped thin aerofoils with porosity gradients, and crystallises the crucial role of trailing edge porosity on the deflection angle. We have also investigated partially porous aerofoils in detail, and found analytic expressions for the lift and drag. These formulae allow careful investigation of the impact of porosity and blade spacing on aerodynamic performance. In particular, we note that the negative effects of porosity on deflection angle, drag and lift are less pronounced for cascades, and these adverse effects can be mitigated further by decreasing the blade spacing. 


\section{List of Symbols}

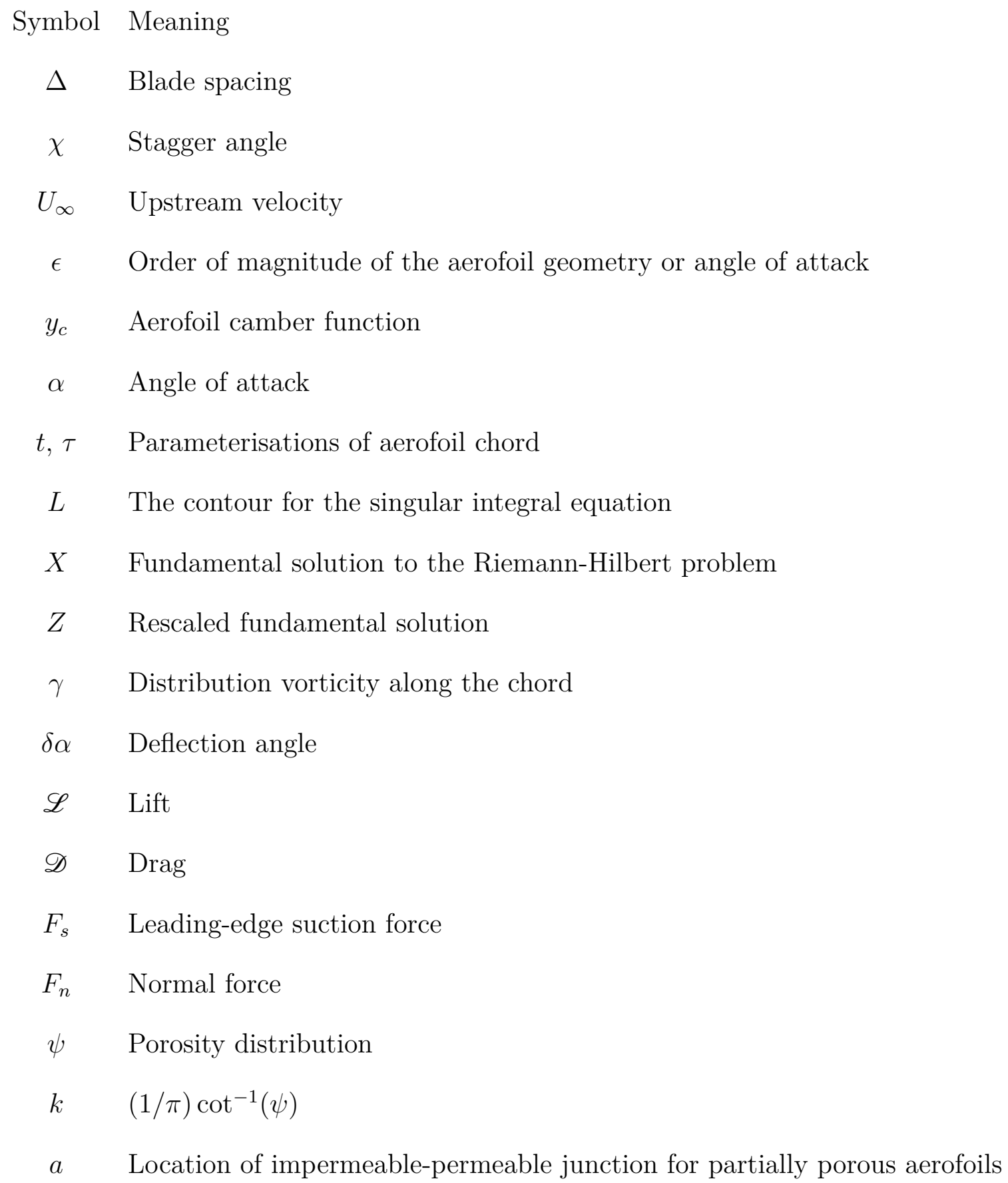




\section{A Alternative Solution Method via Substitution}

We now present an alternative solution to the canonical singular integral equation (4.7) by applying the substitution performed in Antipov (2000). On one hand, the substitution method is an improvement over the method of section 4.3 since it enables us to obtain the exact solution in a more general class of solutions, and is not restricted to the case where $\sum_{k=1}^{2 p} \lambda_{k}=0$ (4.11). On the other hand, this extension is physically meaningless in the context of cascade aerodynamics, and the substitution approach is far more convoluted and consequently obscures the physical meaning encoded into the solution. Nevertheless, we provide some details of the substitution method in this appendix for completeness.

We make the substitutions

$$
\xi=\tanh \left(\frac{\pi t}{\Delta}\right), \quad \zeta=\tanh \left(\frac{\pi \tau}{\Delta}\right)
$$

so that

$$
\begin{aligned}
\operatorname{coth}\left(\frac{\pi(\tau-t)}{\Delta}\right)-1 & =\frac{1-\zeta^{2}}{\xi-\zeta}-\zeta-1, \\
\mathrm{~d} \tau & =\frac{\Delta}{\pi} \frac{\mathrm{d} \xi}{1-\xi^{2}} .
\end{aligned}
$$

These substitutions are equivalent to conformally mapping the period window to a single window where the typical singular integral theory (Muskhelishvili, 1946) can be carried out. Under this substitution, (4.7) becomes

$$
\tilde{A}(\zeta) \tilde{\gamma}(\zeta)+\frac{\tilde{B}(\zeta)}{\mathrm{i} \pi} f_{\tilde{\Gamma}} \tilde{\gamma}(\xi)\left(\frac{1}{\xi-\zeta}-\frac{1}{1-\zeta}\right) \mathrm{d} \xi=\tilde{f}(\zeta)
$$

where

$$
\begin{array}{ccc}
\tilde{\gamma}(\zeta)=\frac{\gamma(t(\zeta))}{1-\zeta^{2}}, & \\
\tilde{A}(\zeta)=\left(1-\zeta^{2}\right) A(t(\zeta)), & \tilde{B}(\zeta)=\left(1-\zeta^{2}\right) B(t(\zeta)), \\
\tilde{\Gamma}=\tanh \left(\frac{\pi \Gamma}{\Delta}\right), & \tilde{f}(\zeta)=f(t(\zeta)) .
\end{array}
$$


We now write

$$
C=\int_{\tilde{\Gamma}} \tilde{\gamma}(\xi) \mathrm{d} \xi
$$

In aerodynamic applications, $C$ corresponds to the circulation around a single aerofoil and must be solved as part of the singular integral problem. In order to proceed with the typical singular integral equation approach (Muskhelishvili, 1946), we must assume that $C$ is known. This enables us to find an expression for $\tilde{\gamma}$ in terms of $C$, which we may then integrate and rearrange to find $C$ and complete the expression for $\tilde{\gamma}$ a posteriori. We now write (4.39) as

$$
\tilde{A}(\zeta) \tilde{\gamma}(\zeta)+\frac{\tilde{B}(\zeta)}{\mathrm{i} \pi} f_{\tilde{\Gamma}} \frac{\tilde{\gamma}(\xi)}{\xi-\zeta} \mathrm{d} \xi=\tilde{F}(\zeta)
$$

where

$$
\tilde{F}(\zeta)=\tilde{f}(\zeta)+\frac{\tilde{B}(\zeta) C}{\mathrm{i} \pi(1-\zeta)}
$$

Now equation (4.40) is in the form of a typical singular integral equation and is therefore directly amenable to the methods of Muskhelishvili (1946). The solution may be written as

$$
\tilde{\gamma}(\zeta)=\tilde{A}^{*}(\zeta) \tilde{F}(\zeta)-\frac{\tilde{A}^{*}(\zeta) \tilde{Z}(\zeta)}{\pi \mathrm{i}} \int_{\tilde{\Gamma}} \frac{\tilde{F}(\xi)}{\tilde{Z}(\xi)(\xi-\zeta)} \mathrm{d} \xi+\tilde{B}^{*}(\zeta) \tilde{Z}(\zeta) P_{\kappa-1}(\zeta)
$$

where

$$
\tilde{Z}(\zeta)=\zeta^{-\kappa / 2} \sqrt{\tilde{A}^{2}(\zeta)-\tilde{B}^{2}(\zeta)} \exp \left[\frac{1}{2 \pi \mathrm{i}} f_{\tilde{\Gamma}} \frac{\log (\tilde{G}(\xi))}{\xi-\zeta} \mathrm{d} \xi\right]
$$

for integer $\kappa$, and

$$
\begin{gathered}
\tilde{A}^{*}=\frac{\tilde{A}(\zeta)}{\tilde{A}^{2}(\zeta)-\tilde{B}^{2}(\zeta)}, \quad \tilde{B}^{*}=\frac{\tilde{B}(\zeta)}{\tilde{A}^{2}(\zeta)-\tilde{B}^{2}(\zeta)}, \\
\tilde{G}(\zeta)=\frac{\tilde{A}(\zeta)-\tilde{B}(\zeta)}{\tilde{A}(\zeta)+\tilde{B}(\zeta)}
\end{gathered}
$$


The final steps of this method involve integrating $\tilde{\gamma}$ to find $C$, and then inverting the substitution (4.36). The solution is equivalent to that obtained in section 4.3.

\section{B Uniqueness of Solutions to the Homogeneous, Periodic Riemann-Hilbert Problem}

We now prove that the solutions of the homogeneous, periodic Riemann-Hilbert problem (4.10) are unique up to a multiplicative constant.

Theorem 2. All solutions of the periodic, homogeneous Riemann-Hilbert problem (4.10) that tend to constants as $|z| \rightarrow \infty$ and share the same classification of special ends, are multiples of (4.11).

Proof. Define $X(z)$ as in (4.11) and let $Y(z)$ also satisfy the Riemann-Hilbert problem (4.10). If we define $\Omega(z)=Y(z) / X(z)$, then $\forall t \in \Gamma$,

$$
\Omega^{+}(t)=\Omega^{-}(t)
$$

since $X$ and $Y$ both satisfy (4.10). Consequently, $\Omega$ is holomorphic everywhere, except possibly at the end points where it may be infinite. However, by our choice of $\lambda_{k}$ at the end points, the degree of infinity of $\Omega$ is always less than one, so corresponds to a constant or a branch point. Since $\Omega$ has only isolated singularities, branch points are not possible, so $\Omega$ is actually holomorphic at the end points. Since $X$ and $Y$ tend to non-zero constants as $|z| \rightarrow \infty, \Omega$ is entire and bounded, so must be constant by Liouville's theorem. Finally,

$$
Y(z)=C X(z)
$$

for some constant $C$. 


\section{Chapter 5}

\section{Scattering by Cascades of Aerofoils with Complex Boundary Conditions}

\subsection{Introduction}

In this chapter we derive analytic solutions for the scattering by cascades where the boundary conditions are not limited to a typical rigid no-flux condition. The method is capable of modelling a range of boundary conditions of practical interest, some of which are detailed below.

Aspirations for lighter and more efficient engines have driven the design of thinner and lighter blades in turbomachinery (Saiz, 2008). As a result, aeroelastic effects such as flutter and resonance must be considered in modern turbomachinery design and testing. The rapid and accurate prediction of the aeroacoustic performance of turbomachinery with consideration of aeroelastic effects is therefore essential in evaluating the appropriateness of potential blade designs. Analytic solutions are excellent candidates for this task (Glegg, 1999; Peake, 1992; Posson et al., 2010b), but are presently limited to rigid blades with no consideration of aeroelastic effects, such as the solution in chapter 3. The present chapter permits the study of compliant blades (Crighton and Leppington, 1970), where blade deforms with a (purely) local response to the pressure gradient across the blade and elastic restoring forces are ignored. This is particularly relevant to marine applications where inertial effects dominate elastic restoring forces and is an important first step towards a more general linearised elastic blade (Cavalieri et al., 2016).

As with the previous chapter, an influential trend in aeroacoustic research is to modify aerofoils with noise reducing technologies. The approach of the present research permits 
porous blades through the assumption of a Darcy-type condition where the seepage velocity through the blade is proportional to the pressure jump across the blade.

In this chapter we extend the analyses of Glegg (1999) and Posson et al. (2010b) to analyse the scattering by a cascade of blades with a range of boundary conditions. The problem is solved with tools from complex variable theory, including the Wiener-Hopf method. Taking a Fourier transform maps the problem into the spectral plane where the Wiener-Hopf analysis is carried out in a similar way to Glegg (1999). An inverse Fourier transform is applied to return the problem to physical space, and contour integration is applied to recover the acoustic field (Posson et al., 2010b). A significant advantage of the presented technique is that the method is identical regardless of the boundary condition - the only effects of modifying the boundary condition are to modify the kernel in the Wiener-Hopf method.

A striking feature of the analysis is that modifications to the boundary conditions do not affect the modal structure of the solution in the far field. In the spectral plane, the only effect of modifying the boundary condition is to vary the locations of the zeros of the Wiener-Hopf kernel. This has a significant effect on the acoustic field in the inter-blade region since the cut-on frequencies of the duct modes are modified to account for energy being absorbed or produced by the blades. The poles of the Wiener-Hopf kernel correspond to the acoustic modes scattered into the far field and are invariant under modifications to the boundary conditions. Consequently, the cut-on frequencies of the acoustic modes are unchanged and the model structure of the upstream and downstream acoustic fields are the same regardless of boundary condition, although the coefficients of these modes do change.

We consider four possible boundary conditions labelled cases 0-III. Physically, case 0 corresponds to rigid blades; case I, porous or compliant blades with no background flow; case II, porous blades with background flow; and case III, a general impedance relation. Mathematically, case 0 corresponds to a Neumann boundary condition; case I, a Robin boundary condition; case II, an oblique derivative boundary condition; and case III, a generalised Cauchy boundary condition.

We begin by presenting a mathematical model for the blade row in section 5.2, including the modelling of the various boundary conditions. We then present some details of the mathematical solution in section 5.3. In section 5.4 we conduct a detailed investigation of the role of blade porosity. In particular, we present a range of results on sound generation and sound transmission. Finally, in section 5.5 we summarise the chapter. 

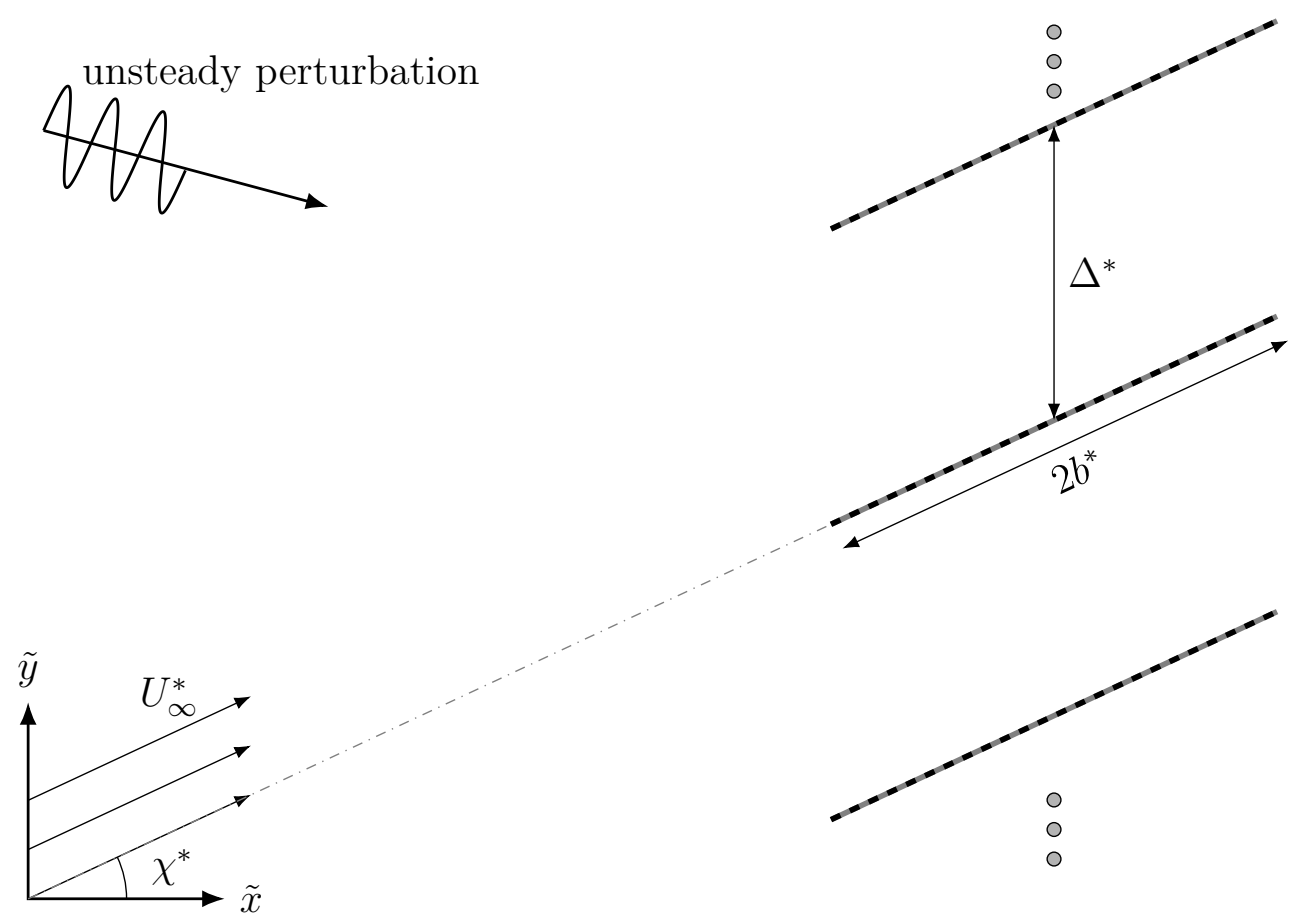

background flow

Fig. 5.1 A rectilinear cascade of flat plates with complex boundaries.

\subsection{Mathematical Formulation}

We consider a rectilinear cascade of blades in a uniform, subsonic flow as illustrated in figure 5.1. As is typical in these analyses (Glegg, 1999; Peake, 1992, 1993), it is useful to rotate the coordinate system so that

$$
\left(x^{*}, y^{*}, z^{*}\right)=\left(\tilde{x} \cos \left(\chi^{*}\right)-\tilde{y} \sin \left(\chi^{*}\right), \tilde{x} \sin \left(\chi^{*}\right)+\tilde{y} \cos \left(\chi^{*}\right), \tilde{z}\right)
$$

and the $x^{*}$ and $y^{*}$ coordinates are tangential and normal to the blades respectively, which have dimensional length $2 b^{*}$. The background flow is tangential to the blades and, in contrast to chapter 3 , may have a spanwise component such that $\boldsymbol{U}_{0}^{*}=\left(U_{\infty}^{*}, 0, W_{\infty}^{*}\right)$, as illustrated in figure 5.2. The blades in the cascade are inclined at stagger angle $\chi^{*}$, and the distance between adjacent blades is $\Delta^{*}$. Consequently, the spacing between blades is simply given by

$$
\left(d^{*}, s^{*}\right)=\Delta^{*}\left(\sin \left(\chi^{*}\right), \cos \left(\chi^{*}\right)\right) .
$$




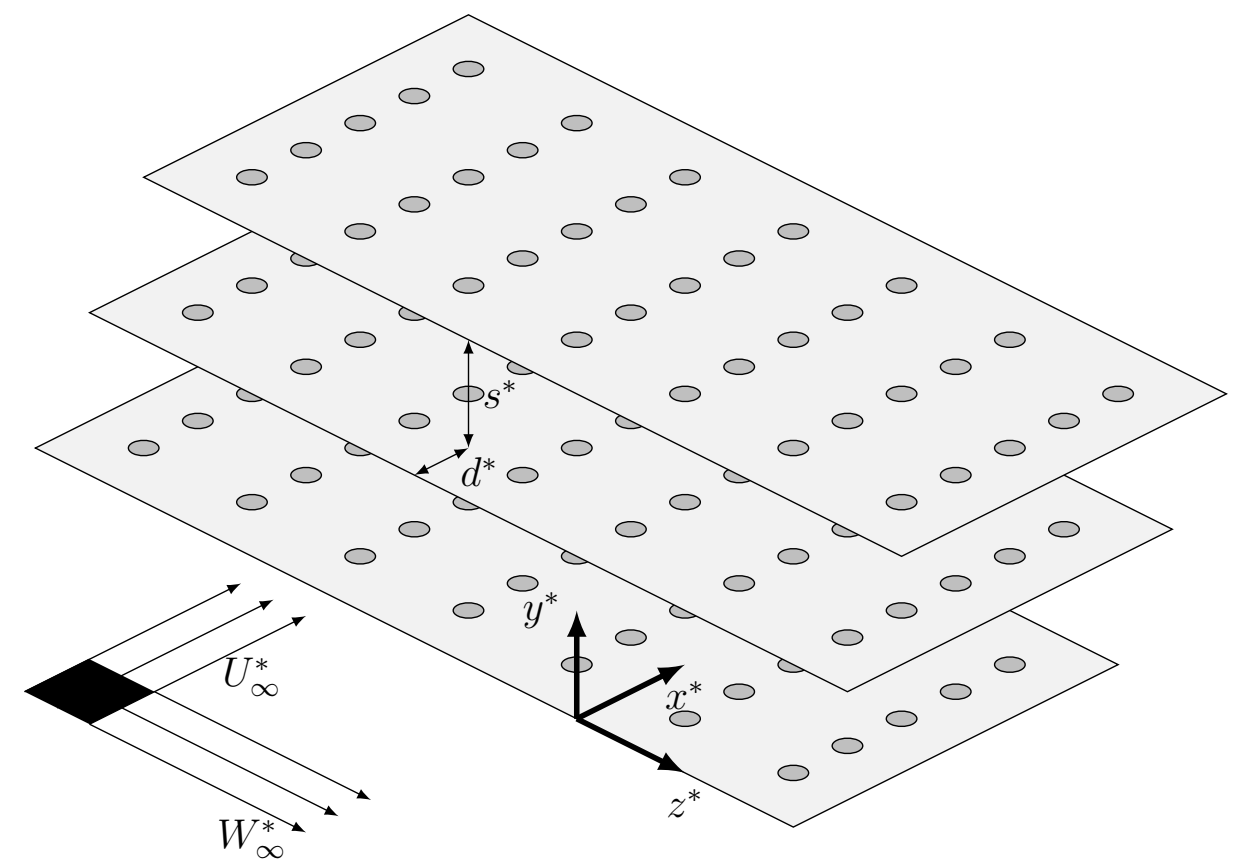

Fig. 5.2 A three-dimensional view of the cascade in the rotated dimensional $\left(x^{*}, y^{*}, z^{*}\right)$ coordinate system. The chordwise and spanwise background velocities are denoted by $U_{\infty}^{*}$ and $W_{\infty}^{*}$ respectively. The complex boundaries are illustrated by the "holes" on each blade, which may represent compliance, porosity, or impedance.

We further assume that a vortical or acoustic wave is incident on the cascade, resulting in a velocity perturbation $\boldsymbol{u}^{*}$ to the mean flow. The Kutta condition is satisfied by ensuring that there is no pressure jump at the blades' trailing edges.

We introduce an acoustic potential function for the scattered field defined by

$$
\nabla \phi^{*}=\boldsymbol{u}^{*}
$$

Consequently, conservation of momentum yields the scattered pressure as

$$
p^{*}=-\rho_{0} \frac{\mathrm{D}_{0} \phi^{*}}{\mathrm{D}_{0} t^{*}},
$$

where the (linearised) convective derivative is defined as

$$
\frac{\mathrm{D}_{0}}{\mathrm{D}_{0} t^{*}}=\frac{\partial}{\partial t^{*}}+\boldsymbol{U}_{0}^{*} \cdot \nabla=\frac{\partial}{\partial t^{*}}+U_{\infty}^{*} \frac{\partial}{\partial x^{*}}+W^{*} \frac{\partial}{\partial z^{*}} .
$$


Accordingly, conservation of mass yields the convected wave equation

$$
\frac{1}{c_{0}^{2}} \frac{\mathrm{D}_{0}^{2} \phi^{*}}{\mathrm{D}_{0} t^{* 2}}-\nabla^{2} \phi^{*}=0
$$

where $c_{0}$ is the isentropic speed of sound.

We suppose that the unsteady perturbation incident on the cascade takes the form

$$
\phi_{i}^{*}=\exp \left[\mathrm{i}\left(k_{x}^{*} x^{*}+k_{y}^{*} y^{*}+k_{z}^{*} z^{*}-\omega^{*} t^{*}\right)\right] .
$$

Since the system is infinite in the spanwise direction, the scattered solution $\boldsymbol{u}^{*}$ must also have harmonic dependence in the $z$-direction. Accordingly, making the following convective transformation and non-dimensionalisations

$$
\begin{gathered}
\phi^{*}\left(x^{*}, y^{*}, z^{*}, t^{*}\right)=U_{\infty}^{*} b^{*} \phi(x, y) \exp \left[\mathrm{i} \omega\left(-M^{2} \delta x+k_{z} z-t\right)\right], \\
d^{*}=b^{*} d, \quad s^{*}=b^{*} \frac{s}{\beta}, \quad \Delta=\sqrt{d^{2}+s^{2}}, \\
x^{*}=b^{*} x, \quad y^{*}=b^{*} \frac{y}{\beta}, \quad z^{*}=b^{*} z, \quad t^{*}=\frac{\omega}{\omega^{*}} t, \\
k_{x}^{*}=\frac{\delta\left(k_{x}-M^{2} \omega\right)}{b^{*}}, \quad k_{y}^{*}=\frac{\omega \beta k_{y}}{b^{*}}, \\
M=U_{\infty}^{*} / c_{0}, \quad \beta=\sqrt{1-M^{2}}, \quad \delta=1 / \beta^{2}, \\
b^{*}
\end{gathered}
$$

reduces $(5.2)$ to

$$
\left(\frac{\partial^{2}}{\partial x^{2}}+\frac{\partial^{2}}{\partial y^{2}}+\omega^{2} w^{2}\right) \phi=0
$$


In terms of these new variables, the scattered pressure (5.1) becomes

$$
p^{*}=-\rho^{*} U^{* 2} p \mathrm{e}^{\mathrm{i}(z-t)}, \quad p=\frac{\partial}{\partial x}\left(\phi \mathrm{e}^{-\mathrm{i} \omega \delta x}\right) \mathrm{e}^{\mathrm{i} \omega x},
$$

where $p$ is the non-dimensional pressure, and the incident perturbation becomes

$$
\phi_{i}^{*}=U^{*} b^{*} \phi^{i}(x, y) \mathrm{e}^{\mathrm{i} \omega\left(-M^{2} \delta x+k_{z} z-t\right)}, \quad \phi_{i}=\exp \left[\mathrm{i}\left(\delta k_{x} x+\omega k_{y} y\right)\right] .
$$

\subsubsection{Boundary Conditions}

We now introduce the boundary conditions for the problem. It is sufficient to specify the behaviour along $y=n s^{ \pm}$for $n \in \mathbb{Z}$. Similarly to chapter 3 , we use $\Delta_{n}$ and $\Sigma_{n}$ to denote the difference and sum of a given quantity either side of the $n^{\text {th }}$ blade or wake.

\subsubsection{Upstream Boundary Condition}

There may be no discontinuities upstream of the blade row. Consequently, we write

$$
\Delta_{n} \phi(x)=0, \quad x<n d .
$$

\subsubsection{Blade Surface Boundary Conditions}

We now introduce several possible blade surface boundary conditions that can be modelled with the present approach. The boundary conditions we consider are the classical impermeable, rigid blade, a porous blade without background flow, a porous blade with background flow, and a general impedance condition. The advantage of this approach is that a spectrum of boundary conditions of practical interest can be modelled without needing to modify the method of solution. As we shall see later, the effect of modifying the boundary condition is to modify the kernel in the ensuing Wiener-Hopf analysis.

\section{Case 0}

When the blade is rigid and impermeable, the no-flux condition is simply

$$
\boldsymbol{u}_{T} \cdot \boldsymbol{n}=0, \quad n d<x<n d+2, \quad y=n s^{ \pm},
$$

where $\boldsymbol{n}$ is the normal vector directed into the blade and $\boldsymbol{u}_{T}$ denotes the total (incident and scattered) velocity field. We sum the contributions of (5.8) either size of each blade 
to obtain

$$
\Sigma_{n}\left[\frac{\partial \phi}{\partial y}\right](x)=-2 w_{0} \exp \left[\mathrm{i}\left(k_{x} \delta(n d+x)+\omega k_{y} n s\right)\right], \quad n d<x<n d+2 .
$$

where $w_{0}=\mathrm{i} \omega k_{y}$ is the non-dimensional amplitude of the normal velocity of the incident perturbation on the $0^{\text {th }}$ blade. This case has been considered in detail in previous research (Glegg, 1999; Posson et al., 2010b), and is detailed further in chapter 3.

\section{Case I}

We now generalise the no-flux condition (5.8) to permit a proportional relationship between the normal velocity and pressure on the surface so that

$$
\boldsymbol{u}_{T} \cdot \boldsymbol{n}=C_{I} p, \quad n d<x<n d+2, \quad y=n s^{ \pm},
$$

for some constant $C_{I}$. Summing the contributions either side of the blade in (5.10) yields

$$
\begin{aligned}
\Sigma_{n}\left[\frac{\partial \phi}{\partial y}\right](x)= & -2 w_{0} \exp \left[\mathrm{i}\left(k_{x} \delta(n d+x)+\omega k_{y} n s\right)\right] \\
& +C_{I} \Delta_{n}[p](x), \quad n d<x<n d+2 .
\end{aligned}
$$

In the absence of background flow $(M=0)$, this condition becomes

$$
\begin{aligned}
\Sigma_{n}\left[\frac{\partial \phi}{\partial y}\right](x)= & -2 w_{0} \exp \left[\mathrm{i}\left(k_{x} \delta(n d+x)+\omega k_{y} n s\right)\right] \\
& +C_{I} \Delta_{n}[\phi](x), \quad n d<x<n d+2 .
\end{aligned}
$$

This boundary condition is capable of modelling a range of scenarios. Early research in aeroelasticity (Crighton and Leppington, 1970) used a boundary condition of the form of (5.12) to analyse the scattering of aerodynamic sound by a compliant plate. In that study, the plate was modelled as possessing inertia, but negligible elastic resistance to deformation. Consequently, the pressure difference across the compliant plate was proportional to the specific mass of the plate multiplied by the acceleration so that, in the notation of the present work, $C_{I}=(-\mathrm{i} \omega m)^{-1}$ where $m$ is the (non-dimensional) mass of the plate per unit area.

Leppington (1977) later showed that the compliant flat plate model is equivalent to that of a rigid screen with periodically arranged circular apertures when the apertures width is small and the wavelength is large compared with the separation. This model has gained popularity as a tool for analysing the aerodynamic scattering of porous 
edges (Ayton, 2016; Jaworski and Peake, 2013; Kisil and Ayton, 2018). In this case, the non-dimensional porosity parameter is

$$
C_{I}=\frac{\alpha_{H} K_{R}}{\pi R^{2}}
$$

where $R$ is the radius of the circular apertures of radius, $K_{R}$ is the Rayleigh conductivity, and the fractional open area is $\alpha_{H}$.

\section{Case II}

In the presence of a background flow, the boundary condition (5.11) becomes

$$
\begin{aligned}
\Sigma_{n}\left[\frac{\partial \phi}{\partial y}\right](x)= & -2 w_{0} \exp \left[\mathrm{i}\left(k_{x} \delta(n d+x)+\omega k_{y} n s\right)\right] \\
& +C_{I I}\left(\mathrm{i} \omega \delta \Delta_{n}[\phi](x)-\Delta_{n}\left[\phi_{x}\right](x)\right), \quad n d<x<n d+2,
\end{aligned}
$$

where $C_{I I}$ is a constant and we have applied (5.5). It is worth pointing out that, unlike $C_{I}$, we do not have an expression for $C_{I I}$ in terms of physical parameters. Further work is required to derive a physically meaningful expression for $C_{I I}$.

\section{Case III}

We may also consider the effects of an impedance boundary condition. In the presence of background flow, the impedance boundary condition is given by Myers (1980)

$$
\boldsymbol{u}_{T} \cdot \boldsymbol{n}=\left(\mathrm{i} \omega+\boldsymbol{U}_{0} \cdot \nabla-\boldsymbol{n} \cdot\left(\boldsymbol{n} \cdot \nabla \boldsymbol{U}_{0}\right)\right) \frac{p}{\mathrm{i} \omega Z} .
$$

The real part of the impedance $Z$ is termed the acoustic resistance and represents the energy transfer of the blade: if $\mathbb{R e}[Z]>0$ then the blades absorb energy whereas if $\mathbb{R e}[Z]<0$ then the blades produce energy. Since the flow is uniform, this condition applied on the upper and lower surfaces of the blades becomes

$$
v_{T}^{ \pm}=\mp\left(-\mathrm{i} \omega+U \frac{\partial}{\partial x}+W \frac{\partial}{\partial z}\right) \frac{p}{\mathrm{i} \omega Z} .
$$

We now sum the upper and lower components of this impedance condition to obtain a condition on the sum of the velocity either side of the blade. In terms of non-dimensional variables, the condition becomes

$$
\Sigma_{n}\left[\frac{\partial \phi}{\partial y}\right](x)=-2 w_{0} \exp \left[\mathrm{i}\left(k_{x} \delta(n d+x)+\omega k_{y} n s\right)\right]
$$




\begin{tabular}{ccccc}
\hline Case & Model & $\mu_{0}$ & $\mu_{1}$ & $\mu_{2}$ \\
\hline $\begin{array}{c}\text { Case 0 } \\
{[1,2]}\end{array}$ & rigid, impermeable & 0 & 0 & 0 \\
Case I & porous, compliant \\
{$[3,4,5,6]$} & (no background flow) & $C_{I}$ & 0 & 0 \\
Case II & $\begin{array}{c}\text { porous } \\
\text { (with background flow) }\end{array}$ & $\mathrm{i} \omega \delta C_{I I}$ & $-C_{I I}$ & 0 \\
Case III & impedance & $-2 \omega^{2}\left(1+W k_{z}\right) C_{I I I}$ & $-2 \mathrm{i} M^{2} \omega C_{I I I}$ & $C_{I I I}$ \\
{$[7,8]$} & & & &
\end{tabular}

Table 5.1 Summary of possible boundary conditions and corresponding $\mu_{0}, \mu_{1}$ and $\mu_{2}$ values for equation (5.15). The references highlight relevant papers, although only $[1,2]$ consider cascade geometries and are restricted to rigid boundaries. The reference numbers correspond to [1] (Glegg, 1999), [2] (Posson et al., 2010b), [3] (Leppington, 1977), [4] (Howe, 1998), [5] (Jaworski and Peake, 2013), [6] (Kisil and Ayton, 2018), [7] (Myers, 1980), and [8] (Brambley, 2009).

$$
+C_{I I I}\left(-2 \omega^{2}\left(1+W k_{z}\right) \Delta_{n}[\phi](x)-2 \mathrm{i} M^{2} \omega \Delta_{n}\left[\phi_{x}\right](x)+\Delta_{n}\left[\phi_{x, x}\right](x)\right)
$$

where $C_{I I I}=U^{3} /(\mathrm{i} \omega Z)$.

\section{Summary of Blade Surface Boundary Conditions}

We may characterise all the modified boundary conditions (5.12), (5.13) and (5.14) in the general form

$$
\begin{aligned}
\Sigma_{n}\left[\frac{\partial \phi}{\partial y}\right](x) & =-2 w_{0} \exp \left[\mathrm{i}\left(k_{x} \delta(n d+x)+\omega k_{y} n s\right)\right] \\
& +\mu_{0} \Delta_{n}[\phi](x)+\mu_{1} \Delta_{n}\left[\phi_{x}\right](x)+\mu_{2} \Delta_{n}\left[\phi_{x, x}\right](x), \quad n d<x<n d+2,
\end{aligned}
$$

where the $\mu_{n}$ are summarised in table 5.1 for the different boundary conditions. Furthermore, in the present analysis we do not allow any added mass and enforce that there is no jump in the normal velocity either side of the plate. Accordingly, we may write

$$
\Delta_{n}\left[\frac{\partial \phi}{\partial y}\right](x)=0, \quad n d<x<n d+2 .
$$




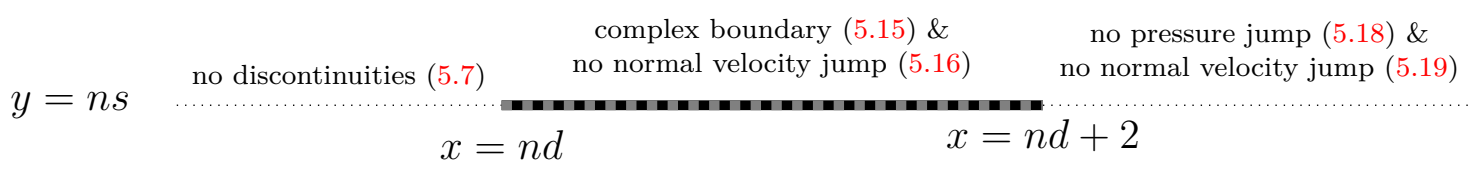

Fig. 5.3 Schematic illustrating where each boundary condition is applied.

\subsubsection{Downstream Boundary Conditions}

Downstream, we require the pressure jump across the wake to vanish:

$$
\Delta_{n}[p](x)=0, \quad x>2+n d .
$$

By employing the pressure definition (5.5) and integrating with respect to $x$, we may write the above condition as

$$
\Delta_{n}[\phi](x)=2 \pi \mathrm{i} P \exp [\mathrm{i} \omega \delta x], \quad x>n d+2,
$$

where $P$ is a constant of integration that will be specified by enforcing the Kutta condition.

Additionally, the normal velocity across the wake must vanish, i.e.

$$
\Delta_{n}\left[\frac{\partial \phi}{\partial y}\right](x)=0, \quad x>n d+2
$$

\subsubsection{Summary of Full Boundary Conditions}

All in all, we have five boundary conditions. In the upstream region we do not permit any discontinuities (5.7). Along each blade we have a relation for the sum of normal velocities either side of the blade (5.15), and do not permit a jump in normal velocity across the blade (5.16). Finally, across the wake we do not permit a jump in pressure (5.18) or normal velocity (5.19). The boundary conditions are illustrated in figure 5.3. This completes the description of the mathematical model.

\subsection{Solution}

We now present the mathematical solution to the Helmholtz equation (5.4) subject to the boundary conditions (5.7), (5.15), (5.16), (5.18) and (5.19). For clarity, we present a "road map" of the solution in figure 5.4. The analysis is similar to that in chapter 3, and therefore we only present the pertinent points. 
As is typical in cascade acoustics problems we employ integral transforms to obtain a solution that is uniformly valid throughout the entire domain (Glegg, 1999; Peake, 1992; Posson et al., 2010b). However, $\phi$ is discontinuous across each blade and wake in the $y$-direction. Therefore, $\partial \phi / \partial y$ possesses non-integrable singularities thus preventing the application of a Fourier transform. Consequently, we must regularise the derivatives of $\phi$ and to remove these non-integrable singularities. To this end, we introduce generalised derivatives (Lighthill, 1958) and write

$$
\frac{\partial^{2} \phi}{\partial y^{2}}=\frac{\tilde{\partial}^{2} \phi}{\tilde{\partial} y^{2}}-\sum_{n=-\infty}^{\infty} \Delta_{n}[\phi](x) \delta^{\prime}(y-n s)-\sum_{n=-\infty}^{\infty} \Delta_{n}\left[\frac{\partial \phi}{\partial y}\right](x) \delta(y-n s),
$$

where $\tilde{\partial}$ represents the partial derivative with discontinuities removed. The second term in (5.20) vanishes because there is zero jump in normal velocity across the blade (5.16) and wake (5.19).

The scattered solution must obey the same quasi-periodicity relation as the incident field (5.3). Consequently, the scattered acoustic potential function in the entire plane may be reduced to that of a single channel in the domain by writing

$$
\phi(x+n d, y+n s)=\phi(x, y) \mathrm{e}^{\mathrm{i} n \sigma^{\prime}},
$$

where the inter-blade phase angle for $\phi$ is $\sigma^{\prime}=k_{x} \delta d+\omega k_{y} s$. Substituting (5.20) into the Helmholtz equation (5.4) and applying the inter-blade phase angle relation (5.21) yields

$$
\frac{\partial^{2} \phi}{\partial x^{2}}+\frac{\partial^{2} \phi}{\partial y^{2}}+\omega^{2} w^{2} \phi=\sum_{n=-\infty}^{\infty} \Delta_{0}[\phi](x-n d) \delta^{\prime}(y-n s) \mathrm{e}^{\mathrm{i} n \sigma^{\prime}}
$$

Physical plane $\quad$ Spectral plane

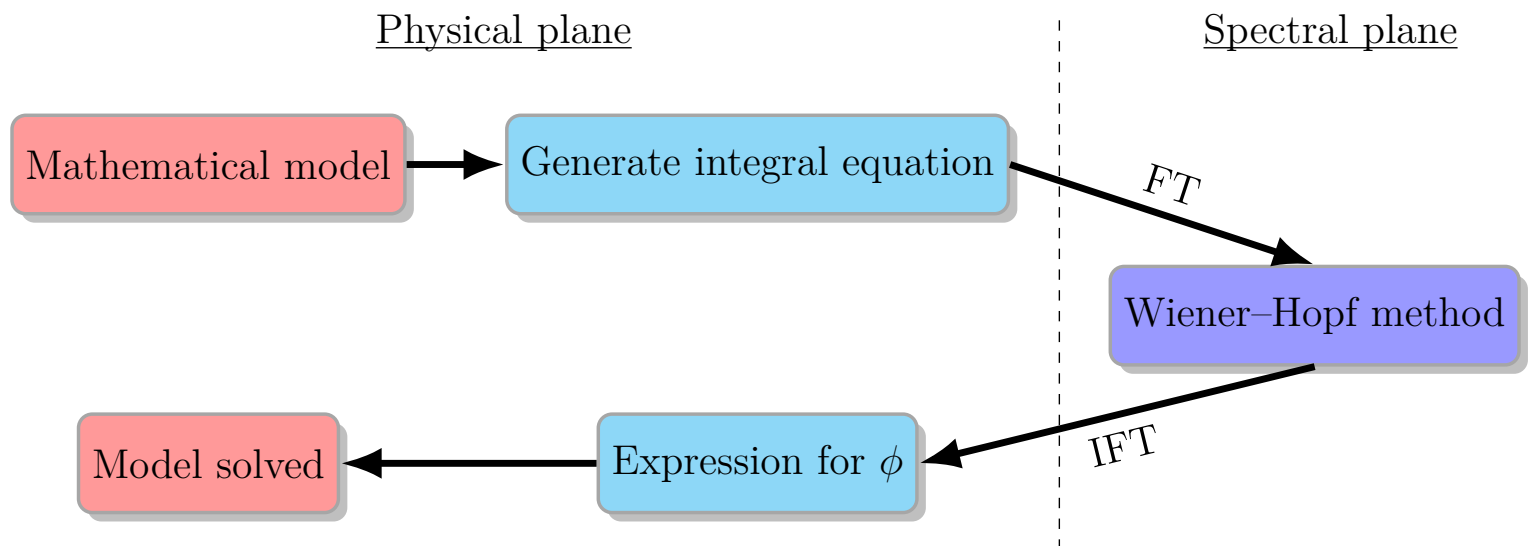

Fig. 5.4 Schematic diagram illustrating the solution method. The abbreviations "FT" and "IFT" stand for "Fourier transform" and "Inverse Fourier transform" respectively. 
We define the Fourier integral transform and its inverse as

$$
\begin{aligned}
& F(\gamma, \eta)=\frac{1}{(2 \pi)^{2}} \int_{-\infty}^{\infty} \int_{-\infty}^{\infty} f(x, y) \mathrm{e}^{\mathrm{i} \gamma x+\mathrm{i} \eta y} \mathrm{~d} x \mathrm{~d} y \\
& f(x, y)= \\
& \int_{-\infty}^{\infty} \int_{-\infty}^{\infty} F(\gamma, \eta) \mathrm{e}^{-\mathrm{i} \gamma x-\mathrm{i} \eta y} \mathrm{~d} \gamma \mathrm{d} \eta
\end{aligned}
$$

Applying the transform to the left-hand side of (5.22) yields

$$
\left(-\gamma^{2}-\eta^{2}+\omega^{2} w^{2}\right) \Phi(\gamma, \eta)=\frac{1}{2 \pi \mathrm{i}} \sum_{n=-\infty}^{\infty} \eta D(\gamma) \mathrm{e}^{\mathrm{i} n\left(\sigma^{\prime}+\gamma d+\eta s\right)},
$$

Similarly to chapter 3 , the problem is now to find $D(\gamma)$ which represents the Fourier transform of the jump in acoustic potential either side of the blade and wake. We invert the Fourier transform to obtain an expression for the acoustic potential in terms of $D$ :

$$
\phi(x, y)=\frac{1}{2 \pi \mathrm{i}} \int_{-\infty}^{\infty} \int_{-\infty}^{\infty} \frac{\mathrm{e}^{-\mathrm{i} \gamma x-\mathrm{i} \eta y}}{\omega^{2} w^{2}-\eta^{2}-\gamma^{2}} \cdot \sum_{n=-\infty}^{\infty} \eta D(\gamma) \mathrm{e}^{\mathrm{i} n\left(\sigma^{\prime}+\gamma d+\eta s\right)} \mathrm{d} \gamma \mathrm{d} \eta
$$

The $\eta$ integral may be performed by closing the contour of integration in an appropriate upper or lower half-plane to obtain

$$
\phi(x, y)=-\frac{1}{2} \int_{-\infty}^{\infty} \sum_{n=-\infty}^{\infty} D(\gamma) \operatorname{sgn}(n s-y) \mathrm{e}^{\mathrm{i} n\left(\sigma^{\prime}+\gamma d\right)+\mathrm{i} \zeta|n s-y|} \mathrm{e}^{-\mathrm{i} \gamma x} \mathrm{~d} \gamma
$$

where $\zeta=\sqrt{\omega^{2} w^{2}-\gamma^{2}}$. The branch cut is defined so that $\operatorname{Im}[\zeta]>0$ when $\gamma$ is in a strip for the Wiener-Hopf method.

We now differentiate (5.25) with respect to $y$ and consider the limits $y \rightarrow 0^{ \pm}$. Summing the contributions from each of these limits yields the integral equation

$$
\Sigma_{0}\left[\frac{\partial \phi}{\partial y}\right](x)=-4 \pi \int_{-\infty}^{\infty} D(\gamma) j(\gamma) \mathrm{e}^{-\mathrm{i} \gamma x} \mathrm{~d} \gamma
$$

where

$$
j(\gamma)=\frac{\mathrm{i} \zeta}{4 \pi} \sum_{n \in \mathbb{Z}} \mathrm{e}^{\mathrm{i} n\left(\sigma^{\prime}+\gamma d\right)+\mathrm{i} \zeta|n s|}=\frac{\zeta}{4 \pi} \cdot \frac{\sin (\zeta s)}{\cos (\zeta s)-\cos \left(\gamma d+\sigma^{\prime}\right)} .
$$

We now solve equation (5.26) subject to the remaining boundary conditions applied on $y=0$ :

$$
\Delta_{0}[\phi](x)=0, \quad x<0,
$$




$$
\begin{aligned}
\Sigma_{0}\left[\frac{\partial \phi}{\partial y}\right](x) & =\mu_{0} \Delta_{0}[\phi](x)+\mu_{1} \Delta_{0}\left[\phi_{x}\right](x)+\mu_{2} \Delta_{0}\left[\phi_{x, x}\right](x) & \\
& -2 w_{0} \exp \left[\mathrm{i} k_{x} \delta x\right] & 0<x<2, \\
\Delta_{0}[\phi](x) & =2 \pi \mathrm{i} P \exp [\mathrm{i} \delta \omega x], & x>2 .
\end{aligned}
$$

The system (5.26, 5.28.a, 5.28.b, 5.28.c) represents an integral equation subject to mixed value boundary conditions. We solve this system with the Wiener-Hopf method in appendix 5.A. The solution for $D$ is given by

$$
\begin{aligned}
D(\gamma) & =\frac{w_{0}}{(2 \pi)^{2} \mathrm{i}\left(\gamma+\delta k_{x}\right) K_{-}\left(-\delta k_{x}\right) K_{+}(\gamma)}+\frac{w_{0} \delta\left(\omega-k_{x}\right) \mathrm{e}^{2 \mathrm{i}\left(\gamma+\delta k_{x}\right)}}{(2 \pi)^{2} \mathrm{i}\left(\gamma+\delta k_{x}\right)(\gamma+\delta \omega) K_{+}\left(-\delta k_{x}\right) K_{-}(\gamma)} \\
& -\sum_{n=0}^{\infty} \frac{\left(\mathscr{A}_{n}+\mathscr{C}_{n}\right) \mathrm{e}^{2 \mathrm{i}\left(\gamma-\theta_{n}^{-}\right)}}{\mathrm{i}(\gamma+\delta \omega)\left(\gamma-\theta_{n}^{-}\right)} \cdot \frac{K_{-}\left(\theta_{n}^{-}\right)}{K_{-}(\gamma)}-\sum_{n=0}^{\infty} \frac{\mathscr{B}_{n}}{\gamma-\theta_{n}^{+}} \cdot \frac{K_{+}\left(\theta_{n}^{+}\right)}{K_{+}(\gamma)}
\end{aligned}
$$

where all new variables are defined in appendix 5.A. Note that the solution is identical to that for the rigid cascade (Glegg, 1999), except the original Wiener-Hopf kernel $j$ is now replaced with the modified kernel $K$. This original kernel is recovered when $\mu_{1}=\mu_{2}=\mu_{3}=0$.

\subsubsection{Inversion of Fourier Transform}

We now invert the Fourier transform of the acoustic field in the previous section. Since $D$ is now known, the Fourier inversion integral in (5.25) can now be computed. Similarly to the analysis in chapter 3 and Posson et al. (2010b), the inversion is performed by splitting the physical domain into five regions as illustrated in figure 5.5.

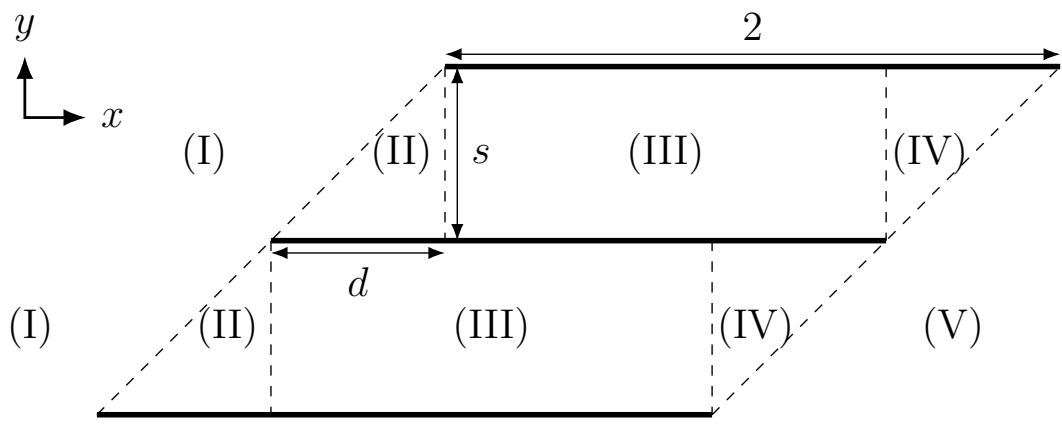

Fig. 5.5 Diagram indicating the different regions in the $(x, y)$-plane which require different areas of contour integration in the Fourier inversion. 
The details can be found in Appendix 5.B and the final results are stated below. All undefined functions are defined in Appendices 5.A and 5.B.

\subsubsection{Upstream Region (I)}

In the upstream region,

$$
\phi(x, y)=\pi \mathrm{i} \sum_{m=-\infty}^{\infty} D^{(1,3)}\left(\lambda_{m}^{+}\right) A^{r}\left(\lambda_{m}^{+}, x, y\right)
$$

\subsubsection{Inter-Blade Upstream Region (II)}

In the inter-blade upstream region,

$$
\begin{aligned}
\phi(x, y)= & -\pi \sum_{n=0}^{\infty} \frac{\mathscr{A}_{n}+\mathscr{C}_{n}}{\theta_{n}^{-}+\delta \omega} A_{d}\left(\theta_{n}^{-}, x, y\right)-\pi \mathrm{i} \sum_{n=0}^{\infty} \mathscr{B}_{n} A_{d}\left(\theta_{n}^{+}, x, y\right) \\
& -\pi \frac{A_{d}\left(-\delta k_{x}, x, y\right)}{K\left(-\delta k_{x}\right)} \cdot \frac{w_{0}}{(2 \pi)^{2}}+\pi \mathrm{i} \sum_{m=-\infty}^{\infty} D^{(1,3)}\left(\lambda_{m}^{+}\right) A_{u}^{r}\left(\lambda_{m}^{+}, x, y\right) .
\end{aligned}
$$

\subsubsection{Inter-Blade Inner Region (III)}

In the inter-blade inner region,

$$
\begin{aligned}
\phi(x, y)= & -\pi \sum_{n=0}^{\infty} \frac{\mathscr{A}_{n}+\mathscr{C}_{n}}{\theta_{n}^{-}+\delta \omega} A\left(\theta_{n}^{-}, x, y\right)-\pi \mathrm{i} \sum_{n=0}^{\infty} \mathscr{B}_{n} A\left(\theta_{n}^{+}, x, y\right) \\
& -\pi \frac{A\left(-\delta k_{x}, x, y\right)}{K\left(-\delta k_{x}\right)} \cdot \frac{w_{0}}{(2 \pi)^{2}} .
\end{aligned}
$$

\subsubsection{Inter-Blade Downstream Region (IV)}

In the inter-blade downstream region,

$$
\begin{aligned}
\phi(x, y)= & -\pi \sum_{n=0}^{\infty} \frac{\mathscr{A}_{n}+\mathscr{C}_{n}}{\theta_{n}^{-}+\delta \omega} A_{u}\left(\theta_{n}^{-}, x, y\right)-\pi \mathrm{i} \sum_{n=0}^{\infty} \mathscr{B}_{n} A_{u}\left(\theta_{n}^{+}, x, y\right) \\
& -\pi \frac{A_{u}\left(-\delta k_{x}, x, y\right)}{K\left(-\delta k_{x}\right)} \cdot \frac{w_{0}}{(2 \pi)^{2}} \\
& -\pi \mathrm{i} \sum_{m=-\infty}^{\infty} D^{(2,4)}\left(\lambda_{m}^{-}\right) A_{d}^{r}\left(\lambda_{m}^{-}, x, y\right)+\pi \mathrm{i} P A_{d}(-\delta \omega, x, y) .
\end{aligned}
$$




\begin{tabular}{cccccc}
\hline Reference & $\begin{array}{c}\text { gap-to- } \\
\text { chord } \\
\text { ratio } \\
\Delta / 2\end{array}$ & $\begin{array}{c}\text { stagger } \\
\text { angle } \\
\chi\end{array}$ & $\begin{array}{c}\text { Mach } \\
\text { number } \\
M\end{array}$ & $\begin{array}{c}\text { reduced } \\
\text { frequency } \\
\omega\end{array}$ & $\begin{array}{c}\text { inter-blade } \\
\text { phase } \\
\text { angle } \\
\sigma\end{array}$ \\
\hline Glegg (1999) & 0.6 & $40^{\circ}$ & 0.3 & $0-40$ & $3 \pi / 4$ \\
Posson et al. (2010b) & $\sec \left(20^{\circ}\right)$ & $20^{\circ}$ & 0.2 & 5 & $3 \pi / 4$
\end{tabular}

Table 5.2 Summary of parameters used in results section.

\subsubsection{Downstream Region (V)}

In the downstream region,

$$
\phi(x, y)=-\pi \mathrm{i} \sum_{m=-\infty}^{\infty} D^{(2,4)}\left(\lambda_{m}^{-}\right) A^{r}\left(\lambda_{m}^{-}, x, y\right)+\pi \mathrm{i} P A(-\delta \omega, x, y)
$$

\subsection{Results}

We now use the analytic solution derived in section 5.3.1 to explore the aeroacoustic performance of a blade row with modified boundary conditions. In particular, we focus on the role of porosity due to its potential to attenuate sound (Ayton, 2016; Jaworski and Peake, 2013). The results show significant sound reductions for modest changes in porosity. We argue that this is attributed to the strong effect of porosity on the unsteady loading: in cascade configurations, the blade loading changes the upstream and downstream flows and therefore influences the intensity of the scattered sound.

The present method is valid for both sound generation and sound transmission analyses, both of which we consider in the following results.

\subsubsection{Sound Generation}

Sound generation is caused when a pressure-free gust (i.e. $k_{x}=\omega$ ) interacts with the blade row, resulting in the production of pressure waves. In order to enable comparison against prior works, we consider cases analysed by Glegg (1999) and Posson et al. (2010b) as defined in table 5.2. 


\subsubsection{Unsteady Lift}

During the solution to the Wiener-Hopf problem associated with the scattering by a blade row with complex boundaries, we observed that the major difference with the rigid case is that the duct modes are modified. Consequently, we expect complex boundary conditions to have a significant effect of the unsteady loading of the blades. In this section we test that hypothesis.

The analytic expression for $D$ (5.29) enables the swift calculation of the unsteady loading on the blades. The unsteady loading is defined as the integral of the unsteady pressure over the blade surface:

$$
C_{p}=\frac{1}{2 \pi w_{0}} \int_{0}^{2} \Delta_{0}[p](x) \mathrm{d} x
$$

Integration by parts and application of the boundary conditions (5.28.a) and (5.28.c) yields the identity

$$
D\left(-\omega \delta M^{2}\right)=\frac{1}{2 \pi} \int_{-\infty}^{\infty} \Delta_{0}[\phi](x) \mathrm{e}^{-\mathrm{i} \omega \delta M^{2} x} \mathrm{~d} x=-\frac{1}{\mathrm{i} \omega 2 \pi} \int_{0}^{2} \frac{\partial}{\partial x}\left(\Delta_{0}[\phi](x) \mathrm{e}^{-\mathrm{i} \omega \delta x}\right) \mathrm{e}^{\mathrm{i} \omega x} \mathrm{~d} x .
$$

Consequently, the normalised unsteady lift (5.32) may be written as

$$
C_{p}=\frac{-\mathrm{i} \omega}{w_{0}} D\left(-\omega \delta M^{2}\right)
$$

The modified boundary conditions have a strong effect on the unsteady loading, as illustrated in figure 5.6. The unsteady loading for a rigid cascade is compared against the loading for a range of porosity parameters, which correspond to the $C_{I I}$ values. The results indicate that the effect of modified boundary conditions is to shift the locations of the channel modes, as indicated by the shifts in the local maximum around $\omega \approx 12$, which has previously been identified with the cut-on frequency of the channel mode (Glegg, 1999). As $C_{I I}$ increases, the pressure jump across the blade must decrease in accordance with Darcy's law (5.13), thus ensuring that the seepage velocity through the blade is proportional to the pressure jump across the blade. This is observed in figure 5.6 , where the effects of increasing the porosity result in an almost uniform reduction in the unsteady lift.

The impact of porosity on unsteady lift appears most pronounced when the frequency is low. In particular, the range $0<\omega<7.62$ shows the largest reduction in lift. After 


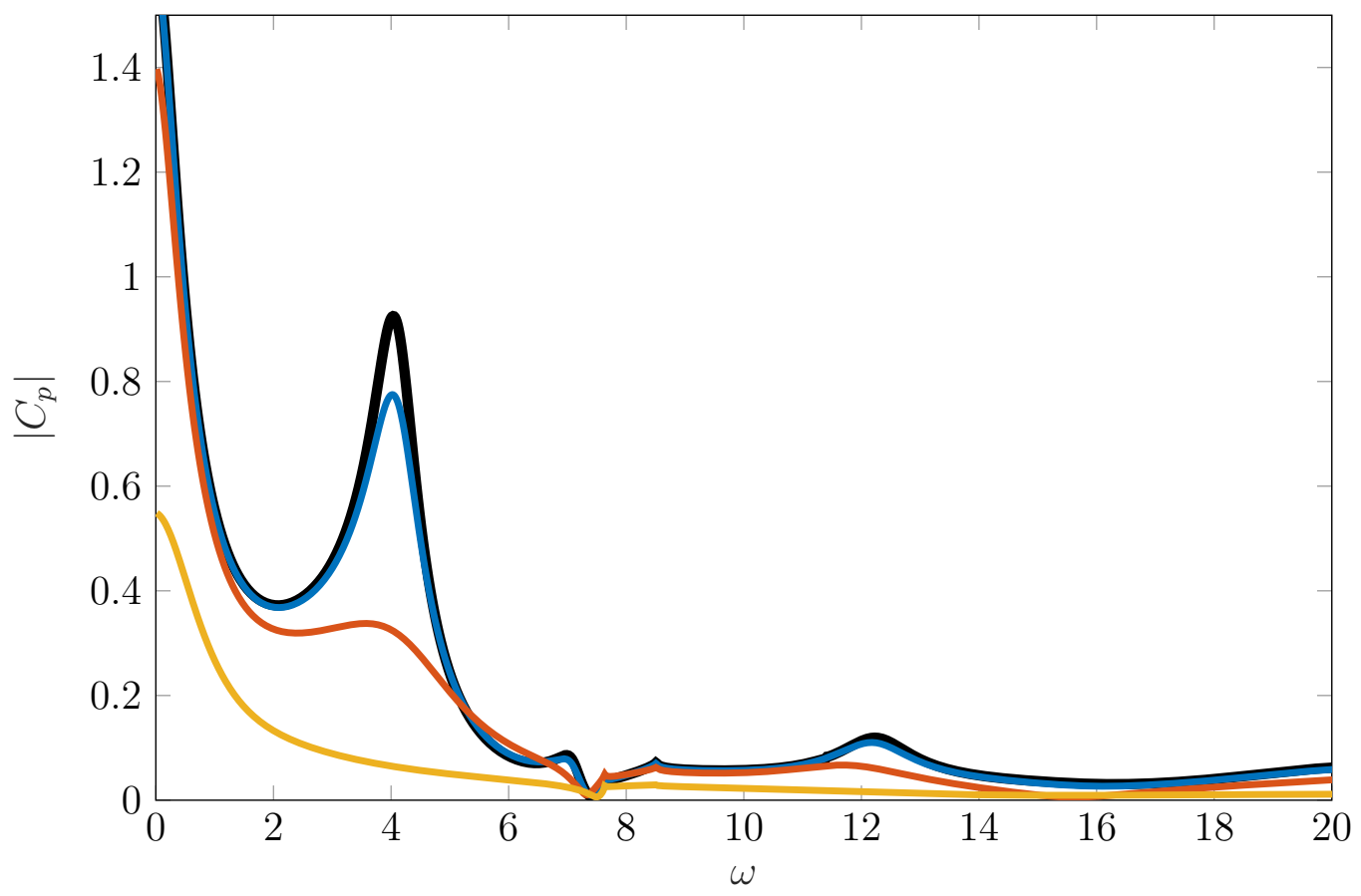

Fig. 5.6 Unsteady lift for a range of frequencies and porosities. The aerodynamic and aeroacoustic parameters are defined in table 5.2 and correspond to those in figure 3 of Glegg (1999). The colours correspond to the porosity parameters $\boldsymbol{C}_{\boldsymbol{I} \boldsymbol{I}}=\mathbf{0}$ (i.e. rigid), $C_{I I}=.01, C_{I I}=.1$ and $C_{I I}=1$. 
the acoustic mode is cut-on at $\omega \approx 7.62$, the reductions in lift are generally smaller, but this is possibly because the lift on the rigid cascade is also reduced.

Another reason for the reduction in unsteady lift is that porosity reduces the build-up of pressure in the inter-vane channel by allowing the flow field to dissipate energy through the blades. Accordingly, modes are less likely to become "trapped" in the inter-blade region, since fluid is now permitted to travel across the blades. Consequently, the unsteady lift is greatly reduced since the cascade produces a lower pressure jump across each blade.

\subsubsection{Sound Power Output}

Analytical expressions for the sound power output are available by a similar method to Glegg (1999). The modal upstream or downstream sound power output for the $m$-th mode is given by

$$
W_{ \pm}(m)=\frac{\omega \pi^{2}}{\Delta} \mathbb{R e}\left[\frac{\left|\zeta_{m}^{ \pm} D\left(\lambda_{m}^{ \pm}\right)\right|^{2}}{\sqrt{\omega^{2} w^{2}-f_{m}^{2}}}\right]
$$

As noted multiple times in this chapter, modifications to the surface boundary conditions do not affect the acoustic modes $\lambda_{m}^{ \pm}$. Consequently, the cut-on frequencies of these upstream and downstream modes are unaffected by porosity, for example. This is observed in figure $5.7 \mathrm{a}$, where the downstream sound power output for the first, second and third modes are illustrated for a large frequency range. Clearly the modes are cut-on at the same frequency, but the magnitude of the sound power output is strongly affected by porosity. For small porosities $\left(C_{I I}=0.01,0.1\right)$ there is little impact on the sound power output of the first mode until the channel modes become cut-on at $\omega \approx 12$. Following this cut-on frequency, we observe a large decrease in the sound power for all modes. Similarly to the unsteady lift, this reduction in sound power output can be attributed to the reduction in the pressure jump across the blade caused by porosity, thereby reducing the scattered sound in the upstream and downstream regions.

We define the sound power level as

$$
L_{W_{ \pm}}(m)=10 \log _{10}\left(\frac{W_{ \pm}(m)}{W_{r} \pm(m)}\right) \mathrm{dB}
$$

where we take the reference sound power $W_{r \pm}$ to be the sound power for a rigid blade row. The reduction in sound power level is illustrated in figure 5.8 for a rage of porosity 
parameters. We observe that even a modest porosity of $C_{I I}=0.1$ is capable of large sound power reductions of $5 \mathrm{~dB}$ for the first mode and $20 \mathrm{~dB}$ for the second mode.

\subsubsection{Sound Transmission}

We now use our model to investigate sound transmission through a cascade of flat plates with complex boundaries. Sound transmission occurs when a sound wave interacts with a cascade of blades, and is reflected and transmitted through the blade row. In order to explore the effects of modified boundary conditions on the transmission and reflection, we follow Bouley et al. (2017) and write the incident acoustic field (5.6) in the form

$$
\phi_{i}=\mathrm{e}^{\mathrm{i} \zeta_{0}^{+} y} \mathrm{e}^{-\mathrm{i} \lambda_{0}^{+} x}, \quad x \leq y d / h,
$$

where the mode is assumed cut-on, so that $\lambda_{0}^{+}$is real. Comparison with the solution in section 5.3.1 shows that the reflected acoustic field takes the form

$$
\phi_{r}=\sum_{m=-\infty}^{\infty} R_{m} \mathrm{e}^{\mathrm{i} \zeta_{m}^{+} y} \mathrm{e}^{-\mathrm{i} \lambda_{m}^{+} x}, \quad x \leq y d / h
$$

and the transmitted acoustic potential takes the form

$$
\phi_{t}=\sum_{m=-\infty}^{\infty} T_{m} \mathrm{e}^{-\mathrm{i} \zeta_{m}^{-} y} \mathrm{e}^{-\mathrm{i} \lambda_{m}^{-}(x-2)}, \quad x \leq 2+y d / h,
$$

where $\boldsymbol{R}$ and $\boldsymbol{T}$ are the vectors of the reflection and transmission coefficients respectively. Note that the mode corresponding to the jump in acoustic potential across the wake $(\delta \omega)$ is not included in this analysis since it does not contribute to the pressure field.

By comparison with (5.30) and (5.31) we obtain the expressions for the transmission and reflection coefficients as

$$
R_{m}=+\frac{\pi \zeta_{m}^{+} D^{(1,3)}\left(\lambda_{m}^{+}\right)}{\sqrt{\omega^{2} w^{2}-f_{m}^{2}}}, \quad T_{m}=-\frac{\pi \zeta_{m}^{-} D^{(2,4)}\left(\lambda_{m}^{-}\right)}{\sqrt{\omega^{2} w^{2}-f_{m}^{2}}} \mathrm{e}^{-2 \mathrm{i} \lambda_{m}^{-}}
$$

We now perform a parametric study on the effects of porosity on the transmission and reflection coefficients. In figure 5.9, we plot the total pressure field and the associated amplitude of the transmission and reflection coefficients. The blades are rigid $\left(C_{I I}=0\right)$ in figures 5.9a.i and 5.9a.ii. In this case, two modes are cut-on, as indicated in figure 5.9a.ii. When the cascade is rigid, the blade row has a strong effect on the reflected and transmitted fields, which can be observed by the significant distortion of the acoustic field in figure 5.9a.i. In figure 5.9b.i, the porosity is increased to the relatively modest 


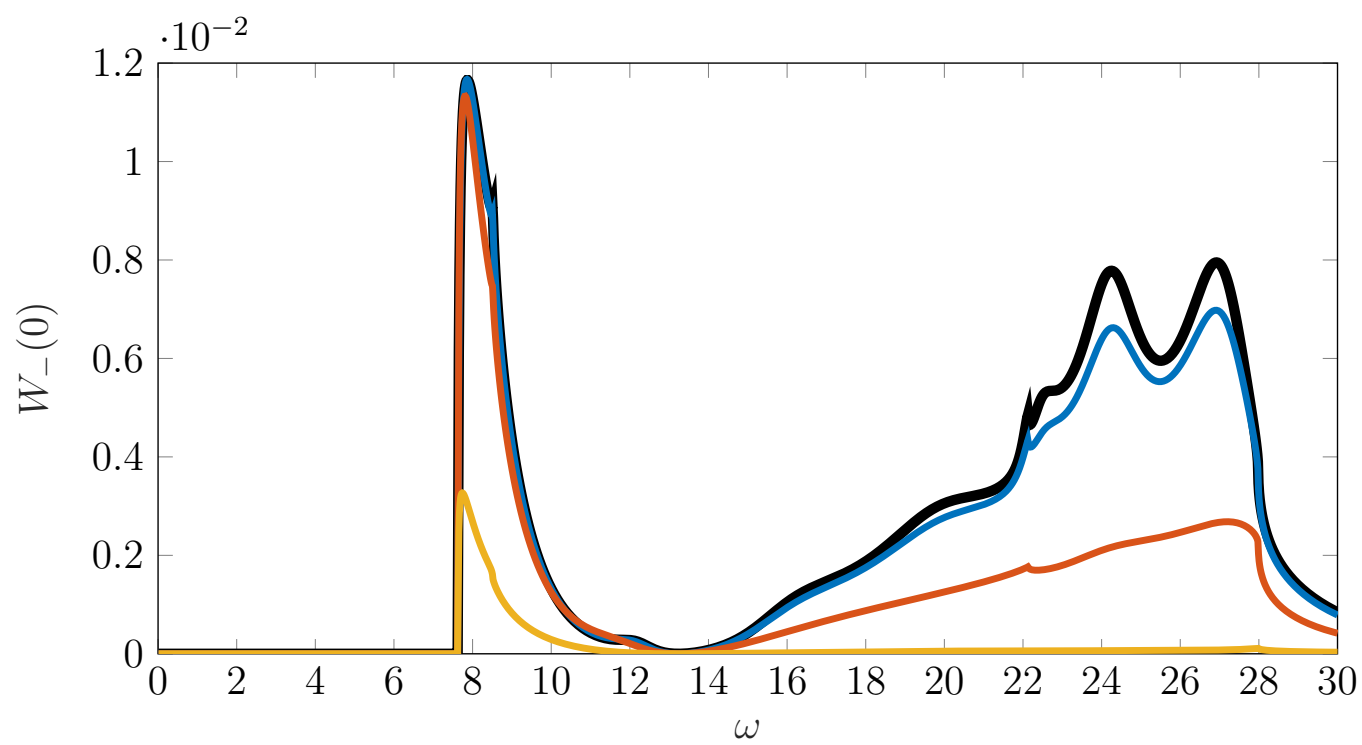

(a)

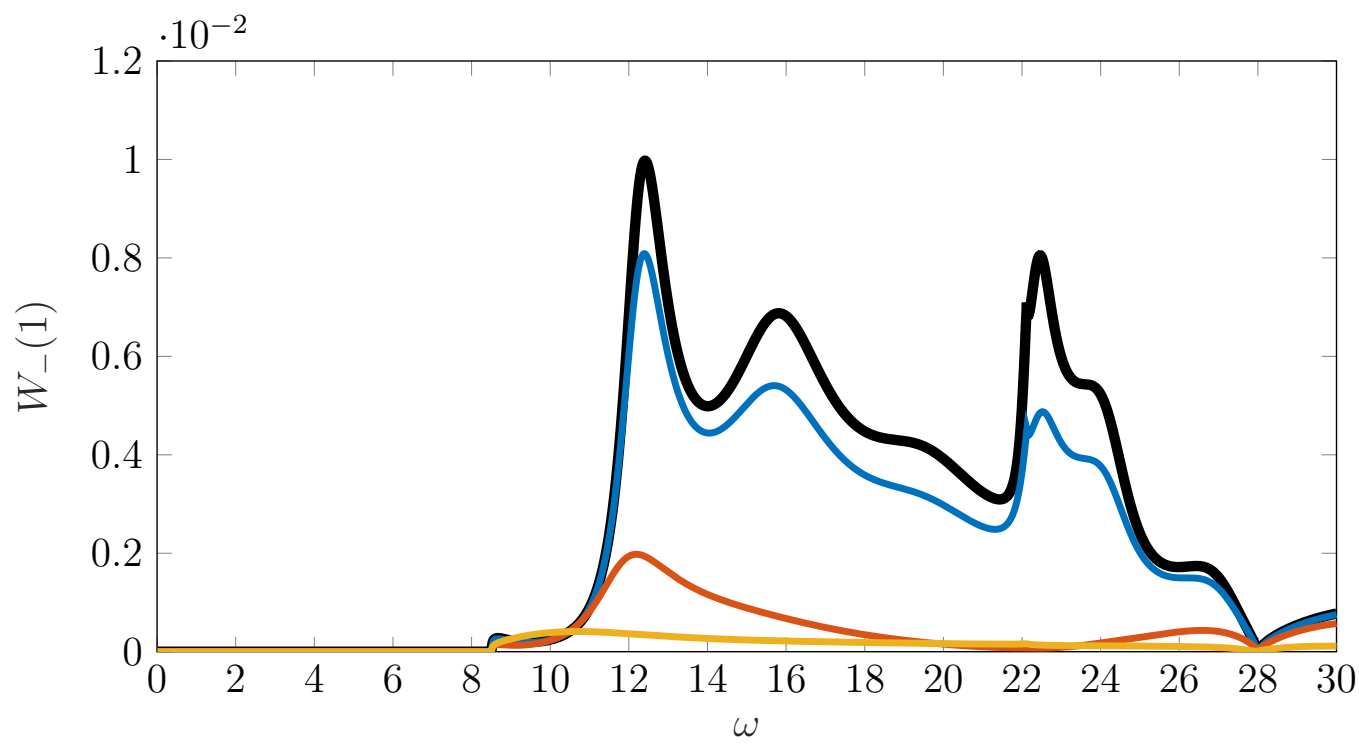

(b)

Fig. 5.7 Modal downstream sound power output for a cascade of porous blades at a range of frequencies for $(\mathrm{a})$ the first mode $(m=0)$ and $(\mathrm{b})$ the second mode $(m=1)$. The aerodynamic and aeroacoustic parameters are defined in table 5.2 and correspond to those in figure 9 of Glegg (1999). The colours correspond to the porosity parameters $C_{I I}=0$ (i.e. rigid), $C_{I I}=0.01, C_{I I}=0.1$ and $C_{I I}=1$. 


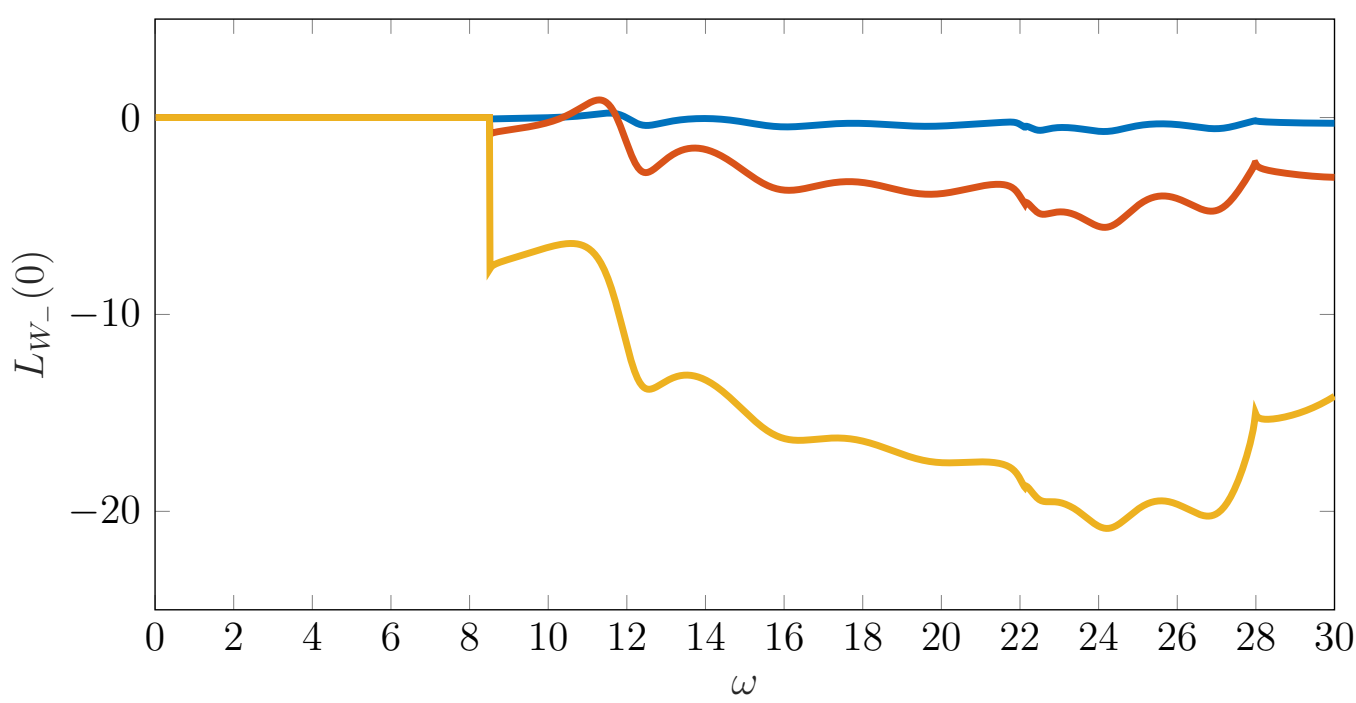

(a)

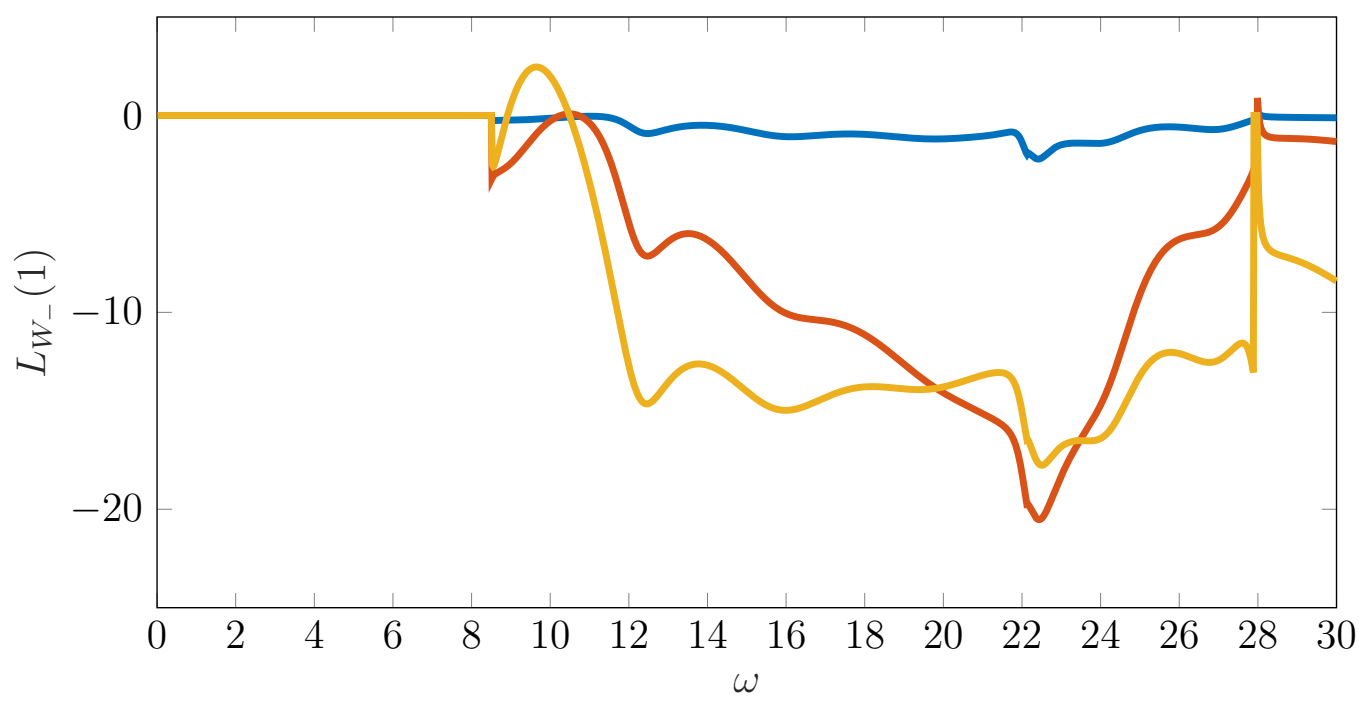

(b)

Fig. 5.8 Modal downstream sound power level for a cascade of porous blades at a range of frequencies for $(\mathrm{a})$ the first mode $(m=0)$ and $(\mathrm{b})$ the second mode $(m=1)$. The aerodynamic and aeroacoustic parameters are defined in table 5.2 and correspond to those in figure 9 of Glegg (1999). The colours correspond to the porosity parameters $C_{I I}=0.01, C_{I I}=0.1$ and $C_{I I}=1$. 
value of $C_{I I}=0.5$. We now observe that the amplitude of the reflected wave substantially decreases, so that the upstream field seems almost unperturbed by the cascade. In contrast, the downstream field is still distorted by the cascade, and the presence of the cut-on modes can still be observed. We note that the two modes are still cut-on, and the porosity does not affect their cut-on frequency. This is consistent with the argument made in the Wiener-Hopf analysis, namely that modifying the boundary conditions does not affect the modal structure of the far-field solution. Finally, when the porosity is increased to $C_{I I}=3$, the acoustic field is essentially unaffected by the blade row, as seen in figure 5.9c.i. This is because the blades are sufficiently porous that the fluid can pass through the blade unhindered. Accordingly, the only significant transmission and reflection coefficient remaining is that of the incident perturbation, as shown in figure 5.9 .

In general, blade porosity has a strong effect on the amplitude of the reflection and transmission coefficients. In figure 5.10a, the amplitudes of the transmission and reflection coefficients are plotted for a range of porosities. We observe a rapid decay in these amplitudes in the region $10^{-2}<C_{I I}<1$. When plotted on a log-scale in figure 5.10b, the behaviour resembles an exponential dependence. This is a promising result for sound reduction technologies, as it suggests that the sound transmission for blade rows can be drastically altered with small amounts of porosity.

\subsection{Conclusions}

We have derived an analytic solution for the scattering of an unsteady perturbation incident on a rectilinear cascade of flat blades with complex boundaries. The analytic nature of the solution means that it is extremely rapid to compute, and offers physical insight into the role played by different boundary conditions. In contrast with previous studies that focussed on the effects of rigid blades (Glegg, 1999; Posson et al., 2010b), the formulation of the present research allows a range of boundary conditions to be studied with minimal effort, such as porosity, compliance and flow impedance. In terms of the spectral plane, the effect of modifying these boundary conditions is to change the locations of the zeros of the Wiener-Hopf kernel, whilst the poles are unchanged. Accordingly, the modal structure of the far-field scattered pressure is invariant under modifications to the flat blades' boundary conditions. Conversely, the modal structure of the near-field region undergoes large deformations since the zeros of the kernel correspond to the duct modes of the inter-blade region. This has a strong effect on the surface pressure fluctuations and unsteady loading, which has implications for the far-field sound. 
(a.i)

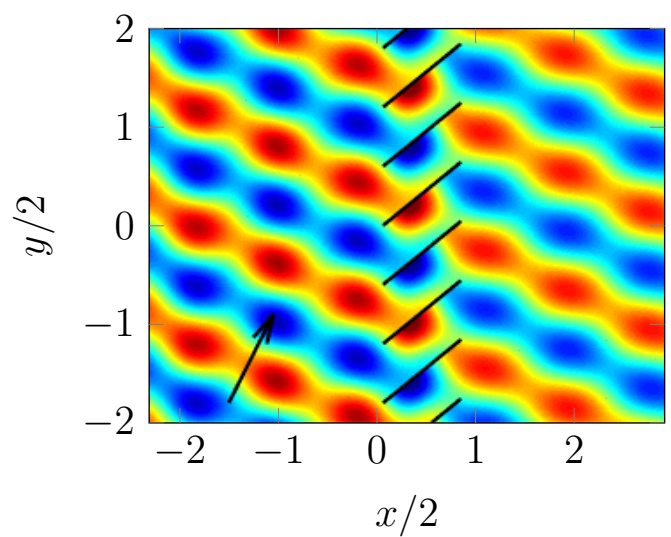

(b.i)

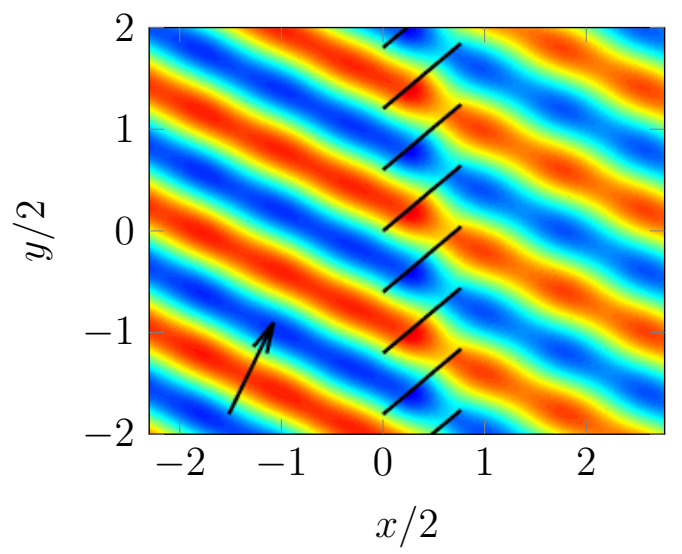

(c.i)

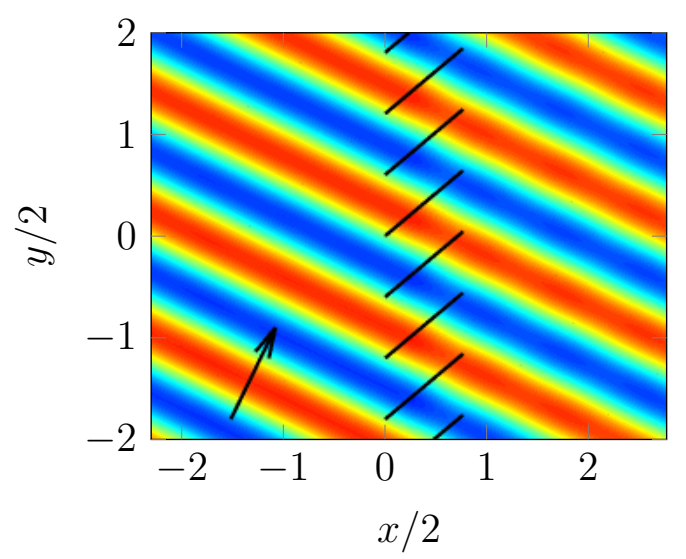

(a.ii)

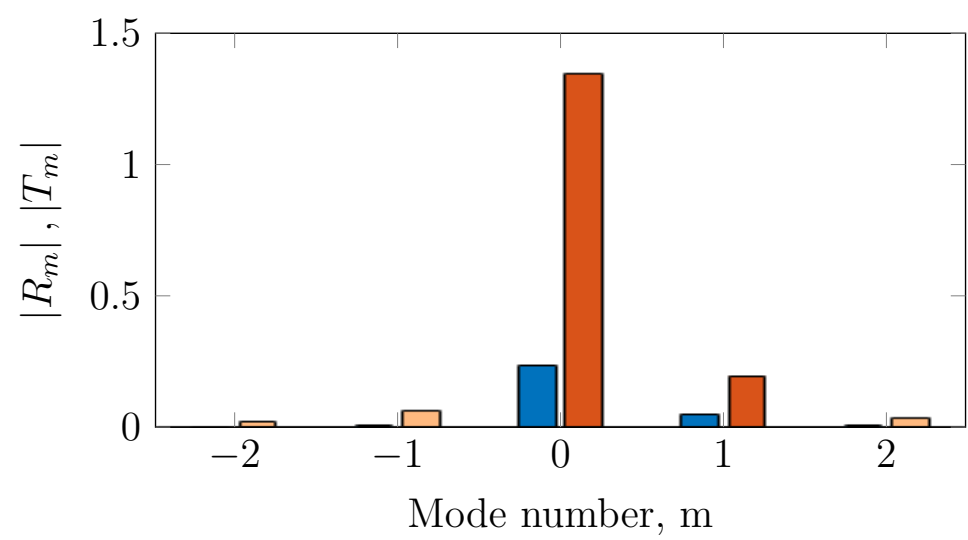

(b.ii)

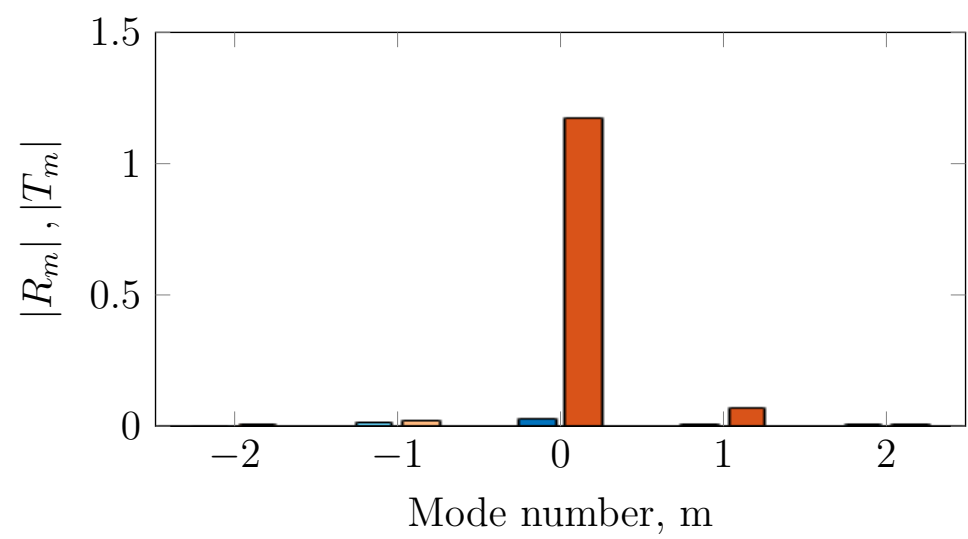

(c.ii)

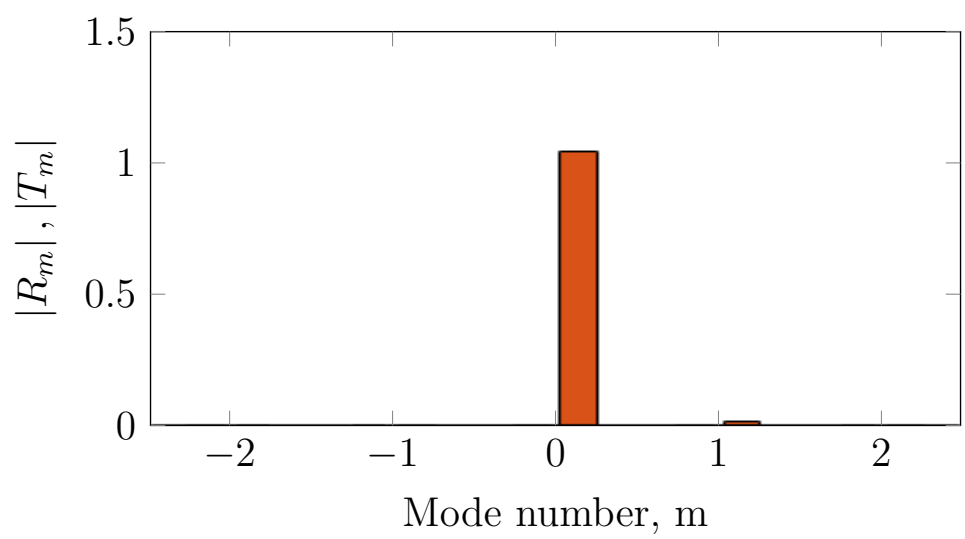

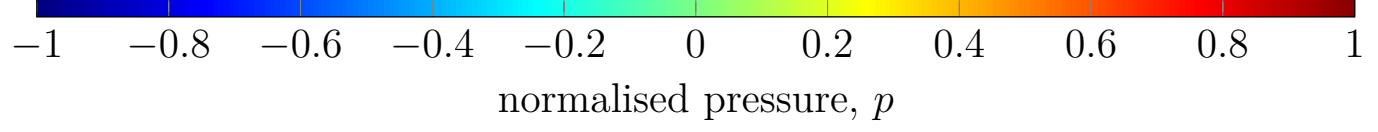

Fig. 5.9 Left: the total (incident and scattered), normalised pressure field. Right: the amplitudes of the normalised reflection (blue) and transmission (orange) coefficients. The $C_{I I}$ values correspond to $C_{I I}=0$ (a), $C_{I I}=0.5$ (b) and $C_{I I}=1$ (c). The darker bars indicate modes that are cut-on whereas the lighter bars are cut-off. The parameters in this case are $\Delta=0.6, \chi=40^{\circ}, M=0.3, \omega=12.5, k_{y}=0.1, k_{x}=3.57$. The arrow indicates the direction of the incident wave. 


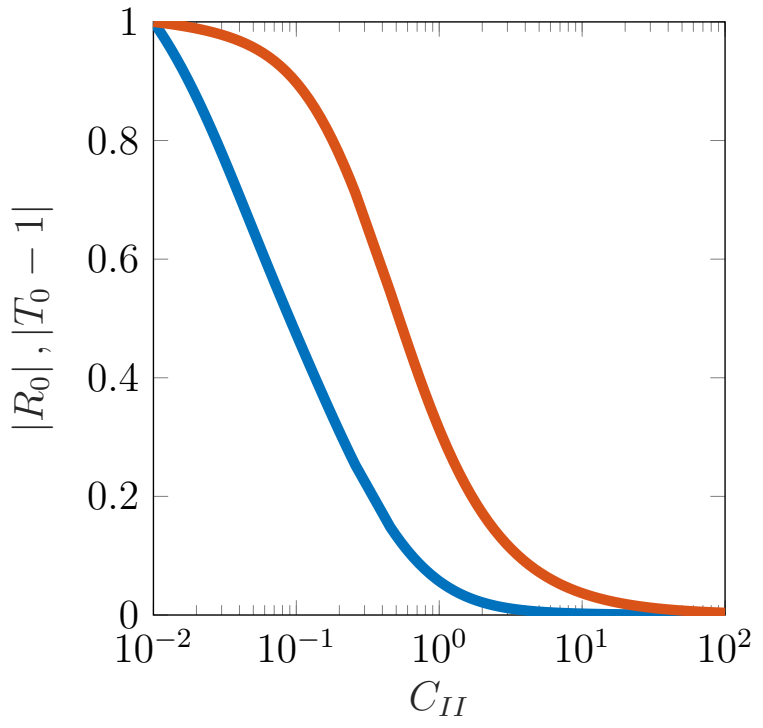

(a)

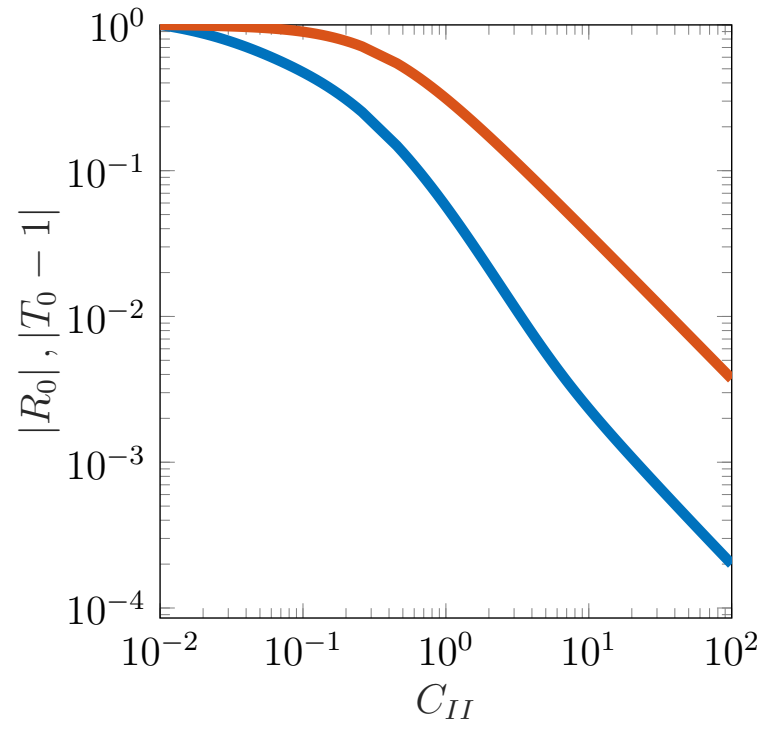

(b)

Fig. 5.10 The first reflection (blue) and transmission (orange) coefficients as a function of porosity coefficient, $C_{I I}$, on (a) a linear scale, and (b) a log scale. The coefficients are normalised by their values for a rigid cascade. The cascade parameters are equivalent to those in figure 5.9.

We have particularly investigated the role of blade porosity in the form of a Darcy-type condition. The results show that substantial reductions in both the unsteady lift and sound power output are possible for even modest values of porosity. At low frequencies, we observe a significant change in the unsteady loading and a moderate effect on the sound power output. Conversely, at high frequencies we observe a significant effect on the sound power output and a small effect on the unsteady loading. Furthermore, the amplitudes of the reflection and transmission coefficients rapidly decrease as the blade porosity is increased. We attribute these considerable reductions to several physical mechanisms associated with porosity. Increasing the blade porosity promotes a compliant relationship between adjacent channels, which prevents the build-up of trapped modes. Moreover, the flow seepage afforded by porous channels permits the dissipation of energy through the blades, thus reducing unsteady pressure fluctuations on the blade. This reduction in pressure fluctuations corresponds to reductions in the scattered pressure and far-field sound. Finally, this study shows that modified boundary conditions have a large impact on gust-cascade interaction noise and offers a useful design tool that can model aeroacoustic and aeroelastic effects. 


\section{List of Symbols}

Symbol Meaning

$\chi \quad$ Stagger angle

$\Delta \quad$ Blade spacing

$d, s \quad$ Horizontal and vertical blade spacing respectively

$\phi \quad$ Non-dimensional acoustic potential function

$\omega \quad$ Non-dimensional temporal frequency

$k_{x}, k_{y} \quad$ Non-dimensional frequencies in the $x$ and $y$ directions respectively

$\beta \quad$ Prandtl-Glauert factor

$\Delta_{n}[f] \quad$ Jump in $f$ across the line $y=n s$

$C_{N} \quad$ Coefficient of the $N$-th derivative of the jump in $\phi$ across the blades

$\gamma \quad$ Spectral parameter in Fourier plane

$\zeta(\gamma) \quad \sqrt{\omega^{2} w^{2}-\gamma^{2}}$

$D \quad$ Fourier transform of $\Delta_{0}[\phi]$

$\mathscr{L}^{ \pm} \quad$ Upper/lower half-plane in $\gamma$-space

$\pm \quad$ Subscript, generally refers to a quantity in $\mathscr{L}^{ \pm}$

$K \quad$ Kernel of Wiener-Hopf problem with modified boundaries

j Kernel of Wiener-Hopf problem with rigid boundaries

$K^{ \pm} \quad$ Multiplicative splitting of the kernel $K$

$\theta_{n}^{ \pm} \quad$ Zeros of $K^{\mp}$

$\lambda_{m}^{ \pm} \quad$ Poles of $K^{\mp}$ 


\section{A Wiener-Hopf Solution}

We now solve the integral equation (5.26) subject to the boundary conditions of no discontinuities upstream (5.7), a modified no-flux condition (5.15) and no pressure jump across the wake (5.18). In a similar way to Glegg (1999), we split this problem into four coupled problems that are amenable to the Wiener-Hopf method. This analysis is different to that of chapter 3: firstly, the modified boundary conditions must be accounted for and we show how to do this in a concise manner. Secondly, chapter 3 was restricted to the case of a pressure-free gust $\left(k_{x}=\omega\right)$, whereas the analysis in the present chapter is for a more general sound wave. Consequently, we present the full solution to the Wiener-Hopf solution in this chapter, although there are similarities with that presented in chapter 3.

We write

$$
\Delta_{0}[\phi](x)=\Delta_{0}\left[\phi^{(1)}\right](x)+\Delta_{0}\left[\phi^{(2)}\right](x)+\Delta_{0}\left[\phi^{(3)}\right](x)+\Delta_{0}\left[\phi^{(4)}\right](x),
$$

and its Fourier transform

$$
D(\gamma)=D^{(1)}(\gamma)+D^{(2)}(\gamma)+D^{(3)}(\gamma)+D^{(4)}(\gamma)
$$

where each $\Delta_{0}\left[\phi^{(n)}\right]$ and $D^{(n)}$ satisfy a semi-infinite integral equation of the form

$$
f^{(n)}(x)=-4 \pi \int_{-\infty}^{\infty} D^{(n)}(\gamma) j(\gamma) \mathrm{e}^{-\mathrm{i} \gamma x} \mathrm{~d} \gamma
$$

for $n=1,2,3,4$. The corresponding boundary conditions are

$$
\begin{aligned}
f^{(1)}(x) & =\mu_{0} \Delta_{0}\left[\phi^{(1)}\right](x)+\mu_{1} \Delta_{0}\left[\phi_{x}^{(1)}\right](x)+\mu_{2} \Delta_{0}\left[\phi_{x, x}^{(1)}\right](x) & & \\
& -2 w_{0} \exp \left[\mathrm{i} \delta k_{x} x\right], & & x>0, \\
f^{(2)}(x) & =\mu_{0} \Delta_{0}\left[\phi^{(2)}\right](x)+\mu_{1} \Delta_{0}\left[\phi_{x}^{(2)}\right](x)+\mu_{2} \Delta_{0}\left[\phi_{x, x}^{(2)}\right](x), & & x<2, \\
f^{(3)}(x) & =\mu_{0} \Delta_{0}\left[\phi^{(3)}\right](x)+\mu_{1} \Delta_{0}\left[\phi_{x}^{(3)}\right](x)+\mu_{2} \Delta_{0}\left[\phi_{x, x}^{(3)}\right](x), & & x>0, \\
f^{(4)}(x) & =\mu_{0} \Delta_{0}\left[\phi^{(4)}\right](x)+\mu_{1} \Delta_{0}\left[\phi_{x}^{(4)}\right](x)+\mu_{2} \Delta_{0}\left[\phi_{x, x}^{(4)}\right](x), & & x<2,
\end{aligned}
$$


and

$$
\begin{array}{cc}
\Delta_{0}\left[\phi^{(1)}\right](x)=0, & x<0, \\
\Delta_{0}\left[\phi^{(1)}\right](x)+\Delta_{0}\left[\phi^{(2)}\right](x)=2 \pi \mathrm{i} P^{(2)} \mathrm{e}^{\mathrm{i} \delta \omega x}, & x>2, \\
\Delta_{0}\left[\phi^{(2)}\right](x)+\Delta_{0}\left[\phi^{(3)}\right](x)+\Delta_{0}\left[\phi^{(4)}\right](x)=0, & x<0, \\
\Delta_{0}\left[\phi^{(3)}\right](x)+\Delta_{0}\left[\phi^{(4)}\right](x)=2 \pi \mathrm{i} P^{(4)} \mathrm{e}^{\mathrm{i} \delta \omega x}, & x>2,
\end{array}
$$

where $P^{(2)}$ and $P^{(4)}$ are two constants of integration that will be specified to enforce the Kutta condition. Summing the four above conditions results in the original boundary conditions and, consequently, we may apply the Wiener-Hopf method to each semiinfinite integral equation and sum the resulting contributions to obtain a solution to the original equations.

\section{A.0.1 Solution to First Wiener-Hopf Equation $-D^{(1)}$}

In this section, we solve the integral equation (5.35) for $n=1$

$$
f^{(1)}(x)=-4 \pi \int_{-\infty}^{\infty} D^{(1)}(\gamma) j(\gamma) \mathrm{e}^{-\mathrm{i} \gamma x} \mathrm{~d} \gamma
$$

subject to (5.36.a) and (5.36.b). Taking a Fourier transform of (5.40.a) in $x$ gives

$$
F_{-}^{(1)}(\gamma)+F_{+}^{(1)}(\gamma)=-4 \pi D_{+}^{(1)}(\gamma) j(\gamma)
$$

where

$$
\begin{gathered}
F_{-}^{(1)}(\gamma)=\frac{1}{2 \pi} \int_{-\infty}^{0} f^{(1)}(x) \mathrm{e}^{\mathrm{i} \gamma x} \mathrm{~d} x, \quad F_{+}^{(1)}(\gamma)=\frac{1}{2 \pi} \int_{0}^{\infty} f^{(1)}(x) \mathrm{e}^{\mathrm{i} \gamma x} \mathrm{~d} x \\
D_{+}^{(1)}(\gamma)=\int_{0}^{\infty} \Delta_{0}\left[\phi^{(1)}\right](x) \mathrm{e}^{\mathrm{i} \gamma x} \mathrm{~d} x .
\end{gathered}
$$


We may employ (5.36.a) to obtain

$$
F_{+}^{(1)}(\gamma)=-\frac{w_{0}}{\pi \mathrm{i}\left(\gamma+\delta k_{x}\right)}+\left(\mu_{0}-\mathrm{i} \mu_{1} \gamma-\mu_{2} \gamma^{2}\right) D^{(1)}(\gamma)
$$

Consequently, the Wiener-Hopf equation (5.41) may be expressed as

$$
F_{-}^{(1)}(\gamma)-\frac{w_{0}}{\pi \mathrm{i}\left(\gamma+\delta k_{x}\right)}=-4 \pi D_{+}^{(1)}(\gamma) K(\gamma)
$$

where

$$
K(\gamma)=j(\gamma)+\frac{1}{4 \pi}\left(\mu_{0}-\mathrm{i} \mu_{1} \gamma-\mu_{2} \gamma^{2}\right)
$$

The multiplicative splitting $K=K_{+} K_{-}$is performed in appendix 5.C. This splitting enables us to write

$$
\frac{F_{-}^{(1)}(\gamma)}{K_{-}(\gamma)}-\frac{w_{0}}{\pi \mathrm{i}\left(\gamma+\delta k_{x}\right) K_{-}(\gamma)}=-4 \pi D_{+}^{(1)}(\gamma) K_{+}(\gamma)
$$

We now additively factorise the left-hand side of (5.45). We apply pole removal (Noble, 1958) to obtain the additive splitting

$$
\frac{1}{\left(\gamma+\delta k_{x}\right) K_{-}(\gamma)}=\underbrace{\frac{1}{\left(\gamma+\delta k_{x}\right) K_{-}\left(-\delta k_{x}\right)}}_{+}+\underbrace{\frac{1}{\left(\gamma+\delta k_{x}\right) K_{-}(\gamma)}-\frac{1}{\left(\gamma+\delta k_{x}\right) K_{-}\left(-\delta k_{x}\right)}}_{-}
$$

where the underbrace \pm denotes that the function is analytic in $\mathscr{L}^{ \pm}$respectively. Therefore, (5.43) becomes

$$
\begin{aligned}
\frac{F_{-}^{(1)}(\gamma)}{K_{-}(\gamma)} & -\frac{w_{0}}{\pi \mathrm{i}\left(\gamma+\delta k_{x}\right) K_{-}(\gamma)}+\frac{w_{0}}{\pi \mathrm{i}\left(\gamma+\delta k_{x}\right) K_{-}\left(-\delta k_{x}\right)} \\
& =-4 \pi D_{+}^{(1)}(\gamma) K_{+}(\gamma)+\frac{w_{0}}{\pi \mathrm{i}\left(\gamma+\delta k_{x}\right) K_{-}\left(-\delta k_{x}\right)}
\end{aligned}
$$

We may now apply the standard Wiener-Hopf argument: since the left- and right-hand sides of (5.46) are analytic in $\mathscr{L}^{\mp}$ respectively, and they agree on a strip, each side defines the analytic continuation of the other. Therefore, equation (5.46) defines an entire function, $E_{1}(\gamma)$. As $|\gamma| \rightarrow \infty$ in the $\mathscr{L}^{-}$, the left-hand side of (5.46) decays. Similarly, as $|\gamma| \rightarrow \infty$ in $\mathscr{L}^{+}$, the right-hand side of (5.46) vanishes. Therefore, $E_{1}(\gamma)$ is bounded in the entire plane and must be constant according to Liouville's theorem. Moreover, since $E_{1}(\gamma)$ decays, this constant must be zero. Finally, we rearrange the right-hand side 
of (5.46) to obtain the solution to the first Wiener-Hopf problem as

$$
D^{(1)}(\gamma)=\frac{w_{0}}{(2 \pi)^{2} \mathrm{i}\left(\gamma+\delta k_{x}\right) K_{-}\left(-\delta k_{x}\right) K_{+}(\gamma)}
$$

\section{A.0.2 Solution to Second Wiener-Hopf Equation $-D^{(2)}$}

In this section we solve the integral equation (5.35) for $n=2$,

$$
f^{(2)}(x)=-4 \pi \int_{-\infty}^{\infty} D^{(2)}(\gamma) j(\gamma) \mathrm{e}^{-\mathrm{i} \gamma x} \mathrm{~d} \gamma
$$

subject to (5.37.a) and (5.37.b).

Taking a Fourier transform of (5.48.a) and applying (5.37.a) yields

$$
\left(\mu_{0}-\mathrm{i} \mu_{1} \gamma-\mu_{2} \gamma^{2}\right) D^{(1)}(\gamma)+F_{+}^{(2)}(\gamma)=-4 \pi\left(D_{-}^{(2)}(\gamma)+D_{+}^{(2)}(\gamma)\right) j(\gamma)
$$

where

$$
F_{+}^{(2)}(\gamma)=\frac{1}{2 \pi} \int_{2}^{\infty} f^{(2)}(x) \mathrm{e}^{\mathrm{i} \gamma x} \mathrm{~d} x=\frac{\mathrm{e}^{2 \mathrm{i} \gamma}}{2 \pi} \int_{0}^{\infty} f^{(2)}(x+2) \mathrm{e}^{\mathrm{i} x \gamma} \mathrm{d} x=\mathrm{e}^{2 \mathrm{i} \gamma} F_{+}^{*(2)}(\gamma),
$$

$D_{+}^{(2)}(\gamma)=\frac{1}{2 \pi} \int_{2}^{\infty} \Delta_{0}\left[\phi^{(2)}\right](x) \mathrm{e}^{\mathrm{i} \gamma x} \mathrm{~d} x=\frac{\mathrm{e}^{2 \mathrm{i} \gamma}}{2 \pi} \int_{0}^{\infty} \Delta_{0}\left[\phi^{(2)}\right](x+2) \mathrm{e}^{\mathrm{i} x \gamma} \mathrm{d} x=\mathrm{e}^{2 \mathrm{i} \gamma} D_{+}^{*(2)}(\gamma)$

$D_{-}^{(2)}(\gamma)=\frac{1}{2 \pi} \int_{-\infty}^{2} \Delta_{0}\left[\phi^{(2)}\right](x) \mathrm{e}^{\mathrm{i} \gamma x} \mathrm{~d} x=\frac{\mathrm{e}^{2 \mathrm{i} \gamma}}{2 \pi} \int_{-\infty}^{0} \Delta_{0}\left[\phi^{(2)}\right](x+2) \mathrm{e}^{\mathrm{i} x \gamma} \mathrm{d} x=\mathrm{e}^{2 \mathrm{i} \gamma} D_{-}^{*(2)}(\gamma)$

Factoring out the $\mathrm{e}^{2 \mathrm{i} \gamma}$ dependence and employing (5.44) transforms the Wiener-Hopf equation (5.51) to

$$
F_{+}^{*(2)}(\gamma)=-4 \pi\left(D_{-}^{*(2)}(\gamma)+D_{+}^{*(2)}(\gamma)\right) K(\gamma)
$$

and we may use the multiplicative splitting of $K$ to write

$$
\frac{F_{+}^{*(2)}(\gamma)}{K_{+}(\gamma)}=-4 \pi\left(D_{-}^{*(2)}(\gamma)+D_{+}^{*(2)}(\gamma)\right) K_{-}(\gamma)
$$


We may use the downstream boundary condition for this problem (5.37.b) to write

$$
D_{+}^{*(2)}(\gamma)=-\frac{P^{*(2)}}{\gamma+\delta \omega}-\frac{1}{2 \pi} \int_{0}^{\infty} \Delta_{0}\left[\phi^{(1)}\right](x+2) \mathrm{e}^{\mathrm{i} \gamma x} \mathrm{~d} x
$$

where $P^{*(2)}=P^{(2)} \mathrm{e}^{2 \mathrm{i} \delta k_{x}}$. To calculate the remaining integral we use the inversion formula for the Fourier transform:

$$
\Delta_{0}\left[\phi^{(1)}\right](x)=\int_{-\infty-\mathrm{i} \tau_{1}}^{\infty-\mathrm{i} \tau_{1}} D^{(1)}(\gamma) \mathrm{e}^{-\mathrm{i} \gamma x} \mathrm{~d} \gamma
$$

By substituting this representation into our desired integral, we obtain

$$
\frac{1}{2 \pi} \int_{0}^{\infty} \Delta_{0}\left[\phi^{(1)}\right](x+2) \mathrm{e}^{\mathrm{i} \gamma x} \mathrm{~d} x=\frac{1}{2 \pi} \int_{0}^{\infty} \int_{-\infty-\mathrm{i} \tau_{1}}^{\infty-\mathrm{i} \tau_{1}} D^{(1)}\left(\gamma_{1}\right) \mathrm{e}^{-\mathrm{i} \gamma_{1}(x+2)} \mathrm{d} \gamma_{1} \mathrm{e}^{\mathrm{i} \gamma x} \mathrm{~d} x
$$

Rearranging the order of integration and computing the resulting $x$-integral results in

$$
\frac{1}{2 \pi} \int_{0}^{\infty} \Delta_{0}\left[\phi^{(1)}\right](x+2) \mathrm{e}^{\mathrm{i} \gamma x} \mathrm{~d} x=\frac{1}{2 \pi \mathrm{i}} \int_{-\infty-\mathrm{i} \tau_{1}}^{\infty-\mathrm{i} \tau_{1}} \frac{D^{(1)}\left(\gamma_{1}\right) \mathrm{e}^{-2 \mathrm{i} \gamma_{1}}}{\gamma_{1}-\gamma} \mathrm{d} \gamma_{1} .
$$

Since $d<2$, we may close the remaining integral in $\mathscr{L}^{-}$. There are no branches in the integrand and the integral consists of the residues of simple poles at $\gamma=-\delta k_{x}, \theta_{n}^{-}$. Consequently, inserting (5.47) into the above integral yields

$$
\begin{aligned}
& \frac{1}{2 \pi \mathrm{i}} \int_{-\infty-\mathrm{i} \tau_{1}}^{\infty-\mathrm{i} \tau_{1}} \frac{1}{\left(\gamma+\delta k_{x}\right) K_{-}\left(-\delta k_{x}\right) K_{+}(\gamma)} \cdot \frac{\mathrm{e}^{-2 \mathrm{i} \gamma_{1}}}{\gamma_{1}-\gamma} \mathrm{d} \gamma_{1} \\
& =\frac{\mathrm{e}^{2 \mathrm{i} \delta k_{x}}}{\left(\gamma+\delta k_{x}\right) K\left(-\delta k_{x}\right)}+\sum_{n=0}^{\infty} \frac{\mathrm{e}^{-2 \mathrm{i} \theta_{n}^{-}}}{\left(\theta_{n}^{-}+\delta k_{x}\right) K_{-}\left(-\delta k_{x}\right) K_{+}^{\prime}\left(\theta_{n}^{-}\right)\left(\gamma-\theta_{n}^{-}\right)},
\end{aligned}
$$

where the derivatives of $K_{ \pm}$evaluated at the duct modes $\theta_{n}^{\mp}$ are given by

$$
\begin{gathered}
K_{ \pm}^{\prime}\left(\theta_{n}^{\mp}\right)=\frac{K^{\prime}\left(\theta_{n}^{\mp}\right)}{K_{\mp}\left(\theta_{n}^{\mp}\right)}=\frac{-1}{4 \pi K_{\mp}\left(\theta_{n}^{\mp}\right)}\left(\frac{\left(\theta_{n}^{\mp} / \zeta\left(\theta_{n}^{\mp}\right)\right) \sin \left(s \zeta\left(\theta_{n}^{\mp}\right)\right)+s \theta_{n}^{\mp} \cos \left(s \zeta\left(\theta_{n}^{\mp}\right)\right)}{\cos \left(s \zeta\left(\theta_{n}^{\mp}\right)\right)-\cos \left(d \theta_{n}^{\mp}+\sigma^{\prime}\right)}\right. \\
\frac{\theta_{n}^{\mp} s \sin \left(s \zeta\left(\theta_{n}^{\mp}\right)^{2}+d \zeta\left(\theta_{n}^{\mp}\right) \sin \left(d \theta_{n}^{\mp}+\sigma^{\prime}\right) \sin \left(s \zeta\left(\theta_{n}^{\mp}\right)\right)\right.}{\left(\cos \left(s \zeta\left(\theta_{n}^{\mp}\right)\right)-\cos \left(d \theta_{n}^{\mp}+\sigma^{\prime}\right)\right)^{2}} \\
\left.\mathrm{i} \mu_{1}+2 \mu_{2} \theta_{n}^{\mp}\right) .
\end{gathered}
$$


Therefore, substitution of (5.54) into (5.53) yields

$$
\begin{aligned}
D_{+}^{*(2)}(\gamma) & =\frac{w_{0} \mathrm{e}^{2 \mathrm{i} \delta k_{x}}}{(2 \pi)^{2} \mathrm{i}\left(\gamma+\delta k_{x}\right) K\left(-\delta k_{x}\right)}-\sum_{n=0}^{\infty} \frac{\mathscr{A}_{n} \mathrm{e}^{-2 \mathrm{i} \theta_{n}^{-}}}{\mathrm{i}\left(\theta_{n}^{-}+\delta \omega\right)\left(\gamma-\theta_{n}^{-}\right)} \\
& -\frac{P^{*(2)}}{\gamma+\delta \omega},
\end{aligned}
$$

where

$$
\mathscr{A}_{n}=\frac{-w_{0}\left(\theta_{n}^{-}+\delta \omega\right)}{(2 \pi)^{2}\left(\theta_{n}^{-}+\delta k_{x}\right) K_{-}\left(-\delta k_{x}\right) K_{+}^{\prime}\left(\theta_{n}^{-}\right)} .
$$

Similarly to chapter 3, we use the notation

$$
\tilde{K}_{-}\left(\gamma, \eta^{-}\right):=\frac{K_{-}(\gamma)}{\gamma-\eta^{-}}
$$

so that substitution of (5.55) into the Wiener-Hopf equation (5.49) yields

$$
\begin{aligned}
\frac{F_{+}^{*(2)}(\gamma)}{4 \pi K_{+}(\gamma)}= & -K_{-}(\gamma) D_{-}^{*(2)}(\gamma)+P^{*(2)} \tilde{K}_{-}(\gamma,-\delta \omega) \\
& +\frac{w_{0} \mathrm{e}^{2 \mathrm{i} \delta k_{x}}}{(2 \pi)^{2} \mathrm{i}} \cdot \frac{\tilde{K}_{-}\left(\gamma,-\delta k_{x}\right)}{K\left(-\delta k_{x}\right)}-\sum_{n=0}^{\infty} \frac{\mathscr{A}_{n} \mathrm{e}^{-2 \mathrm{i} \theta_{n}^{-}}}{\mathrm{i}\left(\theta_{n}^{-}+\delta \omega\right)} \tilde{K}_{-}\left(\gamma, \theta_{n}^{-}\right)
\end{aligned}
$$

We note the additive splitting

$$
\tilde{K}_{-}\left(\gamma, \eta^{-}\right)=\left[\tilde{K}_{-}(\gamma, \eta)\right]_{+}+\left[\tilde{K}_{-}(\gamma, \eta)\right]_{-},
$$

where

$$
\left[\tilde{K}_{-}(\gamma, \eta)\right]_{+}=\frac{K_{-}\left(\eta^{-}\right)}{\gamma-\eta^{-}}, \quad\left[\tilde{K}_{-}(\gamma, \eta)\right]_{-}=\frac{K_{-}(\gamma)-K_{-}\left(\eta^{-}\right)}{\gamma-\eta^{-}}
$$

Substituting these splittings into (5.56) yields

$$
\begin{array}{r}
\frac{F_{+}^{*(2)}(\gamma)}{2 \pi K_{+}(\gamma)}-P^{*(2)}\left[\tilde{K}_{-}(\gamma,-\delta \omega)\right]_{+}-\frac{w_{0} \mathrm{e}^{2 \mathrm{i} \delta k_{x}}}{(2 \pi)^{2} \mathrm{i}} \cdot \frac{\left[\tilde{K}_{-}\left(\gamma,-\delta k_{x}\right)\right]_{+}}{K\left(-\delta k_{x}\right)} \\
+\sum_{n=0}^{\infty} \frac{\mathscr{A}_{n} \mathrm{e}^{-2 \mathrm{i} \theta_{n}^{-}}}{\mathrm{i}\left(\theta_{n}^{-}+\delta \omega\right)}\left[\tilde{K}_{-}\left(\gamma, \theta_{n}^{-}\right)\right]_{+}=-K_{-}(\gamma) D_{-}^{*(2)}(\gamma)+P^{*(2)}\left[\tilde{K}_{-}(\gamma,-\delta \omega)\right]_{-} \\
+\frac{w_{0} \mathrm{e}^{2 \mathrm{i} \delta k_{x}}}{(2 \pi)^{2} \mathrm{i}} \cdot \frac{\left[\tilde{K}_{-}\left(\gamma,-\delta k_{x}\right)\right]_{-}}{K\left(-\delta k_{x}\right)}-\sum_{n=0}^{\infty} \frac{\mathscr{A}_{n} \mathrm{e}^{-2 \mathrm{i} \theta_{n}^{-}}}{\mathrm{i}\left(\theta_{n}^{-}+\delta \omega\right)}\left[\tilde{K}_{-}\left(\gamma, \theta_{n}^{-}\right)\right]_{-} .
\end{array}
$$


In a similar way to section 5.A.0.1, we now apply the typical Wiener-Hopf argument. We enforce the unsteady Kutta condition (Ayton et al., 2016) which restricts the pressure at the trailing edge to be finite. Consequently, the left-hand side of (5.58) decays as $|\gamma| \rightarrow \infty$ in $\mathscr{L}^{+}$and the left-hand side of (5.58) tends to an unknown constant as $|\gamma| \rightarrow \infty$ in $\mathscr{L}^{-}$. Applying analytic continuation and Liouville's theorem determines that this constant must be zero. Accordingly, the coefficient of $\gamma^{-1}$ on the left-hand side of (5.58) must vanish so that

$$
P^{*(2)}=\frac{w_{0} \mathrm{e}^{2 \mathrm{i} \delta k_{x}}}{(2 \pi)^{2} \mathrm{i} K_{+}\left(-\delta k_{x}\right)} \cdot \frac{1}{K_{-}(-\delta \omega)}-\sum_{n=0}^{\infty} \frac{\mathscr{A}_{n} \mathrm{e}^{-2 \mathrm{i} \theta_{n}^{-}}}{\mathrm{i}\left(\theta_{n}^{-}+\delta \omega\right)} \cdot \frac{K_{-}\left(\theta_{n}^{-}\right)}{K_{-}(-\delta \omega)}
$$

Consequently, after substituting in the downstream representation (5.55) and the expression for the constant (5.59), the right-hand side of (5.58) yields

$$
\begin{aligned}
D^{(2)}(\gamma) & =\frac{w_{0}\left(\delta \omega-\delta k_{x}\right) \mathrm{e}^{2 \mathrm{i}\left(\gamma+\delta k_{x}\right)}}{(2 \pi)^{2} \mathrm{i}\left(\gamma+\delta k_{x}\right)(\gamma+\delta \omega) K_{+}\left(-\delta k_{x}\right)} \cdot \frac{1}{K_{-}(\gamma)} \\
& -\sum_{n=0}^{\infty} \frac{\mathscr{A}_{n} \mathrm{e}^{2 \mathrm{i}\left(\gamma-\theta_{n}^{-}\right)}}{\mathrm{i}(\gamma+\delta \omega)\left(\gamma-\theta_{n}^{-}\right)} \cdot \frac{K_{-}\left(\theta_{n}^{-}\right)}{K_{-}(\gamma)} .
\end{aligned}
$$

It should be noted that the poles of $D^{(2)}$ in $\mathscr{L}^{+}$are only at the zeros of $K_{-}$.

\section{A.0.3 Solution to Third and Fourth Wiener-Hopf Equations $-D^{(3)}, D^{(4)}$}

Since the integral equations for $\Delta_{0}\left[\phi^{(3)}\right]$ and $\Delta_{0}\left[\phi^{(4)}\right]$ are coupled, we must solve for them simultaneously. Taking a Fourier transform of (5.35) and applying the boundary conditions for (5.38.a) and (5.39.a) for $n=3,4$ gives

$$
\begin{aligned}
F_{-}^{(3)}(\gamma) & =-4 \pi\left[D_{-}^{(3)}(\gamma)+D_{+}^{(3)}(\gamma)\right] K(\gamma), \\
F_{+}^{*(4)}(\gamma) & =-4 \pi\left[D_{-}^{*(4)}(\gamma)+D_{+}^{*(4)}(\gamma)\right] K(\gamma),
\end{aligned}
$$

where $F_{-}^{(3)}, D_{-}^{(3)}$ and $D_{+}^{(3)}$ are defined in an analogous way to (5.42.a) and (5.42.b), and $F_{+}^{*(4)}, D_{+}^{*(4)}$ and $D_{-}^{*(4)}$ are defined in an analogous way to (5.50.a), (5.50.b) and (5.50.c). Using a similar approach to section 5.A.0.2, the upstream boundary condition (5.38.b) may be expressed as

$$
D_{-}^{(3)}(\gamma)=\frac{1}{2 \pi \mathrm{i}} \int_{-\infty+\mathrm{i} \tau_{0}}^{\infty+\mathrm{i} \tau_{0}} \frac{D^{(2)}\left(\gamma_{1}\right)+D^{(4)}\left(\gamma_{1}\right)}{\gamma_{1}-\gamma} \mathrm{d} \gamma_{1}
$$


Consequently, we may express $D_{-}^{(3)}$ in terms of its poles $\theta_{n}^{+}$as

$$
D_{-}^{(3)}(\gamma)=-\sum_{n=0}^{\infty} \frac{\mathscr{B}_{n}}{\gamma-\theta_{n}^{+}}
$$

where $\mathscr{B}_{n}$ are the residues of $D_{-}^{(2)}\left(\gamma_{1}\right)+D_{-}^{(4)}\left(\gamma_{1}\right)$ at $\gamma=\theta_{n}^{+}$. The residues of $D_{-}^{(4)}$ are currently unknown, but the residues of $D_{-}^{(2)}$ are given by

$$
\begin{aligned}
D_{r, k}^{(2)}=\frac{-\mathrm{e}^{2 \mathrm{i} \theta_{k}^{+}}}{K_{-}^{\prime}\left(\theta_{k}^{+}\right)} & \left\{\frac{w_{0}\left(\delta \omega-\delta k_{x}\right) \mathrm{e}^{2 \mathrm{i} \delta k_{x}}}{(2 \pi)^{2} \mathrm{i}\left(\theta_{k}^{+}+\delta k_{x}\right)\left(\theta_{k}^{+}+\delta \omega\right) K_{+}\left(-\delta k_{x}\right)}\right. \\
& \left.+\sum_{n=0}^{\infty} \frac{\mathscr{A}_{n} \mathrm{e}^{\mathrm{i}\left(\theta_{k}^{+}-\theta_{n}^{-}\right)} K_{-}\left(\theta_{n}^{-}\right)}{\mathrm{i}\left(\theta_{k}^{+}+\delta \omega\right)\left(\theta_{k}^{+}-\theta_{n}^{-}\right)}\right\} .
\end{aligned}
$$

We may now substitute (5.62) into (5.60) to obtain the Wiener-Hopf equation

$$
\frac{F_{-}^{(3)}(\gamma)}{4 \pi K_{-}(\gamma)}+\sum_{n=0}^{\infty} \frac{\mathscr{B}_{n}}{\gamma-\theta_{n}^{+}} K_{+}\left(\theta_{n}^{+}\right)=D_{+}^{(3)}(\gamma) K_{+}(\gamma)-\sum_{n=0}^{\infty} \frac{\mathscr{B}_{n}}{\gamma-\theta_{n}^{+}}\left(K_{+}(\gamma)-K_{+}\left(\theta_{n}^{+}\right)\right)
$$

The edge conditions are identical to those applied in section 5.A.0.1, and we employ the typical Wiener-Hopf argument to obtain

$$
D_{+}^{(3)}(\gamma)=\sum_{n=0}^{\infty} \frac{\mathscr{B}_{n}}{\gamma-\theta_{n}^{+}}\left\{1-\frac{K_{+}\left(\theta_{n}^{+}\right)}{K_{+}(\gamma)}\right\}
$$

Combining this solution with (5.62) yields

$$
D^{(3)}(\gamma)=-\sum_{n=0}^{\infty} \frac{\mathscr{B}_{n}}{\gamma-\theta_{n}^{+}} \cdot \frac{K_{+}\left(\theta_{n}^{+}\right)}{K_{+}(\gamma)}
$$

We proceed to the solution for $D^{(4)}$. In a similar way to section 5.A.0.2, we may invert the Fourier transform for the downstream boundary condition (5.39.b) to write

$$
D_{+}^{*(4)}(\gamma)=-\frac{P^{*(4)}}{\mathrm{i}(\gamma+\delta \omega)}-\frac{1}{2 \pi \mathrm{i}} \int_{-\infty-\mathrm{i} \tau_{1}}^{\infty+\mathrm{i} \tau_{1}} \sum_{n=0}^{\infty} \frac{\mathscr{B}_{n}}{\left(\gamma_{1}-\gamma\right)\left(\gamma_{1}-\theta_{n}^{+}\right)}\left\{1-\frac{K_{+}\left(\theta_{n}^{+}\right)}{K_{+}\left(\gamma_{1}\right)}\right\} \mathrm{e}^{-2 \mathrm{i} \gamma_{1}} \mathrm{~d} \gamma_{1}
$$

This integral can be closed in $\mathscr{L}^{-}$to obtain

$$
D_{+}^{*(4)}(\gamma)=-\frac{P^{*(4)}}{\gamma+\delta \omega}-\sum_{n=0}^{\infty} \frac{\mathscr{C}_{n} \mathrm{e}^{-2 \mathrm{i} \theta_{n}^{-}}}{\mathrm{i}\left(\theta_{n}^{-}+\delta \omega\right)\left(\gamma-\theta_{n}^{-}\right)},
$$


where

$$
\mathscr{C}_{n}=\sum_{k=0}^{\infty} \frac{\mathrm{i}\left(\theta_{n}^{-}+\delta \omega\right)}{\left(\theta_{k}^{+}-\theta_{n}^{-}\right)} \cdot \frac{K_{+}\left(\theta_{k}^{+}\right)}{K_{+}^{\prime}\left(\theta_{n}^{-}\right)} \cdot \mathscr{B}_{1, \Sigma, k} .
$$

After truncation, we may write this system of equations in matrix form

$$
\mathscr{C}=\mathbf{L} \mathscr{B},
$$

where

$$
\{\mathbf{L}\}_{n, m}=\frac{\mathrm{i}\left(\theta_{n}^{-}+\delta \omega\right)}{\left(\theta_{m}^{+}-\theta_{n}^{-}\right)} \cdot \frac{K_{+}\left(\theta_{m}^{+}\right)}{K_{+}^{\prime}\left(\theta_{n}^{-}\right)} .
$$

By applying the notation introduced in (5.57), we may express the Wiener-Hopf equation (5.61) in the form

$$
\begin{array}{r}
\frac{F_{+}^{*(4)}(\gamma)}{4 \pi K_{+}(\gamma)}+P^{*(4)}\left[\tilde{K}_{-}(\gamma,-\delta \omega)\right]_{+}+\sum_{n=0}^{\infty} \frac{\left[\tilde{K}_{-}\left(\gamma, \theta_{n}^{-}\right)\right]_{+}}{\mathrm{i}\left(\theta_{n}^{-}+\delta \omega\right)} \mathscr{C}_{n} \mathrm{e}^{-2 \mathrm{i} \theta_{n}^{-}} \\
=D_{-}^{*(4)}(\gamma) K_{-}(\gamma)-P^{*(4)}\left[\tilde{K}_{-}(\gamma,-\delta \omega)\right]_{-}-\sum_{n=0}^{\infty} \frac{\left[\tilde{K}_{-}\left(\gamma, \theta_{n}^{-}\right)\right]_{-}}{\mathrm{i}\left(\theta_{n}^{-}+\delta \omega\right)} \mathscr{C}_{n} \mathrm{e}^{-2 \mathrm{i} \theta_{n}^{-}} .
\end{array}
$$

Employing the unsteady Kutta condition in (5.66) yields

$$
P^{*(4)}=-\sum_{n=0}^{\infty} \frac{\mathscr{C}_{n} \mathrm{e}^{-2 \mathrm{i} \theta_{n}^{-}}}{\mathrm{i}\left(\theta_{n}^{-}+\delta \omega\right)} \cdot \frac{K_{-}\left(\theta_{n}^{-}\right)}{K_{-}(-\delta \omega)} .
$$

Finally, applying the downstream boundary condition (5.64) and rearranging (5.66) yields

$$
D^{(4)}(\gamma)=-\sum_{n=0}^{\infty} \frac{\mathscr{C}_{n} \mathrm{e}^{2 \mathrm{i}\left(\gamma-\theta_{n}^{-}\right)}}{\mathrm{i}\left(\theta_{n}^{-}+\delta \omega\right)\left(\gamma-\theta_{n}^{-}\right)} \cdot \frac{K_{-}\left(\theta_{n}^{-}\right)}{K_{-}(\gamma)} .
$$

We are now able to calculate the residues of $D^{(3)}$ as

$$
\mathscr{B}_{n}=D_{r, k}-\sum_{m=0}^{\infty} \frac{\mathscr{C}_{n} \mathrm{e}^{2 \mathrm{i}\left(\theta_{n}^{+}-\theta_{m}^{-}\right)}}{\mathrm{i}\left(\theta_{n}^{+}+\delta \omega\right)\left(\theta_{n}^{+}-\theta_{m}^{-}\right)} \cdot \frac{K_{-}\left(\theta_{m}^{-}\right)}{K_{-}^{\prime}\left(\theta_{n}^{+}\right)},
$$

or, in matrix form,

$$
\mathscr{B}=\mathbf{D}_{\mathrm{r}, \mathrm{k}}+\mathbf{F} \mathscr{C}
$$


where

$$
\{\mathbf{F}\}_{n, m}=-\frac{\mathrm{e}^{2 \mathrm{i}\left(\theta_{n}^{+}-\theta_{m}^{-}\right)}}{\mathrm{i}\left(\theta_{n}^{+}+\delta \omega\right)\left(\theta_{n}^{+}-\theta_{m}^{-}\right)} \cdot \frac{K_{-}\left(\theta_{m}^{-}\right)}{K_{-}^{\prime}\left(\theta_{n}^{+}\right)} .
$$

The matrix equations (5.65) and (5.68) may be combined and solved to give the final expressions for $\mathscr{B}_{n}$ and $\mathscr{C}_{n}$.

\section{B Details of Fourier Inversion}

The acoustic field is given by

$$
\phi(x, y)=\frac{1}{2} \int_{-\infty}^{\infty} D(\gamma) A(\gamma, x, y) \mathrm{d} \gamma
$$

where $A=A_{u}+A_{d}$ and

$$
\begin{aligned}
& A_{u}(\gamma, x, y)=\mathrm{e}^{-\mathrm{i} \gamma x} \cdot \frac{\mathrm{e}^{\mathrm{i} d \gamma+\mathrm{i} \sigma^{\prime}} \cos (\zeta y)}{\cos \left(d \gamma+\sigma^{\prime}\right)-\cos (s \zeta)}, \\
& A_{d}(\gamma, x, y)=\mathrm{e}^{-\mathrm{i} \gamma x} \cdot \frac{-\cos (\zeta(y-s))}{\cos \left(d \gamma+\sigma^{\prime}\right)-\cos (s \zeta)} .
\end{aligned}
$$

We calculate the above integral by splitting the physical plane into five separate regions, as illustrated in figure 3.6. Both $A_{a}$ and $A_{b}$ have poles at the acoustic modes $\lambda_{m}^{ \pm}$where the residues are given by

$$
\begin{aligned}
& A_{u}^{r}\left(\lambda_{m}^{ \pm}, x, y\right)=\mp \frac{\zeta_{m}^{ \pm} \mathrm{e}^{\mathrm{i} d \lambda_{m}^{ \pm}+\mathrm{i} \sigma^{\prime}} \cos \left(\zeta_{m}^{ \pm} y\right)}{\Delta \sin \left(s \zeta_{m}^{ \pm}\right) \sqrt{k^{2} w^{2}-f_{m}^{2}}} \mathrm{e}^{-\mathrm{i} \lambda_{m}^{ \pm} x} \\
& A_{d}^{r}\left(\lambda_{m}^{ \pm}, x, y\right)= \pm \frac{\zeta_{m}^{ \pm} \cos \left(\zeta_{m}^{ \pm}(y-s)\right)}{\Delta \sin \left(s \zeta_{m}^{ \pm}\right) \sqrt{k^{2} w^{2}-f_{m}^{2}}} \mathrm{e}^{-\mathrm{i} \lambda_{m}^{ \pm} x}
\end{aligned}
$$

In order to proceed, we split the acoustic potential into four components

$$
\begin{aligned}
\phi(x, y) & =\underbrace{\frac{1}{2} \int_{-\infty}^{\infty}\left(D^{(1,3)}(\gamma)\right) A_{u}(\gamma) \mathrm{e}^{-\mathrm{i} \gamma(x-y d / s)} \mathrm{d} \gamma}_{\phi_{u, u}} \\
& +\underbrace{\frac{1}{2} \int_{-\infty}^{\infty}\left(D^{(1,3)}(\gamma)\right) A_{d}(\gamma) \mathrm{e}^{-\mathrm{i} \gamma(x-y d / s)} \mathrm{d} \gamma}_{\phi_{u, d}}
\end{aligned}
$$




$$
\begin{aligned}
& +\underbrace{\frac{1}{2} \int_{-\infty}^{\infty}\left(D^{(2,4)}(\gamma)\right) A_{u}(\gamma) \mathrm{e}^{-\mathrm{i} \gamma(\phi-\psi d / h)} \mathrm{d} \gamma}_{\phi_{d, u}} \\
& +\underbrace{\frac{1}{2} \int_{-\infty}^{\infty}\left(D^{(2,4)}(\gamma)\right) A_{d}(\gamma) \mathrm{e}^{-\mathrm{i} \gamma(\phi-\psi d / h)} \mathrm{d} \gamma}_{\phi_{d, d}}
\end{aligned}
$$

where $D^{(i, j)}=D^{(i)}+D^{(j)}$.

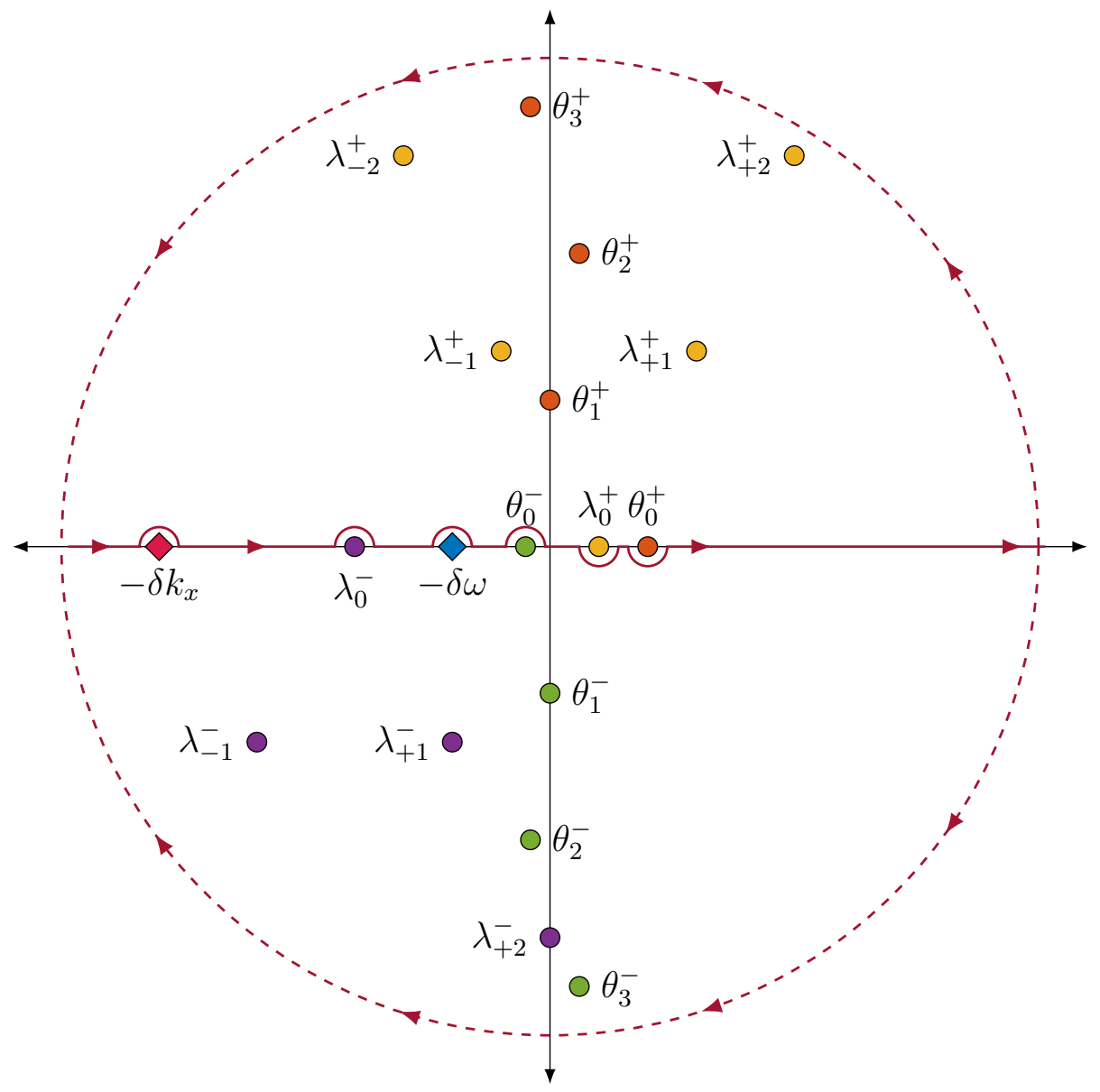

Fig. 5.1 Illustration of the locations of poles in the complex $\gamma$-plane, and the relevant contours of integration.

We first calculate

$$
\begin{aligned}
\phi_{u, u}(x, y) & =\frac{1}{2} \int_{-\infty}^{\infty} D_{0}^{(1,3)}(\gamma) A_{u}(\gamma, x, y) \mathrm{d} \gamma \\
& =2 \pi \int_{-\infty}^{\infty}\left\{D_{0}^{(1,3)}(\gamma) J_{+}(\gamma)\right\} \cdot J_{-}(\gamma) \cdot \frac{\mathrm{e}^{\mathrm{i}(d-\phi) \gamma+\mathrm{i} \sigma} \cos (\zeta \psi)}{\zeta \sin (h \zeta)} \mathrm{d} \gamma
\end{aligned}
$$


Since $J_{+}(\gamma)$ has algebraic growth, inspection of (5.29) shows that $\left|D_{0}^{(1,3)}(\gamma) J_{+}(\gamma)\right| \rightarrow 0$ as $|\gamma| \rightarrow \infty$ except at the poles at $\theta_{n}^{+}$and $-\delta k_{x}$. Consequently, we close the above integral in $\mathscr{L}^{+}$if $x<d$ and in $\mathscr{L}^{-}$if $x>d$. Accordingly, we obtain for $x<d$

$$
\phi_{u, u}(x, y)=-\pi \mathrm{i} \sum_{n=0}^{\infty} \mathscr{B}_{n} A_{u}\left(\theta_{n}^{+}, x, y\right)+\pi \mathrm{i} \sum_{m=-\infty}^{\infty} D^{(1,3)}\left(\lambda_{m}^{+}\right) A_{u}^{r}\left(\lambda_{m}^{+}, x, y\right)
$$

and for $x>d$,

$$
\phi_{u, u}(x, y)=-\pi \sum_{n=0}^{\infty} \frac{\mathscr{A}_{n}+\mathscr{C}_{n}}{\theta_{n}^{-}+\delta \omega} A_{u}\left(\theta_{n}^{-}, x, y\right)-\pi \frac{A_{u}\left(-\delta k_{x}, x, y\right)}{K\left(-\delta k_{x}\right)} \cdot \frac{w_{0}}{(2 \pi)^{2}}
$$

We proceed by considering

$$
\begin{aligned}
\phi_{u, d}(x, y) & =\frac{1}{2} \int_{-\infty}^{\infty} D^{(1,3)}(\gamma) A_{d}(\gamma, x, y) \mathrm{d} \gamma \\
& =2 \pi \int_{-\infty}^{\infty}\left\{D_{0}^{(1,3)}(\gamma) J_{+}(\gamma)\right\} \cdot J_{-}(\gamma) \cdot \frac{-\mathrm{e}^{-\mathrm{i} \gamma x} \cos (\zeta(y-s))}{\zeta \sin (s \zeta)} \mathrm{d} \gamma
\end{aligned}
$$

Using a similar argument to the analysis for (5.69), we close the above integral in $\mathscr{L}^{-}$if $x>0$ in $\mathscr{L}^{+}$if $x<0$. Consequently, for $x>0$ we obtain

$$
\phi_{u, d}(x, y)=-\pi \sum_{n=0}^{\infty} \frac{\mathscr{A}_{n}+\mathscr{C}_{n}}{\theta_{n}^{-}+\delta \omega} A_{d}\left(\theta_{n}^{-}, x, y\right)-\pi \frac{A_{d}\left(-\delta k_{x}, x, y\right)}{K\left(-\delta k_{x}\right)} \cdot \frac{w_{0}}{(2 \pi)^{2}},
$$

and for $x<0$ we obtain

$$
\phi_{u, d}(x, y)=-\pi \mathrm{i} \sum_{n=0}^{\infty} \mathscr{B}_{n} A_{d}\left(\theta_{n}^{+}, x, y\right)+\pi \mathrm{i} \sum_{m=-\infty}^{\infty} D^{(1,3)}\left(\lambda_{m}^{+}\right) A_{d}^{r}\left(\lambda_{m}^{+}, x, y\right),
$$

We now consider

$$
\begin{aligned}
\phi_{d, u}(x, y) & =\frac{1}{2} \int_{-\infty}^{\infty} D_{0}^{(2,4)}(\gamma) A_{u}(\gamma, x, y) \mathrm{d} \gamma \\
& =2 \pi \int_{-\infty}^{\infty}\left\{D^{(2,4)}(\gamma) \mathrm{e}^{-2 \mathrm{i} \gamma} J_{-}(\gamma)\right\} J_{+}(\gamma) \frac{\mathrm{e}^{\mathrm{i}(d+2-x) \gamma+\mathrm{i} \sigma^{\prime}} \cos (\zeta y)}{\zeta \sin (s \zeta)} \mathrm{d} \gamma
\end{aligned}
$$

Following a similar argument to (5.69), $\left|D_{0}^{(2,4)}(\gamma) \mathrm{e}^{-2 \mathrm{i} \gamma} J_{-}(\gamma)\right| \rightarrow 0$ as $|\gamma| \rightarrow \infty$ except at the poles at $-\delta \omega,-\delta k_{x}$ and $\theta_{n}^{ \pm}$. Consequently, we close the integral in $\mathscr{L}^{+}$if $x<d+2$ 
and in $\mathscr{L}^{-}$if $x>d+2$. For $x<d+2$ we obtain

$$
\phi_{d, u}(x, y)=\pi \mathrm{i} \sum_{n=0}^{\infty} B_{n} A_{u}\left(\theta_{n}^{+}\right)
$$

and for $x>d+2$ we obtain

$$
\begin{aligned}
\phi_{d, u}(x, y) & =\pi \sum_{n=0}^{\infty} \frac{\mathscr{A}_{n}+\mathscr{C}_{n}}{\theta_{n}^{-}+\delta \omega} A_{u}\left(\theta_{n}^{-}, x, y\right)+\pi \mathrm{i} P A_{u}(-\delta \omega, x, y) \\
& +\pi \frac{A_{u}\left(-\delta k_{x}, x, y\right)}{K\left(-\delta k_{x}\right)} \cdot \frac{w_{0}}{(2 \pi)^{2}}-\pi \mathrm{i} \sum_{m=-\infty}^{\infty} D^{(2,4)}\left(\lambda_{m}^{-}\right) A_{u}^{r}\left(\lambda_{m}^{-}, x, y\right) .
\end{aligned}
$$

The final integral is

$$
\begin{aligned}
\phi_{d, d}(x, y) & =\frac{1}{2} \int_{-\infty}^{\infty} D^{(2,4)}(\gamma) \mathrm{e}^{-\mathrm{i} \gamma \phi} A_{d}(\gamma, x, y) \mathrm{d} \gamma \\
& =2 \pi \int_{-\infty}^{\infty}\left\{D^{(2,4)}(\gamma) \mathrm{e}^{-2 \mathrm{i} \gamma} J_{-}(\gamma)\right\} J_{+}(\gamma) \frac{-\mathrm{e}^{\mathrm{i}(2-x) \gamma} \cos (\zeta(y-s))}{\zeta \sin (s \zeta)} \mathrm{d} \gamma
\end{aligned}
$$

Using similar arguments to the previous integrals, we close the above integral in $\mathscr{L}^{+}$if $x<2$ and in $\mathscr{L}^{-}$if $x>2$. Consequently, for $x<2$ we obtain

$$
\phi_{d, d}(\phi, \psi)=\pi \mathrm{i} \sum_{n=0}^{\infty} B_{n} A_{d}\left(\theta_{n}^{+}\right),
$$

and for $x>2$ we obtain

$$
\begin{aligned}
\phi_{d, d}(x, y) & =\pi \sum_{n=0}^{\infty} \frac{\mathscr{A}_{n}+\mathscr{C}_{n}}{\theta_{n}^{-}+\delta \omega} A_{d}\left(\theta_{n}^{-}, x, y\right)+\pi \mathrm{i} P A_{d}(-\delta \omega, x, y) \\
& +\pi \frac{A_{d}\left(-\delta k_{x}, x, y\right)}{K\left(-\delta k_{x}\right)} \cdot \frac{w_{0}}{(2 \pi)^{2}}-\pi \mathrm{i} \sum_{m=-\infty}^{\infty} D^{(2,4)}\left(\lambda_{m}^{-}\right) A_{d}^{r}\left(\lambda_{m}^{-}, x, y\right) .
\end{aligned}
$$

Summing the contributions from each integral yields the full Fourier inversion in section 5.3 .1

\section{C Factorisation of Kernel Function}

The kernel function is defined as (5.44)

$$
K(\gamma)=\frac{\zeta \sin (s \zeta)}{4 \pi\left(\cos (s \zeta)-\cos \left(d \gamma+\sigma^{\prime}\right)\right)}+\frac{1}{4 \pi}\left(\mu_{0}-\mathrm{i} \mu_{1} \gamma-\mu_{2} \gamma^{2}\right)
$$


We seek a multiplicative factorisation of this function into parts that are analytic in $\mathscr{L}^{ \pm}$respectively. Consequently, we restrict our attention to the upper half plane and a corresponding factorisation for the lower half plane can be constructed by an appropriate symmetry argument.

\section{C.1 Factorisation of Poles of $K$}

Previous work (Glegg, 1999; Peake, 1992) has factorised the poles of $K$ into the form:

$$
\cos (s \zeta)-\cos \left(d \gamma+\sigma^{\prime}\right)=E(\gamma) \prod_{m=-\infty}^{\infty}\left(1-\frac{\gamma}{\lambda_{m}^{+}}\right) \prod_{m=-\infty}^{\infty}\left(1-\frac{\gamma}{\lambda_{m}^{-}}\right)
$$

where $E(\gamma)$ is an entire function that contains no zeros, and

$$
\lambda_{m}^{ \pm}=-f_{m} \sin (\chi) \pm \cos (\chi) \zeta\left(f_{m}\right), \quad \quad f_{m}=\frac{\sigma^{\prime}-2 \pi m}{\Delta} .
$$

\section{C.1.1 Asymptotic Behaviour of Poles}

We note that the asymptotic behaviour of these poles is

$$
\begin{aligned}
\lambda_{m}^{+} & \sim \lambda_{R}^{(0)+} m+\lambda_{R}^{(2)+}+o(1), \\
\lambda_{-m}^{+} & \sim \lambda_{L}^{(0)+} m+\lambda_{L}^{(2)+}+o(1),
\end{aligned}
$$

as $n \rightarrow \infty$ where

$$
\begin{aligned}
\lambda_{R}^{(0)+} & =2 \pi \frac{d+\mathrm{i} s}{\Delta^{2}}, & \lambda_{R}^{(2)+} & =-\frac{d+\mathrm{i} s}{\Delta^{2}} \sigma^{\prime}, \\
\lambda_{L}^{(0)+} & =2 \pi \frac{-d+\mathrm{i} s}{\Delta^{2}}, & \lambda_{L}^{(2)+} & =\frac{-d+\mathrm{i} s}{\Delta^{2}} \sigma^{\prime} .
\end{aligned}
$$

The subscripts $R$ and $L$ indicate that the pole is in the right- or left-hand side of $\mathscr{L}^{+}$ respectively.

\section{C.2 Factorisation of Zeros of $K$}

We now outline the procedure for factorising the zeros of $K$. In contrast to previous analyses for rigid plates (Glegg, 1999; Peake, 1992), no analytic factorisation is available. A numerical root finding algorithm is sufficient to find the locations of these roots, but we also require some knowledge about their asymptotic behaviour. The reason for this is that during the Wiener-Hopf method we must know the asymptotic behaviour of 
the factorised kernel function. This asymptotic behaviour is inextricably linked to the asymptotic behaviour of the kernel's zeros and poles.

We focus on the zeros located in the first quadrant of the complex $\gamma$-plane. These roots are labelled as $\theta_{n, R}^{+}$. The asymptotic behaviour of the roots in the other quadrants can be determined by a similar procedure.

Recall the definition of the branch cut of $\zeta$ :

$$
\zeta=\sqrt{k^{2} w^{2}-\gamma^{2}}=\mathrm{e}^{i \psi_{1} / 2} \mathrm{e}^{i \psi_{2} / 2}\left|k^{2} w^{2}-\gamma^{2}\right|^{1 / 2}
$$

where

$$
\psi_{1}=\arg (k w-\gamma), \quad \psi_{2}=\arg (k w+\gamma),
$$

and

$$
\pi / 2<\psi_{1}<5 \pi / 2, \quad-\pi / 2<\psi_{2}<3 \pi / 2
$$

Since $\theta_{n, R}^{+}$are in the first quadrant, we have

$$
\zeta\left(\theta_{n, R}^{+}\right) \sim \mathrm{i} \theta_{n, R}^{+}, \quad \text { as } n \rightarrow \infty
$$

This leads us to determine the following asymptotic behaviours:

$$
\begin{gathered}
\sin \left(s \zeta\left(\theta_{n, R}^{+}\right)\right) \sim-\frac{1}{2 \mathrm{i}} \mathrm{e}^{s \theta_{n, R}^{+}}, \\
\cos \left(s \zeta\left(\theta_{n, R}^{+}\right)\right) \sim \frac{1}{2} \mathrm{e}^{s \theta_{n, R}^{+}}, \quad \cos \left(d \theta_{n, R}^{+}+\sigma^{\prime}\right) \sim \frac{1}{2} \mathrm{e}^{-\mathrm{i}\left(d \theta_{n, R}^{+}+\sigma^{\prime}\right)} .
\end{gathered}
$$

We now substitute these representations into (5.70) to obtain asymptotic expansions for the roots $\theta_{n, R}^{i+}$. Each case must be considered separately, although the asymptotic behaviours are similar.

\section{Case I Boundary Condition}

For the no-mean-flow boundary condition, the asymptotic behaviour of the roots obeys

$$
\theta_{n, R}^{+} \sim \mu_{0}\left(1-\exp \left[-\mathrm{i}\left((d-\mathrm{i} s) \theta_{n, R}^{+}+\sigma^{\prime}\right)\right]\right)
$$


We seek an asymptotic expansion of the first quadrant roots of the classical form

$$
\theta_{n, R}^{+} \sim \sum_{m=0}^{\infty} a_{R}^{(k)} \theta_{R}^{(k)}(n)
$$

where $\theta_{R}^{(k+1)}(n)=o\left(\theta_{R}^{(k)}(n)\right)$ as $n \rightarrow \infty$. In (5.73) we require the linear and exponential terms to match. However, since $\left|\theta_{n, R}^{+}\right| \rightarrow \infty$ as $n \rightarrow \infty$, the exponential term will grow at a faster rate than the linear term. Consequently, the real part of the argument of the exponential must be asymptotically small compared to the imaginary part. We therefore expand the roots into real and imaginary parts as $\theta_{n, R}^{+}=\theta_{n, R}^{r+}+\mathrm{i} \theta_{n, R}^{i+}$ and write

$$
d \theta_{n, R}^{i+}-s \theta_{n, R}^{r+}=G(n)
$$

where $G(n)=o\left(\theta_{R}^{(0)}(n)\right)$. Rearranging yields

$$
\theta_{n, R}^{+}=\theta_{n, R}^{+}+\mathrm{i} \theta_{n, R}^{i+}=\left(1+\mathrm{i} \frac{s}{d}\right) \theta_{n, R}^{r+}-\mathrm{i} \frac{G(n)}{d} .
$$

Since the arguments of the left- and right-hand sides of equation (5.73) must match, we obtain an expression for the imaginary part of the argument of the exponential:

$$
\sigma^{\prime}+d \theta_{n, R}^{r+}+s \theta_{n, R}^{i+}=\pi-\arctan \left(\frac{s}{d}\right)-2 n \pi+o(1) .
$$

Applying the asymptotic expansion (5.74) and taking the leading order terms of (5.75) and (5.76) yields

$$
\begin{aligned}
\theta_{R}^{(0)}(n) & =n, \\
a_{R}^{(0)} & =\frac{2 \pi(d+\mathrm{i} s)}{\Delta^{2}} .
\end{aligned}
$$

We may now substitute the expansion for $\theta_{n, R}^{+}$so far into (5.73) to obtain

$$
\frac{2 \pi(d+\mathrm{i} s)}{\Delta^{2}} n+o(n) \sim \mu_{0}\left(1+\exp \left[G(n)+\mathrm{i} \arctan \left(\frac{s}{d}\right)\right]\right) .
$$

We now match leading order terms to obtain

$$
\frac{2 \pi}{\Delta} n=\mu_{0} \exp [G(n)]
$$


so that

$$
G(n)=\log (n)+\log \left(\frac{2 \pi}{\mu_{0} \Delta}\right)
$$

Similarly, taking the leading order terms in (5.75) and (5.76) yields

$$
\begin{aligned}
\theta_{R}^{(1)}(n) & =\log (n), \\
a_{R}^{(1)} & =\mathrm{i} \frac{d+\mathrm{i} s}{\Delta^{2}} .
\end{aligned}
$$

and

$$
\begin{aligned}
\theta_{R}^{(2)}(n) & =1 \\
a_{R}^{(2)} & =\frac{d+\mathrm{i} s}{\Delta^{2}} \cdot\left(\pi-\sigma^{\prime}+\mathrm{i} \log \left(\frac{2 \pi}{\mu_{0} \Delta}\right)-\arctan \left(\frac{s}{d}\right)\right) .
\end{aligned}
$$

Substitution of $(5.77,5.78,5.79)$ into the asymptotic expansion $(5.74)$ yields

$$
\theta_{n, R}^{+} \sim \frac{d+\mathrm{i} s}{\Delta^{2}}\left(2 \pi n+\mathrm{i} \log (n)+\pi-\sigma^{\prime}+\mathrm{i} \log \left(\frac{2 \pi}{\mu_{0} \Delta}\right)-\arctan \left(\frac{s}{d}\right)+o(1)\right) .
$$

Similar analysis yields the asymptotic behaviour for the roots in the second quadrant as

$$
\theta_{n, L}^{+} \sim \frac{-d+\mathrm{i} s}{\Delta^{2}}\left(2 \pi n-\mathrm{i} \log (n)-\pi+\sigma^{\prime}-\mathrm{i} \log \left(\frac{2 \pi}{\mu_{0} \Delta}\right)-\arctan \left(\frac{s}{d}\right)+o(1)\right) .
$$

\section{Case II Boundary Condition}

For the Darcy-type boundary condition, the asymptotic behaviour of the roots obeys the equation

$$
1 \sim-\mathrm{i} \mu_{1}\left(1-\exp \left[-\mathrm{i}\left((d-\mathrm{i} s) \theta_{n, R}^{+}+\sigma^{\prime}\right)\right]\right)
$$

We assume an asymptotic expansion of the roots of the form (5.74). Similar reasoning to the previous sections yields that the leading order terms are also given by

$$
a_{R}^{(0)}=\frac{2 \pi(d+\mathrm{i} s)}{\Delta^{2}}, \quad a_{L}^{(0)}=\frac{2 \pi(-d+\mathrm{i} s)}{\Delta^{2}} .
$$


We may now solve (5.82) directly to find the coefficients of the next two orders of the asymptotic expansion as

$$
\begin{array}{ll}
a_{R}^{(1)}=0, & a_{L}^{(1)}=0, \\
a_{R}^{(2)}=\frac{d+\mathrm{i} s}{\Delta^{2}}\left(\mathrm{i} \log \left(1+\frac{1}{\mathrm{i} \mu_{0}}\right)-\sigma^{\prime}\right), & a_{L}^{(2)}=\frac{-d+\mathrm{i} s}{\Delta^{2}}\left(-\mathrm{i} \log \left(1-\frac{1}{\mathrm{i} \mu_{0}}\right)+\sigma^{\prime}\right) .
\end{array}
$$

\section{Case III boundary condition}

For the case III boundary condition, the asymptotic behaviour of the roots obeys

$$
1 \sim-\mu_{2} \theta_{n, R}^{+}\left(1-\exp \left[-\mathrm{i}\left((d-\mathrm{i} s) \theta_{n, R}^{+}+\sigma^{\prime}\right)\right]\right) .
$$

Similar analysis to the previous sections possesses an identical asymptotic expansion (up to the terms considered) and we have, at leading order,

$$
a_{R}^{(0)}=\frac{2 \pi(d+\mathrm{i} s)}{\Delta^{2}}, \quad a_{L}^{(0)}=\frac{2 \pi(-d+\mathrm{i} s)}{\Delta^{2}} .
$$

Substitution of (5.85) into (5.84) yields the coefficients of the next two orders of the asymptotic expansion as

$$
\begin{aligned}
a_{R}^{(1)} & =0, & a_{L}^{(1)} & =0, \\
a_{R}^{(2)} & =\frac{\sigma^{\prime}(-d-\mathrm{i} s)}{\Delta^{2}}, & a_{L}^{(2)} & =\frac{\sigma^{\prime}(-d+\mathrm{i} s)}{\Delta^{2}} .
\end{aligned}
$$

\section{C.3 Full Factorisation of Kernel, $K$}

We propose a multiplicative splitting of $K$ of the form:

$$
K(\gamma)=K_{+}(\gamma) K_{-}(\gamma)
$$

where

$$
\begin{aligned}
& K_{-}(\gamma)=\mathrm{e}^{E(\gamma)} \frac{\prod_{m=1}^{\infty}\left(1-\gamma / \theta_{m, R}^{+}\right)\left(1-\gamma / \theta_{m, L}^{+}\right)}{\prod_{m=-\infty}^{\infty}\left(1-\gamma / \lambda_{m}^{+}\right)}, \\
& K_{+}(\gamma)=\mathrm{e}^{-E(\gamma)} \frac{\prod_{m=1}^{\infty}\left(1-\gamma / \theta_{m, R}^{-}\right)\left(1-\gamma / \theta_{m, L}^{-}\right)}{\prod_{m=-\infty}^{\infty}\left(1-\gamma / \lambda_{m}^{-}\right)} K(0) .
\end{aligned}
$$


The entire function $E$ is included to ensure that $K_{ \pm}$has algebraic growth in $\mathscr{L}^{ \pm}$ respectively. Previous works for rigid blades (Glegg, 1999; Peake, 1992) have derived the form of the entire function $E$ and shown that it is a polynomial. In the remainder of this section, we will show that $E$ is in fact a constant for the present problem.

\section{Asymptotic Behaviour of Proposed Factorisation}

We focus on $K_{-}$since the asymptotic behaviour of $K_{+}$follows in an analogous manner. We first establish the existence and growth of some relevant products.

Proposition 1. The infinite product

$$
P_{1}=\prod_{m=1}^{\infty}\left(\frac{a_{R}^{(0)} m+a_{R}^{(2)}}{\theta_{m, R}^{+}}\right) \cdot\left(\frac{a_{L}^{(0)} m+a_{L}^{(2)}}{\theta_{m, L}^{+}}\right)
$$

exists.

Proof. We use the asymptotic expansions (5.80) and (5.81) to obtain

$$
\begin{aligned}
& \frac{a_{R}^{(0)} m+a_{R}^{(1)}}{\theta_{m, R}^{+}} \sim 1-\frac{a_{R}^{(1)} \log (m)}{a_{R}^{(0)} m}+o\left(m^{-1}\right), \\
& \frac{a_{L}^{(0)} m+a_{L}^{(1)}}{\theta_{m, L}^{+}} \sim 1-\frac{a_{L}^{(1)} \log (m)}{a_{L}^{(0)} m}+o\left(m^{-1}\right),
\end{aligned}
$$

respectively. Substitution into the product (5.87) yields

$$
P_{1}=\prod_{m=1}^{\infty}\left(1-A \frac{\log (m)}{m}+o\left(m^{-1}\right)\right)
$$

where

$$
A=\frac{a_{R}^{(1)}}{a_{R}^{(0)}}+\frac{a_{L}^{(1)}}{a_{L}^{(0)}}
$$

and application of (5.77) and (5.78) shows that $A=0$. Consequently, the products (5.88) and therefore (5.87) exist via the comparison method.

Proposition 2. The infinite product

$$
P_{2}(\gamma)=\prod_{m=1}^{\infty}\left(\frac{\theta_{m, R}^{+}-\gamma}{a_{R}^{(0)} m+a_{R}^{(2)}-\gamma}\right) \cdot\left(\frac{\theta_{m, L}^{+}-\gamma}{a_{L}^{(0)} m+a_{L}^{(2)}-\gamma}\right)
$$


tends to unity as $\gamma \rightarrow \infty$ in $\mathscr{L}^{-}$.

Proof. Consider the log-derivative of $P_{2}(\gamma)$ :

$$
\begin{aligned}
L(\gamma) & =\frac{\mathrm{d} \log \left(P_{2}(\gamma)\right)}{\mathrm{d} \gamma} \\
& =\sum_{m=1}^{\infty}\left(\frac{\theta_{m, R}^{+}-\left(a_{R}^{(0)} m+a_{R}^{(2)}\right)}{\left(a_{R}^{(0)} m+a_{R}^{(2)}-\gamma\right)\left(\theta_{m, R}^{+}-\gamma\right)}+\frac{\theta_{m, L}^{+}-\left(a_{L}^{(0)} m+a_{L}^{(2)}\right)}{\left(a_{L}^{(0)} m+a_{L}^{(2)}-\gamma\right)\left(\theta_{m, L}^{+}-\gamma\right)}\right) \\
& =\sum_{m=1}^{\infty} f_{m}(\gamma) .
\end{aligned}
$$

Recall (5.80) and $\operatorname{Im}[\gamma]<0$ and $\operatorname{Im}\left[a_{R}^{(0)}, a_{L}^{(0)}\right]>0$. Therefore, $\exists M_{1}>0$ s.t. $\forall m_{1}>M_{1}$,

$$
\begin{aligned}
& \operatorname{Im}\left[\theta_{m_{1}, R}^{+}-\gamma\right], \operatorname{Im}\left[a_{R}^{(0)} m_{1}+a_{R}^{(2)}-\gamma\right]>m_{1} \operatorname{Im}\left[a_{R}^{(0)}\right], \\
& \operatorname{Im}\left[\theta_{m_{1}, L}^{+}-\gamma\right], \operatorname{Im}\left[a_{L}^{(0)} m_{1}+a_{L}^{(2)}-\gamma\right]>m_{1} \operatorname{Im}\left[a_{L}^{(0)}\right] .
\end{aligned}
$$

Furthermore, (5.80) shows that $\exists M_{2}>0$ s.t. $\forall m_{2}>M_{2}$,

$$
\begin{aligned}
& \left|\theta_{m_{2}, R}^{+}-\left(a_{R}^{(0)} m_{2}+a_{R}^{(2)}\right)\right|<C_{R} \log \left(m_{2}\right), \\
& \left|\theta_{m_{2}, L}^{+}-\left(a_{L}^{(0)} m_{2}+a_{L}^{(2)}\right)\right|<C_{L} \log \left(m_{2}\right),
\end{aligned}
$$

for some $C_{L}$ and $C_{R}$. Therefore, we have a uniform bound on $f_{m}(\gamma)$ for $m>M=\max \left(M_{1}, M_{2}\right)$ :

$$
\left|f_{m}(\gamma)\right|<C \frac{\log (m)}{m^{2}}
$$

for some $C$. Consequently, the series $L(\gamma)$ converges uniformly in $\mathscr{L}^{-}$.

Now note that $P_{2}(0)$ exists by proposition 1 . Since the log-derivative of $P_{2}$ is uniformly convergent, we may adapt (14.2.7) of Tao (2016) and claim that $P_{2}$ itself converges uniformly as $\gamma \rightarrow \infty$ in $\mathscr{L}^{-}$. Therefore, we may exchange the orders of limit and product and write

$$
\lim _{\substack{\gamma \rightarrow \infty \\ \gamma \in \mathscr{L}^{-}}} P_{2}(\gamma)=\prod_{m=1}^{\infty} \lim _{\substack{\gamma \rightarrow \infty \\ \gamma \in \mathscr{L}^{-}}}\left(\left(\frac{\theta_{m, R}^{+}-\gamma}{a_{R}^{(0)} m+a_{R}^{(2)}-\gamma}\right) \cdot\left(\frac{\theta_{m, L}^{+}-\gamma}{a_{L}^{(0)} m+a_{L}^{(2)}-\gamma}\right)\right)=1 .
$$


We now calculate the asymptotic behaviour of $K_{-}$by comparison with the product

$$
P(\gamma)=\prod_{m=1}^{\infty} \frac{\left(1-\gamma / \theta_{m, R}^{+}\right) \mathrm{e}^{\gamma / \theta_{m, R}^{+}}\left(1-\gamma / \theta_{m, L}^{+}\right) \mathrm{e}^{\gamma / \theta_{m, L}^{+}}}{\left(1-\gamma /\left(a_{R}^{(0)} m+a_{R}^{(2)}\right)\right) \mathrm{e}^{\gamma / a_{R}^{(0)} m}\left(1-\gamma /\left(a_{L}^{(0)} m+a_{L}^{(2)}\right)\right) \mathrm{e}^{\gamma / a_{L}^{(0)} m}}
$$

This function may be written as

$$
P(\gamma)=\exp \left(\gamma \sum_{m=1}^{\infty} \frac{1}{\theta_{m, R}^{+}}-\frac{1}{a_{R}^{(0)} m}-\frac{1}{\theta_{m, L}^{+}}-\frac{1}{a_{L}^{(0)} m}\right) P_{1} P_{2}(\gamma)
$$

The product $P_{1}$ is known to exist by proposition 1 . Applying proposition 2 yields

$$
P(\gamma) \sim B \gamma^{\alpha} \exp \left(\gamma \sum_{m=1}^{\infty} \frac{1}{\theta_{m, R}^{+}}-\frac{1}{a_{R}^{(0)} m}+\frac{1}{\theta_{m, L}^{+}}-\frac{1}{a_{L}^{(0)} m}\right),
$$

for some constant B. By applying the asymptotic behaviour of the Gamma function (Peake, 1992, B7 \& B8), we derive the relation

$$
\prod_{n=1}^{\infty}\left(1-\frac{\gamma}{a m+b}\right) \exp \left[\frac{\gamma}{a m}\right] \sim C \exp \left[\frac{\gamma}{a}(\mathscr{E}-1-\log (-a))+\left(\frac{\gamma}{a}-\frac{b}{a}-\frac{1}{2}\right) \log (\gamma)\right]
$$

where $\mathscr{E}$ is the Euler--Mascheroni constant and

$$
C=\frac{-b}{\sqrt{2 \pi}} \Gamma\left(\frac{b}{a}\right)(-a)^{\frac{b}{a}-\frac{1}{2}}
$$

This representation may be substituted into the denominator of (5.91). Rearranging yields the asymptotic behaviour

$$
\begin{aligned}
\prod_{m=1}^{\infty} & \left(1-\gamma / \theta_{m, R}^{+}\right) \mathrm{e}^{\gamma / \theta_{m, R}^{+}}\left(1-\gamma / \theta_{m, L}^{+}\right) \mathrm{e}^{\gamma / \theta_{m, L}^{+}} \sim B_{2} \gamma^{\alpha} \exp \left(\gamma \sum_{m=1}^{\infty} \frac{1}{\theta_{m, R}^{+}}+\frac{1}{\theta_{m, L}^{+}}\right) \\
& \times \exp \left[\frac{\gamma}{a_{R}^{(0)} a_{L}^{(0)}}\left((\mathscr{E}-1)\left(a_{R}^{(0)}+a_{L}^{(0)}\right)-a_{L}^{(0)} \log \left(a_{R}^{(0)}\right)+a_{R}^{(0)} \log \left(a_{L}^{(0)}\right)\right)+\right. \\
& \left.\left(\gamma\left(\frac{1}{a_{R}^{(0)}}+\frac{1}{a_{L}^{(0)}}\right)-\left(\frac{a_{R}^{(2)}}{a_{R}^{(0)}}+\frac{a_{L}^{(2)}}{a_{L}^{(0)}}\right)-1\right) \log (\gamma)\right],
\end{aligned}
$$

for some constant $B_{2}$. We also note that

$$
\prod_{m=-\infty}^{\infty}\left(1-\gamma / \lambda_{m}^{+}\right) \mathrm{e}^{\gamma / \lambda_{m}^{+}} \sim B_{3} \gamma \exp \left(\gamma \sum_{m=1}^{\infty} \frac{1}{\lambda_{m}^{(0)+}}+\frac{1}{\lambda_{-m}^{(0)+}}\right)
$$




$$
\begin{aligned}
& \times \exp \left[\frac{\gamma}{\lambda_{m}^{(0)+} \lambda_{-m}^{(0)+}}\left((\mathscr{E}-1)\left(\lambda_{m}^{(0)+}+\lambda_{-m}^{(0)+}\right)-\lambda_{-m}^{(0)+} \log \left(\lambda_{m}^{(0)+}\right)+\lambda_{m}^{(0)+} \log \left(\lambda_{-m}^{(0)+}\right)\right)\right. \\
& \left.+\left(\gamma\left(\frac{1}{\lambda_{m}^{(0)+}}+\frac{1}{\lambda_{-m}^{(0)+}}\right)-\left(\frac{\lambda_{m}^{(2)+}}{\lambda_{m}^{(0)+}}+\frac{\lambda_{-m}^{(2)+}}{\lambda_{-m}^{(0)+}}\right)-1\right) \log (\gamma)\right]
\end{aligned}
$$

for some constant $B_{3}$. Noting that $\lambda_{m}^{(0)+}=a_{R}^{(m)}$ and $\lambda_{-m}^{(0)+}=a_{L}^{(m)}$, we obtain

$$
\frac{\prod_{m=1}^{\infty}\left(1-\gamma / \theta_{m, R}^{+}\right)\left(1-\gamma / \theta_{m, L}^{+}\right)}{\prod_{m=-\infty}^{\infty}\left(1-\gamma / \lambda_{m}^{+}\right)} \sim B_{4} \gamma^{\alpha-1} \exp \left[-\left(\frac{a_{R}^{(2)}}{a_{R}^{(0)}}+\frac{a_{L}^{(2)}}{a_{L}^{(0)}}\right) \log (\gamma)\right]
$$

for some constant $B_{4}$. Consequently, the entire function $E$ in (5.86.a) is a constant. 



\section{Chapter 6}

\section{Potential Flow Through Cascades with Multiple Aerofoils per Period}

\subsection{Introduction}

The analyses in this thesis have, thus far, been restricted to cascades consisting of blades with thin profiles and at small angles of attack. These restrictions allowed us to find analytic solutions for the potential flow through cascades of rigid and porous aerofoils in chapters 2 and 4 respectively. However, the blades used in realistic turbomachinery stages possess large amounts of camber in order to turn the flow through a desired angle. The studies in previous chapters were also limited insofar as they were restricted to analysing a single cascade in isolation and were unable to account for interference effects between adjacent cascades. Such effects may be important in rotor-stator interactions where adjacent blade rows are tightly spaced. Additionally, the work in previous chapters was limited to uniform flows and could not be used to analyse the behaviour of singularities in the flow or the effects of the motion of the blades. The rapid motion of rotors is clearly essential to their modelling, and is not accessible using the methods of previous chapters. In this chapter, we address these deficiencies and derive solutions for the potential flow through cascades where (a) the blades are of arbitrary geometry, (b) there are an arbitrary number of blades per period window, and (c) the flow may involve point vortices and moving blades. A possible arrangement that is amenable to the methods of the present section is illustrated in figure 6.1.

Essential tools for constructing these flows are conformal mappings. As outlined in section 1.2.3, conformal mappings enable us to relate simple canonical domains to complex, physical domains. Since Laplace's equation is invariant under such mappings, it is sufficient to construct the complex potential in the simple domain, and then map the 


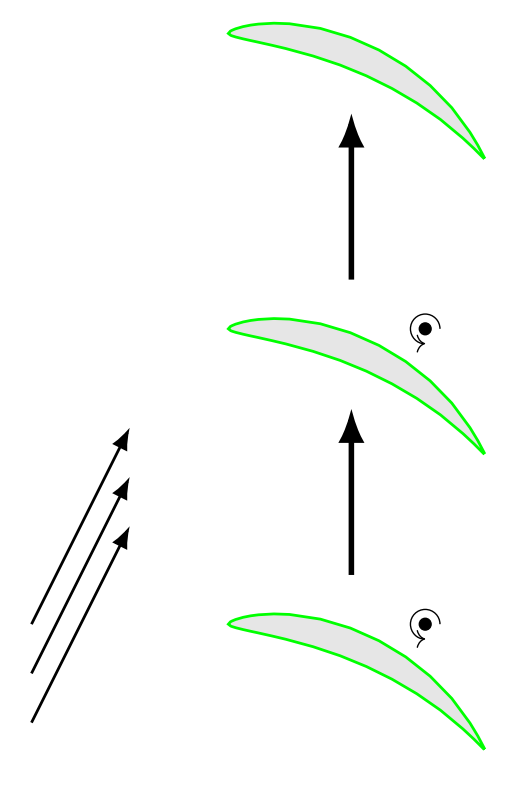

rotor

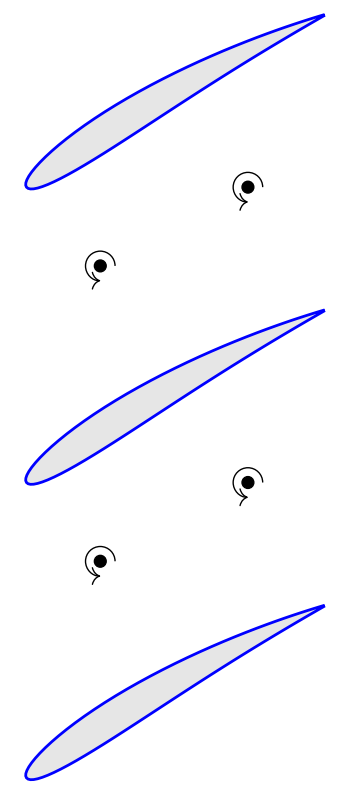

stator

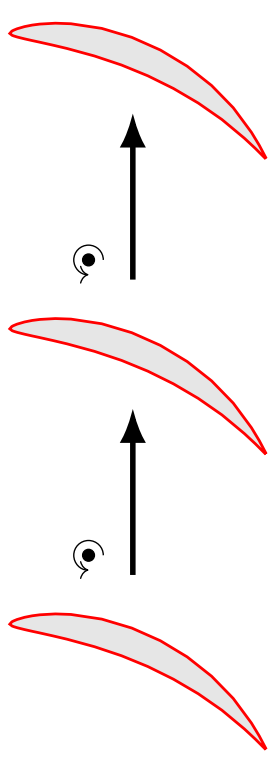

rotor

Fig. 6.1 An example of a possible rotor-stator configuration amenable to the methods outlined in this chapter. The upstream arrow indicates an incoming mean flow (at arbitrary angle of attack), the spirals indicate point vortices, and the vertical arrows indicate the motion of the blades. The arrangement is periodic in the vertical direction.

solution to the target domain of interest. Consequently, we also study periodic conformal mappings that may be used to map a circular domain to the desired target domain.

In section 1.2.3.2 we introduced the transcendental Schottky-Klein prime function. We demonstrated that the prime function is a fundamental object in multiply connected domains, partly because the five canonical conformal mappings can be expressed in terms of the prime function. Accordingly, the prime function is an important building block for the potential flow solutions in this chapter. The prime function has previously been applied to solve potential flows in non-periodic domain by Crowdy (2010), and we now extend that analysis to consider potential flows through periodic domains. In particular, we present two new "canonical" conformal mappings for periodic domains which we will use to construct the potential flow. We also show that we recover the non-periodic solution in the limit where the period is large.

The layout of this chapter is as follows. In section 6.2, we present two new "canonical" periodic conformal mappings. We also present a new periodic Joukowski mapping that enables a range of relevant aerofoil shapes to be analysed. In section 6.3 , we present analytic solutions for a range of flows through periodic domains, including point vortices, 

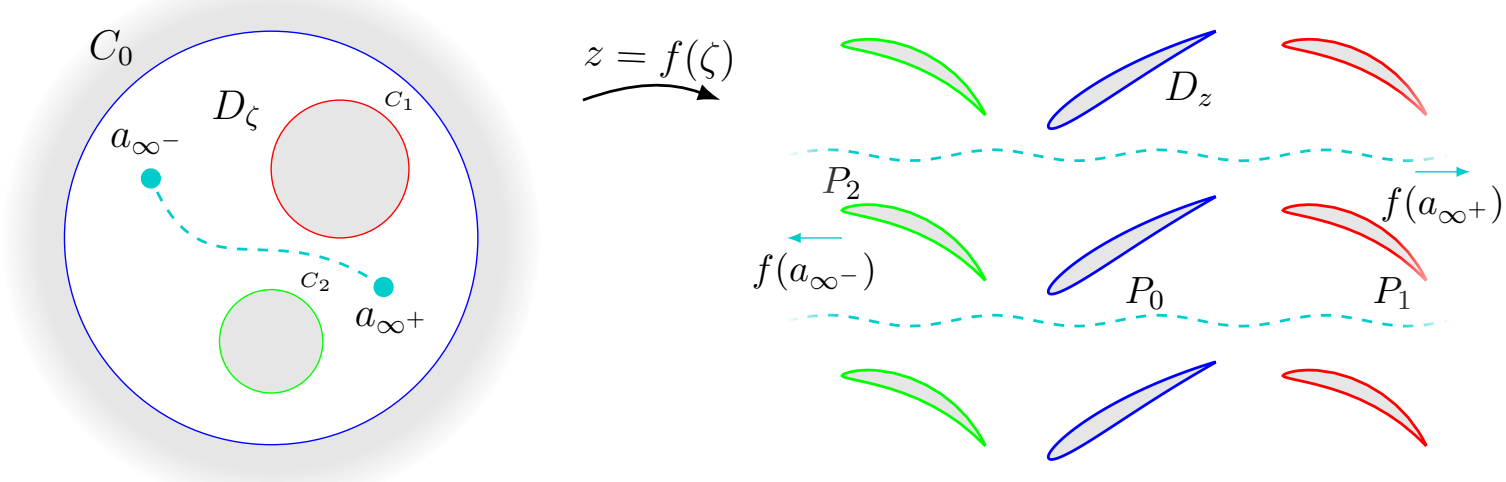

Fig. 6.2 An example of a periodic conformal mapping from a canonical circular domain to the physical target domain in figure 6.1. In this case, there are three objects per period window, so $M=2$. The dashed line indicates the branch cut.

uniform flows, and the effect of moving objects. Finally, in section 6.4 we summarise the chapter.

\subsection{Periodic Conformal Mappings}

Periodic conformal mappings have been applied in various contexts within fluid dynamics. Following Baddoo and Crowdy (2019), we divide all periodic conformal mappings into three cases with respect to the boundedness of the target period window: the target period window may be unbounded in two directions (case I), unbounded in only one direction (case II), or bounded (case III). Case I mappings have found relevance in the study of superhydrophobic surfaces (Crowdy, 2011) where the mappings were used to derive frictional slip lengths for grooved surfaces. Additionally, these mappings have been applied to find analytic solutions for free boundary problems, including von Kármán streets of hollow vortices (Crowdy and Green, 2011) for both simply connected and doubly connected domains, and arrays of bubbles in Hele-Shaw cells (Vasconcelos, 1993, 2015). Case II mappings have been used to study the interaction of a vortex street with a shear flow by Crowdy and Nelson (2010), and free surface Euler flows by Crowdy (2000). Case III mappings have been applied to study steady capillary waves on an annulus by Crowdy (1999), and to derive effective slip lengths for superhydrophobic surfaces by Crowdy (2017).

It is often difficult to construct the desired mapping, particularly in case I domains. Whilst constructive formulae are available for simply connected geometries in case II 
and case III (Floryan, 1985; Floryan and Zemach, 1993), case I mappings have not been investigated in detail until relatively recently (Baddoo and Crowdy, 2019). The only previous example of a case I mapping is a mapping from the unit disc to a periodic array of slits, which has found prominence in turbomachinery studies (Evers and Peake, 2002; Robinson and Laurmann, 1956; Wade, 1967). We will show later that this mapping is, in fact, a special case of the new canonical periodic mappings derived in this section. In this section, we present several new periodic conformal mappings, many of which are valid for an arbitrary number of objects per period window.

In general, we wish to relate a canonical circular domain $D_{\zeta}$ to a periodic physical target domain $D_{z}$ as illustrated in figure 6.2. As discussed in section 1.2.3, a canonical circular domain of connectivity $M+1$ is defined as the unit disc with $M$ excised circles. We assume that the points $a_{\infty^{ \pm}} \in D_{\zeta}$ are mapped to downstream and upstream infinity respectively. Moreover, we assume that the circular domain has a branch cut which connects $a_{\infty^{ \pm}}$. This branch cut is responsible for the periodicity of the mappings in this section. We label the discs in the circular domain as $C_{j}$, and the images of these discs in the physical domain as $P_{j}$, as illustrated in figure 6.2.

\subsubsection{Two Canonical Periodic Conformal Mappings}

We now consider two special periodic conformal mappings. These mappings are given special attention because they map the canonical circular domain to a periodic array of slits with constant imaginary part. These mappings will later be used to construct the complex potential for a flow through a periodic domain, which we also require to have constant imaginary part on each boundary.

We first consider the "circular arc maps" (Crowdy and Marshall, 2006; Nehari, 1952) that were introduced in section 1.2.3. The effect of these mappings are to transplant the $M+1$ discs to $M+1$ finite-length circular arc slits, each of which are centered at the origin in the $z$-plane. The mappings take the functional form

$$
T\left(\zeta ; a_{\infty^{-}}, a_{\infty^{+}}\right)=\frac{\omega\left(\zeta, a_{\infty^{-}}\right) \omega\left(\zeta, 1 / \overline{a_{\infty^{+}}}\right)}{\omega\left(\zeta, 1 / \overline{a_{\infty^{-}}}\right) \omega\left(\zeta, a_{\infty^{+}}\right)},
$$

where $a_{\infty^{-}}$is mapped to the origin and $a_{\infty^{+}}$is mapped to infinity. The function $\omega(\cdot, \cdot)$ is the Schottky-Klein prime function introduced in section 1.2.3.2. The effect of such a mapping is illustrated in figure 6.3 for $M=2$. 

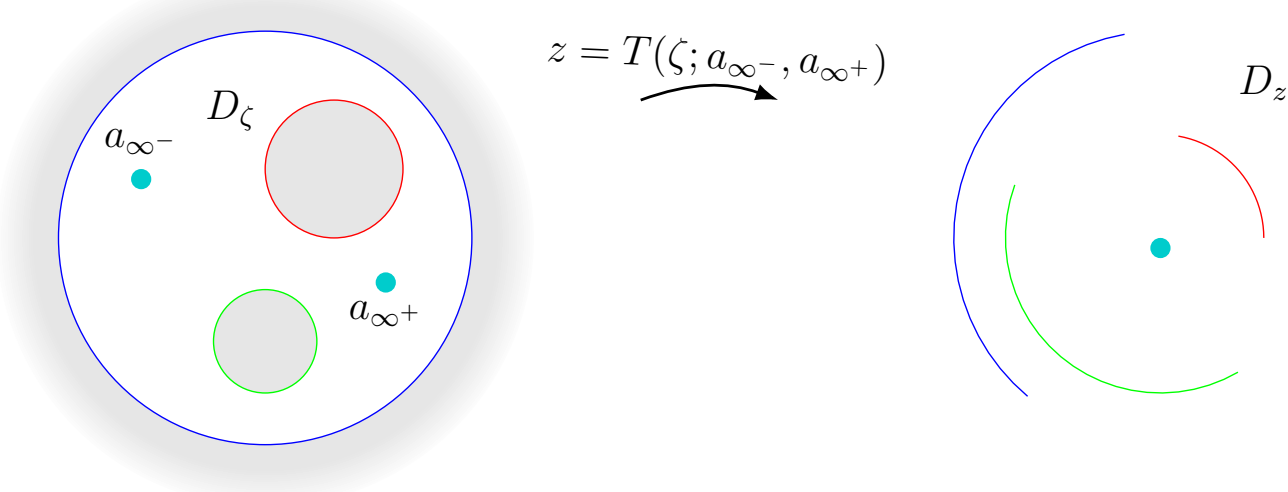

Fig. 6.3 A circular arc map for $M=2$.

We now take the logarithm of the circular arc slit map and rescale by a pure imaginary factor such as

$$
\begin{aligned}
H\left(\zeta ; a_{\infty^{-}}, a_{\infty^{+}}\right) & =\frac{1}{2 \pi \mathrm{i}} \log \left(T\left(\zeta ; a_{\infty^{-}}, a_{\infty^{+}}\right)\right) \\
& =\frac{1}{2 \pi \mathrm{i}} \log \left(\frac{\omega\left(\zeta, a_{\infty^{-}}\right) \omega\left(\zeta, 1 / \overline{a_{\infty^{+}}}\right)}{\omega\left(\zeta, a_{\infty^{+}}\right) \omega\left(\zeta, 1 / \overline{a_{\infty^{-}}}\right)}\right) .
\end{aligned}
$$

Since circular arcs have constant radius, taking a logarithm transplants these circular arcs to lines with constant real part. Rescaling by an imaginary factor then rotates the system so that the slits have constant imaginary part. In particular, the period of the mapping is real so that the branch cut is oriented horizontally. Accordingly, the branch cut in $D_{\zeta}$ is arranged such that it passes between the branch points at $a_{\infty^{-}}$and $a_{\infty^{+}}$, as illustrated in figure 6.4. In hydrodynamical terms, $H$ corresponds to the complex potential induced by two vortices of equal but opposite strength located at $a_{\infty^{-}}$and $a_{\infty^{+}}$. The mapping $H$ is the first canonical periodic conformal mapping. Note that the images of the discs are slits with constant imaginary parts.

We now present the analytic form of the radial slit map, which was introduced in 1.2 .3 , as

$$
R\left(\zeta ; a_{\infty^{-}}, a_{\infty^{+}}\right)=\frac{\omega\left(\zeta, a_{\infty^{-}}\right) \omega\left(\zeta, 1 / \overline{a_{\infty^{-}}}\right)}{\omega\left(\zeta, a_{\infty^{+}}\right) \omega\left(\zeta, 1 / \overline{a_{\infty^{+}}}\right)}
$$

Crowdy and Marshall (2006) showed that this mapping transplants each boundary circle in the circular domain to a radial slit pointing at the origin. Similarly to the circular $\operatorname{arc}$ map $T$, the point $a_{\infty^{-}}$is mapped to the origin whereas the point $a_{\infty^{+}}$is mapped to 

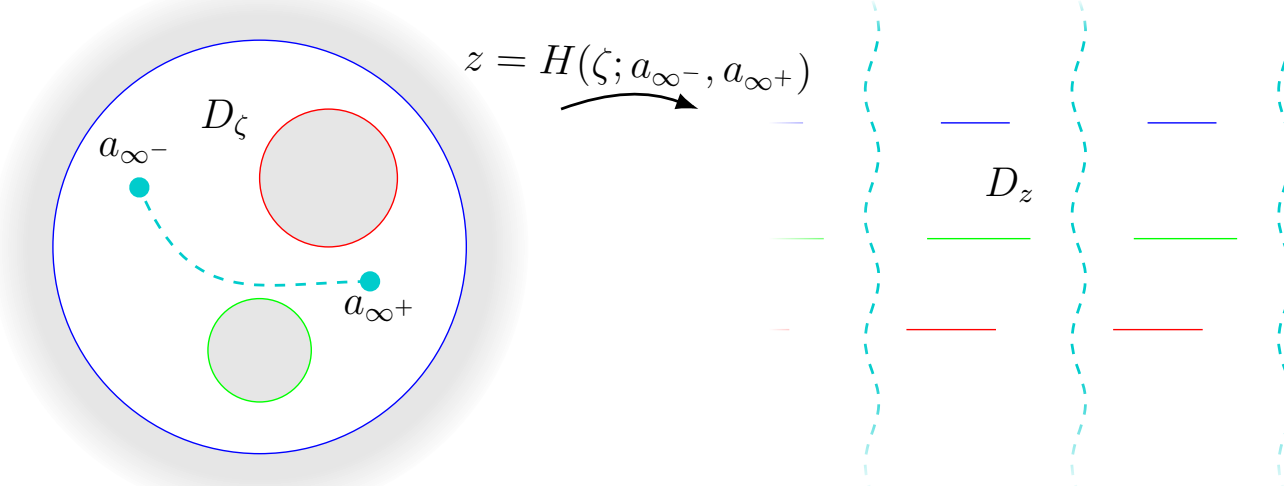

Fig. 6.4 The effect of the conformal mapping $H$ on a canonical circular domain for $M=2$.

infinity. The geometric effect of the radial slit map is illustrated in figure 6.5 in the case $M=2$.
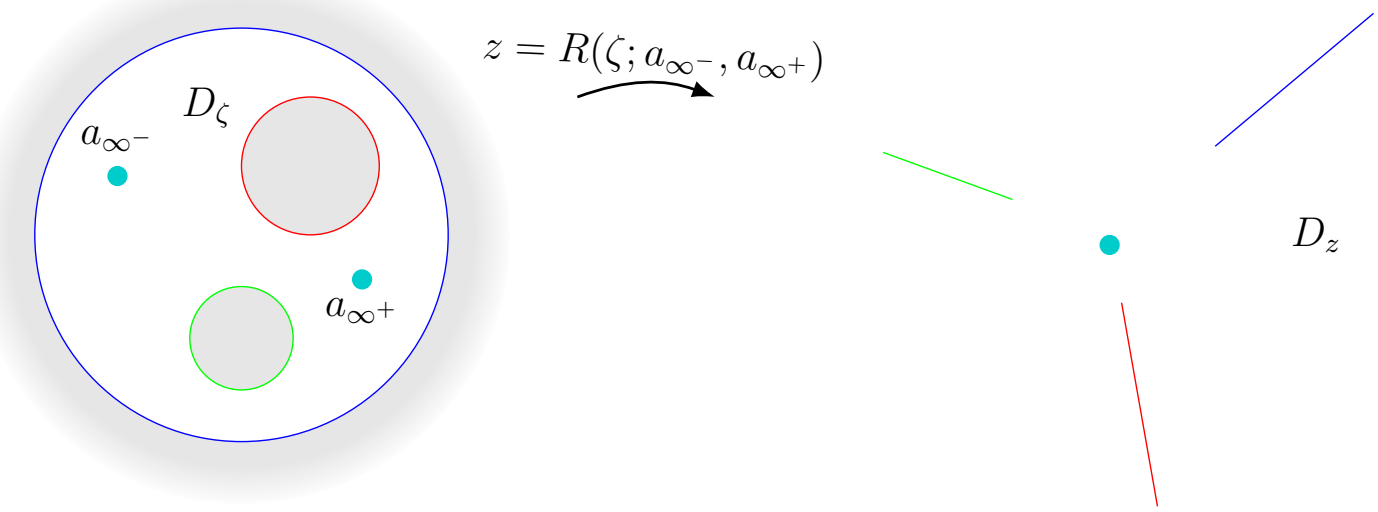

Fig. 6.5 A radial slit map for $M=2$.

Similarly to the canonical mapping $H$, we take a logarithm of this radial slit map and write

$$
\begin{aligned}
V\left(\zeta ; a_{\infty^{-}}, a_{\infty^{+}}\right) & =\frac{1}{2 \pi} \log \left(R\left(\zeta ; a_{\infty^{-}}, a_{\infty^{+}}\right)\right) \\
& =\frac{1}{2 \pi} \log \left(\frac{\omega\left(\zeta, a_{\infty^{-}}\right) \omega\left(\zeta, 1 / \overline{a_{\infty^{-}}}\right)}{\omega\left(\zeta, a_{\infty^{+}}\right) \omega\left(\zeta, 1 / \overline{a_{\infty^{+}}}\right)}\right) .
\end{aligned}
$$

This new composite mapping sends each disc to a slit with constant imaginary part, as illustrated in figure 6.6. As the argument of $V$ winds around the branch point $a_{\infty^{-}}$ in the clockwise direction, $V$ increases by i. Conversely, winding around $a_{\infty^{+}}$in the clockwise direction decreases $V$ by i. Consequently, and in contrast to $H$, the period is now oriented in the vertical direction. In hydrodynamical terms, $V$ corresponds to the 
complex potential induced by a source-sink pair with the source located at $\zeta=a_{\infty^{-}}$and the sink located at $\zeta=a_{\infty^{+}}$(Crowdy, 2013).

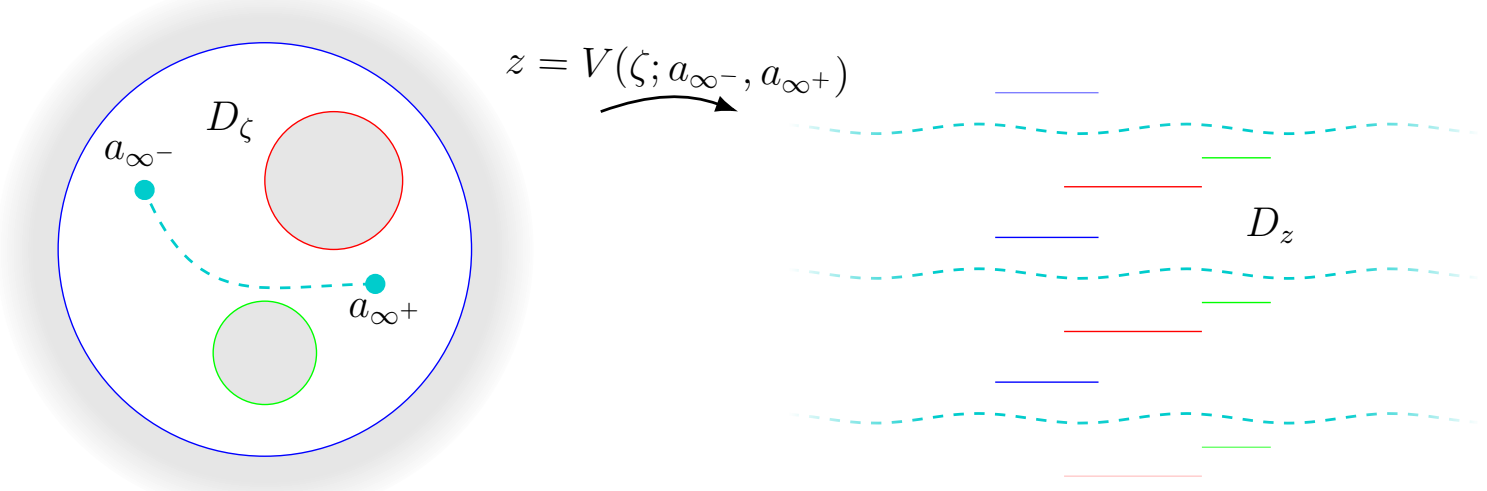

Fig. 6.6 The effect of the conformal mapping $V$ on a canonical circular domain for $M=2$.

We now combine the canonical slit maps to produce a new map. Since $H$ has period 1 and $V$ has period i, the mapping

$$
S\left(\zeta ; a_{\infty^{-}}, a_{\infty^{+}}\right)=\mathrm{e}^{\mathrm{i} \chi}\left(\cos \left(\chi-\frac{\pi}{2}\right) H\left(\zeta ; a_{\infty^{-}}, a_{\infty^{+}}\right)-\sin \left(\chi-\frac{\pi}{2}\right) V\left(\zeta ; a_{\infty^{-}}, a_{\infty^{+}}\right)\right)
$$

has period i, except the slits no longer have constant imaginary part but rather make an angle of $\chi$ with the horizontal axis. Expanded in terms of the prime function, $S$ may be expressed as

$$
S\left(\zeta ; a_{\infty^{-}}, a_{\infty^{+}}\right)=\frac{1}{2 \pi}\left(\log \left(\frac{\omega\left(\zeta, a_{\infty^{-}}\right)}{\omega\left(\zeta, a_{\infty^{+}}\right)}\right)+\mathrm{e}^{2 \mathrm{i} \chi} \log \left(\frac{\omega\left(\zeta, 1 / \overline{a_{\infty^{-}}}\right)}{\omega\left(\zeta, 1 / \overline{a_{\infty^{+}}}\right)}\right)\right)
$$

The effect of $S$ as a conformal mapping is illustrated in figure 6.7. The mapping $S$ could be used as a first approximation to the mapping in figure 6.2, although the slits mapped by $S$ must all be at the same angle. In hydrodynamical terms, $S$ represents the complex potential induced by a vortex spiral (Crowdy, 2013).

\subsubsection{The Periodic Joukowski Mapping}

In this section, we show that the new canonical periodic conformal mappings may be used to construct conformal mappings for periodic arrays of "Joukowski-type" aerofoils. For simplicity, we consider the case $M=0$, although the general strategy could be applied to cases where there are multiple objects per period window. 


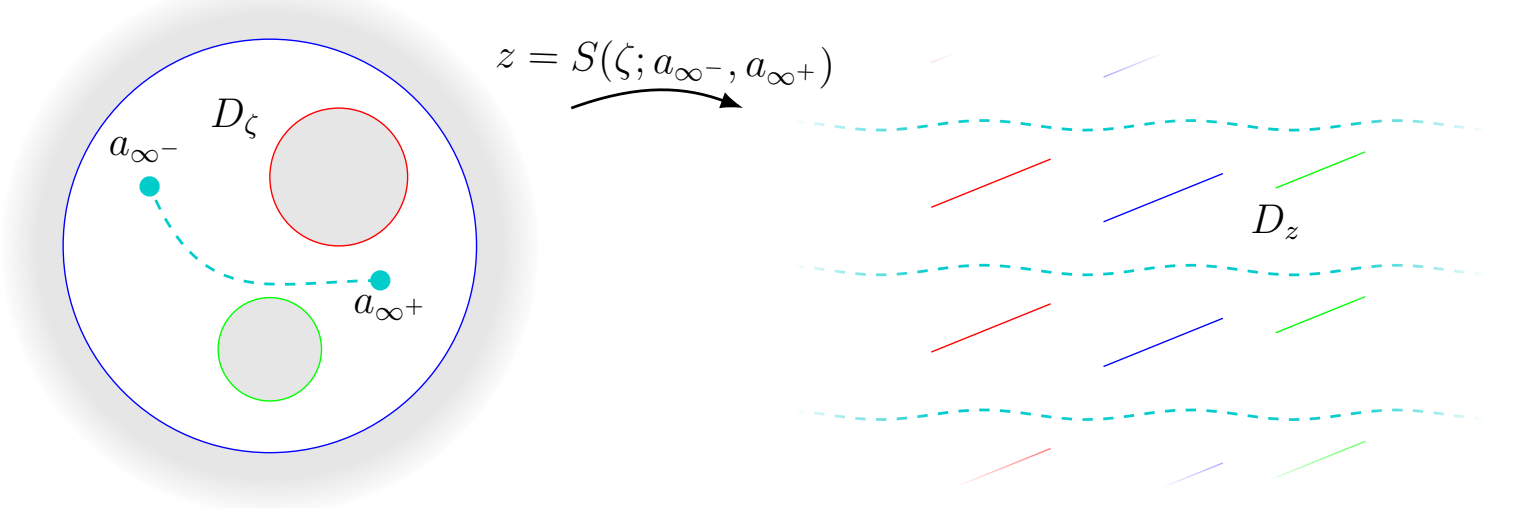

Fig. 6.7 The effect of the conformal mapping $S$ on a canonical circular domain for $M=2$. The slits are inclined at an angle of $\chi$ to the horizontal.

In the case where there is a single object per period window $(M=0)$, the mapping $S$ becomes

$$
S\left(\zeta ; a_{\infty^{-}}, a_{\infty^{+}}\right)=\frac{1}{2 \pi}\left(\log \left(\frac{\zeta-a_{\infty^{-}}}{\zeta-a_{\infty^{+}}}\right)+\mathrm{e}^{2 \mathrm{i} \chi} \log \left(\frac{\zeta-1 / \overline{a_{\infty^{-}}}}{\zeta-1 / \overline{a_{\infty^{+}}}}\right)\right),
$$

which is precisely the mapping used in the previously mentioned turbomachinery studies (Evers and Peake, 2002; Robinson and Laurmann, 1956; Wade, 1967). This mapping is, in fact, a periodic analogue of the Joukowski transform: as we send $a_{\infty^{-}}, a_{\infty^{+}} \rightarrow 0$, we obtain

$$
S(\zeta ; 0,0)=C\left(\zeta+\frac{1}{\zeta}\right)
$$

for constant $C$. Note that the above mapping is no longer periodic since there is no branch cut in the $\zeta$-plane. In particular, the branch points at $a_{\infty^{-}}$and $a_{\infty^{+}}$have joined to form a simple pole.

The Joukowski transform (first presented in Joukowski (1910)) is a popular conformal mapping that is often used in aerodynamic applications. The mapping relates the unit disc to a class of "Joukowski-type" aerofoils. Its popularity stems from: (i) the fact that the mapping can be expressed in a simple form, (ii) it is invertible, (iii) and it can generate a large range of aerofoil geometries. In this section we exploit the new periodic slit mapping $S$ to map the unit disc to a periodic array of Joukowski aerofoils.

It is illustrative to begin with a brief discussion of the non-periodic Joukowski mapping. The mapping (6.2) maps the interior of the unit disc to the exterior of a slit ranging 


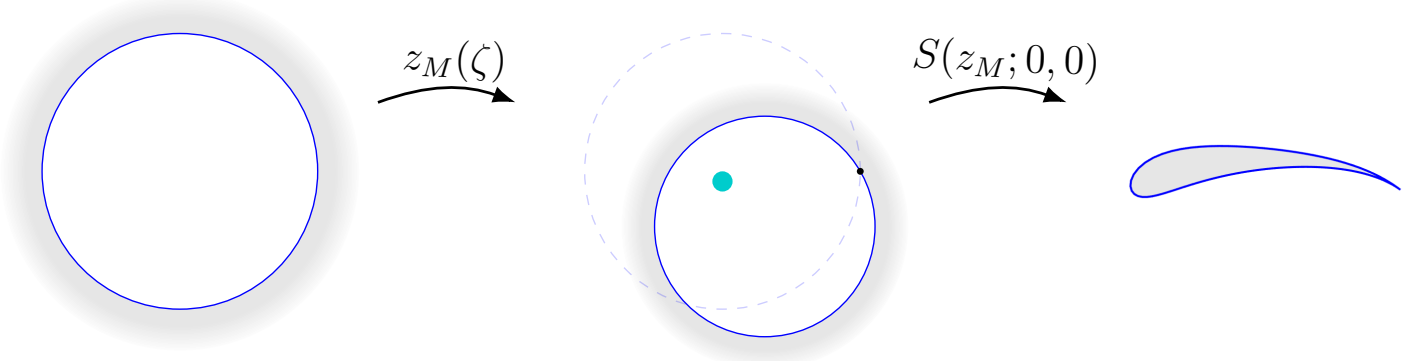

Fig. 6.8 The procedure for constructing the non-periodic Joukowski mapping.

between \pm 1 . It is of particular importance to note that the derivative of the mapping (6.2) has a simple pole at the pre-image of the edges of the slit at $\zeta= \pm 1$. Consequently, the image of any curve that passes through the points $\zeta= \pm 1$ will also have a cusp at $S( \pm 1 ; 0,0)$. When the curve is chosen to be a circle, the image of the curve possesses a blunt leading edge and a cusped trailing edge: it is a Joukowski aerofoil. Moreover, the velocity potential in the circle can be computed using standard techniques. Consequently, we perturb the unit disc by a Möbius map of the form

$$
z_{M}(\zeta)=(z-1) r \mathrm{e}^{\mathrm{i} \theta}+1
$$

for some angle $\theta$ and scale factor $r$. This Möbius map is chosen since it maps the unit disc to a new disc that passes through +1 . The circle must enclose the pre-image of infinity (in this case $\zeta=0$ ) otherwise the region $D_{z}$ is bounded. We now apply the Joukowski map (6.2) to the new perturbed disc to obtain a Joukowski-type aerofoil. This procedure is illustrated in figure 6.8 .

We now employ a similar strategy to derive the periodic Joukowski mapping. We first note that the zeros of the derivative of $S$ are not, in general at $\zeta= \pm 1$. Since the image of the circle under the Möbius map must pass through an edge, we write

$$
z_{M, P}=\left(z-\zeta_{t}\right) r \mathrm{e}^{\mathrm{i} \theta}+\zeta_{t}
$$

where

$$
\left[S_{\zeta}\left(\zeta, a_{\infty^{+}}, a_{\infty}^{-}\right)\right]_{\zeta=\zeta_{t}}=0
$$

In an analogous way to the non-periodic case, the circle $z_{M, P}\left(C_{0}\right)$ must enclose both of the branch points. We now apply the periodic slit map to the transformed circle to obtain 

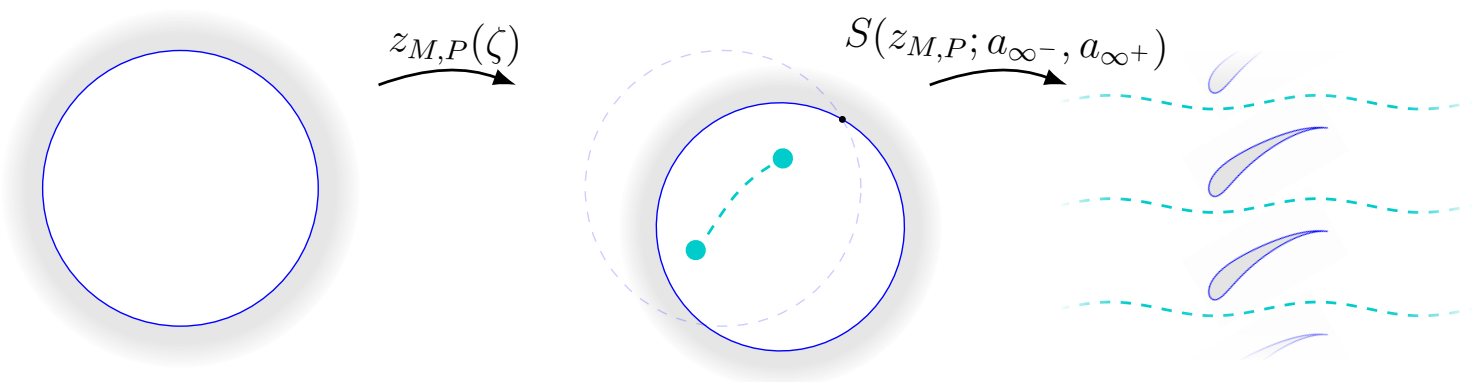

Fig. 6.9 The procedure for constructing the periodic Joukowski mapping.

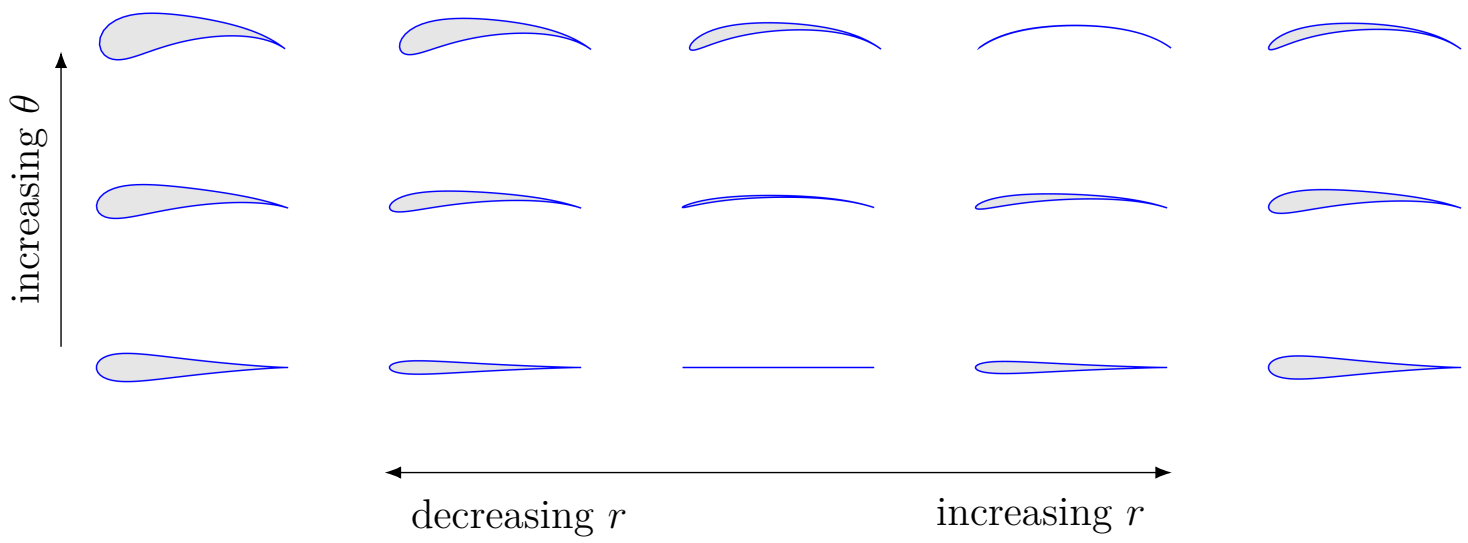

Fig. 6.10 Several examples of Joukowski-type aerofoils that are amenable to the periodic Joukowski formula. The dependence of the aerofoil shape on the parameters of the Möbius mapping (6.3) are illustrated by the arrows.

a periodic array of Joukowski-type aerofoils. This mapping procedure is illustrated in figure 6.9.

A range of aerofoil geometries are amenable to the periodic Joukowski mapping, some of which are illustrated in figure 6.10. We note that every aerofoil has a blunt leading edge and a cusped trailing edge. Evidently, a large range of interesting geometries are available, including large cambers and thickness that are not permissible in the thin aerofoil approximation of chapter 2. The aerofoil geometry is controlled by varying the radius and angle of intersection of the modified circle in the Joukowski transformation. Varying the radius controls the thickness of the aerofoil, whereas varying the angle of intersection controls the camber. 

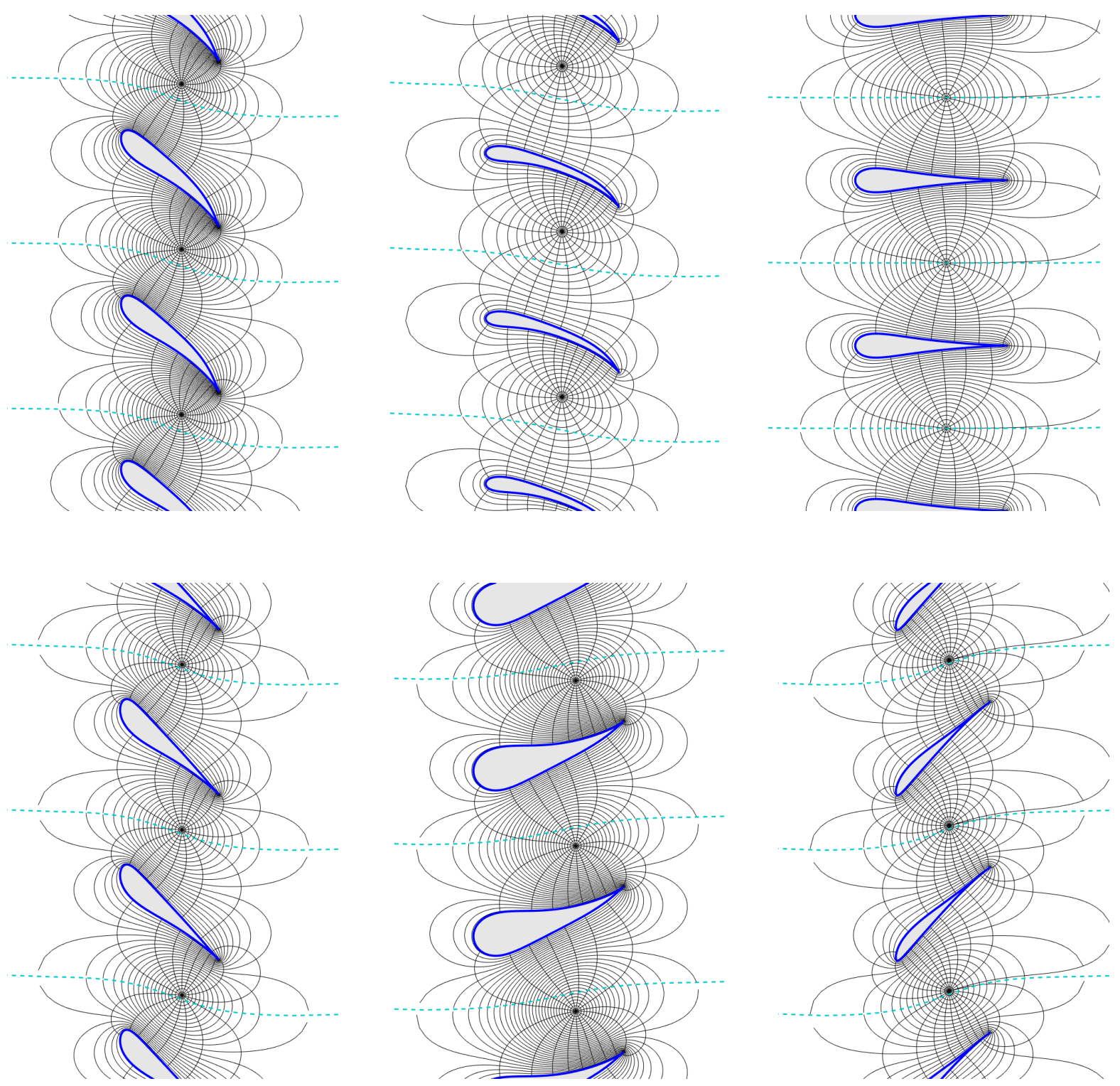

Fig. 6.11 Several examples of cascades of Joukowski-type aerofoils. The black lines are the images of a polar grid centered at the origin in the $z_{M, P}$-plane. 


\subsubsection{The Periodic Schwarz-Christoffel Formula}

Although the extended Riemann mapping theorem guarantees the existence of a conformal mapping between two domains of the same connectivity, it is often difficult to construct such a mapping in practice. Schwarz-Christoffel (S-C) formulae are useful tools for generating such mappings, as they represent constructive tools that furnish a conformal mapping to a desired target domain. Typically, S-C mappings provide a conformal mapping between a canonical domain (taken to be circles in the present work) and a polygonal domain, although there are extensions available for polycircular arc domains (Crowdy et al., 2011), gear-like regions (Goodman, 1960), and curved regions (Henrici, 1986). Although the mappings are explicit, every S-C mapping is subject to a family of accessory parameters that must, in general, be determined numerically. A great deal of work has been devoted to solving this "parameter problem", and a comprehensive review is available in Driscoll and Trefethen (2002). In particular, the MatLaB program sc-toolbox (https://github.com/tobydriscoll/sc-toolbox) allows the rapid computation of (usually simply connected) S-C mappings through the use of several novel numerical algorithms (Driscoll, 1996, 2005).

Historically, Schwarz-Christoffel mappings were typically restricted to simply connected domains. A major advance came about in the early 2000s when two groups of researchers independently extended the S-C mapping formulae to consider multiply connected domains (Crowdy, 2005, 2007; DeLillo et al., 2004). The latter has an advantage over the former insofar as the mapping formula is written explicitly in terms of the aforementioned Schottky-Klein prime function. Further work has been done to solve the parameter problem in the multiply connected domains Kropf (2012).

Recent work in Baddoo and Crowdy (2019) has further extended the original S-C mapping to permit target domains that are periodic. In a similar way to other work by Crowdy (Crowdy, 2005, 2007), the mapping formula is phrased in terms of the SchottkyKlein prime function. Consequently, the formula is valid for any number of objects per period window. An example of such a mapping with three objects per period window is illustrated in figure 6.12. The mapping formula is given by (6.3) in Baddoo and Crowdy (2019) as

$$
z=f(\zeta)=A \int_{1}^{\zeta} S_{P}\left(\zeta^{\prime} ; a_{\infty^{+}}, a_{\infty}^{-}\right) \prod_{j=0}^{M} \prod_{k=1}^{n_{j}}\left[\omega\left(\zeta^{\prime}, a_{k}^{(j)}\right)\right]^{\beta_{k}^{(j)}} \mathrm{d} \zeta^{\prime}+B
$$

where $\beta_{k}^{(j)}$ represent the turning angles at each vertex and $a_{k}^{(j)}$ represent the unknown preimages of each vertex. The subscript corresponds to the $k$-th vertex and the superscript 


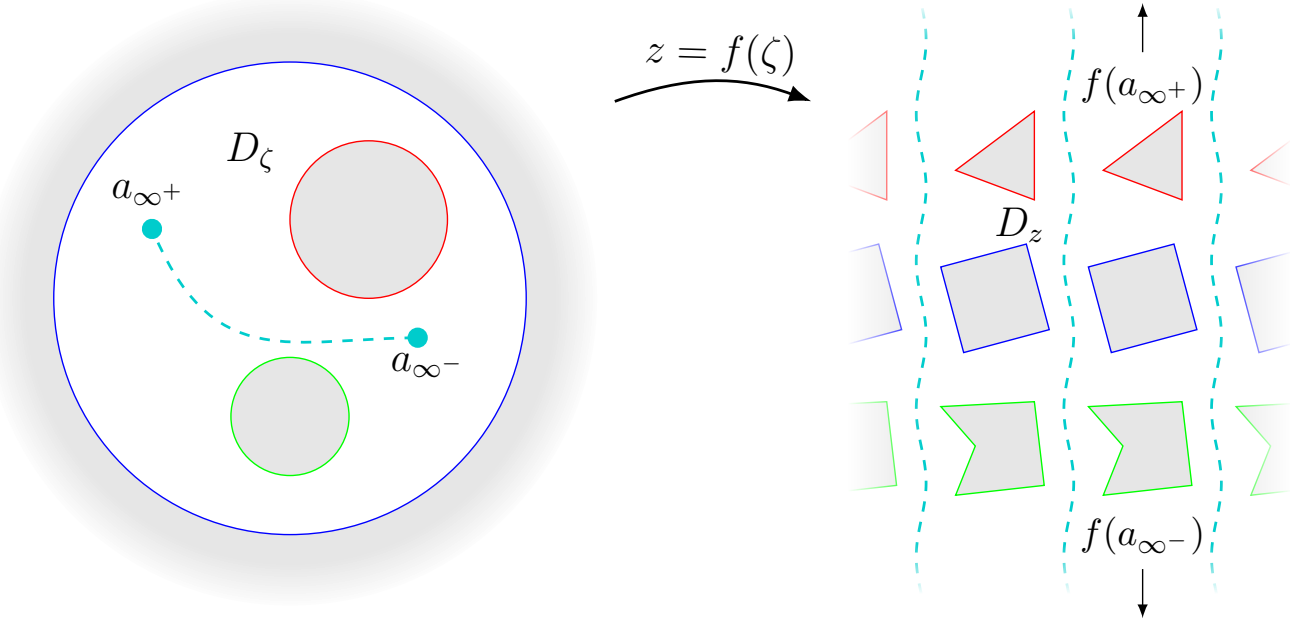

Fig. 6.12 An example of a periodic Schwarz-Christoffel mapping with real period.

corresponds to the $j$-th circle. The constants $A$ and $B$ represent the translation and scaling of the map. The function $S_{P}$ possesses simple poles at $a_{\infty^{ \pm}}$so that, when integrated, they become branch points at $a_{\infty^{ \pm}}$. In particular, $S_{P}$ is given by

$$
S_{P}\left(\zeta ; a, a_{\infty^{+}}, a_{\infty^{-}}\right)=\frac{S_{B}(\zeta)}{\omega\left(\zeta, a_{\infty^{+}}\right) \omega\left(\zeta, a_{\infty^{-}}\right) \omega\left(\zeta, 1 / \overline{a_{\infty^{+}}}\right) \omega\left(\zeta, 1 / \overline{a_{\infty^{-}}}\right)}
$$

and $S_{B}$ is defined as

$$
S_{B}(\zeta)=\frac{\omega_{\zeta}(\zeta, a) \omega(\zeta, 1 / \bar{a})-\omega_{\zeta}(\zeta, 1 / \bar{a}) \omega(\zeta, a)}{\prod_{j=1}^{M} \omega\left(\zeta, \gamma_{1}^{(j)}\right) \omega\left(\zeta, \gamma_{2}^{(j)}\right)},
$$

where $a \in D_{\zeta}$ is a constant in the circular domain and $\gamma_{1,2}^{(j)}$ are constants defined in Baddoo and Crowdy (2019).

The original S-C mapping is recovered in the limit where $a_{\infty^{ \pm}} \rightarrow a$ and the two branch points combine to form a simple pole at $\zeta=a$, thereby rending the map non-periodic. Further work is required to solve the parameter problem for the periodic $\mathrm{S}-\mathrm{C}$ mapping, but a similar approach to that employed by Driscoll (2005) will be applicable, at least in the case where there is only a single object per period window.

\subsection{The Calculus of Flows in Periodic Domains}

In this section we adapt the work of Crowdy (2010) to enable the calculation of complex potentials for flows in periodic domains with multiple objects per period window. Constructing the complex potential for a simply connected domain is straightforward 
upon use of the Milne-Thomson circle theorem (Batchelor, 2000). However, problems in multiply connected domains are not amenable to the same tools, and the complex potential must be phrased in terms of the Schottky-Klein prime function. In this section, we focus on solutions for complex potential induced by (1) point vortices, (2) a uniform flow and (3) moving objects. The analytic solutions for point vortices and moving objects are identical to the non-periodic solution by Crowdy (2010), and do not require modification. Nevertheless, these solutions have not previously been applied to periodic domains and, therefore, we reiterate the original solutions by Crowdy and provide new illustrative examples. Conversely, the solution for uniform flow must be modified to account for the periodicity of the domain. In this case, we will show that we recover the original solution by Crowdy (2010) in the limit where the period tends to infinity.

\subsubsection{Point Vortices}

A fundamental object in constructing potential flows in multiply connected domains is the hydrodynamic Green's function $G_{0}$, which satisfies

$$
\begin{array}{rrr}
\nabla^{2} G_{0}(\zeta, \beta) & =-\delta(\zeta-\beta), & \zeta \in D_{\zeta}, \\
\operatorname{Im}\left[G_{0}(\zeta, \beta)\right] & =c_{j}, & \zeta \in C_{j}, \quad j=0, \cdots, M .
\end{array}
$$

Crowdy and Marshall (2005) showed that the hydrodynamic Green's function takes the form

$$
G_{0}(\zeta, \beta)=\frac{1}{2 \pi \mathrm{i}} \log \left(\frac{\omega(\zeta, \beta)}{|\beta| \omega(\zeta, 1 / \bar{\beta})}\right),
$$

where $\omega$ is the Schottky-Klein prime function introduced in section 1.2.3.2. In hydrodynamical terms, $G_{0}$ is the complex potential for a unit circulation point vortex. An advantage of writing $G_{0}$ in the form of (6.6) is that, since it is phrased in terms of the Schottky-Klein prime function, (6.6) is valid for circular domains of arbitrary connectivity. Moreover, $G_{0}$ has -1 circulation around $C_{0}$ and zero circulation around $C_{j}$, $j=1, \cdots, M$. The circulations around each circle may be changed by introducing the modified hydrodynamic Green's function

$$
G_{j}(\zeta, \beta)=\frac{1}{2 \pi \mathrm{i}} \log \left(\frac{\omega(\zeta, \beta)}{|\beta| \omega\left(\zeta, \theta_{j}(1 / \bar{\beta})\right)}\right),
$$


where $\theta_{j}$ are the Möbius mappings

$$
\theta_{j}(\zeta)=\delta_{j}+\frac{q_{j}^{2} \zeta}{1-\overline{\delta_{j}} \zeta},
$$

and $q_{j}$ and $\delta_{j}$ are the conformal moduli described in section 1.2.3. The modified function $G_{j}$ has unit circulation around $C_{j}$ and zero circulation around $C_{i}, i=0, \cdots j-1, j+$ $1, \cdots M$. We will use this fact later to construct flows with specified circulations around each object, thus satisfying the Kutta condition. Accordingly, we may construct the complex potential induced by $n$ vortices by writing

$$
\begin{aligned}
W_{\kappa}(\zeta) & =\sum_{k=1}^{n} \kappa_{k} G_{j_{k}}\left(\zeta, \beta_{k}\right) \\
& =\frac{1}{2 \pi \mathrm{i}} \sum_{k=1}^{n} \kappa_{k} \log \left(\frac{\omega\left(\zeta, \beta_{k}\right)}{\left|\beta_{k}\right| \omega\left(\zeta, \theta_{j_{k}}\left(1 / \overline{\beta_{k}}\right)\right)}\right)
\end{aligned}
$$

In many applications it is desirable to give each object a specific circulation. Consequently, we may place point vortices at one of the pre-images of infinity to remove the circulations around each body. For example, we may remove the circulations by placing appropriately tuned vortices at $a_{\infty^{-}}$.

$$
\begin{aligned}
W_{\kappa}(\zeta) & =\sum_{k=1}^{n} \kappa_{k}\left(G_{j_{k}}\left(\zeta, \beta_{k}\right)-G_{j_{k}}\left(\zeta, a_{\infty^{-}}\right)\right) \\
& =\frac{1}{2 \pi \mathrm{i}} \sum_{k=1}^{n} \kappa_{k} \log \left(\left|\frac{a_{\infty^{-}}}{\beta_{k}}\right| \cdot \frac{\omega\left(\zeta, \beta_{k}\right)}{\omega\left(\zeta, a_{\infty^{ \pm}}\right)} \cdot \frac{\omega\left(\zeta, \theta_{j_{k}}\left(1 / \overline{a_{\infty^{-}}}\right)\right)}{\omega\left(\zeta, \theta_{j_{k}}\left(1 / \overline{\beta_{k}}\right)\right)}\right) .
\end{aligned}
$$

In figure 6.13, we plot the streamlines induced by point vortex in a periodic domain with three objects per period window $(M=2)$. In figure $6.13 \mathrm{a}$, the vortex induces -1 circulation around the image of $C_{0}$ and zero circulation around every other slit. The circulation is then deleted via the placement of a point vortex at both the pre-images of infinity in figure $6.13 \mathrm{~b}$. This has a significant effect on the flow field upstream of the cascade, whereas the downstream field is relatively unchanged. The behaviour of the flow field local to the point vortex is also unchanged by the modifications to the circulation.

The trajectories of point vortices may also be analysed using this framework. The Kirchhoff-Routh path function (Saffman, 1993) provides analytic expressions for the trajectories of point vortices. The path function has also been extended to multiply connected domains (Crowdy and Marshall, 2005), but further work is required to ensure that the same formulation is applicable to the periodic domains of this chapter. 


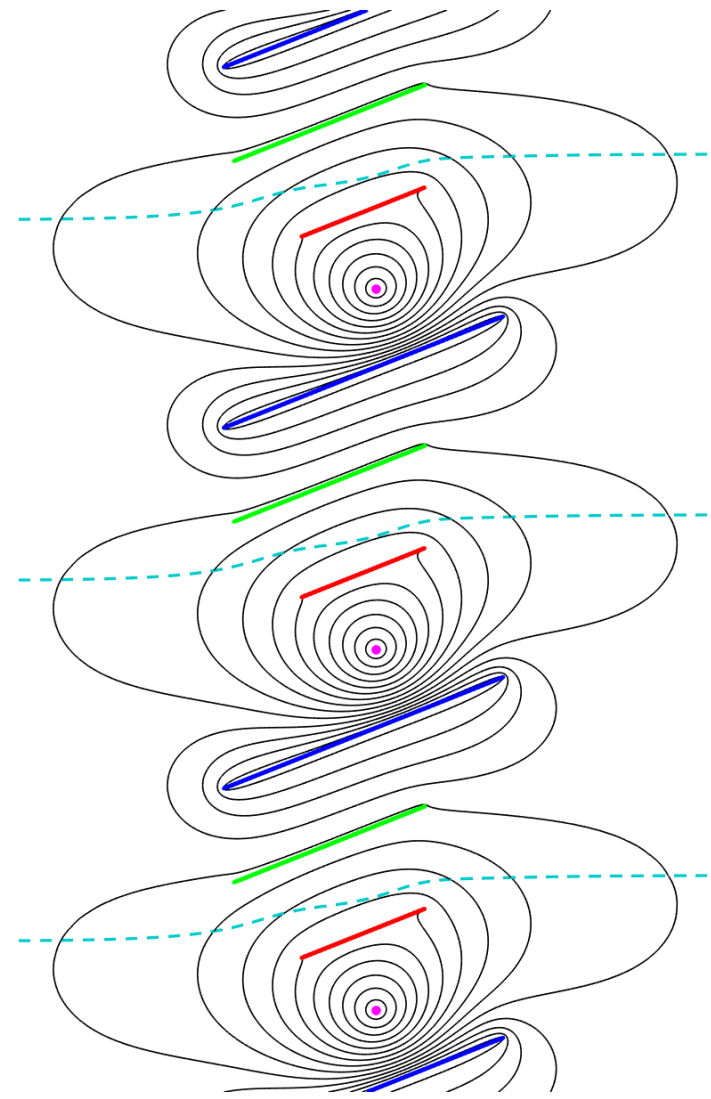

(a)

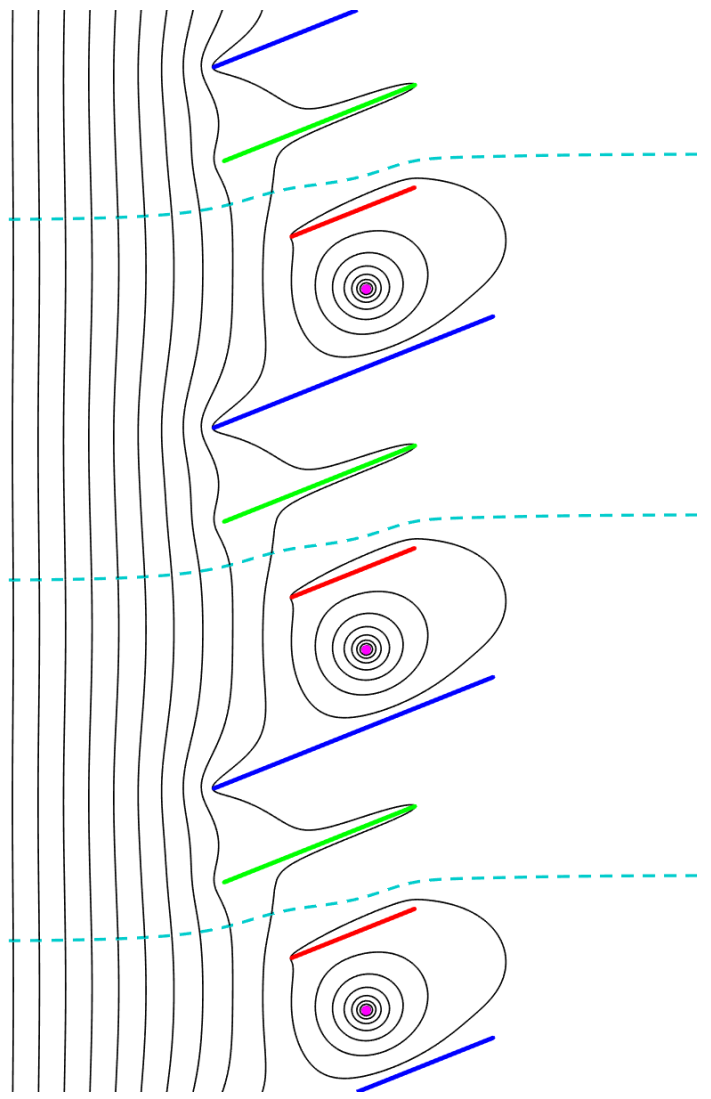

(b)

Fig. 6.13 Streamlines for a vortex induced flow with zero circulation around each $C_{j}$, $j=1,2$ and (a) -1 circulation around $C_{0}$, (b) 0 circulation around $C_{0}$. In this case there are three objects per period window and $M=2$. The point vortex is located at the purple circle and the branch cut is illustrated in the dashed line.

\subsubsection{Uniform Flow}

We now construct the complex potential for a uniform flow through the periodic arrangement of objects. The complex potential in a period window in the physical z-plane, $w_{U}(z)$, satisfies

$$
\begin{array}{rlrl}
\nabla_{z}^{2} w_{U}(z) & =0, & z \in D_{z}, \\
\operatorname{Im}\left[w_{U}(z)\right] & =c_{j}, & z \in P_{j}, & j=0, \cdots, M, \\
\frac{\mathrm{d} w_{U}}{\mathrm{~d} z} & \sim U \mathrm{e}^{-\mathrm{i} \alpha}, & \text { as }|z| \rightarrow \infty,
\end{array}
$$


where $U$ is the magnitude of the uniform flow at infinity, $c_{j}$ are some constants, and $\alpha$ is the angle of attack. A periodic conformal mapping $f(\zeta)$ must posses a branch cut in the circular $\zeta$-plane. Accordingly, $f(\zeta)$ has a pair of branch points at $a_{\infty^{ \pm}}$, and we have the following asymptotic behaviours:

$$
f(\zeta) \rightarrow \mp A \log \left(\zeta-a_{\infty^{ \pm}}\right)+\text {locally analytic function, }
$$

as $\zeta \rightarrow a_{\infty}^{ \pm}$for some constant $A$.

We write the complex potential in the $\zeta$-plane as

$$
w_{U}(z)=w_{U}(f(\zeta))=W_{U}(\zeta)
$$

Consequently, in the $\zeta$-plane, equations (6.10.a), (6.10.b), and (6.10.c) become

$$
\begin{aligned}
& \nabla_{\zeta}^{2} W_{U}(\zeta)=0, \quad \zeta \in D_{\zeta} /\left\{a_{\infty^{ \pm}}\right\}, \\
& \operatorname{Im}\left[W_{U}(\zeta)\right]=c_{j}, \quad \zeta \in C_{j}, \quad j=0, \cdots, M, \\
& \frac{\mathrm{d} W_{U}}{\mathrm{~d} \zeta} / \frac{\mathrm{d} f}{\mathrm{~d} \zeta} \sim U \mathrm{e}^{-\mathrm{i} \alpha}, \quad \text { as } \zeta \rightarrow a_{\infty^{ \pm}}
\end{aligned}
$$

Substitution of (6.11) into (6.12.c) yields

$$
\frac{\mathrm{d} W_{U}}{\mathrm{~d} \zeta} \sim \mp \frac{U A \mathrm{e}^{-\mathrm{i} \alpha}}{\zeta-a_{\infty^{ \pm}}}
$$

as $\zeta \rightarrow a_{\infty^{ \pm}}$. Consequently, $W_{U}$ must possess logarithmic branch points at $\zeta=a_{\infty^{ \pm}}$, in addition to being holomorphic (6.12.a) and taking constant imaginary values on each boundary (6.12.c). Using an approach analogous to that of section 6.2 in Crowdy (2010), we can construct this complex potential using the two canonical periodic mappings derived in section 6.2. The two canonical slit maps $H$ and $V$ have the asymptotic behaviours

$$
\begin{aligned}
H\left(\zeta ; a_{\infty^{+}}, a_{\infty^{-}}\right) & \sim \mp \frac{1}{2 \pi \mathrm{i}} \log \left(\zeta-a_{\infty^{ \pm}}\right), \\
V\left(\zeta ; a_{\infty^{+}}, a_{\infty^{-}}\right) & \sim \mp \frac{1}{2 \pi} \log \left(\zeta-a_{\infty^{ \pm}}\right),
\end{aligned}
$$

as $\zeta \rightarrow a_{\infty^{ \pm}}$. Moreover, both $H$ and $V$ have constant imaginary value on each $C_{j}$, $j=0, \cdots, M$. Consequently, we may take real, linear combinations of the two canonical 
slit maps to generate a new mapping that possesses the correct singularities at the pre-images of infinity, $a_{\infty^{ \pm}}$, whilst maintaining a constant imaginary value on each circle. The function

$$
W_{U}(\zeta)=2 \pi U\left(\cos (\alpha) V\left(\zeta ; a_{\infty^{+}}, a_{\infty^{-}}\right)+\sin (\alpha) H\left(\zeta ; a_{\infty^{+}}, a_{\infty^{-}}\right)\right)
$$

indeed has constant imaginary part on each boundary circle, and has the asymptotic behaviour described in (6.13). In terms of the prime function, we may expand $W_{U}$ into the form

$$
W_{U}(\zeta)=A U\left(\mathrm{e}^{-\mathrm{i} \alpha} \log \left(\frac{\omega\left(\zeta, a_{\infty^{-}}\right)}{\omega\left(\zeta, a_{\infty^{+}}\right)}\right)+\mathrm{e}^{\mathrm{i} \alpha} \log \left(\frac{\omega\left(\zeta, 1 / \overline{a_{\infty^{-}}}\right)}{\omega\left(\zeta, 1 / \overline{a_{\infty^{+}}}\right)}\right)\right) .
$$

It may be shown by analytic continuation and Liouville's theorem that (6.14) is unique in satisfying (6.12.a), (6.12.b), and (6.12.c). Moreover, $W_{U}(\zeta)$ is single-valued as $\zeta$ loops around any of the circles $C_{j}$, which implies that each object has zero circulation (Crowdy, 2006). It should be noted that the form of $W_{U}$ is identical to that of the combined canonical slit map $S$, defined in (6.1), up to a multiplicative factor.

In the case where there is a single object per period window, we have $M=0$ and (6.14) becomes

$$
W_{U}(\zeta)=A U\left(\mathrm{e}^{-\mathrm{i} \alpha} \log \left(\frac{\zeta-a_{\infty^{+}}}{\zeta-a_{\infty^{-}}}\right)+\mathrm{e}^{\mathrm{i} \alpha} \log \left(\frac{\overline{a_{\infty^{+}}}}{\overline{a_{\infty^{-}}}} \cdot \frac{\overline{a_{\infty^{-}}} \zeta-1}{\overline{a_{\infty^{+}}} \zeta-1}\right)\right) .
$$

In figure 6.14 we plot the streamlines for the uniform flow through a periodic array of slits. In these figures there is no circulation around any object and, consequently, the outlet angle is the same as the inlet angle. Clearly the no-flux condition is satisfied in figures $6.14 \mathrm{a}$ and $6.14 \mathrm{~b}$ since the streamlines do not pass through any of the slits. It is noted that the velocity field associated with these streamlines possess a singularity at the sharp corners of the slits at each leading and trailing edge. As will be detailed later, this can be corrected by enforcing the Kutta condition.

\subsubsection{Comparison to Solution for Non-Periodic Flows}

We now show that the solution for uniform flow through a periodic domain (6.14) collapses to the solution for uniform flow through a non-periodic domain (Crowdy, 2010) in the appropriate limit. We take the limit $a_{\infty^{+}} \rightarrow a_{\infty^{-}}$, which corresponds to the two branch points in the conformal mapping coalescing to form a simple pole. Consequently, in this limit there is no branch cut and the mapping is not periodic. Taylor expanding the 


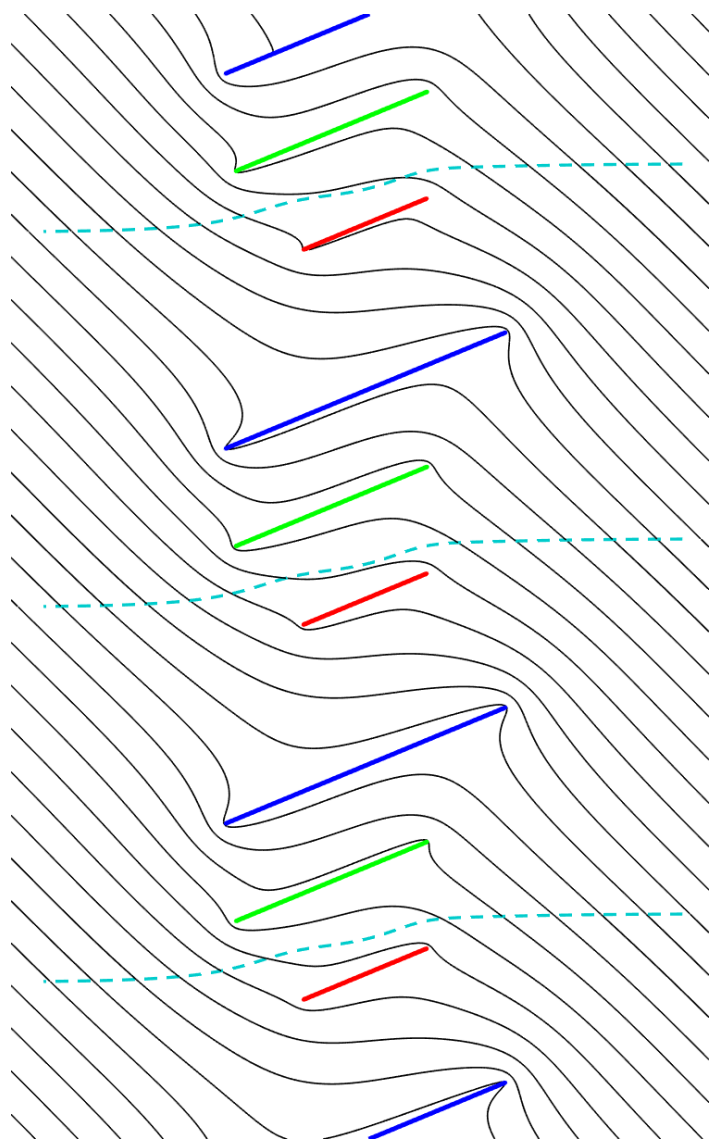

(a)

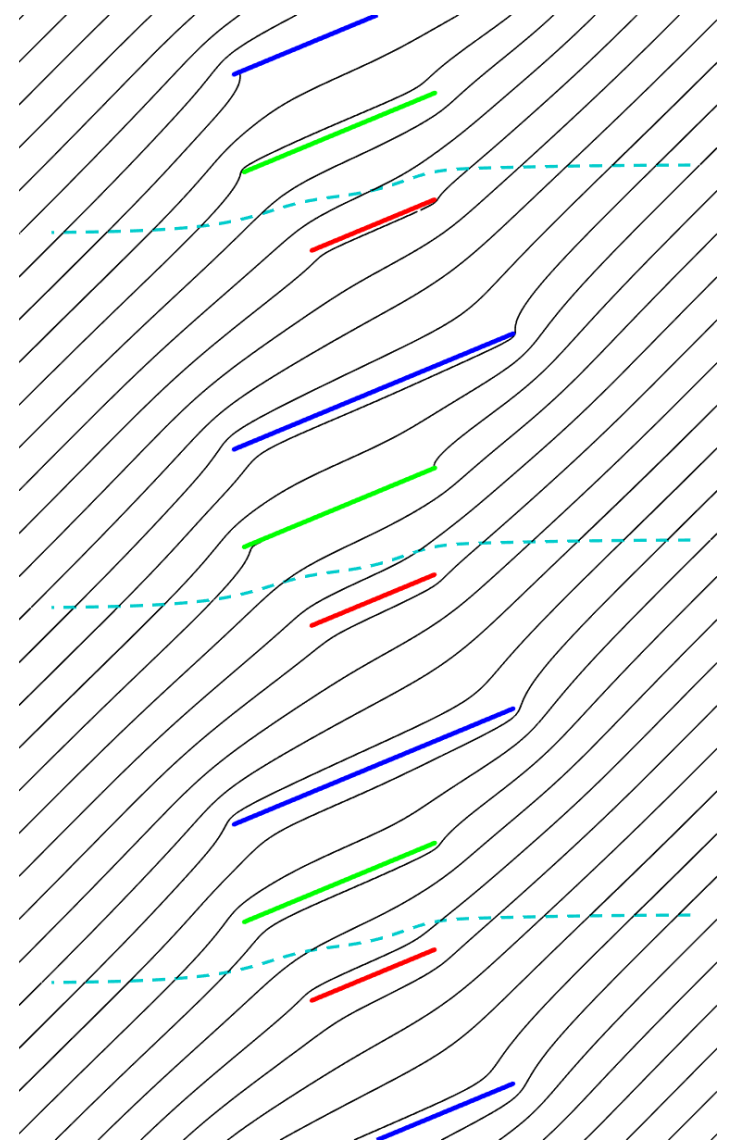

(b)

Fig. 6.14 Streamlines for uniform flow through a periodic arrangement of slits at angle of attack (a) $\alpha=-\pi / 4$, (b) $\alpha=\pi / 4$. In this case there are three objects per period window and $M=2$. There is zero circulation around every object. The branch cut is illustrated by the dashed line.

logarithms in (6.14) yields

$$
\begin{aligned}
\log \left(\frac{\omega\left(\zeta, a_{\infty^{+}}\right)}{\omega\left(\zeta, a_{\infty^{-}}\right)}\right) \sim\left(a_{\infty^{-}}-a_{\infty^{+}}\right) & \frac{\omega_{\alpha}\left(\zeta, a_{\infty^{-}}\right)}{\omega\left(\zeta, a_{\infty^{-}}\right)} \\
& =2 \pi \mathrm{i}\left(a_{\infty^{-}}-a_{\infty^{+}}\right)\left[\frac{\partial G_{0}}{\partial \beta}(\zeta, \beta)\right]_{\beta=a_{\infty^{-}}}, \\
\log \left(\frac{\omega\left(\zeta, 1 / \overline{a_{\infty^{-}}}\right)}{\omega\left(\zeta, 1 / \overline{a_{\infty^{+}}}\right)}\right) \sim\left(a_{\infty^{-}}-a_{\infty^{+}}\right) & \frac{1}{{\overline{a_{\infty^{-}}}}^{2}} \frac{\omega_{\beta}\left(\zeta, 1 / \overline{a_{\infty^{-}}}\right)}{\omega\left(\zeta, 1 / \overline{a_{\infty^{-}}}\right)} \\
& =2 \pi \mathrm{i}\left(a_{\infty^{-}}-a_{\infty^{+}}\right)\left[\frac{\partial G_{0}}{\partial \bar{\beta}}(\zeta, \beta)\right]_{\beta=a_{\infty^{-}}},
\end{aligned}
$$


subject to addition of an arbitrary constant, where the subscript $\beta$ indicates the derivative with respect to the second argument. Substitution of (6.16.a) and (6.16.b) into (6.14) and rescaling $A=\tilde{A} /\left(a_{\infty^{+}}-a_{\infty^{-}}\right)$yields

$$
W_{U}(\zeta) \sim 2 \pi \mathrm{i} \tilde{A} U\left[\mathrm{e}^{\mathrm{i} \alpha} \frac{\partial G_{0}}{\partial \bar{\beta}}-\mathrm{e}^{-\mathrm{i} \alpha} \frac{\partial G_{0}}{\partial \beta}\right]_{\beta=a_{\infty^{-}}},
$$

as $a_{\infty^{+}} \rightarrow a_{\infty^{-}}$, which is equivalent to the complex potential for uniform flow through a non-periodic system as detailed in section 6.2 of Crowdy (2010).

\subsubsection{Moving Objects}

In many applications the objects are moving, in addition to being in a background flow. For example, if we wish to model a sets of rotors and stators then the rotors cannot be accurately modelled as being stationary. In natural flight, birds may utilize pitch and heave motions in approximately periodic configurations to enhance their lift. In industrial applications, stirrers perturb fluid to achieve optimal mixing (Crowdy, 2008a). In each of these cases, the standard no-flux boundary condition changes, and we must accordingly introduce extra terms in our analysis.

The kinematic boundary condition states that the normal velocity of fluid on a rigid surface must move at the same velocity as that surface. Consequently, we write

$$
\mathbf{u} \cdot \mathbf{n}=\mathbf{U}_{j} \cdot \mathbf{n} \quad \text { on } P_{j}
$$

where $\mathbf{n}$ represents the normal direction, $\mathbf{u}$ represents the fluid velocity, and $\mathbf{U}_{j}$ represents the prescribed velocity of that boundary. We note that the dot product may be rewritten in terms of complex notation as $\mathbf{a} \cdot \mathbf{b}=\mathbb{R e}[\bar{a} b]$ where $a$ is the obvious complexification of $\mathbf{a}$. We also note that the tangent vector $\mathbf{t}$ may be written in complex form as $\mathrm{d} z / \mathrm{d} s$, where $s$ is the arc length. Therefore, the normal vector $\mathbf{n}$ may be written as $-\mathrm{id} z / \mathrm{d} s$ and the kinematic boundary condition (6.17) may be written as

$$
\mathbb{R e}\left[\bar{u}(s) \times-\mathrm{i} \frac{\mathrm{d} z}{\mathrm{~d} s}\right]=\mathbb{R e}\left[\bar{U}_{j}(s) \times-\mathrm{i} \frac{\mathrm{d} z}{\mathrm{~d} s}\right] .
$$

We introduce the complex potential due to the kinematic condition as $w_{B}$ and therefore write the kinematic condition as

$$
\mathbb{R e}\left[\frac{\mathrm{d} w_{B}}{\mathrm{~d} z} \times-\mathrm{i} \frac{\mathrm{d} z}{\mathrm{~d} s}\right]=\mathbb{R e}\left[\bar{U}_{j}(s) \times-\mathrm{i} \frac{\mathrm{d} z}{\mathrm{~d} s}\right] .
$$


The first term may be simplified by the chain rule to obtain

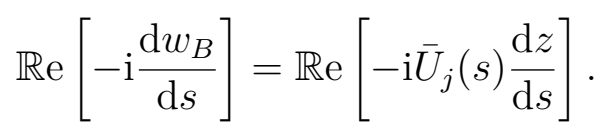

In the present work, we only consider rigid bodies. Accordingly, the only possible motions are rotations and translations. Therefore, every point $z \in P_{j}$ may be expressed as

$$
z=c_{j}(t)+\eta_{j}(s) \mathrm{e}^{\mathrm{i} \vartheta_{j}(t)}
$$

The velocity of each moving object may therefore be written as

$$
U_{j}(s)=\dot{c}_{j}(t)+\mathrm{i} \dot{\vartheta}_{j}(t) \eta_{j}(s) \mathrm{e}^{\mathrm{i} \vartheta j(t)}=\dot{c}_{j}(t)+\mathrm{i} \dot{\vartheta}_{j}(t)\left(z-c_{j}(t)\right)
$$

Therefore, the kinematic condition (6.18) becomes

$$
\begin{aligned}
\mathbb{R e}\left[-\mathrm{i} \frac{\mathrm{d} w_{B}}{\mathrm{~d} s}\right] & =\mathbb{R e}\left[-\mathrm{i}\left\{\dot{\bar{c}}_{j}(t)-\mathrm{i} \dot{\vartheta}_{j}(t)\left(\bar{z}-\bar{c}_{j}(t)\right)\right\} \frac{\mathrm{d} z}{\mathrm{~d} s}\right] \\
& =\mathbb{R e}\left[-\mathrm{i} \dot{\bar{c}}_{j}(t) \frac{\mathrm{d} z}{\mathrm{~d} s}-\dot{\vartheta}_{j}(t)\left(\bar{z}-\bar{c}_{j}(t)\right) \frac{\mathrm{d} z}{\mathrm{~d} s}\right] .
\end{aligned}
$$

Noting that

$$
\frac{\mathrm{d}}{\mathrm{d} s}\left|z-c_{j}\right|^{2}=2 \mathbb{R e}\left[\frac{\mathrm{d} z}{\mathrm{~d} s}\left(\bar{z}-\bar{c}_{j}\right)\right]
$$

we may integrate the kinematic condition (6.19) with respect to arc length $s$ to get

$$
\mathbb{R e}\left[-\mathrm{i} w_{B}\right]=\mathbb{R e}\left[-\mathrm{i} \dot{\bar{c}}_{j}\left(z-c_{j}\right)-\frac{\dot{\vartheta}_{j}}{2}\left|z-c_{j}\right|^{2}\right]+d_{j}, \quad \text { for } z \in P_{j}
$$

for a constant $d_{j}$ which will be chosen to comply with a compatibility condition to be defined later.

We write $W_{B}(\zeta)=w_{B}(z)$ and translate (6.20) into the canonical circular domain to obtain the condition

$$
\mathbb{R e}\left[-\mathrm{i} W_{B}\right]=\mathbb{R e}\left[-\mathrm{i} \dot{\bar{c}}_{j}\left(z(\zeta)-c_{j}\right)-\frac{\dot{\vartheta}_{j}}{2}\left|z(\zeta)-c_{j}\right|^{2}\right]+d_{j} \equiv \phi_{j}(\zeta, \bar{\zeta}), \quad \text { for } \zeta \in C_{j} .
$$


In other words, the problem is to find an analytic function $-\mathrm{i} W_{B}$ such that the real part of $-\mathrm{i} W_{B}$ equals $\phi_{j}(\zeta, \bar{\zeta})$ on the boundary of each circle $C_{j}$. This is a form of the modified Schwarz problem, the solution to which has been given by Crowdy (2008b) for a domain of arbitrary connectivity as

$$
W_{B}(\zeta)=\frac{1}{2 \pi} \oint_{\partial D_{\zeta}} \phi_{j}\left(\zeta^{\prime}, \overline{\zeta^{\prime}}\right)\left(\mathrm{d} \log \left(\omega\left(\zeta^{\prime}, \zeta\right)\right)+\mathrm{d} \log \left(\bar{\omega}\left(\overline{\zeta^{\prime}}, 1 / \zeta\right)\right)\right)+E
$$

where $E$ is an arbitrary real constant. In particular, $W_{B}$ has zero circulation around each circle. The boundary data must furthermore satisfy the compatibility condition

$$
\oint_{\partial D_{\zeta}} \phi_{j}(\zeta(s), \overline{\zeta(s)}) \frac{\partial \sigma_{j}}{\partial n} \mathrm{~d} s=0, \quad j=1, \cdots, M
$$

where $s$ represents the arc length, $\partial / \partial n$ represents the normal derivative, and $\sigma_{j}$ represent the harmonic measures (Crowdy, 2008b; Crowdy et al., 2016).

The integral in (6.22) is taken over every boundary circle and can therefore be decomposed into contributions from each circle. Consequently, the solution to the modified Schwarz problem may also be expressed as

$$
\begin{aligned}
W_{B}(\zeta) & =\frac{1}{2 \pi} \oint_{C_{0}} \phi_{0}\left(\zeta^{\prime}, \overline{\zeta^{\prime}}\right)\left(\mathrm{d} \log \left(\omega\left(\zeta^{\prime}, \zeta\right)\right)+\mathrm{d} \log \left(\bar{\omega}\left(\overline{\zeta^{\prime}}, 1 / \zeta\right)\right)\right) \\
& -\frac{1}{2 \pi} \sum_{j=1}^{M} \oint_{C_{j}} \phi_{j}\left(\zeta^{\prime}, \overline{\zeta^{\prime}}\right)\left(\mathrm{d} \log \left(\omega\left(\zeta^{\prime}, \zeta\right)\right)+\mathrm{d} \log \left(\bar{\omega}\left(\overline{\theta_{j}}(1 / \zeta), 1 / \zeta^{\prime}\right)\right)\right)+E,
\end{aligned}
$$

where $\theta_{j}$ are the Möbius mappings defined in (6.7).

In the case $M=0$ there is a single object per period window and the solution to the modified Schwarz problem is given by the Poisson formula (Ablowitz and Fokas, 2003):

$$
W_{B}(\zeta)=\frac{1}{2 \pi} \oint_{C_{0}} \phi_{0}\left(\zeta^{\prime}, \overline{\zeta^{\prime}}\right) \cdot \frac{\zeta^{\prime}+\zeta}{\zeta^{\prime}-\zeta} \cdot \frac{\mathrm{d} \zeta^{\prime}}{\zeta^{\prime}}+E
$$

Furthermore, in the case $M=1$ there are two objects per period window, and, when the canonical circular domain is the annulus $\rho<|z|<1$, the solution is given by the Villat formula (Akhiezer, 1990; Crowdy, 2008b)

$$
\begin{aligned}
W_{B}(\zeta) & =\frac{1}{2 \pi} \oint_{C_{0}} \phi_{0}\left(\zeta^{\prime}, \overline{\zeta^{\prime}}\right) \cdot\left(1-2 K\left(\frac{\zeta^{\prime}}{\zeta}\right)\right) \cdot \frac{\mathrm{d} \zeta^{\prime}}{\zeta^{\prime}} \\
& +\frac{1}{2 \pi} \oint_{C_{1}} \phi_{1}\left(\zeta^{\prime}, \overline{\zeta^{\prime}}\right) \cdot 2 K\left(\frac{\zeta^{\prime}}{\zeta}\right) \cdot \frac{\mathrm{d} \zeta^{\prime}}{\zeta^{\prime}}+E
\end{aligned}
$$




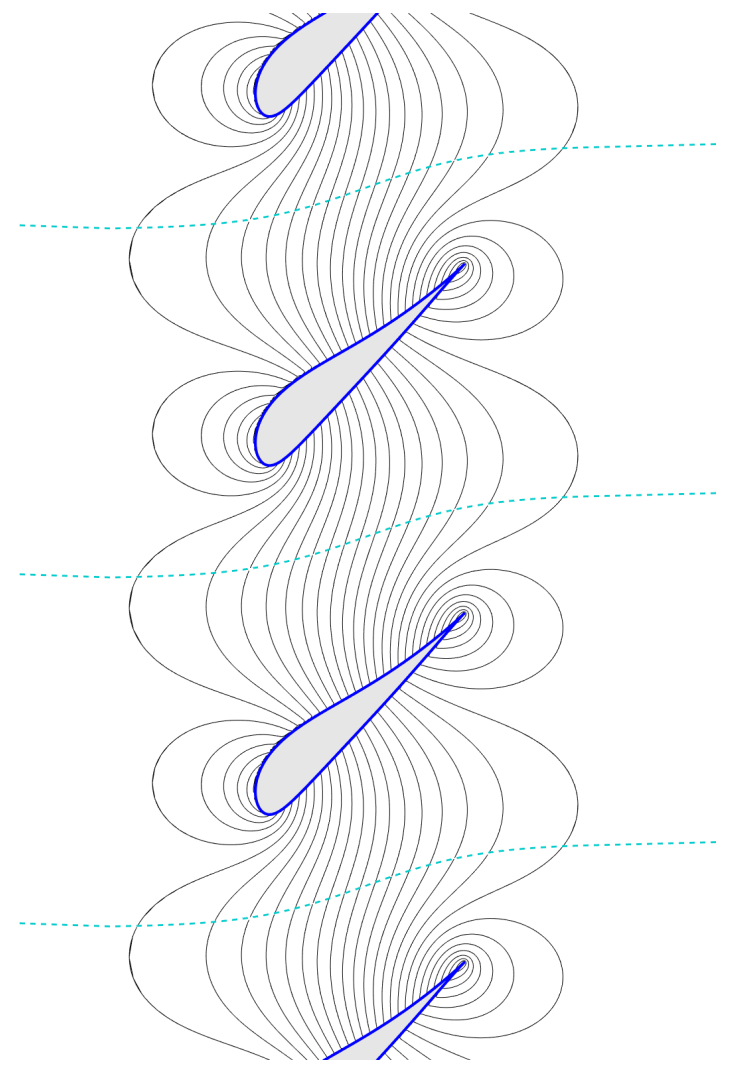

(a)

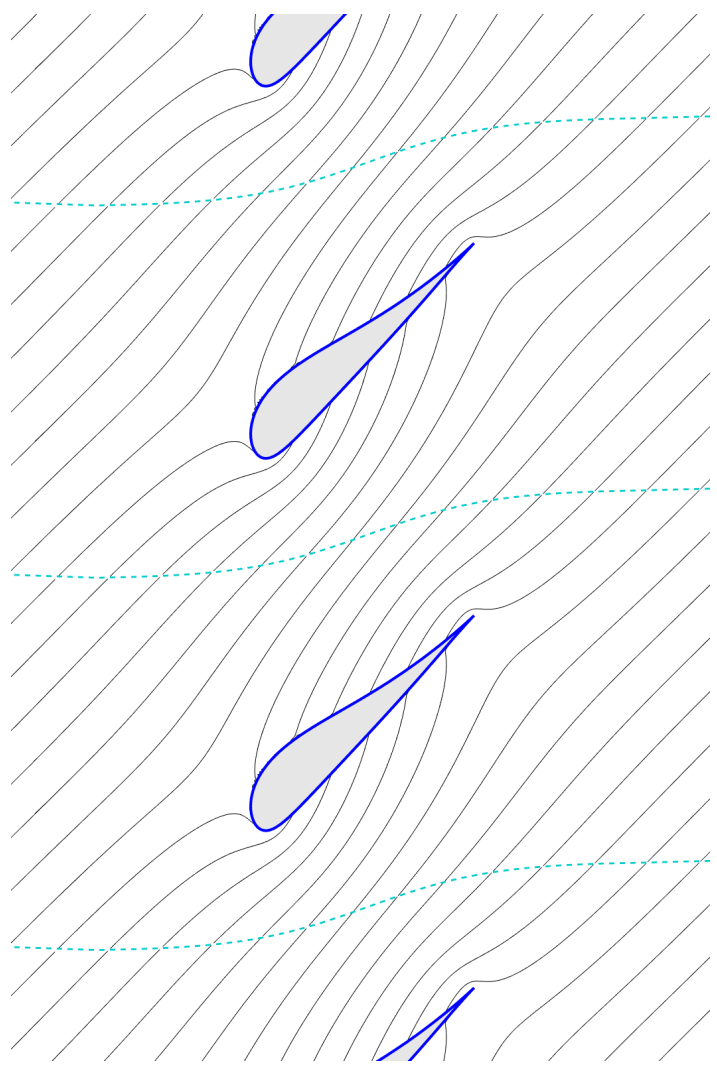

(b)

Fig. 6.15 The potential flow induced by a cascade of moving Joukowski aerofoils. The direction of motion is indicated by the arrow. In figure (a) there is no background uniform flow, whereas in figure (b) there is a background uniform flow.

where

$$
\begin{aligned}
K(\zeta) & =\frac{\zeta P_{\zeta}(\zeta)}{P(\zeta)} \\
P(\zeta) & =(1-\zeta) \prod_{j=1}^{\infty}\left(1-\rho^{2 j} \zeta\right)\left(1-\frac{\rho^{2 j}}{\zeta}\right)
\end{aligned}
$$

In figure 6.15 we plot the streamlines induced by a cascade of Joukowski aerofoils that are moving in the vertical direction. Two cases are considered: with and without a background flow. In contrast to previous examples, the streamfunction is no longer constant along the aerofoils' surfaces since the motion of the blades implies that the rigid surface does not define a streamline. Note that the velocity is infinite at the trailing edges since the conformal mapping has vanishing derivative there. We now address this issue by applying the Kutta condition. 


\subsubsection{The Kutta Condition}

Thus far we have been careful to ensure that the circulation around each object vanishes. However, this means that the flow is infinite in the $z$-plane wherever the objects have sharp corners. Accordingly, in this section we regularise the flow at these sharp corners by enforcing the Kutta condition.

We introduce the complex potential

$$
\begin{aligned}
W_{\Gamma}(\zeta) & =-\sum_{k=0}^{M} \Gamma_{k} G_{k}\left(\zeta, a_{\infty^{+}}\right) \\
& =-\frac{1}{2 \pi \mathrm{i}} \sum_{k=0}^{M} \Gamma_{k} \log \left(\frac{\omega\left(\zeta, a_{\infty^{+}}\right)}{\left|a_{\infty^{+}}\right| \omega\left(\zeta, \theta_{k}\left(1 / \overline{a_{\infty^{+}}}\right)\right)}\right)
\end{aligned}
$$

which represents the complex potential of a vortex placed at the pre-image of downstream infinity, $a_{\infty^{+}}$. The circulation induced by $W_{\Gamma}$ on the circle $C_{j}$ is $\Gamma_{j}$, and we choose the $\Gamma_{j}$ specifically so that the flow is regular at a particular corner. We may regularise the flow at a maximum of $M+1$ corners since we have $M+1$ Green's functions to manipulate.

We write the full complex potential as

$$
W(\zeta)=W_{\kappa}(\zeta)+W_{U}(\zeta)+W_{B}(\zeta)+W_{\Gamma}(\zeta)
$$

where the contributions are induced by point vortices, a background uniform flow, the motion of the objects, and the circulations around the objects respectively. In order to satisfy the Kutta condition at the $J$ corners $\zeta_{j}$, we enforce

$$
\frac{\mathrm{d} W}{\mathrm{~d} \zeta}\left(\zeta_{j}\right)=0, \quad j=1, \cdots, J
$$

We set $\Gamma_{j}=0$ if we do not regularise the flow on the $j$-th object. Rearranging (6.24) yields

$$
\begin{aligned}
\frac{1}{2 \pi \mathrm{i}}\left(\frac{\omega_{\zeta}\left(\zeta_{j}, a_{\infty^{+}}\right)}{\omega\left(\zeta_{j}, a_{\infty^{+}}\right)}-\frac{\omega_{\zeta}\left(\zeta_{j}, \theta_{k}\left(1 / \overline{a_{\infty^{+}}}\right)\right)}{\omega\left(\zeta_{j}, \theta_{k}\left(1 / \overline{a_{\infty^{+}}}\right)\right)}\right) \Gamma_{k} & \\
& =\frac{\mathrm{d} W_{\kappa}}{\mathrm{d} \zeta}\left(\zeta_{j}\right)+\frac{\mathrm{d} W_{U}}{\mathrm{~d} \zeta}\left(\zeta_{j}\right)+\frac{\mathrm{d} W_{B}}{\mathrm{~d} \zeta}\left(\zeta_{j}\right), \quad j=1, \cdots, J .
\end{aligned}
$$

It is straightforward to invert this linear system to obtain numerical values for the circulations. 
In periodic configurations, the effect of applying the Kutta condition is to modify the deflection angle obtained by the cascade, as illustrated in figure 6.16. The Kutta condition is not enforced in figures 6.16a and 6.16c and, therefore, the circulation around each object is zero. Consequently, the flow velocity is infinite at both the leading and trailing edges of each slit. In the absence of vortices, neglecting the Kutta condition implies that the outlet angle of the flow is identical to the inlet angle, such as in figure 6.16a. Applying the Kutta condition changes the flow angle at downstream infinity, and accordingly changes the outlet angle of the flow as seen in figure 6.16b. We also illustrate an example where there are point vortices in the flow in figures 6.16c and 6.16d. Applying the Kutta condition results in a drastic change in the outlet angle in figure 6.16d, and the effect of the point vortices on the global properties of the flow is substantially reduced.

We also plot the streamlines for a uniform flow past a cascade of Joukowski aerofoils in figure 6.17. This problem is intractable with the methods of chapter 2, which were restricted to thin aerofoils at small angles of attack. In contrast, the present method is, in principle, not restricted to a particular aerofoil geometry, although the appropriate conformal map must be determined. Moreover, we are now able to include point vortices in the flow, and model the motion of the blades.

\subsection{Conclusions}

In this chapter, we have derived analytic solutions for the potential flow through a cascade where there are multiple objects per period window. To facilitate this solution, we derived two new canonical periodic conformal maps that each map a circular domain to a periodic array of slits. These maps are expressed in terms of the transcendental Schottky-Klein prime function and, accordingly, they are valid for period windows of arbitrary connectivity. In the case where there is just a single object per period window, we used these canonical mappings to derive a periodic analogue of the Joukowski mapping. The canonical mappings were also used to construct the complex potential for a uniform flow through a periodic array of objects. We also applied previous work on non-periodic domains by Crowdy (2010) to our new periodic problems to construct flows with point vortices and moving objects. Critically, all the solutions presented in this chapter are valid for an arbitrary number of objects per period window. 


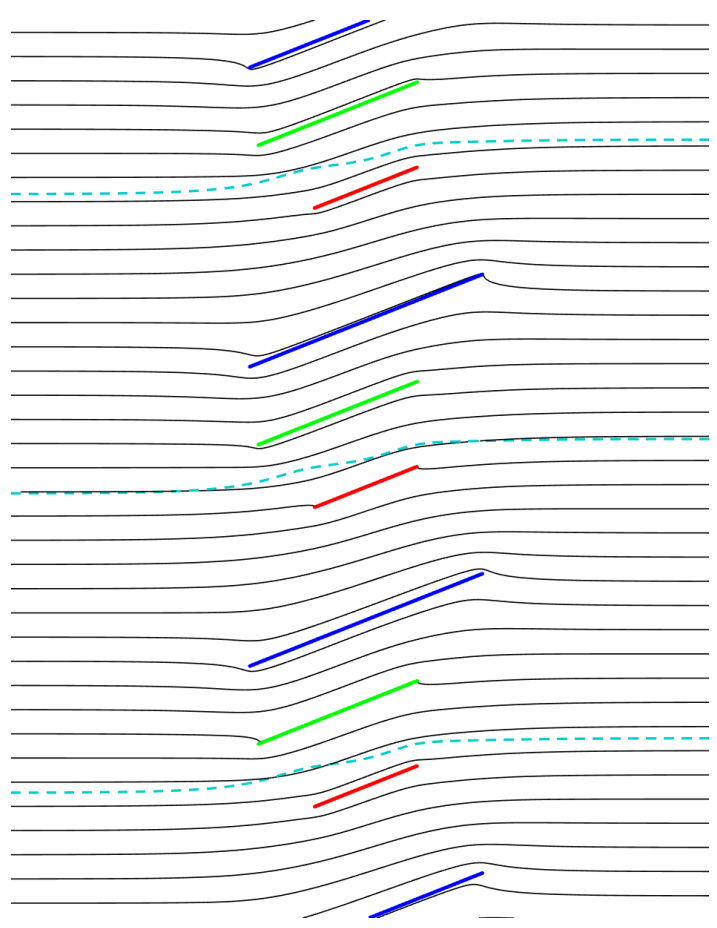

(a)

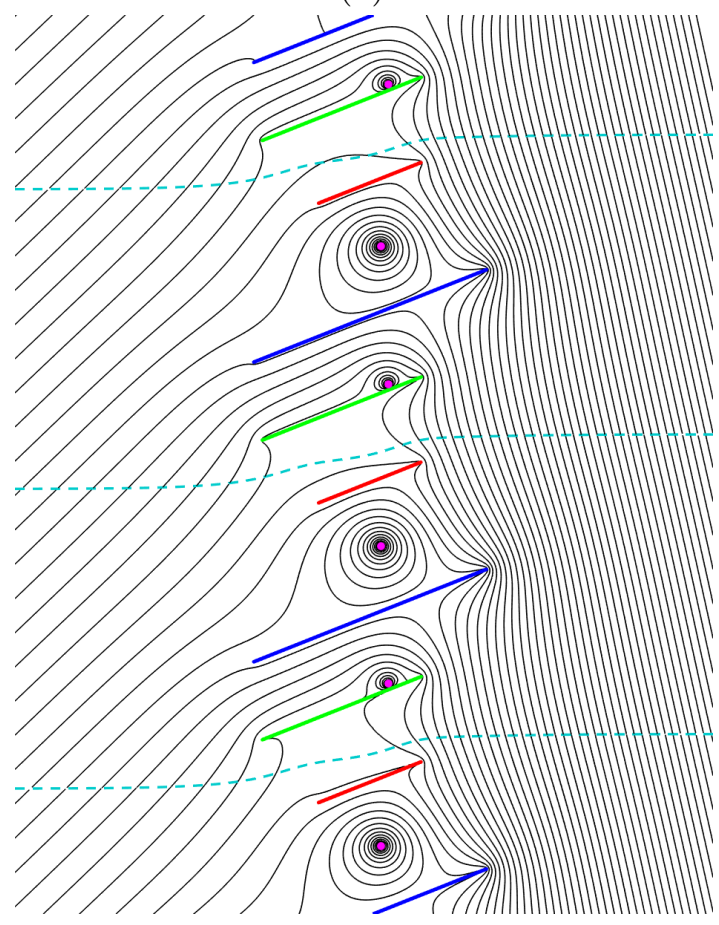

(c)

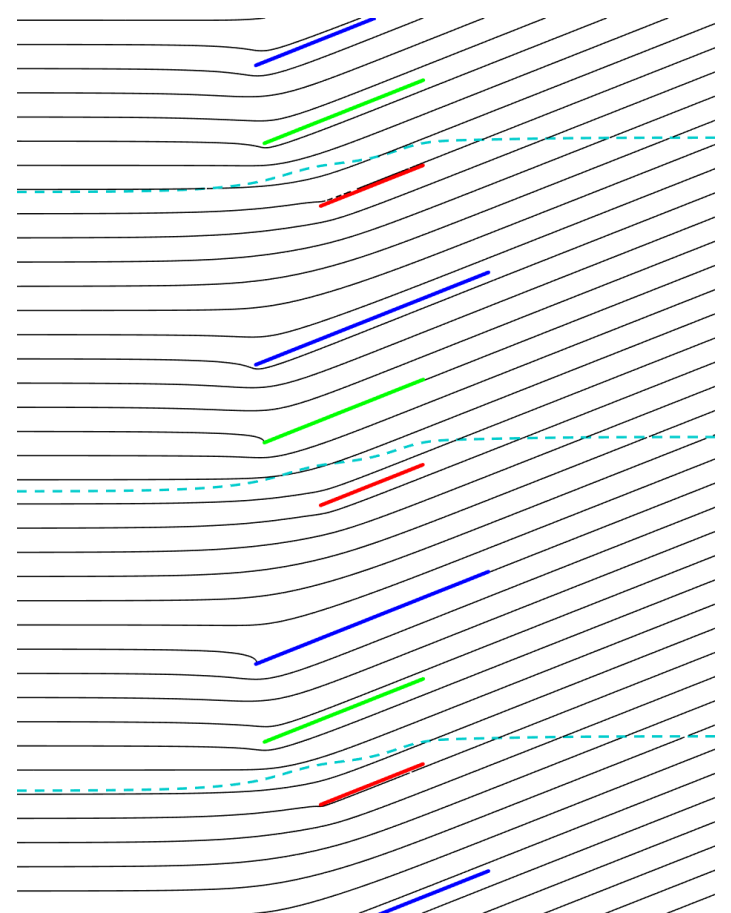

(b)

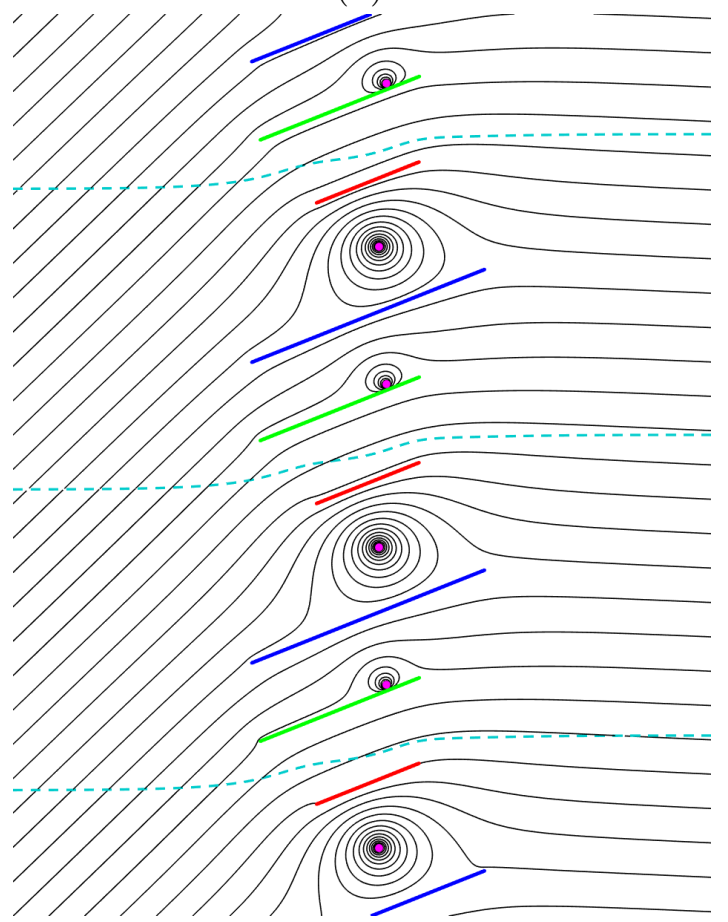

(d)

Fig. 6.16 Illustration of the Kutta condition applied to a cascade of slits. In figures (a) and (c), there is no regularisation at the trailing edges, whereas in figures (b) and (d), the Kutta condition is applied at each trailing edge. Point vortices are located at the purple circles and the branch cut is illustrated by the dashed line. 

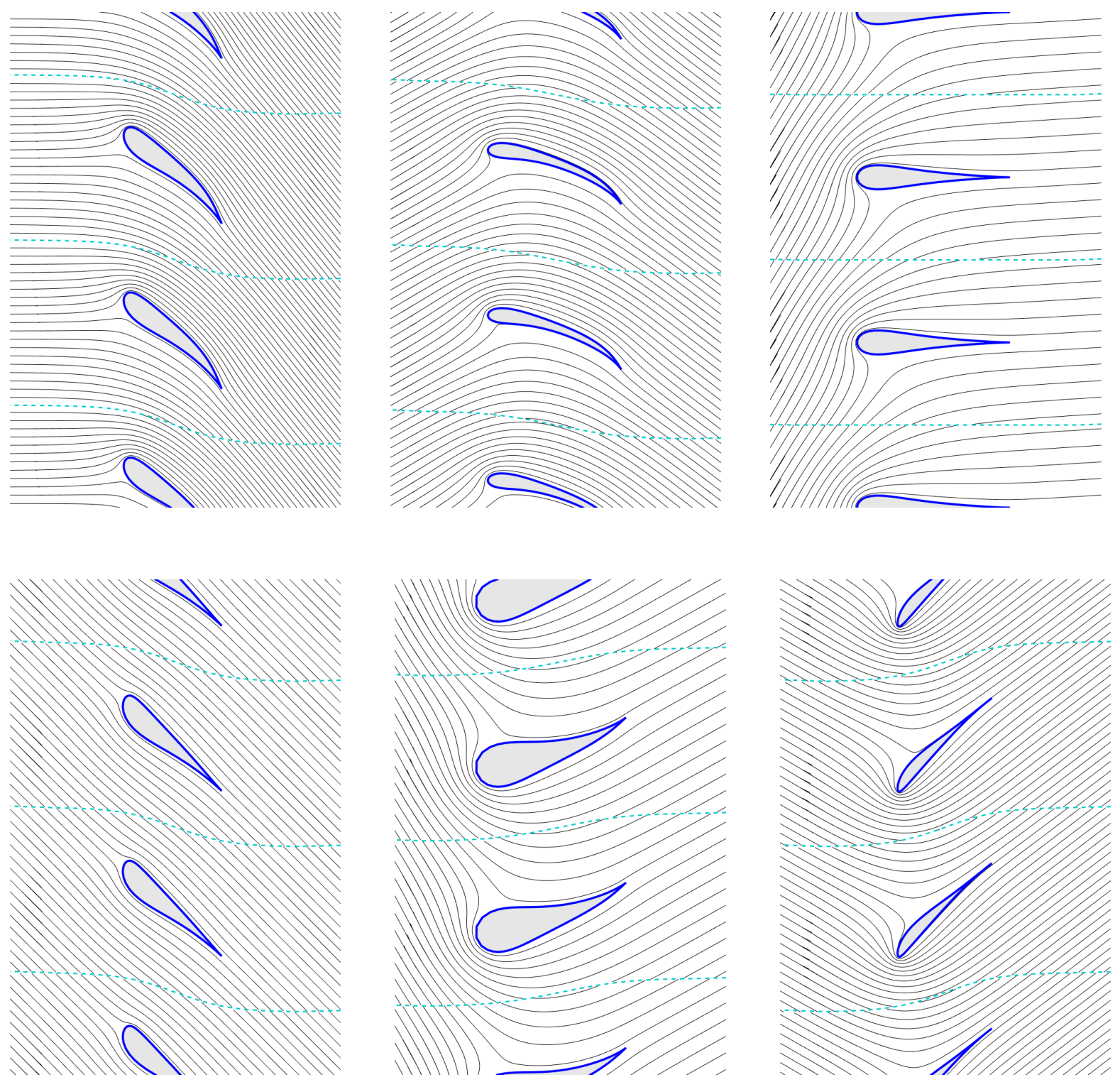

Fig. 6.17 Several examples of the flow past cascades of Joukowski-type aerofoils where the Kutta condition is enforced. 


\title{
List of Symbols
}

\author{
Symbol Meaning \\ $z \quad$ Coordinate in physical domain \\ $\zeta \quad$ Coordinate in canonical circular domain \\ $f \quad$ Conformal mapping relating $z$ and $\zeta$ \\ $\chi \quad$ Stagger angle \\ $\omega(\cdot, \cdot) \quad$ Schottky-Klein prime function \\ $a_{\infty^{ \pm}} \quad$ Pre-images of downstream and upstream infinity \\ $T(\cdot ; \cdot, \cdot) \quad$ Circular arc map \\ $R(\cdot ; \cdot, \cdot) \quad$ Radial slit map \\ $H(\cdot ; \cdot, \cdot) \quad$ First canonical periodic slit map \\ $V(\cdot ; \cdot, \cdot) \quad$ Second canonical periodic slit map \\ $S(\cdot ; \cdot, \cdot) \quad$ Combined canonical periodic slit maps \\ $G_{j}(\cdot, \cdot) \quad$ Modified hydrodynamic Green's function \\ $C_{j} \quad$ Discs in the canonical circular domain \\ $P_{j} \quad$ Image of disc $C_{j}$ in the physical domain \\ $D_{\zeta} \quad$ Canonical circular domain \\ $D_{z} \quad$ Physical domain \\ $W_{\kappa} \quad$ Velocity potential for point vortices \\ $W_{U} \quad$ Velocity potential for uniform flow \\ $W_{B} \quad$ Velocity potential for motion of objects \\ $\Gamma_{j} \quad$ Circulation around disc $j$ \\ $\beta \quad$ Vortex location \\ $\alpha \quad$ Angle of attack
}




\section{Chapter 7}

\section{The Quasi-Periodic Compact Green's Function}

\subsection{Introduction}

In this chapter we investigate sound generation by cascades with multiple aerofoils per period window. This topic has received scant attention in the literature, possibly due to the non-trivial topology associated with the geometry of the cascade. Nevertheless, the modelling of interference effects between adjacent cascades is essential for the accurate modelling of rotor-stator interaction noise. We now leverage the potential flows solutions of chapter 6 to analyse the acoustics of cascades with multiple objects per period window. Similarly to chapter 6 , our solutions are, in principle, valid for cascades with an arbitrary number of aerofoils per period window. The aerofoils themselves may also have arbitrary geometric profiles.

The compact Green's function (Howe, 2003) is a powerful tool for modelling the production of sound. By assuming that the length scale of the scattering objects is small in comparison to the acoustic wavelength, the Green's function can be approximated using the so-called Kirchhoff vectors. These quantities essentially represent a potential flow through the system and are therefore amenable to methods of complex variable theory derived in the previous chapter. In periodic domains, the acoustic potential can be written in terms of the quasi-periodic Green's function. In the compact regime, we are able to derive an approximation for the quasi-periodic Green's function that is written in terms of quantities derived in chapter 6 .

The structure of this chapter is as follows. In section 7.2 we present the quasiperiodic Green's function in the context of acoustics. In section 7.3 we derive a compact approximation to the quasi-periodic Green's function in the absence of background flow. 


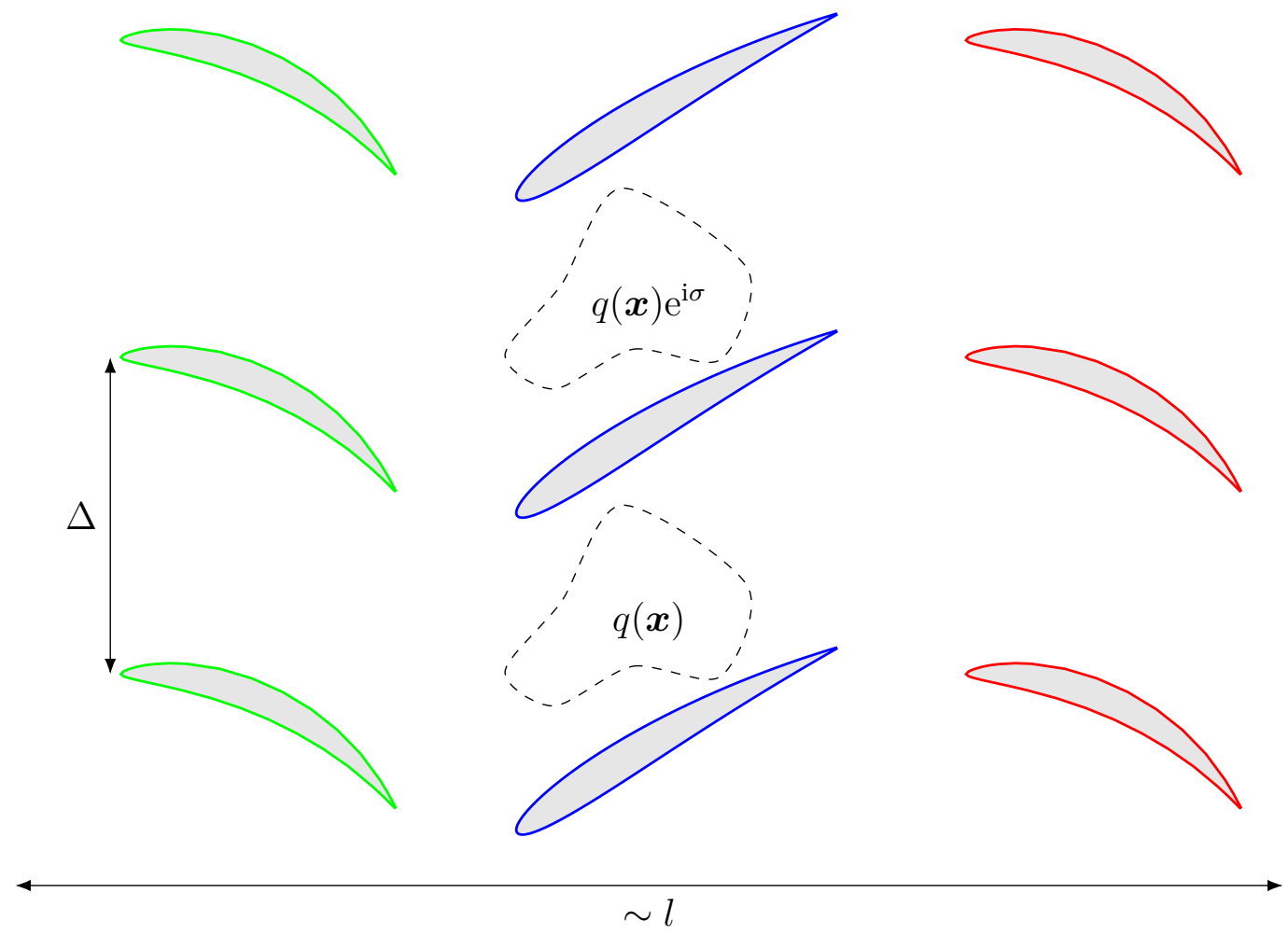

Fig. 7.1 A cascade with multiple aerofoils per period window with quasi-periodic acoustic sources. The length of the period is $\Delta$ and each period contains a source, $q$. The difference in phase between adjacent period windows is $\sigma$. The characteristic horizontal length scale of the cascade is $l$.

In section 7.4 we generalise this approach to account for a low Mach number background flow. Finally, in section 7.5 we summarise the chapter.

\subsection{Mathematical Modelling}

We consider an unbounded, periodic domain where each period window consists of an arrangement of aerofoils and a source. The horizontal length scale of the arrangement is $l$, and the spatial period is $\Delta$, which is oriented in the vertical direction. The system is illustrated in figure 7.1. We generally wish to solve the inhomogeneous Helmholtz equation for the acoustic potential

$$
\kappa_{0}^{2} \phi(\boldsymbol{x})+\nabla_{x}^{2} \phi(\boldsymbol{x})=q(\boldsymbol{x})
$$

where $\kappa_{0}=\omega / c_{0}$ and $\omega$ is the frequency of the fluctuating source $q$. We suppose further that the sources in adjacent period windows possess a phase shift of $\sigma$, as illustrated in 
figure 7.1. Consequently, the acoustic potential must satisfy the quasi-periodicity relation

$$
\phi(\boldsymbol{x}+m \boldsymbol{\Delta})=\mathrm{e}^{\mathrm{i} m \sigma} \phi(\boldsymbol{x}), \quad m \in \mathbb{Z},
$$

where $\boldsymbol{\Delta}=(0, \Delta)$.

We suppose that we can find a Green's function, $G$, such that

$$
\kappa_{0}^{2} G(\boldsymbol{x}, \boldsymbol{y})+\nabla_{y}^{2} G(\boldsymbol{x}, \boldsymbol{y})=\delta(\boldsymbol{x}-\boldsymbol{y}),
$$

where $\boldsymbol{x}$ is fixed and $G$ is effectively a function of $\boldsymbol{y}$. If $G$ can be found, then the divergence theorem determines the acoustic potential as

$$
\begin{aligned}
\phi(\boldsymbol{x}) & =\oint_{\partial D_{z}}\left\{G(\boldsymbol{x}, \boldsymbol{y}) \frac{\partial \phi}{\partial n}(\boldsymbol{y})-\phi(\boldsymbol{y}) \frac{\partial G}{\partial n}(\boldsymbol{x}, \boldsymbol{y})\right\} \mathrm{d} y \\
& +\iint_{D_{z}} G(\boldsymbol{x}, \boldsymbol{y}) q(\boldsymbol{y}) \mathrm{d} A_{y},
\end{aligned}
$$

where the normal derivative is directed into the fluid. The contour $\partial D_{z}$ represents the boundary of every object in the fluid, as illustrated in figure 7.2a. Due to the quasiperiodicity relation (7.1), we may reduce the integrals in $D_{\zeta}$ to integrals on a single period window $D_{0}$, as illustrated in figure 7.2b. Consequently, (7.3) becomes

$$
\begin{aligned}
\phi(\boldsymbol{x}) & =\sum_{m=-\infty}^{\infty} \mathrm{e}^{\mathrm{i} m \sigma} \oint_{\partial D_{0}}\left\{G(\boldsymbol{x}, \boldsymbol{y}+m \boldsymbol{\Delta}) \frac{\partial \phi}{\partial n}(\boldsymbol{y})-\phi(\boldsymbol{y}) \frac{\partial G}{\partial n}(\boldsymbol{x}, \boldsymbol{y}+m \boldsymbol{\Delta})\right\} \mathrm{d} y \\
& +\sum_{m=-\infty}^{\infty} \mathrm{e}^{\mathrm{i} m \sigma} \iint_{D_{0}} G(\boldsymbol{x}, \boldsymbol{y}+m \boldsymbol{\Delta}) q(\boldsymbol{y}) \mathrm{d} A_{y} .
\end{aligned}
$$

We now interchange the orders of summation and integration and write

$$
\begin{aligned}
\phi(\boldsymbol{x}) & =\oint_{\partial D_{0}}\left\{G^{q}(\boldsymbol{x}, \boldsymbol{y}) \frac{\partial \phi}{\partial n}(\boldsymbol{y})-\phi(\boldsymbol{y}) \frac{\partial G^{q}}{\partial n}(\boldsymbol{x}, \boldsymbol{y})\right\} \mathrm{d} y \\
& +\iint_{D_{0}} G^{q}(\boldsymbol{x}, \boldsymbol{y}) q(\boldsymbol{y}) \mathrm{d} A_{y}
\end{aligned}
$$

where $G^{q}$ is the quasi-periodic Green's function defined by

$$
G^{q}(\boldsymbol{x}, \boldsymbol{y})=\sum_{m=-\infty}^{\infty} \mathrm{e}^{\mathrm{i} m \sigma} G(\boldsymbol{x}, \boldsymbol{y}+m \boldsymbol{\Delta})
$$




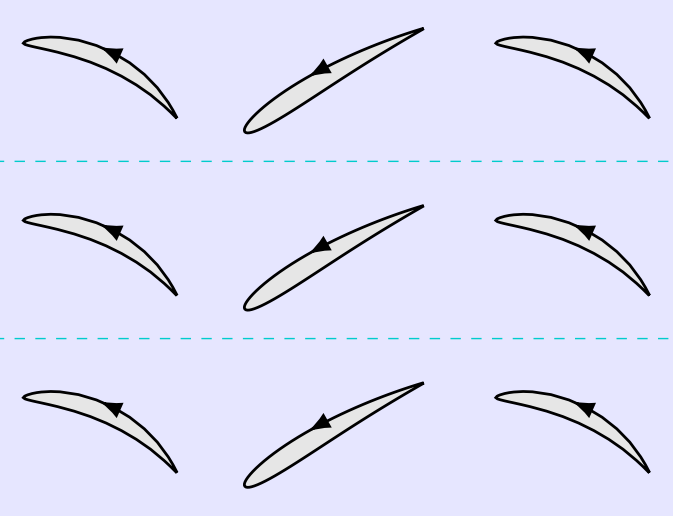

(a) The area $D_{z}$ and contour $\partial D_{z}$.
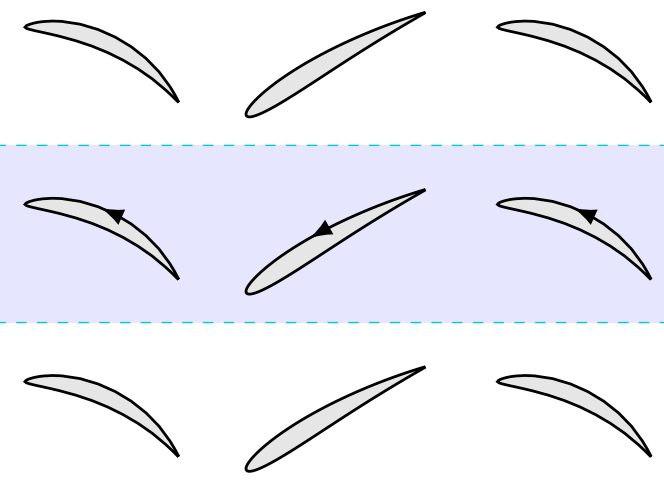

(b) The area $D_{0}$ and contour $\partial D_{0}$.

Fig. 7.2 An illustration of the reduction of the integrals to a single period window. The areas $D_{z}$ and $D_{0}$ are indicated by the blue shading and the contours $\partial D_{z}$ and $\partial D_{0}$ are indicated by the arrows.

Equivalently, $G^{q}$ is the solution to

$$
\nabla_{y}^{2} G^{q}(\boldsymbol{x}, \boldsymbol{y})+\kappa_{0}^{2} G^{q}(\boldsymbol{x}, \boldsymbol{y})=\sum_{m=-\infty}^{\infty} \mathrm{e}^{\mathrm{i} m \sigma} \delta(\boldsymbol{x}-(\boldsymbol{y}+m \boldsymbol{\Delta}))
$$

Therefore, $G^{q}$ is the Green's function for a periodic array of sources with a phase shift of $\sigma$ between each period window.

\subsection{Compact Approximation without Background Flow}

We now seek an approximation for $G^{q}$. Using the reciprocity property of acoustic Green's functions (Howe, 1998), we place the observer at $\boldsymbol{y}$ and the sources at $\boldsymbol{x}+m \boldsymbol{\Delta}$ for $m \in \mathbb{Z}$. We further suppose that $G^{q}$ satisfies the no-flux condition

$$
\frac{\partial G^{q}}{\partial n_{y}}(\boldsymbol{x}, \boldsymbol{y})=0, \quad \boldsymbol{y} \in \partial D_{z}
$$

in addition to the forced Helmholtz equation (7.4). Our approximation must be valid in the compact limit $\left(\kappa_{0} l \ll 1\right)$ when the sources are in the far field $(|\boldsymbol{x}| \rightarrow \infty)$ and the observer is close to the cascade $(|\boldsymbol{y}| \sim l)$. We write the full quasi-periodic compact Green's function as

$$
G^{q}(\boldsymbol{x}, \boldsymbol{y}) \sim G_{0}^{q}(\boldsymbol{x}, \boldsymbol{y})+G_{1}^{q}(\boldsymbol{x}, \boldsymbol{y})
$$


where $G_{0}^{q}$ is the free space quasi-periodic Green's function for an array of cylindrically spreading waves and $G_{1}^{q}$ is a first order correction to be chosen to satisfy the boundary conditions on each aerofoil in the cascade. The free space Green's function for a source at $\boldsymbol{x}$ and observer at $\boldsymbol{y}$ is given by

$$
G_{0}^{q}(\boldsymbol{x}, \boldsymbol{y})=-\frac{\mathrm{i}}{4} H_{0}^{(1)}\left(\kappa_{0}|\boldsymbol{x}-\boldsymbol{y}|\right)
$$

where $H_{0}^{(1)}$ is the zero-order Hankel function of the first kind. The quasi-periodic Green's function is therefore obtained by summing the source distributions from each period window, with a phase shift of $\sigma$. Consequently, the free space quasi-periodic Green's function is given by

$$
G_{0}^{q}(\boldsymbol{x}, \boldsymbol{y})=-\frac{\mathrm{i}}{4} \sum_{m=-\infty}^{\infty} \mathrm{e}^{\mathrm{i} \sigma m} H_{0}^{(1)}\left(\kappa_{0}|\boldsymbol{x}+m \boldsymbol{\Delta}-\boldsymbol{y}|\right)
$$

Equation 7.6 is referred to as the spatial representation of the quasi-periodic Green's function. However, the series in (7.6) converges extremely slowly, and the form generally obscures the behaviour of $G_{0}^{q}$ as $|X| \rightarrow \infty$. There are many strategies available to speed-up the numerical computation of $G_{0}$, as outlined by Linton (1998), one of which we will apply later. We now expand $G_{0}^{q}$ in our desired asymptotic regime. We first note that (Olver et al., 2010, Eq. 10.17.5)

$$
H_{0}^{(1)}(z) \sim \sqrt{\frac{2}{\pi z}} \exp \left[\mathrm{i}\left(z-\frac{\pi}{4}\right)\right]
$$

as $|z| \rightarrow \infty$. By combining (7.7) with the relations

$$
\begin{aligned}
|\boldsymbol{x}+m \boldsymbol{\Delta}-\boldsymbol{y}| & \sim|\boldsymbol{x}+m \boldsymbol{\Delta}|-\frac{(\boldsymbol{x}+m \boldsymbol{\Delta}) \cdot \boldsymbol{y}}{|\boldsymbol{x}+m \boldsymbol{\Delta}|} \\
\frac{1}{|\boldsymbol{x}+m \boldsymbol{\Delta}-\boldsymbol{y}|} & \sim \frac{1}{|\boldsymbol{x}+m \boldsymbol{\Delta}|},
\end{aligned}
$$

we may approximate the free space quasi-periodic Green's function as

$$
G_{0}^{q}(\boldsymbol{x}, \boldsymbol{y}) \sim \frac{-\mathrm{i}}{2 \sqrt{2 \pi}} \sum_{m=-\infty}^{\infty} \frac{\mathrm{e}^{\mathrm{i} \sigma m}}{\sqrt{|\boldsymbol{x}+m \boldsymbol{\Delta}|}} \exp \left[\mathrm{i} \kappa_{0}\left(|\boldsymbol{x}+m \boldsymbol{\Delta}|-\frac{(\boldsymbol{x}+m \boldsymbol{\Delta}) \cdot \boldsymbol{y}}{|\boldsymbol{x}+m \boldsymbol{\Delta}|}\right)-\mathrm{i} \frac{\pi}{4}\right] .
$$

The above series converges, as proved by Bruno and Reitich (1992). 
Since $|\boldsymbol{y}| \sim l$ and $\kappa_{0} l \ll 1$, we have $\left|\kappa_{0} \boldsymbol{y}\right| \ll 1$. Consequently, we may write

$$
\exp \left[-\mathrm{i} \kappa_{0} \frac{(\boldsymbol{x}+m \boldsymbol{\Delta}) \cdot \boldsymbol{y}}{|\boldsymbol{x}+m \boldsymbol{\Delta}|}\right] \sim 1-\mathrm{i} \kappa_{0} \frac{(\boldsymbol{x}+m \boldsymbol{\Delta}) \cdot \boldsymbol{y}}{|\boldsymbol{x}+m \boldsymbol{\Delta}|},
$$

so that (7.8) becomes

$$
G_{0}^{q}(\boldsymbol{y}, \boldsymbol{x}) \sim \frac{-\mathrm{e}^{\mathrm{i} \pi / 4}}{2 \sqrt{2 \pi}} \sum_{m=-\infty}^{\infty} \mathrm{e}^{\mathrm{i} \sigma m} \frac{\exp \left[\mathrm{i} \kappa_{0}|\boldsymbol{x}+m \boldsymbol{\Delta}|\right]}{\sqrt{|\boldsymbol{x}+m \boldsymbol{\Delta}|}}\left(1-\mathrm{i} \kappa_{0} \frac{(\boldsymbol{x}+m \boldsymbol{\Delta}) \cdot \boldsymbol{y}}{|\boldsymbol{x}+m \boldsymbol{\Delta}|}\right) .
$$

We now choose $G_{1}^{q}$ so that the full quasi-periodic Green's function, $G^{q}$, satisfies the no-flux boundary condition (7.5). The appropriate choice of $G_{1}^{q}$ is

$$
G_{1}^{q}(\boldsymbol{y}, \boldsymbol{x}) \sim \frac{-\mathrm{e}^{\mathrm{i} \pi / 4}}{2 \sqrt{2 \pi}} \sum_{m=-\infty}^{\infty} \mathrm{e}^{\mathrm{i} \sigma m} \frac{\exp \left[\mathrm{i} \kappa_{0}|\boldsymbol{x}+m \boldsymbol{\Delta}|\right]}{\sqrt{|\boldsymbol{x}+m \boldsymbol{\Delta}|}} \mathrm{i} \kappa_{0} \frac{(\boldsymbol{x}+m \boldsymbol{\Delta}) \cdot \boldsymbol{\varphi}^{*}(\boldsymbol{y})}{|\boldsymbol{x}+m \boldsymbol{\Delta}|} .
$$

where $\varphi$ satisfies

$$
\begin{array}{ll}
\nabla_{y}^{2} \boldsymbol{\varphi}^{*}(\boldsymbol{y})=\mathbf{0}, & \boldsymbol{y} \in D_{z}, \\
\frac{\partial \boldsymbol{\varphi}^{*}}{\partial n}(\boldsymbol{y})=\boldsymbol{n}_{y}, & \boldsymbol{y} \in \partial D_{z}, \\
\left|\boldsymbol{\varphi}^{*}(\boldsymbol{y})\right| \rightarrow 0 & |\boldsymbol{y}| \rightarrow \infty, \quad \boldsymbol{y} \nVdash \boldsymbol{\Delta} .
\end{array}
$$

The first condition (7.9.a) ensures that $G_{1}^{q}$ satisfies the homogeneous form of the Helmholtz equation (7.2) up to $\mathcal{O}\left(\left(\kappa_{0} l\right)^{2}\right)$ so that the full Helmholtz equation (7.2) is satisfied by the full Green's function, $G^{q}$. The second condition (7.9.b) ensures that the full quasi-periodic compact Green's function satisfies the no flux condition (7.5). The final condition (7.9.c) states that the distortion of the flow produced by the cascade should be small when the observer is far from the cascade. Additionally, the causality principle implies that each $\varphi_{i}$ must have vanishing circulation around every aerofoil (Howe, 1998).

Consequently, the full compact quasi-periodic Green's function may be expressed as

$$
G^{q}(\boldsymbol{y}, \boldsymbol{x}) \sim \frac{-\mathrm{e}^{\mathrm{i} \pi / 4}}{2 \sqrt{2 \pi}} \sum_{m=-\infty}^{\infty} \mathrm{e}^{\mathrm{i} \sigma m} \frac{\exp \left[\mathrm{i} \kappa_{0}|\boldsymbol{x}+m \boldsymbol{\Delta}|\right]}{\sqrt{|\boldsymbol{x}+m \boldsymbol{\Delta}|}}\left(1-\mathrm{i} \kappa_{0} \frac{(\boldsymbol{x}+m \boldsymbol{\Delta}) \cdot \boldsymbol{Y}}{|\boldsymbol{x}+m \boldsymbol{\Delta}|}\right)
$$


where $\boldsymbol{Y}$ are the Kirchhoff vectors (Howe, 2003) defined by

$$
\boldsymbol{Y}(\boldsymbol{y}) \equiv \boldsymbol{y}-\varphi^{*}(\boldsymbol{y})
$$

The Kirchhoff vectors satisfy

$$
\begin{aligned}
\nabla_{y}^{2} \boldsymbol{Y}(\boldsymbol{y}) & =\mathbf{0}, & \boldsymbol{y} \in D_{z}, \\
\frac{\partial \boldsymbol{Y}}{\partial n}(\boldsymbol{y}) & =\mathbf{0}, & \boldsymbol{y} \in \partial D_{z}, \\
\boldsymbol{Y}(\boldsymbol{y}) & \rightarrow \boldsymbol{y}, & |\boldsymbol{y}| \rightarrow \infty, \quad \boldsymbol{y} \nVdash \boldsymbol{\Delta} .
\end{aligned}
$$

Condition (7.11.b) follows from

$$
\frac{\partial \boldsymbol{Y}}{\partial n}=\frac{\partial \boldsymbol{y}}{\partial n}-\boldsymbol{n}=n_{j} \frac{\partial \boldsymbol{y}}{\partial y_{j}}-\boldsymbol{n}=\mathbf{0}
$$

The solutions of (7.11.a-7.11.c) have a hydrodynamic interpretation: $Y_{j}$ is the velocity potential for uniform flow in the $j$-direction satisfying a no-flux condition. In chapter 6 we derived analytic forms for the complex potential satisfying these requirements. Using these analytic forms, we may write

$$
\begin{aligned}
& Y_{1}(\boldsymbol{y})=\mathbb{R e}\left[A\left(\log \left(\frac{\omega\left(\zeta(\boldsymbol{y}), a_{\infty^{-}}\right)}{\omega\left(\zeta(\boldsymbol{y}), a_{\infty^{+}}\right)}\right)+\log \left(\frac{\omega\left(\zeta(\boldsymbol{y}), 1 / \overline{a_{\infty^{-}}}\right)}{\omega\left(\zeta(\boldsymbol{y}), 1 / \overline{a_{\infty^{+}}}\right)}\right)\right)\right], \\
& Y_{2}(\boldsymbol{y})=\mathbb{R e}\left[A \mathrm{i}\left(-\log \left(\frac{\omega\left(\zeta(\boldsymbol{y}), a_{\infty^{-}}\right)}{\omega\left(\zeta(\boldsymbol{y}), a_{\infty^{+}}\right)}\right)+\log \left(\frac{\omega\left(\zeta(\boldsymbol{y}), 1 / \overline{a_{\infty^{-}}}\right)}{\omega\left(\zeta(\boldsymbol{y}), 1 / \overline{a_{\infty^{+}}}\right)}\right)\right)\right],
\end{aligned}
$$

where $\zeta$ is the conformal mapping from the physical domain to a canonical circular domain and $\omega(\cdot, \cdot)$ is the Schottky-Klein prime function (see section 1.2.3.2). All other undefined quantities are defined in chapter 6 . An example of the Kirchhoff vectors are plotted in figure 7.3 for a cascade with three aerofoils per period window. The Kirchhoff vector $Y_{1}$ is the velocity potential for flow in the horizontal direction whereas $Y_{2}$ is the velocity potential for flow in the vertical direction. Note that the contours intersect each aerofoil at a right angle, thus indicating that the no-flux condition (7.11.b) is satisfied. Moreover, observing the upstream and downstream behaviour in figure 7.3 indicates that condition (7.11.c) is satisfied. We also note that, by formulating the solution in terms 


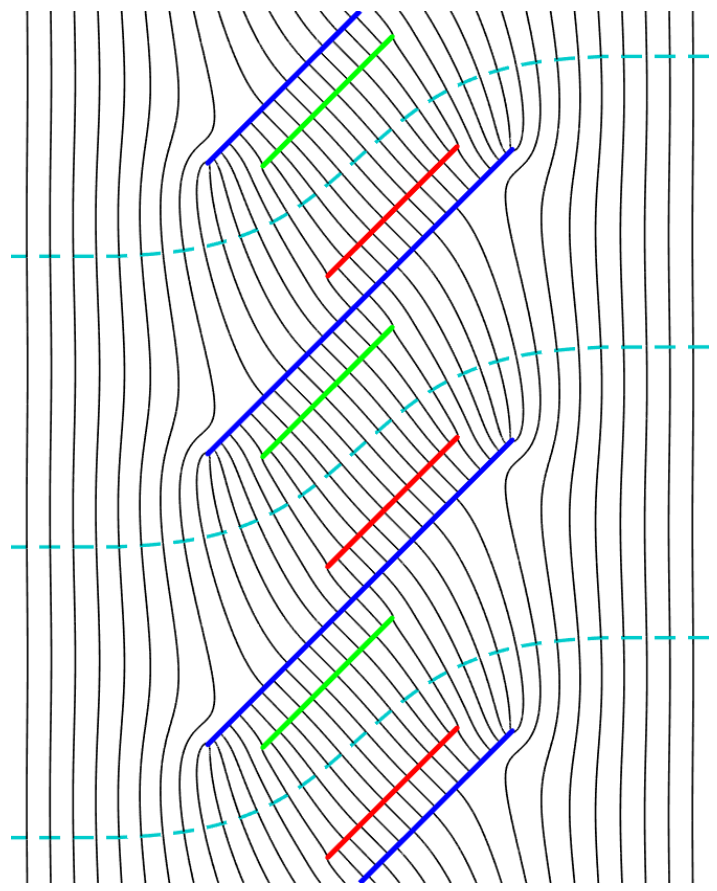

(a) $Y_{1}$

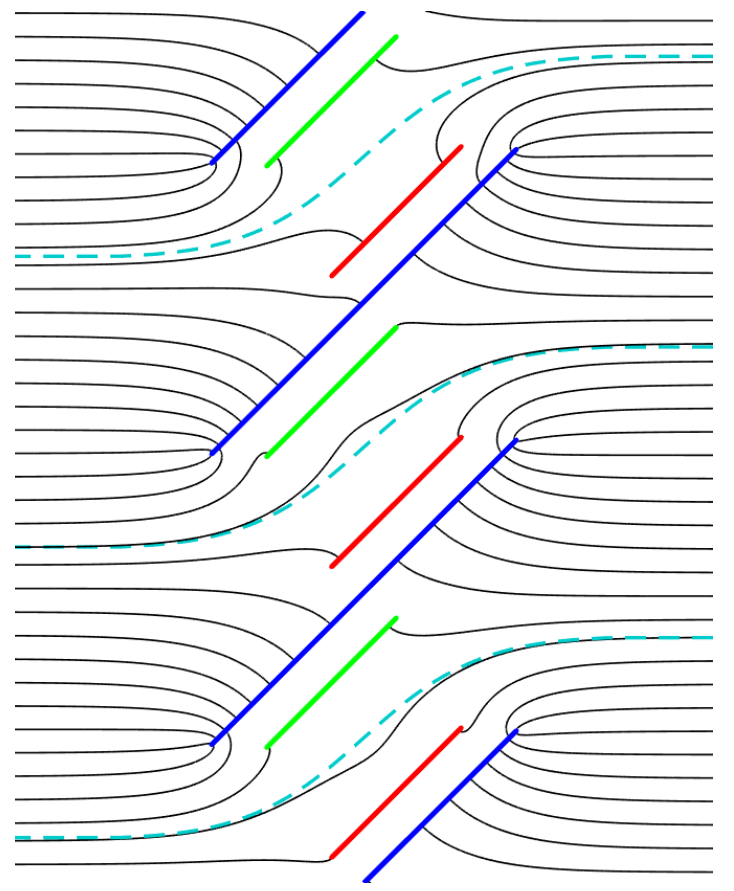

(b) $Y_{2}$

Fig. 7.3 The contours of the Kirchhoff vectors for a cascade with three aerofoils per period window.

of the prime function, the Kirchhoff vectors in (7.12.a) and (7.12.b) are valid for any number of objects per period window.

By reversing the asymptotic approximations made in this section, we may also write the compact, quasi-periodic Green's function (7.10) in the symmetric form

$$
G^{q}(\boldsymbol{x}, \boldsymbol{y}) \sim \frac{-\mathrm{i}}{4} \sum_{m=-\infty}^{\infty} \mathrm{e}^{\mathrm{i} \sigma m} H_{0}^{(1)}\left(\kappa_{0}|\boldsymbol{X}-\boldsymbol{Y}+m \boldsymbol{\Delta}|\right)
$$

where

$$
\boldsymbol{X}(\boldsymbol{x}) \equiv \boldsymbol{x}-\varphi^{*}(\boldsymbol{x})
$$

Alternatively, we may write (7.13) as

$$
G^{q}(\boldsymbol{x}, \boldsymbol{y}) \sim G_{0}^{q}(\boldsymbol{X}, \boldsymbol{Y})
$$

In other words, the compact approximation to the quasi-periodic Green's function is simply the original free space quasi-periodic Green's function where the arguments are replaced by the Kirchhoff vectors. This final expression is particularly significant 
since it means that all the convergence properties of the $G_{0}^{q}$ may be translated to our compact approximation. For example, $G_{0}^{q}$ may be equivalently expressed in the spectral representation (Linton, 1998)

$$
G_{0}^{q}(\boldsymbol{x}, \boldsymbol{y})=-\frac{1}{2 \Delta} \sum_{m=-\infty}^{\infty} \frac{\mathrm{e}^{-\zeta_{m}\left|x_{1}-y_{1}\right| / \Delta} \mathrm{e}^{\mathrm{i} \sigma_{m}\left|x_{2}-y_{2}\right| / \Delta}}{\zeta_{m}},
$$

where

$$
\sigma_{m}=\sigma+2 \pi m, \quad \zeta_{m}=\sqrt{\sigma_{m}-\Delta^{2} \kappa_{0}^{2}}
$$

We assume that the frequency is not at a Wood anomaly (when one or more scattered waves propagate in the $(0,1)$ direction, (Wood, 1902)), so $\zeta_{m} \neq 0$. The advantage of the spectral representation (7.14) is that the behaviour as $|\boldsymbol{x}| \rightarrow \infty$ is clear. Moreover, the series in (7.14) converges exponentially, so is more appropriate for numerical computations. However, it is not immediately obvious that (7.14) possesses the correct distribution of monopoles, but this can be proved rigorously (Bruno and Reitich, 1992).

Applying the spectral representation (7.14) to our compact approximation allows us to write

$$
G^{q}(\boldsymbol{x}, \boldsymbol{y}) \sim-\frac{1}{2 \Delta} \sum_{m=-\infty}^{\infty} \frac{\mathrm{e}^{-\zeta_{m}\left|X_{1}-Y_{1}\right| / \Delta} \mathrm{e}^{\mathrm{i} \sigma_{m}\left|X_{2}-Y_{2}\right| / \Delta}}{\zeta_{m}}
$$

\subsection{Compact Approximation with Background Flow}

We now extend the quasi-periodic compact Green's function to account for a background low Mach number, irrotational flow. If we assume that the Mach number is sufficiently low such that $\left(M^{2} \ll 1\right)$, the acoustic potential, $\phi$ now satisfies the inhomogeneous convected wave equation:

$$
\frac{1}{c_{0}^{2}} \frac{\mathrm{D}_{0}^{2} \phi}{\mathrm{D}_{0} t^{2}}(\boldsymbol{x})-\nabla_{x}^{2} \phi(\boldsymbol{x})=-q(\boldsymbol{x}),
$$

where

$$
\frac{\mathrm{D}_{0}}{\mathrm{D}_{0} t}=\frac{\partial}{\partial t}+\boldsymbol{U}_{0} \cdot \nabla_{x}
$$

is the material derivative with respect to the background flow $\boldsymbol{U}_{0}=\left(U_{1}, U_{2}\right)$. 
In this case, the frequency domain Green's function satisfies the equation (Howe, 1998)

$$
\left(\nabla_{y}^{2}+\kappa_{0}^{2}-2 \mathrm{i} \kappa_{0} \boldsymbol{M}_{0} \cdot \nabla_{y}\right) G=\delta(\boldsymbol{x}-\boldsymbol{y})
$$

where $\boldsymbol{M}_{0}=\boldsymbol{U}_{0} / c_{0}$. Since the flow is at low Mach number it is effectively incompressible and we may write the components of the mean flow as

$$
U_{i}=\boldsymbol{U}_{\infty} \cdot \nabla \Phi_{i}
$$

where

$$
\boldsymbol{U}_{\infty}=U_{\infty}(\cos (\alpha), \sin (\alpha))
$$

is the background velocity far upstream and $\Phi_{i}$ corresponds to the velocity potential for potential flow through the cascade in the $y_{i}$ direction far upstream. Note that we have not set the downstream angle of the background flow as this will in general be determined by the steady Kutta condition.

We follow Taylor (1978) and use the substitution

$$
G(\boldsymbol{x}, \boldsymbol{y})=\tilde{G}(\boldsymbol{x}, \boldsymbol{y}) \exp \left[\mathrm{i} \kappa_{0} \boldsymbol{M}_{0} \cdot \boldsymbol{\Phi}(\boldsymbol{y})\right]
$$

to transform the convected Helmholtz equation (7.16) to

$$
\nabla_{y}^{2} \tilde{G}(\boldsymbol{x}, \boldsymbol{y})+\kappa_{0}^{2} \tilde{G}(\boldsymbol{x}, \boldsymbol{y})=\delta(\boldsymbol{x}-\boldsymbol{y}) \exp \left[-\mathrm{i} \kappa_{0} \boldsymbol{M}_{0} \cdot \boldsymbol{\Phi}(\boldsymbol{x})\right]
$$

Consequently, we may follow an analogous procedure to section 7.3 to obtain an expression for the symmetric quasi-periodic compact Green's function in background flow as

$$
G^{q}(\boldsymbol{x}, \boldsymbol{y})=-\frac{1}{2 \Delta} \exp \left[-\mathrm{i} \kappa_{0} \boldsymbol{M}_{0} \cdot(\boldsymbol{\Phi}(\boldsymbol{x})-\boldsymbol{\Phi}(\boldsymbol{y}))\right] \sum_{m=-\infty}^{\infty} \frac{\mathrm{e}^{-\zeta_{m}\left|X_{1}-Y_{1}\right| / \Delta} \mathrm{e}^{\mathrm{i} \sigma_{m}\left|X_{2}-Y_{2}\right| / \Delta}}{\zeta_{m}} .
$$

The above expression shows that, in the compact limit, the effect of background flow is to change the phase of the Green's function. If the cascade is loaded, it will produce lift and therefore the inlet angle is different to the outlet angle. Consequently, $\boldsymbol{\Phi}$ will be different in the upstream and downstream regions, and therefore the background flow will induce a different phase shift in the upstream and downstream regions. This phenomenon is not observed for isolated bodies since $\boldsymbol{\Phi}$ must be the same in the upstream and downstream regions. 


\subsection{Conclusions}

In this chapter we have derived a compact approximation to the quasi-periodic Green's function. The approximation is written in terms of the Kirchhoff vectors, which represent the velocity potential for a uniform flow through the cascade. Moreover, we have presented analytic forms for the Kirchhoff vectors in terms of the Schottky-Klein prime function. Accordingly, the solutions in this chapter are valid for any number of aerofoils per period window. Importantly, we have shown that the compact approximation to the quasi-periodic Green's function may be written as the free space quasi-periodic Green's function where the arguments are replaced by the Kirchhoff vectors. Consequently, rapid numerical algorithms for the free space quasi-periodic Green's function (such as Bruno and Fernandez-Lado (2017)) may be leveraged for our compact approximation.

However, the method is not without its limitations. Since we assumed that the cascade is acoustically compact, the range of admissible frequencies is quite narrow, and will not be appropriate for all cascade interactions. As with chapter 6, the appropriate conformal map must be determined in order to calculate the Kirchhoff vectors, and further work is required for the rapid and reliable computation of these mappings. 


\title{
List of Symbols
}

\author{
Symbol Meaning \\ $\phi \quad$ Acoustic potential function \\ $q \quad$ Source terms \\ $\omega \quad$ Frequency of source \\ $l \quad$ Characteristic horizontal length scale of the cascade \\ $\Delta \quad$ Spatial length of period \\ $\kappa_{0} \quad \omega / c_{0}$ (reduced frequency) \\ $\sigma \quad$ Phase shift between adjacent period windows \\ G Green's function \\ $G^{q} \quad$ Quasi-periodic Green's function \\ $G_{0} \quad$ Free space Green's function \\ $G_{0}^{q} \quad$ Free space quasi-periodic Green's function \\ $\tilde{G} \quad$ Transformed Green's function for convected wave equation \\ $\boldsymbol{Y} \quad$ Kirchhoff vector \\ $\omega(\cdot, \cdot) \quad$ Schottky-Klein prime function \\ $\boldsymbol{U}_{0} \quad$ Background flow vector \\ $\boldsymbol{U}_{\infty} \quad$ Upstream background flow vector \\ $\alpha \quad$ Angle of attack
}




\section{Chapter 8}

\section{Conclusion}

During this thesis we have derived analytic solutions for flows through cascades. We have devoted efforts to three scenarios of practical interest, namely cascades with a) aerofoils with realistic geometry, b) porous aerofoils, and c) multiple aerofoils per period window. Each scenario has been investigated from both aerodynamic (steady) and aeroacoustic (unsteady) perspectives to provide a holistic analysis. We have employed a range of tools to solve these problems, with a particular emphasis on complex variable methods. The main tools have been Riemann-Hilbert problems, the Wiener-Hopf method and multiply connected conformal mappings and we also made use of the compact Green's function.

The solutions presented in this thesis offer physical insight into the roles played by aerofoil shape, blade spacing and the aerofoil surface boundary condition. For example, we derived an explicit asymptotic relation that relates the angle of attack, aerofoil camber and blade spacing to the deflection angle for a cascade in a potential flow in chapter 2. In chapter 3 we showed that aerofoil geometry has a significant effect on gust-cascade interaction noise, and that angle of attack and camber control the upstream and downstream cut-on frequencies respectively. In chapter 4 we showed that that the adverse aerodynamic losses caused by porosity can be mitigated by reducing the blade spacing, and in chapter 5 we showed that gust-cascade interaction noise can be greatly reduced by introducing aerofoil porosity and therefore permitting flow seepage through the blades. The analytic nature of the solutions means that they are are extremely rapid to compute and therefore could provide important design tools for designers seeking to optimise the aerodynamic and aeroacoustic performance of cascades.

In the remainder of this conclusion we suggest directions of future research for the problems encountered in each chapter. 


\subsection{Future Work by Chapter}

\section{Chapter 2}

In this chapter we extended thin aerofoil theory to derive an asymptotic solution for the potential flow past a cascade of thin aerofoils. This is only the first step in a full generalisation of thin aerofoil theory to cascades, and there is a plethora of further work to be done in generalising other aspects of thin aerofoil theory. For example, the inclusion of small amplitude unsteady disturbances is a straightforward extension of the analysis presented in chapter 2. Analytic forms of aerodynamic transfer functions could be derived, such as the Theodorsen, Sears, Küssner and Wagner functions (Bisplinghoff et al., 1996). Another natural extension of this work is to take into account three-dimensional effects. One way to achieve this is to consider cascades with wings of finite span, where the wing circulation satisfies a periodic singular integro-differential equation (von Kármán and Burgers, 1936).

\section{Chapter 3}

In this chapter we derived an analytic solution for gust-cascade interaction noise whilst accounting for aerofoil geometry and angle of attack. The model is restricted to aerofoils with small thickness and mean loading of size $\mathcal{O}(\epsilon)$ and is valid for non-dimensional frequencies such that $k \epsilon \ll 1$. This range includes the important first few blade passing frequencies for turbulence-OGV interaction noise within a typical high bypass ratio aeroengine, which is a dominant source of broadband noise. Nevertheless, these restrictions should be relaxed in future research. Work is currently underway in applying the model to predict the acoustic feedback from an aero-engine turbine during unsteady combustion (Guzman Inigo et al., 2019, 2018). The model could also be integrated into a broadband fan noise prediction model Posson et al. (2010a) to better account for three-dimensional effects in a realistic axial fan.

\section{Chapter 4}

In this chapter we extended the analysis of chapter 2 to consider cascades of aerofoils with porosity gradients. The porosity was modelled using a Darcy-type condition, where the flow through the wing is assumed to be proportional to the pressure jump across the wing. As indicated in chapter 4, the Darcy formulation is only valid when the pore-scale Reynolds number is small. Accordingly, the analysis is only valid for small 
porosities. Future work will be devoted to applying the Ergun model (Ergun, 1952) which is applicable to high Reynolds number flows.

The analysis in this chapter is restricted to cascades with small stagger, since the SIE for general stagger does not have an analytic solution. However, recent work (Baddoo et al., 2019a,b) has developed a numerical scheme to solve SIEs that could be leveraged for a cascade of porous aerofoils. The method is based on expanding the solution function $\gamma$ into a series of weighted Jacobi polynomials, and determining the coefficients of the series using a spectral collocation method. The parameters of the Jacobi polynomials are determined by asymptotic analysis at the endpoints of the aerofoils. The convergence of the method is spectral, i.e. exponentially fast, and enables a rapid solution without the evaluation of singular integrals. This approach is analogous to the series expansion in Chebyshev polynomials conducted in chapter 2, where the Chebyshev polynomials are replaced with the appropriate Jacobi polynomials.

\section{Chapter 5}

In this chapter we investigated the scattering of cascades with modified boundary conditions. We devoted efforts to analysing a Darcy-type condition. However, there is little theoretical support for this model. Moreover, as mentioned above, the Darcy flow model assumes a Stokes flow through the wing, which will generally not be valid in aeroacoustic applications. Nevertheless, this chapter is a valuable first step towards more sophisticated analyses of the aeroacoustics of cascades of porous aerofoils. Similarly to chapter 3, future work should focus on integrating this analysis into a broadband fan noise prediction model, such as that in Posson et al. (2010a). Future work will focus on more detailed analysis of elastic plates (Ayton, 2016; Cavalieri et al., 2016) in order to explore the important effects of flutter and resonance.

\section{Chapter 6}

In this chapter we presented analytic solutions to the potential flow through periodic domains where there is an arbitrary number of objects in each period window. However, the solutions are only useful when the appropriate conformal map is available. These conformal mappings are not always trivial to construct and, accordingly, further work is required in solving the "parameter problem" (see Driscoll and Trefethen (2002)) for the periodic Schwarz-Christoffel mapping (Baddoo and Crowdy, 2019). An approach analogous to that employed in Driscoll (2005) is a promising path forwards in this regard.

The work in this chapter could certainly be applied to discrete vortex models for turbomachinery. In particular, a periodic extension of the analysis in Michelin and 
Llewellyn Smith (2009) would provide a powerful tool for analysing turbomachinery aerodynamics. In that study, the unsteady motion of a rigid body in inviscid flow was analysed with results comparing well to numerical and experimental studies. The discrete vortex shedding was analysed using the approach of (Brown and Michael, 1954), which enables the Kutta condition to be satisfied at each sharp corner. This method is computationally inexpensive, and could be applied using the techniques of the present chapter. The method could also be adapted to consider more sophisticated hybrid models consisting of point vortices and vortex sheets, such as in Darakananda and Eldredge (2019). Analytic expressions for the trajectories of point vortices could also be derived by adapting the work of Crowdy and Marshall (2005).

\section{Chapter 7}

In this chapter we presented a compact approximation to the quasi-periodic Green's function. Further applications of the new Green's function are multifarious, and several possible applications are detailed in Howe (1998) such as vibrating cascades, vortex sound, and acoustic scattering. Further work is also required to satisfy the Kutta condition in unsteady flow by vortex shedding at the trailing edges. 


\section{References}

Abbott, I. H. and Von Doenhoff, A. E. (1959). Theory of Wing Sections: Including a Summary of Airfoil Data, volume 11. Dover Publications. [Cited on page 25.]

Ablowitz, M. J. and Fokas, A. S. (2003). Complex Variables: Introduction and Applications, volume XXXIII. Cambridge University Press. [Cited on pages 10, 12, 13, 26, 27 , and 244.]

ACARE (2011). Flightpath 2050 - Europe's vision for aviation. Technical report, European Commission, Brussels. [Cited on page 2.]

Akhiezer, N. I. (1990). Elements of the theory of elliptic functions. In Transl. Math. Monogr. American Mathematical Society, Providence, vol 79 edition. [Cited on page 244.]

Antipov, Y. A. (2000). Galin's problem for a periodic system of stamps with friction and adhesion. Int. J. Solids Struct., 37(15):2093-2125. [Cited on page 172.]

Ayton, L. J. (2016). Acoustic scattering by a finite rigid plate with a poroelastic extension. J. Fluid Mech., 791:414-438. [Cited on pages 6, 156, 157, 182, 189, and 265.]

Ayton, L. J. (2017). An analytic solution for gust-aerofoil interaction noise including effects of geometry. IMA J. Appl. Math., 82(2):280-304. [Cited on page 63.]

Ayton, L. J. (2018). Analytic solution for aerodynamic noise generated by plates with spanwise-varying trailing edges. J. Fluid Mech., 849:448-466. [Cited on page 8.]

Ayton, L. J., Gill, J. R., and Peake, N. (2016). The importance of the unsteady Kutta condition when modelling gust-aerofoil interaction. J. Sound Vib., 378:28-37. [Cited on pages 10, 71, 107, and 206.]

Ayton, L. J. and Peake, N. (2013). On high-frequency noise scattering by aerofoils in flow. J. Fluid Mech., 734:144-182. [Cited on pages 22, 62, and 63.]

Ayton, L. J. and Peake, N. (2015). On high-frequency sound generated by gust-aerofoil interaction in shear flow. J. Fluid Mech., 766:297-325. [Cited on page 8.] 
Baddoo, P. J. and Ayton, L. J. (2017). An analytic solution for gust-cascade interaction noise including effects of realistic aerofoil geometry. In 23rd AIAA/CEAS Aeroacoustics Conf., page 3216, Reston, Virginia. American Institute of Aeronautics and Astronautics. [Cited on page iii.]

Baddoo, P. J. and Ayton, L. J. (2018a). An analytic solution for gust-cascade interaction noise including effects of realistic aerofoil geometry: inter-blade region. In 2018 AIAA/CEAS Aeroacoustics Conf. AIAA Aviat. Forum, page 2957, Atlanta, Georgia. American Institute of Aeronautics and Astronautics. [Cited on page iii.]

Baddoo, P. J. and Ayton, L. J. (2018b). Potential flow through a cascade of aerofoils: direct and inverse problems. Proc. R. Soc. A Math. Phys. Eng. Sci., 474(2217):20180065. [Cited on pages iii and 88.]

Baddoo, P. J. and Ayton, L. J. (2019a). A calculus for flows in periodic domains [UNDER REVIEW]. Theor. Comput. Fluid Dyn. [Cited on page iii.]

Baddoo, P. J. and Ayton, L. J. (2019b). Acoustic scattering by cascades with complex boundary conditions: compliance, porosity and impedance [UNDER REVIEW]. $J$. Fluid Mech. [Cited on page iii.]

Baddoo, P. J. and Ayton, L. J. (2019c). Analytic solutions for acoustic scattering by blade rows with complex boundaries: porosity, compliance and impedance. In Proc. 23rd Int. Congr. Acoust. [Cited on page iii.]

Baddoo, P. J. and Ayton, L. J. (2019d). The compact Green's function for multiple bodies. In AIAA/CEAS Aeroacoustics Conf., page 2470, Delft. [Cited on page iii.]

Baddoo, P. J. and Ayton, L. J. (2020). An analytic solution for gust-cascade interaction noise including effects of realistic aerofoil geometry [IN PRESS]. J. Fluid Mech. [Cited on page iii.]

Baddoo, P. J. and Crowdy, D. G. (2019). Periodic Schwarz-Christoffel mappings with multiple boundaries per period. Proc. R. Soc. A Math. Phys. Eng. Sci., 475(2228). [Cited on pages iii, 20, 225, 226, 234, 235, and 265.]

Baddoo, P. J., Hajian, R., and Jaworski, J. W. (2019a). A Jacobi spectral collocation method for the steady aerodynamics of porous aerofoils. In AIAA Aviat. 2019 Forum, number Jue, pages 2019-2959, Dallas, Texas. American Institute of Aeronautics and Astronautics. [Cited on page 265.]

Baddoo, P. J., Hajian, R., and Jaworski, J. W. (2019b). Unsteady aerodynamics of porous aerofoils [UNDER REVIEW]. J. Fluid Mech. [Cited on page 265.]

Bae, Y. and Moon, Y. J. (2011). Effect of passive porous surface on the trailing-edge noise. Phys. Fluids, 23(12):126101. [Cited on page 150.] 
Baker, H. F. (1897). Abelian functions: Abel's theorem and the allied theory of theta functions. Cambridge University Press. [Cited on page 14.]

Balsa, T. F. (1977). Potential flow interactions in an array of cylinders in cross-flow. $J$. Sound Vib., 50(2):285-303. [Cited on page 21.]

Batchelor, G. K. (2000). An Introduction to Fluid Dynamics. Cambridge University Press. [Cited on pages 24 and 236.]

Batchelor, G. K. and Proudman, I. (1954). The effect of rapid distortion of a fluid in turbulent motion. Q. J. Mech. Appl. Math., 7(1):83-103. [Cited on pages 18 and 61.]

Bhimarasetty, A. and Govardhan, R. N. (2010). A simple method for potential flow simulation of cascades. Sadhana, 35(6):649-657. [Cited on page 38.]

Bisplinghoff, R. L., Ashley, H., and Halfman, R. L. (1996). Aeroelasticity. Dover Publications. [Cited on page 264.]

Bouley, S., François, B., Roger, M., Posson, H., and Moreau, S. (2017). On a twodimensional mode-matching technique for sound generation and transmission in axialflow outlet guide vanes. J. Sound Vib., 403:190-213. [Cited on pages 85, 86, and 193.]

Brambley, E. J. (2009). Fundamental problems with the model of uniform flow over acoustic linings. J. Sound Vib., 322(4-5):1026-1037. [Cited on page 183.]

Brenner, H. (1980). Dispersion resulting from flow through spatially periodic porous media. Philos. Trans. R. Soc. A Math. Phys. Eng. Sci., 297(1430):81-133. [Cited on page 21.]

Bromwich, T. J. I. (2005). An Introduction to the Theory of Infinite Series. American Mathematical Soc. [Cited on page 57.]

Brown, C. E. and Michael, W. H. (1954). Effect of leading-edge separation on the lift of a delta wing. J. Aeronaut. Sci., 21(10):690-694. [Cited on page 266.]

Bruno, O. P. and Fernandez-Lado, A. G. (2017). Rapidly convergent quasi-periodic Green functions for scattering by arrays of cylinders - including Wood anomalies. Proc. R. Soc. A Math. Phys. Eng. Sci., 473(2199):20160802. [Cited on page 261.]

Bruno, O. P. and Reitich, F. (1992). Solution of a boundary value problem for the helmholtz equation via variation of the boundary into the complex domain. Proc. $R$. Soc. Edinburgh Sect. A Math., 122(3-4):317-340. [Cited on pages 255 and 259.]

Cavalieri, A. V., Wolf, W. R., and Jaworski, J. W. (2016). Numerical solution of acoustic scattering by finite perforated elastic plates. Proc. R. Soc. A Math. Phys. Eng. Sci., 472:20150767. [Cited on pages 6, 147, 157, 175, and 265.] 
Chen, K., Liu, Q., Liao, G., Yang, Y., Ren, L., Yang, H., and Chen, X. (2012). The sound suppression characteristics of wing feather of owl (Bubo bubo). J. Bionic Eng., 9(2):192-199. [Cited on page 147.]

Clancy, L. J. (1978). Aerodynamics. Wiley. [Cited on page 25.]

Clark, I. A., Daly, C. A., Devenport, W., Alexander, W. N., Peake, N., Jaworski, J. W., and Glegg, S. (2016). Bio-inspired canopies for the reduction of roughness noise. $J$. Sound Vib., 385:33-54. [Cited on page 6.]

Crighton, D. G. (2001). Asymptotic factorization of Wiener-Hopf kernels. Wave Motion, 33(1):51-65. [Cited on page 10.]

Crighton, D. G. and Leppington, F. G. (1970). Scattering of aerodynamic noise by a semi-infinite compliant plate. J. Fluid Mech., 43(4):721-736. [Cited on pages 157, 175, and 181.]

Crowdy, D. G. (1999). Exact solutions for steady capillary waves on a fluid annulus. J. Nonlinear Sci., 9(6):615-640. [Cited on page 225.]

Crowdy, D. G. (2000). A new approach to free surface Euler flows with capillarity. Stud. Appl. Math., 105(1):35-58. [Cited on page 225.]

Crowdy, D. G. (2005). The Schwarz-Christoffel mapping to bounded multiply connected polygonal domains. Proc. R. Soc. A Math. Phys. Eng. Sci., 461(2061):2653-2678. [Cited on pages 14, 20, and 234.]

Crowdy, D. G. (2006). Analytical solutions for uniform potential flow past multiple cylinders. Eur. J. Mech. B/Fluids, 25(4):459-470. [Cited on page 240.]

Crowdy, D. G. (2007). Schwarz-Christoffel mappings to unbounded multiply connected polygonal regions. Math. Proc. Cambridge Philos. Soc., 142(2):319-339. [Cited on pages 14 and 234.]

Crowdy, D. G. (2008a). Explicit solution for the potential flow due to an assembly of stirrers in an inviscid fluid. J. Eng. Math., 62(4):333-344. [Cited on page 242.]

Crowdy, D. G. (2008b). The Schwarz problem in multiply connected domains and the Schottky-Klein prime function. Complex Var. Elliptic Equations, 53(3):221-236. [Cited on pages 14 and 244.]

Crowdy, D. G. (2010). A new calculus for two-dimensional vortex dynamics. Theor. Comput. Fluid Dyn., 24(1-4):9-24. [Cited on pages 20, 224, 235, 236, 239, 240, 242, and 247.]

Crowdy, D. G. (2011). Frictional slip lengths for unidirectional superhydrophobic grooved surfaces. Phys. Fluids, 23(7):72001. [Cited on page 225.] 
Crowdy, D. G. (2013). Analytical formulae for source and sink flows in multiply connected domains. Theor. Comput. Fluid Dyn., 27(1-2):1-19. [Cited on page 229.]

Crowdy, D. G. (2016). Uniform flow past a periodic array of cylinders. Eur. J. Mech. B/Fluids, 56:120-129. [Cited on page 21.]

Crowdy, D. G. (2017). Effective slip lengths for immobilized superhydrophobic surfaces. J. Fluid Mech., 825:R2. [Cited on page 225.]

Crowdy, D. G., Fokas, A. S., and Green, C. C. (2011). Conformal mappings to multiply connected polycircular arc domains. Comput. Methods Funct. Theory, 11(2):685-706. [Cited on page 234.]

Crowdy, D. G. and Green, C. C. (2011). Analytical solutions for von Kármán streets of hollow vortices. Phys. Fluids, 23(12):126602. [Cited on page 225.]

Crowdy, D. G., Kropf, E. H., Green, C. C., and Nasser, M. M. S. (2016). The SchottkyKlein prime function: A theoretical and computational tool for applications. IMA J. Appl. Math., 81(3):589-628. [Cited on pages 14 and 244.]

Crowdy, D. G. and Luca, E. (2014). Solving Wiener-Hopf problems without kernel factorization. Proc. R. Soc. A Math. Phys. Eng. Sci., 470(2170):20140304-20140304. [Cited on page 10.]

Crowdy, D. G. and Marshall, J. (2005). Analytical formulae for the Kirchhoff-Routh path function in multiply connected domains. Proc. R. Soc. A Math. Phys. Eng. Sci., 461(2060):2477-2501. [Cited on pages 236, 237, and 266.]

Crowdy, D. G. and Marshall, J. (2006). Conformal mappings between canonical multiply connected domains. Comput. Methods Funct. Theory, 6(1):59-76. [Cited on pages 14, $15,16,20,226$, and 227.]

Crowdy, D. G. and Nelson, R. (2010). Steady interaction of a vortex street with a shear flow. Phys. Fluids, 22(9):096601. [Cited on page 225.]

Curle, N. (1955). The influence of solid boundaries upon aerodynamic sound. Proc. $R$. Soc. London. Ser. A. Math. Phys. Sci., 231(1187):505-514. [Cited on page 6.]

Darakananda, D. and Eldredge, J. D. (2019). A versatile taxonomy of low-dimensional vortex models for unsteady aerodynamics. J. Fluid Mech., 858:917-948. [Cited on page 266.]

Deift, P. (2000). Orthogonal Polynomials and Random Matrices: a Riemann-Hilbert Approach. American Mathematical Society. [Cited on page 10.]

DeLillo, T. K., Elcrat, A. R., and Pfaltzgraff, J. A. (2004). Schwarz-Christoffel mapping of multiply connected domains. J. d'Analyse Math., 94(1):17-47. [Cited on page 234.] 
Dittmar, J. H. (1972). Methods for reducing blade passing frequency noise generated by rotor-wake-stator interaction. Technical report, NASA. [Cited on page 60.]

Driscoll, T. A. (1996). Algorithm 756; a MATLAB toolbox for Schwarz-Christoffel mapping. ACM Trans. Math. Softw., 22(2):168-186. [Cited on page 234.]

Driscoll, T. A. (2005). Algorithm 843: Improvements to the Schwarz-Christoffel toolbox for MATLAB. ACM Trans. Math. Softw., 31(2):239-251. [Cited on pages 234, 235, and 265.]

Driscoll, T. A. and Trefethen, L. N. (2002). Schwarz-Christoffel Mapping. Cambridge University Press, Cambridge. [Cited on pages 234 and 265.]

EASA (2019). Type-certificate data sheet for Trent 1000 series engines. Technical report. [Cited on pages 3 and 4.]

Erdogan, F. and Gupta, G. D. (1972). On the numerical solution of singular integral equations. Q. Appl. Math., 29(4):525-534. [Cited on pages 18, 22, and 45.]

Ergun, S. (1952). Fluid flow through packed columns. Chem. Eng. Prog., 48(2):89 - 94. [Cited on pages 148, 150, 164, and 265.]

Evers, I. and Peake, N. (2002). On sound generation by the interaction between turbulence and a cascade of airfoils with non-uniform mean flow. J. Fluid Mech., 463:25-52. [Cited on pages $4,22,60,65,226$, and 230.]

Falcão, A. F. O. (1975). Three-dimensional potential flow through a rectilinear cascade of blades. Ingenieur-Archiv, 44(1):27-41. [Cited on page 22.]

Fang, J. and Atassi, H. M. (1993). Compressible flows with vortical disturbances around a cascade of loaded airfoils. In Unsteady Aerodyn. Aeroacoustics, Aeroelasticity Turbomachines Propellers, pages 149-176. Springer New York, New York, NY. [Cited on pages 89,92 , and 94.$]$

Ffowcs Williams, J. E. and Hawkings, D. L. (1969). Sound generation by turbulence and surfaces in arbitrary motion. Philos. Trans. R. Soc. A Math. Phys. Eng. Sci., 264(1151):321-342. [Cited on page 7.]

Floryan, J. M. (1985). Conformal-mapping-based coordinate generation method for flows in periodic configurations. J. Comput. Phys., 62(1):221-247. [Cited on page 226.]

Floryan, J. M. and Zemach, C. (1993). Schwarz-Christoffel methods for conformal mapping of regions with a periodic boundary. J. Comput. Appl. Math., 46(1-2):77-102. [Cited on page 226.]

Fokas, A. S. (2008). A Unified Approach to Boundary Value Problems. Society for Industrial and Applied Mathematics. [Cited on page 21.] 
Gakhov, F. D. (1966). Boundary Value Problems. Pergamon Press. [Cited on pages 18, 28, 29, 148, and 151.]

Gea-Aguilera, F., Gill, J., Zhang, X., and Nodé-Langlois, T. (2016). Turbulence-cascade interaction noise using an advanced digital filter method. In 23rd Int. Congr. Sound Vib., number July, pages 1-8. [Cited on pages 5 and 92.]

Geyer, T., Sarradj, E., and Fritzsche, C. (2010). Measurement of the noise generation at the trailing edge of porous airfoils. Exp. Fluids, 48(2):291-308. [Cited on pages 6 and 147.]

Geyer, T. F. and Sarradj, E. (2014). Trailing-edge noise of partially porous airfoils. In 20th AIAA/CEAS Aeroacoustics Conf., Reston, Virginia. American Institute of Aeronautics and Astronautics. [Cited on page 157.]

Glauert, H. (1926). The elements of airfoil and airscrew theory. Cambridge University Press. [Cited on page 46.]

Glegg, S. A. L. (1999). The response of a swept blade row to a three-dimensional gust. J. Sound Vib., 227(1):29-64. [Cited on pages 4, 5, 8, 18, 19, 59, 60, 65, 71, 72, 76, 88, 89, 98, 100, 101, 108, 110, 175, 176, 177, 181, 183, 185, 187, 189, 190, 191, 192, 194 , $195,196,200,213$, and 218.]

Glegg, S. A. L. and Devenport, W. J. (2017). Aeroacoustics of low Mach number flows: fundamentals, analysis, and measurement. Academic Press,. [Cited on page 92.]

Goldstein, A. W. and Jerison, M. (1947). Isolated and cascade airfoils with prescribed velocity distribution. Technical report, NACA. [Cited on page 34.]

Goldstein, M. E. (1978). Unsteady vortical and entropic distortions of potential flows round arbitrary obstacles. J. Fluid Mech., 89(03):433. [Cited on pages 60 and 65.]

Goodman, A. W. (1960). Conformal mapping onto certain curvilinear polygons. Univ. Nac. Tucum'an Rev. Ser. A, 13(20):6. [Cited on page 234.]

Gostelow, J. P. (1984). Cascade Aerodynamics. Pergamon Press, 1 edition. [Cited on page 38.]

Grace, S. M. (2016). Influence of model parameters and the vane response method on a low-order prediction of fan broadband noise. Int. J. Aeroacoustics, 15(1-2):131-143. [Cited on page 5.]

Graham, R. R. (1934). The silent flight of owls. J. R. Aeronaut. Soc., 38(286):837-843. [Cited on pages 5, 147, and 167.]

Guzman Inigo, J., Baddoo, P. J., Morgans, A. S., and Ayton, L. J. (2019). Noise generated by entropic and compositional inhomogeneities interacting with a cascade of airfoils. In AIAA/CEAS Aeroacoustics Conf., page 2526. [Cited on pages 80 and 264.] 
Guzman Inigo, J., Duran, I., and Morgans, A. (2018). A model for the sound generated by entropy disturbances interacting with isolated blades. In 2018 AIAA/CEAS Aeroacoustics Conf., Reston, Virginia. American Institute of Aeronautics and Astronautics. [Cited on page 264.]

Hajian, R. and Jaworski, J. W. (2017). The steady aerodynamics of aerofoils with porosity gradients. Proc. R. Soc. A Math. Phys. Eng. Sci., 473:20170266. [Cited on pages 19, $147,148,150,154,155,157$, and 170.]

Hall, K. C. (1997). Exact solution to category 3 problems: turbomachinery noise. In Second Comput. Aeroacoustics(CAA) Work. Benchmark Probl., pages 41-43. [Cited on pages 85 and 86.$]$

Hall, M. and Thwaites, B. (1965). On the calculation of cascade flows. Technical report, Aeronautical Research Council. [Cited on page 21.]

Henrici, P. (1986). Applied and Computational Complex Analysis, Volume 3: Discrete Fourier Analysis, Cauchy Integrals, Construction of Conformal Maps, Univalent Functions. Wiley. [Cited on page 234.]

Herrig, L., Emery, J., and Erwin, J. (1957). Systematic two-dimensional cascade tests of NACA 65-series compressor blades at low speeds. Technical report, NASA. [Cited on page 38.]

Hixon, R., Sescu, A., and Allampalli, V. (2010). Towards the prediction of noise from realistic rotor wake/stator interaction using CAA. Procedia IUTAM, 1:203-213. [Cited on page 5.]

Howe, M. S. (1998). Acoustics of fluid-structure interactions. Cambridge University Press. [Cited on pages 183, 254, 256, 260, and 266.]

Howe, M. S. (2003). Theory of vortex sound. Cambridge University Press. [Cited on pages 20, 251, and 257.]

Hughes, C. (2013). NASA Collaborative Research on the Ultra High Bypass Engine Cycle and Potential Benefits for Noise, Performance, and Emissions. Technical report, NASA. [Cited on page 3.]

Hultgren, L. S. (2010). Emerging importance of turbine noise. Int. J. Aeroacoustics, 10(1):i-iv. [Cited on page 4.]

Ioakmidis, N. I. and Theocaris, P. S. (1977). Array of periodic curvilinear cracks in an infinite isotropic medium. Acta Mech., 28(1-4):239-254. [Cited on pages 148, 152, and 170.]

Iosilevskii, G. (2011). Aerodynamics of permeable membrane wings. Eur. J. Mech. B/Fluids, 30(5):534-542. [Cited on pages 19, 147, 157, and 161.] 
Iosilevskii, G. (2013). Aerodynamics of permeable membrane wings. Part 2: Seepage drag. Eur. J. Mech. B/Fluids, 39:32-41. [Cited on pages 147, 157, 161, 162, and 164.]

Jaworski, J. W. and Peake, N. (2013). Aerodynamic noise from a poroelastic edge with implications for the silent flight of owls. J. Fluid Mech., 723:456-479. [Cited on pages 6, 8, 147, 157, 182, 183, and 189.]

Jaworski, J. W. and Peake, N. (2020). Aeroacoustics of Silent Owl Flight. Annu. Rev. Fluid Mech. [Cited on page 5.]

Joukowski, N. (1910). Über die Konturen der Tragflächen der Drachenflieger. Zeitschrift für Flugtechnik und Mot., 1:281--284. [Cited on page 230.]

Katz, J. and Plotkin, A. (2009). Low-Speed Aerodynamics. Cambridge University Press, Cambridge. [Cited on page 161.]

Kerschen, E. J. and Myers, M. R. (1987). Perfect gas effects in compressible rapid distortion theory. AIAA, 25(3):504-507. [Cited on page 66.]

Kisil, A. and Ayton, L. J. (2018). Aerodynamic noise from rigid trailing edges with finite porous extensions. J. Fluid Mech., 836:117-144. [Cited on pages 6, 147, 156, 157, 182, and 183.]

Kisil, A. V. (2015). The relationship between a strip Wiener-Hopf problem and a line Riemann-Hilbert problem. IMA J. Appl. Math., 80(5):1569-1581. [Cited on pages 10 and 11.]

Koch, W. (1971). On the transmission of sound waves through a blade row. J. Sound Vib., 18(1):111-128. [Cited on page 4.]

Krenk, S. (1975). On quadrature formulas for singular integral equations of the first and the second kind. Q. Appl. Math., 33(3):225-232. [Cited on page 46.]

Kropf, E. (2012). Numerical computation of Schwarz-Christoffel transformations and slit maps for multiply connected domains. PhD thesis. [Cited on page 234.]

Kuethe, A. M. and Chow, C.-Y. (1998). Foundations of Aerodynamics: Bases of Aerodynamic Design. Wiley, 5 edition. [Cited on pages 23, 38, and 51.]

Lawrie, J. B. and Abrahams, I. D. (2007). A brief historical perspective of the Wiener-Hopf technique. J. Eng. Math., 59(4):351-358. [Cited on page 8.]

Leclaire, P., Horoshenkov, K. V., and Cummings, A. (2001). Transverse vibrations of a thin rectangular porous plate saturated by a fluid. J. Sound Vib., 247(1):1-18. [Cited on page 147.]

Lehigh University (2014). The Stealthy Owl Wing. https://www2.lehigh.edu/news/stealthy-owl-wing. [Cited on page 6.] 
Leppington, F. G. (1977). The effective compliance of perforated screens. Mathematika, 24(02):199. [Cited on pages 181 and 183.]

Liao, J. C. (2007). A Review of Fish Swimming Mechanics and Behaviour in Altered Flows. Philos. Trans. R. Soc. Lond. B. Biol. Sci., 362(1487):1973-1993. [Cited on page 21.]

Lifanov, I. K., Matveev, A. F., and Molyakov, I. M. (2009). Flow around permeable and thick airfoils and numerical solution of singular integral equations. Russ. J. Numer. Anal. Math. Model., 7(2):109-144. [Cited on page 150.]

Lighthill, M. J. (1952). On sound generated aerodynamically I. General theory. Proc. $R$. Soc. London. Ser. A. Math. Phys. Sci., 211(1107):564-587. [Cited on page 6.]

Lighthill, M. J. (1954). On sound generated aerodynamically II. Turbulence as a source of sound. Proc. R. Soc. London. Ser. A. Math. Phys. Sci., 222(1148):1-32. [Cited on page 6.]

Lighthill, M. J. (1958). An Introduction to Fourier Analysis and Generalised Functions. Cambridge Univ. Press. [Cited on pages 72 and 185.]

Linton, C. (1998). The Green's function for the two-dimensional Helmholtz equation in periodic domains. J. Eng. Math., 33(4):377-401. [Cited on pages 255 and 259.]

Michelin, S. and Llewellyn Smith, S. G. (2009). An unsteady point vortex method for coupled fluid-solid problems. Theor. Comput. Fluid Dyn., 23(2):127-153. [Cited on page 265.]

Mikhlin, S. G. (1957). Integral equations and their applications to certain problems in mechanics, mathematical physics, and technology. Pergamon Press. [Cited on pages 148, 151, and 170.]

Moore, M. N. J. (2017). Riemann-Hilbert problems for the shapes formed by bodies dissolving, melting, and eroding in fluid flows. Commun. Pure Appl. Math., 70(9):18101831. [Cited on page 10.]

Moreau, S. (2019). Turbomachinery noise predictions: present and future. Acoustics, 1(1):92-116. [Cited on page 3.]

Muskhelishvili, N. I. (1946). Singular Integral Equations: Boundary Problems of Function Theory and their Application to Mathematical Physics. Dover Publications. [Cited on pages $11,12,18,19,22,26,36,58,148,151,152,153,157,172$, and 173.]

Myers, M. K. (1980). On the acoustic boundary condition in the presence of flow. $J$. Sound Vib., 71(3):429-434. [Cited on pages 182 and 183.]

Myers, M. R. and Kerschen, E. J. (1995). Influence of incidence angle on sound generation by airfoils interacting with high-frequency gusts. [Cited on pages 61, 63, 65, and 92.] 
Myers, M. R. and Kerschen, E. J. (1997). Influence of camber on sound generation by airfoils interacting with high-frequency gusts. J. Fluid Mech., 353:221-259. [Cited on page 63.]

Nehari, Z. (1952). Conformal mapping. McGraw-Hill, New York. [Cited on pages 16 and 226.]

Noble, B. (1958). Methods Based on the Wiener-Hopf Technique For the Solution of Partial Differential Equations. Pergamon Press, Belfast, Northern Ireland. [Cited on pages $8,103,123$, and 202.]

Olver, F. W. J., Lozier, D. W., Boisvert, R. F., and Clark, C. W. (2010). NIST Handbook of Mathematical Functions. Cambridge University Press, Cambridge. [Cited on pages 47, 50, and 255.]

Peake, N. (1992). The interaction between a high-frequency gust and a blade row. $J$. Fluid Mech, 241:261-289. [Cited on pages 4, 8, 10, 19, 101, 175, 177, 185, 213, 218, and 220.]

Peake, N. (1993). The scattering of vorticity waves by an infinite cascade of flat plates in subsonic flow. Wave Motion, 18(3):255-271. [Cited on pages 4, 19, 108, and 177.]

Peake, N. and Kerschen, E. J. (1995). A uniform asymptotic approximation for highfrequency unsteady cascade flow. Proc. R. Soc. A Math. Phys. Eng. Sci., 449(1935):177186. [Cited on page 4.]

Peake, N. and Kerschen, E. J. (1997). Influence of mean loading on noise generated by the interaction of gusts with a flat-plate cascade: Upstream radiation. J. Fluid Mech., 347:315-346. [Cited on pages 4, 59, 60, 63, and 65.]

Peake, N. and Kerschen, E. J. (2004). Influence of mean loading on noise generated by the interaction of gusts with a cascade: Downstream radiation. J. Fluid Mech., 515:99-133. [Cited on pages 4, 59, 60, 63, and 65.]

Peake, N. and Parry, A. B. (2012). Modern challenges facing turbomachinery aeroacoustics. Annu. Rev. Fluid Mech., 44(1):227-248. [Cited on pages 1 and 4.]

Posson, H., Moreau, S., and Roger, M. (2010a). On the use of a uniformly valid analytical cascade response function for fan broadband noise predictions. J. Sound Vib., 329(18):3721-3743. [Cited on pages 264 and 265.]

Posson, H., Roger, M., and Moreau, S. (2010b). On a uniformly valid analytical rectilinear cascade response function. J. Fluid Mech., 663:22-52. [Cited on pages xvi, 4, 5, 18, 19, $59,60,71,72,81,85,86,88,175,176,181,183,185,187,189$, and 196.$]$

Rienstra, S. W. (1992). A note on the Kutta condition in Glauert's solution of the thin aerofoil problem. J. Eng. Math., 26(1):61-69. [Cited on pages 18, 22, and 46.] 
Rienstra, S. W. (2007). Acoustic scattering at a hard-soft lining transition in a flow duct. J. Eng. Math., 59(4):451-475. [Cited on page 8.]

Robinson, A. and Laurmann, J. A. (1956). Wing Theory. Cambridge University Press. [Cited on pages 22, 161, 226, and 230.]

Rolls-Royce (2019). Trent 1000. https://www.rolls-royce.com/products-and-services/civilaerospace/airlines/trent-1000.aspx\#section-technology. [Cited on page 3.]

Saffman, P. G. (1993). Vortex Dynamics. Cambridge University Press, Cambridge. [Cited on page 237.]

Saiz, G. (2008). Turbomachinery Aeroelasticity Using a Time-Linearised Multi Blade-row Approach. PhD thesis, Imperial College London. [Cited on pages 4 and 175.]

Schmueser, D. and Comninou, M. (1979). The periodic array of interface cracks and their interaction. Int. J. Solids Struct., 15(12):927-934. [Cited on pages 148, 152, and 170.]

Schulten, J. (1982). Sound generated by rotor wakes interacting with a leaned vane stator. AIAA J., 20(10):1352-1358. [Cited on page 22.]

Smith, S. N. (1973). Discrete frequency sound generation in axial flow turbomachines. Aeronaut. Res. Counc. Reports Memo., (3709):1-58. [Cited on page 85.]

Spurr, R. A. and Allen, H. J. (1947). A theory of unstaggered airfoil cascades in compressible flow. Natl. Advis. Comm. Aeronaut. NACA, page 14. [Cited on page 22.]

Tao, T. (2016). Analysis II, volume 38 of Texts and Readings in Mathematics. Springer, Singapore. [Cited on page 219.]

Taylor, K. (1978). A transformation of the acoustic equation with implications for windtunnel and low-speed flight tests. Proc. R. Soc. A Math. Phys. Eng. Sci., 363:271-281. [Cited on page 260.]

The Economist (2015). Flying's new gear. https://www.economist.com/sites/default/files/ images/print-edition/20160102_STC450.png. [Cited on page 2.]

Theodorsen, T. and Garrick, I. E. (1979). General potential theory of arbitrary wing sections. Technical Report December, NASA. [Cited on page 21.]

Thwaites, B. (1960). Incompressible Aerodynamics: an Account of the Theory and Observation of the Steady Flow of Incompressible Fluid Past Aerofoils, Wings, and Other Bodies. Dover Publications. [Cited on pages 18, 22, 34, 40, 42, and 147.]

Tsai, C.-T. (1992). Effect of airfoil thickness on high-frequency gust interaction noise. [Cited on page 62.]

Van Dyke, M. (1964). Perturbation methods in fluid mechanics. Academic press, New York. [Cited on pages 25 and 137.] 
Vasconcelos, G. L. (1993). Exact solutions for a stream of bubbles in a Hele-Shaw cell. Proc. R. Soc. A Math. Phys. Eng. Sci., 442(1915):463-468. [Cited on page 225.]

Vasconcelos, G. L. (2015). Multiple bubbles and fingers in a Hele-Shaw channel: complete set of steady solutions. J. Fluid Mech., 780:299-326. [Cited on page 225.]

Verdon, J. M. (1993). Review of unsteady aerodynamic methods for turbomachinery aeroelastic and aeroacoustic applications. AIAA J., 31(2):235-250. [Cited on page 5.$]$

von Kármán, T. and Burgers, J. M. (1936). General aerodynamic theory - Perfect fluid. Vol II of Aerodynamic Theory. Julius Springer, Berlin. [Cited on page 264.]

Wade, R. B. (1967). Linearized theory of a partially cavitating cascade of flat plate hydrofoils. Appl. Sci. Res., 17(3):169-188. [Cited on pages 54, 226, and 230.]

Weidenfeld, M. and Manela, A. (2016). On the attenuating effect of permeability on the low frequency sound of an airfoil. J. Sound Vib., 375:275-288. [Cited on page 150.]

Wiener, N. and Hopf, E. (1931). Über eine klasse singulärer integralgleichungen. Sitzungsberichte der Preuß. Akad. der Wissenschaften, Phys.-math. Klasse, 31:696-706. [Cited on page 7 .]

Wood, R. W. (1902). On a remarkable case of uneven distribution of light in a diffraction grating spectrum. Proc. Phys. Soc. London, 4(21):396-402. [Cited on page 259.]

World Health Organisation (2011). Burden of disease from environmental noise - Quantification of healthy life years lost in Europe. Eur. Comm. [Cited on page 1.]

Zhou, X. (2005). The Riemann-Hilbert problem and inverse scattering. SIAM J. Math. Anal., 20(4):966-986. [Cited on page 10.] 
